
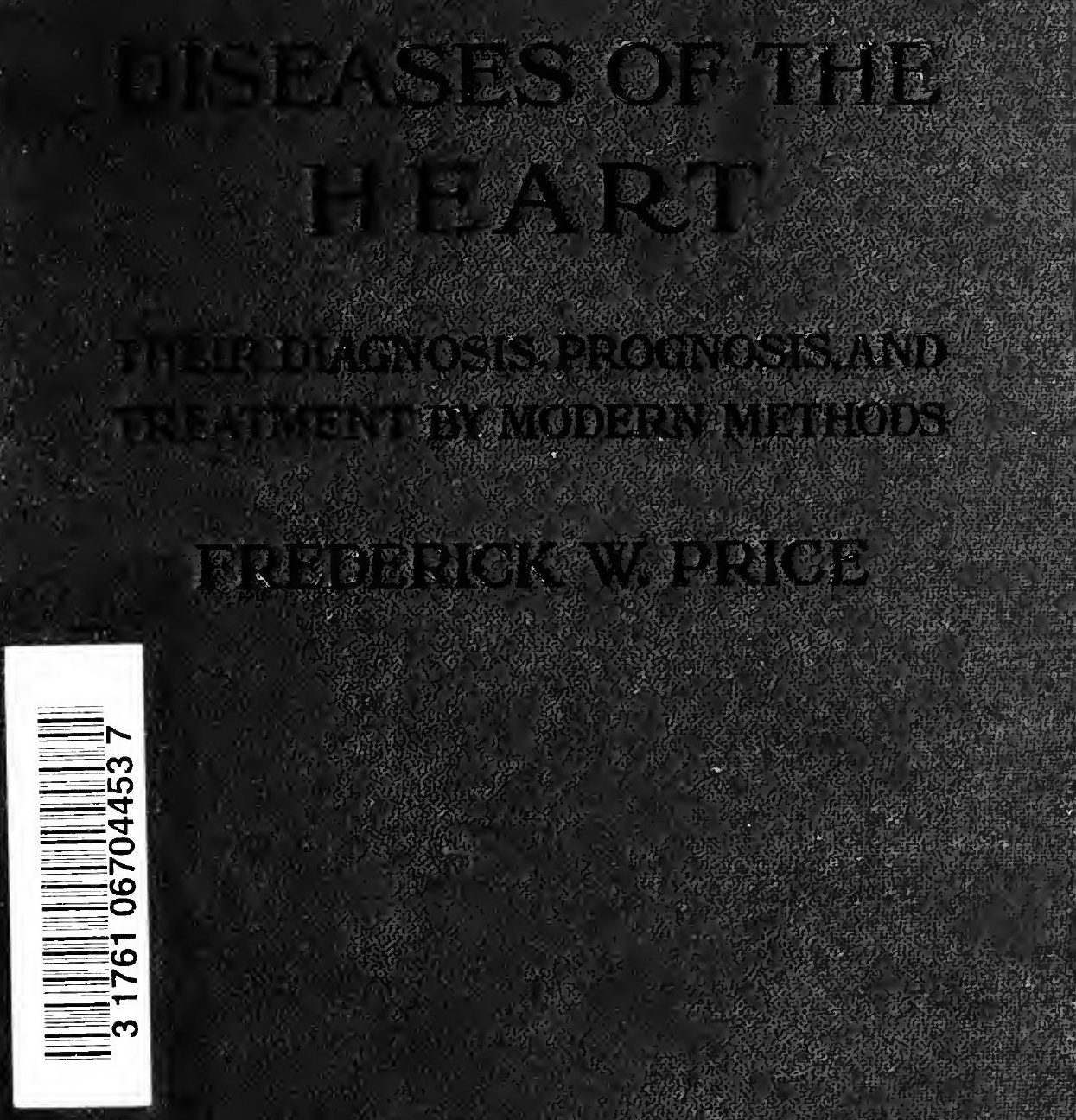

DSORO MUDICAL
PUGHCATIONS 1.7. 


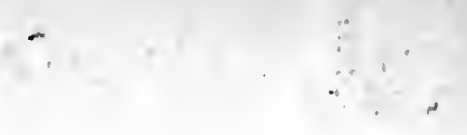






\section{Digitized by the Internet Archive in 2007 with funding from Microsoft Corporation}


DISEASES OF THE HEART 
PUBLISHED BY THE JOINT COMMITTEE OF HENRY FROWDE AND HODDER \& STOUGHTON AT THE OXFORD PRESS WAREHOUSE

FALCON SQUARE, LONDQN, E.C.1. 



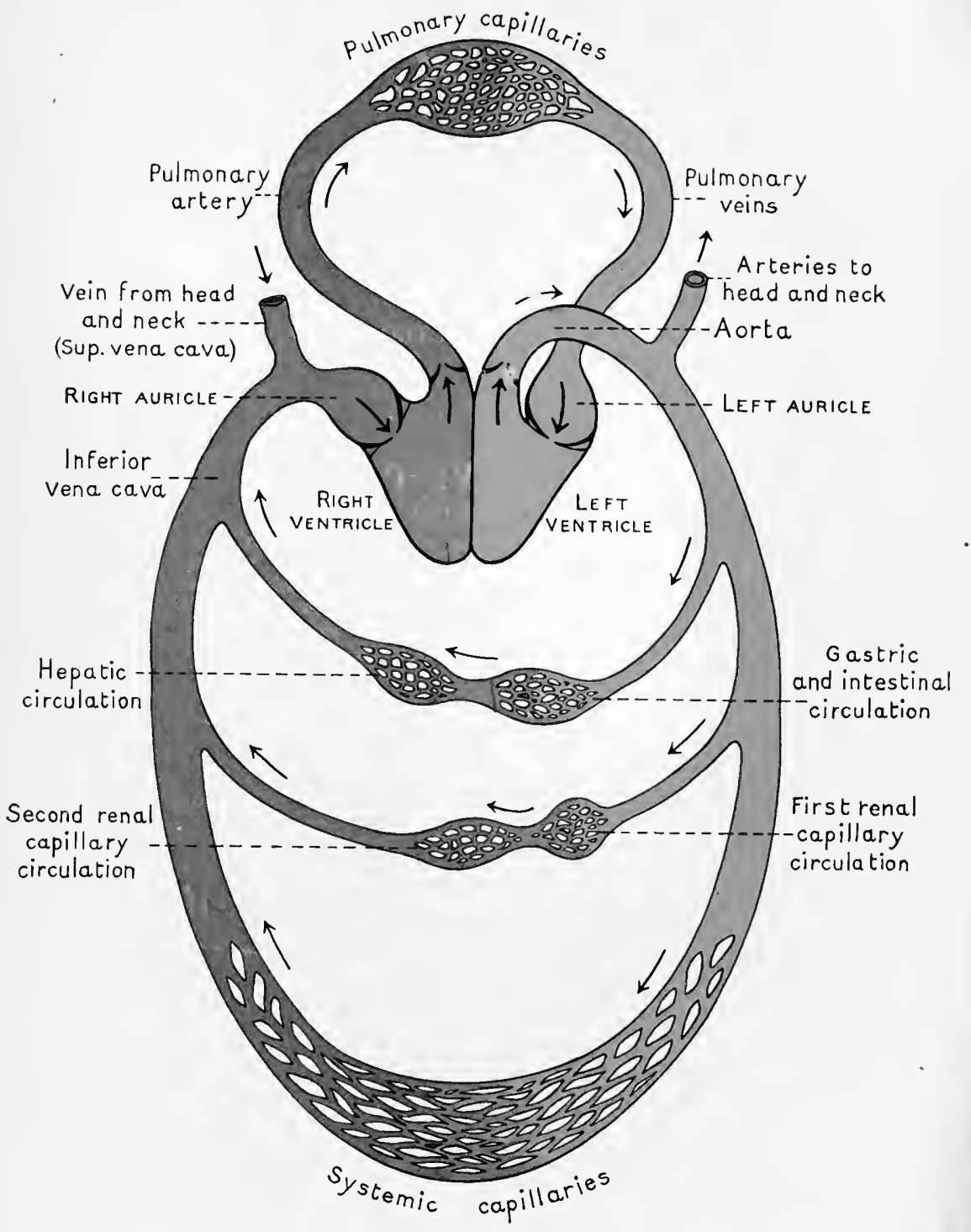

DIAGRAMMATIC SCHEME OF THE CIRCULATION. 
MU:

$P$

DISEASES OF THE HEART

\section{THEIR DIAGNOSIS, PROGNOSIS, AND TREATMENT BY MODERN METHODS}

WITH A CHAPTER ON THE ELECTRO-CARDIOGRAPH

BY

FREDERIGK W. PRICE

M.D., F.R.S. (EDIn.)

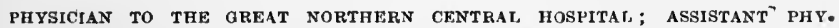
SICIAN TO THE NATIONAL HOSPITAL FOR DISEASES OF THE HEART, LONDON. LATE, LECTURER ON POLYGRAPHIC METHOIS AT THE MFDICAL GRADUATES COLIEGE AND POLYCLINIC, PHYSICIAN AND HONORARY PATHOLUGIST TO THE MOUNT VERNON HOSPITAL FOR CONSUMPTION AND DISEASES OF THF CHEST, AND EXAMINER IN MEDICINE AT THE UNIVERSITY OF ST, ANDREWS

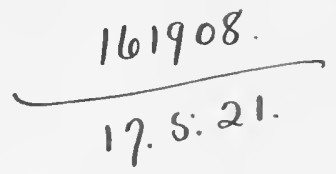

LON DON

HENRY FROWDE

Oxford University Press
HODDER \& STOUGH'ION

Warwick Square, E.C. 


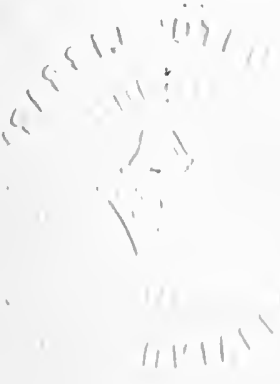

PRINTED IN GREAT BRTAIN

BУ HAZELL, WATSON AND VINEY, LD.,

'LONDON AND AYLESBURY. 
THIS BOOK IS DEDICATED, WITH GRATITUDE AND AFFECTION,

TO

SIR JAMES ORMISTON AFFLECK,

M.D., LL.D., F.R.C.P.E., F.R.S.E., Consulting Physician to the ROYAL INFIRMARY OF EDINBURGH,

WHO TAUGHT ME THE FIRST PRINCIPLES OF THE PRACTICE OF MEDICINE, AND WHOSE PRECEPTS AND EXAMPLE HAVE BEEN A CONTINUED SOURCE OF INSPIRATION AND ENCOURAGEMENT TO ME IN MY PROFESSIONAL LABOURS 



\section{P R E F A C E}

THE object of this book is to present, in as concise a form as possible, information which will be of service in the diagnosis, prognosis, and treatment of diseases of the heart by modern methods. I have dealt with the subject from the point of view of the clinician, and have carefully avoided matter of merely theoretical interest.

It is generally recognised that within recent years there has been a great advance in our knowledge of cardiac disorders, and this advance has been of so practical a character and of such vital importance in the diagnosis, prognosis, and treatment of these diseases, that it is the duty of every clinician to make himself acquainted with its nature and scope. Many of the problems in the study of diseases of the heart which have baffled clinicians for generations have been practically solved, while others are in process of solution. This progress has been mainly due to the introduction of what are called graphic methods in the examination of the cardiac mechanism. By means of the clinical polygraph and the electro-cardiograph we are now able to analyse the cardiac action in a manner which was never possible before. The expert knowledge and use of these instruments, however, are for practical purposes frequently not necessary; for, while it is true that most of our recent knowledge in the domain of cardiac disorders has been gained by means of them, all that is necessary in a large proportion of cases is the use of a Dudgeon's sphygmograph. Indeed, in a 
not inconsiderable proportion of cases even the sphygmograph is not essential.

For some years now $\mathrm{I}$ have devoted my attention more particularly to the study of cardiology, and in the following pages I have attempted to place before the reader the more important results of modern investigations on this subject. In order to secure the aim which I have had in view, the greatest care has been exercised to include only what is likely to be of practical valuc. The literature of the subject is already immense, but in large measure scattered and fragmentary. I have, however, taken full advantage of the writings of other workers in the field, although in a book of this character it is impossible to acknowledge my indebtedness to all of them. At the same time, I cannot refrain from expressing my sense of obligation and gratitude to Mackenzie, Cushny, Wenkebach, Lewis, Ritchie, Hay, and Davenport Windle for assistance derived from a study of their researches on this subject.

It is not intended, however, to confine the scope of the book to a description of the usefulness of graphic methods and other modern developments in the study of cardiology. It aims at including within its compass also an account of such knowledge of the diagnosis, prognosis, and treatment of cardiac disorders as was in our possession prior to the introduction of these more recent methods; here, again, the aim has been strictly practical. With regard to this part of the subject, I wish to express my indebtedness to the writings of the late Dr. G. A. Gibson and Sir William Broadbent.

By including, therefore, information with regard to recent advances in the study of cardiac disorders as well as an account of our. knowledge prior to this, and by discussing both from the point of view of the clinician, I hope to enable the medical practitioner to take the fullest possible advantage of modern methods in the diagnosis, prognosis, and treatment of diseases of the heart. 
Treatment has been fully discussed, and in this connection a good deal of what has been written is based upon the results of a series of investigations in which I was associated with Sir James Mackenzie, Professor Cushny, and other workèrs at the Mount Vernon Hospital for Diseases of the Chest. The electro-cardiograph cannot be regarded as an instrument available to the general practitioner, and as this book is intended primarily for him, I have included clinical electro-cardiography in one chapter at the end of the book.

Some of the matter in this volume has appeared in print elsewhere or has been given as lectures or addresses to post-graduate audiences in London and other places. This applies particularly to an opening address which I had the honour of delivering before the Toronto Academy of Medicine, and which was published in the British Medical Journal of March 12th, 1913. Among other papers are those read by me before the annual meetings of the British Medical Association, the Royal Society of Medicine, and the International Congress of Medicine, as well as some which have appeared in the British Medical Journal, the Lancet, and the Practitioner.

I cannot adequately express my indebtedness to Sir James Mackenzie, in association with whom I have worked for some years, and without whose inspiration and encouragement this book would not have been written. It is unnecessary to point out to those acquainted with the subject that a large portion of this book is necessarily founded upon his work. I desire to thank very cordially Dr. Davenport Windle for his helpful criticism, his invaluable assistance with regard to the polygraphic records, and in addition for a considerable number of tracings. I wish also to express my thanks to Sir Thomas Horder, my friend and former colleague, for his care in reading the proof-sheets, and for the generous disposal of his time in offering many hints directed towards making the book as useful as possible. It is a pleasant duty to express my sense of gratitude to 
Drs. Chapman Gibbes, R. O. Moon, and Russell Wells, my colleagues at the National Hospital for Diseases of the Heart, for kindly allowing me the use of the electrocardiographic records of some of their patients at that Institution, and to Professor Arthur Keith for certain illustrations.

133, Harley Street,

Frederick W. Price.

LoNdon, W.

February, 1918. 


\section{CONTENTS}

\section{CHAPTER I}

Anatomical and Physiological Considerations .

CHAPTER II

Physical Signs: their Interpretation and Significance $\quad . \quad \cdot \quad \cdot \quad \cdot \quad \cdot \quad \cdot 23$

\section{CHAPTER III}

The Arterial Pulse . $\quad$ • $\quad$ • $\quad$ • $\quad$ • $\quad$ • 55

\section{CHAPTER IV}

BLOOD-PRESSURE • $\quad$ • $\quad$ • $\quad$ • $\quad$ • $\quad$ • $\quad 69$

\section{CHAPTER V}

The Venous Pulse-the Ink Polygraph-PolyGRAPHIC RECORDS $\quad$. $\quad$ • $\quad$. 88

\section{CHAPTER VI}

X-ray Examination-Graphic Records of Heart SOUNDS-THE ELECTRO-CARDIOGRAPH • • 113 


\section{CHAPTER VII}

Heart Failure : its Symptomatology $\quad$ • 115

\section{CHAPTER VIII}

Prognosis in Cardiac Affections $\quad \cdot \quad \cdot \quad \cdot 125$

\section{CHAP'TER IX}

Treatment in Cardiac Affections . $\quad$. 130

CHAPTER $\mathbf{X}$

Functional Disorders of the Heart . $\quad$ • 158

CHAPTER XI

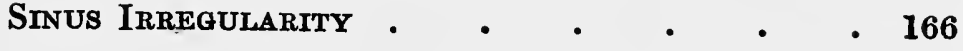

CHAPTER XII

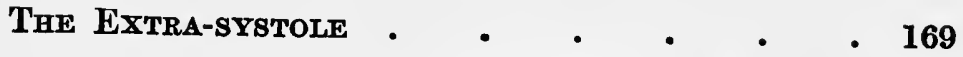

CHAPTER XIII

Heart-BLOCK

CHAPTER XIV

Púlsus Alternans 
CHAPTER XV

Auricular Fibrillation

CHAPTER XVI

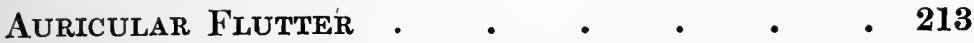

CHAPTER XVII

Paroxysmal Tachycardia . • • • • • 224

\section{CHAPTER XVIII}

Carditis : the Rheumatic Infection of the Heart in Childhood

- 231

\section{CHAPTER XIX}

ENDOCARDITIS • • • • • • • • • • 234

CHAPTER XX

Chronic Valvular Disease $\quad$ • • • • 256

\section{CHAPTER XXI}

Diseases of the Myocardium and Coronary ARteries $. \quad . \quad . \quad . \quad . \quad . \quad .294$

\section{CHAPTER XXII}

Affections of the Pericardium • $\quad$ • $\quad$ - 330 


\section{CHAPTER XXIII}

Less Common Diseases of the Heart and PeriCARDIUM

\section{CHAPTER XXIV}

Congenital Heart Disease $\quad$ - $\quad$ - 356

\section{CHAPTER XXV}

Primary Cardiac Overstrain- " Soldier's Heart" 374

CHAPTER XXVI

Angina Pectoris-Pseudo-angina • $\quad$ - 380

\section{CHAPTER XXVII}

Clinical Electro-cardiography $\quad$ - $\quad$ - 398

INDEX $\quad$ - $\quad$ - $\quad$ - $\quad$ - $\quad .1487$ 


\section{DISEASES OF THE HEART}

\section{CHAPTER I}

ANATOMICAL AND PHYSIOLOGICAL CONSIDERATIONS

Position of the Heart. - The heart, covered by the pericardium, lies obliquely in the middle mediastinum, being inclined from above downwards, forwards, and to the left (Fig. 1). It is situated nearer to the front than the back of the chest, and two-thirds of it lie to the left of the middle line. Anteriorly, the pleuræ and lungs cover the large vessels and the whole of the heart, with the exception of a small portion of the right ventricle. Posteriorly, the heart is in relation with the structures which lie in the posterior mediastinum, occupying a position in front of the 5 th, 6 th, 7 th, and 8 th dorsal vertebræ. The base of the heart is directed upwards and to the right, and the apex downwards and to the left. The highest point which the organ normally reaches is on a level with the upper margin of the third left costal cartilage, close to the left border of the sternum; from this point its boundaries run outwards and downwards on both sides. The aorta arises behind the left half of the sternum, opposite the third costal cartilage; the highest part of the arch being at the level of the third dorsal vertebra.

The position of the heart varies a good deal according to the age of the individual, and other conditions. In the child the organ is situated at a somewhat higher level, 
is proportionatcly larger and broader, and is farther to the left of the middle line, than in the adult. In old age the organ is situated at a somewhat lower level. With forced breathing the heart moves downwards with inspiration, and upwards with expiration ; and its position may be altered to the extent of $3-5$ c.m. with a change of position from one side to the other.

The anterior aspect of the heart lies behind and to the right and left of the sternum, and is formed almost entirely by the right auricle and ventricle, the left auriclc appearing only at its left upper part, and the left ventricle as a narrow strip along the left border; the greater portion of the anterior surface being formed by the right ventricle (Fig. 1). The whole of the right border of the heart is formed by the right auricle; indeed, the whole of the organ which is situated to the right of the sternum is formed by that chamber. The left border and the apex are formed by the left ventricle. The upper border is formed by the right auricle and its appendix, the conus pulmonalis, and the tip of the left auricular appendix. The lower border is formed by the right ventricle, and the apical portion of the left. The furrow between the ventricles runs from the third to the fifth left costal cartilages, just behind their junction with the ribs. The posterior surface of the heart is formed entirely by the auricles.

The Precordium.-That part of the thoracic wall which lies in front of the heart is called the precordium. Usually the precordium is characterised by a certain degree of flattening over the region of the sternum, and a slight elevation on either side of it, which is rather greater on the left than on the right side.

The Apex-beat.-The movement of the heart at each contraction is communicated to the chest-wall, and can be seen and felt over a limited area. This impact upon the chest-wall is called the apex-beat, and may be defined as that part of the area of pulsation, distinctly palpable, which is lowest and farthest to the left; it is situated just 
above the anatomical apex of the heart. In normal circumstances, it is the left ventricle which gives rise to the clinical apex-beat, and the apex-beat. is then a definite forward thrust, lasting about $\frac{1}{3}$ second, and occurring about $\frac{1}{10}$ second before the carotid, and $\frac{1}{5}$ second before the radial, pulse. It consists of three phases, namely, $(a)$ a sudden and short shock, representing the pre-sphygmic

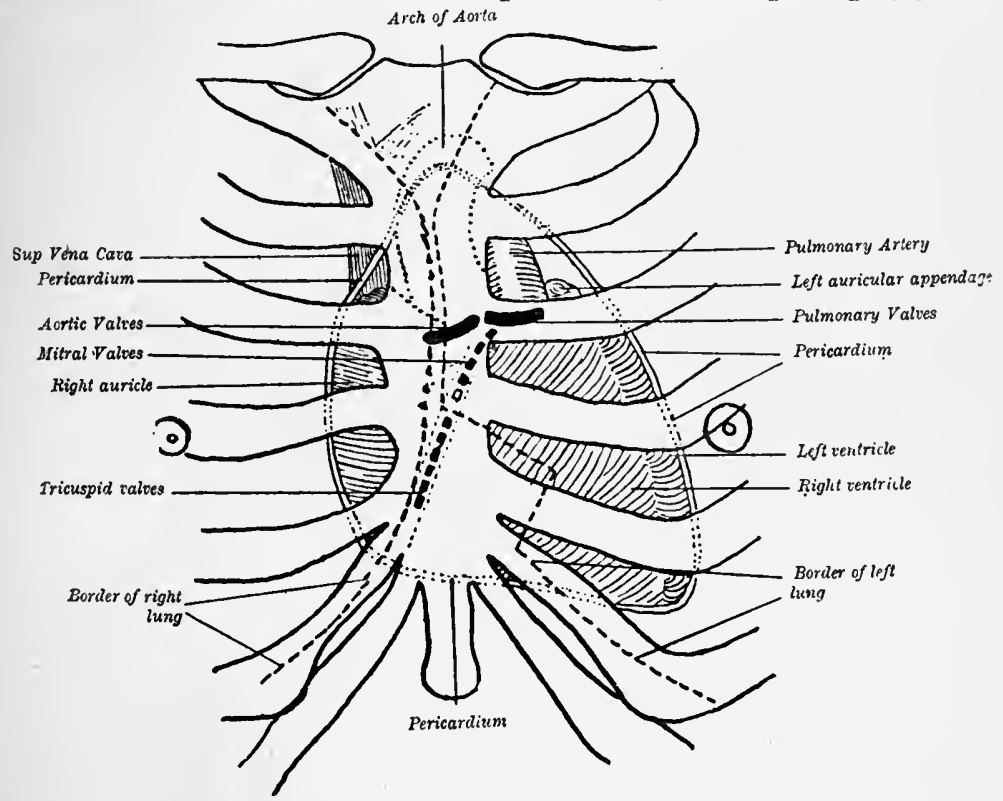

Fig. 1. The position of the heart in the chest (Keith).

period; $(b)$ bulging of the heart into the intercostal space, the apex pressing against the chest-wall, corresponding with the sphygmic period; and $(c)$ a backward movement or falling away from the intercostal space, corresponding with the post-sphygmic interval. When the right ventricle is much enlarged, however, that chamber may displace the left ventricle backwards, so that the apex-beat is formed by the right, instcad of the left, ventricle; in this event, there may be a more diffuse systolic recession over the lower part of the precordium, the out-thrust being diastolic in time. 
In the adult, the apex-beat is normally in the 5th intercostal space, between the left parasternal line and the left mammary line. It is usually lower in the aged and higher in the young; being not infrequently situated in the sixth intercostal space and also relatively nearer the middle line in old age, while in children it is frequently found in the fourth intercostal space and also relatively farther from the middle line. Normally the apex-beat is confined to one intercostal space, and has a diameter not exceeding one inch in any direction.

A tracing of the apex-beat, or of the movements of any other portion of the superficial surface of the heart, may be recorded by means of the cardiograph. At the present time, however, the usual custom is to employ instead the receiver of the ink polygraph.

Orifices and Valves of the Heart.-The aortic and mitral orifices are much more deeply situated than the pulmonary and tricuspid orifices, which lic comparatively near the chest-wall. The aortic orifice is situated behind the left half of the sternum, at the level of the lower border of the third costal cartilage; the mitral orifice behind the left half of the sternum, at the level of the third intercostal space and fourth costal cartilage; the pulmonary orifice behind the junction of the third left costal cartilage with the sternum; and the tricuspid orifice behind the sternum, at the level of the fourth intercostal space and fifth costal cartilage (Fig. 1). The aortic valve has three cusps, one situated anteriorly and two posteriorly, the left posterior being connected with the anterior mitral cusp; the mitral valve has two cusps, the larger being situated anteriorly and to the right and being named the aortic flap, and the other posteriorly and to the left; the pulmonary valve has three cusps, two being situated anteriorly and one posteriorly; while the tricuspid valve has three cusps, one in front (the infundibular), one to the right (the external), and the largest behind and to the left (the septal). 
The character of the sounds relating to the different orifices is ascertained in four areas. These are not situated immediately over the valves from which they derive their names, but are those areas over which the sounds of the respective valves are best heard; in other words, the anatomical and clinical surface-markings of the valves do not correspond (Figs. 1 and 2). The aortic area is situated over the inner end of the second right costal cartilage, at

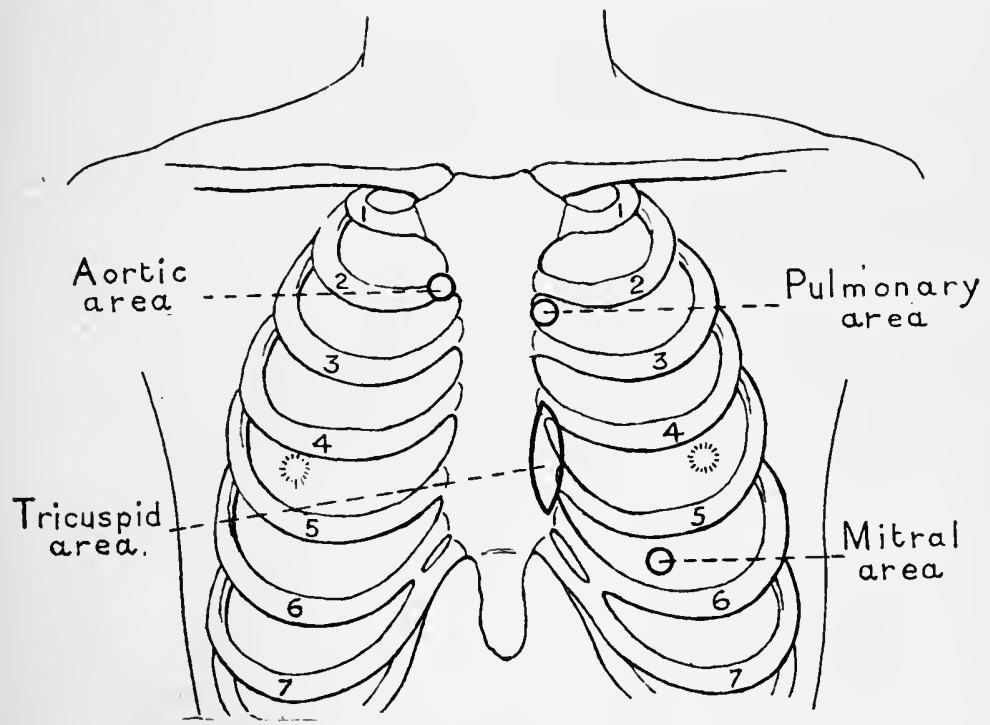

FIG. 2. Diagram to show the areas over which the sounds ot the heart are best heard.

which point the aorta is nearer the surface than at any other. The mitral area is situated over the apex of the heart. This may not mean the visible or palpable apexbeat, because, as already noted, in enlargement of the right ventricle the clinical apex-beat may not be produced by the left ventricle. In any doubtful case, therefore, we should determine its position by percussing the left border of the heart. The pulmonary area is situated over the inner end of the second left intercostal space, at which point the pulmonary valve is near the surface. The 
tricuspid area is rather larger in extent than the other areas, and is situated in the region of the lower extremity of the sternum and the adjacent extremities of the 4 th, 5th, and 6th left costal cartilages.

Structure of the Heart.-The structure of the heart consists of (1) the serous layer of the pericardium, (2) the muscular wall or myocardium, and (3) the endocardium or lining membrane. The myocardium is composed of striated muscle-fibres, with intervening connective tissue, which contains blood-vessels, lymphatics, and nerves. It should be noted that the myocardium is very vascular.

Blood-supply of the Heart.--The heart is supplied with blood by the right and left coronary arteries, which break up into a network of capillaries around the muscle-fibres. The coronary arteries arise from the root of the aorta. Their origin is in relation with two of the aortic cusps, the right coronary artery arising opposite the anterior cusp, and the left opposite the left posterior cusp.

Innervation of the Heart.-The nerves which supply the heart are derived from the vagus, and from the sympathetic system, the fibres in each case being both afferent and efferent. The efferent fibres of the vagus constitute the inhibitory nerves of the heart, while the afferent or sensory fibres pass to the medulla oblongata. The efferent fibres of the sympathetic system arise in the anterior roots of the second and third dorsal nerves, and are the accelerator or augmentor nerves of the heart.

Sino-auricular Node.-The sino-auricular node is a node of specialised tissue, discovered by Keith and Flack, at the junction of the superior vena cava with the auricle, immediately beneath the epicardium. In it are nervefibres and ganglion cells, which are connected with the vagus and sympathetic nerves.

Auriculo-ventricular Node and Bundle.-The auriculo-ventricular node is a small node of specialised tissue which is situated in the septal wall of the right 


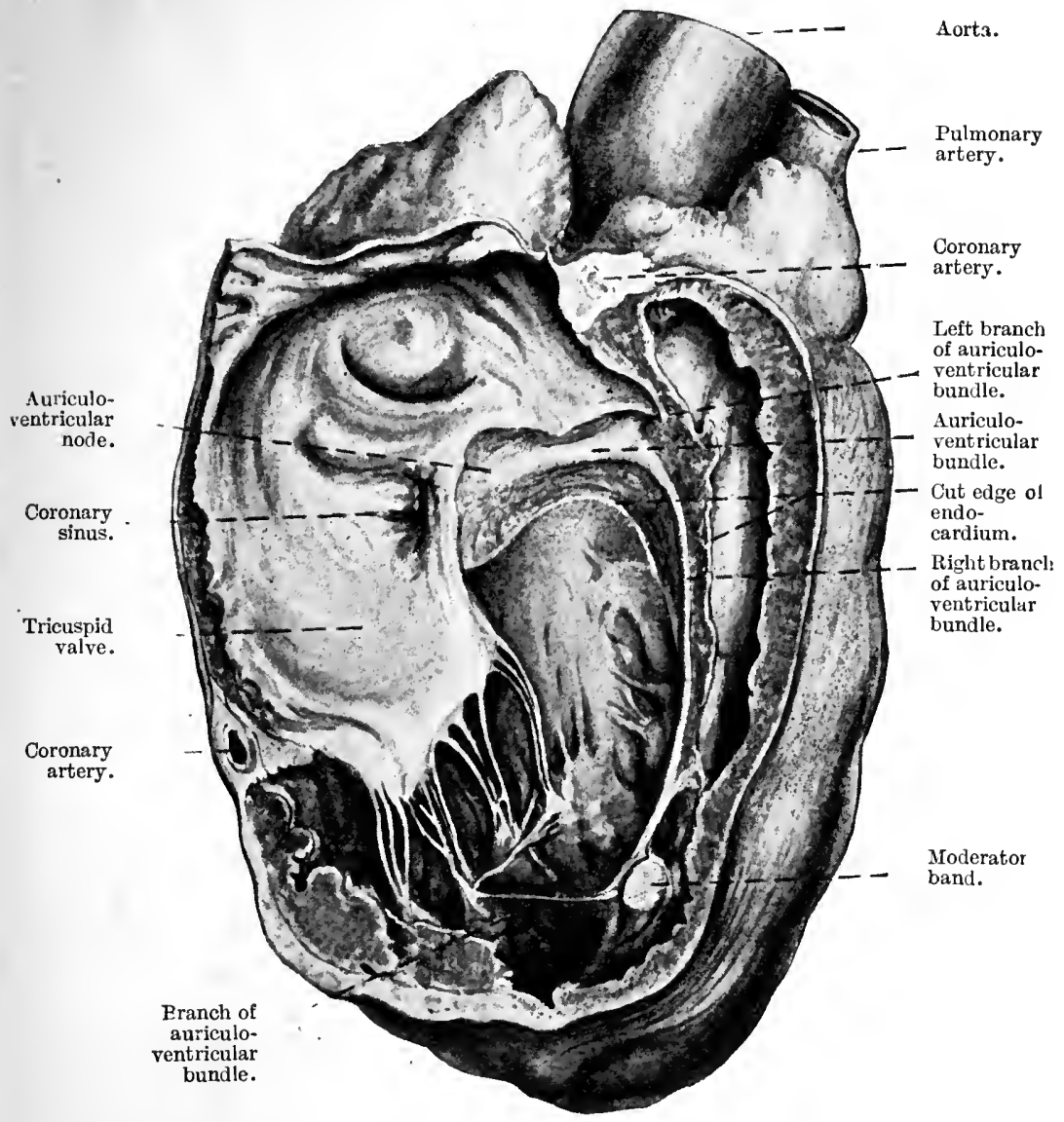

Fic. 3. Dissection showing the origin of the auriculo-ventricular bundle, and the course of the right branch in the right ventricle. The anterior and lateral walls of the right ventricle have been removed as well as a portion of the tricuspid valve. A slight dissection has been made which exposes the bundle arising in the auriculo-ventricular node, an ill-defined structure in the right auricle, near the opening of the coronary sinus. The main stem runs from the node to the auriculo-ventricular septum, where it divides into two branches, the left branch going through to the left ventricle, as shown in Fig. 4, the right branch going down in the muscle of the ventricle near the endocardium as a narrow rounded band. In the specimen from which the dissection was made, it could be traced to the moderator band, which is shown cut in the drawing. Here it divided into numerous fine branches, one of which is seen passing across the cavity as a fine thread (Mackenzie). 
auricle, near to the coronary sinus, immediately above the

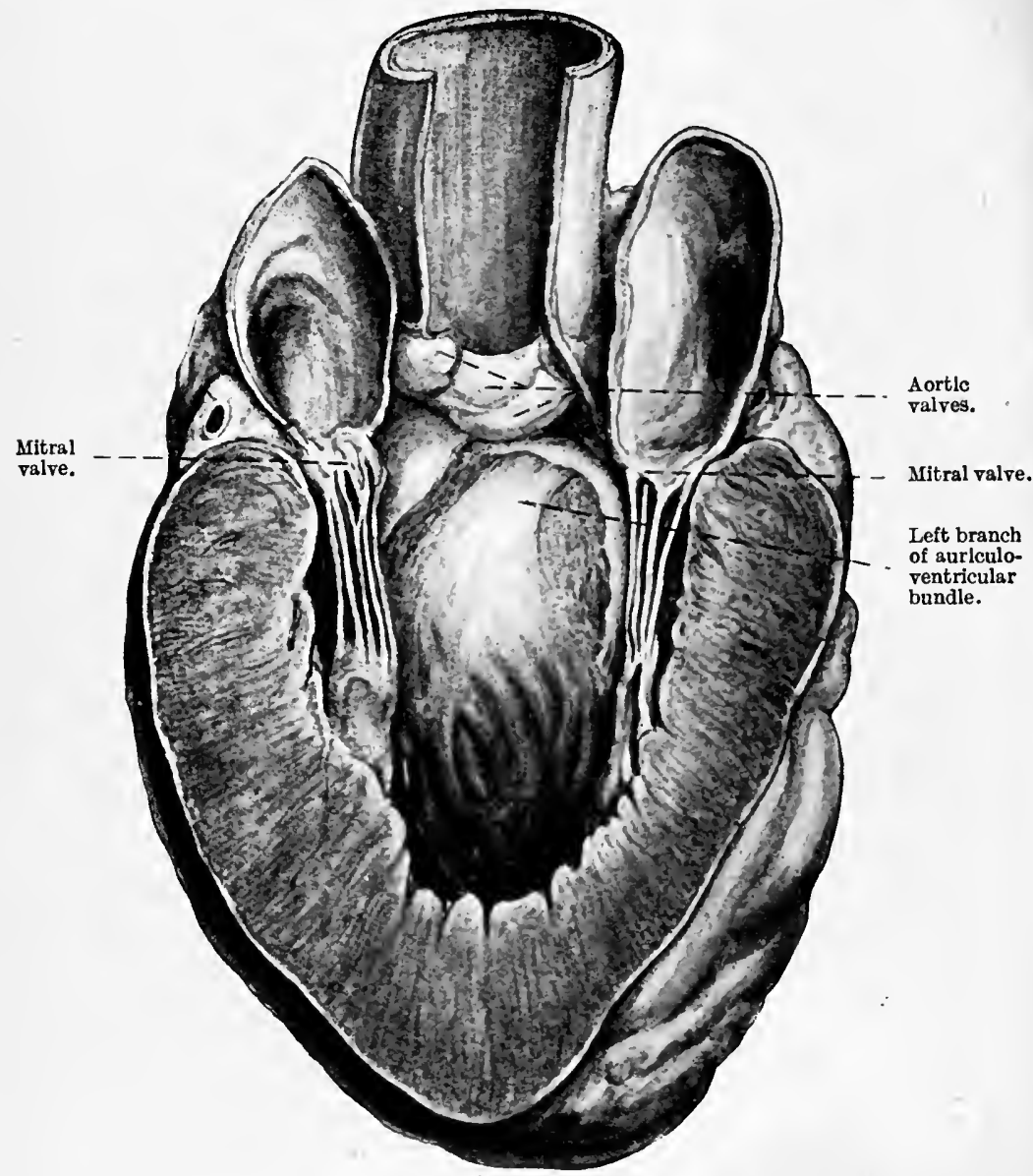

FrG. 4. Dissection showing the left branch of the auriculo-ventricular bundle. The posterior wall of the left ventricle has been slit open, the incision passing up through the left auricle and aorta. The cavity of the left ventricle is exposed, and a very slight dissection made to show the left branch of the bundle entering the ventricle immediately below the septal cusp of the aortic valve. The bundle widens out into a thin band lying immediately under the endocardium, and as it passes down the ventricle it sends branches into the muscle, until it can no longer be recognised as a separate and distinct structure (Mackenzie).

insertion of the median cusp of the tricuspid valve (Fig. 3). 
The auriculo-ventricular bundle of Stanley Kent and His is a bundle of tissue connecting the auricles and ventricles (Figs. 3 and 4). It arises from the auriculoventricular node, passes forwards in the inter-auricular septum, then turns downwards, and at the upper margin of the inter-ventricular septum divides into two branches, the right and left scptal divisions, one of which goes to the

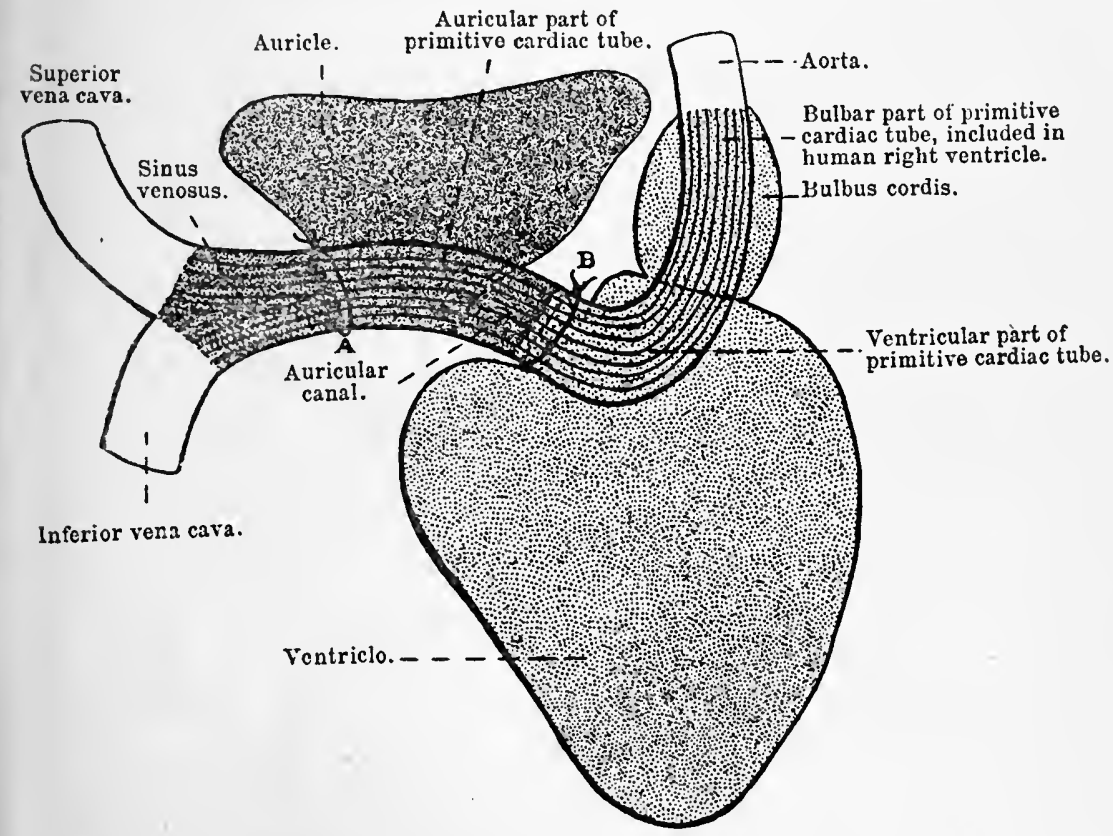

FIG. 5. Diagram of the primitive vertebrate heart, showing the development of the auricle and ventricle from the primitive cardiac tube. $A$, position of the first Stannius ligature; $B$, position of second Stannius ligature (Keith).

right and the other to the left ventricle, each ending in the ventricular musculature by widespread subendothelial ramifications-the Purkinje fibres. Nervefibres and ganglion cells are present in the auriculoventricular bundle, and its blood-supply is mainly derived from a special branch of the right coronary artery.

The Primitive Cardiac Tube.-Early in fotal life the 
heart consists of a single tube, which is continuous in front with the two primitive aortæ, and behind with the veins (Fig. 5). The venous or postcrior extremity is the most exeitable part of the tube, the result being that the wave of contraction commences there. The remainder of the tube, however, possesses the powcr of initiating the wave of contraction, though in a lesser degrec, and if any of this portion become more cxcitable than the sinus or venous extremity the wave of contraction commenees at that part.

In the adult mammalian heart the primitive cardiac tube has become modified and has ceased to exist as a tube. The sinus venosus, instead of remaining as a separate structure, has become incorporated in the auricle, its area being bounded by the orifices of the two venæ cavæ, the eoronary sinus, and the inter-auricular septum. The sino-auricular node is belicved to represent part of the sinus venosus, and there is reason to believe further that the auriculo-ventricular node and bundlc arc also remains of the primitive cardiae tube.

The remains of the primitive cardiac tube are morc excitable than the auricular or ventricular tissue, and the remains of the sinus venosus at the orifices of the great veins are the most excitable part. The stimulus for contraction, therefore, arises normally at this point, and there is reason to believe that it begins in the sino-auricular node; for this reason the node has been called by Erlanger the "pace-maker" of the heart; the rhythm of the sinus governs the rhythm of the remaining segments of the heart, the impulse travelling as a wave of contraction which spreads peristaltically over the other portions of the heart at rates of speed which vary in different parts. From the sino-auricular node the stimulus spreads rapidly over the auricles, and auricular systole takes place. It then travels through the auriculo-ventricular node, along the auriculo-ventricular bundle, and is so distributed to the ventricles. Normally, therefore, stimulation and con- 
traction of the sinus, the auricles, and the ventrieles occur in the order named, the ventricle contracting only in response to stimuli received from the auricle through the auriculo-ventricular bundle. It is scarcely necessary to point out that relaxation proceeds in the same order $c \mathrm{~s}$ contraction. As will be noted later, by means of a specialized function, called conductivity, the eardiac muscle has the power of conveying the stimulus for contraction from fibre to fibie. The stimulus travels so rapidly that the contraction of the ventricle commences almost immediately after the completion of the aurieular systole. But while normally the stimulus for contraction arises in the sinus part of the auriele, if any other portion of the remains of the primitive cardiac tube become more exeitable the stimulus for contraction arises at that point; for this reason the different parts of the hcart are capable of starting an independent eontraction.

We know that the junctional tissues possess the power of originating the stimulus for contraction for the following reasons: firstly, if a Stannius ligature be applied, or an incision made, so as to sever completely the connection between the sinus and the auricle (Fig. 5), the sinus continues beating. After a period, the aurieles and ventricles commence to beat again, independently of, and at a different rate from, the sinus. At first the auriculo-ventricular rate is slow, but gradually increases, until a fair rate is attained, though this is less than that of the sinus. If a second ligature be applied, or an incision made, so as to sever completely the connection between the auricles and ventricles (Fig. 5), the sinus and auricles continue to beat. After a period the ventricles again begin to beat, independently of, and at a different rate from, the sino-auricular portion of the heart. The ventricular rate is at first rapid, but gradually lessens until the ventricles cease beating. After a time the ventricles recommence to beat, at first very slowly, but steadily increasing in rate until a uniform rate is attained, 
which is lower, however, than that of the rest of the heart.

Secondly, the gradual compression of the auriculoventricular bundle by means of a clamp with a fine screw adjustment produces various degrees of heart-block. If compression be gentle, it causes merely a delay in the passage of the stimulus from auricles to ventricles; the interval between the auricular and ventricular response being increased without an alteration of the normal sequence. If stronger compression be applied, some of the impulses are not transmittcd from the auricle to the ventricle. There may be merely an occasional block, or, on the other hand, only every sceond or every third or fourth impulse may reach the ventricle. If the compression be still further increased, no stimulus whatever will reach the ventricle from the auricle, and the ventricles remain at rest until they commence to beat independently of, and at a rate different from, the rest of the heart.

Thirdly, varying degrees of heart-block occur in diseased conditions of the auriculo-ventricular junctional tissues, as are manifested in the experimental compression of the auriculo-ventricular bundle already mentioned. In heartblock there exists some defect of the auriculo-ventricular bundle, which prevents, in varying degrees, the transmission of the wave of contraction from auricle to ventricle. Complete heart-block occurs when a lesion of any kind completely severs the physiological connection between the auricle and the ventricle.

What is known as a " reversed rhythm" may occur when, owing to the fact that the ventricle is the most irritable part of the heart, the stimulus for contraction arises there, proceeding thence to the auricle, so that each contraction of the auricle follows, instead of precedes, ventricular systole.

Fundamental Functions of the Heart Musclefibres.-Gaskell has described five fundamental functions of the fibres of the heart muscle, namely, stimulus pro- 
duction, excitability, conductivity, contractility, and tonicity; contractility being the most important.

Mackenzie has defined these functions as follows : by stimulus production is meant the power which the musclefibres possess of originating a stimulus which can excite the heart to contract : by excitability is meant the power to receive a stimulus : by contractility is meant the power of contracting when stimulated : by conductivity is meant the power of conveying a stimulus from fibre to fibre: and by tonicity is meant that function of the heart muscle which keeps the heart during diastole in a state of slight tonic contraction.

The functions of stimulus production and excitability are most highly developed at the sinus part of the auricle. The rate of the heart depends upon these functions, an increase of their activity resulting in an increase of the frequency of the cardiac action. The activity of the function of stimulus production varies a good deal, as, for instance, the slight acceleration of the heart-rate during inspiration, and the corresponding slowing which occurs during expiration, in the young. When excitability is increased, the heart has also a greater tendency to respond to abnormal or irregular stimuli; hence extrasystoles are more likely to occur. While diminished excitability may lessen the heart-rate, it should be remembered that slowing of the ventricular rate may be due to other causes, such as depressed conductivity. When stimulus production and excitability are equal, the rhythm of the heart is regular. The pulsus alternans is probably an indication of depressed contractility of the heart. The rate at which the wave of contraction travels from fibre to fibre varies in different parts of the heart. Thus, the impulse spreads in the auricles and ventricles more quickly than it does from auricle to ventricle. In this connection, it may be noted that conductivity is observed by estimating the time between the systole of the auricles and ventricles. A lowered state of tonicity results in 
dilatation of the heart and of the auriculo-ventricular orifices.

Immediately after the muscle-fibres of the heart have contracted, they cannot again be stimulated; in other words, excitability has for the moment disappeared. This is called the refractory stage. Restoration of excitability, however, at once recommences, and steadily increases during diastole. When stimulated, the cardiac muscle either does not contract at all, or it contracts to the fullest possible extent at the time, whether the stimulus be weak or strong. There is, therefore, no relation between the strength of the stimulus and the strength of the contraction ; it is a case of "all or none."

The longer the time that has elapsed since the previous contraction, the weaker is the stimulus required to bring about a further contraction. Further, the greater the degree of excitability of the muscle-fibres, the weaker is the stimulus required, and the carlier in the refractory period will the heart contract. The contractility of the muscle-fibres is measured by the degree of contraction. The amount of contraction does not depend upon the strength of the stimulus employed, but varies according to the time at which the stimuli are applicd. When a contraction occurs early in diastole, the contraction in the succeeding cardiac cycle is weaker than that preceding it. Within certain limits, the degree of contractility depends upon the length of the preceding diastole; the greater the period of rest, the more perfect and full is the recovery. It can, therefore, be readily understood that the greater the heart-rate the greater is the possibility of eardiac failure. The circulation is carried on most efficiently when the cardiac rate is exactly that which allows the myocardium to recover its full contractility; a rate over or under this being a disadvantage. Similar laws apply to each of the other special functions of the heart muscle. In the case of conductivity, for example; when conduction occurs, this function has been 
exercised to the fullest extent possible at the time of stimulation. The refractory period follows immediately after the conduction of a stimulus, and during this brief period the muscle-fibres cannot again conduct a stimulus, conductivity being completely exhausted. Restoration, however, quickly commences, and ultimately the function is restored. The extent of the relaxation between the contractions depends upon the degree of tone present, and upon the rate of the heart-beat. With a slower rate, there is, of course, more time for full relaxation.

There should be a sufficient period of rest after each function has been exercised. Within certain limits, the greater the period of rest the more perfect and full is the recovery of each special function. In heart failure one or more of these special functions are at fault, and, if this occur to two or more simultaneously, the different functions are not necessarily equally affected. From what has been said, the value of rest in heart failure can be readily understood.

The heart has the power of rhythmically contracting and dilating, due to an inherent power possessed by the cardiac musculature, independent of any extrinsic nervous influence. Nevertheless, the activity of the various functions of the muscle-fibres is under nervous control. Both the inhibitory fibres of the vagus and the accelerator fibres of the sympathetic are normally in a state of tonic activity, and the cardiac centres are in a state of continuous slight excitation.

There are reasons for believing that, besides decreasing the rate of the heart, the vagus nerve depresses the functions of excitability, contractility, conductivity-either by acting on the auriculo-ventricular bundle, or by diminishing the irritability of the ventricle itself-and tonicity; and that the accelerator nerve, besides increasing the rate of the heart, increases the force of contraction, and conductivity. Stimulation of the central part of the divided vagus nerve is followed by a fall of blood-pressure, 
and simultaneously by a retardation of the beats of the heart. Inhibition of the heart is due to stimulation of the vagus as a diastolic effect; aceeleration is due to stimulation of the sympathetic as a systolie effect.

The Cardiac Cycle and Sounds.-As has already been noted, the movements of the heart begin at the cardiac orifices of the great veins, passing thenee to the auricles,

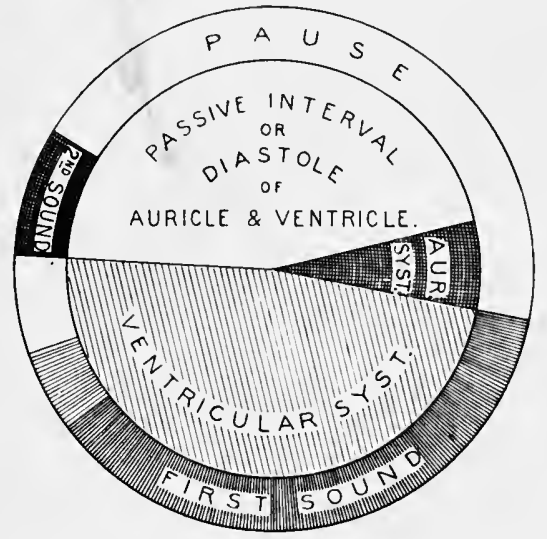

FIG. 6. Diagrammatic representation of events constituting a cardiac cycle. The inner circle indicates the movements of the heart; the outer shows the relation of the sounds and pauses to these movements. and thence to the ventricles; the contraction of the organ taking the form of a wave which originates in the sinus and spreads peristaltieally over the whole heart. Each cardiac cyele may thus be divided into three periods, namely, (1) the systole of the auricles, (2) the systole of the ventricles, and (3) the rest of the whole heart (Fig. 6).

Passing to consider the eardiac cycle in detail : The sequence of events commences with a slight flickering or quivering contraction of the venæ cavæ and pulmonary veins near the heart. This movement is immediately followed by a sudden and rapid contraction of the auricles, during which time the auriculo-ventricular valves open and the blood is propelled into the ventricles, after which there is a short interval. This is followed by relaxation of the auricles, which become refilled with blood, and the commencement of the contraction of the ventricles; the contraction of the ventricles follows that of the aurieles almost immediately, not exceeding 
one-tenth of a second. The ventricular contraction commences at the base, and thence travels to the apex, and is more prolonged and forcible than that of the auricles. The contraction of the ventricles increases the pressure within their cavities, and directly the pressure within the ventricles becomes greater than that within the auricles the auriculoventricular valves close, and their closure prevents any reflux of blood into the auricles. The apposition of the edges of the valves is aided by the attachment of the chordæ tendineæ of the musculi papillares. Further, the contraction of the circular fibres at the base of the heart constricts the auriculo-ventricular orifices and approximates the valves at their origin. The auriculo-ventricular valves being closed, the pressure of the ventricles speedily increases until it exceeds that in the aorta and pulmonary artery, when the semilunar valves open and the contents of the ventricles are discharged into these vessels.

The contraction of the ventricle is followed by a somewhat rapid relaxation, and, as the ventricular muscle relaxes, the pressure inside those chambers rapidly falls. When the pressure in the aorta and pulmonary artery becomes greater than that in the ventricles, the semilunar valves close sharply. When the stage of complete ventricular relaxation is reached and the pressure inside the ventricles has become less than that inside the auricles, the auriculo-ventricular valves open, and the blood flows from the auricles into the ventricles and slowly fills them. The ventricles remain at rest for some time, when they are passively filling with blood flowing from the auricles, the propelling force being the systemic bloodpressure, the muscular movement, and the aspiration of the thorax. This period of relaxation and rest of the ventricles is called the diastole. Its beginning is marked by the closure of the semilunar valves, which remain closed until the commencement of the following ventricular systole. During the whole of diastole, the ventricles have become increasingly distended by the influx 
of blood, this distension being suddenly increased by the forcible contraction of the auricles driving the blood through the open auriculo-ventricular valves into the ventrieles. Then follows the contraction of the ventricles. It will be seen, therefore, stated briefly, that there occur in rapid succession auricular systole, ventricular systole, and ventrieular diastole; and that the auricular diastole eommenees during the ventricular systole, and that the ventricular diastole continues during auricular systole. The diastole of the aurieles coineides with the commencement of the ventricular systole, the diastole of the ventricles with the commencement of the pause. The two active phases (systole and diastole) are followed by the state of rest.

There are three phases in the systole of the ventricles, namely, (a) the period during which all four valves are elosed ; this is ealled the pre-sphygmic period. The pressure within the ventricles has eaused the auriculo-ventrieular valves to elose, but it is not yet greater than that in the aorta and pulmonary artery, so that the semilunar valves remain elosed. (b) The period during which the semilunar valves are open ; this is called the sphygmic or pulse-period. The pressure within the ventricle having risen above that in the aorta and pulmonary artery, the semilunar valves are foreed open and the blood flows from the ventrieles into these vessels. (c) The period between the closure of the semilunar valves and the opening of the auriculo-ventricular valves; this is called the post-sphygmic period.

It should be noted that the sphygmic or pulse-period in a tracing of the cardiac apex, carotid pulse, or radial pulse, does not refer to the actual time of occurrence of ventricular systole, but to a period in the tracing; and, on account of the distance from the heart, the sphygmic or pulse-period will necessarily be later in a radial tracing than the same period in a tracing taken at the cardiac apex or over the carotid. The periods of time which elapse 
between the apex-beat and the carotid pulse, and between the carotid and radial pulses, have each been found to be one-tenth of a second; the pulse-period, therefore, in a radial tracing commenees about one-tenth of a second behind the same period in a tracing of the carotid, and about one-fifth of a second behind that in a cardiogram.

Assuming that in normal health the human heart beats about 72 times per minute, each cardiac cycle, therefore, is completed in about eight-tenths of a second, this time being divided up in the following manner. Systole of auricle, one-tenth of a second ; systole of ventricle, threetenths of a second; diastole, four-tenths of a second.

Each beat of the heart is aceompanied by two distinet sounds, and followed by a pause corresponding with diastole (Fig. 6). The first sound is heard best near the apex of the heart, and is longer and lower in piteh than the second. It occurs along with the ventrieular systole, and has been compared to the syllable lubb. It continues through an appreciable period of systole, but not to its termination. It is followed by a short interval. The second sound is sharper, clearer, and shorter, and has been compared to the syllable dupp. It occurs at the end of systole, is synchronous with the elosure of the aortic and pulmonary valves, and is best heard over the base of the heart. The second sound is followed by a longer interval. Clinically, systole commences with the beginning of the first sound and terminates immediately before the second sound, while diastole commences with the beginning of the second sound and ends immediately before the first sound. Regarding the cycle as a whole, its rhythm may be described-to borrow a musical expression-as that of triple time, the accent falling on the first beat in the mitral and tricuspid areas, and on the second beat in the aortic and pulmonary areas, the third beat, representing the long pause, being silent.

In the opinion of some writers, a third sound of the heart is audible in certain individuals, the area of maxi- 
mum intensity being over the apex-beat. It occurs in the diastolic portion of each cardiae cyele, a short time after the seeond sound, being lower in piteh and tone than the second sound. I cannot say that I have so far met with this condition. In the opinion of some, the cause of the third sound is that at the moment of opcning of the aurieulo-ventrieular valves there is such a rush of blood from the upper into the lower chambers of the heart as to cause the cusps to float baek and to block the passage of blood from auricle to ventricle.

During early years the first sound on the left side of the heart is relatively much louder than on the right. It is somewhat lower in piteh and longer in duration in the mitral than in the trieuspid area. At the same period of life the aortie second sound is not so loud as the pulmonary seeond, whereas during later years there is a progressive diminution of the intensity of the pulmonary second sound. The aortie second sound also becomes less distinct, but in this case the diminution is less, and ultimately it becomes louder than the pulmonary. Up to the age of 25 or 30 , the seeond sound in the pulmonary area is louder than the second sound in the aortic area. At the apex and to the left of it, it is believed that only the aortic element of the seeond sound is audible.

The most stable part of the cardiac eycle is the ventricular systole, and the most variable is the diastole. The rate of the heart-beat mainly depends upon the duration of the diastole. When the heart beats unusually quickly, the duration of the diastole is shortened by a greater degree than that of the systole; in other words, when the period of the cardiac cycle decreases it is the long pause which is particularly shortened.

The Cardiac Rate. - The normal cardiac rate is about 72 beats per minute. It should be remembered, however, that it has a eonsiderable range of variation in perfectly healthy individuals ; in some it is always frequent, even up to 120 per minute, whereas in others it is always 
infrequent. It is affected by age, sex, meals, change of posture, physical exertion, and mental excitement.

According to Starling, the average rate at different ages is as follows:-

Age in years.

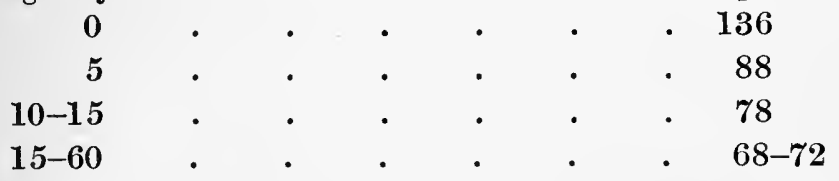

The increase in rate when the individual assumes the

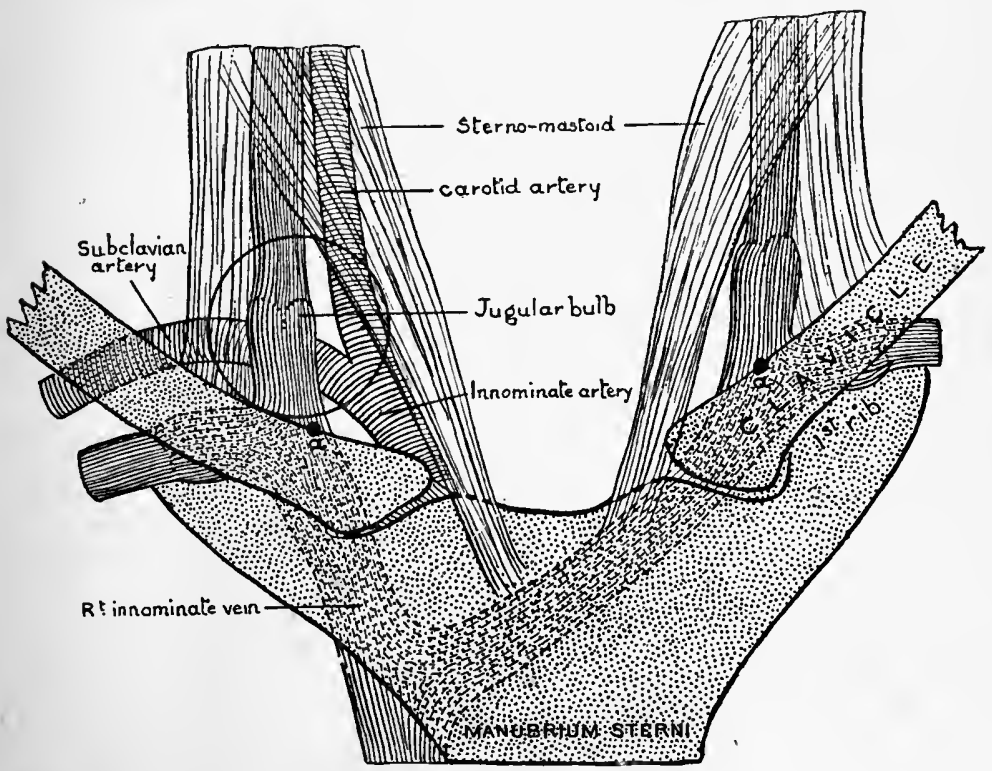

Fic. 7. Shows the relation of the internal jugular vein to the carotid and subclavian arteries, and to the sterno-mastoid muscle. The circle represents the position of the receiver in taking a tracing, and is seen to cover not only the jugular vein, but also portions of the carotid and subclavian arteries. The spot at $a$ is one inch from the internal end of the clavicle (Keith).

upright posture after lying down is normally not more than 20 per minute. The increase in rate induced by physical exertion varies in healthy individuals; it may be marked on severe physical exertion, even when the 
individual is in training, and not infrequently there is a considerably increased rate, say up to 120-140 per minute, on moderate physical exertion. It is difficult, therefore, to decide when an inerease of rate is pathological.

The Jugular Veins.-The internal jugular vein has a valve about one inch above the sternal end of the clavicle, the part of the vein immediately below being referred to as the jugular bulb (Fig. 7). This is situated behind the space which separates the sternal and clavicular heads of the sterno-mastoid muscle. It is over the jugular bulb that the open mouth of the small cup or receiver is usually placed in taking a tracing of the jugular vein.

The external jugular vein usually has a valve where it erosses the sterno-mastoid muscle, but there is none below this point; for this reason slight pulsation just above the clavicle may be normally present. 


\section{CHAPTER II}

PHYSICAL SIGNS : THEIR INTERPRETATION AND. SIGNIFICANCE

\section{Inspection and Palpation}

Changes in the Form of the Precordium.- Undue prominence or bulging of the precordium may be due to a variety of causes. It may be occasioned by conditions other than enlargement of the heart, as, for example, scoliosis, tumour or abscess of the parictes, pleural effusion, malignant disease of the lung, mediastinal tumour, aneurism above or behind the heart, and pericardial effusion. In pericardial effusion, bulging of the intercostal spaces as well as of the ribs may oecur. While heart disease may cause prominence of the precordium, it does so comparatively seldom, except in children and in cases where the disease originated when the patient was young and the bones were yielding. When so caused, the heart is very large, and the most common cause of this in children is adherent perieardium.

Flattening or depression of the precordium may be congenital, or the result of pressure (especially in eertain occupations), retraction of the lung, or a previous attack of pericarditis.

Changes in the Apex-beat.-In studying the apex-beat, we should note its position, its extent, and its character. The normal apex-beat has already been described.

Displacement of the apex-beat may be due to (1) extrinsic causes, (2) congenital causes, or (3) diseases of the heart or pericardium. 
With regard to extrinsic causes, the apex-beat may be displaced upwards, owing to displacement of the contents of the abdominal cavity in such conditions as ascites, meteorism, and tumours, in spinal curvature. It may be displaced downwards in cardioptosis, emphysema, and pneumothorax, and, in the opinion of some, there is downward and outward displacement in aneurism of any part of the arch of the aorta. Displacement to the left may occur in right-sided pleural effusion and pneumothorax, and in left-sided adherent pleura, chronic interstitial pneumonia, and fibroid pulmonary tuberculosis; while displacement to the right may be found in precisely similar conditions on the other side of the chest. When the apex-beat is displaced to the right, the pulsation which is evident to the right side of the sternum is due to the right auricle and ventricle, the apex of the heart in such cases being usually behind the sternum. It is of great importance to be on the look-out for these extrinsic causes of displacement of the apex-beat.

With regard to congenital causes, displacement of the apex-beat may be due to what is known as dextro-cardia -a reversal of the normal position of the heart, so that the apex-beat is on the right instead of the left side of the thorax-or to other developmental errors.

In diseases of the heart or pericardium, displacement of the apex-beat may be due to dilatation or hypertrophy of the heart, or to both, or to pericardial effusion. In dilatation and hypertrophy of the right heart, as the breadth of the organ is increased to a greater degree than its length, the apex-beat is displaced chiefly to the left and only slightly downwards. In dilatation and hypertrophy of the left ventricle, as the long axis of the heart is increased, the apex-beat is displaced chiefly downwards and only slightly outwards. In pericardial effusion the apex-beat is often displaced upwards.

The extent of the apex-beat may be increased by conditions extrinsic to the heart, as, for instance, in those. 
who have thin parietes, in retraction of the left lung, during or immediately following physical exertion or mental excitement, and also in cardiac hypertrophy. The area of the apex-beat may be diminished or completely obliterated in stout and in emphysematous subjects.

The force of the apex-beat may be increased in cases in which the chest-wall is thin, in retraction of the left lung, and when the heart's action is excited-as, for example, during and immediately following muscular exertion or mental excitement, and in the earlier stages of endocarditis or pericarditis, and in hypertrophy from whatever cause. In cardiac hypertrophy, the apex-beat has a "heaving" or "thrusting" character, that"is, it is slower and longer than normal, and this sign is the most characteristic feature of hypertrophy. A sharp slapping impact is described by some observers in cases of irritability of the heart. The impulse may be diminished in force, or may be altogether absent, if the thoracic wall be unduly thick, in pleural thickening, emphysema, when air or liquid is present in the pericardial sac, when the apex-beat lies behind a rib, in weak cardiac action, such as exists in anæmia, pyrexia, acute myocarditis, in cardiac failure resulting from acute pericarditis, valvular disease, or fibroid degeneration, in pericardial effusion, and in cardiac dilatation. From this enumeration of causes it will be seen that diminution or disappearance of the apex-beat is not necessarily indicative of disease. In cardiac dilatation the apex-beat is diffuse, and often difficult or even impossible to locate, but if it can be determined it is found to be tapping in character. As was pointed out in Chapter $\mathbb{I}$, when an enlarged right ventricle displaces the left ventricle backwards, so that the apex-beat is formed by the right, instead of the left, ventricle, there may be a more diffuse systolic recession over the lower part of the precordium, the out-thrust being diastolic in time. 
Pulsations.-Pulsation in an abnormal position of the anterior aspect of the chest may sometimes be observed.

A diffuse pulsation over most of the intercostal spaces of the precordial region may be present in persons with thin thoracic parictes, and especially when in addition there is retraeted lung; in that area in which the pulsation is due to the right ventricle the pulsation is diastolic in time.

Systolic pulsation in the aortic area is, in the opinion of some, oceasionally to be noted in aortic incompetence, while diastolic pulsation, resulting from closurc of the aortic valves, is rarely observed.

Pulsation in the pulmonary area, synchronous with the apex-beat, may be due to the pulmonary artery (second left intereostal space), or to dilatation of the conus artcriosus (third left intcreostal space).

Pulsation, diastolic in time, in the 3rd, 4th, and 5th intercostal spaces between the margin of the stcrnum and the parasternal line on the left side, or in the 4 th and 5 th intercostal spaces on the right side, may sometimes be observed in enlargement of the right ventricle.

It should also be remembered that pulsation in an abnormal position of the anterior aspect of the chest may be due to displacement of the heart, as a result of abdominal or pulmonary disease, transposition of the heart, or aneurism of the aortic arch. Pulsation due to ancurism is at first always above the level of the third rib, but later may be over a considerable area of the chest-wall. If the ascending aorta be affected, the pulsation is mostly to the right of the sternum; if the transverse, over the manubrium sterni; if the descending, the pulsation is more to the left. The pulsation is synchronous with the apex-beat, and is expansile in character.

Two remaining causes of pulsation in the region of the precordium may be noted; these are pulsating empyema and new-growths of marked vascularity. 
Pulsation in the epigastrium may sometimes be observed. We must first determine the time of the pulsation. If it be synchronous with the apex-beat, the cause may be displacement of the heart downwards or to the right, from one of the causes already mentioned, or pulsation of the liver itself in tricuspid regurgitation. If it be diastolic, it is due to the right ventricle, the out-thrust of that chamber of the heart being diastolic in time. If the pulsation occur immediately after the apex-beat, it may be caused by the transmission of the pulsation of a normal abdominal aorta by the liver, or by an abdominal tumour lying in front of it, by a neurotic aorta, or by an aneurism of the aorta; in the last case, the pulsation is expansile in character. If there be any doubt in determining whether the pulsation be expansile or not, a small flag, made as light as possible, may be attached on either side of the tumour; when the tumour is expansile, it will be seen that the free extremities of the flags recede from each other as the tumour fills. This method may be employed in order to determine the timerelation of a particular pulsation, one flag being placed over the apex-beat and another over the pulsation in the epigastrium.

Systolic Recession.-Systolic recession of the apex-beat may sometimes be observed in certain cases, the apexthrust being replaced by an indrawing during cardiac systole. This indrawing may be due to an enlarged right ventricle displacing the left ventricle backwards, so that the clinical apex-beat is formed by the right, instead of the left, ventricle; or it may be indicative of adhesion of the pericardium to the chest-wall. Systolic recession of the intercostal spaces on either side of the sternum may be due to the right ventricle, apart from pericardial adhesions, or to pericardial adhesions. Systolic recession of the sternum itself is in all probability indicative of pericardial adhesions. Systolic recession is sometimes to be noted in the epigastrium. A slight degree is not 
infrequently present in healthy persons. It is usually present in enlargement of the right ventricle, and in adhesion between the pericardium and the chest-wall. Systolic recession of the lateral and posterior walls of the left side of the chest is occasionally to be noted in chronic adhesive pericarditis, but it has also been observed in marked enlargement of the heart.

Diastolic Shock.--By diastolic shock is meant the shock felt by the hand at the closure of the aortic or pulmonary valve. This follows immediately on the true impulse, and is supposed to be due to the recoil of the vessel-wall after the systolic distension. It is an important physical sign of an aneurism; but it may also be present over the aortic area if there be dilatation of the vessel and super-normal blood-pressure, and over the pulmonary area when there is an increase of pressure within the pulmonary circuit, in the latter case being better felt when the lung is retracted.

Pulsation at the Root of the Neck.-Pulsation at the root of the neck may occur in the episternal notch, or external to the sterno-mastoid. In the former, it is usually systolic, and may be due to (1) chlorosis or other forms of anæmia, (2) fusiform dilatation or aneurism of the aorta, the pulsation in the latter case being expansile in character, (3) an abnormally large thyroidea ima artery, and (4) an abnormal origin of the right subclavian from a point to the left of the middle line.

Pulsation outside the sterno-mastoid may be arterial or venous. These can be easily distinguished from one another if the following facts be borne in mind. Arterial pulsation is palpable as well as visible, while venous pulsation is visible only-unless hypertrophy of the auricle or the ventricular form of venous pulse be present; venous pulsation is diffuse, wavy, and the collapse of the vein is more conspicuous than the expansion; and, lastly, venous pulsations are normally twice as frequent as those of the arteries, a fact which may be noted merely with. 
the aid of the naked eye. Arterial pulsation is due to the carotid artery, and may be caused, among other things, by temporary muscular or mental excitement, and as the result of exophthalmic goitre, or hypertrophy of the left ventricle-especially if aortic incompetence be present.

Venous pulsation may be best observed by the naked eye in the external or internal jugular veins, the former just above the clavicle, and the latter as a large wavy movement over the side of the neck between the angle of the jaw and the sterno-mastoid muscle; but it may also be noted in the axillary and cephalic veins.

Pulsation in the jugular veins is usually best seen when the individual is recumbent. Slight pulsation may be normally present, but this cannot be said to be the case when pulsation is found to extend far up the neck. When the venous pulse is observed by the naked eye, it may be one of two types, (1) that in which two waves are apparent, this being called the "physiological," "negative," "double," or auricular form of venous pulse; and (2) when only one wave is visible, this being designated the " positive," " single," or ventricular form of venous pulse. In the first of these, on comparing the venous with the carotid pulse the vein will be found to fill and collapse twice as frequently as the carotid, the two waves in the jugular vein being termed the $a$ and $v$. waves respectively. The first wave is synchronous with auricular systole, the second wave occurring towards the end of the ventricular systole. In the ventricular form of venous pulse, the $v$ wave occurs during the period of ventricular systole. The vast majority of cases are due to auricular fibrillation; a small proportion are due to a greatly distended right auricle, or to some forms of paroxysmal tachycardia. In complete heart-block, jugular pulsation may be sometimes noted as occurring at a much more frequent rate than that of the radial or carotid arteries.

Distension or pulsation of the jugular veins may be 
due to stasis in the right auricle from any cause, such as valvular disease, or affections of the lungs which cause embarrassment of the pulmonary circulation, compression of the superior vire eava by a mediastinal tumour or other causes, or constriction of the opening of the superior vena cava into the heart by chronic adhesive pericarditis. The distended vein may show pulsation, even of marked degrec. In order to determine whether tricuspid regurgitation is present or othcrwise, the following plan is advisable. In regurgitation, when the vein is cmptied from below by means of the finger, and its upper extremity is kept closed by pressure, it rapidly refills from below; when, on the other hand, there is no regurgitation, it either remains empty or at the most refills slowly. Sometimes, especially in ehronic heart disease in which sclerosis of the vein is present, owing to chronie venous stasis the veins resemble knotted cords, there being no pulsation, due to their valves being elosed.

Pulsation of the internal jugular vein may be graphically recorded by means of the elinical polygraph, this being a better method than examination by the naked eye.

Exaggerated pulsation of the arteries, e.g. the carotid, brachial, and temporal arteries, is not infrequently to be noted in aortic incompetence, exopthalmic goitre, and anæmia, espeeially in the first-named.

The phenomenon of the capillary pulse may be observed in aortic regurgitation, but is occasionally also seen in other conditions in which the diastolic blood-pressure is subnormal, e.g. in pyrexia and anæmia. In this condition, if any part of the skin normally pale bc made red by pressure $o:$ friction, there is an alternative flushing and pallor with each systole and diastole of the heart. The phenomenon may be observed close to the lunules at the base of the nails, especially when the free border of the nail is pressed downwards; it may also be noted along an erythematous streak made by drawing the finger-nail across the forehead, or over an area of skin to which 
slight friction has been applied, or during gentle compression of the lips or gums by a glass slide.

Pulsation in the superficial veins (" centripetal venous pulse") is sometimes visible to the naked eye, especially in those of the upper part of the anterior chest-wall, the back of the hands in the dependent position, and in the feet. This is due to the arterial pulse-wave passing on through the capillaries to the veins; it is met with when the cardiac action is forcible and the arterioles are greatly relaxed, e.g. in aortic incompetence and febrile states, and also in aneurismal varix-in which condition an artery and a vein communicate.

Thrills.-Thrills may be felt on palpation of the precordium, and may also be palpable over the large arteries at the root of the neck. They may be due to pericardial friction-as in simple pcricarditis, and in tuberculous, cancerous, or gummatous deposits-inflammation of the pleuro-pericardial membrane, valvular disease, or to aneurism.

If due to pericarditis or pleurisy, the area of maximum intensity does not absolutely correspond with that due to valvular disease, they are not exactly synchronous with either systole or ciastole, they oftcn have a toand-fro character, and they give the impression to the hand of being superficial.

Those due to pericarditis can be distinguished from those due to pleurisy by the fact that the latter will be either much reduced or disappear when the patient stops breathing, and increased by deep respiration.

In studying thrills, we should determine (1) their pos:tion in the cardiac cycle, and (2) their area of maximum intensity.

With regard to the former, thrills may be systolic, diastolic, or, in the case of those produced at the auriculoventricular orifices, presystolic in time. If a thrill commence with the apex-beat and continue during the period of ventricular contraction, it is called systolic; if it occur 
while the ventrieles are relaxed, it is termed either diastolic or presystolie-the latter if it occur near the close of diastole, when the aurieles have entered upon systole. The area of maximum intensity of thrills is important, because a thrill having its origin in a particular orifice may also be palpable over another area; the point to determine is the area of maximum intensity.

An aortic systolic thrill indieates aortic obstruetion or aneurism. In the case of the former, the thrill may be felt over the whole precordium, and sometimes indeed all over the ehest and the large vessels of the neek. An aortie diastolic thrill is rarely present, and when it is it indieates ineompetenee. Its maximum intensity is not infrequently at a lower level than the aortic area, and may even be felt towards the lower part of the sternum and in the neighbourhood of the apex-beat. A mitral systolic thrill is not of eommon oceurrenee. When present it signifies the existence of mitral regurgitation, but some narrowing of the valve is probably also necessary to produce it. A mitral presystolie or diastolic thrill is indicative of mitral stenosis; the former is the more eommon, and on palpating the precordium there is a sensation eommunieated to the hand resembling that produeed by the purring of a eat. The area of maximum intensity of both mitral presystolie and diastolie thrills is at the apex, or somewhat nearer the parasternal line. Examination for the charaeteristic thrill and murmur of mitral stenosis should be condueted with the patient oceupying an absolutely reeumbent posture, sitting up and inelined forwards, standing with the arms extended, and also after gentle exereise. A thrill deteeted in these circumstanees is almost pathognomonie of mitral stenosis. It must, however, be carefully distinguished from mere vibration of the ehest-wall which may be occasioned by a forcibly aeting heart when the ribs are rigid; in this case, by separating the fingers and placing them in the intercostal spaees the osseous vibrations are not felt. Some writers 
also believe that in certain cases of aortic regurgitation a presystolic thrill may be felt at the apex.

A pulmonary systolic thrill is sometimes found in pulmonary stenosis; it is also occasionally met with in certain diseases-especially in exophthalmic goitre-and is rarely the result of movement of the conus arteriosus or pulmonary artery during the systole of the right ventricle. A pulmonary diastolic thrill indicates incompetence of that valve. When the ductus arteriosus is permanently patent, a prolonged thrill may be palpable over the pulmonary area ; it occupies both systole and diastole, commencing shortly after the beginning of ventricular systole and extending into a considerable part of the diastole; indeed, it may persist almost throughout the whole cardiac cycle. A prolonged thrill occupying both systole and diastole may also be met with in arterio-venous aneurism.

Tricuspid thrills are exceedingly rare. A systolic thrill indicates regurgitation, but it has never been met with in the experience of the writer. Diastolic and presystolic thrills indicate obstruction, and present the same features as those which occur in mitral stenosis.

Occasionally a systolic thrill is felt over an aneurism of the aorta or of one of the larger vessels, and may also be found in dilatation of the aorta when this reaches as high as the upper limit of the manubrium sterni.

\section{Percussion}

By percussion of the heart we are able to ascertain the position and size of the organ, the extent of the area uncovered by lung, and the existence or otherwise of any morbid conditions in the immediate neighbourhood of the heart or great vessels. My custom is to employ the mediate or indirect method, using the back of the middle phalanx of the forefinger of the left hand as the pleximeter, and the tip of the right middle finger as the plessor. There are certain cardinal rules which should always be observed when percussion is being employed. The pleximeter 
should be placed in firm apposition with the chest-wall; percussion should be performed from the wrist and finger joints, not from the elbow; and the percussing finger should strike the pleximeier as nearly as possible at a right angle. In defining the boundaries between resonant and less resonant or non-resonant organs, percussion should be performed from the former towards the latter. Furthermore, the long axis of the pleximeter should be parallel with the border of the organ the boundary of which is being defined, and the line of percussion should be at right angles to that boundary. When percussing, we should always compare rib with rib and interspace with interspace, and both the tone and sense of resistance should be noted. As we percuss towards the cardiac area we note at what points the lung resonance begins to diminish. This area is called the area of deep or relative impairment, and its outline affords fairly accurate information regarding the position and size of the heart. In determining it, considerable strength of percussion should be employed.

There is some difficulty in determining the upper border of the area of deep or relative impairment, because the roots of the great vessels at the base of the heart diminish the lung resonance, thus producing a note which cannot readily be distinguished from that obtained over the heart itself. It is possible, however, to determine that part of the upper border which lies to the left of the roots of the great vessels, by selecting a vertical line at a sufficient distance from the middle plane so as to be to the left of these vessels, but not so far as to go beyond the left border of the heart. These requirements are met by choosing the left parasternal line or a line rather internal to it.

In ascertaining the right border of the heart, the upper boundary of the deep hepatic impairment in the right mammary line should first of all be made out, after which percussion should be carried out from right to left along a line which is above the upper border of hepatic impair- 
ment until the comparative impairment of the heart is reached. It may be impossible, when the lungs are somewhat voluminous, to make out an area of impaired resonance to the right of the sternum, and, that being the case, no reliance can be placed upon the note obtained over the sternum, as the sternum acts as a sounding-board. In determining the left cardiac border, we can percuss from left to right in the fourth interspace, and only light percussion should be employed in outlining this border. It is of very great importance to percuss accurately the left border of deep cardiac impairment in cases in which the apex-beat is faint or absent; I have seen several cases in which'a dilated heart associated with myocardial degeneration was not detected because of an omission in this respect. We cannot define the lower border of the heart by percussion, as it rests upon the liver, and both organs give the same percussion note. But the boundary between the two organs may be ascertained in the following way: Having determined the upper border of deep hepatic impairment, and also the right and left borders of the heart, a line should be drawn from the angle formed by the junction of the line of deep hepatic impairment and that of the right border of the heart to the cardiac apex.

In an average healthy chest the upper border of comparative impairment in the left parasternal line is at the level of the third rib or upper border of the third interspace; the right border at the level of the fourth rib is just to the right of the right lateral sternal line, or about $1 \frac{1}{2}$ to 2 inches from the middle of the sternum; while the left border at the level of the fourth rib is rather internal to the mammary line, or about $3 \frac{1}{2}$ inches from the middle of the sternum. The line of impairment of the upper border inclines slightly downwards from the left parasternal line across the sternum to the right, and curves more and more, until, as already indicated, it reaches a point about $1 \frac{1}{2}$ to 2 inches from the middle of 
the sternum at the level of the fourth right costal cartilage. The left border runs outwards and downwards in a crescentic line from the highest point noted, until, as already indicated, it reaches a point about $3 \frac{1}{2}$ inches from the middle line at the level of the fourth rib.

The area of the heart which is uncovered by the lungs and lies directly against the chest-wall is called the area of superficial or absolute cardiac impairment, and in determining it light percussion should be employed. To ascertain the upper border of this area we should percuss downwards between the left sternal and left parasternal lines; while percussion at the lcvel of the fourth intercostal space or fifth rib should be employed to determine the right and left borders of the area, in the case of the former beginning to the right of the sternum and in the case of the latter from the left mammary line-both towards the middle line.

In an average healthy chest the form of the area of superfieial cardiac impairment is somcwhat triangular, the left side of the triangle being convex outwards. When respiration is stationary or quiet, the upper border of this area is at the level of the upper border of the fourth costal cartilage. The left border commences at this point, and then proceeds outwards and downwards, crossing the fourth intercostal space and the corresponding rib, to the fifth intercostal space, when it curves slightly inwards until it reaches the sixth rib, at which point the areas of superficial cardiac and hepatic dulness merge. The left border of the superficial cardiac impairment at its upper end is rather more than half an inch within the border of the deep cardiac impairment, while at its lower end the two approximate more closely, and almost merge into one another at the apex-beat. The right border of superficial cardiac impairment corresponds with the midsternal line, but cannot be defined with accuracy, on account of the sternum acting as a sounding-board; indeed, a difference in the percussion note cannot with 
certainty be noted until the left lateral sternal line is reached, at which point the area of impairment extends from the fourth to the sixth costal cartilage. The lower border of superficial cardiac impairment cannot be determined by percussion, but corresponds with the actual lower border of the heart. The superficial cardiac impairment extends vertically from the upper border of the fourth costal cartilage to the level of the sixth chondrosternal articulation, a distance of about 2 inches, while its breadth at the level of the fifth costal cartilage is $1 \frac{1}{2}$ inches. On forced inspiration the size of the area of superficial cardiac impairment is lessened, and on forced expiration it is increased in extent.

An increase in the area of the relative or deep impairment may be caused by disease of the heart or pericardium, and by morbid conditions in the immediate neighbourhood. Thus, cardiac hypertrophy and dilatation, especially the latter, may occasion it. Enlargement of the left side of the heart chiefly affects the vertical area, and the percussion outline becomes more nearly conica?; while enlargement of the right side of the heart chiefly affects the transverse area of impairment, and the percussion outline becomes more nearly square. If the area of relative impairment extend beyond the left mammary line, but not to the left of the apex-beat, provided the heart is not displaced to the left as a whole, and in the absence of pulmonary or pleural disease, in all probability enlargement of the left ventricle is present. If the area of deep cardiac impairment be displaced to the right, provided there is an absence of pulmonary and pleural disease, and we can exclude pericardial effusion, it may be inferred that the right heart is enlarged.

In cases of pericardial effusion, whether due to pericarditis, hydro-pericardium, or hæmo-pericardium, the extent of the deep cardiac impairment may be considerably increased, this increase in the area taking place in all_directions. When the effusion is considerable, the 
form of the increased area of cardiac impairment is characteristic, its outline as a rule closely resembling that of a pear hanging downwards by its stalk, the upper limit being higher than normal. When the left border of the area of relative impairment is outside the apex-beat, in the absence of any morbid condition of the lung or pleura, the cause will be found to be pericardial effusion, in which case the right border will be found to be displaced considerably to the right, even as far out as the right parasternal line. If the impairment in the left parasternal line be found to extend upwards into the second interspace or higher, and we are sure that the heart as a whole is not displaced upwards by pressure from the abdomen, and that disease of the lung or pleura, and the presence of aneurism, new-growth, and enlarged glands can with certainty be excluded, the condition is in all probability due to pericardial effusion or great cnlargement of the right ventricle. An increase in the area of relative cardiac impairment may be due to lesions of the lungs or pleuræ.

In aneurismal dilatation of the ascending aorta, there is an area of impairment above and continuous with that of the heart, extending outwards to the right of the sternum at the level of the sccond rib and adjacent intercostal spaces, the note over the manubrium sterni being less resonant than normal, amounting in some cases to dulness. Rarely, the heart is displaced forwards by an aneurism or new-growth in the posterior mediastinum.

The area of relative impairment may be diminished or even abolished in cases where the heart is abnormally small, and also in emphysema, pneumothorax, and pneumopericardium. If the area of relative cardiac impairment be found not to be diminished in emphysematous subjects, we may infer that the heart is enlarged.

The area of absolute or superficial impairment does not give such valuable information as that of the relative impairment, although it frequently gives more informa- 
tion as to the condition of the lungs or pleuræ than of the heart. Nevertheless, it becomes modified in some cardiac affections, and is altered by the same conditions which modify the area of relative impairment; thus, in dilatation and hypertrophy it is often increased to some extent, and still more so in pericarditis with considerable effusion. The greatest increase, however, is due to collapse or fibrosis of the lung, or to pleuritic adhesions. In young children an increase in the size of the area may be due to the presence of a large thymus gland. The area may be diminished in emphysema, pneumothorax, and pneumopericardium.

Lastly, it should be remembered that the area of cardiac impairment is altered by changes in the position of the heart, which may be due to its displacement by extrinsic morbid conditions, or to developmental errors, such as dextro-cardia. Pleural effusion pushes the heart towards the sound side of the chest, while fibrosis of the lung draws it to the affected side. In dextro-cardia the area of impairment is the mirror image of what is found in normal conditions. The area of cardiac impairment may be situated higher than usual when the heart is displaced upwards in cases of ascites or large abdominal tumours.

\section{Auscultation}

It is well to auscultate first over the mitral area, and we may accurately ascertain the time of the sounds in the cardiac cycle by simultaneously palpating the apexbeat.

Modifications of the normal sounds of the heart may occur as follows: (1) alteration in loudness, length, character, or rhythm; and (2) adventitious sounds may be heard, either occurring along with the heart sounds or replacing them altogether, or during the pauses.

Alteration in Loudness, Length, Character, or Rhythm.-The heart sounds are increased in intensity in 
retraction of the lung, during and immediately after physical exertion or mental excitement, and also when the cardiac action is increased from any cause, as in the cardiac neuroses-especially in Graves' disease. They are found to be diminished in intensity in emphysema, and in the presence of fluid or air in the pericardial sac. There may be considerable variation of the first sound in health, more so in the mitral than in the tricuspid area. In stout people it is often very faint and short; in thin subjects it is often short, high in pitch, and ringing in quality; while in individuals of robust type it is often of long duration, low in pitch, and rumbling.

If the second sound be louder than the first in the mitral or tricuspid area, we may assume either that the first sound is diminished in intensity, or that the second sound is accentuated. If the first sound be louder than the second over the base of the heart, we may assume either that the first sound is increased in intensity, or that the second sound is diminished.

In hypertrophy of the left ventricle, the first sound in the mitral area is frequently long, sustained, low in pitch, and muffled. In dilatation of the left ventricle, and in certain stages of febrile conditions when the cardiac action is excited and the blood-pressure is subnormal, it is often accentuated, considerably shortened, sharp, clear, and high in pitch. The first sound may be short and diminished in intensity when the contractile force of the ventricle is reduced, as, for example, in fatty degeneration of the myocardium, and in the later stages of febrile conditions; this feature is of diagnostic importance in the case of the former, while in the latter condition it is of considerable prognostic significance, the outlook being grave when the first sound is so weak as to be almost inaudible. In pure mitral stenosis the first sound is altered in a characteristic manner; it becomes short, sharp, and clear, like a sudden snap; it may resemble the second sound so closely as to be mistaken for it, 
especially when the second sound is not well heard at the apex.

An increase in the loudness of the first sound in the tricuspid area is not so common as in the mitral area. It may occur, however, with increase of pressure within the pulmonary circuit; this takes place when there is obstruction to the passage of blood into the left chambers of the heart, or to its onward course through the lungs, such as occurs in chronic valvular disease of the left side of the heart, in emphysema, and in fibrosis of the lung. But when the increase of pressure in the pulmonary circuit goes beyond a certain point, the safetyvalve function of the tricuspid valve comes into play, the consequent tricuspid regurgitation causing a diminution in the intensity of the first sound; this accounts for the fact that not infrequently with an accentuated pulmonary second sound the tricuspid first sound is either faint or replaced by a murmur. Diminished intensity of the first sound in the tricuspid area occurs in all conditions causing weakness of the right ventricular wall.

The second sound in the aortic or pulmonary area may be increased in intensity, an indication that the respective valve has been closed with unusual force. Aortic accentuation may occur in nephritis-especially the chronic forms of the disease, arterio-sclerosis, super-normal blood-pressure, and fusiform or saccular dilatation of the vessel near its orifice. In cases of dilatation of the vessel beyond the ring, the second sound is sometimes not only accentuated but resembles the echo produced when a cork is drawn from an empty bottle. Pulmonary accentuation occurs when there is increased blood-pressure in the pulmonary circulation, due to causes already mentioned. It indicates hypertrophy of the right ventricle, and when the force of the ventricular contraction fails the accentuation of the pulmonary second sound lessens. The second sound may be diminished in intensity, an indication that the 
valve has been closed with less force than normal; this occurs when the blood-pressure is subnormal, or the valves are stiff. Absence of the second sound is an indication that a marked valvular defect is present. The second sound may become shorter when the heart's action is feeble and rapid.

In ancurismal dilatation altered cardiac sounds are commoner than murmurs. The first sound tends to become toneless, while the second sound is often accentuated.

The first or the second sound may be reduplicated under certain conditions, the sound instead of being single being represented by two short sounds. As a rule, the interval betwcen the two elements of the double sound is incomplete, but it is sometimes distinct. Reduplication of the first or the second sound is probably the result of the valves, whether auriculo-ventricular or semilunar, closing sooner on one side of the heart than on the other. In the case of the mitral and tricı spid valves, the cause is asynchronism of the contraction of the respective ventricles; while in the case of the aortic and pulmonary valves, the cause is a variation in the normal relation of blood-pressure in the aorta and pulmonary arteries, an increase of pressure acting on a particular valve, causing an earlier closure of that valve.

Reduplication of the first sound is usually best heard rather to the inner side of the cardiac apex, and is as a rule the result of high pressure in the systemic circulation, as, for example, in renal disease. A variety of reduplication of the first sound is what is termed the gallop rhythm. In such cases there are three sounds audible at the apex ; the accent is variable, but is usually on the second element of the triplet. This condition sometimes indicates failing heart muscle, as, for instance, in the advanced stage of Bright's disease, and is sometimes of very unfavourable import. In certain cases it is due to asynchronism of the two ventricles, as when the right 
main division of the auriculo-ventricular bundle is damaged and the right ventricle contracts a little later than the left.

Reduplication of the second sound is heard most distinctly at the base of the heart, and may be audible at this situation only. Reduplication of the aortic second sound is caused by the same conditions as bring about its accentuation. Reduplication of the pulmonary second sound means an increase of pressure in the pulmonary circulation, due to causes already mentioned; it is very characteristic of mitral stenosis, but is occasionally to be noted in healthy individuals.

The conditions which may give rise to a triple rhythm, in other words to the presence of three sounds, are (1) doubling of the first sound; (2) doubling of the second sound; or, when heard at the apex, (3) a very short diastolic bruit.

In cardiac hypertrophy the short pause may be lengthened, and the long pause may be shortened. When the cardiac action is very rapid, the diastolic pause may become shortened to approximately the same interval of time as that between the first and second sounds, and the cardiac sounds occur at uniform intervals, like the ticking of a watch. This is called the tic-tac heart beat, embryocardia, or fœtal rhythm. It is met with when the vitality has been greatly impaired, as, for example, by long-continued super-normal blood-pressure, such as is common in renal disease, when it is of grave omen. The systolic interval may also become shortened; this may be the result of the systole being rapidly accomplished, or may occur in great cardiac weakness when there is incomplete contraction of the ventricle and the systole becomes abortive.

Adventitious Sounds.-These are divided into two groups, namely, (1) those which are exocardial in origin, and (2) those which are endocardial in origin. The latter group are called murmurs or bruits. 
Exocardial sounds may be due either to pericardial friction, or to a localised pleurisy near the heart causing frietion. Unlike endocardial murmurs, the different portions of the friction sounds do not absolutely coincide either in rhythm or duration with any period of the cardiac eycle, being rather later than any of them and usually heard during portions of more than one period; the area of maximum intensity does not absolutely correspond with that of any of the valvular areas; the friction sounds are as a rule eondueted either not at all or only to a slight extent, and there is no definite sclective direetion of prop gation; they are of a rubbing quality; they give the impression of being produced immediately bencath the stethoscope, while additional pressure with the stethcscope usually eauses an increase in their intensity; and, further, their position, intensity, and eharacter may alter in a few hours.

Pericardial friction oceurs when there is any roughening of the piricardium, as in simple periearditis, and in tubercular, cancerous, or gummatous deposits.

Pleuro-perieardial friction is caused by inflammation affecting the adjacent surfaces of the parietal pericardium and the pleura. It has a double rhythm, being systolic and diastolic in time. It can be distinguished from pericardial friction by the fact that the pleuro-pericardial friction is either much reduced or ceases when the patient stops breathing, while it is increased by respiration. Further, pleuritic frietion usually disappears with the cffusion of fluid, whereas in pericarditis with effusion friction is not abolished unless the quantity of fluid is great, and even then it may persist.

Murmurs or Bruits.-Murmurs or bruits may be eardiac, arterial, or venous.

Cardiac murmurs are classified as (1) organic, and (2) inorganic or functional. In the former, the valves are involved, and a murmur may result either from obstruction to the onward flow of blood, or from leakage backwards 
through an incompetent valve, the former being known as obstructive, and the latter as regurgitant, bruits. In the case of inorganic or functional murmurs, the valves themselves are healthy; these murmurs include, among others, (1) bruits due to anæmia, (2) those due to relative incompetence of one of the valves (vide pages 258 and 259), (3) cardio-pulmonary murmurs, and (4) murmurs which may be present when there is excitation of the cardiac action, e.g. in Gravcs' disease.

In every case in which a murmur over the precordium is audible, the question whether the murmur is organic or functional should be carefully considered, the latter being of frequent occurrence.

In investigating a murmur, the following points should be carefully noted: (1) the time of its occurrence; (2) the point of maximum intensity; (3) the direction of selective propagation - by which is meant that, instead of murmurs being audible equally well at all points of the chest-wall from their points of maximum intensity, they are better heard at a distance in some directions than in others ; and (4) its character. The point of maximum intensity and the direction of selective propagation are of great value in differentiating the bruits that occupy the same position in the cardiac cycle.

It may be well to point out at the outset that if a murmur be audible over more than one valvular area, it must not be inferred that more than one valve is necessarily affected. In order to be assured that this is the case, we must satisfy ourselves that there is either an increase in the intensity, or an alteration in the character, of the murmur, as the second area is approached; in other words, when more than one valve is affected, murmurs have separate points of maximum intensity.

Time of Occurrence of Murmurs. - With regard to the time of occurrence, endocardial murmurs are always definitely associated with the different phases of the cardiac cycle. The time in the cardiac cycle may be ascertained 
by comparing the time of the murmur with the carotid pulse, the sounds of the heart, or with the time of occurrence of the apex-beat. The last, however, is not reliable, as the clinical apex-beat may be inverted.

Murmurs may be produced at any part of the cardiac cycle during which the blood is flowing. Those occurring during the systole of the ventricle are termed systolic, or, according to the late Sir William Gairdner, ventriculosystolic. They may occupy the whole or a part of the period from the commencement of the first sound to that of the second sound (Fig. 8). Those occurring during

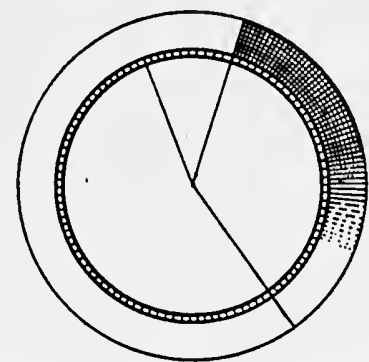

Fig. 8. Shows the rhythm of a systolic murmur.

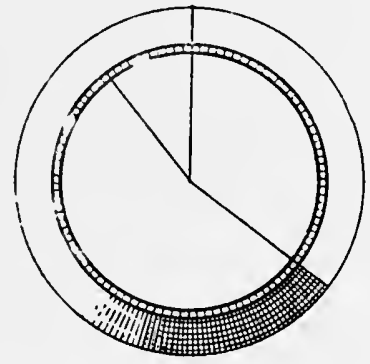

Fig. 9. Shows the rhythm of a diastolic murmur.

ventricular diastole are called either diastolic or presystolic; they may occupy the whole or a part of the period from the commencement of the second sound to that of the first. Diastolic murmurs are those which accompany, replace, or follow the second sound, and were called by Gairdner ventriculo-diastolic (Fig. 9). A feature of diastolic murmurs is that they are diminuendo in type. Presystolic murmurs can only be produced at the auriculoventricular orifices. They occur at the end of ventricular diastole, during the systole of the auricle, and immediately preceding ventricular systole (Fig. 10). They were called by Gairdner auriculo-systolic. A distinctive feature of presystolic murmurs is that they run up to and unite with the first sound, becoming louder as they proceed, 
and being cut short at their loudest. It is believed by some, however, that when in mitral or tricuspid stenosis there is a delay between the auricular and ven-

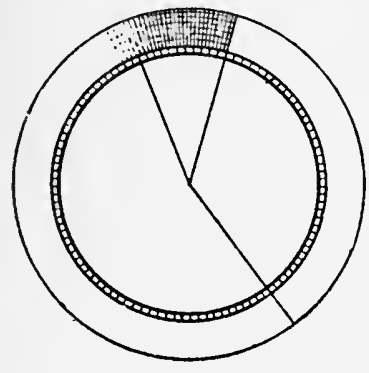

Fra. 10. Shows the rhythm of a presystolic murmur.

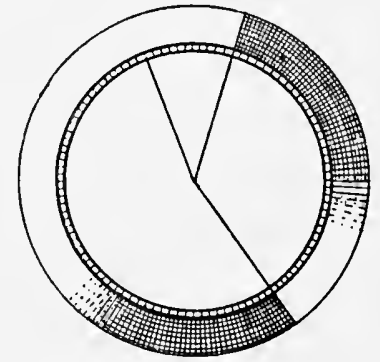

FIG. 11. Shows a combination of systolic and dias. tolic murmurs.

tricular contractions, a slight interval between the auricular systolic thrill or murmur and first sound may be noted. Presystolic murmurs denote obstruction of

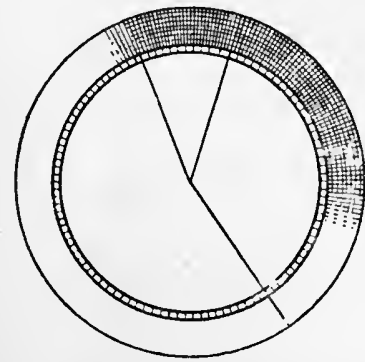

Frg. 12. Shows a combination of presystolic and systolic murmurs.

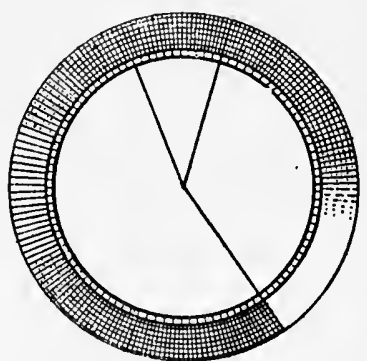

FIG. 13. Shows a combina. tion of presystolic, systolic, and diastolic murmurs.

the orifices, but most writers believe that in ccrtain cases of aortic incompetence a presystolic murmur is audible at the cardiac apex (Flint's murmur).

Systolic murmurs at the aortic and pulmonary orifices may denote obstruction, since these valves ought to be fully open during ventricular systole. It should be noted, 
however, that a diagnosis of aortic or pulmonary stenosis should not be made from the mere presence of a systolic murmur having its point of maximum intensity in the aortic or pulmonary area respectively, as the murmur may arise from other causes; the differential diagnosis is discussed under aortic and pulmonary stenosis.

Murmurs at the auriculo-ventricular orifices which are systolic in time denote regurgitation, since these valves should be closed during the systole of the ventricles. It should be noted, however, that mitral and tricuspid systolic murmurs may be due to what is called relative incompetence of the valves - that is, incompetence in which the valves themselves are healthy-and other causes, as well as to organic disease.

When diastolic murmurs occur at the aortic and pulmonary area, they indicate an escape of blood backwards from the arteries into the ventricles, and, therefore, denote incompetence of the respective valves. It must be remarked, however, that a pulmonary diastolic bruit indicating pulmonary incompetence is rare, this being due to the fact that, while the typical position of an aortic diastolic bruit is over the aortic area, it is frequently loudest in the pulmonary area. Relative incompetence of the aortic valve is almost unknown.

When diastolic murmurs occur at the auriculo-ventricular orifices, they indicate some interference with the passage of the blood from the auricle into the ventricle; they, therefore, denote obstruction. In the opinion of some writers, however, a mitral systolic and diastolic murmur in children may be present in an almost pure regurgitant lesion, sometimes complicated by an adherent pericardium, the diastolic murmur occurring practically in the absence of obstruction.

It will thus be seen that in mitral and tricuspid stenosis there may be a presystolic, or diastolic, murmur, or both. In each case, the murmur is caused by the onward flow of blood through a narrowed orifice, the presystolic 
being due to the systole of the auricle driving blood through the narrowed orifice, and the diastolic to the passive inflow of blood from auricle to ventricle. In this connection, it is necessary to point out that the terms "presystolic" and "auriculo-systolic" are not infrequently erroneously regarded as synonymous. The term "presystolic" should be applied to a murmur only because it immediately precedes the first sound. The bruit is usually due to the contraction of the auricle driving the blood through the narrowed mitral orifice. In the absence of auricular systole, however, blood will flow from the left auricle to the ventricle from the time of commencement of ventricular diastole until the pressure is equal in the two chambers; so that a bruit wholly ventriculo-diastolic in time will be present. If in these cases the cardiac rate be frequent, the next ventricular systole becomes due before the pressure in the two chambers has become equal, and before the sound produced by the flow of blood has terminated ; the end portion of this bruit may accuratcly be termed "presystolic." If, on the other hand, the cardiac rate be infrequent, the pressure between the two chambers will become equal before the next ventricular systole, and an interval will separate the bruit from the following first sound. It will thus be evident that while an auriculo-systolic murmur disappears with the onset of auricular fibrillation, and it is impossible for both to coexist, a bruit presystolic in time may be present in that condition.

In cases of auricular fibrillation and rapid cardiac action, in which a murmur occupies the whole interval between the second and first sounds, the latter part of the murmur can be distinguished from a presystolic murmur due to the systole of the auricle by noting that, (1) during one of the long pauses, or when the heart rate becomes slow, a silence will be detected between the termination of the murmur and the first sound, and (2) the murmur is not crescendo in type. 
The late G. A. Gibson described a prolonged murmur, which occupies both systole and diastole. It commences shortly after the beginning of the first sound, and extends into a considerable part of the diastole; indeed, it may persist almost throughout the whole cardiac cycle. Gibson believed it to be indicative of a patcnt ductus arteriosus. A prolonged murmur occupying both systole and diastole may also be met with in arterio-venous aneurism.

Point of Maximum Intensity and Direction of Selective Propagation of Murmurs.-With regard to the direction of selective propagation of a murmur, it may be noted that sound is eonveyed better in the direction of a moving current.

An aortic systolic murmur may have its point of maximum intensity in the aortic area, or over the manubrium sterni, or to the left of the stcrnum-in which case a diagnosis should be made from a pulmonary systolic murmur. The murmur, however, may be heard all over the chest and even at the back when it indicates aortic stenosis, the direction of selective propagation being upwards along the vessels of the neek. The point of maximum intensity of an aortic diastolic bruit is very variable, the typieal position being over the inner end of the second right intercostal cartilage. Frequently, however, it is the second left space, or immediately over the sternum. More rarely the point of maximum intensity is at a lower level than this, approaching even the level of the apex-beat, and in my experience is often nearer the left than the right border of the sternum. The murmur has a tendency to be propagated downwards towards the lower end of the sternum, and is often heard over a wide area.

Murmurs due to mitral stenosis are best heard at the apex or between this point and the left border of the sternum. Their locality is almost always very limited, and the area may not be larger than one inch in diameter. This 
should be particularly noted, because it accounts for the fact that this lesion is so often unrecognised. Occasionally, however, the murmur is propagated over a considerable area, even as far as the right border of the heart; but there is an absence of direction of selective propagation. As a rule, a mitral diastolic murmur is audible over a larger area than a presystolic, although this is not invariably the case. A mitral systolic bruit is best heard at or near the apex-beat, and its direction of selective propagation is towards the left axilla and the angle of the scapula. Occasionally it can be heard over the whole chest. The area of slight mitral systolic murmurs is frequently comparatiyely limited. Pulmonary systolic and diastolic murmurs almost always have their point of maximum intensity over the inner end of the second left intercostal space. A pulmonary systolic murmur is usually audible outwards in the second left interspace, and upwards towards the left clavicle; occasionally it is heard over a considerable area of the chest, and, indeed, even over the whole chest. In the vast majority of cases, however, it is not audible over the vessels of the neck, and as a rule it has no direction of selective propagation. A pulmonary diastolic murmur may be audible over a considerable area, its direction of selective propagation being downwards along the left border of the sternum. As has been indicated, a double murmur over the pulmonary area may have its origin in the aortic valve. Tricuspid murmurs are generally best heard over the tricuspid area, and the systolic murmur is conducted towards the right, but usually its area of conduction is limited.

Character of Murmurs.-Murmurs are usually one of three kinds, namely, (1) rough and grating, common in stenosis, (2) soft and blowing, much more common in incompetence, and (3) musical, the most common being an aortic diastolic bruit. Musical murmurs are apt to be found in cases in which semi-detached fragments of valve 
move about in the blood-stream, in loose chordæ tendineæ, and perforation of the valvular cusps.

The loudness of murmurs varies greatly, having no direct ratio to the gravity of the lesion, a loud murmur being often of far less serious import than one which is almost inaudible. If the degree of loudness have any significance at all, the seriousness of the lesion may be said to be in inverse ratio to this, since this implies failure of the cardiac muscle, and when the patient is extremely enfcebled not infrequently no murmur is audible, but becoming so as the patient improves. A returning murmur, therefore, is of good omen.

Obstructive murmurs having their origin at the auriculoventricular orifices, and, in the opinion of some writers, also pulmonary and aortic regurgitant murmurs, are usually louder in the sitting or standing posture, and become fcebler and may disappear altogether when the patient lies down. The opposite holds good with regurgitant murmurs having their origin in the auriculo-ventricular orifices. The reason for the variation in intensity is probably to be looked for in the effect of gravitation. In any doubtful case, therefore, the patient should be examined lying down, as well as in the sitting or standing posture.

Inorganic or functional murmurs as a group present certain characteristics. They are almost invariably systolic in time, are often of short duration, and tend merely to accompany and not to replace the cardiac sound. The point of maximum intensity is much more frequently in the pulmonary area, or rather below or external to it; sometimes it is in the mitral area, and rarely in the aortic or tricuspid area. Functional murmurs tend to be audible over the greater part or even the whole of the precordium, but their area of conduction outside the precordium is limited, and their direction of selective propagation does not coincide with that of organic limits ; mitral systolic bruits are comparatively rarely audible in 
the axilla. Functional bruits are usually soft and blowing, but are occasionally loud and rough in character. Their loudness not infrequently shows considerable variation from time to time. They are louder after exertion, and, indeed, may only be audible then. They vary with the phases of respiration, and with changes of posture, being usually louder when the individual lies down, feebler or even absent in the sitting or standing posture.; in any doubtful case, therefore, the patient should be examined during the various phases of respiration, and lying down, as well as in the sitting or standing posture. They are often audible over the larger arteries, even without stethoscopic pressure, and the "bruit du diable" is frequently present as well.

Cardio-pulmonary murmurs are due to the heart beating strongly against the lung, and not to any cardiac disease. These murmurs are not absolutely synchronous with systole or diastole; the point of maximum intensity does not coincide with any of the valves; they are usually heard best over the margins of the lungs; and they are more distinct during expiration and disappear when the breath is held after deep inspiration.

In aneurismal dilatation, murmurs may or may not be present; in the former case, they do not necessarily indicate co-existing valvular disease. They may be systolic or diastolic, the former being of more common occurrence.

The murmurs which may be found in the various forms of congenital morbus cordis will be discussed under their appropriate headings.

Auscultation of Arteries.-If a stethoscope be applied very lightly over the carotid or subclavian artery a systolic and a diastolic sound are usually audible; while over the abdominal aorta or femoral artery a systolic sound only, almost toneless, and over the other arteries no sound at all, is audible. If greater pressure be exerted, and also with only very light pressure in persons suffering from 
anæmia, aortic regurgitation, or Graves' disease, as well as by transmission from aneurism, or disease of the heart, as in aortic stenosis, a soft murmur is found to accompany the first sound over the carotid and subclavian artery. In aortic incompetence a diastolic murmur may be audible over the carotid and femoral arteries; with light pressure over the femoral artery, in place of the almost toneless sound, a more distinct sound, closely resembling a cardiac sound, is to be heard, while if greater pressure be exerted a systolic bruit is audible.

Auscultation of Veins.-On applying the stethoscope very lightly over the internal jugular vein at the root of the neck, especially over the jugular bulb just above the clavicle, and usually bettcr heard over the right side, on standing and with the head turned to the opposite side, a continuous, humming, rushing, or roaring sound may be frequently heard in anæmia; this sound has been referred to as the venous hum, or " bruit du diable"-from a French toy, called "diabolo," which makes a similar noise. 


\section{CHAPTER III}

\section{THE ARTERIAL PULSE}

ThE arterial pulse gives information regarding the action of the left ventricle, and is a valuable aid in diagnosis and prognosis. But, while this is so, it should be remembered that, taken by itself, it is not a sufficient guide, and, for this reason, conclusions should never be arrived at from an examination of the pulse alone.

The trained finger can gather much information regarding the pulse. Thus, we may note the rate, the volume, the force, and general character of the pulsewave, the rhythm, the state of the vessel wall, the arterial blood-pressure, and the symmetry of the two pulses. The pulse of the radial artery at the wrist is usually employed, because of its convenient situation; but the brachial, the carotid, the femoral, or the posterior tibial artery behind the internal malleolus may also be palpated by the finger.

The finger, however, should be supplemented by instrumental methods, and by means of the sphygmograph we can record the pulse-wave graphically, the tracing obtained being called a sphygmogram. As the pulse-wave is due to ventricular systole, there is a correspondence between the ventricular and arterial pressures during the time that the aortic valves are open, and a sphygmographic tracing in this way affords information regarding variations in the aortic pressure. By this means, likewise, we are able to determine the period during which the semilunar valves are open. While sphygmograms are not altogether free from errors due to the instrument, they afford valuable information in a large proportion of cases of 
heart disease; indeed, the sphygmograph alone enables us to take full advantage of the recent advances in knorvledge of cardiac disorders, which have been mainly due to the introduction of the clinical polygraph and electro-cardiograph, in a considerable proportion of cases. Of the

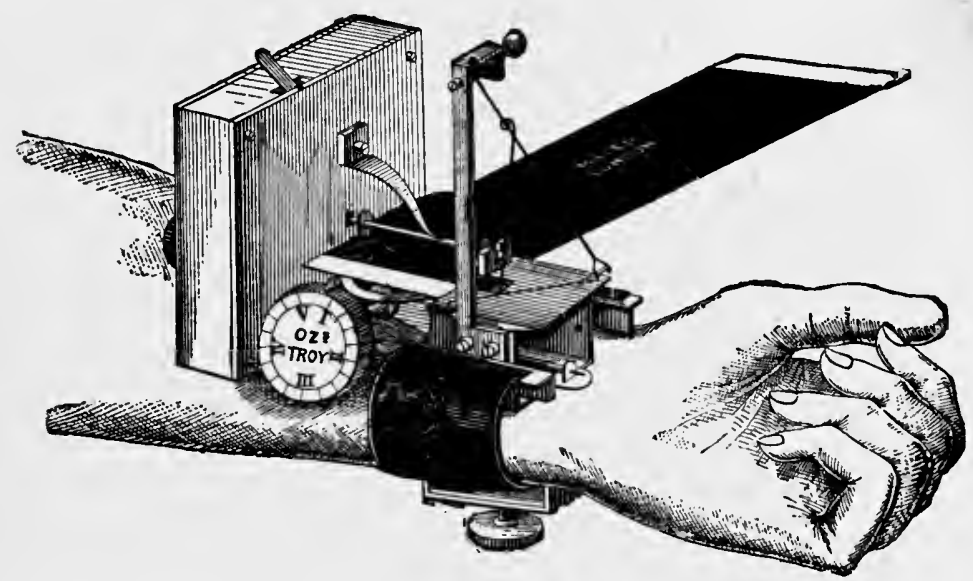

Fra. 14. Dudgeon's sphygmograph, applied to the radial artery.

various sphygmographs in use, Dudgeon's is perhaps the most serviceable (Fig. 14). Von Jacquet's sphygmograph is designed on the same plan as the Dudgeon. In applying the sphygmograph, it is important to see that the spring is placed precis ly over the artery.

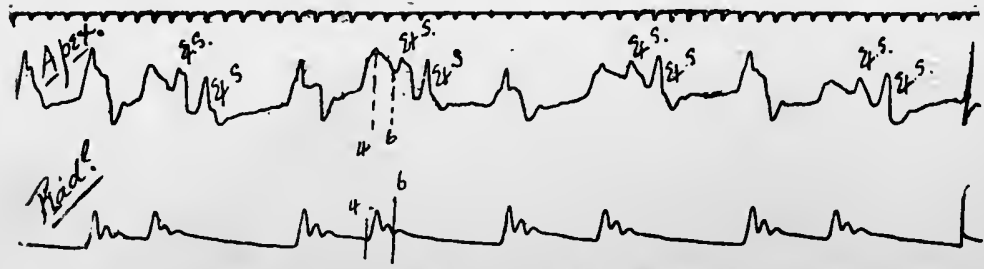

Fir. 15. Simultaneous tracings of the apex-beat and of the radial pulse, from a case with extra-systoles. The extra-systoles, eight in number. failed to send pulse-waves to the wrist.

Rate of the Pulse.-With regard to the pulse-rate, it should be noted that the rate of the radial pulse does 
not necessarily represent the ventricular rate, for not infrequently in irregular action of the heart many beats are so feeble that the pulse-waves do not reach the wrist (Fig. 15); the ventricular rate, therefore, should in these cases be counted at the apex, either by palpation or auscultation.

Volume of the Pulse. - By the volume of the pulse is meant the range of movement of the vessel wall which is to be noted during the passage of a pulse-wave; it depends upon the vigour of ventricular systole, and the state of the vessel wall, being larger when the latter is soft and yielding.

Character of the Pulse-wave. -Figures 16-19 represent normal sphygmograms, each of which is made un of the following constituent parts : an upstroke, more or less vertical; a rather sharp apex, quickly followed by a small wave, called he tidal or pre-dicrotic wave, which ends in the dicrotic or aortic notch; thi in

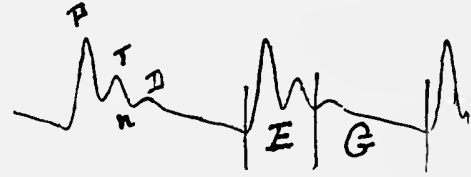

FiG. 16. A normal sphygmogram. $P$ is the percussion wave, $T$ the tidal or pre-dicrotic wave, $N$ the dicrotic notch, and $D$ the dicrotic wave. The space $E$ represents the systolic portion, and the space $G$ the diastolic. turn being succeeded by the dicrotic wave, after which
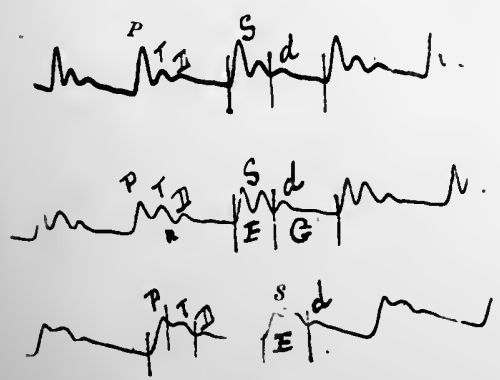

FIas. 17, 18, and 19. Normal sphygmograms.

finger. That part of the pulse-wave which extends from the commencement of the upstroke to the com-

the curve gradually falls, the fall being sometimes interrupted by slight secondary oscillations, called post-dicrotic waves, which are of no practical importance. The tidal wave is best felt with light, and the dicrotic wave with greater, pressure of the 
mencement of the tidal wave is called the primary or "percussion" wave. It corresponds to the expansion of the artery due to the increase of pressure resulting from ventricular systole. The height of the percussion wave depends upon the vigour of the ventricular systole,
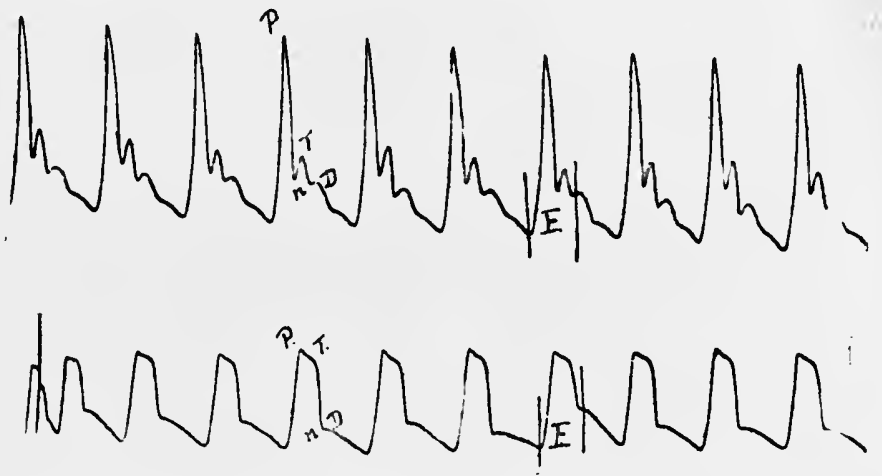

Figs. 20 and 21. Sphygmograms showing that the height of the percussion wave is largely instrumental. The lettering is the same as in Fig. 16. In Fig. 20, that part of the percussion wave which is above $T$ is instrumental. Fig. 21 was taken from the same case after preventing fling of the recording lever, by increasing the tension of the spring.

and the condition of the vessel wall, although at the same time it is largely affected by the instrument employed (Figs. 20 and 21); the height is also greater when the vessel wall is soft and yielding. How nearly the upstroke of the pulse-wave approaches the perpendicular is an indication of the quickness or abruptness of the ventricular systole. The upstroke is longer and more perpendicular than normal when the ventricle propels an unusually large volume of blood into the vessel, in the presence of relaxed arterioles and a subnormal blood-pressure; while the upstroke is shorter and less perpendicular than normal under conditions the opposite of these, and also in aortic stenosis. The dicrotic or aortic notch marks the end of the ventricular systole, and the point at which the aortic valves close. For graphic'purposes the arterial pulse may be divided into two portions : 
namely, the systolic portion, which commences with the beginning of the percussion wave and ends at the bottom of the dicrotic notch, and represcnts the time during which the ventricle is in systole and the aortic valves are open; and the diastolic, which commences at the bottom of the dicrotic notch and ends at the beginning of the next percussion wave, and represents the time during which the ventricle is in diastole and the aortic valves are closed (Figs. 16-19).

The dicrotic wave is the most constant of the oscillations on the downstroke. It is best marked in pulses of low tension, with soft and

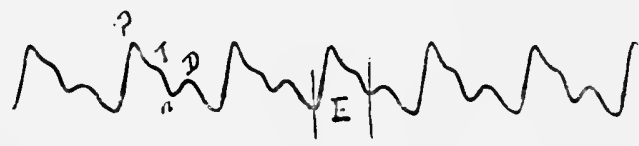

FIG. 22. Sphygmogram showing dicrotism.

yielding vessel-walls, provided there is no aortic regurgitation, and is small when the blood-pressure is high. Di-

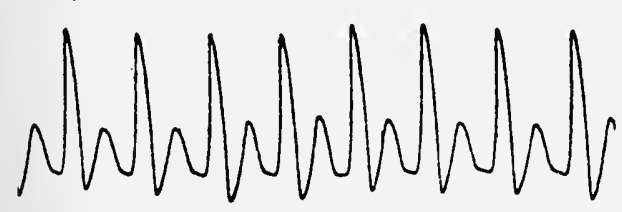

crotism is found especially in febrile states. When the wave is very pronounced, the pulse is called pulsus $d i$ -

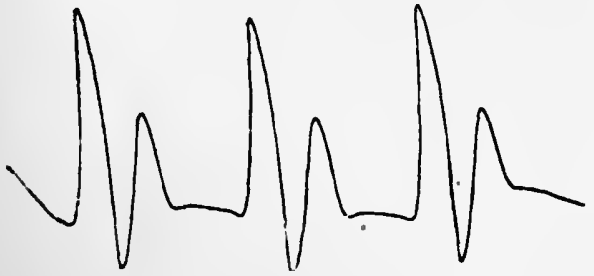
croticus (Fig. 22). It may be so pronounced as to be perceptible to the finger, two bcats being felt instead of

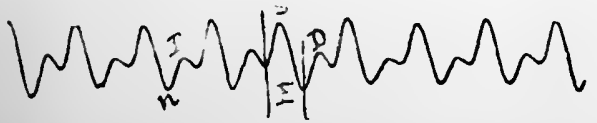

FIGs. 23, 24, and 25. Sphygmograms showing hyper-dicrotism. Fig. 25 was taken from a case of tachycardia; the dicrotic wave is caught by the percussion wave.

base line, the pulse is called hyper-dicrotic (Figs. 23, 24, and 25). 
The tidal or predicrotic wave is best marked in certain pulses of high tension and in aortic regurgitation, while in pulses of very low pressure it may be absent. In certain pulses of high pressure, the tidal wave may be more

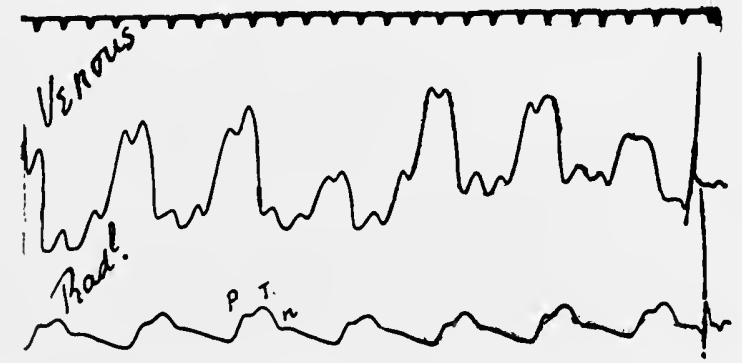

Fic. 26. Anacrotic pulso, from a case of aortic stenosis.

forcible than the percussion wave, and as a result the latter part appears merely as an indentation in a slow slanting upstroke, the apex of which is formed by the tidal wave; this is called an anacrotic pulse (Figs. 26 and 149). Or, the tidal wave may be felt separately from the primary

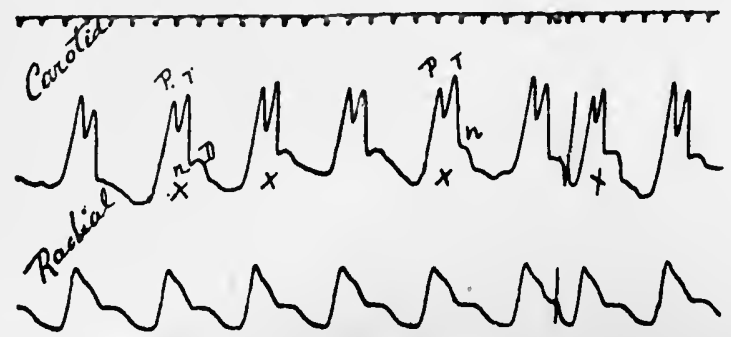

FIG. 27. Pulsus bisferiens. The points marked $X$ show the true outline; that is, the tidal wave is higher than the percussion wave. Note that the sphygmogram of the radial artery is normal.

impact-that is to say, the summit is bifurcate, due to the predicrotic fall being followed by a rise equal to or above that of the percusion wave; this is called pulsus bisferiens (Figs. 27, 150 and 151). Both the anacrotic pulse and pulsus bisferiens are found more especially in aortic stenosis. The former, while characteristic of aortic stenosis, 
is not pathognomonic, as it may occur in other circumstances; it may be the result, for example, of a persistently high blood-pressure. It is of more diagnostic value in aortic stenosis than is the pulsus bisferiens.

When the beat is felt to be quick, sharp, and abrupt, and the collapse sudden, the pulse is called pulsus celer (Fig. 28); this is

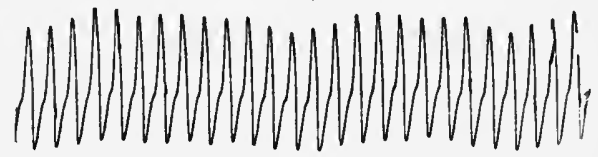
met with, as a rule, Fí. 28. Pulsus celer, from a case of tachyin low blood-prescardia with very low blood-pressure.

sure, and is apt to occur in febrile states. A special form of this pulse, which is found in cases of aortic incompetence, is the "collapsing," the "water-hammer," or Corrigan's pulse (Figs. 142, 143 and 144); it will be described in Chapter XX. A pulse presenting the opposite

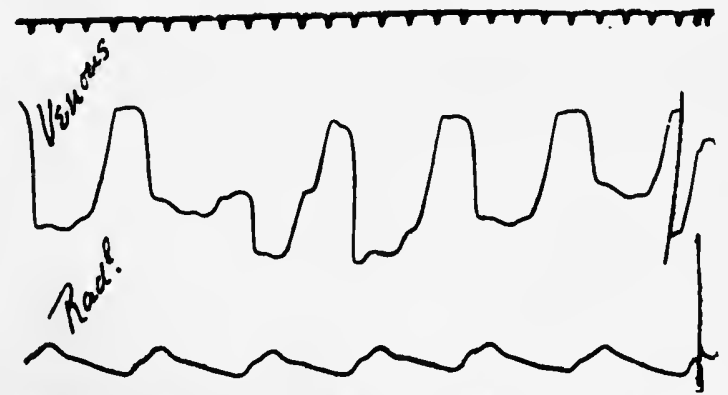

FIG. 29. Simultaneous tracings of the jugular and radial pulses. Sphygmogram shows the pulsus tardus.

features to those of pulsus celer is called pulsus tardus (Fig. 29); this is almost invariably associated with supernormal blood-pressure, being apt to occur in aortic stenosis and arterio-sclerosis, but it may also be found in aneurism.

In pulses of normal blood-pressure, the wave due to the systole of the ventricle is well maintained, and the fall during diastole is gradual, with a dicrotic notch more or less well marked. A pulse of low blood-pressure exhibits a well-developed dicrotic wave, the aortic notch approaches the base line, and the tidal wave is small or absent (Fig. 
22). A pulse of high blood-pressure exhibits a moderate upstroke, a blunted apex, a small dicrotic wave, a welldeveloped tidal wave (Fig. 30), and it may rarely be

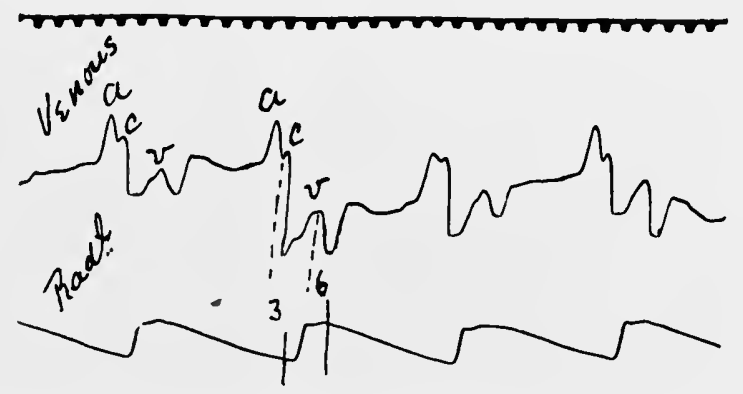

FIG. 30. Simultaneous tracings of the jugular and radial pulses. Sphygmogram shows a pulse of high blood-pressure. The position of the dicrotic notch is shown by the downstroke 6 .

either an anacrotic pulse or pulsus bisferiens. It should be remembcred, however, that besides arterial pressure many factors, such as the rate of the pulse, the condition of the aortic valve and arterial wall, and instrumental defects, affect sphygmographic tracings, so that we should excrcise considcrable caution i l making in-

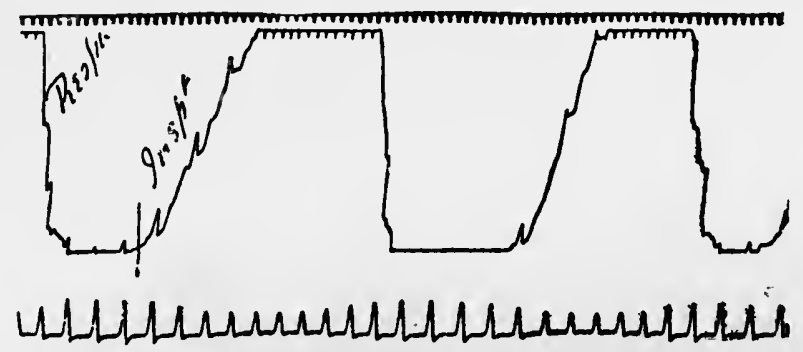

FIa. 31. The pulsus paradoxus.

ferences with regard to the blood-pressure from tracings alone.

When the pulse-waves, instead of increasing in size, which they normally do during each inspiration, become smaller, the pulse is called pulsus paradoxus (Fig. 31); 
this is met with more especially in chronic adhesive pericarditis in which extra-pericardial adhesions exist, but it has also been observed in pericardial effusion and in other morbid conditions, and even in normal people.

The characteristic features of the pulse which may be found in the various forms of heart disease will be discussed under their appropriate headings.

Rhythm of the Pulse.-The pulse may be regular in time-by which is meant that the intervals between the beats arc of equal size-or volume, or both; or it may be irregular in either or both. The subject of cardiac irregularity will be discussed in Chapter X. It may, however, be advisable to describe here what is meant by the terms " intermittent" pulse or a "dropped beat," "pulsus bigeminus," "pulsus trigeminus," and " coupling of the beats."

Intermission of the pulse is a form of irregularity which is not uncommon. By it is meant a condition in which the normal rhythm of the pulse is interrupted, either occasionally or more frequently, either at regular or irregular intervals, by an abnormally long pause during which no wave is felt at the wrist, a beat being missed (Fig. 32). It is of great importance to understand how intermission of the pulse is caused, especially from the point

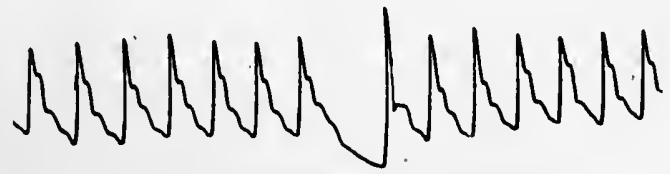

FIQ. 32. Intermission of the pulse.

of view of prognosis. By far the commonest cause is a premature contraction of the ventricle (" extra-systole") which is so feeble that the wave fails to reach the wrist (Fig. 33); the next most common cause is partial heartblock (Fig. 34), while the condition is occasionally due to sinus irregularity, and very rarely to sino-auricular block.

The differential diagnosis between extra-systole and 
partial heart-block is fully discussed in Chapter XII. It is sufficient to note here that a correct diagnosis can as a rule be readily made solely by the aid of palpation and auscultation. When extra-systole is responsible for an intermission of

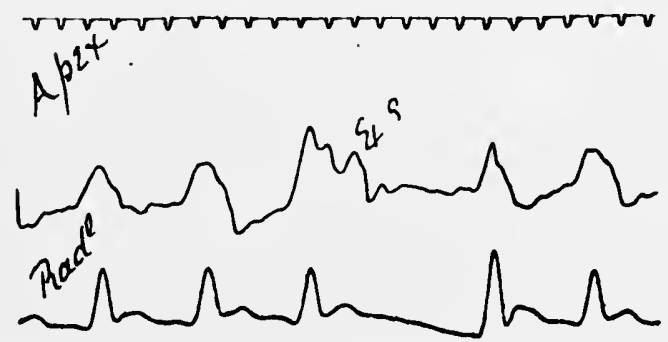

FiG. 33. Simultaneous tracings of the apex-beat and of the radial pulse, showing intermission of the pulse, due to a ventricular extra-systole failing to send a wave to the wrist.

the pulse, a premature beat can be detected at the cardiac apex, and, on auscultation, one or two sounds will, in the great majority of cases, be audible during the early part of the pause, because the ventricle has contracted. In

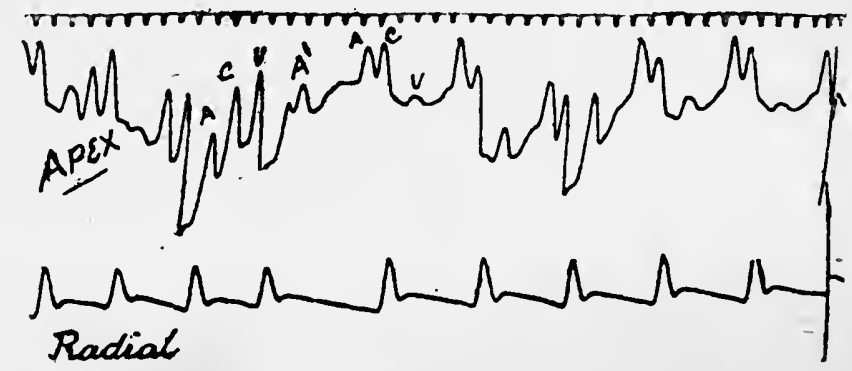

FIG. 34. Simultaneous tracings of the apex-beat and of the radial artery, showing intermission of the pulse, due to partial heart-block, the ventricle failing to respond to the stimulus of the auricle at $A^{\prime}$.

partial heart-block, on the other hand, there will be an absence of an apex-thrust and of heart sounds during the pause, because the ventricle has failed to contract. 'The differential diagnosis also between occasional dropped beats due on the one hand to sinus irregularity and on 
the other to partial heart-block is discussed in Chapter XIII.

The term pulsus bigeminus should, in my opinion, be restricted to that condition in which the pulse-beats

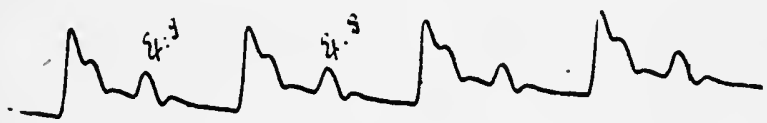

Fig. 35. Pulsus bigeminus, due to a single extra-systole with its succeeding compensatory pause occurring regularly after each normal beat.

occur in pairs, and in which the paired beats fall at regular intervals. It should, however, be noted that some writcrs include under this term the coupling of the beats which
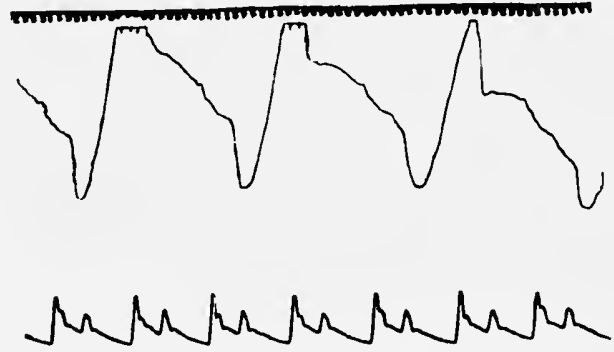

FiG. 36. Shows the same as Fig. 35 .

is sometimes to be found in auricular fibrillation, in which the paired beats occur at irregular intervals. Using the term in the more restricted sense, the commonest cause is

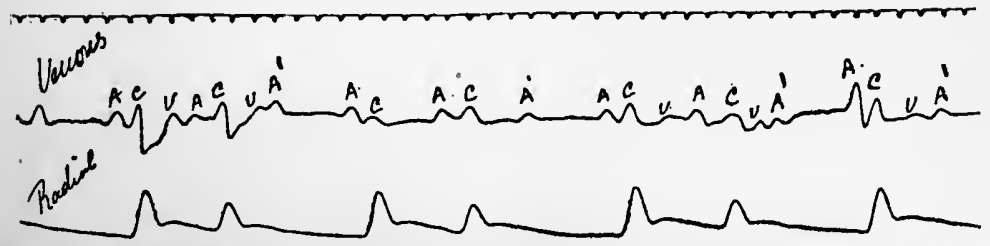

Fra. 37. Pulsus bigeminus, due to partial heart-block, the ventricle failing to respond to the stimulus from the auricle at $A^{\prime}$.

extra-systole; either a single extra-systole with its succeeding compensatory pause occurring regularly after each normal beat (Figs. 35 and 36 ), or such occurring 
after every two normal beats, the premature contraction in the latter case, however, being so feeble that no wave reaches the wrist. The next most common cause

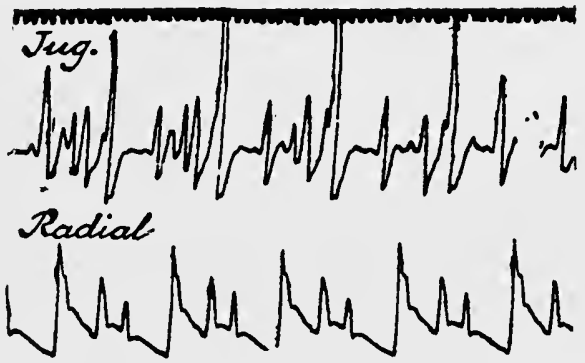

FIG. 38. Simultaneous tracings of the jugular and radial pulses, showing pulsus trigeminus, due to a single extra-systole with its succeeding compensatory pause occurring after every two normal beats.

is partial heart-block, when the ventricle fails to respond to every third bcat of the aur cle (Fig. 37). A rare cause is sino-auricular block, in which the auricle fails to respond to every third impulse generated at the sinus.

By pulsus trig, minus is meant a condition in which the pulse-beats are grouped in threes. The causes of this condition are similar to those of pulsus bigeminus (Figs.

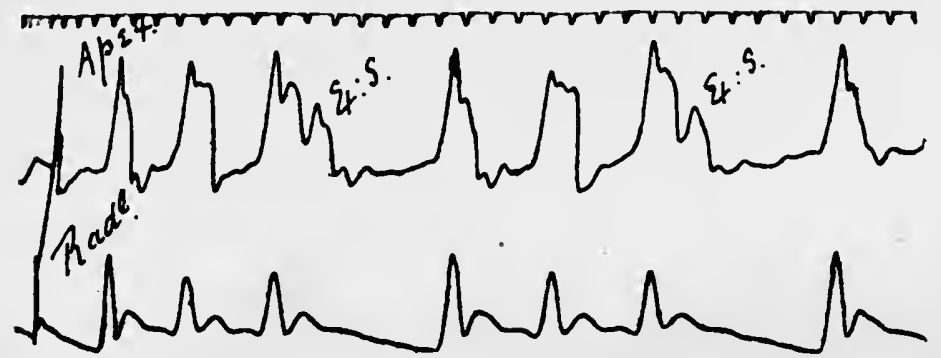

FIG. 39. Simultaneous tracings of the apex-beat and of the radial pulse, showing pulsus trigeminus, due to a single extra-systole with its succeeding compensatory pause occurring regularly after every three normal beats and failing to send a wave to the wrist.

38 and 39). As pulsus bigeminus and pulsus trigeminus are met with in conditions other than extra-systole, they are not pathognomonic of the latter condition. The 
distinguishing features between pulsus bigeminus or pulsus trigeminus due on the one hand to extra-systole and to partial heart-block on the other hand may be established, with a view to differential diagnosis, in the same way as in the case of an intermittent pulse.

In my opinion the term coupling of the beats should be restricted to that condition in which the pulse-beats occur regularly in pairs, for long or short periods, and in which the paired beats fall at irregular intervals. This is sometimes met with in auricular fibrillation, and may occur independently of the action of digitalis (Fig. 116), although it is usually due to it (Fig. 117).

Condition of the Vessel-wall.-Let us now consider the condition of the vessel-wall as revealed by digital examination. In health, this is soft and yielding, but, when degeneration is present, it may show general thickening, or irregular patches of induration, or, if calcification exist, may even resemble a rigid tube. Examination of the brachial artery ought not to be neglected in any case of suspected arterio-sclerosis. The elbow should be extended, when the artery, if sclerosed, can be felt to be thickened, and may be rolled under the finger against the bone.

The Arterial Blood-pressure. - The maximum bloodpressure may be estimated by the force which is necessary to obliterate the pulse at the wrist when the fingers are placed upon the radial artery; if the pulse be easily obliterated, it is spoken of as "soft," while if the contrary be the case it is referred to as a "hard" pulse. The minimum blood-pressure may be estimated by rolling the vessel from side to side under the finger between the beats. If the minimum blood-pressure be low, the vesselwall is not palpable at all; while, if it be high, it may be felt to be even as hard as a piece of whipcord. We must be on our guard, however, to avoid confusing the latter condition with thickening of the vessel-wall. Another method of estimating the minimum blood-pressure 
is to palpate the artery first with light, and then with increasing, pressure-a pulse of low minimum pressure being more easily felt with light palpation, while, in the case of a pulse with a high minimum pressure, the more firmly the vessel is compressed the stronger does the

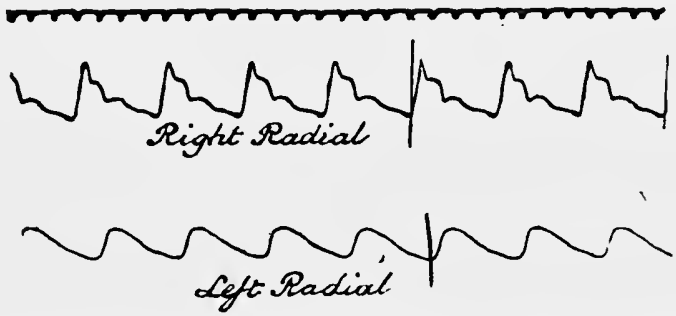

FIG. 40. Simultaneous tracings of the right and left radial pulses, from a case of aneurism of the thoracic aorta, showing a marked difference in the character of the two pulses. The seeming delay in the left radial is seen to be due to the slow rise of the wave in the left artery compared with the abrupt rise in the right (Mackenzie).

pulse apparently become. The characteristic features of the sphygmograms of pulses of normal, low, and high blood-pressure have already been described.

Symmetry of the two Pulses.-A difference in the two radial pulses may sometimes be found in aneurism of the thoracic aorta, but it should be remembered that this also occurs in abnormalities of the radial artery itself. In aneurism, the pulse on one side of the body may exhibit a smaller amplitude, a more gradual upstroke, and a less pointed apex (Fig. 40). 


\section{CHAPTER IV}

\section{BLOOD-PRESSURE}

By the blood-pressure is meant the pressure that the blood exerts against the wall of the vessel in which it is contained. The term, therefore, includes endocardial pressure, arterial pressure, capillary pressure, and venous blood-pressurc. We are here concerned only with arterial blood-pressure. This depends upon (1) the force of the contraction of the left ventricle; (2) the volume of blood which the left ventricle propels into the already full arteries; (3) the elasticity of the middle coats of the large arteries ; and (4) the peripheral resistance.

An increase in the output of the heart causes a rise of blood-pressure, provided the size of the arterioles remains unchanged; but increase of the cardiac rate alone does not raise the blood-pressure. Fluctuations in the volume of blood do not constitute an important factor, as they are compensated for by the vaso-motor system; the value of venesection in super-normal blood-pressure is, therefore, much smaller than would naturally be supposed, for the resultant fall of arterial pressure is quickly restored, as the blood-vessels contract and accommodate themselves to the altered volume of blood within them, fluid being also withdrawn from the tissues. The yellow elastic tissue which constitutes a portion of the middlc coat of the large arteries undergoes expansion at each systole, and recoils during each diastole. This recoil converts an intermittent into a continuous force, and results in a continuous outflow of blood into the vessels; in this way the elasticity of the vessel-wall results in 
equalisation of the pressure during systole and diastole; loss of this elasticity resulting in greater fluctuation in blood-pressure. The elasticity of the vessel-wall is greatest at about the normal pressure, diminishing with an increase of pressure. The peripheral resistance is chiefly the result of contraction of the muscular coats of the small artcries, these vessels being under the control of the ccntral nervous system. The muscularity of the arterioles is a very important factor in the regulation of the arterial blood-pressure, and in this connection it may be noted that the splanchnic area exerts the greatest influcnce on the gencral blood-pressure. The vaso-motor system regulates both the general blood-pressure and the local blood-supply. These functions are carried on by (1) the vaso-motor centrc, situated in the floor of the fourth ventricle, with probably subsidiary centres in the grey matter of the spinal cord; (2) efferent nerves, which are able to induce constriction or dilatation of the vessels; and (3) afferent nerves, which convey impulses to the vaso-motor centre, and call for a general rise or fall of blood-pressure. It is probable that the calibre of the artcrioles may be influenced by chemical stimuli, and it is now commonly believed that in the first stage of super-normal blood-pressure structural changes in the vessel-wall are not necessarily present. The regulation of the diastolic pressure is mainly due to peripheral resistance.

The arterial pressure synchronous with the systole of the ventricle is called the systolic blood-pressure, that synchronous with the diastole is called the diastolic bloodpressure, while the difference between the two is called the pulse-pressure. The mean arterial pressure is the mathematical mean between the systolic and diastolic pressures.

There are three methods of obtaining information regarding the arterial blood-pressure in man-namely, (1) by means of the trained finger ; (2) by means of sphygmo- 
graphic tracings; and (3) by means of special instruments, termed sphygmomanometers. The first two have already been dealt with in Chapter III.

The Sphygmomanometer Method.-The introduction of the sphygmomanometer has greatly stimulated interest in the subject of blood-pressure during the last few years, and has proved of great value in clinical medicine. The results are more reliable, and, indeed, not infrequently the practitioner will find cases of super-normal bloodpressure which might otherwise have been overlooked; further, the results can be expressed in terms of mathematical formulæ, which can be kept for reference, and in this way the practitioner is enabled to compare the records while patients are undergoing treatment with the aim of reducing tension. A word of warning, however, is necessary: it should be remembered that the sphygmomanometer may exhibit fallacies and possesses limitations, and there is undoubtedly a danger of overestimating its value in elinical work, when we rely upon its readings alone as an indication of the patient's condition. Bloodpressure measurements should be considered in conjunction with other elinical data; in other words, hard-andfast conclusions should not be drawn from blood-pressure readings alone. The normal blood-pressure in all probability has a wider range of variation in different individuals than is generally supposed, and, if this be so, great caution should be exercised in arriving at the conclusion that a blood-pressure outside what is generally supposed to be the normal limits is necessarily indicative of a diseased state. At the same time it should be remembered that the detection of super-normal blood-pressure in its earliest stages is of great practical importance, because it is in these stages that therapeutic measures may be successful. In considering the question whether a rather high pressure reading is normal to the individual, or is indicative of a pathological departure, it is important that enquiries should be made with regard to three points-namely, 
(1) what was the patient's usual blood-pressure previously ; (2) are there any symptoms other than a supposed abnormal blood-pressure, such as headache, dyspnœa, or other indications of cardiac failure, and what is the condition of the heart, arteries, and kidneys; and (3) what is the result of treatment; if there be a fall in the pressure readings, together with a coincident improvement in the general health of the individual, during treatment, it may be inferred that the higher pressure reading is pathological.

In some prolonged and detailed sphygmomanometer observations which I conducted on different patients at the Mount Vernon Hospital, I found that in not a very small percentage of cases considerable fluctuations in the systolic blood-pressure occurred from day to day, in one case amounting to $26 \mathrm{~mm}$. of mercury. I am not now referring to the variations associated with meals, physical exertion, or mental states, but to diurnal variations which appeared to be quite independent of these. Now, if it be proved that fluctuations occur in normal individuals from day to day, we should be very careful in coming to conclusions with regard to the action of drugs on the blood-pressure in man; further, it ought not to be forgotten that in disease any changes observed during or after the administration of a drug may be due to the natural course of the malady. As a rule, however, apart from variations associated with physiological conditions, such as meals, change of posture, physical exertion, sleep, and sensory stimulation, the diurnal pressure readings are practically constant.

In estimating the blood-pressure by means of the sphygmomanometer, the patient should be in the recumbent posture, absolutely quiescent, with the elbow in a state of flexion, and the muscles of the upper arm completely relaxed. It is advisable that the observer should wait some little time after adjusting the armlet, and also inform the patient what is about to be done 
before taking a reading, in order that he may not be taken by surprise, as mental perturbation may result in a temporary rise of blood-pressure. It is important that the daily observations of the same individual should be taken at the same hour, and under similar conditions, such as the question of posture, and the time-relationship to food and exercise. It is generally better to take the measurements an hour before a meal, and not less than an hour after exercise. It is advisable to take the average of several readings at the same sitting, the consecutive readings having sufficient intervals. I am accustomed to confine my observations to cases with a regular heart action, or with only an occasional irregularity - that is, where there are periods of regularity of sufficient length during which to take measurements; in cases in which there is a constant variation in the strength of the pulsebeats, it is impossible to obtain a reading which accurately represents the condition.

Among the more reliable sphygmomanometers are Martin's modification of the Riva-Rocci, Hill and Barnard's, Oliver's compressed-air hæmomanometer, and Pachon's sphygmometric oscillometer. Probably the most accurate instruments are Erlanger's, and G. A. Gibson's sphygmomanometers, by means of which records of both maximum and minimum pressures may be obtained; they are, however, only suitable for hospital practice, on account of their cost and size. In my own experience the best. single instrument for ordinary practice is Martin's modification of the Riva-Rocci (Fig. 41), in connection with which there is now an unspillable mercurial manometer.

An armlet, not less than $20 \mathrm{~cm}$. wide, containing an air-bag, is fixed round the upper arm. An india-rubber bulb, provided with a screw-valve, is connected to the armlet on the one hand, and to a mercurial manometer on the other, by means of rubber-tubing. The mercurial manometer indicates the amount of pressure within the air-bag encircling the arm, this being read in millimetres 
by the difference in the height of the two columns of mercury. An aneroid may be substituted for the mercurial manometer, and is more convenient for consultations away from home, on account of its smaller size; but it needs correction from time to time by comparison with a mercurial manometer.

With the screw-valve closed, the air-bag in the armlet

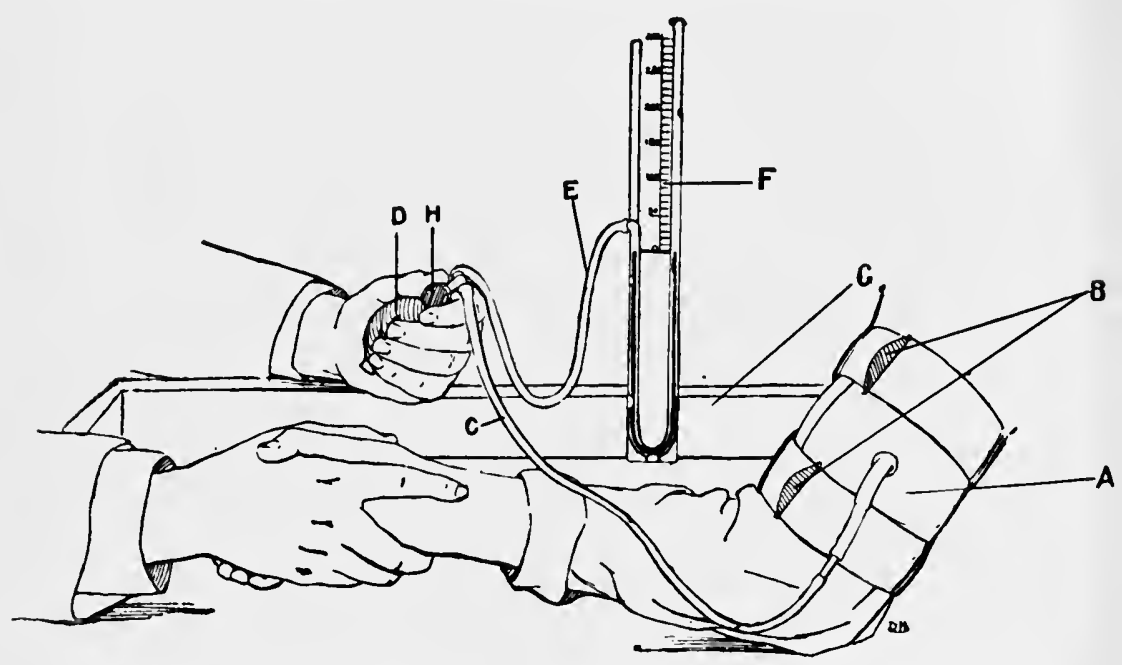

FIa. 41. Martin's modification of the Riva-Rocci sphygmomanometer. $A$, armlet ; $B$, straps of armlet ; $C$, rubbər-tubing leading to armlet; $D$, india-rubbor bulb; $E$, rubber-tubing leading to mercurial manomoter; $F$, morcurial manometer; $G$, box; $H$, screw-valve.

is inflated by compressing the india-rubber bulb, while a finger of the other hand is applied to the patient's radial pulse; the inflation is continued until the pressure in the air-bag is sufficient to cause the disappearance of the radial pulse. This-the obliteration pressure-is generally believed to correspond with the systolic arterial pressure. Two methods may be employed: (1) the pressure within the bag is gradually raised until the radial pulse disappears, at which point a reading on the manometer or in the aneroid is made; and (2) the pressure 
within the bag is raised until it is from 10 to $12 \mathrm{~mm}$. above the point at which the pulse is no longer palpable, and it is then gradually reduced by unscrewing the screw of the bulb, and a reading is made at the point at which the radial pulse-beats first reappear. The latter method gives a slightly lower reading, and most observers accept it as the more accurate.

Until recently my practice has been to estimate the systolic pressure only, as I had some doubt as to the value of the readings obtained of the diastolic pressure. Recent work, especially that of MacWilliam and Spencer Melvin, has, however, modified my opinion. As the pressure within the bag is increased, oscillations of the mercury in the manometer connected with the air-bag may be detected; these gradually increase in magnitude with the rising pressure, until a point is reached at which they rapidly increase and reach a maximum; as the pressure is still further raised the oscillations diminish in size, at first slowly, until at the obliteration pressure they either do so rapidly - and, if so, small oscillations are still apparent above the obliterative pressure-or disappear altogether. A reading of the lowest level at the period of maximum oscillation should be taken; the pressure within the airbag is then raised until the oscillations are diminished, and then lowered until the maximum oscillations reappear, when a second reading is taken. The mean between these two readings is then estimated. It is believed by some that this (or, on palpation of the radial artery and gradually reducing the pressure in the air-bag, when the maximum wave is felt) represents the diastolic pressure ; others arc of opinion that the maximum oscillations represent the mean pressure.

A more accurate method of determining both the systolic and the diastolic pressures, more particularly the latter, is Korotkoff's ausculatory method, first introduced in 1905, instead of the use of the finger. The bell of a binaural stethoscope, the diameter of which should not 
be more than $25 \mathrm{~mm}$., is lightly placed over the brachial artery at the bend of the elbow, from 1 to $2 \mathrm{~cm}$. below the band encircling the upper arm. The pressure within the air-bag is then steadily raised until it is from 10 to $12 \mathrm{~mm}$. above the point at which the pulse is no longer palpable, at which point no sound is audible over the vessel. The air in the bag is then allowed to cscape gradually, and at the point at which the lessened pressure in the bag allows blood to pass at each cardiac systole a sound rescmbling a thud or click becomes audible. This is indicative of the systolic pressure, and its commencement is usually 5-10 $\mathrm{mm}$. above the point at which the reappearance of the pulse is felt at the radial artery. If the air in the bag be further allowed to escape, the sound becomes louder and may somewhat resemble a murmur. Later, it becomes less loud, until a point is reached at which the sound becomes rather suddenly fainter and its character changes from a clear to a duller sound; in the opinion of some, including MacWilliam and Melvin, this change in the degree of loudness and character is the index of the diastolic pressure. Still later, the sound may quickly cease, or it may continue even up to a further lowering of the pressure in the bag of $30 \mathrm{~mm}$. In the opinion of others, the disappearance of the sound represents the diastolic pressure.

Some authorities hold that the sphygmomanometer reading is a composite one of the blood-pressure plus the resistance of the arterial wall, and, that being so, that the armlet reading may be affected by thickening and calcification of the arterial wall, and, therefore, these conditions should be taken into consideration; they believe that the reading when determined by obliteration of the pulsation beyond the armlet may be too high as representing the systolic blood-pressure, because the arterial wall does not readily collapse. In the opinion of others, however, the elasticity of the vessel-wall is of small importance, so long as a wide armlet is employed. 
The following may be regarded as the normal standard of systolic blood-pressure, as measured in the brachial artery, while at rest: In children under ten, $100 \mathrm{~mm}$.; in early manhood, from 100 to $120 \mathrm{~mm}$.; in middle life, from 125 to $135 \mathrm{~mm}$.; above sixty years of age, 145 to $150 \mathrm{~mm}$. It has been suggested that if the figure 1 is prefixed to the age of individuals of 15 years or upwards the result will give the normal systolic blood-pressure; the reading in the case of a man of 50 years, for example, would be $150 \mathrm{~mm}$. This is sufficiently accurate to be of value clinically, though it should be noted that the figure is rather too high in the case of older people. In the female, the systolic pressure is generally from 10 to 20 $\mathrm{mm}$. lower than in the male. MacWilliam and Melvin found that the range of diastolic pressure, as estimated by the auscultatory method, in healthy young adults (examined in the sitting position) was $50-80 \mathrm{~mm}$., the average being 65 ; these readings are considerably lower than those usually accepted.

\section{Variations in Blood-Pressure under Physiological Conditions}

There are variations in the blood-pressure under physiological conditions, among these being mental states, sensory stimulation, sleep, change of posture, meals, and physical exertion.

Those of nervous, anxious temperament, as well as those whose mode of life necessitates considerable nervous strain, have a tendency to exhibit higher readings, especially in the later period of life; while in those of an equable and placid temperament, and those whose lives are less harassed, the opposite is found.

Sensory stimulation causes a rise in the arterial pressure, especially in the diastolic. The effect of pain varies in different individuals; in some it results in a rise of pressure, in others it results in a fall, while in others again there is no effect produced. If an individual 
unaccustomed to the use of tobacco smoke a cigar, the arterial pressure first becomes raised $10-25 \mathrm{~mm}$., but falls 30 or even $50 \mathrm{~mm}$. in from fifteen minutes to half-anhour; if the individual, on the other hand, be a smoker, there is only a very slight rise in pressure. In sleep there is a fall in the minimum blood-pressure, and probably a slight fall in the maximum pressure also.

Opinions as to the effects of the digestion upon the systemic blood-pressure vary, some holding that there is a constant rise, others that there is a constant fall, while others again are of opinion that there is no alteration. In all probability, the maximum and the pulse-pressures are increased, and the minimum pressure may be also to a less extent. It is probable that the change in pressure commences within a quarter-of-an-hour after a meal, the highest reading being increascd in about an hour, after which it gradually returns to the normal, which is reached in about two hours.

During exercise the maximum and minimum pressures of the systemic circulation are raised, and the pulse-rate is also increased. Immediately after exercise, the arterial pressure may be super-normal, normal, or even subnormal ; in the first case, a lowering quickly supervenes, that of the diastolic pressure usually occurring before the systolic. The arterial pressure generally returns to the normal in about half-an-hour; it is, however, usually better to postpone taking a measurement for at least one hour after exercise.

Variations in Blood-pressure under Pathological Conditions

\section{Subnormal Arterial Pressure (Hypotension)}

The chief factors in the causation of subnormal arterial pressure are, (1) a diminution of the ventricular output, and (2) lowering of the vaso-motor tone; splanchnic stasis being usually associated with the latter. It should 
be noted that a subnormal blood-pressure is more often an indication of derangement of the vaso-motor centre than of cardiac impairment.

Etiology.-Subnormal blood-pressure may be met with in acute cardiac disease which is not accompanied by a pronounced degree of cyanosis, in some cases of chronic valvular disease, in Graves' disease, Addison's disease, shock, collapse, after extensive bleeding, after severe vomiting, in diarrhœa, dysentery, cholera, chronic wasting diseases, acute infectious diseases-particularly enteric fever, influenza, diphtheria, and pneumonia-in gastric disorders, cerebral thrombosis, neurasthenia, the excessive usc of tobacco, acute mania, certain cases of sleeplessness, in some cases in which there is an unduly slow pulse, and after prolonged rest in the recumbent posture.

Treatment.-The following therapeutic measures are recommended by various writers. In acute cases, rest in bed may be advisable; after the acute stage has passed, great care should be taken to avoid over-cxertion, and gentle and graduated excrcise, with perhaps massage in certain cases, and an adequate amount of rest after exercise and meals, are indicated. The clothing should be light, and heavy woollen underclothing is better avoided. A tepid or cold sponge bath in the morning, followed by suitable exercises, and residence in a bracing climate are to be commended. The proportion of those articles of food which especially stimulate the cardio-vascular system, such as meat and meat-extractives, should be increased; roasted meat being of greater value than meat which has been boiled, while meat soups and gravies should also be given. Cod-liver oil, malt, glycerophosphates, phosphorus, iron, and arsenic may be tried. Digitalis, and to a less extent strophanthus, have gained a reputation in the treatment of subnormal blood-pressure, but, as will be noted later, I found no evidence in favour of the view that digitalis increases the blood-pressure in man. Strychnine also has a considerable reputation in the treat- 
ment of this condition, especially when present in diphtheria or other infectious febrile conditions. Pituitary extract is belicved to increase the blood-pressure. It may be given in tabloid form, each containing two grains, one of which may be administered four times daily, the dose being increased in suitable cases. The drug may also be used in the form of "vaporoles" for intra-muscular injection, 1 c.c. being given twice daily. In either case, and especially the latter, the effects of this drug should be carefully watched. Adrenalin, eserine, and ergot are among other drugs which may be used in the treatment of this condition; and the administration of formic acid and watery extract of muscle has recently been introduced.

\section{Super-normal Arterial Pressure (Hypertension)}

In pathological hypertension the blood-pressure is persistently above the normal of the individual; temporary super-normal blood-pressure is found, for example, in the nervous excitement incident to medical examination. The importance of the detection of super-normal bloodpressure in its earliest stages from the therapeutic standpoint has been already noted.

Etiology. - The condition may be met with in individuals who habitually indulge in over-eating, in the various forms of chronic rheumatism and gout, lead-poisoning, diabetes, nephritis - especially the chronic interstitial form-uræmia, intra-cranial hæmorrhage, arterio-sclerosis, hypertrophy of the left ventricle from other causes, during the paroxysms of angina pectoris, in other painful affections-such as neuralgia and the lightning pains of locomotor ataxyin the attacks of idiopathic epilepsy and bronchial asthma, in migraine, meningitis, melancholia, some cases of sleeplessness, and in the early stages of some acute diseases.

The readings of the systolic blood-pressure in chronic interstitial nephritis are among the highest found; 
although it should be noted that increased arterial pressure is not present in all cases of this disease, as, for example, when the left ventricle is failing. A marked increase in the arterial pressure is not so common in the chronic parenchymatous form of nephritis, while it is only moderately high in acute nephritis. Some of the highest readings are to be found in intra-eranial hæmorrhage. In the nodular form of atheroma the arterial pressure may be raised only slightly, if at all. In the diffuse or generalised form, however, there is usually a considerable rise in the arterial pressure, although the accessible arteries, such as the temporal or radial, may not be unduly palpable; the systolie reading may reach even $250 \mathrm{~mm}$. or more. In most cases of aneurism the arterial pressure is either normal or only slightly above the normal : it is often super-normal in the cyanosis of cardiac failure, and in the dyspnœa of emphysema and heart disease. The systolic pressure is usually inereased in aortic incompetenee, while the diastolic is lowered; the pulse-pressure is as a rule not less than $60-70 \mathrm{~mm}$., and is not infrequently as high as $80-90 \mathrm{~mm}$.

Symptomatology.-There may be a complete absence of subjective symptoms for some years; the patient, however, is apt to suffer from headache, giddiness, tinnitus aurium, insomnia, and palpitation. There is evidence of hightension pulse, and it may be thickening of the vessel-wall, hypertrophy of the left ventricle, reduplication of the first sound over the septum venticulorum, and accentuation of the aortic seeond sound. There may also be a certain degree of cardiac dilatation. Renal symptoms may be absent for years, or the patient may sufter from frequent micturition at night, with transient slight albuminuria, while the presenee of hyaline casts in the urine may oceasionally be noted. Later on, the history may show great diversity. In some cases, coronary disease, with its consequent results, may develop; in others, cardiac dilatation may follow hypertrophy, and the clinical pieture 
resembles that of chronic cardiac failure occurring in valvular disease, a mitral systolic murmur being present; in others again, uræmia may develop; while cerebral symptoms such as transient hemiplegia, monoplegia, or aphasia, vertigo, Adams-Stokes' syndrome, and cerebral hæmorrhage; bronchitis and emphysema ; and gangrene of the extremities, from endarteritis or embolism, may also follow this condition.

Treatment.-It is important first of all to review the etiology of the condition. I would especially urge the importance of attending to the condition of the tecth and gastro-intestinal canal, particular cnquiry as to the prescnce of pyorrhœea alvcolaris and chronic intestinal autointoxication being instituted. Another point which should be noted at the outset is that much harm may be done, especially in the case of nervous subjects, by letting the patient know that his arterial pressure is above the normal, and especially by informing him as to the reading on each occasion. On the other hand, it is of importance to allay the fears of these patients, as far as possible.

Treatment is mainly prophylactic, hygienic, and dietetic, the actual spccific treatment being of far less value. In a series of investigations which $I$ conducted at the Mount Vernon Hospital I was impressed with the futility of drugs to lower permanently super-normal blood-pressure in the great majority of cases, and my custom now is to place greater reliance on forms of treatment other than by drugs, among the most important being rest, exercise, diet, and free elimination.

The value of rest, both physical and mental, in the management of high blood-pressure is great, and is not sufficiently appreciated. 'It is sometimes necessary to keep the patient in bed, the period varying according to the nature of the case. Rest, especially when combined with hepatic stimulation and suitable purgatives, may be sufficient. Exercise, -provided it be moderate and regulated, also ranks high as a therapeutic measure, and this 
applies with suitable discrimination to any period of life. A cardinal rule in all cases should be that exercise should not be carried to the point of inducing increased frequency of the pulse or respiration, or indications of cardiac distress. It is much better to abstain from exercise soon after a meal, when rest should be cnjoined for a period of half-an-hour to an hour. Walking is an excellent form of exercise; quiet cycling, golf, and horse-riding are useful in some cases; while special forms of exercises, such as the Swedish form, and slow brcathing exercises, are not infrequently of value. Sudden exertion, hurry, straining at stool, and mental strain of every kind should be carefully avoided.

Some writers lay great emphasis upon the necessity for drastic regulation of the diet, and many patients are in consequence rendered miserable by the deprivations to which they are subjected in this respect. Whilst I am of opinion that regulation of the diet may be too drastic, I believe that the value of a regulated diet of moderate latitude may be great, and it is very important for the patient to avoid dietary excesses of all kinds, both in regard to the quantity and character of the food. In choosing a diet, therefore, for a patient suffering from super-normal blood-pressure, we should see that the number and bulk of the meals are limited, and that those articles of food which especially stimulate the cardio-vascular system are proportionally reduced; thus, the quantity of beef and mutton should be diminished below the proportion ordinarily taken, while the allowance of white meat, such as chicken and fish, may be correspondingly increased. In cases of moderately increased arterial pressure, my usual custom is to recommend a fair helping of meat once daily on four or five days in the week, fish and chicken being substituted on the remaining days. When the increase in blood-pressure is severe, the amount of beef and mutton should be decreased still further. Some authorities go further than this and recommend a reduction also in the 
quantity of veal, poultry, and fish. Meat which has been boiled and is taken without the broth is preferable to roasted meat taken with the gravy. Meat extractives, such as meat soups and gravies, should certainly be largely diminished or totally excluded from the dietary. An adequate supply of carbohydrate foods may be allowed, while the proportion of green vegetables and the simpler fruits should be increased. All food should be eaten slowly and thoroughly masticated. Some physicians, and especially Continental physicians, attach great importance to the diminution or exclusion of chlorides from the diet. I cannot say that I have been convinced on this point. If this view be correct, we should remember that the deprivation of the salt usually added at table is not enough ; food stuffs which before being cooked have the smallest proportion of salt should, therefore, be chosen. The late Sir William Robcrts rccommended chloride of potassium in place of chloride of sodium as a table-salt, but some have found it disagrceable to the taste. An exclusively milk diet for a few days, combined with rest, is useful in some cases. Tea and coffee in strict moderation may be allowed in most cases, but with regard to alcohol, my view is that it is better left alone altogether. If, however, the increase in arterial pressure be not considerable, a moderate amount may be allowed; and even in cases in which the increase of pressure is of severe grade, if the patient be made unhappy by being deprived of all alcoholic beverages, it becomes a question whether a certain allowance of alcohol should not be made, preferably avoiding wines and liqueurs.

The quantity of fluid taken with meals should be diminished, but, on the other hand, it is advisable to take a considerable quantity of fluid from one to two hours before meals. My usual custom is to order the patient to take two or three tumblers of hot water, preferably soft or distilled, before breakfast and also before the midday meal, and perhaps in addition a tumblerful before going 
to bed. A preference is given by some to the mildly alkaline waters. Strict moderation with regard to the use of tobacco should be enjoined. The teeth should receive careful attention, and any abnormal condition should be rectified. If dyspepsia be present, it should be thoroughly treated.

Purgatives are certainly useful in the treatment of super-normal arterial pressure, and it should be noted that a slight laxative action of the bowels each day is of greater value than an occasional purge. In choosing an aperient, saline purgatives, as they induce fluid motions, are the most suitable, and should be administered in hot water some time before breakfast. It is advisable also to administer some mercurial preparation once a week at bed-time. Some writers are of opinion that the administration of mercury results additionally in vascular relaxation, and, if this be the case, minute doses of calomel may advantageously be given two or three times a day for a few weeks occasionally. Sulphur is also supposed by some to reduce the blood-pressure, as well as to act on the bowels. The functions of the skin should be attended to, and in this connection a warm equable climate and woollen underclothing, especially in winter, are to be commended. Cold baths are to be avoided; but a warm bath, the temperature of which is gradually lowered, may be taken daily, and once or twice a week a Turkish bath, perhaps followed by massage, may be allowed. A regular course of balneological treatment is sometimes beneficial, and different kinds of baths, among these being the Aixles-Bains douche, are in fashion in this country, and especially on the Continent. Some physicians believe in the efficacy of electrical treatment. Venesection undoubtedly reduces high blood-pressure, but in my investigations at the Mount Vernon Hospital I found that, while it certainly acts rapidly, its effects are only transient ; at the same time, it is undoubtedly of great value as a purely temporary measure. In one of the cases 
observed, the blood-pressure fell about $30 \mathrm{~mm}$. after the withdrawal of $30 \mathrm{oz}$. of blood, but began to rise again in the course of a few days.

With regard to appressor remedies, it is not right, even if it were possible, to lower super-normal blood-pressure by drugs the action of which is to diminish the force of the cardiac action; we must, therefore, resort to drugs which are supposed to cause dilatation of the blood-vessels, especially of the peripheral vessels; among these are amyl nitrite, isobutyl nitrite, nitroglycerine, sodium nitrite, erythrol tetranitrate, and mannitol nitrate. I must say, however, that in my investigations at the Mount Vernon Hospital, I had very disappointing results from the administration of drugs in severe degrees of supernormal blood-pressure; indecd, I found them valueless in almost every case, not only as to any permanent effect produced, but also with regard to their temporary results. As examples, I may mention that I tried erythrol tetranitratc in large doses, samples of guarantecd pure sodium nitrite (obtained from Mr. Martindale) up to $10 \mathrm{gr}$. doses, iodide of potassium up to $1 \mathrm{drm}$. doses, and nitroglycerine up to $\frac{1}{50}$ gr. doses, each three times a day. In many cases I took measurements every few minutes for several hours after individual doses. Even in cases of slight super-normal blood-pressure, the results of the administration of these drugs wcre not infrequently negative. In one case I gave a large dose of erythrol tetranitrate, and took measurements every ten minutes for three hours afterwards ; the result being negative. If these appressor drugs be used, we should see that those the action of which is slowest and most prolonged are chosen, and administer them in increasing doses until the physiological effects are obtained, while they may be continued in rather smaller doses for considerable periods in suitable cases. Amyl nitrite, isobutyl nitrite, and nitroglycerine exhibit the most pronounced, but most transient, effect. Some authorities believe that the hippurates and benzoates are 
useful, and Oliver recommends the following combination of the slowly acting vaso-dilators : sodium nitrite, gr. $\frac{1}{2}$; erythrol tetranitrate, gr. $\frac{1}{8}$; mannitose nitrate, gr. $\frac{1}{4}$; ammon. hippurate, gr. 1 ; in chocolate tabloid form (B. W. \& Co.). If there be indications of cardiac dilatation or cardiac failure, some physicians prefer to administer one of the digitalis series of drugs along with the vaso-dilators. The iodides have a great reputation in the treatment of arterio-sclerosis, but, in the investigations already mentioned, in no case was their administration followed by a reduction of blood-pressure. In some cases a single dose of 20-30 grains of one of the iodides at nighttime is better than smaller doses three times a day. When the usual preparations cannot be borne, one of the iodide compounds, such as iodipin or iodoglidine, may be tried. 


\section{CHAPTER V}

THE VENOUS PULSE-THE INK POLYGRAPH--

POLYGRAPHIC RECORDS

By means of the clinical polygraph we are able to obtain records of the movements of both the auricles and ventricles, and also to make out the time-relations of the contraction of these chambers; unlike the sphygmograph, which affords information regarding the movements of the left ventricle alone. The clinical polygraph is a convenient and suitable instrument for the general prac-

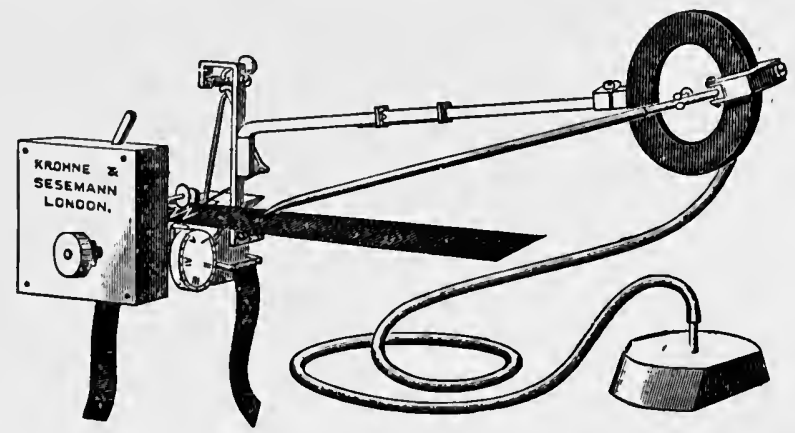

FIG. 42. The clinical polygraph, consisting of a tambour attached to a Dudgeon's sphygmograph.

titioner ; its practical use may be learned in a comparatively short time, and its cost is within the reach of most.

A tambour and lever may be fitted on to a Dudgeon's (Fig. 42), or Jacquet's sphygmograph, so that two records can be taken simultaneously; smoked paper is employed, and the time-marker registers one-fifth of a second. I myself, however, invariably recommend "Mackenzie's ink 
polygraph" (Fig. 43) to my pupils, as being the most useful. ${ }^{1}$ This does away with the trouble of blackening

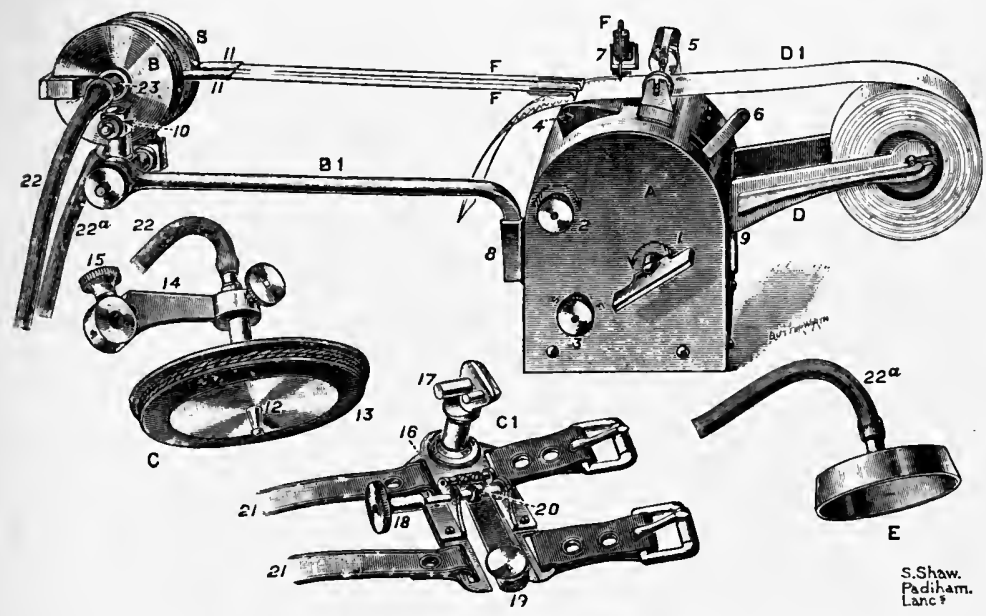

FrG. 43. Mackenzie's ink polygraph. The body $A$ contains the paper rolling and time-marking movements. Attached to the body are two sockets ; one in front (8) for holding the rod $B 1$ which supports the writing tambours $(B B)$ with their levers and pens $F F$, and the other behind (9) for holding the bracket $D$ which supports the paper roll $D 1$. At the end of the body are three-keys. The large one (1) is for winding the paper-rolling movement; the top smaller stud-key (2) is for winding the time-marker movement; and the bottom one (3) regulates the speed of the paper passing through the rollers, the direction required being indicated by the letters $F$ and $S$ (fast and slow). On the top are the writing table (4), friction rollers and tension spring (5) for passing along the paper. Behind these, to the right, is the start-and-stop lever (6), and to the left the fork (7) carrying the time-marker pen $F$. This fork vibrates at the rate of 300 per minute, an equivalent to $1 / 5$ of a second. The wrist tambour is in two parts : First, the attachment $C 1$, consisting of a splint (16) which is strapped on to the wrist (Fig. 44), and is fitted with a spring tongue and button (19) which is placed precisely over the radial artery, and also an eccentric (20) and regulating screw (18) for regulating the pressure on the artery. Second, the tambour $C$, with supporting arm (14), rubber membrane (13), and compression disc and peg (12). The two parts are held together by the clamping screw (15) in the supporting arm binding on to the pillar (17). $E$ is a shallow cup or receiver for placing over the apex-beat, the carotid artery, or the jugular vein. The writing tambours have inlets (23) for attaching rubber-tubing $(22,22 a)$ which connect them with the wrist tambour and with the receiver.

1 The instrument supplied by Mr. Shaw, Church Street, Padiham, Lancashire, at the very reasonable price of twelve guineas, is, in my opinion, undoubtedly the most satisfactory. 
and varnishing the paper, and also enables us to take records of any length.

By means of this instrument it is possible to take a simultaneous tracing on the same recording surface of any two of the following: the radial pulse, the apex-beat, the carotid pulse, the jugular pulse, the liver pulse, and the respiratory curve; further, a time-marker registers $\frac{1}{5}$ sec. on the same recording surface. The usual plan is to take simultaneous tracings of the arterial and venous pulses. With regard to the former, the radial artery is usually chosen (Fig. 44), although the carotid is sometimes employed. As a rule a tracing of the radial or carotid artery is more reliable than that of the apexbeat, because, in contrast with the latter, it is possible to determine with certainty the systolic and diastolic portions. Sometimes, however, $\varepsilon s$, for example, when it is difficult or impossible to obtain a satisfactory tracing of the radial artery, it is advisable to take a tracing of the apex-beat instead. With regard to the venous pulse, the jugular vein is almost always employed; very rarely the liver. The venous pulse affords information regarding the action of the auricle.

Detailed instructions are supplied with Mackenzie's ink polygraph. The clock-work of the time-marker should be wound up before taking each tracing, and in applying the sphygmograph it is important to see that the spring is placed precisely over the artery. The head of the patient should be comfortably supported by pillows, and slightly turned away from the operator, while care should be taken that the sterno-mastoid muscle is in a state of relaxation. If the excursion of the lever recording the jugular pulse be found to be too great, the patient may be further propped up in bed, while, if the opposite be the case, the head should be placed at a lower level than the body. The open mouth of the small cup or receiver is. placed over the internal jugular vein, just above the clavicle and 1 to $1 \frac{1}{2}$ inch external to the sterno-clavicular 
joint, the right side of the neck being preferable; in this way a tracing of the internal jugular vein is obtained (Fig. 7). If a satisfactory tracing be not possible in this position, the receiver should be placed just above the clavicle and in the mid-clavicular line, opposite the point of junction of the external jugular and subclavian veins. If a tracing of the carotid artery be desired, the receiver should be applied over the vessel, just internal to the sternomastoid, at the level of the thyroid cartilage or hyoid bone,

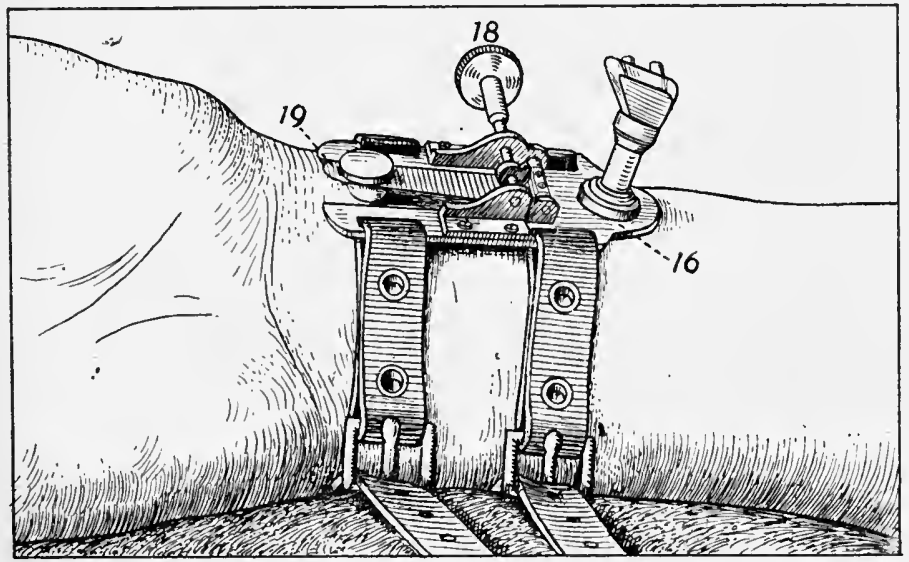

FIG. 44. The attachment of the wrist tambour of the Mackenzie ink polygraph strapped on to the right wrist.

the head of the patient being turned somewhat to the opposite side, and the chin slightly elevated; the pressure exercised should be rather more than in the case of the jugular vein. If a tracing of the apex-beat be desired, the receiver should be placed over the area of maximum impulse; if the excursion of the lever be found to be too great, the receiver should be moved rather towards the axilla. Further instructions with regard to obtaining a tracing of the apex-beat will be described later.

A special form of receiver is used for taking a tracing of the liver pulse; it is larger, and contains a small air-hole (Fig. 45). In order to take the liver pulse, the 
receiver should be placed on the skin of the abdomen so that the lower border of the receiver is one or two inches below the lower margin of the liver. The receiver should then be steadily pressed backwards and upwards, and then the upper margin of the receiver should be applied to the skin. The air-hole should then be stopped with a finger, when excursions of the needle will be noted, these being partly due to pulsations of the liver and partly to the movements of respiration; if the patient cease breathing, the latter will be eliminated. In taking tracings

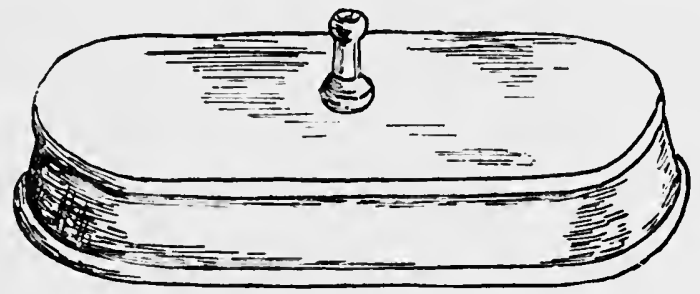

FIG. 45. The liver receiver (half size).

of the apex-beat, or of the carotid or jugular pulse, or of the liver pulse, care should be taken that the whole margin of the receiver is in contact with the skin; but while the pressure should be sufficient for this purpose it should not be such as will affect pulsation. The receiver should bc hcld steadily, in order to avoid the disturbing cffect of fine oscillations.

The respiratory movements may be recorded by substituting a small india-rubber bag for the receiver, and attaching the bag to the chest by means of a bandage. Another method is the following: The small cup or receiver should be disconnected from the rubber-tubing, and the latter is connected with an additional piece of rubber-tubing by means of an L-shaped piece of glasstubing. A piece of tape is then attached to the end of this additional piece of rubber-tubing, and another to the far end of the glass-tubing. The two pieces of tape are then tied round the chest, at just sufficient 
tension to stretch slightly the rubber-tubing during respiration.

When taking a polygraphic tracing, before starting and also on stopping the clock-work, we should see that the levers record the position by upright lines; this should also be done from time to time during the tracing; in this way ordinates or index-marks are obtained. When the radial artery is employed, it is helpful, for the purpose of analysis, if a combined record of the carotid and radial arteries be taken for a few cycles, either at the beginning or at the end of the tracing.

A tracing of the arterial pulse is called a sphygmogram, a tracing of the apex-beat is called a cardiogram, and a tracing of the venous pulse is called a phlebogram. The features of a normal sphygmogram have been described in Chapter III. It may, however, be noted here that the sphygmic or pulse-period commences with the beginning of the percussion wave and ends at the bottom of the dicrotic notch.

The Cardiogram. - A cardiogram is simply a record of the mechanical displacement of the organ in relation to the chest-wall, and is not indicative of the ventricular pressure. If the receiver be placed over the left ventriclc the record is called a positive cardiogram, while if the receiver be placed over the right ventricle it is called a negative or inverted cardiogram. It should be remembered that when in enlargement of the right ventricle that chamber displaces the left ventricle, and the clinical apex-beat is formed by the right, instead of the left, ventricle, a cardiogram over the actual apex-beat will be a negative or inverted one; otherwise, the significance of an inverted cardiogram will be misunderstood.

In taking a negative cardiogram in normal circumstances, the receiver should be placed over the precordium to the right of the apex, or over the epigastrium in the angle between the left costal margin and the xiphisternum. The result is sometimes unsuccessful when 
the patient is lying down, but is generally obtained when he sits up.

In cases of heart failure, it is sometimes possible to

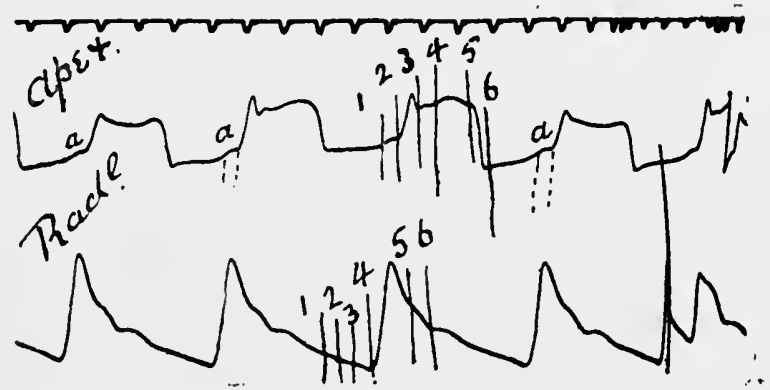

FIG. 46. Simultaneous tracings of the apex-beat and of the radial pulse, showing a positive cardiogram. At 1 , the commencement of auricular systole; at 2, the commencement of ventricular systole; at 3 , the commencement of the carotid pulse; at 4 , the commencement of the radial pulse ; at 5 , closure of the semilunar valves; at 6 , opening of the tricuspid valves. The space between 2 and 3 represents the pre-sphygmic period, the space between 3 and 5 the sphygmic period, and the space between 5 and 6 the post-sphygmic period. Note that the radial pulse begins $1 / 5$ sec. after the commencement of ventricular systole.

observe the gradual transition type; as the right heart becomes smaller, there is a gradual return from the negative to the positive form of eardiogram.

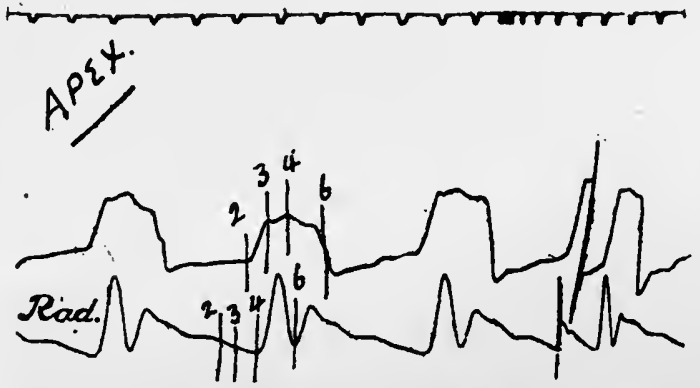

Fra. 47. Simultaneous tracings of the apex-beat and of the radial artery, showing a positive cardiogram.

On analysis, a positive eardiogram is found to consist of the following parts (Figs. 46-51). (a) An ascending limb, representing the pre-sphygmic period. Its commence: 
ment is indicated by the vertical stroke 2, and exactly corresponds with the summit of the $a$ wave in a tracing of the jugular vein, and is synchronous with the commence-

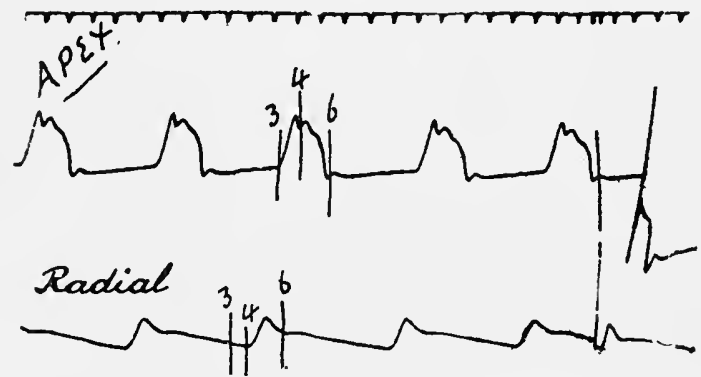

Fig. 48. Simultaneous tracings of the apex-beat and of the radial artery. showing a positive cardiogram, with a different outline from that of Fig. 47.

ment of the first sound; while its termination, which is usually abrupt, is represented by the vertical stroke 3, and corresponds with the commencement of the carotid pulse. (b) A fairly level line-the systolic plateau; but,

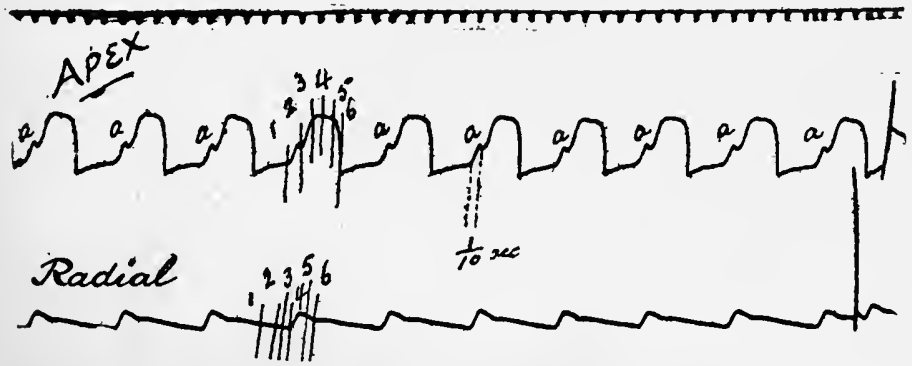

FIG. 49. Simultaneous tracings of the apex-beat and of the radial artery, showing a positive cardiogram, with a pronounced $a$ wave. The $A s-V s$ interval (space $1-2$ ) is $1 / 10$ sec.

instead of this, the line may continue to rise, or it may sometimes rapidly descend, representing the sphygmic or pulse-period. Its commencement is indicated by the vertical stroke 3 , and its termination by the vertical stroke 5 , the termination practically corresponding with the dicrotic 
notch of the carotid pulse. (c) A fall, either a slanting or a more vertical descent, representing the post-sphygmic period. Its commencement is represented by the vertical stroke 5 , and the lowest point by the vertical stroke 6 ;

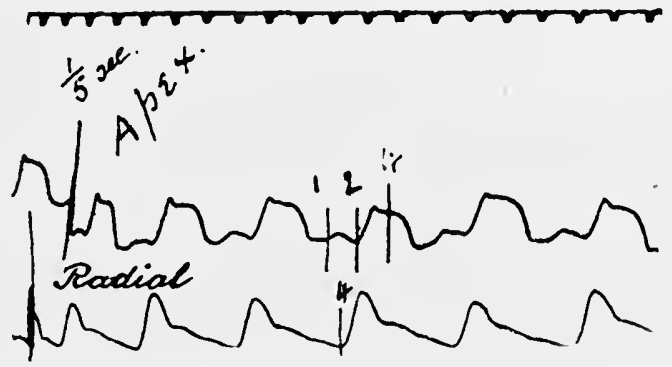

FIG. 50. Simultaneous tracings of the apex-beat and of the radial artery, showing a positive cardiogram with a pronounced $a$ wave. The $A s-V s$ interval (space 1-2) is increased, being $1 / 5$ sec.

the latter eoincides with the apex of the $v$ wave in the phlebogram. (d) During the period in which the blood flows from the auricles into the ventricles and slowly fills them, the heart may push against the intereostal space,

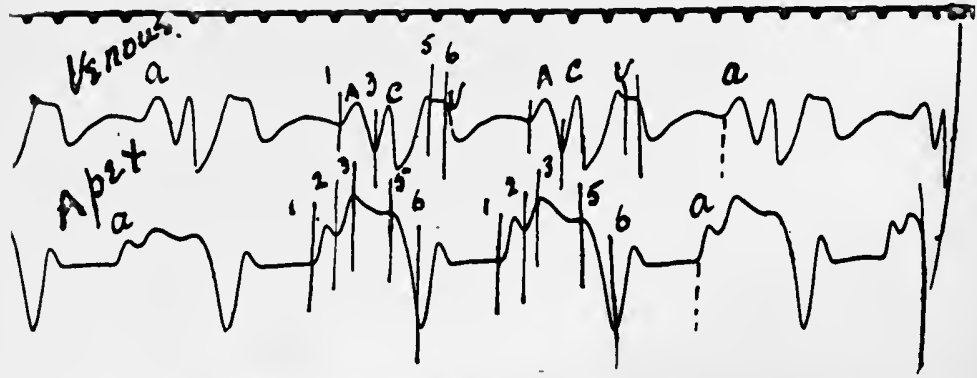

Fic. 51. Simultaneous tracings of the apex-beat and of the jugular vein. Note that the $a$ wave occurs at exactly the same time in both.

giving rise to a gradual ascent in the cardiogram; or filling of the ventricles may have no effect upon the interspace, in which event the level of the tracing remains much the same until the next ventricular systole. In some cases, a slight abrupt rise is found immediately 
preceding ventricular systole, due to the contraction of the auricle causing a sudden increase in the contents of the ventricle. The positive wave is called $a$, and its commencement is represented by the vertical stroke 1 .

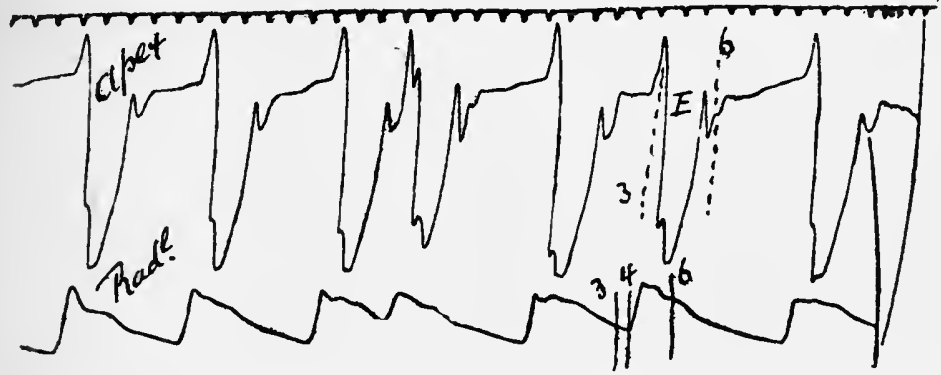

FIG. 52. Simultaneous tracings of the clinical apex-beat and of the radial artery, from a case of greatly dilated heart, showing a negative cardiogram.

Normally it precedes the commencement of the wave due to the ventricular systole by about $\frac{1}{10}$ sec. (Fig. 49), an increase of this interval (Fig. 50) being sometimes indicative of a delay in the passage of the stimulus from

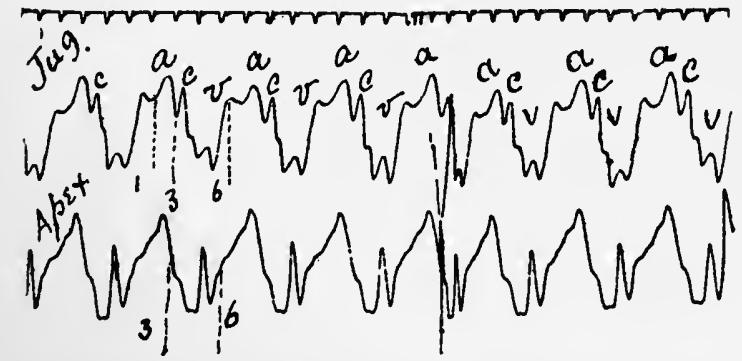

Fig. 53. Simultaneous tracings of the apex-beat and of the jugular vein, from a case of aortic regurgitation. The cardiogram is negative.

auricle to ventricle. The $a$ wave may be absent even when other indications of auricular systole are present. There is a period of about one-tenth of a second each between the vertical strokes 2 and 3 , and 3 and 4 .

A negative cardiogram is characterised by the following features (Figs. 52-55): Instead of an ascending limb 
and a flattened elevation, there is a slight rise, followed by a great depression-the former being due to the shock of the contracting ventricle, and the latter to the ventricles diminishing in size, on account of pouring out

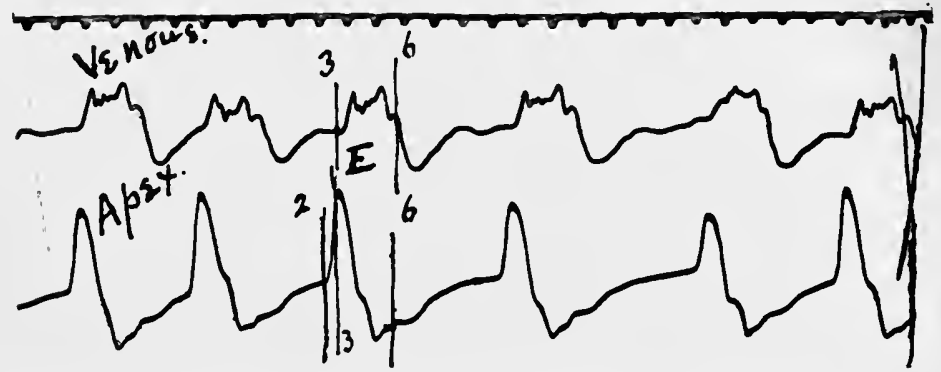

FIG. 54. Simultaneous tracings of the apex-beat and of the jugular vein, from a case of greatly enlarged heart. The cardiogram is negative.

their blood as soon as the semilunar valves have opened. The $a$ wave may be present.

The Phlebogram.-If a tracing of the jugular pulse taken from a normal individual be studied, in each

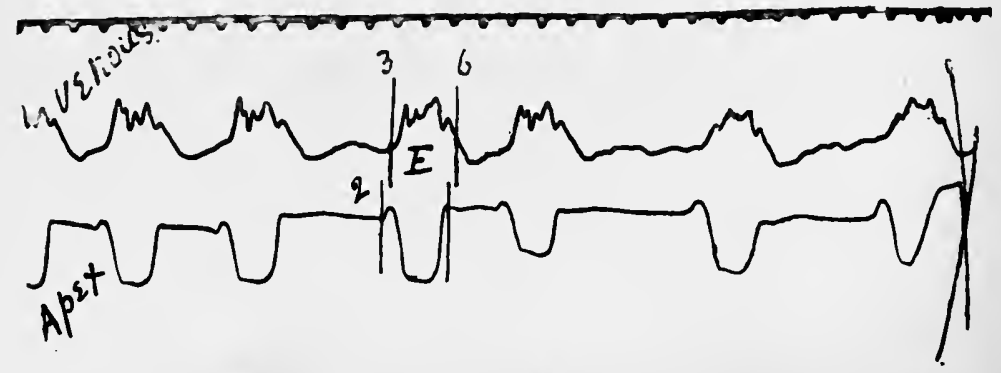

FIG. 55. Same as Fig. 54, after moving the receiver a little to the right.

cardiac cycle three main waves are seen (Fig. 58). They indicate a rise of pressure within the vein. The first wave in point of time is due to the contraction of the auricle, and is called the $a$ wave. Its commencement is an indication of the beginning of auricular systole. Following the first wave is a fall, called $x$. The fall $x$ is temporarily interrupted by the second wave. It has 
been already noted that the semilunar valves open at an early stage of ventricular systole. A wave of increased pressure passes along the arterial system, reaching the carotid artery about ${ }_{10}^{1}$ sec. before the radial artery.

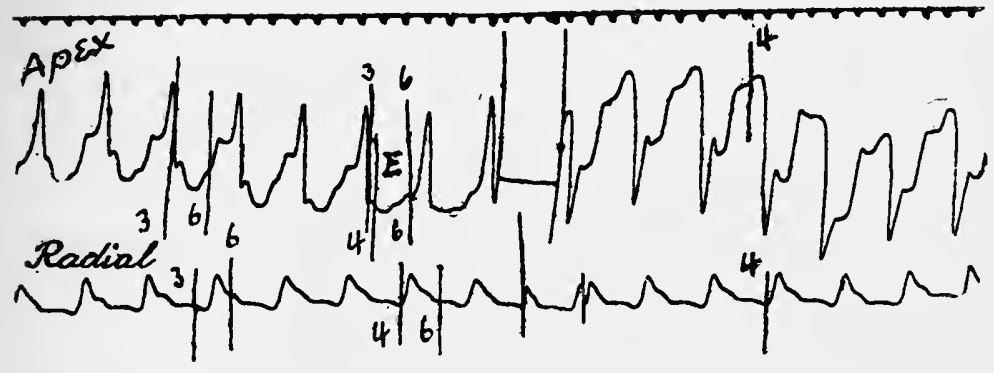

FIG. 56. Simultaneous tracings of the apex-beat and of the radial artery, from a case of greatly enlarged heart. The first part of the tracing was taken a little to the right of the clinical apex-beat, and shows a negative cardiogram. The second part of the tracing was taken from the clinical apex-beat, and shows a positive cardiogram.

This wave of increased pressure in the carotid artery is represented by the second wave in the tracing of the jugular vein, and is called the $c$ wave, being synchronous

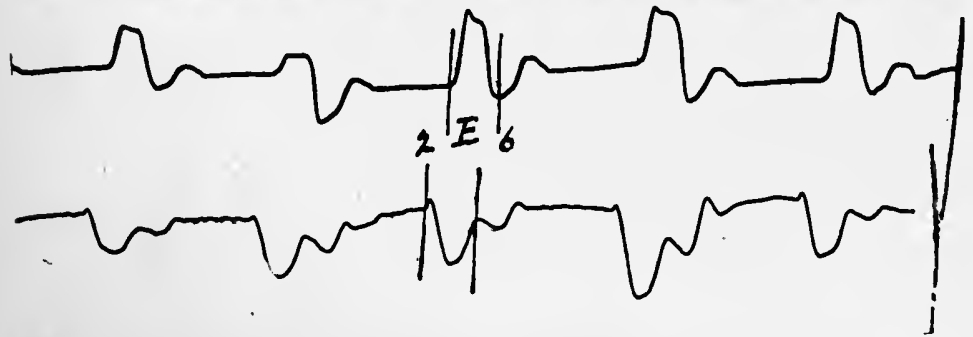

FrG. 57. Simultaneous tracings of the apex-beat, from a case of greatly enlarged heart. The upper tracing was taken from the clinical apexbeat, and the lower from a spot $1^{\prime \prime}$ to the right of this. The curves are opposed during the sphygmic period $E$.

with the primary or "percussion" wave of the arterial pulse at the same level of the neck. After the $c$ wave the fall $x$ is continued until towards the termination of ventricular systole, when the third wave occurs; the 
onflowing blood experiences an increasing difficulty in entering the auricle, leading to a fulness of the jugular vein, this being indicated by a wave in the venous tracing. The third wave is called the $v$ wave. It terminates at about the time of opening of the tricuspid valves, and is then followed by a depression, which is called $y$. The pressure within the ventricle falls rapidly during diastole, the blood contained in the auricle passes into the ventricle, and as a result the auricular pressure falls. From the point of time corresponding with the bottom of the $v$ wave to the beginning of the next auricular systole, the venous tracing shows a line which is either horizontal, or which slopes upwards to the commencement of the following $a$ wave.

It must be pointed out that it is of the utmost importance to observe that the $a$ wave is present in each cardiac cycle, and that its position is constant from cardiac cycle to cardiac cycle. These features distinguish the $a$ wave from the multiple undulations which may be present in cases of auricular fibrillation in which there is infrequent cardiac action. Sometimes the $c$ wave is divided into two component parts, and the $a$ wave more rarely so.

The time-distance between the beginning of the $a$ wave and the commencement of the $c$ wave is called the $a-c$ interval. It is of great clinical importance; for it is an index of the $A s-V s$ interval, that is, the interval separating the commencement of auricular and ventricular contraction, and is a measure of the function of conductivity of the heart. In normally acting hearts the $a-c$. interval is almost constant, and does not measure more than one-fifth of a second (Fig. 58), being about 0.15 to 0.20 sec. in duration. In rapidly-acting hearts there is a tendency for it to become shortened. If, therefore, in rapidly-acting hearts the $a-c$ interval measure one-fifth of a second, or, if in hearts acting at the normal or less than the normal rate it exceed one-fifth of a second 
(Figs. 91-94)-particularly if there be a change in the $a-c$ interval from beat to beat (Fig. 93)-it may be inferred that there is a prolongation of the $A s-V s$ interval, and a delay in the conduction of the stimulus, this indicating that the function of conductivity is depressed; this is called "depressed conductivity," and probably indicates some affection of the auriculo-ventricular bundle. The $a-c$ interval is longer than the corresponding $A s-V s$ interval in a cardiogram by one-tenth of a second.

The time of commencement of the $v$ wave varies, this

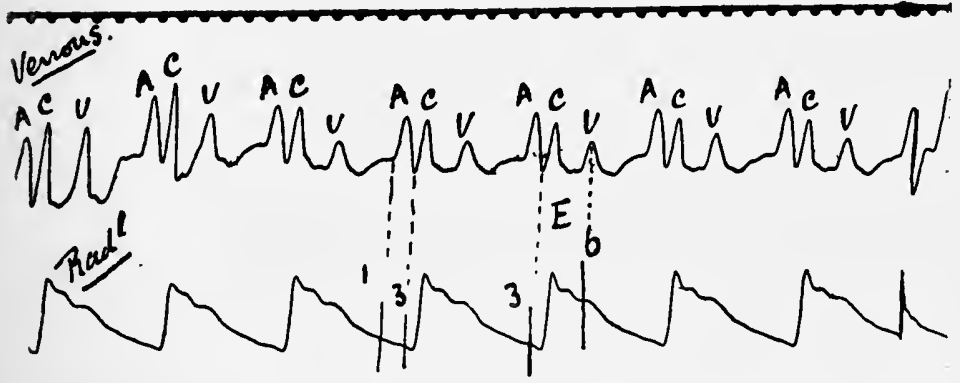

Fig. 58. Simultaneous tracings of the jugular and radial pulses, showing the auricular form of venous pulse. Note that the $a-c$ interval is $1 / 5 \mathrm{sec}$, and that the summit of the $v$ wave coincides with the dicrotic notch of the radial pulse.

in all probability depending largely upon the degree of engorgement of the right heart; it may begin during the systolic plateau, or towards its termination. The earlier the $v$ wave commences in the negative phase $x$, the greater the degree of engorgement of the right side of the heart; when the engorgement is marked, there may be no fall in the tracing during the period $E$ (Figs. 59 and 60 ). The termination of the $v$ wave, on the other hand, does not vary; it never extends beyond the end of systole; when, therefore, the duration of the $v$ wave increases, it does so by commencing earlier in the sphygmic period. The summit of the $\mathrm{v}$ wave is of great importance as a time-mark, being synchronous with the opening of the auriculo-ventricular 
valves. It corresponds approximately with the bottom of the aortic notch in the radial pulse.

Sometimes the $v$ wave is divided into two component parts, there being a notch or angle between them, that

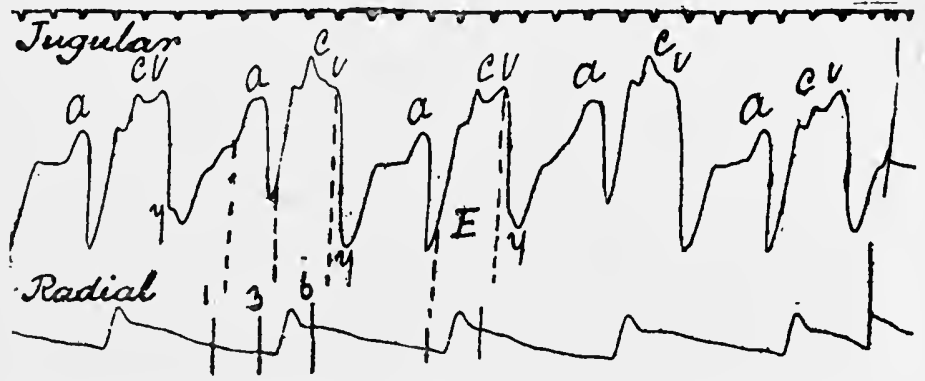

FIG. 59. Simultaneous tracings of the jugular and radial pulses, from a case with great distension of the right side of the heart, showing the auricular form of venous pulse, in which there is no fall in the tracing during the period $E$. Compare this with Fig. 58.

part which occurs earlier being called $v^{1}$, and the other $v^{2}$ (Figs. 61 and 62). The angle between the two component parts, in other words the beginning of $v^{2}$, coincides in point

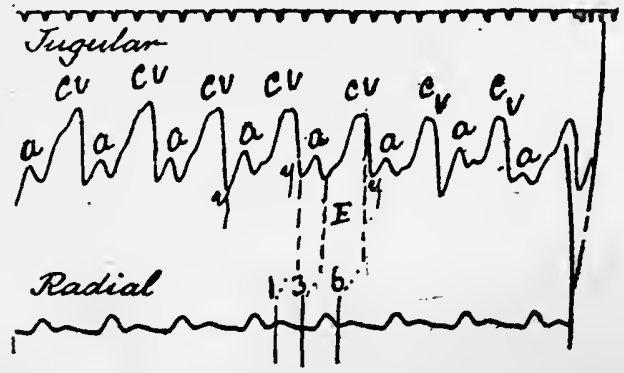

FIG. 60. Shows the same as Fig. 59.

of time with the closure of the semilunar valves, and is rcpresented in tracings by the vertical stroke 5 ; while the apex of $v^{2}$ coincides in point of time with the opening of the auriculo-ventricular valves, and is represented by the vertical stroke 6. 
The negative phase $y$ begins with the opcning of the auriculo-ventricular valves. This phase tends to diminish more and more as diastole becomes shortened with increasing frequency of the cardiac rate; the $v$ wave

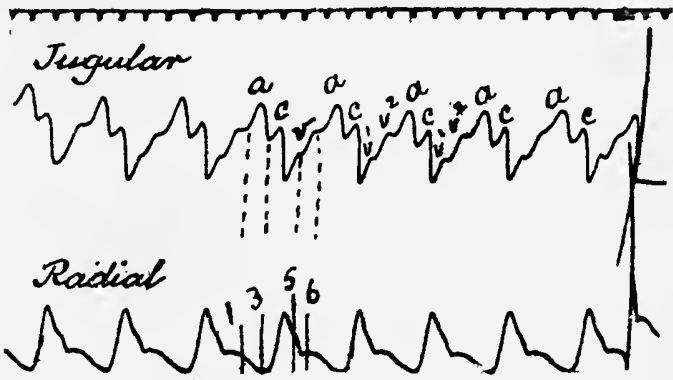

FrG. 61. Simultaneous tracings of the jugular and radial pulses, showing the auricular form of venous pulse. There is a notch in the upstroke of the $v$ wave which coincides in point of time with the closure of the semilunar valves (5). The space between this notch and the apex of $v 2(6)$ is the post-sphygmic period.

approaches more and more to the following $a$ wave, and when the cardiac rate is unusually frequent $v$ and $a$ may even coincide and superimpose (Figs. 63 and 64).

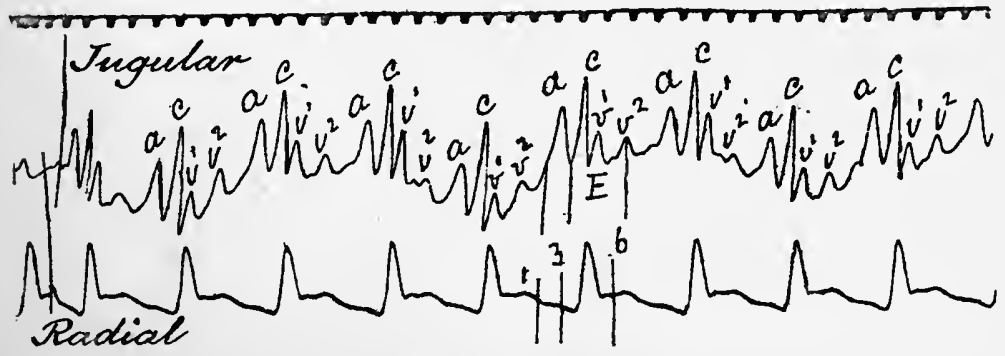

Fra. 62. Simultaneous tracings of the jugular and radial pulses, showing the auricular form of venous pulse. The $v$ wave is divided into two component parts.

The $\boldsymbol{h}$ or $\boldsymbol{b}$ Wave.-Occasionally, more especially in relatively slow-acting hearts, there may be small waves due to stasis. Hirschfelder and A. G. Gibson described a fourth wave, occurring in the diastolic period of each cycle, shortly after the opening of the tricuspid valves, 
and between the $v$ wave and the succeeding $a$ wave; it has been called the $h$ or $b$ wave (Fig. 65). It disappears when the pulse-rate becomes more rapid. Its supposed cause is that at the moment of opening of the auriculo-ventricular valves there is such a rush

Texpulare

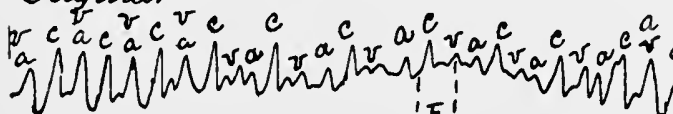

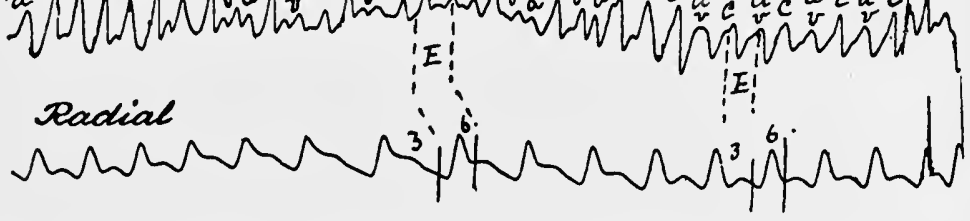

FIG. 63. Simultaneous tracings of the jugular and radial pulses. With the slower rate, $a, c$, and $v$ are separate; with the faster rate, $v$ and $a$ coincide and superimpose.

of blood from the upper into the lower chambers of the heart as to cause the cusps to float back and to block the passage of blood from auricle to ventricle. It

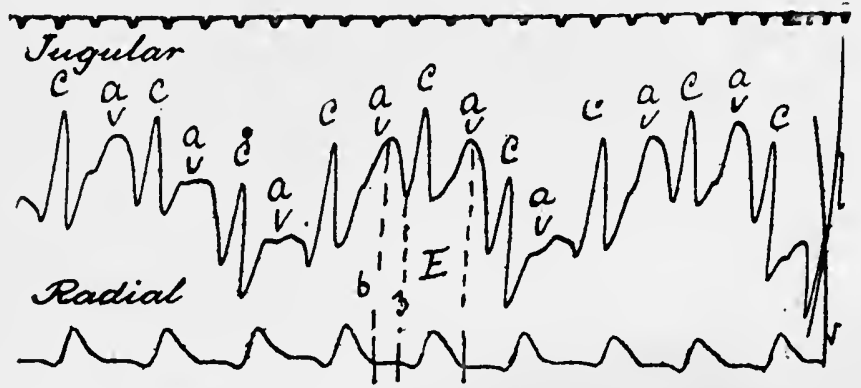

FIG. 64. Simultaneous tracings of the jugular and radial pulses. The $v$ and $a$ waves coincide and superimpose.

should be noted that the relation of this wave to the preceding $c$ or $v$ wave is comparatively constant. This is important from the point of view of diagnosis; for the wave is occasionally seen in cases of auricular fibrillation, when it may exhibit some resemblance to an $a$ wave, and, further, it may also fall exactly midway between two, $a$ waves, and then it is necessary to distinguish the con- 
dition from partial heart-block, in which every second impulse from the auricle is blocked. A differential diagnosis is possible in both of these. In auricular fibrillation, the $h$ or $b$ wave may be distinguished from an $a$ wave by the constancy of its relation to the preceding systole,

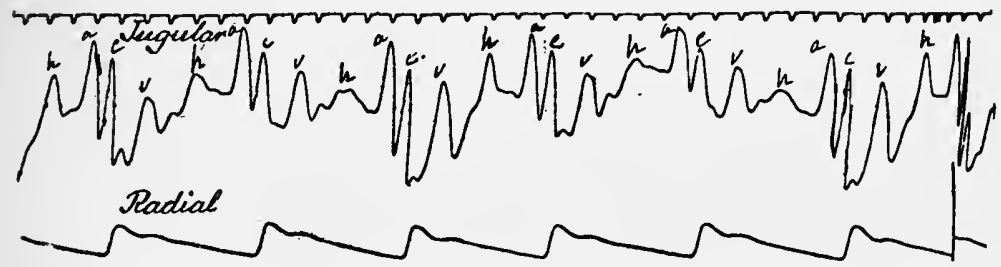

FIG. 65. Simultaneous tracings of the jugular and radial pulses, showing the auricular form of venous pulse, with the $h$ or $b$ wave.

while the distance which separates it from the $c$ or $v$ wave of the following systole will be found to vary in different cardiac cycles; in the second case, also, if the cardiac rate be altcred, as, for example, by physical exertion, the $h$ or $b$ wave presents the same features, in contrast with the $a$ wave of partial heart-block.

We are now able to note the relation of the different events in the cardiac cycle as recorded in a sphygmogram, cardiogram, tracing of the carotid pulse, and phlebogram :

At 1, commencement of the $a$ wave in the phlebogram, and, if present, in the cardiogram.

At 2, summit of the $a$ wave in the phlebogram, and commencement of the ascending limb in the cardiogram.

At 3, commencement of the carotid pulse, of the $c$ wave in the phlebogram, and summit of the ascending limb in the cardiogram.

At 4, commencement of the radial pulse, and summit of the $c$ wave in the phlebogram.

At 5, commencement of $v^{2}$-if the wave be divided into two component parts-sudden drop in the cardiogram, and dicrotic notch in the carotid pulse.

At 6, lowest point in the cardiogram, summit of the $v$ wave-or of $v^{2}$ if the $v$ wave be divided into two com- 
ponent parts-in the phlebogram, and in the radial curve the bottom of the aortic notch. There is an interval of about $\frac{1}{10}$ sec. each between the vertical strokes 1 and 2,2 and 3 , and 3 and 4 .

The Auricular and Ventricular Forms of Venous Pulse.-When in a venous pulse the $a$ wave is un-

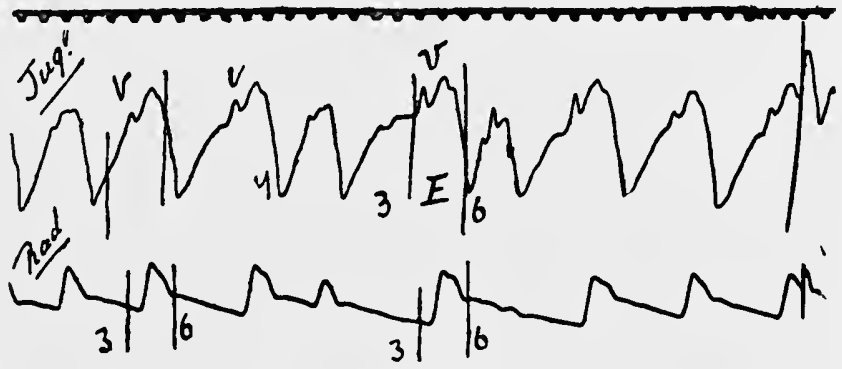

FIs. 66. Simultaneous tracings of the jugular and radial pulses, showing the ventricular form of venous pulse, from a case of auricular fibrillation.

doubtedly present, the form of pulse is called the auricular or negative form of venous pulse. In this form of pulse, the $a$ wave is present in each cardiac cycle, and its position is constant from cardiac cycle to cardiac cycle. The presence of the $a$ wave informs us that the auricle is con-

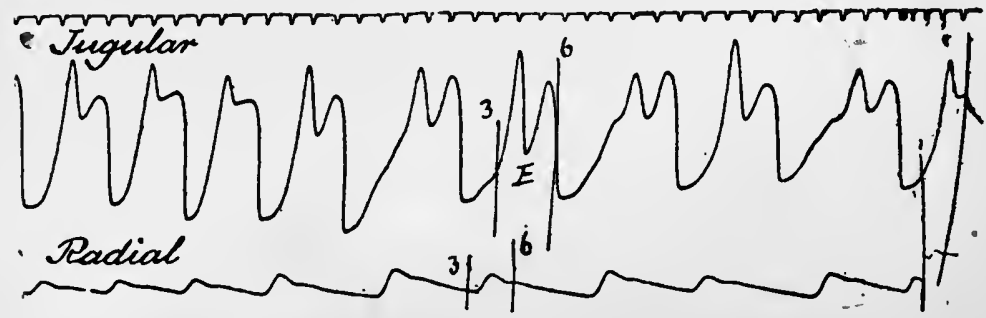

FIG. 67. Shows the same as Fig. 66, from a case of auricular fibrillation.

tracting normally. In what circumstances does the auricular form of venous pulse occur? It is present in two classes-(1) in all normal individuals ; (2) in a large proportion of cases of organic heart disease-a point which - should be particularly noted. 
When in a venous curve there is no sign of the presence of the $a$ wave, the type of pulse is called the ventricular or positive form of venous pulse, in contradistinction to the auricular form of pulse. It signifies that the auricle

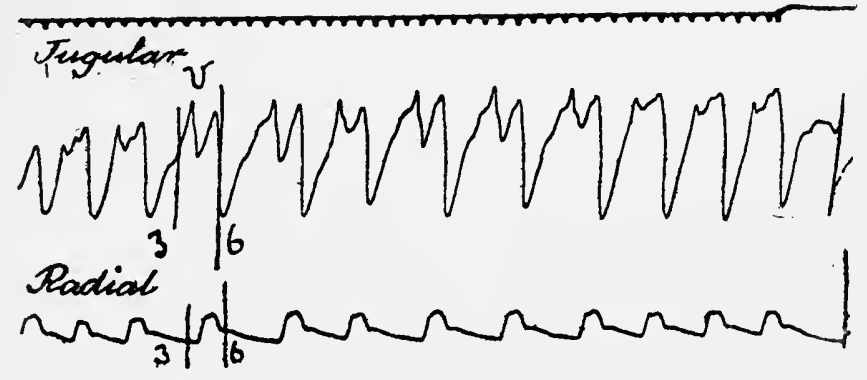

Fia. 68. Shows the same as Fig. 66, from a case of auricular fibrillation.

is not contracting in a normal manner. The vast majority of cases are due to auricular fibrillation (Figs. 66-68); a small proportion are due to a greatly distended right auricle (Fig. 69), or to some forms of paroxysmal tachy-

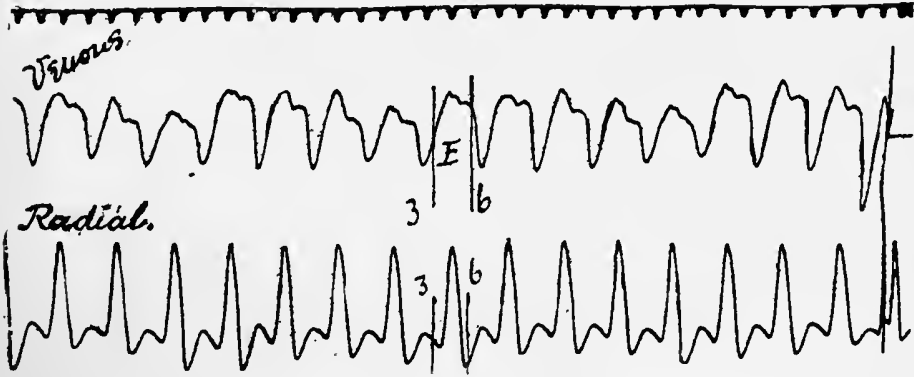

FIG. 69. Simultaneous tracings of the jugular and radial pulses, , showing the ventricular form of venous pulse, from a case of mitral disease, with great distension of the right side of the heart and tricuspid regurgitation.

cardia. It will thus be seen that all cases of heart disease, whether valvular or myocardial-and it may be here mentioned whether cardiac failure be present or not - may be divided into two groups-(1) those cases in which the auricular form of venous pulse is present, and 
(2) those cases in which the ventricular form of venous pulse is present.

Analysis of Polygraphic Records.-By taking simultaneous tracings of the radial or carotid pulse or of the apex-beat on the one hand, and of the jugular pulse on the other, it is possible to establish the time of appearance in the cardiac cycle of each wave in the venous tracing, and thus to arrive at an interpretation of the venous-pulse tracing. If when the radial artery be employed we take a combined record of the carotid and radial pulses for a few cycles, either at the beginning or at the end of the combined tracing of phlebogram and sphygmogram, we are enabled to note a point in the tracing of the radial artery which is synchronous with the time of commencement of the carotid pulse in the neck, this point being usually $\frac{1}{10}$ sec. prior to the commencement of the radial beat. This point is then transferred to the phlebogram, and in this way we are able to obtain a point in the phlebogram which represents the beginning of the sphygmic period, and can, therefore, identify the $c$ wave in the phlebogram.

It will be well, perhaps, to give a detailed interpretation of a normal polygraphic record. This can be best done if one of the two following methods of procedure be carried out.

If one point of a pair of compasses be inserted into a tracing at the point which indicates the commencement of the upstroke of a radial beat to the right of one of the ordinates, say at $A$ (Fig. 70), and the other point at the commencement of the upstroke of any other radial pulse to the left of the ordinate, say at $B$, measure the distance between these two points; having done so, place one point of the compasses at the commencement of the upstroke of the carotid beat which occurs within the same cardiac cycle as the point marked $A$ (this point being marked $C$ ), and measure from this point the same distance as between $A$ and $B$. In this way a point. 
(marked $D$ ) will be obtained in the phlebogram which represents the beginning of the sphygmic period, and, therefore, the point at which the commencement of the $c$ wave would be expected. It will be noted that the point $\boldsymbol{D}$ coincides with the commencement of a wave, which we may rightly assume is the $c$ wave. Having fixed the $c$ wave in the phlebogram, if in a phlebogram there be found to be three waves in each cardiac cycle, and one of these waves be found to be directly

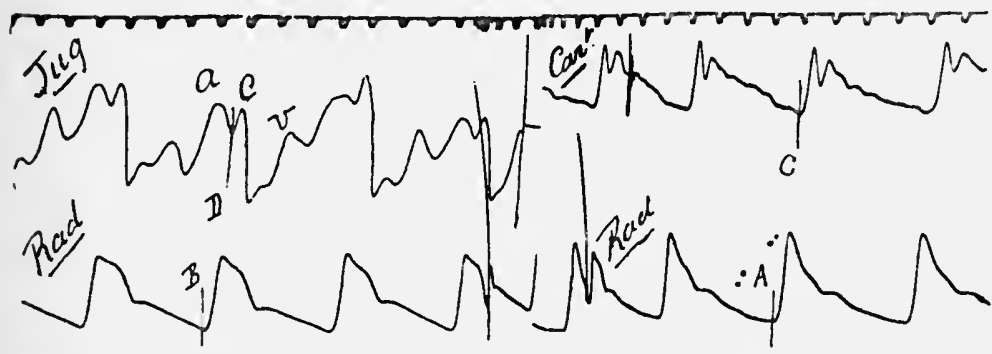

FIG. 70. Simultaneous tracings of the jugular and radial pulses, and of the carotid and radial pulses; for the purpose of analysis, see text.

in front of the $c$ wave, we can assume that this is the $a$ wave.

The second method, and the one which I myself usually adopt, is the following: The combined record of the carotid and radial pulses at the beginning or end of the combined record of phlebogram and sphygmogram is dispensed with, and in the simultaneous radial and venous curves I transfer the point in the radial curve which represents the time of commencement of the carotid pulse in the neck to the venous curve, and in this way obtain the point which represents the onset of the ventricular systole in the venous curve. Taking a piece of polygraphic record in which ordinates are present, measure one-tenth of a second in front of the commencement of the upstroke of any radial beat, say at $A$ (Fig. 71). Place one point of a pair of compasses on the tracing at this point (marked $\boldsymbol{A}$ ), and the other point on an ordinate 
of the radial tracing, say at $B$; measure the distance between these two points; having done so, place one point of the compasses on the ordinate marked $C$ of the

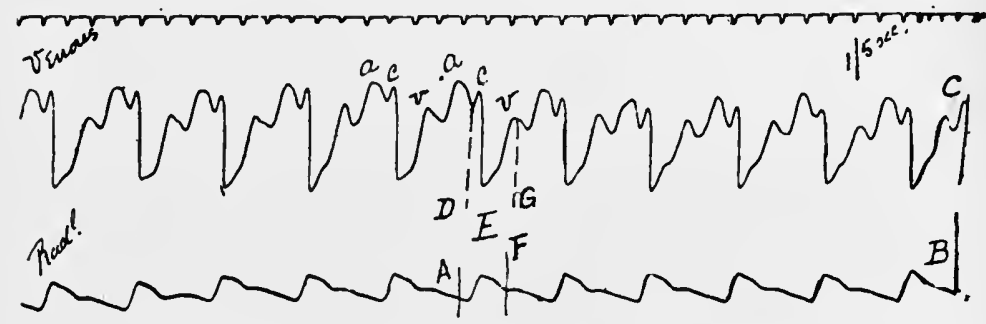

FiG. 71. Simultaneous tracings of the jugular and radial pulses; for the purpose of analysis, see text.

tracing of the jugular vein, and measure from this point the same distance as between $A$ and $B$. We thus get a point (marked $D$ ) which is the commencement of the sphygmic period in the venous tracing, and, therefore, the point at which the beginning of the $c$ wave would be expected. It will be now noted that the point referred to as $\boldsymbol{D}$ coincides with the commencement of a wave, which we may rightly assume to be the $c$ wave.

Having by either of these methods identified the $c$ wave in the phlebogram, we should next determine the end of the sphygmic period in the venous tracing, because of the possibility of mistaking the $v$ wave for the $a$ wave; or, in cases in which these two waves are suspected of coinciding-as when the heart is acting rapidly or when the $a-c$ interval is increased. The termination of ventricular systole is represented in a phlebogram by the apex of the $v$ wave, in a tracing of the carotid pulse by a point lying a little (usually one-tenth of a second) in front of the bottom of the dicrotic depression, and in a tracing of the radial beat practically by the dicrotic notch itself. We may determine the end of the sphygmic period in the phlebogram by measuring the distance between a point which is one-tenth of a second in front of the commence- 
ment of the upstroke of any radial beat (say at $A$ ) and the bottom of the dicrotic notch of the radial beat (marked $F$ ), and measuring the same distance from the commencement of the $c$ wave in the phlebogram (marked $D$ ). This will be found to fall at the summit of a wave (marked $G$ ), which must, therefore, be the $v$ wave.

We have now divided the phlebogram into two portions, namely, (1) that during which the semilunar valves are open, and (2) that during which they are closed. It will be seen that there is a wave immediately preceding the $c$ wave, and it is reasonable to suppose that this is the $a$ wave, due to auricular systole. As has been already pointed out, it is of the utmost importance to observe whether this wave is present in each cardiac cycle, and whether its position is constant from cardiac cycle to cardiac cycle, in order to distinguish it from undulations which may be present in tracings taken from cases of auricular fibrillation. If we now measure the distance from the commencement of the wave $a$ to the commencement of the wave $c$, we determine the $a-c$ interval.

Liver Pulse.-In a tracing obtained from the liver, the $c$ wave is, of course, absent. The liver pulse, like the

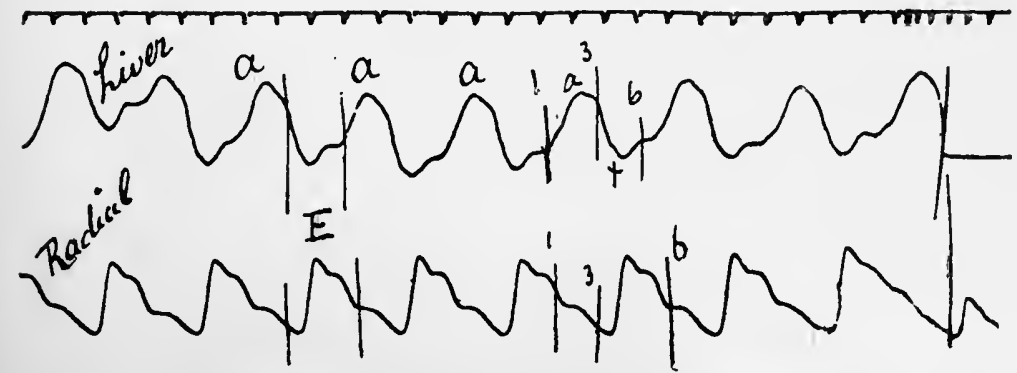

FIG. 72. Simultaneous tracings of the liver and radial pulses. The liver pulse is of the auricular form.

pulse of the jugular vein, may be one of two types-(1) the auricular form, in which the $a$ wave is present (Fig. 72), and (2) the ventricular form, in which the $a$ wave is 
absent (Fig. 73). When the liver pulse is of the auricular

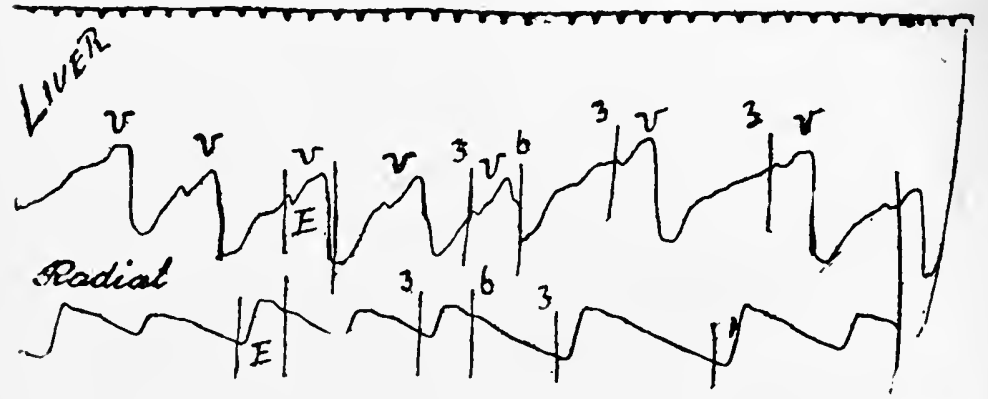

FiG. 73. Simultaneous tracings of the liver and radial pulses. The liver pulse is of the ventricular form.

type, according to Mackenzie, we may infer that tricuspid stenosis in all probability exists. 


\section{CHAPTER VI}

\section{X-RAY EXAMINATION-GRAPHIC RECORDS OF HEART SOUNDS-THE ELECTRO-CARDIOGRAPH}

X-Ray Examination.-Examination by the Röntgen rays sometimes affords useful information in the diagnosis of diseases of the heart. It may be of service in determining the position, size, and shape of the organ, and the effect of respiration on these; and it may furnish information regarding systole and diastole, and the changes in size and shape brought about by them. This method of examination is of special value in the diagnosis of morbid conditions in the immediate neighbourhood of the heart. As the shadow thrown upon the screen or plate by the heart or blood vessels is actually larger than these, correction is necessary, and this is done by means of an instrument called $: \mathrm{n}$ orthodiagraph. The reader is referred to special text-books on the subject.

Graphic Records of Heart Sounds.-Within recent years efforts have been made to record the heart sounds graphically, and with varying success. Among others may be mentioned the method devised by Huerthle, by means of the use of the microphone, in conjunction with an electro-magnet, the vibrations being finally recorded by means of an ordinary registering tambour. Einthoven also employed this method, catching the sounds from a specialised stethoscope upon a microphone, the vibrations in this case being recorded directly by means of a string electrometer. In both these methods, it is essential that the microphone be kept free from the slightest vibration. 
Neither Weiss nor Frank uses the microphone. In the case of both of these observers, the vibrations of a membrane set in motion by the heart sounds are utilised, being magnified and recorded photographically. Crehore's method for the registration of the heart sounds by means of the micrograph is laborious, but is said to be exceedingly delicate.

The Electro-cardiograph.-As in the case of the clinical polygraph, by means of the electro-cardiograph we are able to obtain records of the movements of both the auricles and ventricles, and also to make out the timerelations of the contraction of these chambers. It has been pointed out in the preface that as the electro-cardiograph cannot be regarded as an instrument available to the general praetitioner, and as this book is intended primarily for hin, clinical electro-cardiography is included in one chapter at the end of the book. 


\section{CHAP'TER VII}

HEART FAILURE : ITS SYMPTOMATOLOGY

IN considering heart disease it is of fundamental importance that we should have a true coneeption of the principles which underlic heart failure. It should be understood that the essential cause of eardiae failure lies in the heart muscle, and is due to changes in the heart muscle which render it unable to maintain an effieient circulation, one or morc of its five fundamental functions being impaired. Now, if this view of the causation of heart failure be correct, valvular defects, diseased conditions of the blood-vessels, and disturbances of the cardiac mechanism-sueh as auricular fibrillation-should be regarded from the point of view of the relation which they bear to the myocardium, and not so much as speeifie affections in themselves. Let us take the case of a valvular lesion: First of all, it can be readily understood that it is a mechanieal disadvantage to the heart muscle in its work. But it should always be remembered that there are as a rule along with the valvular lesion coincident changes in the cardiac museulature, the aorta, or eoronary arteries. In all cases of valvular disease, therefore, it is of the utmost importance that we should endeavour to ascertain whether the lesion whieh has invaded the valve has also affected the myocardium, or blood-vessels, or both. These morbid changes of the museulature of the heart may not be limited to one chamber, but may affect all the chambers. They may occur as an acute condition, which later on may become chronic; or as a primary chronic affection. As an example of the former, may be cited 
acute infections of the endocardium of the mitral valve by the rheumatic poison. While theoretically it may be true that in every case of acute endocarditis there is also acute myocarditis, we should always endeavour to ascertain whether the latter is present in a definite or even marked degrec. Now, it is well understood that an attack of rheumatic endocarditis is usually followed by organisation, resulting in the formation of fibrous tissue, which tends to contract as life advanees, and that structural changes ultimately give rise to permanent stenosis, or incompetence, or both. Similarly, in acute myocarditiseither coineident with, or independent of acute endoearditis-the eondition is often followed by permanent structural changes, which, as in the case of endocarditis, may not declare themselves for even many years afterwards.

As an example of a primary chronic affection, may be cited degenerative processes affecting the aortic valve, which come on insidiously, and ultimately give rise to a valvular defect. The question which should always be asked is whether there are also coincident or secondary changes in the cardiac musculature, aorta, or coronary arteries, and, if so, in what degree? As a rule such changes are present.

Another point of great importance is whether the lesion (valvular, myocardial, or vascular) is progressive. In this connection, it may be mentioned that degenerative processes show a greater tendency to be progressive than those changes which follow acute endocarditis.

Now, with regard to the investigation as to whether the myocardium is involved and to what extent, and whether the lesion which is present is progressive, while the results of physical examination-such as evidence of enlargement of the heart-are important, there are other criteria, which are by no means sufficiently appreciated. Of these, the most important of all is the question of the functional efficiency of the heart. Is this impaired, and, if 
so, to what extent, and is the degree of impairment increasing? Mackenzie has shown that the best way to answer these questions is to ascertain how the heart responds to effort; in other words, the amount of physical exertion in which the patient can indulge without experiencing symptoms of cardiac distress.

In conducting this inquiry, it is essential at the outset that two facts should be borne in mind. The first is that the natural standard of the heart's strength varies in individuals, each individual knowing the kind and amount of exertion in which he can normally indulge without producing symptoms of cardiac distress, and being aware that there is a departure from this, and of its extent. Our enquiry should aim at finding out whether the normal standard of a patient has decreased, and, if so, to what extent, and whether it is still decreasing.

The second fact which should be borne in mind is that symptoms of cardiac distress on exertion may be due to causes other than heart disease. Further, the cardiac muscle may be merely temporarily exhausted, and this may occur whether the heart is diseased or otherwise. But, provided extrinsic and temporary causes-such as anæmia, sleeplessness, temporary physical or mental overstrain, deficient tone due to lack of physical exercisecan be excluded, the fact of a departure from the normal standard of an individual, and its degree, may be taken as fairly indicative of the presence and degree of myocardial disease. Further, if the symptoms of cardiac distress on exertion be increasing - in spite of an adequate period of rest and the employment of other remedial methods-and provided again that we can exclude extrinsic and temporary causes to account for this, we may be satisfied that the lesion is progressing, and its rate of progress may be inferred by the rate of increase in the severity of the symptoms.

In the great majority of cases, the first indications of heart failure are symptoms of cardiac distress on exer- 
tion, and it is only afterwards that objective signs-such as increased pulse-rate, dilatation, or dropsy-appear. These symptoms appear as the result of less and less cffort, until ultimately some or all of them are present even during rest, and objective signs also become evident.

Some of the symptoms of cardiac failure are of much grcater importance than others. The commonest are breathlessness, palpitation, fatigue, a sense of tightness across the chest, and precordial pain on exertion.

The evidences of cardiac failure in chronic valvular d.scase and myocardial degencration as a rule appear gradually; in rhcumatic carditis, acute myocarditis in diphthcria, and the rapid cffusion of fluid into the pericardial sac, they are usually rapid in onset; while in the casc of rupture of a valve from over-exertion, and the complete and abrupt closure of the lumen of a coronary artery or one of its main branches, they are usually abrupt.

Breath!cssness or dyspnca is one of the earliest, and a very important, symptom of cardiac failure, the amount of exertion which the patient can undergo without experiencing shortness of breath being of great value in cstimating the state of integrity of the heart muscle. It should be borne in mind, however, that dyspnœa on exertion is a symptom common to many affections besides heart disease, such as anæmia, pulmonary affections, and displacement of the contents of the abdominal cavity. The degree of dyspnoa in heart disease may be so slight that the patient himself may only observe it on over-exertion. Later, it is induced by less and less effort, until ultimately - when cardiac failure is marked-intense dyspnœa is brought on by such slight forms of exertion as a change of position in bed ; it may be present, indeed, without any effort of any kind. The degree of dyspncea may amount to orthopnœa; that is, the patient may be unable to lie down.

A peculiar form of breathlessness which may occur. in heart disease is what is often termed "cardiac 
asthma." This condition is characterised by paroxysms of breathlessness, the attack coming on suddenly, with a sense of suffocation - the intensity of which may be extreme-compelling the patient to sit up in bed, and to breathe in a very laboured fashion; wheezing sounds may be present, and the patient may cough up frothy sputum. After a time, it may be a few hours, the attack quickly subsides. These attacks are frequently nocturnal, and the patient is apt to suffer from what are called " night starts" - that is, he starts up with a sensation of suffocation; this is apt to occur when he is falling off to sleep. When a patient suffers from paroxysms of breathlessness, we should always be careful to exclude renal disease, bronchial asthma, and hysterical dyspnœea.

The syndrome known as Cheyne-Stokes' respiration may occur as a symptom in cardiac failure, and is usually of serious import; it should be distinguished from other forms of periodic respiration, such as are occasionally found in children during sleep, and also in meningitis. It may be present for days, or even for some weeks, and yet ultimately disappear.

While ordinary dyspnœe on exertion may occur in all forms of heart disease, it is more pronounced in mitral than in aortic disease; in mitral disease, indeed, it is usually the earliest symptom noted by the patient. Cardiac asthma and Cheyne-Stokes' respiration are most apt to occur in myocardial degeneration, especially with super-normal blood-pressure.

Palpitation may be a symptom of cardiac failure; but while this symptom may occur in organic disease of the heart, it should be remembered that it is more frequently met with apart from organic disease.

A common symptom of cardiac failure is a sense of exhaustion during or after physical or mental effort; this may even be experienced in slight or very temporary heart failure; in the more serious cases other phenomena also are present. 
Pain in the precordium is a frequent symptom of cardiac failure, being of more common occurrence in aortic disease and in myocardial degeneration than in mitral disease. The most characteristic feature of pain due to heart disease is that it is brought on by exertion-though it should be noted that the pain may not come on until some hours after the exertion which has induced it. It may be constant, dull, and aching; or recurring and sharp; or may present the characteristic features of angina pectoris. Its severity varies greatly. While pain in the precordium may occur in organic disease of the heart -and, indeed, is present some time or other during the course of most cases of the severer forms of cardiac disease-it should be remembered that it is also frequently met with apart from organic disease, especially in females.

I he site of the pain is frequently in the neighbourhood of the apex-beat, but it may be over any part of the precordial arca. The pain of angina pectoris, on the other hand, is almost always over the sternum; only rarely is it on one side of the chest or the other.

Accompanying pain in the precordium, or occurring independently of it, there may be a sense of constriction or oppression of the chest, and tenderness in the precordium may also be associated with it.

Cerebral symptoms, due to a diminished supply of blood to the brain, may be indicative of cardiac failure; they are more generally met with in aortic disease and in myocardial degeneration than in mitral disease. Giddiness is not an uncommon manifestation of cerebral anæmia, being most frequently met with in elderly people with arterio-sclerosis and myocardial degeneration. It may be slight and transient, or severe enough to cause the patient to fall. It should be noted that attacks of giddiness so frequently complained of in old people have no necessary relation to cardiac failure.

Syncope or fainting attacks also may occur in patients 
suffering from cerebral anæmia depending upon cardiac causes. These attacks are preceded as a rule by a preliminary sensation of great weakness, giddiness, loss of sight, and, it may be, nausea and vomiting; while during the attack the face becomes of a greenish pallor, the patient breaks out into a clammy sweat, respiration almost or completely ceases, and the pulse at the wrist is very feeble or even imperceptible; the return to consciousness is gradual. While syncope may, and frequently does, occur in heart disease, especially in aortic disease and myocardial degeneration, it is probably as frequently due to some temporary and functional derangement. It may be occasioned by purely reflex disturbances, is apt to occur in crowded and badly ventilated rooms, while it is caused in some individuals by unpleasant sights or certain odours. It is more common in females. In those who are subject to attacks of faintness, it will be found that the normal increase in the pulse-rate on assuming the erect posture is not infrequently increased, as the result of vaso-motor derangement.

Attacks closely resembling petit mal may occur in elderly people, espccially in those with arterio-sclerosis and myocardial degeneration, and in the early stages of heart-block, and it is sometimes extremely difficult to distinguish them from fainting attacks. The distinguishing features of the latter have already been described.

Adams-Stokes' syndrome may occur when the bloodsupply to the brain is completely arrested. The most common cause of this is heart-block, but it is occasionally met with in bradycardia due to other causes.

Among other symptoms of cerebral anæmia which may occur are lack of power of concentration, early fatigue on mental effort, impairment of memory, headache, sleeplessness, disagreeable dreams, nervousness, loss of emotional control, mental irritability, twitchings, and, in severe cases, delirium, hallucinations, or even insanity.

In heart disease, especially in affections of the mitral 
valve, there is often a persistent dusky flush over the cheeks, while later therc may be lividity of the lips, ear-tips and chceks, and clubbing of the finger-tips may be present in chronic cases. In aortic disease, on the contrary, the face is generally pallid and anxious, though in some cases the colour may be high; while, later, when relative incompetence of the mitral valve has become established, the facics resembles that which is characteristic of mitral cases. An icteric change of the skin may also be present in cardiac failure.

Loss of flesh may be present in cardiac failure, and, when heart disease occurs in the early years of life, development may be arrested.

Digestive disturbances are not uncommon in, and may give early indications of, cardiac failure, being due either to deficient blood-supply or chronic venous congestion of the alimentary organs, usually the latter. These may take the form of loss of appetite, a sense of fulness and oppression after meals, pain in the epigastrium and between the shoulder-blades, nausea, vomiting, gastric and intestinal flatulence, and constipation or diarrhœa. Hæmatemesis and melæna are of rare occurrence, and, if severe, are usually due to associated hepatic disease. When tricuspid regurgitation and venous engorgement have become established, there may be tenderness over the liver, which may be palpable, having a sharp edge and smooth surface, and may pulsate. Later, jaundice and ascites may develop. The spleen may be increased in size, though it is generally smaller than normal, unless there is infarction or chronic septicæmia. There may be menorrhagia or metrorrhagia, due to congestion of the uterine mucous membrane.

Edema is an important manifestation of cardiac failure, and is more liable to occur in mitral than in aortic lesions. It usually commences in the most dependent parts, and generally the patient first notices some puffiness round the ankles in the evening. Even in severe 
and prolonged cases, the œedema is often confined to the lower extremities; but, occasionally, especially in children, it is first observed in the face, and, when this is the case, renal disease is often suspected. Towards the final stages it may become general, even when the kidneys are unaffected. Edema may involve the serous membranes, with resultant ascites, hydrothorax, and occasionally hydropericardium. In mitral stenosis, it is not very infrequently limited to the peritoneal cavity.

The urine may be scanty, high-colourcd, exhibit an excess of urates, and may contain albumin, or even blood.

Affections of the respiratory system, such as bronchitis and broncho-pneumonia, are liable to occur in heart disease, especially in mitral affections. Hæmoptysis is not uncommon ; mitral disease, indeed, especially stenosis, is one of the commonest causes of blood-spitting, and the heart should invariably be examined when a patient presents himself with a history of hæmoptysis. Hæmoptysis occurring in chronic valvular disease may be the result of rupture of capillaries or pulmonary infarction. There may be chronic venous congestion and odema of the lungs, particularly of the bases, and, later, hypostatic pneumonia. More rarely, pulmonary infarction may be present. The existence of pleural effusion should always be borne in mind in cases of dyspnœa, for not infrequently $\mathrm{I}$ have seen it completely overlooked; when present, it is generally bilateral. Acute suffocative œdema is rarely met with; in these cases breathlessness comes on suddenly, and is accompanied by the appearance of frothy mucus which wells out of the mouth and nose in quantities, the patient usually dying within a few hours of the beginning of the attack.

Embolism, as well as arterial and venous thrombosis, may be an expression of cardiac failure.

With regard to the pulse, while increased frequency is the rule, bradycardia is occasionally to be noted in cardiac 
failure; the volume and force of the pulse may become diminished. The arterial pressure varies, and is, therefore, not a reliable indication of cardiac failure; it may be subnormal, but, on the other hand, it may be even supernormal until shortly before death. The apex-beat is usually displaced outwards, may be diffuse, and difficult or even impossible to locate. On percussion, the area of cardiac impairment is generally increased transversely to the right, or the left, or both. On auscultation, the cardiac sounds are, as a rule, found to be weaker and may be almost imperceptible, and galop de rhythme may be present.

Distension and pulsation of the jugular veins and other indications of tricuspid incompetence (to be described later) may be present.

Lastly, sudden death may occur, even without previous indications of cardiac failure. 


\section{CHAPTER VIII}

\section{PROGNOSIS IN CARDIAC AFFECTIONS}

Prognosis in cardiac affections is a subject of the utmost importance, and often presents great difficulties. From the nature of the ailment, the reasons for the importance of prognosis are not far to seek. Among these are the fact that the patient and his friends are naturally anxious for, and have the right to, an opinion, based upon a full consideration of all the circumstances of the case, as to the probable duration of life, the ability of the patient to lead within limits a fairly normal existence, and perhaps also his capacity for employment. This is a very natural anxiety, which if unrelieved may have a most injurious effect upon the general health and well-being of the patient. It is a misfortune that in the popular view there is a tendency to group together all classes of cardiac affections as necessarily of serious import, the result being that in a considerable proportion of cases quite needless anxiety may be occasioned.

Now, in trying to form a prognosis in any given case of cardiac disorder, the following points, among others, require consideration : the family history, age, sex, occupation, mode of life, social conditions, the locality, the nature, extent, and mode of origin of the lesion, its duration, the condition of the myocardium, whether the lesion is stationary or progressive, the degree of cardiac failure, the risk of sudden death, the immediate cause of the present attack, the number, if any, of previous attacks, the state of the patient's general health, the existence or 
otherwise of complications, and finally the response to treatment.

With regard to the family history, we should particularly note whether the other members of the family have suffered from any form of cardiac-vascular disease, the average period of longevity, and the constitutional strength of the family.

The question of age is one of considerable importance, the prognosis of heart disease being less favourable at the extremes of life. This in early life is owing to the fact that the heart is less able to undergo compensatory changes during the period of active growth, while any form of cardiac affection has a deleterious effect upon the general physical and mental development; moreover, rheumatic endocarditis is not infrequently accompanied by pericarditis, and there is also the risk of recurrence of rheumatic infection of the organ. Sudden death, on the other hand, is of rare occurrence. At the other extreme of life, the eapacity of the heart for compensatory changes is much diminished, while degenerative processes, and associated disease in other viseera, are not infrequently present.

Taking all cases of cardiac affections into account, the prognosis is better in females than in males. Among other reasons for this is the fact that the former are less subject to physical and mental strain, in them mitral disease is relatively more common than aortic disease, and coronary disease and myocardial degeneration are relatively of less frequent occurrence. On the other hand, mitral stenosis is much more common, and the strain at puberty is greater in females than in males, and, further, the risks of pregnancy and parturition have to be considered. Any occupation or mode of life involving undue physical or mental strain or exposure, and unhealthy habits, such as over-eating or over-drinking, render the prognosis less favourable. The prognosis is considerably modified for the worse also if the patient be unable, when this is 
found to be necessary, to change an unfavourable occupation for one more suitable; or if he be compelled to live under unsatisfactory hygienic conditions as to food, fresh air, and the opportunity for rest so necessary in those suffering from cardiac affections; or, if after suitable treatment has been instituted, a return has to be made to an unfavourable environment.

There appears to be no doubt that taking cases as a whole, the prognosis of aortic is less favourable than that of mitral disease, and lesions affecting both valves graver than when one only is involved. It is a matter of no small difficulty to give the order of relative gravity of lesions affecting the different valves, but so far as my observations of four varieties of chronic valvular disease are concerned, I should place in order of gravity aortic incompetence, mitral stenosis, aortic stenosis, and mitral regurgitation. With regard to aortic stcnosis, however, a qualification is necessary; for although when due to a previous attack of acute endocarditis the prognosis is better than that of mitral stenosis, when degenerative processes are responsible it is not so good, owing to the frequent association of myocardial degeneration.

Among the points which may be of assistance in trying to form an estimate of the extent of the lesion are how far the character of the pulse is modified, and the size of the heart. It should be noted that the severity of pain in the precordium is not necessarily indicative of the nature or severity of the morbid changes present; for the pain may be severe in patients who are the subjects of slight lesions, and, on the other hand, may be of trifling severity in those who suffer from grave lesions.

Taking cardiac cases as a whole, when the lesion is due to a previous attack of acute endocarditis the prognosis is not so serious as when due to degenerative processes; because in the latter case the lesion is apt to come on after middle life and to be progressive, and to be asso- 
ciated with myocardial degeneration. It should, however, not be forgotten that when the lesion is due to a previous attack of acute endocarditis the patient is liable to recurrent attacks and consequent further crippling of the valve. If the lesion have existed for a considerable time and the health of the patient be still good, the outlook is correspondingly hopeful.

In any estimate of the prognosis of a case of cardiac disorder, it is of the utmost importance that the condition and degree of the integrity of the myocardium, and also whether the lesion is stationary or progressive, should be determined ; both of these subjects have been discussed in the preceding chapter.

The degree of cardiac failure is, of course, of great importance, and its estimation is dealt with elsewhere. It may, however, here be pointed out that the nature and severity of the subjective symptoms, the rate-both while at rest and after physical excrtion-the volume and force of the pulse, the strength of the apex-beat and of the impulse of the right ventricle, the existence or otherwise of secondary dilatation, the character of the first sound, whether murmurs have diminished in intensity, the respiratory rate, the extent of œdema, and the size of the liver, have all to be noted.

With reference to the risk of the occurrence of sudden death in cardiac affections, a fatal issue in this way is most likely to occur in fatty or fibroid degeneration of the myocardium; it is also of not uncommon occurrence in angina pectoris; while of the valvular diseases it is most frequent in aortic incompetence. Another cause of sudden death is complete and abrupt closure of the lumen of a coronary artery, or of one of its main branches.

With regard to the immediate cause of the present attack, inquiry should be made as to whether this can be attributed to some temporary cause, such as a period of undue physical or mental strain, or some such intercurrent malady as acute bronchitis, or whether the symptoms 
have gradually appeared without any such cause; in the latter event, the prognosis is less favourable.

The prognosis is also influenced by the state of the patient's general health, and the existence or otherwise of complications, among the latter being anæmia, bronchitis, super-normal blood-pressure, and renal disease. The bearing upon prognosis of the supervention of auricular fibrillation, or of auricular flutter, or the occurrence of pulsus alternans, in any case of valvular or myocardial disease, will be considered in the chapters on these affections.

Finally, the prognosis in cardiac affections must be considered in the light of response to treatment. For example, given a case of auricular fibrillation, with indications of severe cardiac failure, it is impossible to say what the future will be until full doses of digitalis have been administered for a week or two; if there be no response the outlook is unfavourable, whereas in proportion to the rapidity and completeness of the response so is the outlook favourable.

Having now reviewed the various points which may arise in the consideration of the prognosis of most cardiac affections, these need not be again referred to when the prognosis of individual cardiac affections comes under discussion. 


\section{CHAPTER IX}

\section{TREATMENT IN CARDIAC AFFECTIONS}

Importance of Treatment in Cardiac Affections.-In few departments of medicine is careful and skilful treatment of so great importance as in that of cardiac disordcrs, and in few is it so wcll rewarded. For although we cannot rectify the actual lesion in the case of a damaged valve, or myocardium, it is none the less true that in a large proportion of cases it is possible by the adoption of proper and adequatc therapeutic measures to prolong life for many years, and in most cases to save patients from much suffering. Moreover, the work which has been done in recent years in the realm of cardiac disorders has resulted in a great advance with regard to what can be accomplished in the large class of patients who are the subjects of auricular fibrillation. At the outset, therefore, I would emphasise the fact that the practitioner will be amply rewarded for any time spent and patience exercised in considering and putting into practice suitable therapeutic measures for any given case of heart disease which may come under his care.

It is desirable to devote one chapter to the consideration of the therapeutic measures which may be applicable to any form of cardiac disorder, in order to save much needless repetition when we come to consider the treatment of the individual cardiac affections.

Objects of Treatment.-If the view advanced in Chapter VII that the essential cause of cardiac failure lie in the heart muscle, certain points of fundamental importance with regard to the management of any case 
of heart disease can readily be grasped. We should focus our attention on the heart muscle. On the one hand, every factor which has an influence in strengthening it should be taken advantage of, while, on the other hand, everything which may throw an undue strain upon it should be considered carefully and in detail. Our objects should be: (1) To avoid everything which may add to the gravity of the already existing lesion, or embarrass the heart ; $(2)$ to do everything in our power to promote the efficiency of the myocardium; (3) to treat cardiac failure when this be present; and (4) to treat symptoms as they arise.

Instructions regarding Mode of Life.-Given a cardiac affection, whether valvular, myocardial, or arterial, one of the most unfortunate of things we have to face is the fact that such a large proportion of patients endeavour, or are compelled, to live beyond the limits of the heart's strength ; a constant strain is thus thrown upon the organ. It is of the utmost importance that the patient should acquire the habit of always living within the limits of the heart's strength, and should on no account attempt to go beyond it. The medical attendant will have done the greatest service to his patient if he succeed in helping him in this respect. In this matter, in my opinion, it is best to be perfectly frank with the patient. He should be told in as judicious and tactful a way as possible that there is a cardiac defect which cannot be renoved, and that he must not attempt to live as though his heart were normal. At the same time, he should be made to understand that, if only he will be content to curtail his physical and mental activities in sufficient degree so as to keep within the boundary of the heart's limited strength, he will be amply rewarded. What instructions, then, should be given to a patient suffering from heart disease? How far should he be allowed to live his ordinary life? It is of vital importance that there should be an abundance of rest in his daily life. 
There should be no shortage of physical and mental recreation, an absence of undue muscular or mental strain, and, as far as possible, a generous allowance of holidays. It is not enough to regulate the amount of physical exertion only; the importance of attending to the amount of mental work in these cases is not sufficiently appreciated. Severe mental exertion, and every form of excitcment, anxiety, worry, and emotional strain should, as far as possible, be avoided. On the other hand, care should be taken not to restrict unnecessarily the normal pursuits of a patient; otherwise he is apt to become unduly nervous and introspective. Mental occupation helps him to avoid brooding over his condition. These points should be borne in mind in selecting an occupation for a patient; or, if he have already chosen one, it may be found necessary to advise him to change his calling.

With regard to the amount and kind of physical exertion which a patient suffering from heart disease may undertake without harmful effects, it is impossible to lay down hard-and-fast rules, because the constitution and temperament of different patients differ greatly. One patient, for example, can cycle but cannot play tennis, without untoward results, while in the case of another the reverse may hold good. It is possible, however, to enunciate a cardinal principle which will be found to be of inestimable service in these cases. It is, that any exertion which the patient may undergo should not be attended or followed by breathlessness, palpitation, fatigue, a sense of tightness across the chest, or precordial pain. On the other hand, any exertion short of producing these symptoms is usually not harmful, provided violent and sudden effort be always avoided. When exercise is taken, it should be entered upon gradually, and in going up-hill or upstairs it is better for the patient to proceed more slowly than when walking on the level. But, while undue physical exertion should be avoided, a sufficient amount 
of exercise, on the other hand, is in every way desirable. As a rule, walking on the flat is preferable to hill-climbing, but in some cases a carefully regulated plan of hill-climbing is beneficial. In either case, the exertion should not produce any of the symptoms of cardiac distress already noted. When the cardiac affection is progressive, the amount of effort, physical and mental, must be correspondingly reduced.

If the patient suffer from an unduly excitable nervous system, this should receive careful attention; the bromides are often extremely useful, but their long-continued use is not to be encouraged. The ammonium salt is less depressant than the potassium salt, and may be given in doses of 10 to 20 grains, three times a day after food. It is difficult to exaggerate the importance of sleep, and invariably enquiry should be made regarding its amount and character, and if any defect be found it should be energetically and skilfully treated on the lines laid down later on.

A patient with a cardiac affection should lead a quiet and carefully regulated life, avoiding excesses of every kind, more especially in the use of tea, coffee, alcohol, tobacco, and in sexual intercourse. It is better on the whole for the patient to abstain from alcohol altogether; but, if he have been accustomed to alcohol, and its deprivation be a great hardship, a strictly moderate amount of light wine, or well-diluted spirit, taken with meals, may be allowed.

Diet.-With regard to diet, the intake of highly nitrogenous food should be restricted, because it increases the resistance in the peripheral circulation. The three meals of the day should be evenly balanced, as a large meal causes too great a variation in the vascular system, to which the heart does not readily adjust itself. In most cases, the daily amount of fluid should be somewhat restricted, our aim being to lessen the amount of work done by the left ventricle, by diminishing its output. 
The meals should be as dry as the patient will take them, a sufficient amount of fluid being taken between meals. The food should be nutritious, but at the same time easily assimilable and not likely to cause indigestion. A diet of carbohydrates is bulky, and is apt to cause flatulence and hyperchlorhydria, while a diet consisting of highly nitrogenous food increases the resistance in the peripheral circulation and is equally to be avoided; while, therefore, the diet should be a carefully mixed one, the albuminous elemer.t should predominate. Anything which causes flatulent distension of the stomach or bowels should be avoided, since it, by direct pressure, embarrasses the heart, and is one of the commonest causes of palpitation. Articles of food which the paticnt knows by experience to cause indigestion should, of course, not be taken. In the opinion of most, a salt-free diet is of value as an aid in preventing senile changes in the arteries, and in the treatment of arterio-sclerosis and super-normal bloodpressure.

The patient should be carefully instructed to eat slowly and thoroughly masticate the food, and, where necessary, the teeth should receive attention in order to allow of this. Constipation should be avoided, for the retention of fæcal matter in the large intestine gives rise to flatulent distension. In order to obtain an efficient removal of waste products, not only the bowels, but the functions of the liver and kidneys, and the action of the skin, should be regulated.

A gravelly or sandy soil, where the rainfall is below, and the sunshine is above, the normal, is best for those suffering from heart affections. It is important to try to prevent attacks of bronchitis and other affections of the lungs. Woollen underclothing should be worn, the extremities especially being warmly clad, and as far as possible the patient should avoid exposure to inclement weather; in this connection, it may be preferable for him to winter abroad, or at some dry and sheltered place in this country. 
It is important also to guard against rheumatism in valvular disease, especially when of mitral origin, and particularly so in children or young adults. Woollen or flannel underclothing should be worn both summer and winter, and damp feet and damp clothes are to be avoided. The difficulty attending the diagnosis of rheumatic carditis, and the importance of this condition, are dealt with elsewhere. In all cases of heart disease, prophylactic measures should be adopted with regard to the risks of infection by influenza or other intercurrent diseases. An attack of bronchitis or other affection of the lungs, influenza, or other febrile disease, should be most carefully and energetically treated, and a sufficient amount of rest during convalescence should be enjoined. When exertion is again allowed, it should not be such as will produce any symptoms of cardiac distress; if it do, the amount should be diminished. Any existing anæmia in patients suffering from heart disease requires to be effectively treated by a generous supply of red meat, and iron with arsenic. Patients who exhibit a gouty tendency should have their diet regulated, and take an occasional dose of blue pill, followed by a saline aperient.

In adults who are the subjects of heart disease, a course of such tonics as iron, arsenic, phosphorus, strychnine, or quinine, may be taken from time to time; while in children, cod-liver oil, the syrup of phosphate of iron, or the syrup of iodide of iron are of especial advantage.

Rest.-Needless to say, rest is of cardinal importance in the treatment of cardiac failure ; it is difficult, indeed, to exaggerate its value. It is not sufficiently appreciated that cardiac rest is the objective of all therapeutic measures. I have no doubt that not infrequently improvement thought to be due entirely to some other therapeutic agency is, in reality, partly or wholly the result of bodily rest. The importance of resting the heart will be better understood when it is remembered that after cardiac 
systole, the power of contraction of the heart is for a brief period abolished, and when the diastole is correspondingly prolonged the succeeding contraction is more powerful ; in other words, the contractile power of the heart is in direct ratio to the period of rest. While bodily rest is indicated in all cases of cardiac failure, it is, in my opinion, of greater value in those cases in wlich auricular fibrillation is not present than when it is ; indeed, in the former group of eases rest easily holds the first place among the therapeutic agencies at our disposal.

In determining the degree to which exertion should be curtailed, a cardinal prineiple, as has already been pointed out, is that the exertion in which the patient indulges should fall short of producing symptoms of cardiac distress. When there are indications of progressive exhaustion of the heart's strength, the amount of effort should be proportionately reduced, and when the symptoms of cardiac failure are pronounced it will be necessary to order the patient to keep io his bed.

Systematic Exercises.-Systematic and graduated exercises are sometimes useful in cardiac affections, provided they be judiciously and carcfully employed, and their effects watched. They are of special value in cases of slight cardiac failure due to the heart muscle being flabby and lacking in tone, as in fatty infiltration ; in these cases they may prove a valuable aid to treatment, but it is essential that the myocardium should be fairly sound. Graduated exercises are contra-indicated in acute affections of the heart, in considerable myocardial degeneration, in cases of severe failure-as, for example, when there is severe dyspnca on exertion, dropsy, enlarged liver, or angina pectoris-and in cases in which the heart failure is progressive. In my opinion, simple movements are, certainly in most cases, better than the resistance exercises as practised at Nauheim, and of the former I am inclined to favour Swedish exercises. Exercises may be indulged in even when the patient is confined to bed, 
but in all cases should stop short of producing any indications of cardiac distress.

Baths and Spa Treatment.-It is commonly known that immersion of the body in water may exercise a decided influence on the circulation. It is, further, believed by some that certain waters at Nauheim, on account of their ingredients, possess a specific therapeutic value in cardiac affections, but I am very sceptical with regard to this. Patients undoubtedly often derive very great benefit from a stay at Nauheim and at similar Spas, but this appears to be due to the rest, the change of air, the regular mode of life and exercise, the careful dieting, and other factors. Given the same conditions of life, equally good results would be obtainable elsewhere. Spa treatment is particularly serviceable in patients who have indulged in excesses of various kinds.

Massage.-Massage is useful in many cases. It may be employed when the patient is confined to bed, but in which absolute rest is not necessary, while, on the other hand, ordinary muscular exercise is not advisable.

\section{Drugs}

The proper use of drugs in the treatment of heart disease is a subject of great importance, and has for a long time been the subject of much attention.

It is, unfortunately, true that the reputation of many drugs used in the treatment of cardiac affections does not rest upon trustworthy evidence; by this I mean proof that the drug is a definite causal factor in the improvement of the patient. When a combination of various therapeutic agencies is employed, care should be taken to avoid crediting the administration of a particular drug with results which are in reality due to some other agency, more especially rest, or to the natural course of the malady. In this connection, it should be remembered that many of our beliefs with regard to the action of drugs in disease 
rest only upon experiments upon animals, and animals which are in a normal state of health. Now, it should not be forgotten that the experimentalist, in order to obtain his results within a few hours, usually enploys extremely large doses ; and, further, that the reaction of a diseased heart to drugs is influcneed by the nature of the lesion. For these and other reasons, although it may be proved that a particular drug has a ccrtain action upon a healthy animal, it does not necessarily follow that it has the same action on a human being suffering from some morbid eondition.

Two examples of the faet that the reputation of the actions of many drugs is not reliable may be mentioned. It has been believed for generations that the internal administration of aconite diminishes the heart rate. This belief is expressed in text-books; but, if search be made for convineing evidenee upon which the belief rests, sueh will not be found. A few years ago I conducted an investigation with regard to this matter. ${ }^{1}$ The eases chosen were threc cases of mitral disease, one of combined aortie and mitral disease, one of malignant endocarditis with a high temperature, four of pulmonary tubereulosis, and seven of searlet fever. All the patients were kept in bed. The pulse was counted nine times a day, at exaetly the same hour each day, for some days before, during, and after the administration of the drug. First of all, samples of tineture of aconitc obtained from several firms of chemists were employed; these, on being tested at the University College Pharmacological Laboratory, were in each ease found to be quite inert. Afterwards specimens of aconitina prepared in the laboratory were used, commencing with very small, and gradually inereasing to full, doses. On comparing the reeords of the times during which the drug was being administered with those when it was not, it was found that in no ease was there any reduction in the

1 See a paper read before the Royal Society of Medicine, June 1911. 
pulse-rate. The second example I wish to refer to is the belief hitherto held without questioning by the profession at large that the internal administration of digitalis increases the blood-pressure in man by constricting the peripheral vessels. The result has been that therapeutists are afraid of administering the drug in cases of cardiac failure in which there is super-normal blood-prcssure or degeneration of the arterial wall. Some therapeutists have tried to get over the difficulty by prescribing along with digitalis some appressor, such as sodium nitrite or nitro-glycerine, in order to dilate the vessels and so lessen the arterial tension. It should not be forgotten, however, that the effeets of appressors are but transitory, while the rise in the peripheral resistanee-if it be brought about by digitalis - is practically continuous. Others prefer to employ strophanthus in these eases, because of the prevailing view that it has little or no effect in constricting the peripheral vessels. Now the belief that digitalis raises the blood-pressure by causing arterial constriction restswith the exception of Sir Lauder Brunton's observations by means of the sphygmograph upon himself-so far as I am aware, entirely upon experiments upon animals which were in normal health. But it has been shown, from investigations conducted at the bedside, ${ }^{1}$ that, judged by the methods in use for observing the blood-pressure clinically, the internal administration of digitalis does not raise the blood-pressure in man.

A problem which has been notoriously a source of difficulty and perplexity to the clinician is the extraordinary difference in the rcsults of the administration of digitalis or one of its allies in persons who are suffering from identically the same lesions, and complaining of precisely the same symptoms. As long as the drug has been in use in the treatment of cardiac affections, it has been

1 See papers in the British Medical Journal, September 21, 1912, and September 13, 1913, and Transactions of the Seventeenth International Congress of London. 
known that the results of its administration have been astonishingly varied; in eome eases of cardiac failure it is of very great value, while in others it is quite ineffective. As an example of this, let us suppose three patients are suffering from preeisely the same degree of eardiac failure and from identical lesions, and the same dosage of digitalis is employed in each case. In one, perhaps, the results are extraordinarily good, the drug seeming to act like a eharm; in the second, the beneficial effect produeed may be only moderate or slight; while in the third case, absolutely no good result whatever can be detected. What is the cause of this variability? Is the drug caprieious in its action or uncertain in its strength? Or, is it due to some partieular preparation being aetive while another preparation is inert? This has been thought to be the ease by some, and for this reason speeial preparations have been prepared and advertised as being more uniformly eertain in their action. These opinions have been proved to be wrong. It is of great importance to the praetitioner to realise that this problem of the variability in the results of the administration of digitalis has been to a great extent solved. Put briefly, those cases of cardiac failure which show wonderfully good results are in the vast majority of instances cases of auricular fibrillation.

The Digitalis Group of Drugs.-This includes, among others, digitalis, strophanthus, squills, convallaria majalis, and apoeynum. The special indieations which are commonly aceepted for the administration of digitalis are increased pulse-rate-say over 80 per minute-weakness of the contractile foree of the heart musele, cardiac dilatation, and anasarca, the cardinal indication being failure of tonieity. The drug is more generally useful in mitral than in aortic cases, especially with scanty, high-coloured urine, and with dropsy; in all probability, the explanation of this is that auricular fibrillation is much more common in mitral lesions. The drug is often useful when 
mitral regurgitation has become super-added to aortic disease, and also in many cases of fibroid degeneration of the myocardium, but in the latter group of cases the effects should be carcfully watched.

The question is often asked whether digitalis should be given in cases of aortic incompetence. There are divided opinions on this point. A supposed disadvantage of the employment of the drug in aortic incompetence is that slowing of the pulse-if it occur-by prolonging diastole, allows more time for the distension of the left ventricle by the regurgitant current of blood. It should be pointed out, however, that slowing of the pulse may occur independently of the use of digitalis. It is further urged that the drug tends to cause sudden death in this affection. Patients have undoubtedly been known to dic during its administration, but it does not necessarily follow that digitalis is responsible for this, for sudden death is apt to occur in aortic disease whether patients are taking the drug or not. In order to prove the contention that sudden death is due to the use of digitalis, there should be no doubt that a relatively larger proportion of people suffering from aortic disease die when taking the drug than in the case of those in which it is not being used. I am not convinced on this point. To be on the safe side, however, the drug should be given cautiously in this condition, and the patient should be kept in bed when considerable doses are being administered; while in those cases in which auricular fibrillation is not present, the drug should be stopped as soon as the acute symptoms have disappeared. Some authorities adopt the same precautions in cardiac failure associated with mitral stenosis, or suspected marked fatty degeneration. Others, again, prefer strophanthus to digitalis in aortic disease.

Digitalis is, as a rule, contra-indicated in cases of undue slowness of the pulse; in these cases, if given at all, it should be cautiously administered. The same precautions apply in the presence of a forcible apex-beat and 
bounding arteries. It is possible to do harm in cases of diminished conductivity, though the drug is not necessarily contra-indicated in these cases. Some writers recommend that prior to the administration of digitalis there should be free purgation. In cases of super-normal bloodpressure, Balfour believed in dilating the arterioles, and those who share this view administer one of the vasodilators along with digitalis or strophanthus. I myself am not optimistic with regard to the efficacy of vasodilators, except perhaps in cases of slight or moderate supei-normal blood-pressure; and, as I have already pointed out, my investigations have shown that there is no risk attending the administration of digitalis in those cases in which there is degeneration of the walls of the blood vessels, or when there is super-normal blood-pressure.

But, while the special indications for digitalis are increased pulse-rate, weakness of the contractile force of the heart muscle, dilatation, and dropsy, the results of its administration when these are present are varied. The remarks which I am now about to make on this subject are based mainly upon a series of investigations in which I was associated with Sir James Mackenzie, Pro. fessor Cushny, and other workers, at the Mount Vernon Hospital for Consumption and Diseases of the Chest, dealing with the action of digitalis and some of its allies on the human heart. ${ }^{1}$ These investigations showed that the response to these drugs differs according to the cardiac action and character of the rhythm; those cases of cardiac failure which show wonderfully good results are, in the vast majority of instances, cases of auricular fibrillation - whether the lesion be valvular, or myocardial, particularly when subsequent to acute rheumatism or chorea.

1 See a paper entitled " Recent Advances in Cardiac Therapeutics" in the British Medical Journal of October 7, 1911, and another entitled "Recent Advances in the Diagnosis, Prognosis, and Treatment of Heart Disease" in the British Medical Journal of March 8, 1913. 
If auricular fibrillation be present, and accompanied by a rapid pulse, whether dropsy be present or not, the results of the administration of digitalis in the majority of cases are very, and in some cases extraordinarily good. In these cases, the administration of the drug is followed by a rapid fall in the pulse-rate (Figs. 74 and 123-128), and a concomitant improvement in the general symptoms. Two exceptions are: some cases of marked myocardial degeneration, and (2) cases in which there is accompanying pyrexia. In the latter, apparently the response to digitalis, if present at all, is as a rule very slight, whether auricular fibrillation be present or not. With regard to dosage : as a rule, a certain amount of improvement may be obtained after a time by the administration of small doses of five minims of the tincture three times a day; but, on the other hand, the full effects of the drug are produced in a much shorter

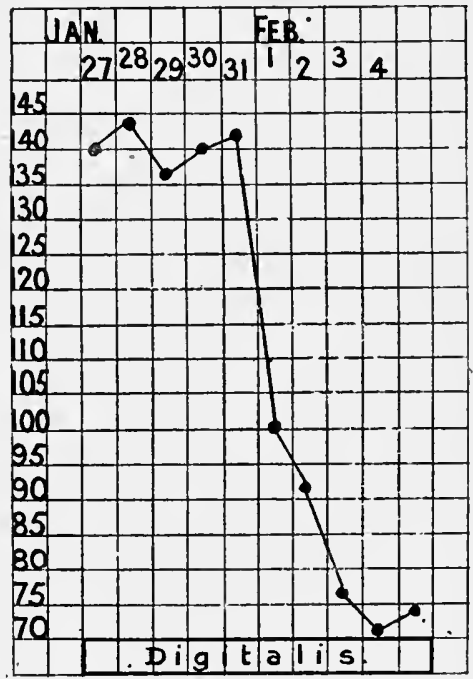

FIG. 74. Chart showing typical result on the cardiac rate of the administration of digitalis in a case of auricular fibrillation with tachycardia. A drachm of the tincture per diem was commenced on January 27 th.

period by large doses. It would, therefore, appear to be desirable to commence with full doses, and to go on with these until some physiological reaction is obtained; and then, if required, to continue the administration, but to regulate the amount until it is discovered what is the optimum dose in a given case. A good method is to begin with a drachm of the tincture per dism, or in urgent cases even one-and-a-half or two drachms per dism, and to continue until there is nausea or vomiting, diarrhœa, 
headache, an unduly slow pulse, or what is called " coupling of the beats" (Fig. 117).

In some cases, the action of digitalis on the heart is manifested before its effect on the stomach; in others, nausea, vomiting, headache, or diarrhœa occurs first; in others again, the cffects on the circulatory and digestive systems are coincident. A physiological reaction usually occur's within a week, sometimes in three or four days; the time, however, varies greatly. The drug should then be stopped until these symptoms have passed away, which is gencrally in a few days. In the great majority of cases the patient relapses, and the indication then is to find out what dose suits him best-that is, the dose sufficient to control the action of the heart, without at the same time giving rise to any toxic symptoms ; this may require weeks or even months of careful observation, and much perseverance will generally be required. Onc method is to bcgin with half the original dose, and this can be increased or dim:nished according as the ratc of the heart increases or diminishes. In this conncction, help may frequently be obtaired from the patient himself, who is often able to say what dose suits him bcst; in other words, the patient's own scnsations are often a good guide, and should be taken into account, as well as the pulse-rate. The dosage necessary in individual cases may exhibit a wide variation; it may bc as small as a drachm of the tincture per week, or as large as 45 minims or even more per diem. Having discovered this dose, it will be necessary, in the great majority of cases, for the patient to continue the drug for the remainder of his life. In the course of time, variations in the dose may be found to become necessary. The cumulative action of digitalis is apparently not nearly so important as has been believed; at the same time, the pulse ought to be watched. "Coupling of the beats" is a danger signal, and we should either stop the drug for a few days or diminish the dose. By this mode of treatment, we are enabled to save many lives and also 
bring comparative comfort to people who would otherwise be the subjects of much misery.

When auricular fibrillation is not accompanied by a rapid pulse, no marked result usually follows the administration of digitalis ; indeed, it is often difficult to be certain how far the improvement may be due to the rest in bed and other measures adopted, apart from the effect of the drug.

If the case be not one of auricular fibrillation, exceptionally the administration of the drug induces fibrillation; but, apart from these cases, as a rule, though not always, the beneficial results will be only moderate, almost negligible, or altogether absent-unless dropsy is present. My custom is to commence here also with large doses, and to continue until there is nausea or vomiting, diarrhœa, headache, an unduly slow pulse, extra-systolcs, or partial heartblock. In some cases, extra-systoles or partial heartblock are at first only observed after exertion; after the temporary acceleration produced by the exertion has subsided, the pulse-rate becomes slower than before the exertion, if the individual be under the influence of the drug, and it is during this period of slowing that extrasystoles or partial heart-block appear. When they have appeared, the drug should be stopped, or the dosage reduced. If improvement in cases without auricular fibrillation occur, it can usually be maintained without continuing the drug; sometimes, however, it is advisable after stopping the drug for a few days to resume with smaller, but yet sufficiently large doses. Here again, the patient's own sensations should be taken into account.

In our investigations, it was found that there was no fundamental difference between the action of digitalis, strophanthus, and squills. As a rule, however, it required nearly twice the dosage of tincture of strophanthus or of squills as of digitalis, but the two former showed considerable variation in the matter of dosage; the effect of their administration by the mouth appears to be 
uncertain. In some cases, strophanthus and squills seemed to have less tendency to induce digestive disturbances, and in a few cases the latter succeeded where the administration of digitalis or strophanthus was followed by. gastro-intestinal symptoms or headache. On the whole, however, from a therapeutic standpoint, digitalis proved to be the most reliable of the three drugs; it is, in the great majority of cases, the most valuable; the others should be regarded as substitutes, which may be tried especially when digitalis appears to induce toxic symptoms.

In no case in which the tincture of digitalis failed did any of the other preparations of that drug succeed. In this connection, it should be remembered that the tincture contains all the glucosides, and that there is no evidence that the more recent preparations of the drug are either pure substances, or are more reliable than the tincture. Nativelle's digitalin granules ( $\frac{1}{240}$ grain, also $\frac{1}{60} \overline{0}$ grain) are a convenient form of the drug. We found that samples of tincture of digitalis supplied by different firms of chemists proved to be very constant in strength; it is advisablc, however, whenever possible, to employ physiologically standardised preparations. The fresh infusion of digitalis is also reliable. The preparations of strophanthus obtained from chemists arc not nearly so constant in strength as those of digitalis. It was found that the active principles of digitalis, strophanthus, and squills not infrequently underwent decomposition when the tincture was freely diluted with water, and this was especially so in the case of strophanthus. For this reason, the tinctures should be dispensed either undiluted, or diluted only with alcoholic preparations, and afterwards taken in water.

Intravenous injections of strophanthin were employed by other workers at the Mount Vernon Hospital, and the results obtained were the same as those following the administration of digitalis and strophanthus by the mouth, 
but the results were much more rapid, sometimes taking place within a few hours. These injeetions may be used in urgent eases of eardiac failure, when it may be advisable to obtain a more speedy result than is possible by the administration of one of the digitalis series of the drugs by the mouth. We may employ one dose of $\frac{1}{100}$ of a grain, or two or three doses of $\frac{1}{2} 50$ of a grain every two hours; this may be followed by the administration of tineture of digitalis by the mouth when advisable. Some prefer intra-muscular injeetions of strophanthin. Hypodermic injeetions of digitalin also are resorted to by some in eases of emergency, especially if nausea and vomiting be present. $\Lambda$ s has been already noted, aconitc had no effeet upon the pulse-rate.

Caffcine has acquired a certain reputation in the treatment of eardiac affections, but this is, in the main, the result of its diuretic aetion, which is of great value. It should not, however, be regarded as a substitute for digitalis, for the action of the respective drugs on the heart is not the same. Apart from its diuretic aetion, eaffeine may be tried when digitalis, strophanthus, and squills have failed, particularly where there is no dilatation. It does not slow the pulse, and, therefore, bradyeardia is no contra-indication to its use.

Diuretin, agurin, and theocin-sodium-acetate have a more constant and marked effeet upon the kidneys than caffeine. They may be used in eonjunetion with one of the digitalis group.

Strychnine has aequired a great reputation in eases of cardiae failure when very rapid effeets are required, but there appears to be no absolutely reliable experimental or elinical evidenee that in medieinal doses the drug has any effeet upon the heart or blood vessels ; nevertheless, many are of opinion that the prevailing belief is supported by clinical experience. The aetion of the drug is probably an indirect one as far as the heart is concerned, its essential action being that of a respiratory stimulant. 
Difjusible stimulants (aleohol, ammonia, and ether) are of value in some cases of eardiae affections, particularly during temporary attacks of eardiac failure, febrile cases, and for the relief of urgent symptoms. With regard to alcohol, it is often a good thing to begin with a small quantity of wine with food. Ultimately, stimulants in larger quantities may be employed, and of these old brandy is the best.

Atropine is supposed to paralyse the eardio-inhibitory terminations, and cases of heart-block have been recorded in which the writers have stated that in their opinion the drug was the means of increasing the ventricular rate. If this be so, it should be of service in Adams-Stokes' syndrome. One-hundredth of a grain may be given hypodermically, and repeated in half an hour if necessary. I am bound to say, however, that $I$ have never yet seen any good result follow its administration in these cases.

Some believe that iron and arsenic are eardiac tonics, and, therefore, do not restrict the use of these drugs to cases of heart disease in which there is co-existing anæmia, but employ them in the early stages of cardiae failure. The late Sir William Broadbent believed that phosphorus was of greater value than arsenic. Iron, arsenic, strychnine, and quinine are often serviceable during convalescence.

The bromides are useful in cases of heart disease in which there is exeitability or irritability of the nervous system, and in sleeplessness. The iodides are said to be of value in gouty conditions, in super-normal blood-pressure, in arterio-sclerosis, in angina pectoris, and, most of all, when there is reason to believe that valvular, myocardial, or arterial disease is the result of syphilis, and they may be taken for long periods of time; in the last group of cases they should be given in full doses (15 to 20 grains three times a day) for some weeks. Mercury may with advantage be administered along with the iodides when the syphilitic infection is recent. With regard to arteriosclerosis, while iodides may exert a beneficial influence in 
the earlier stages of the disease, it is practically certain that they are valueless in the later stages.

The nitrites may be tried in cases of increased bloodpressure, and in angina pectoris-in the latter even if unattended with high pressure. Nitrite of sodium, erythrol tetranitrate, nitroglycerine, and nitrite of amyl are most commonly employed.

Purgatives are of value when there are indications of distension of the right chambers of the heart, and dropsy, and also in cases of super-normal blood-pressure ; salines, administered from half-an-hour to an hour before breakfast, being preferred. Diuretics should be given in cases in which the urine is scanty, especially if dropsy be present. The best diuretics are digitalis, squills, caffeine, diuretin, agurin or theocin sodium acetate, scoparium, and the acetate, citrate, or bitartrate of potassium.

Oxygen.- This is sometimes of value in cases of cardiac failure, the chief indications for its use being dyspnœa, cyanosis, and angina pectoris, particularly the two former. On the whole, I have been disappointed with the results of its administration, though occasionally substantial good has apparently attended its use. Leonard Hill has introduced a new method of giving it, by which the patient inhales almost pure oxygen. The patient's head is enclosed in a mask, the oxygen entering the mask from a cylinder, and the séance lasting from 10 to 20 minutes at a time.

Blood-letting.- This is of considerable value in a certain class of cases, and I am inclined to think that this mode of treatment is often not used when it might be employed with great advantage. It should be employed when there are indications of great distension of the right chambers of the heart-as, for example, severe dyspnœea, cyanosis, noticeable turgescence of the veins of the neck, and increased area of impairment of the percussion note to the right, especially when the radial pulse is small and weak, whilc, at the same time, there is forcible pulsa- 
tion of the right ventricle in the epigastrium. As a rule, venesection should not be performed in aortic incompetence; in these cases it often does harm. My custom is to choose the median basilic vein, and to withdraw a good quantity of blood-occasionally even up to 20 or $30 \mathrm{oz}$. ; sometimes, however, 8 to $10 \mathrm{oz}$. is sufficient. If venesection be not practicable, or be objected to by the patient or his friends, six to twelve leeches over the right ventricle or liver are a good substitute. When blood-letting is employed, it should be accompanied by dry diet and free purgation.

\section{TREATMENT WHEN CARDIAC FAILURE IS PRESENT}

The amount of physical or mental effort should be reduced. In the slighter degrees of cardiac failure, it may be sufficient if the patient remain in bed for a couple of hours every afternoon or one day a weck, although it is usually better to commence treatment by keeping him continuously in bed for a short time. When heart failure is marked, the amount of exertion should be reduced to a minimum, the patient being kept in bed for a period which may extend even to some months. When subject to nocturnal attacks of dyspnœa, he may be most comfortable when supported by high pillows or a bedrest, or he may not be able to breathe with comfort even in bed, and may prefer to sit up in a large easy-chair, leaning forwards and supporting himself with his arms on the back of another chair. A crossbar, suspended by a rope from a bracket on the wall or from the roof, is often useful as an aid to the movements of the body. In certain cases of cardiac failure, exercise in moderation, provided careful and detailed instructions be given as to its nature and exent, is of value.

In cases of cardiac failure unaccompanied by nausea and vomiting, light solids form the best diet, and, as a rule, agree better than fluid food, being less likely to 
cause flatulence; the necessary mastication is also an advantage. The daily amount of fluid taken, particularly at meal times, should be reduced. Specific instruction should invariably be given in regard to thorough mastication, and great care should be exercised to see that the patient does not take a larger quantity of food than he can comfortably digest. The food should be easily digestible, and those articles of food which are found not to agree with the digestive organs should be avoided. When cardiac failure is extreme, the amount of food should be considerably restricted, and only very small quantities at a time, at fairly frequent intervals, should be allowed. Biscuits, small thin sandwiches of potted meat, or small quantities of milk are not infrequently all that can be comfortably digested. Occasionally a pure milk diet, or a modified Salisbury diet, is best. Strict attention to the condition of the bowels is indicated, and, if necessary, resort should be made to the employment of means for procuring sleep. Other therapeutic measures appropriate to the condition should be employed until the requisite effects are obtained; the indications for, and the manner of employment of, each class of these in cardiac failure have already been fully described. When there are indications of distension of the right heart, free purgation, the administration of diuretics, and bloodletting are indicated. Special symptoms should be treated on the lines laid down later on. The return of convalescence should be gradual, and the greatest care should be exercised. Convalescence from severe cardiac failure is sometimes aided at first by gentle massage, and, later, by carefully regulated and gradually increasing exercisesat first passive, and later active-before the patient is allowed to get up. The movements should always stop short of producing symptoms of cardiac distress, and should be followed by a period of absolute rest. 


\section{Treatment of Symptoms}

Rest, and other measures and remedies previously described in detail, should be employed in the treatment of symptoms.

Sleeplessness.-The ventilation of the bedroom should receive attention, and, while the patient should be kept warm, the bedclothes should not be too heavy. It should be remembered that insomnia may be due to dyspnœa, and that in these cases the patient is sometimes troubled with " night starts" as he is falling off to sleep; the dyspnœa should be treated on the lines to be laid down later. Similarly, if super-normal blood-pressure be present, this should reeeive attention. It may be necessary to employ hypnotics in the insomnia of heart disease. I generally begin with the bromides, and usually choose the ammonium salt, on aecount of its less depressant effect, in doses from ten to twenty grains three times a day. Instead of the bromides, sueh mild hypnotics as trional, veronal, medinal, urethane, sulphonal, or chloralamide may be tried. Chl ralamide acts nearly as well as chloral hydras in some, though by no means in all, cases, and is supposed to be safer. Paraldehyde is a rather more powerful hypnotic, and is an extremely valuable drug in many cases, espeeially when there is dyspnœa. Its unpleasant taste may be partly concealed when administered in an almond mixture, or as an emulsion; or it may be given in capsules, each containing $\mathbf{4 0}$ minims. I have found a drachm of tincture of henbane, in a little hot brandy and water, sometimes succeed when these drugs were not successful. If these remedies fail, chloral hydras may be tried. This drug has the reputation of being a dangerous remedy in heart disease, especially in degeneration of the myocardium, but I have used it very extensively and have never found it so. It produces a refreshing sleep, and is especially useful when nocturnal dyspnœa and super-normal blood-pressure are present. 
It may be given in doses of 5-10 grains by the mouth, gradually increasing to 15 or even 20 grains if necessary, the dose being repeated in two hours, and the effects carefully watched all the time.

If chloral fail, resort should be had to opium or morphia, which are especially useful when there are accompanying restlessness and dyspnœa. The hypodermic administration of morphia is much more efficacious in cardiac failure than when given orally, and there are disadvantages attending the latter method. It is well to commence with very small doses, and gradually to increase them until relief is obtained, the effects being carefully watch $c$ d. I generally commence with $\frac{1}{8}$ grain, gradually and carefully increasing to $\frac{1}{4}$ grain if necessary; while, later, it may be necessary to increase the dose even still further. Cyanosis is not an absolute contra-indication to the administration of chloral, opium, or morphia. Speaking generally, these drugs should not be administered in those cases in which there is much bronchial secretion, or œdema of the lungs, or in Bright's disease; but in individual cases they may be tried if the first dose be extremely small, and the dosage be subsequently increased with the greatest caution. Further, when there is much bronchial secretion, or œdema of the lung , a sharp lookout must be kept to see whether the administration of the drug increases them; in such a contingency, atropine or strychnine should be administered in combination with the morphia.

Dyspnœa.-If bronchitis, or gastric or intestinal flatulence be present, they should receive attention; and, similarly, fluid in the abdomen may require to be withdrawn If there be indications of congestion or œdema of the lungs, dry-cupping is indicated, the best time, as a rule, being when the patient desires to go to sleep. The exclusion of hydrothorax as a cause of dyspnoea should always be a matter of consideration, and, when present, paracentesis thoracis should be performed. Oxygen. 
diffusible stimulants, such as ammonia and ether, and strychnine may be tried in dyspnœa. Strychnine undoubtedly has an action on the respiratory centre, and in patients who suffer from " night-starts" and nocturnal dyspnœa may be given in full doses in the evening. Venesection or leeching is sometimes of value. For paroxysmal dyspnœa, vaso-dilators-such as the inhalation of amyl-nitrite, or the internal administration of nitroglycerine, erythrol tetranitrate, or sodium nitriteare sometimes efficacious. When these various measures fail, chloral may be tried, and, if this bc not successful, opium or morphia. The dosage of these drugs and the contra-indications to their use have already been noted under the head of sleeplessness.

Faintness.-Among temporary measures are plenty of fresh air, fanning, warmth to the feet, cold to the head, and diffusible stimulants, such as ammonia and ether.

Edema.--The measures which are of particular value in the treatment of œdema are the restriction of the amount of daily flurd, free purgation, the administration of diuretics, and, in the opinion of most, a salt-free diet. A watery diarrhœa is not necessarily a contra-indication to purgation, bccause it is frequently due to a passive congestion of the intestinal mucous membrane. Besides the usual purgatives, calomel and colocynth pill, or pill hydrargyri, followed by Epsom salts or some other saline in the morning, may be given once or twice a week for a time. The infusion of digitalis has a greater reputation than any other preparation of that drug in the treatment of dropsy, but I am not sure that this is warranted. If digitalis fail, theocin-sodium-acetate, agurin, or diuretin may be added to it. Many people prefer a combination of drugs, and in many cases this method of treatment appears to be more efficacious. Baillie's or Guy's pill, which consists of mercury, digitalis, and squills, is a wellknown combination. It is particularly useful in persistent dropsy due to cardiac failure; indeed, when given along 
with saline aperients it forms the most useful diuretic in many cases of odema of heart disease. Among various prescriptions of this combination are:

R Pilulæ Hydrargyri

Pulveris Digitalis

Pulveris Scillæ

Extracti Hyoscyami, $\overline{\boldsymbol{a}}$. gr. i

Ft. Pil. One three times a day after meals.

R Pilulæ Hydrargyri gr. ii Pulveris Digitalis gr. $\frac{1}{2}$ Pulveris Scillæ gr. i

Ft. Pil. One three times a day after meals.

Another good combination is 5 grains of citrate of caffeine, 20 grains of citrate of potash, and half-an-ounce of infusion of digitalis. It is always best, when possible, to give mercury before commencing the administration of a diuretic, as it appears to start the diuresis.

In early dropsy, bandaging of the lower extremities is often very useful, and this may be combined with massage. When the œdema does not disappear in spite of the measures mentioned, the mechanical removal of fluid should be considered. The means which may be employed are Southey's tubes, acupuncture, and scarification, the first two being better than the third. Whatever method be employed, strict aseptic precautions should be exercised. With regard to acupuncture, the dependent limbs and scrotum may be punctured by means of a sharp broad surgical needle (which is grasped by the finger and thumb from a quarter to half-an-inch from the point), and then wrapped in sterili ed dressings to drain. If ascites or pleural effusion do not yield to other treatment, and cause embarrassment, the fluid should be withdrawn. With regard to the former, Southey's tubes or tapping may be employed; in the latter case, tapping is necessary.

Cough. - The cause of the cough, which may be œdema of the lungs or hydrothorax, should after investigation 
be suitably treated, and relief may be sometimes obtained by the adninistration of hot drinks, and the application of a large mustard and linseed poultice over the bases of the lungs. A linctus may be necessary.

Hæmoptysis.-Hæmoptysis is only rarely a serious accompaniment of heart disease, and may be combated by rest, together with the administration of calomel and saline purgatives. It may be necessary, however, to supplement these measures by the administration of morphia. Venesection or leeching is advocated by some writers in severe cases.

Pulmonary CEdema.-Dry-cupping is sometimes beneficial, and for acute pulmonary odema prompt venesection is indicated.

Vomiting.-Milk is, in the majority of cases, the best food, to which sodium citrate (15 grains to each $10 \mathrm{ozs}$.) may be added. If milk be not tolerated, peptonised milk, whey, albumen water, milk gruel, koumiss, or strong meat essences may be sipped, the patient not being allowed any solid food. If one of the digitalis series of drugs be indicated for the general condition, and the patient be peculiarly intolerant of digitalis, strophanthus, squills, or one of the other substitutes may be tried. A brisk purge should be administered, and bismuth with hydrocyanic acid may be given. Iced champagne is sometimes useful, and counter-irritation over the epigastrium is occasionally of some help for the relief of vomiting. If, in spite of these measures, the vomiting still continue, all food, and even water and drugs, by the mouth should be stopped, and resort be had to rectal feeding. The great advantage in these cases of rectal feeding with milk pancreatised for at least twenty-four hours is not sufficiently appreciated.

Palpitation.-The possible causes of palpitation should be carefully considered and adequately treated; among these, gastric and intestinal dyspepsia, and toxic agents, are especially to be noted. Rest is indicated in some cases, and a belladonna plaster applied to the precordium 
is sometimes efficacious. If these measures fail, the bromides may be given a trial.

Cardiac Pain. - As in the case of palpitation, we should first of all consider the possible causes of cardialgia. Rest is especially indicated when it is obviously due to overstrain or dilatation. Iodide of potassium is sometimes efficacious, even when the pain is not associated with super-normal blood-pressure. A bclladonna plaster applied to the precordium is sometimes beneficial; while sometimes-especially in acute inflammatory conditions-counter-irritation in the form of a mustard leaf, blister, leeching, hot applications, or the use of an ice-bag or Leiter's coil, affords relief. If these mcasures fail, the bromides may be administered, and in the even: of their not being successful it may be necessary to resort to opiates, nepenthe or tincture of opium being tried at first, while if the pain still continue morphia may become necessary.

Tachycardia. - We should first of all review the etiology of the condition, with a view to treatment. The treatment of tachycardia in organic heart disease has, for the most part, been dealt with in the general consideration of cardiac failure. It has been pointed out that, as a rule, the best remedy for cases unaccompanied by auricular fibrillation is prolonged rest, and for cases accompanied by auricular fibrillation digitalis or one of its allies. The question of toxic agents, such as tea and tobacco, febrile states, neurasthenia, and hysteria, as possible causes of tachycardia should always be considered. The bromides, with belladonna, pushed to the limits of tolerance, appear to be of value in some cases of tachycardia.

Having now discussed the various therapeutic measures which may be applicable to any form of cardiac disorder, these need not be again referred to when we come to consider the treatment of the individual cardiac affections : in this way much repetition will be saved. 


\section{CHAPTER X}

\section{FUNCTIONAL DISORDERS OF TIIE HEART}

By funetional disorders of the heart are meant those which oecur independently of any struetural ehange; they are merely disturbanees of funetion, no organic lesion being necessarily present. It should be remembered, however, that any type of functional disorder may be found in assoeiation with organic disease, whether valvular or myocardial, especially if eardiac failure be present; and, further, that a persistent funetional disorder may terminate in organie disease, although not necessarily so. Some writers, on the other hand, deny the existence of functional disorders, being of opinion that in every case some struetural change exists.

Among functional disorders may be included palpitation, eardiac pain-ineluding pseudo-angina, tachycardia, paroxysmal tachyeardia, bradyeardia, the various types of eardiac irregularity, "irritable heart," and " cardiac weakness." Some of these will be dealt with in subsequent chapters.

\section{Palpitation}

By palpitation is meant the conseiousness of the eardiac impact against the chest-wall, and with this there is frequently, but not always, an increase in rate. The term, as used here, does not include the sensations which may be present in extra-systoles, auricular fibrillation, auriculàr flutter, or paroxysmal tachycardia; it will further be. 
restricted to those cases in which the consciousness of the cardiac impact occurs in attacks.

Etiology.-While palpitation may occur in organic disease of the heart-valvular or myocardial-it should be remembered that it is probably more frequently and most characteristically met with apart from organic disease. It is more common in females than in males. The condition is often reflex in origin, as, for example, when the result of dyspepsia-particularly those forms of it which are attended with flatulent distension of the stomach or colon, and in uterine disorders. Among other causes are excessive indulgence in the use of tea, coffee, alcohol, or tobacco; conditions which tend to produce exhaustion or undue excitability of the nervous system, among which may be enumerated puberty, the period of menstruation, the menopause, neurasthenia, hysteria, mental excitement or overstrain, exhausting illnesses, and following the acute fevers. The immediate cause of an attack is frequently emotion or excessive physical exertion.

Symptomatology.-The attack may last from a few seconds to a few hours. It is usually, though not always, gradual in onset, and it passes off gradually. The subjective symptoms vary from a sensation of a gentle impact in the slightest attacks, to the consciousness of a violent one against the chest-wall, accompanied by a sensc of great distress, and even associated with actual pain in the precordium in the severer forms of the attack. In some instances there is a sense of faintness, and more rarely the fear of impending death. Complaint is sometimes made of flushing of the face, tinnitus aurium, a sensation of throbbing in the head, and giddiness.

The pulse-rate is, as a rule, increased, though in some cases there is an absence of tachycardia; it is increased by physical exertion, which is not the case in paroxysmal tachycardia. The pulse may be of moderate or only small volume, and its character may resemble that of the 
pulsus eeler. The large arteries, such as the carotid, may exhibit marked pulsation. The apex-beat is found to be in the normal position, and its area and foree are generally much inereased. On percussion, the area of eardiac impairment shows no increase in size, unless valvular or myocardial disease be present, while on auscultation, the cardiae sounds are found to be abnormally loud, elear, and ringing in character, and the second sound at the base much exaggerated and may have a metallic character. Excepting in cases of organic disease of the heart or anæmia, as a rule no murmur is audible, although occasionally an apieal systolic murmur, soft in character, may be heard during the attack.

In dyspeptic patients, complete or partial relief may coincide with the eruetation of a large quantity of gas. A sense of exhaustion is usually complained of after the paroxysm has passed off.

Prognosis. - The prognosis is good, if no organic disease be present, although it may be difficult to eradicate the cause of the eondition.

Treatment.-This has been considered in Chapter IX.

\section{Cardiac Pain}

As has already been pointed out, pain in the precordium may not only occur in organic disease of the heart, but is also frequently met with apart from organic disease, especially in females. It is often reflex in origin, as, for example, when the result of dyspepsia-particularly those forms of it which are attended with flatulent distension of the stomach or colon, and in uterine disorders. Among other causes are primary cardiac overstrain ; toxic conditions, such as occur in influenza and diphtheria, and as the result of excessive indulgence in the use of tea, coffee, tobacco, or alcohol; neurasthenia, hysteria, emotional states, and sexual excess.

Treatment.-This has been discussed in Chapter IX. 


\section{TACHYCARDiA}

By the term tachycardia is meant frequent action of the heart. It will here be used in a restrictcd sense, not including the frequent action which is often present during attacks of palpitation-which has already been described, or that due to an abnormal rhythm-which will be discussed later on.

Etiology.-The condition may be physiological. It has already been pointed out in Chapter I that in some perfectly healthy individuals the normal cardiac rate is always frequent, even up to $\mathbf{1 2 0}$ per minute or more ; and, further, that the increase in rate induced by physical exertion, even when the individual is in training, is not

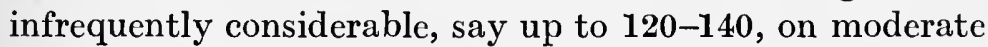
physical exertion. It is difficult, therefore, to decide when an increase of the cardiac rate in an individual is pathological; in this connection, a record of the past history is of value.

The pathological form of tachycardia may be the result of most forms of cardiac disease, primary cardiac overstrain, states of exhaustion, acute and subacute inflammatory conditions, pyrexia, tumour or clot in the medulla oblongata, the pressure of a tumour upon the pneumogastric nerves, involvement of the vagi in bulbar paralysis or in multiple neuritis, some forms of spinal disease, Graves' disease, neurasthenia and emotional states, reflex irritation from uterine or ovarian disease, the use of certain drugs, such as atropine, alcohol, strychnine, or tobacco, and as a sequel of influenza.

In the pathological form of the condition, the increase in the cardiac rate may only be induced by change of posture or other forms of physical exertion ; or it may be persistently above the normal.

Treatment.-This has been described in Chapter IX. 


\section{Bradycardia}

The term bradycardia may be used in two different senses, namcly, it may be restrieted to infrequent action of the heart as a whole, the aurieular rate being diminished pari passu with that of the ventricles; or it may be employed to denote infrequeney of the pulse-rate at the wrist, including that due to extra-systole, partial or complete auriculo-ventricular block, ${ }^{1}$ auricular fibrillation, or auricular flutter. The importance of this latter group of cases as causes of an infrequent pulse must be emphasised. The term will be employed here in the former scnse. The condition may be rccognised either by means of the polygraph, or the electro-cardiograph.

Etiology.-Bradycardia may be physiological, especially in males ; in some perfectly healthy individuals the normal pulse is always infrcquent, a pulse-rate of 50 not being very uncommon, and, indeed, cases have been recorded of a pulse-rate of 40 in a healthy individual. Further, it must be noted that as age advances the rate of the heart normally becomes less frequent.

The pathological form of the condition, which is found more frequently in males, may be the result of some form of eardiac disease-especially aortic stenosis and fatty degeneration of the myocardium, coronary disease, and primary cardiac overstrain; states of collapse or exhaustion, and during the convalescence of acute infectious diseases, especially influenza, diphtheria, pneumonia, typhoid, and typhus ; toxic conditions, such as jaundice, diabetes, uræmia, influenza, and diphtheria, and the use of certain drugs, such as digitalis, muscarine, opium, coffee, and tobacco; hypothyroiaism; affeetions of the nervous system, such as meningitis, cerebral tumour, or apoplexy, diseases of the medulla, diseases or injuries of the cervical

1 It may perhaps be right to point out that some writers limit the use of the term "bradycardia" to cases of partial and complete heart-block. It may also be noted that heart-block has been termed "false bradycardia." 


\section{FUNCTIONAL DISORDERS OF THE HEART 163}

part of the spinal cord, neurasthenia, hysteria, severe pain, epilepsy, melancholia, and general paralysis of the insane ; diseases of the digestive system, such as chronic dyspepsia, gastric dilatation, ulcer, and cancer; in the puerperal state; and occasionally in diseases of the urinary or respiratory system, and in sunstroke.

Sino-auricular block is a very rare cause of bradycardia.

\section{Cardiac Irregularity}

Irregular action of the heart in persons who may or may not exhibit evidence of organic disease of the heart is a subject of the utmost importance. It is a problem that has notoriously been a source of difficulty and perplexity to the clinician. It was known that cardiac irregularity might signify serious impairment or disease of the heart on the one hand, or that it might be of little or no importance; for it was found that some cases lived to the normal span of life without developing symptoms of cardiac failure, while others died, perhaps soon or only after a lapse of some years. The difficulty arose from the fact that until recently it was impossible to determine the significance of the irregularity in any given case. All this is now changed. Owing to the introduction of the clinical polygraph and of the electrocardiograph, the problem has for the most part been solved; for it is now possible to classify the vast majority of cases of cardiac irregularity, and it is known what each type signifies. We have clear and certain grounds, therefore, for purposes of diagnosis, and prognosis, and also for lines of treatment.

All cases of cardiac irregularity, apart from comparatively rare exceptions, fall into one of six groups.: What is called sinus irregularity-a common type ; (2) irregularity due to extra-systole, likewise common ; (3) to depressed conductivity; (4) to the pulsus alternans; (5) associated with auricular fibrillation, which accounts for approximately 50 per cent. of all cases of persistent 
irregularity of the heart; and (6) with auricular flutter. Sino-auricular block is a rare cause.

\section{"Irritable Heart"}

This term may be used in two ways, namely, (1) the " irritable heart of soldiers," first described by Da Costa and Myers, and (2) a condition which is sometimes met with during adolescence. The first of these will be discussed in Chapter XXV. The second condition is met with in badly developed subjects, and is often associated with an unstable ncrvous system and not infrequentlydyspepsia ; it may or may not have becn brought on by excessive or injudicious physical exertion.

Symptomatology.-These may include palpitation, shortness of breath, and precordial uneasiness, or even pain on cxcrtion ; there may be giddiness or faintness. The patient is usually uncomfortably aware of the cardiac action, especially on exertion. The peripheral arteries give the impression of being lax, while thc upstroke of a tracing of the radial artery is found to be quick, abrupt, and high, the dicrotic wave being large. If examined while the patient is at rest, the blood-pressure is usually not above the normal.

The apex-beat may sometimes give the impression of being displaced somewhat downwards and outwards, on account of the shape of the chest; it is unduly visible and palpable, tumultuous, but not heaving, in character. Similarly, the area of transverse diameter of the heart may give the impression of being somewhat increased.: The first sound is shortencd and sharp, while the second sound at the apex is accentuated. A systolic murmur is sometimes audible, often having its point of maximum intensity in the pulmonary area.

Diagnosis.-It is necessary to diagnose this condition from cardiac hypertrophy; in the latter condition, the blood-pressure is above the normal, the apex-thrust is 
slower and longer than normal, and the first sound is long, sustained, low in pitch; and muffled.

Prognosis.-This is good, the patient improving as physical and mental development proceeds.

Treatment.-Judicious, systematic, and carefully regulated exercise, breathing exercises, and all agencies which tend to mental development are of value. The patient should rest before and after meals, all food should be thoroughly masticated, the amount of fluid taken with food should be restricted, and digestive disorders treated if present. Hydro-therapeutic measures have been found to be of use. If the patient be unduly excitable or suffer from insomnia, the bromides may be used.

\section{"Weak Heart"}

The term "weak heart" or "cardiac weakness" is sometimes employed. I cannot agree with its use, and, in my opinion, the only way in which the term might be justified is in the following circumstances : I have already pointed out that the natural standard of the heart's strength varies in individuals, each knowing the kind and amount of exertion in which he can normally indulge without producing cardiac distress, and being aware when there is a departure from this, and of its extent. The term "weak heart" might be legitimately employed to denote those cases in which the standard of the heart's strength in a particular individual is below the normal. Cases other than these should, in my opinion, be classed in one or other of the cardiac affections described in this volume. 


\section{CHAPTER XI}

\section{SINUS IRREGULARITY}

IT has already been pointed out that excitability of the heart is most marked in the remains of the sinus venosus at the orifices of the great veins, and that the rhythm of the sinus governs the rhythm of the remaining segments of the heart. Further, it has been shown that the heart possesses the power of contracting and dilating independently of any extrinsie nervous influence; but, at the same time, the activity of the various functions of the muscle-fibres is under nervous control.

While normally the rhythm of the sinus is a fairly regular one, in by no means a small pereentage of individuals the rhythm is not regular. In all probability this irregularity is of vagus origin, due to an unusual susceptibility of the vagus centre to impulses from distant parts and these impulses being transmitted reflexly to the heart. The chief charaeteristic of sinus irregularity is the variation in the length of the diastolic period of the cardiac cycle, the systolic period remaining constant. The irregularity, which may be slight or marked, diminishes and may even disappear with an increase, while it is apt to increase or return with a diminution of, the cardiac rate. It is usually, though not invariably, abolished within half-anhour of the administration of a hypodermic injection of atropine.

Etiology.-Sinus irregularity is much more frequently met with in the young, being indeed fairly common in young adults; but it may occur at all ages. There is a greater tendency to sinus irregularity in children after a 
febrile illness, and it may be first noticed then. There is usually a definite relationship to respiration. The irregularity is most marked during slow respiration, and may indeed only be noted on deep breathing. The rate of the heart usually gradually quickens during full inspiration, and gradually lessens during full expiration ; but while generally the rate of the pulse waxes and wanes with respiratory movements, in some cases there is an abrupt slowing of one or two beats with expiration. Sinus irregularity may occur during the administration of digitalis, and the act of swallowing even may produce it-the heart-rate during the act being quickened for a few beats, followed by a reduction in the rate.
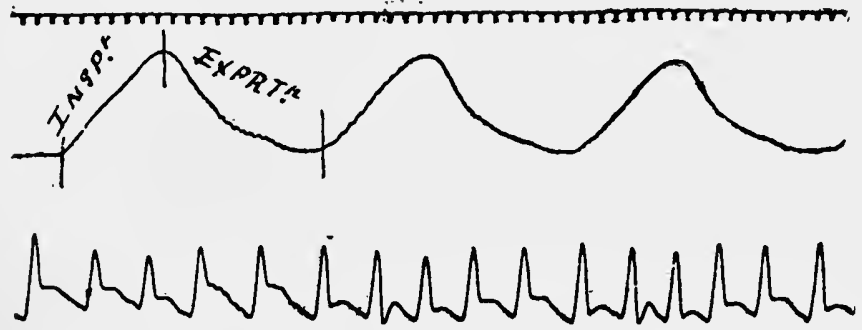

Fig. 75. Simultaneous tracings of the respiration and the radial pulse, from a healthy boy aged 14 , showing sinus irregularity.

Symptomatology.-Usually there are no subjective symptoms of the condition, but very rarely the abnormal pause between any two beats of the heart may be so long that symptoms of cerebral anæmia, such as giddiness or syncope, may occur. On auscultation, the interval between the first and second sounds is found to be constant, while the varying length of the diastolic interval can be detected. The latter can be noted also in a tracing of the radial artery, although the actual variations in the length of the diastole may not be numerous, while the pulse-beats are of equal or nearly equal size (Figs. 75-77). A polygraphic tracing shows that a ventricular contraction follows each auricular contraction in the usual way, 
and that the whole heart is affected by the irregularity (Figs. 76 and 77).

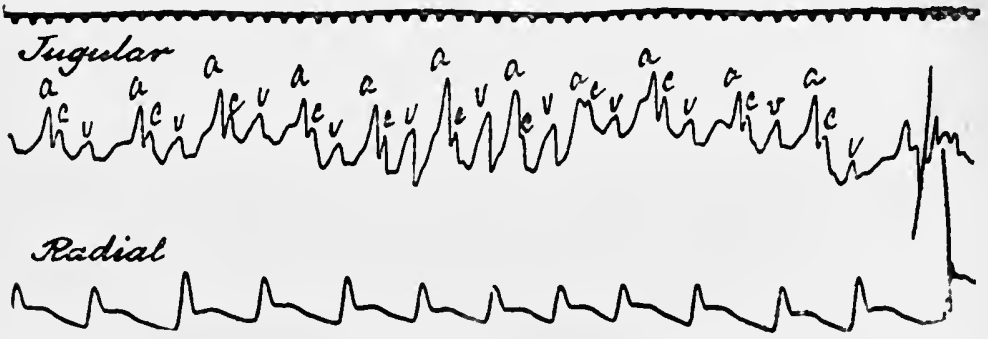

FIG. 76. Simultaneous tracings of the jugular and radial pulses, from a healthy boy aged 7 , showing sinus irregularity.

Diagnosis.-A diagnosis may be made by auscultation, or by a study of the sphygmogram, or of a polygraphic record. When irregularity of the cardiac action is found to be definitcly related to respiration, it is in all probability of the sinus type.
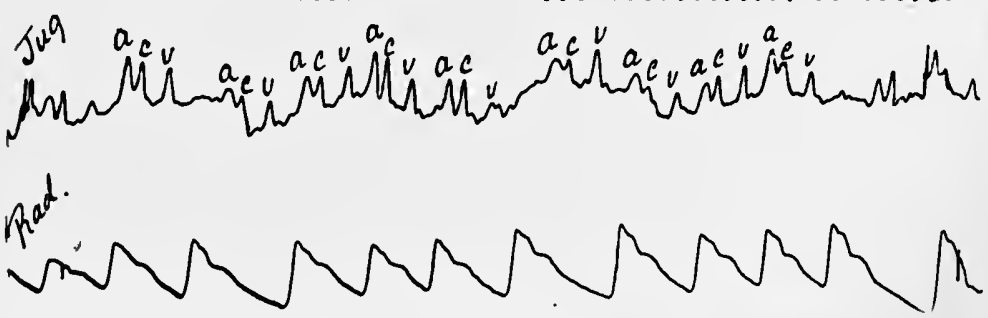

Fig. 77. Simultaneous tracings of the jugular and radial pulses, from an adult showing sinus irregularity.

Prognosis.-There is no reason to suppose that sinus irregularity is either indicative of an impaired heart, or that it adds to the gravity of any diseased cardiac condition which may be present. The condition itself, therefore, may be entirely disregarded, and the prognosis based upon any other abnormal signs which may exist.

Treatment. - No treatment of this condition is required. 


\section{CHAP'TER XII}

THE EXTRA-SYSTOLE

By extra-systole is meant the premature contraction of the auricle (auricular extra-systole), or of the ventricle (ventricular extra-systole), or of both chambers together (nodal cxtra-systole), while the fundamental or sinus rhythm is otherwise maintained in most cases. Usually the cxtra-systole is followed by a long pause (compensatory pause). Rarely the premature contraction occurs between two normal beats (interpolated extrasystole). Two or more premature contractions of the auricle or ventricle may follow one another (multiple extra-systoles).

It is of great importance to be able to recognise the presence of extra-systoles, because they constitute one of the most frequent causes of irregularity of the pulse, and may also be accompanied by subjective symptoms which cause the patient much distress and even alarm.

It has been shown that, while normally the stimulus for contraction arises in the sinus part of the auricle, if any other portion of the remains of the primitive cardiac tube become more excitable the stimulus for contraction arises at that point, and, for this reason, the different parts of the heart are capable of independent contraction. In abnormal conditions, the auricle, the auriculo-ventricular node, or the auriculo-ventricular bundle, may become temporarily more excitable than the sinus, in 
which case the stimulus for contraction starts at that particular point, the result being that a contraction takes place before the anticipated time. If the stimulus start in the auricle, there is a premature contraction of that chamber; if in the auriculo-ventricular node, there is a premature contraction of both auricle and ventricle together; while if it start in the ventricle, there is a prematurc contraction of the ventricle. In each case, the sinus rhythm remains unaltered. It will thus be seen that an extra-systole indicates that some portion of the heart below the sinus is temporarily more excitable than the sinus.

Extra-systoles may appear at long, or at frequent ir-

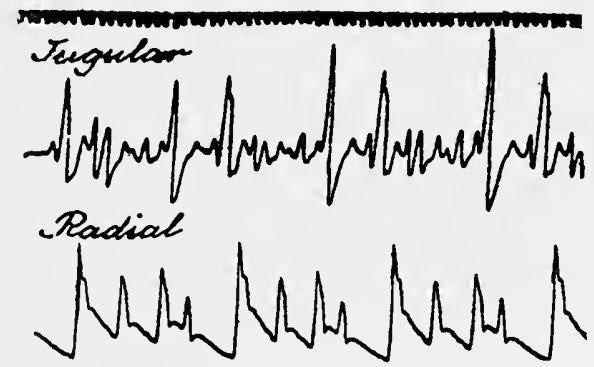

Fra. 78. Simultaneous tracings of the jugular and radial pulses, showing an extra-systole after each fourth beat.

regular intervals, or regularly after each beat, or after every second, third, or more normal beats (Fig. 78). Of the three varieties, the auricular and ventricular are the most important from the clinical point of view; the ventricular is the most common; all of them, however, may occur in the same subject.

The Ventricular Variety of Extra-Systole.-We may take this variety for the purpose of illustration. While the chambers of the heart are contracting in the normal fashion, a premature contraction of the ventricle takes place, this arising independently of a stimulus from the auricle, which maintains its usual rhythm and contracts as the result of the normal stimulus from the 
sinus. The exact time at which the premature contraction of the ventricle takes place is either immediately before the systole of the auricle (Fig. 79), or-as is usually the case-synchronously with it (Figs. 80-82), or

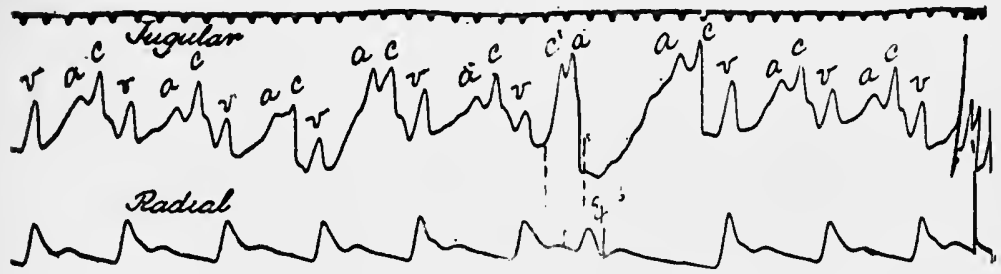

FIG. 79. Simultaneous tracings of the jugular and radial pulses, showing a ventricular extra-systole at $E x . s$. The usual rhythm of the auricle is maintained. The extra-systole in the jugular tracing is represented by the wave $c^{\prime}$, immediately before the systole of the auricle. The compensatory pause is complete.

after it, but prior to that point of time at which it would have occurred if this had been in response to a stimulus received from the auricle in the normal way (Fig. 83). In most cases, the succeeding impulse from

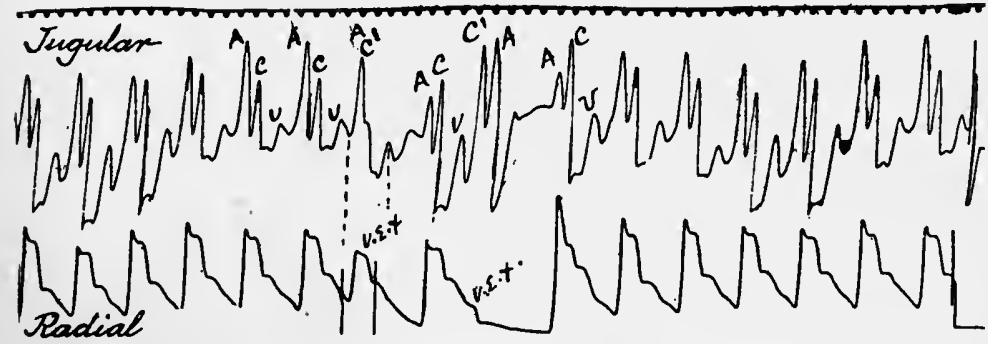

Frg. 80. Simultaneous tracings of the jugular and radial pulses, showing two ventricular extra-systoles $(v \cdot e . x)$. The first is synchronous with, while the second precedes, the rhythmic contraction of the auricle.

the auricle fails to cause a contraction of the ventricle, in all probability due either to the auricular impulse reaching the ventricle when that chamber is either contracting or in the refractory stage following contraction, or to the auriculo-ventricular bundle failing to transmit the impulse, the ventricle not contract- 
ing until it receives the next normal impulse from the auricle; for this reason, the pause which follows the premature contraction is abnormally long, being called the compensatory pause. When this prolonged diastole

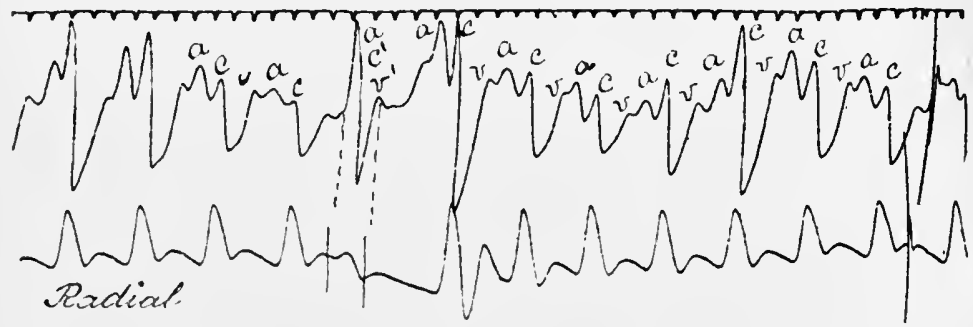

F1G. 81. Simultaneous tracings of the jugular and radial pulses. The extra-systole is synchronous with the systole of the auricle $\left(a c^{\prime}\right)$. The compensatory pause is complete.

and the preceding shortened diastole together equal in time two normal eardiac eycles, the compensatory pause is said to be complete. Sinee during the prolonged pause the ventricle has had an unusually long period in which

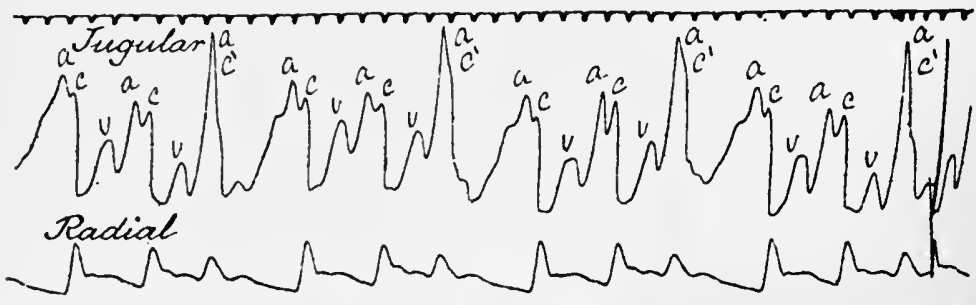

FIG. 82. Simultaneous tracings of the jugular and radial pulses, showing ventricular extra-systole after each second beat. The extra-systole is synchronous with the rhythmic auricular contraction. There is a large single wave $\left(a c^{\prime}\right)$ in the jugular tracing.

to fill and recuperate, the pulse-beat immediately following a compensatory pause has often a greater amplitude than on other occasions. Sometimes, more especially when the cardiac rate is slow, a ventricular extra-systole occurs so early in the cardiac cycle that the ventricle does contract in response to the normal stimulus which is transmitted from the auricle immediately after the 
premature contraction ; in this way a premature contraction is interpolated between two normal beats. This form of the extra-systole is called "interpolated" (Fig. 84).

On auscultation, in the vast majority of cases two heart

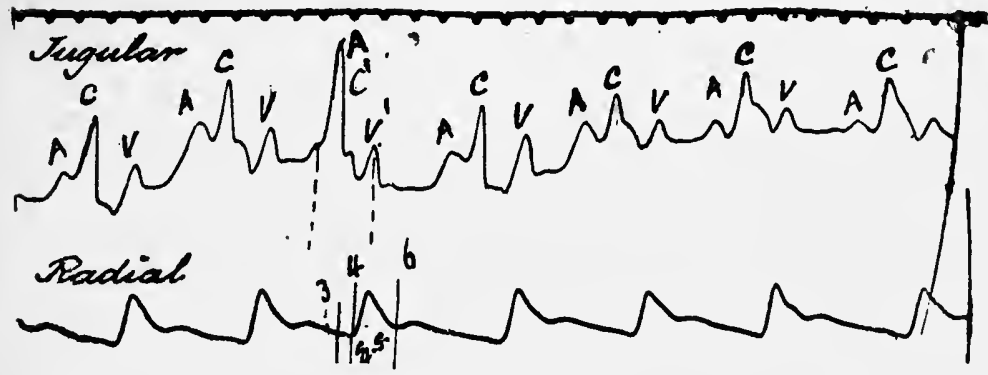

FIG. 83. Simultaneous tracings of the jugular and radial pulses, showing an extra-systole which occurs after a rhythmic auricular contraction.

sounds are synchronous with each extra-systole, these forming. with those of the preceding normal contraction a group of four sounds. Sometimes, however, the extrasystole occurs early in diastole, and before the cardiac

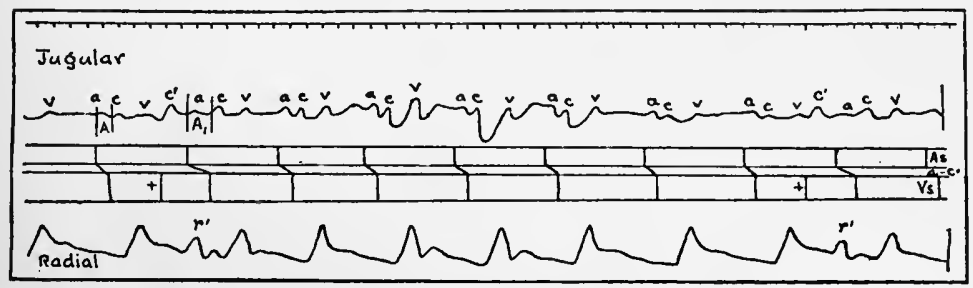

FIG. 84. Simultaneous tracings of the jugular and radial pulses, showing an interpolated ventricular extra-systole $\left(c^{\prime}\right.$ and $\left.r^{\prime}\right)$, represented in the diagram by the downstroke + . The downstrokes in the space As represent the auricular waves $(a)$ in the jugular, and the downstrokes in the space $V_{8}$ represent the carotid waves $c$, and the slanting lines connecting them represent the $a-c$ interval (Mackenzie).

muscle has had sufficient time to recover fully from the preceding systole, in which event the premature contraction is so feeble as to fail to open the aortic valves, and only the first sound is heard; in this way a group of three sounds becomes audible. Very rarely the prema- 
ture contraction is so feeble as not to produce any cardiac sounds whatever.

The amplitude of the pulse-wave of an extra-systole is not so great as that of a contraction occurring at a normal

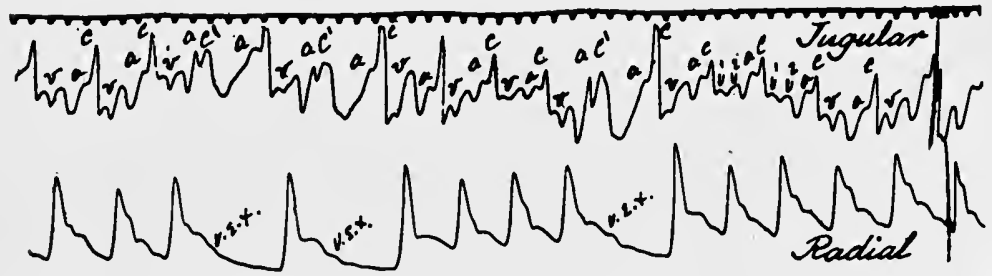

FIG. 85. Simultaneous tracings of the jugular and radial pulses, taken while the patient was at rest, showing frequent ventricular extrasystoles.

interval, because the heart muscle has not had time to recover from its previous cffort. The earlier an extrasystole occurs in the diastole, the more feeble it is; it may be so feeble that no wave is felt at the wrist, so that there is simply an abnormally long pause, resulting in what is called a " dropped beat" or an "intermittent" pulse (Fig. 33) ; as has been already noted, an extra-systole which fails to reach the wrist is, indeed, the most usual causc of an intcrmittent pulse. If a single extra-systole with its succecding compensatory pause occur regularly after each normal beat, then the beats at the cardiac apex occur in pairs, as also do the pulse-beats, the condition being called pulsus bigeminus (Figs. 35 and 36). Similarly, if a single extra-systole with its succeeding compensatory pausè occur after every two normal beats, then the apex-beat occurs in threes, as also do the pulse-beats, the condition being known as pulsus trigeminus (Fig. 38); but if the premature contraction is so feeble that no wave reaches the wrist, the pulse-beats are paired. Ventricular extra-systoles frequently disappear with a quickened pulse-rate due to physical exertion (Figs. 85 and 86 ), or after the administration of a hypodermic injection of atropine. 
If a polygraphic record of a case of ventricular extrasystole be analysed, it will be found that, as the normal rhythm of the auricle proceeds as usual, the $a$ wave appears at regular intervals; each $a$ wave is followed by a $c$ wave,

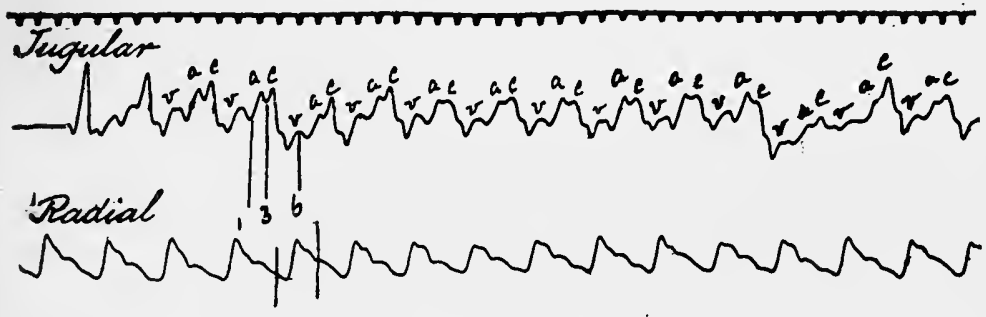

FiG. 86. Simultaneous tracings of the jugular and radial pulses, taken from the same patient as Fig. 85, immediately after exertion. With the quickened pulse-rate the extra-systoles have disappeared.

except when an extra-systole occurs, when the $c$ wave either precedes the $a$ wave, or-as is more commonly the case-occurs synchronously with it, in which case the amplitude of the wave is correspondingly increased.

Auricular Variety of Extra-Systole.-In this variety,

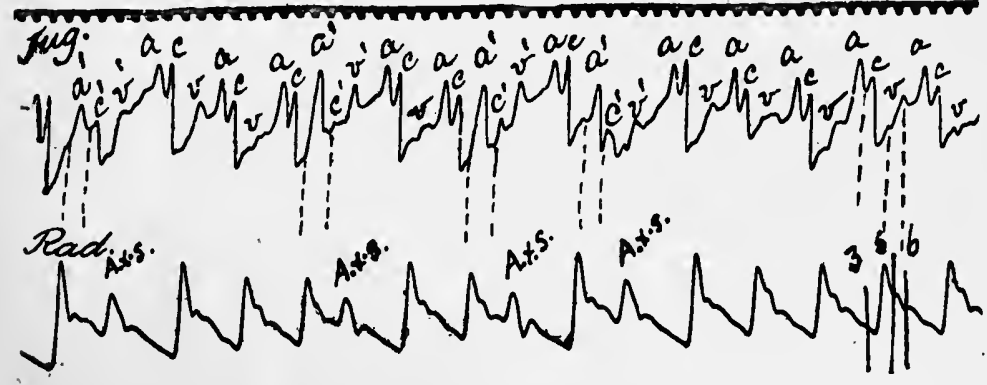

FIG. 87. Simultaneous tracings of the jugular and radial pulses, showing auricular extra-systoles, marked $A . X . S$. in the sphygmogram, and $a^{\prime} c^{\prime} v^{\prime}$ in the jugular tracing. The $a-c$ interval of the premature beat is more than 1/5 sec.; with the rhythmic beats it is normal.

there is a premature contraction of the auricle, the stimulus arising in the wall of the auricle prior to the normal stimulus from the sinus. The premature contraction of the auricle is usually followed by a premature contraction of the ventricle, following the usual transmis- 
sion of the stimulus from the auricle along the auriculoventricular bundle. Not infrequently, in the case of extrasystole the stimulus from auricle to ventricle travels slowly, so that the $a-c$ interval is lengthened (Figs. 87 and 88), and the prematurity of the ventricular systole is conse-

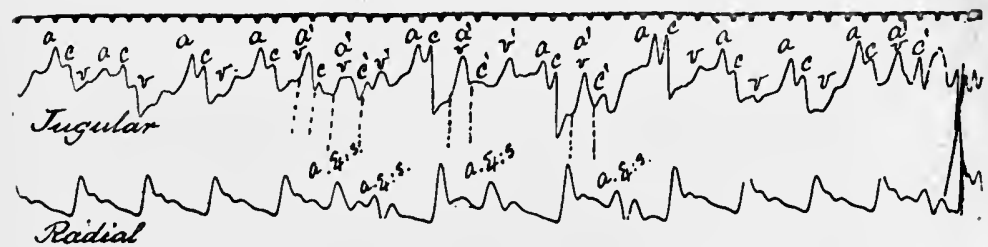

Fig. 88. Simultaneous tracings of the jugular and radial pulses, showing four extra-systoles. The $a-c$ interval of the premature beats is lengthened. The compensatory pauses are not complete.

quently not so great as that of the auricle; in this way, the compensatory pause is shortened. In some instances, the contraction which immediately follows the extra-systole takes place rather earlier than the normal interval, this being probably due to the unusual length of the rest period; in this way there is further encroachment upon the compensatory pause. This is less marked in the case of the second contraction, and it disappears within a few cycles. Sometimes there are variations in the rate of transmission of these auricular premature contractions to the ventricle.

Occasionally the stimulus from the auricle does not reach the ventricle at all, and no premature contraction of this chamber takes place, in which event the premature contraction of the auricle is not followed by a premature contraction of the ventricle, the condition being known as " blocked auricular extra-systoles." In these cases, a long pause is to be noted in the sphygmogram. If in blocked auricular extra-systoles a single extra-systole. occur regularly after each normal beat, and the stimulus from the auricle do not reach the ventricle, infrequency of the pulse-rate may result. It should further be noted that sometimes the stimulus from the auricle reaches the 
ventricle on some occasions, but not on others, resulting in marked irregularity of the pulse.

As in the case of the ventricular variety, an auricular extra-systole is followed by a lengthened pause; but, with rare exceptions, this compensatory pause is not complete. In some cases, however, the compensatory pause is complete; the sinus rhythm remains unaltered, and at the usual interval the sinus sends a stimulus to the auricle, but as the wall of that chamber is in a refractory state therc is no response, the auricle waiting for the succeeding stimulus from the sinus, which arrives after the usual interval.

If a polygraphic record of a case of auricular extrasysto e be analysed, it will be found that each $c$ wave is preceded by an $a$ wave, while there is a premature $a$ wave and a premature $c$ wave, sometimes with an increased $a-c$ interval, and an alteration of the relative position of the beats following the extra-systole.

Nodal or Auriculo-Ventricular Variety of ExtraSystole.-In this variety the stimulus for premature contraction originates in some part of the auriculo-ventricular junctional tissue, travelling upwards into the auricle and downwards into the ventricle, and giving rise to a premature and simultaneous contraction of both auricle and

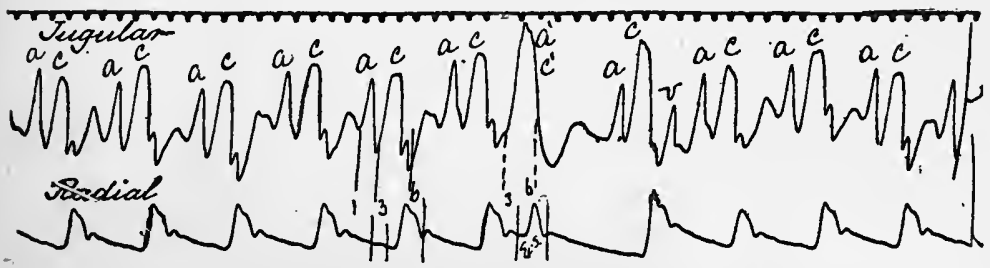

FrG. 89. Simultaneous tracings of the jugular and radial pulses, showing nodal extra-systoles, marked $e x . s$ in the sphygmogram, and $a^{\prime} c^{\prime}$ in the jugular tracing. The premature ventricular contraction practically coincides with that of the auricle.

ventricle. The premature ventricular contraction more or less coincides with that of the auricle, but this is by no means absolute; the contraction of both chambers may be absolutely synchronous (Fig. 89), or the ventricular 
systole may begin after, or before (Fig. 90) that of the auricle. When the auricular eontraction occurs before that of the ventricle, the period of time between the

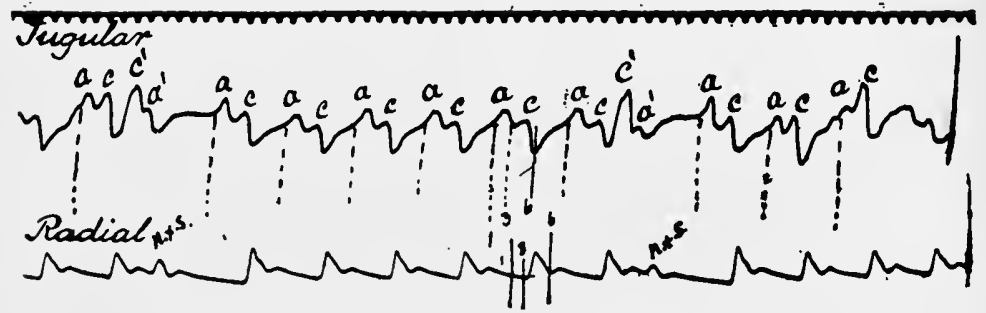

FiG. 90. Simultaneous tracings of the jugular and raclial pulses, showing two nodal extra-systoles (n.x.s.). Both auricle and ventricle contract prematurely, but the contraction of the ventricle precedes that of the auricle.

contraction of the upper and lower chambers is shortened. The eompensatory pause may or may not be eomplete.

In a tracing of the jugular vein from a casc of nodal cxtra-systole, therc is prematurity of the $a$ wave, and also of the $c$ wave; when the premature contraction of the chamber is synehronous both waves are combined, in which case the amplitude of the wave is increased.

In the ventricular and nodal varieties of extra-systoles, in eontrast with the "double" venous pulsation in the jugular vein which may be visible in the case of the auricular variety, there is usually an unduly large "single" pulsation coincident with the premature contraction; this is due to the contraction of the upper and lower chambers of the heart oecurring synehronously, the result being that the auriele, instead of discharging its contents into the ventricle, does so into the venæ cavæ.

Multiple extra-systoles of aurieular origin may occur, and may give rise to short paroxysms of tachycardia, with a regular pulse, or to irregularity of the pulse rhythm when the response of the ventricle to auricular contraction is at irregular intervals. Multiple extra-systoles of ventricular origin may also occur (Figs. 194, 195 and 197); as a rule these only persist for a few cardiac cycles. 
Etiology.-Extra-systoles may be met with at any age, but they are much more frequent in the middle-aged and elderly than in the young. They are rare during the first decade of life; the only cases I can recall at the moment being two, one that of a girl, aged eight, who was under my care at the Mount Vernon Hospital suffering from early pulmonary tuberculosis; she developed tonsillitis, and during this period extra-systoles appeared, and subsequently continued during the time she was under observation at the hospital-a period of many weeks. Extrasystoles are of more common occurrence in men than in women, and are also more usual in the subjects of heart disease than in those who are not, and are more frequent in myocardial degeneration than in valvular disease. But it should be remembered that there is a large group of cases in which extra-systoles are prcsent without any other manifest cardiac abnormality. They are to be found, for example, in persons with a history of rheumatic infection, the victims of tobacco poisoning, in digestive disturbances, super-normal blood-pressure, neurasthenia, and as a result of the administration of certain poisons, especially digitalis and aconitine; while in a considerable proportion of cases there is no ascertainable cause.

Subjective Symptoms.-An individual in whom extrasystoles occur may be quite unconscious of their presence. On the other hand, he may experience certain sensations, which may cause worry and anxiety, particularly if he have retired to bed, and for this reason it is most important that the nature of the complaint should be recognised. The patient may experience a sensation of fluttering in the chest when a premature beat occurs; or he may be conscious of the long pause and complain that the "heart stops"; or the contraction of the heart following the pause may be accompanied by the consciousness of a thud or shock in the region of the heart, sometimes followed by a feeling of exhaustion. The patient may 
complain of a sensation of a " eateh in the breath," or of "gripping in the throat." Very rarely, during a prolonged pause, faintness, sweating, and aetual syncope may take plaee, and the anxiety of the patient may be great; the Adams-Stokes' syndrome may even be simulated.

Diagnosis.-Extra-systoles may be reeognised with eertainty by means of the polygraph or the electro-cardiograph. But it is important for the practitioner to recognise that in the great majority of cases a correct diagnosis can be made simply from palpation and auscultation. The normal rhythm of the heart is disturbed by the oceurrence of a premature beat in the radial pulse and at the eardiae apex, followed by an abnormally long pause. The auseultatory signs have already been noted; they are of great diagnostie value. When, on palpation of the radial artery, an apparently otherwise regular pulse is sometimes interrupted by an unusually long pause, it should suggest either an extra-systole which is so feeble that no wave is felt at the wrist, or partial heart-bloek. When extrasystole is responsible for this unusually long pause, a premature beat can be detected at the cardiac apex, and on auscultation one or two heart sounds will, in the great majority of cases, be audible during the early part of the pause, because the ventricle has contracted; whereas in the case of partial heart-block, there will be absence of an apex-thrust and of heart sounds during the pause, because the ventricle has failed to contract. Further, in these eases of extra-systole, a wave is usually present in a sphygmographie traeing, and a premature contraction is always visible in a traeing of the apex-beat. These three features are almost invariably sufficient to distinguish between an intermittent pulse due to an extra-systole which fails to reach the wrist and an intermittent pulse due to partial heartbloek. A polygraphie traeing or an electro-eardiogram will place the matter beyond all possibility of doubt. It may here be noted that it is necessary to exclude sinus 
irregularity, and also sino-auricular block, in cases of an unusually long pause in the radial pulse.

As pulsus bigeminus and pulsus trigeminus are met with in conditions other than extra-systole, they are not, therefore, pathognomonic of premature contraction. Pulsus bigeminus is found, for example, when the ventricle fails to respond to every third beat of the auricle. The distinguishing features between pulsus bigeminus or pulsus trigeminus due to extra-systole and either of these conditions due to partial heart-block may be established, with a view to differential diagnosis, in the same way as in the case of an intermittent pulse.

If an extra-systole occur regularly after each normal beat, and the extra-systole fail to reach the wrist on each occasion, the rate of the arterial pulse being halved, the case may be wrongly diagnosed as one of infrequency of the pulse-rate due to heart-block. The differential diagnosis is the same as has just been discussed.

With regard to the differential diagnosis of the varieties of extra-systoles, inspection of the jugular vein may be of service. Further, with the exception of cases in which an extra-systole alternates with the normal beat, or follows pairs of normal beats, it is usually possible to distinguish between auricular and ventricular extrasystoles simply from a tracing of the radial artery; all that is necessary is to note the length of the compensatory pause. If this be not complete, it may be inferred that the extra-systole is probably auricular in origin, provided an interpolated extra-systole can be excluded. It cannot be assumed, however, that when the compensatory pause is complete the extra-systole is necessarily ventricular in origin, as this sometimes also occurs in the case of an auricular extra-systole. Needless to say, the varieties of extra-systoles may be distinguished by analysing the polygraphic, or electro-cardiographic, curves. With regard to the former of these, among the distinguishing features are the following:-In the ventricular type of 
extra-systole, the sequence of the $a$ waves is unaltered; there is a premature eontraction of the ventricle; the compensatory pause is complete ; there is increased amplitude of the radial pulse after each extra-systole; and the relative position of the beats following the extra-systoles is unaltered.

In the case of the auricular variety of extra-systole, there is a premature appearance of the $a$ wave in the phlebogram, usually followed by a premature contraction of the ventricle; there may be an increase of the $a-c$ interval following the premature contraction of the auricle; the compensatory pause is usually incomplete; there is an alteration in the relative position of the beats following the extra-systole; while oeeasionally there is a long pause in the sphygmogram, due to the stimulus for contraction passing from auriele to ventriele following the premature eontraction of the auriele being blocked.

In the nodal variety, the $a$ and $c$ waves in the phlebogram are premature, and the two waves may be combined ; the $a-c$ interval, if present, is diminished ; while the compensatory pause may or may not be complete. When it is found that the $a$ and $c$ waves are premature and absolutely synchronous, we may infer that the extra-systole is of the nodal variety.

Prognosis.-The question of prognosis of extra-systoles is very important. Unfortunately the lay mind has come to attach an unneccssary significance to them. Furthermore, it is important that the medical practitioner should understand that extra-systoles constitute one of the most frequent causes of irregularity of the pulse, and that an extra-systole which fails to reach the wrist is the commonest cause of an "intermittent" pulse. When extrasystoles are considered by themselves-that is, without reference to the conditions with which they may be associatedthere is so far no evidence for supposing that they are either indicative of an impaired heart or that they add to the gravity of any existing morbid condition. While 
it is true that extra-systoles may precede the occurrence of other forms of cardiac irregularity, such as the complete irregularity associated with auricular fibrillation, it should be borne in mind that these might have arisen independently. The prognosis, therefore, should be based entirely upon the associated conditions.

Treatment.-Our first duty is to reassure the patient; this may be done with absolute confidence. The etiology of extra-systole should be reviewed, the general health should receive attention, and if cardiac failure be present it should be treated on the lines laid down elsewhere. Some hold the opinion that digitalis is of value for the irregularity itself, but $I$ have never found it so. The bromides are sometimes of benefit for the subjective symptoms, especially in nervous subjects. 


\section{CHAPTER XIII}

\section{HEART-BLOCK}

\section{Auriculo-ventricular Block-Adams-Stokes'}

SYNDROME

IT has been noted that the stimulus for eontraction is eonveyed from fibre to fibre by means of a speeialised function of the eardiac muscle-fibres ealled conductivity, and that the $a-c$ interval in a polygraphic tracing is a measure of this function.

There are three degrees of depressed conductivity: (1) The stimulus from the auricle to the ventricle may be merely delayed-that is, there is merely a prolongation of the interval which separates the eommeneement of contraction of the auriele and that of the ventriele, each stimulus of the auricle reaching the ventriele, which duly responds. (2) The stimulus does not always reach the ventricle-in other words, the ventricle does not always respond to the stimulus from the auricle. The first two degrees are ealled "partial heart-bloek." And (3) no impulses reach the ventricle from the auricle, so that the auricles and ventricles beat independently of each other; the former in response to stimuli received from the sinus, and at an approximate rate of $\mathbf{7 2}$ per minute; and the latter as the result of stimuli arising in its own wall, at about 30 per minute, because this is the normal ventricular rate; this is called " complete heart-block, ; "disassociation of the auriculo-ventricular rhythm," "ven-) tricular rhythm," or the "paroxysmal form of brady- 
cardia." Rarely complete heart-block is associated with auricular fibrillation or auricular flutter.

First Degree of Depressed Conductivity.-This grade of heart-block may give rise to irregularity of the pulse, on account of variations in the length of the $a-c$ interval.

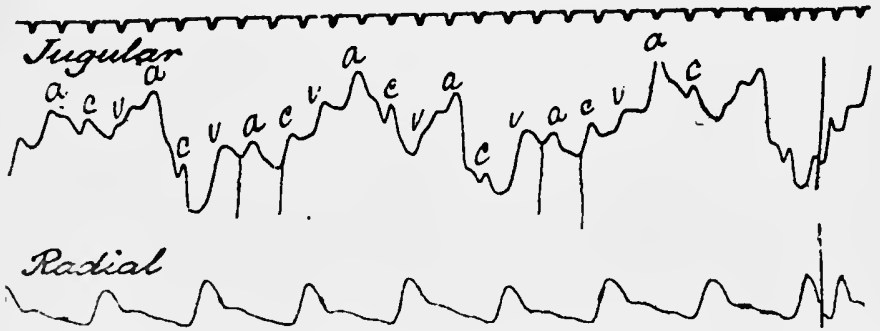

FIG. 91. Simultaneous tracings of the jugular and radial pulses, from a case of myocardial degeneration. The $a-c$ interval is mor $\theta$ than $1 / 5 \mathrm{sec}$.

Some writers believe that contraction of the auricle produces a faint muffled sound, and that consequently when there is a slight delay between the auricular and ventricular contractions there may be a reduplication of the first sound, while if the delay be considerable, so that the contraction of the auricles falls in early diastole, there may be

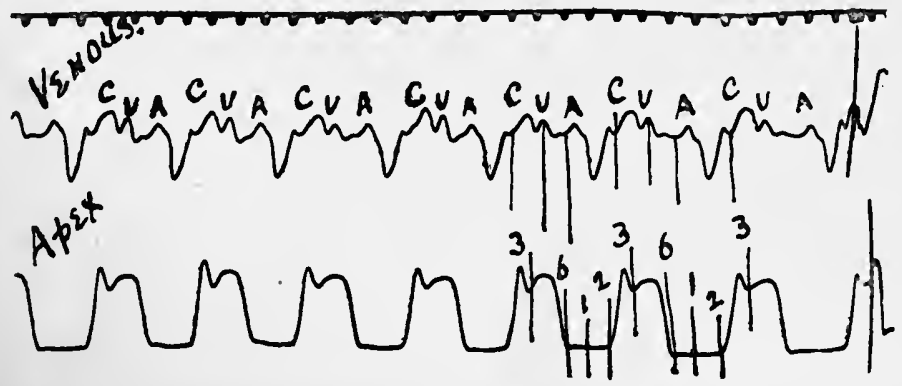

FrG. 92. Simultaneous tracings of the jugular pulse and of the apex beat, from a case of mitral stenosis. The $a-c$ interval is $2-5$ sec.

a reduplication of the second sound. It is also believed by some that in cases of mitral stenosis unaccompanied by auricular fibrillation a slight interval between the auriculo-systolic thrill and apex-beat, as well as between the auriculo-systolic murmur and first sound, is to be 
noted. I am bound to say that I have never been able to detect these phenomena. In a polygraphic tracing, the

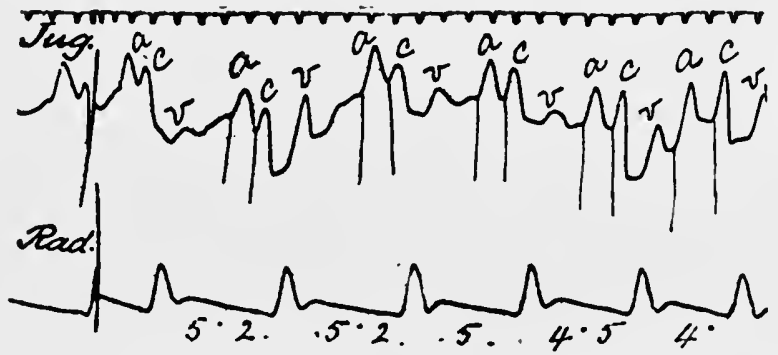

FiG. 93. Simultaneous tracings of the jugular and radial pulses, from a case of mitral stenosis, showing irregularity of the radial pulse, due to variation in the length of the $a-c$ interval.

$a-c$ interval is more than one-fifth of a second (Figs. 91-94).

Second Degree of Depressed Conductivity.-In this condition what are callcd "dropped bcats" occur (Fig. 94). These may be only occasional, or they may be more frequent. Or, each third or fourth impulse may fail to

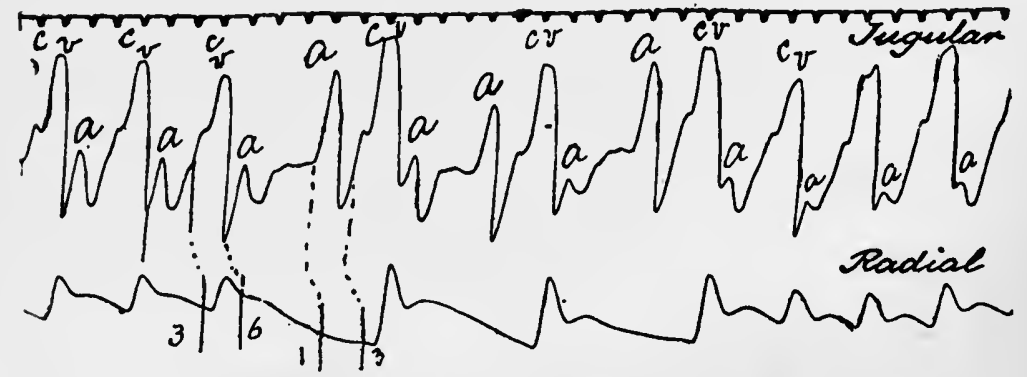

Frg. 94. Simultaneous tracings of the jugular and radial pulses, from a case of aortic and mitral disease, showing partial heart-block. There are three blocked auricular impulses. The $a-c$ interval is $1 \cdot 5 / 5$ sec.

reach the ventricle; in the former case the ventricular beats aie grouped in twos (Fig. 95), and in the latter they are grouped in threes, and in this way a bigeminal or trigeminal pulse results. Or, every other impulse may fail to reach the ventricle, giving rise to halving of the 
ventricular rate, the condition being spoken of as 2:1 rhythm (Fig. 96); or only each third or fourth auricular

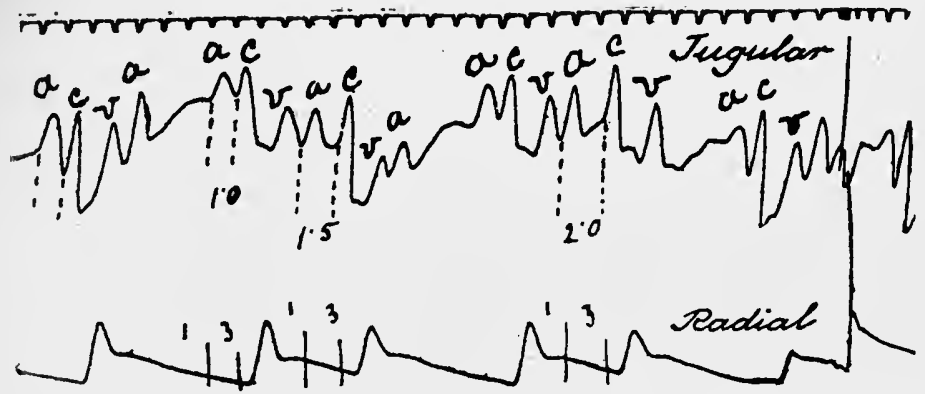

FIG. 95. Simultaneous tracings of the jugular and radial pulses, showing partial heart-block in which each third stimulus from the auricle fails to reach the ventricle, resulting in pulsus bigeminus.

impulse may be transmitted to the ventricle, resulting in 3:1 (Fig. 97) or 4:1 heart-block. In some cases certain ratios alternate.

It might naturally be supposed that the length of the prolonged pause during a dropped beat would be equal to two regular pulse-beats ; such, however, is usually not

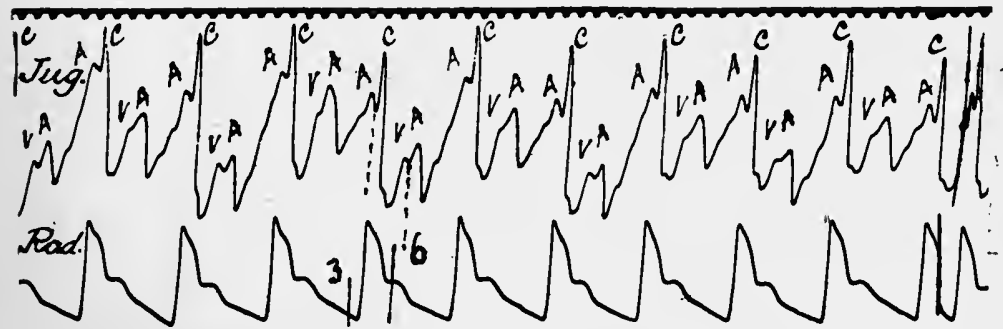

Fic. 96. Simultaneous tracings of the jugular and radial pulses, showing continuous 2:1 auriculo-ventricular block. The pulse-rate is 42 per minute.

the case, for the following reason. There are variations in the $A s-V s$ interval in association with dropped beats, there being a progresssive increase of the $A s-V s$ interval preceding (Fig. 98) and a progressive shortening of the interval following each dropped beat; the former is due to the increasing difficulty the impulses from the auricle 
have in reaching the ventricle, and the latter to the rest which the ventricle has experienced during the pause. The result of the progressive increase of the $A s-V s$ interval prior to, and of the progressive shortening of the $A s-V s$
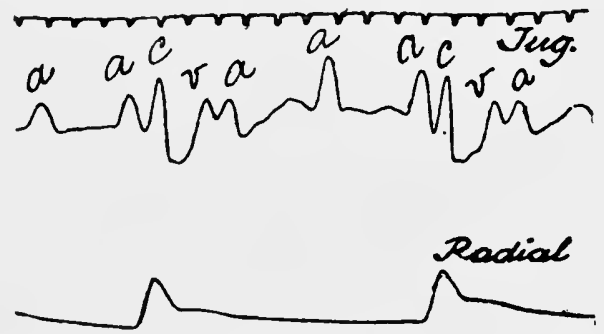

F1G. 97. Simultaneous tracings of the jugular and radial pulses, showing continuous $3: 1$ auriculo-ventricular block.

interval after, the dropped beat is that the long pause becomes shortened. When, however, there are no variations in the $A s-V s$ interval in association with a dropped beat, the length of the prolonged pause is equal to two regular pulse-beats.

2:1 heart-block gives risc to infrequency of the pulse-

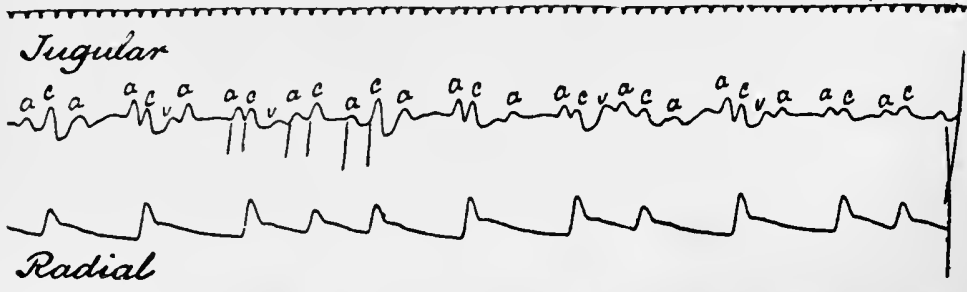

FIG. 98. Simultaneous tracings of the jugular and radial pulses, showing partial heart-block. Note the progressive increase in the $a-c$ interval of three successive rhythmic beats.

rate, the rhythm being regular. This grade of heartblock is sometimes continuous, but usually the ventricle responds from time to time to successive stimuli received from the auricle, the pulse-rate, therefore, being usually above 36 per minute.

On analysis of a polygraphic tracing from_a case of partial 
heart-block, it will be found that the $a$ wave is present at uniform intervals, and is of more frequent occurrence than the $c$ or $v$ waves-it may be even twice, three times, or more times as frequent, according to the degree of block; but, unlike complete heart-block, each $c$ wave is preceded by an $a$ wave (Figs. 94-98).

Partial heart-block of neurogenic origin is sometimes lessened in degree or abolished by the administration of a hypodermic injection of atropine.

Complete Heart-block.- Here the pulse-rate is slow, not infrequently between 20 and 30 beats per minute, and usually not more than 36 , though some cases have been recorded in which the rate has been considerably higher - even up to 60 beats per minute; it is little influenced by physical exertion, emotion, or pyrexia, nor, as a rulc, by atropine. The beats are usually full and strong, and,

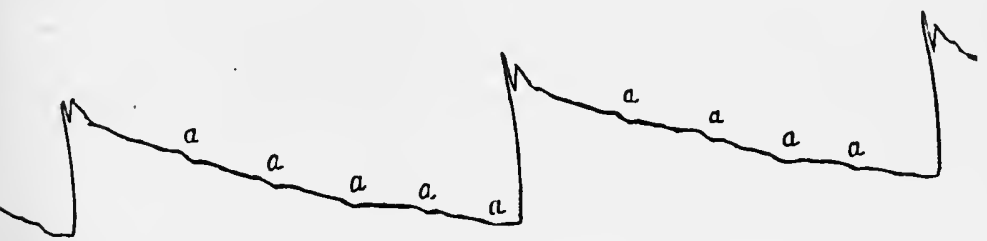

FIG. 99. Sphygmogram from Webster's case of complete heart-block, showing small waves occurring at sixteen regular intervals on the descending limb of the pulse-beat (Mackenzie).

as a rule, the rhythm is regular, the pulse being irregular, however, when extra-systoles are present. It may be noted that in the case of extra-systole occurring with comp'ete heart-block there is usually no compensatory pause following the premature contraction, the reason for this being that no impulses reach the ventricle from the auricle, and after the extra-systole the stimulus for contraction is developed in the muscle-fibres of the ventricle itself. This rule is not absolute, however, for the period following the extra-systole may be either slightly greater or less than in the case of the normal beats. In a tracing of the radial artery, small waves occurring at 
regular intervals on the descending limb of the pulse-beat, probably due to auricular systole, may sometimes be noted (Fig. 99). The systolic blood-pressure is generally high, and during the long pause there is a considerable drop in pressure; the mean blood-pressure is low. There is usually evidenee of hypertrophy of the left ventricle.

On inspection of the neek, regular pulsations in the jugular veins, at considerably more frequent intervals than those of the radial or earotid arteries, due to auricular systolc, may usually be noted; and in some instances a large pulsation in the jugular vein is seen from time to time when the auricular and ventricular contractions happen to coincide.

On auseultation, a first and second sound are found accompanying each contraetion of the ventricle, and, in the opinion of some, faint muffled sounds may oecasionally be heard during the long pauses, due to the eontractions of the auricle; personally I have never been able to detect them. Some writers too have noted an accentuation of the first sound, synchronous with the large pulsation in the jugular vein, when the auricular and ventrieular contractions coincide. In the case of a lesion of either main division of the auriculo-ventricular bundle, the gallop rhythm may be audible, due to asynchronism of the two ventricles.

If a polygraphic tracing be analysed, the disassociation of the auricular and ventricular rhythms is manifest. The $a$ waves oceur at uniform intervals, and are more numerous than the $c$ or $v$ waves, and the time-relation of the $a$ and $c$ waves is a constantly varying one-the $a$ waves at one time preceding, at another following, and at some times again eoinciding with the $c$ waves (Figs. 100-105). When an $a$ wave falls during the limits of ventricular systole, its amplitude is increased.

Etiology.-Heart-block is more common in males than in females. Most of the cases fall within two groups : (1) Patients, usually young adults, who have previously 
suffered from a rheumatic infection of the heart; and (2) patients, usually elderly people, who are suffering from myocardial degeneration. In the former group, the

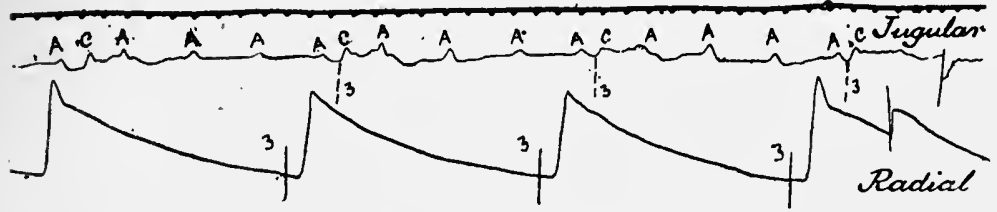

FIG. 100. Simultaneous tracings of the jugular and radial pulses, showing complete auriculo ventricular block. The auricular rate is 107, and the ventricular rate 22 , per minute;

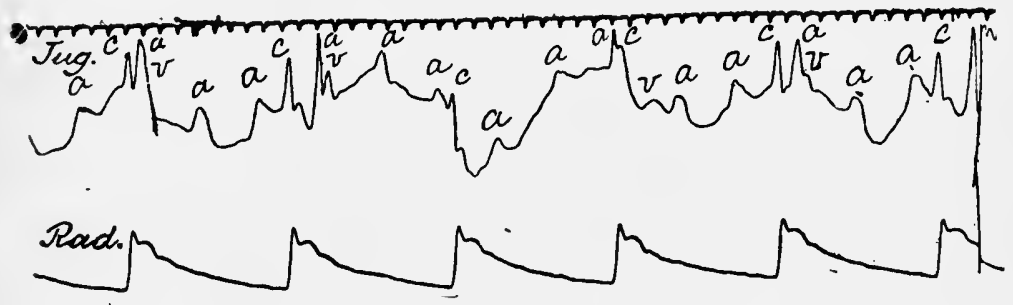

FIG. 101. Complete auriculo-ventricular block. The auricular rate is 88 , and the ventricular rate 30 , per minute.

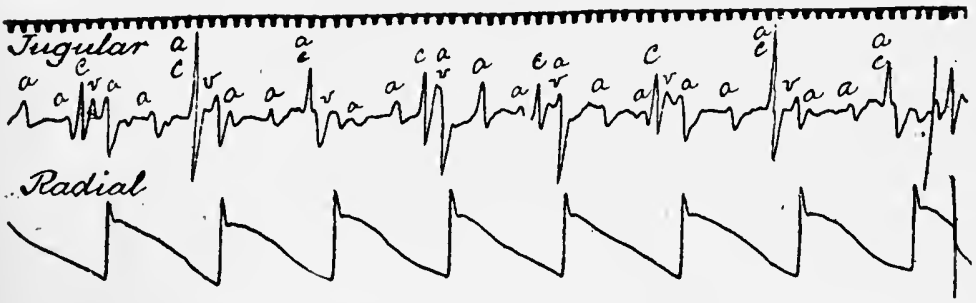

FIG. 102. Simultaneous tracings of the jugular and radial pulses, from a woman aged 25 , with severe heart failure, showing complete auriculoventricular block. The auricular rate is 88 , and the ventricular rate 30 , per minute.

block is almost always of slight degree; but in our investigations at the Mount Vernon Hospital it was noted that in these cases the higher degrees of heart-block were sometimes induced by full doses of one of the digitalis series of drugs. In the latter group, there is a past 
history of syphilis in a considerable proportion $o^{f}$ cases. The milder grades of heart-b'ock may also be found in

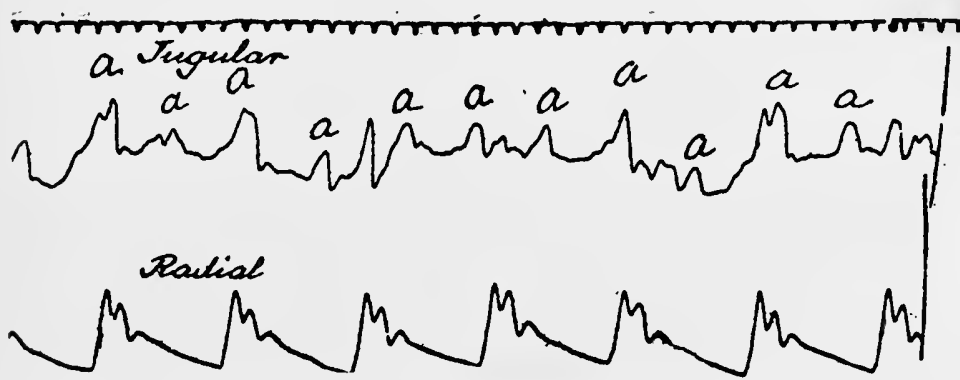

FIG. 103. Simultancous tracings of the jugular and radial pulses, showing complete auriculo-ventricular block. The ventricular rate is 42 per minute.

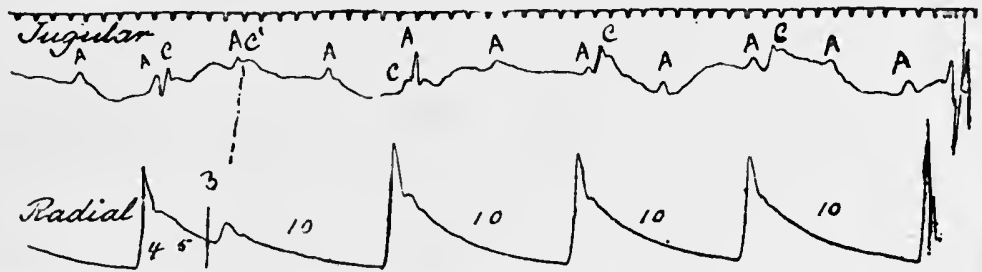

FIG. 104. Simultaneous tracings of the jugular and radial pulses, show. ing complete auriculo-ventricular block, with an extra-systole at 3 . There is no compensatory pause following the premature contraction.
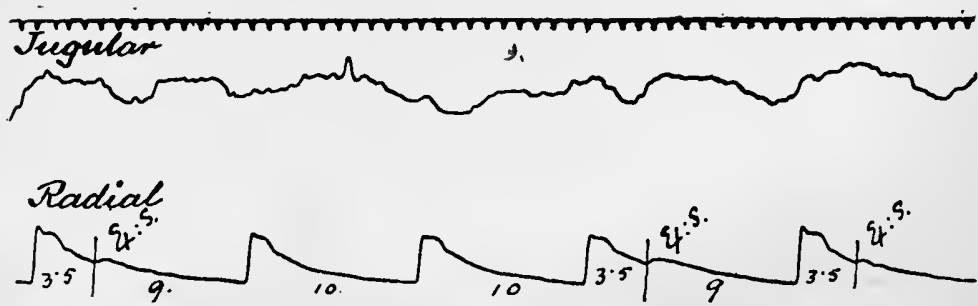

FIG. 105. Simultaneous tracings of the jugular and radial pulses, showing complete auriculo-ventricular block, with three extra-systoles. The periods following the extra-systoles are less than in the case of the rhythmic beats.

patients suffering from infective diseases, such as acute rheumatism, influenza, diphtheria, and pneumonia; the condition, however, is usually temporary in these diseases. 
Morbid Anatomy.-Sclerotic changes, gummata, or acute inflammatory changes of the auriculo-ventricular junctional tissues are found in most cases of heart-block; when the condition occurs during the course of infectious disease, acute inflammatory changes are those most frequently found. Cases have been recorded, however, in which there was an absence of any lesion of the auriculoventricular bundle, but in some of these the vagus was found to be affected. A temporary or permanent lesion of the bundle, or of either main division, or of both together, may occur. A permanent lesion of the right main division appears to be much more frequent than that of the left. It should be remembered that in heart-block the morbid condition is rarely limited to the junctional tissues, the myocardium being usually affected, while valvular disease may also co-exist.

Symptomatology.-Patients suffering from heart-block may complain of symptoms due to the associated cardiac lesions - whether valvular or myocardial-which are almost invariably present, as well as of those due to the block itself; when, however, the valves and myocardium are normal, there may be a complete absence of subjective symptoms.

The symptoms of valvular and myocardial disease will be dealt with later; the commonest of these are dyspnœea, palpitation, fatigue, a sense of tightness across the chest, and precordial pain, on exertion.

With regard to the symptoms due to the block itself, reference has already been made to the pulse, the bloodpressure, the auscultatory phenomena, and the polygraphic records. In most cases, symptoms due to the ventricular bradycardia are present. In the severe grades of partial heart-block and in complete heart-block, there may be marked weakness and fatigue; and, owing to temporary anæmia of the brain, brief attacks of faintness, giddiness, or even temporary loss of consciousness. Not infrequently there are temporary periods of excessive 
slowing, or cessation of ventricular systole for prolonged intervals. Cheyne-Stokes' respiration may be present, and the Adams-Stokes' syndrome may occur, the latter being characterised by apoplectiform or epileptiform attacks associated with marked infrequeney of the pulse. It should be noted that Adams-Stokes' syndrome and heart-block are not synonymous terms, the former being a purely clinical term, whercas by the latter is simply meant an abnormal cardiac mechanism in which the impulses from the auricle do not reach the ventricle. Complete heart-block may occur without Adams-Stokes' syndrome; and the latter may oceur in conditions in which the pulse-rate is infrequent from causes other than heartblock, or in conditions in which there is temporary cessation of the action of the hcart. In heart-block Adams-Stokes' syndrome is particularly apt to occur during the period in which complete heart-block is developing; at this stage there may be intermittent periods of complete heart-block, and the ventricle does not immediately take up its own rhythm after the stimulus to contraction from auricle to ventricle has ceased, so that there is a prolonged pausc. When complete heart-block has become permanently established, the ventriele apparently becomes accustomed to the condition, and these pauses are not so likely to occur, the Adams-Stokes' syndrome being met with more rarely. The attacks, however, may occur after complete heart-block has become permanently established if the ventricular rate be below 30 ; there may be pauses of unusual length in these cases, as the ventricular rate may fall temporarily even as low as 6 per minute.

In Adams-Stokes' syndrome the patient, as a rule, has no knowledge of an impending attack. During the seizure there may be marked pallor or cyanosis, and deep or even stertorous breathing. Usually the convuls.ons are confined to the lace and upper limbs, the tongue is not bitten, and urine is not passed involuntarily. There may be repeated seizures. 
Diagnosis.-Diminished conductivity and the various grades of auriculo-ventricular heart-block are readily rccognised by means of the polygraph or electro-cardiograph, since these instruments afford separate records of the movements of both the upper and lower chambers of the heart. The first grade can only be recognised by means of either of these instruments, and a lesion of either main branch of the auriculo-ventricular bundle by means of the electrocardiograph. Fortunately, however, they are not necessary for the recognition of most of the other cases-palpation and auscultation furnishing all the evidence required. As an example of this may be mentioned the differential diagnosis between occasional dropped beats, pulsus bigeminus, and pulsus trigeminus, due on the one hand to extra-systoles and on the other to partial heartblock; this has already been dealt with in Chapter XII. It is necessary to remember that sometimes sinus irregularity may closely simulate occasional dropped beats due to partial heart-block, and that the length of the prolonged pause is not sufficient for differential diagnosis; for, as has been pointed out, in the latter condition the length of the pause is often less than two regular pulse-beats, on account of variations in the $A s-V s$ intervals. A polygraphic or electro-cardiographic record of sinus irregularity, however, shows that, unlike partial heart-block, the auricle, as well as the ventricle, has failed to contract.

The higher grades of heart-block give rise to infrequency of the pulse-rate. When the ventricular rate suddenly falls to half its former rate, in all probability the case is one of $2: 1$ heart-block.

2:1 heart-block must be distinguished from the condition in which an extra-systole occurs after each normal beat and fails to reach the wrist on each occasion. This can be done in the same way as in the case of a less severe grade of heart-block. The distinction between polygraphic records of 2:1 heart-block, and of the normal rhythm with an $h$ or $b$ wave falling exactly 
midway between two $a$ waves, has been dealt with in Chapter V.

It is sometimes difficult to distinguish between partial and complete heart-block without the aid of the polygraph or electro-cardiograph. Most cases with a ventricular rate of 36 or under, however, are cases of complete heartblock. Also, the presence of more rapid pulsations in the jugular veins than the rate of the radial pulse, the presence of small waves occurring at regular intervals on the descending limb of a sphygmogram and auscultatory phenomena afford help in differential diagnosis. A polygraphic or electro-cardiographic tracing may be taken to determine disassociation of the auricular and ventricular rhythms.

Adams-Stokes' syndrome is easily recognised; marked infrequency of the pulse-rate, rapid pulsations in the jugular veins, and the symptoms already described furnishing a characteristic clinical picture.

Prognosis. -The occurrence of partial heart-block during the course of infective diseases is a sign, and may be the only sign, of myocardial involvement. Persistent heart-block of mild degree also is indicative of myocardial damage.

With regard to persistent heart-block of severe degree, there are two questions of importance in considering the prognosis: namely, (1) whether there be involvement of the heart muscle as a whole, and whether this involvement be progressive; and (2) is the patient subject to syncopal attacks, and, if so, their frequency? The first question has been fully dealt with in Chapter VII; and with regard to the latter, it has been pointed out that the particular period of danger is during the period of development of complete heart-block.

When there are no symptoms of heart failure, and when also the patient does not suffer from syncopal attacks, the span of life may be prolonged for many years, and he may be able to live a fairly active life without incon- 
venience. But, when indications of cardiac failure are present, and especially if these be progressive, and when the patient is. subject to recurrent syncopal attacks, especially if severe and frequent, life is always in danger; and, apart from the risk of a fatal termination during an attack, death may occur with the usual clinical picture of cardiac failure. A few syphilitic cases have been recorded in which energetic anti-syphilitic treatment appears to have been rewarded with recovery.

Treatment.-When there is reason to suspect that syphilis is the cause, appropriate and energetic antisyphilitic treatment should be employed. When heartblock occurs during the course of infective diseases, rest in bed is indicated, and the cause, such as rheumatism, should be adequately treated. Persistent heart-block of mild degree requires no treatment in itself. But, as there is usually valvular or myocardial disease or both, it is of great importance that the patient should live strictly within the limits of the heart's strength, all the more so in cases of persistent heart-block of severe degree. Accordingly, the daily life of the patient should be most carefully regulated, and undue physical or mental strain should be scrupulously avoided. If the patient bc subject to fits, he should be warned to avoid anything known to predispose to the attacks, and, as far as possible, he should be protected from the risks of falling. Unfortunately, there is no drug which undoubtedly accelerates the ventricular rate, and, therefore, none is efficacious during an attack. Atropine may be given a trial, since cases of Adams-Stokes' syndrome have been recorded in which the writers have stated that in their opinion the drug was the means of increasing the pulserate and giving relief to the symptoms; 1/100 gr. may be given hypodermically, and repeated in half an hour if necessary. I myself have never seen any good result follow its administration in this disease. Strychnine, oxygen, and amyl nitrite have been administered, but 
there is no reason to suppose that they are of any value. Digitalis may be tried in cases of complete heart-block in which there is dropsy or some other indication for its use, for the drug does not slow the ventricle when that chamber is contracting independently of the auricle. 1

\section{Sino-AURICUlar Block}

Sino-auricular block is a very rare condition. I have only seen one case. It may cause irregularity of the pulse. In this condition, the stimulus for contraction arises at the normal site, but occasionally or more frequently it is not transmitted to the auricle, in which event the auricle and ventricle fail to contract, so that there is an abnormally long pause; as already mentioned, this condition is one of the causes of an "intermittent" pulse or " dropped" beats. The length of the abnormally long pause is generally rather less than the duration of two cardiac cycles. A polygraphic or electro-cardiographic tracing reveals the fact that the auricle and ventricle have not contracted during the abnormally long pause. 


\section{CHAPTER XIV}

\section{PULSUS ALTERNANS}

By pulsus alternans is meant a condition of the pulse in which, while the rhythm is perfectly regular, a large beat and a small bcat alternate. It is probably indicative of depressed contractility.

In the most typical cases of pulsus alternans, stimulation and contraction of the sinus, the auricles, and the ventricles occur in the order named, the ventricles contracting only in response to stimuli received from the auricles, and ventricular systole following the auricular systole at the normal interval (Fig. 106). Sometimes,

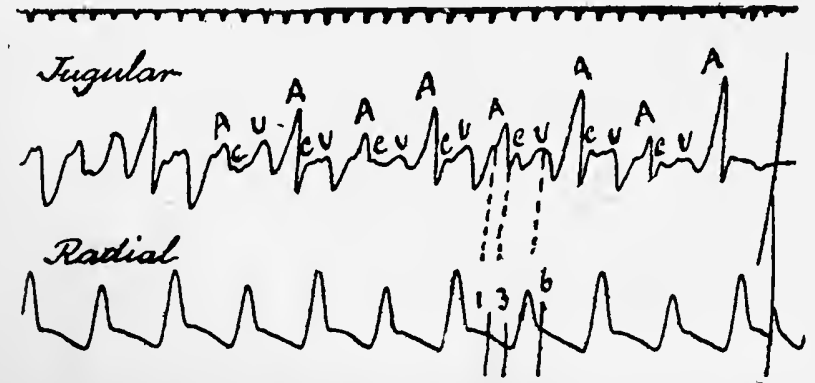

FIG. 106. Simultaneous tracings of the jugular and radial pulses, showing continuous pulsus alternans, in which stimulation and contraction of the sinus, auricles, and ventricles occur in the order named.

however, pulsus alternans is associated with conditions, such as auricular flutter (Fig. 107), or paroxysms of auricular extra-systoles, in which the mechanism of the cardiac action is abnormal. The condition may be continuous, or it may be only observed on an increase of the 
pulse-rate, such as follows exercisc, or only during a few cycles immediately following an extra-systole (Fig. 108).

Etiology.-Excluding those cases that occur in association with an abnormal cardiac action, such as auricular

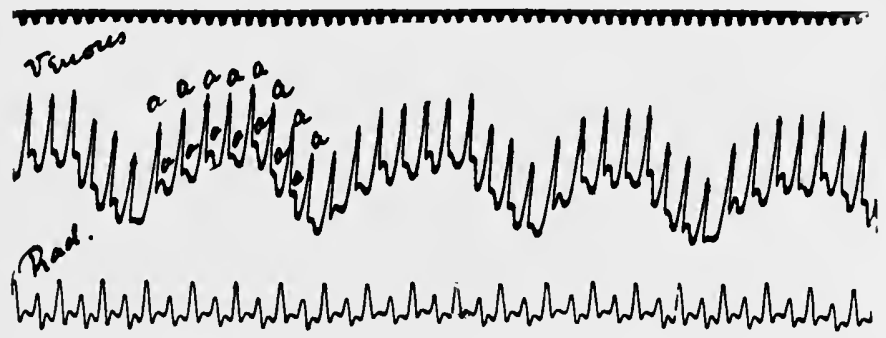

FIG. 107. Simultaneous tracings of the jugular and radial pulses, showing pulsus alternans associated with an attack of auricular flutter, in which the ratio between the auricular and ventricular rate is con. tinuously $2: 1$.

flutter, in the great majority of cases patients showing pulsus alternans arc past middle age, and arc the subjects of myocardial degencration; the condition, however, is sometimes found in younger people with post-rheumatic

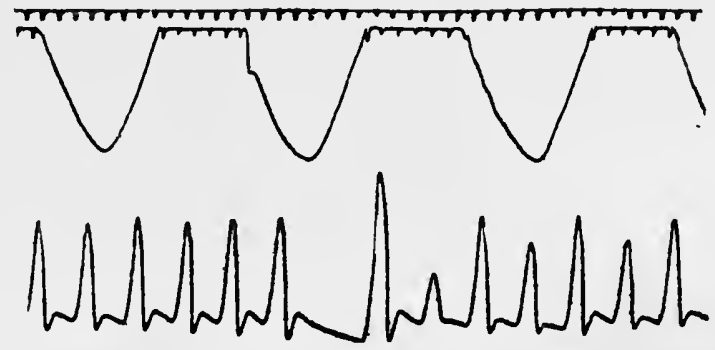

FIG. 108. Tracing of the radial pulse, showing pulsus alternans, immediately following an extra-systole which failed to send a pulse-wave to the wrist.

valvular disease, and occasionally it has been noted in pneumonia and other acute illnesses.

Symptomatology.-The pulsus alternans in itself causes no subjective symptoms. When associated with myocardial disease, evidences of cardiac failure are almost invari- 
ably present. Many patients suffer from severe dyspnœa on cxertion, and cardiac asthma, while not uncommonly Cheyne-Stokes' breathing occurs at night when the patient is asleep. A close relationship has been shown to exist between the pulsus alternans and attacks of angina pectoris. It requires long practice to detect pulsus alternans by the finger. In this connection, it may be noted that the difference in strength of the pulse-beats is made more pronounced when the brachial artery is compressed by the finger or the cuff of the sphygmomanometer. Sometimes the small beats are so feeble that no waves are transmitted to the wrist; the condition may, therefore, bc a cause of infrequency of the pulse.

Diagnosis.-As the pulsus alternans is a sign of great prognostic importance, we should always be on the

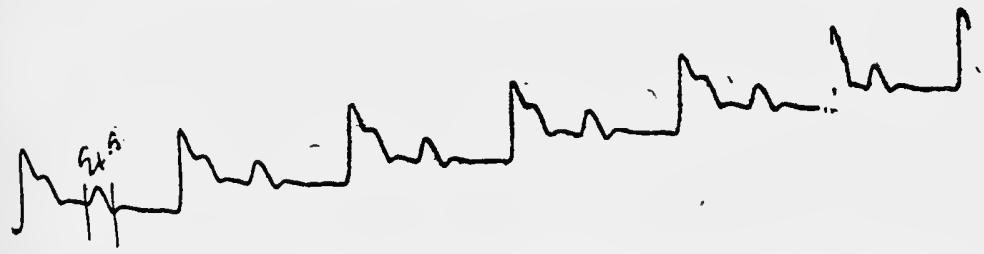

FIG. 109. Tracing of the radial pulse, showing pulsus bigeminus, due to a single extra-systole with its succeeding compensatory pause occurring regularly after each normal beat.
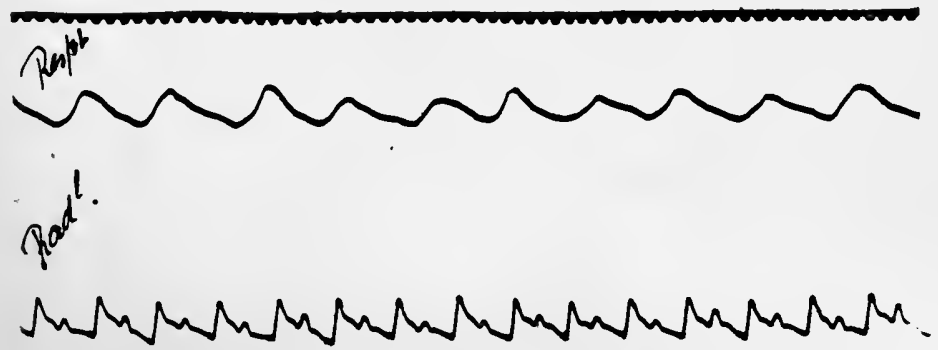

FIG. 110. Shows the same as Fig. 109.

look-out for it, especially after exertion. In the great majority of cases, it is necessary to take a tracing of the radial artery in order to recognise the condition. It is necessary to distinguish it from pulsus bigeminus due to 
extra-systoles (Figs. 109 and 110). The differential diagnosis can be made with certainty; in pulsus alternans the pause between the large and the small beat is equal to that between the small and the large beat, whereas in the case of extra-systole the pause following the small
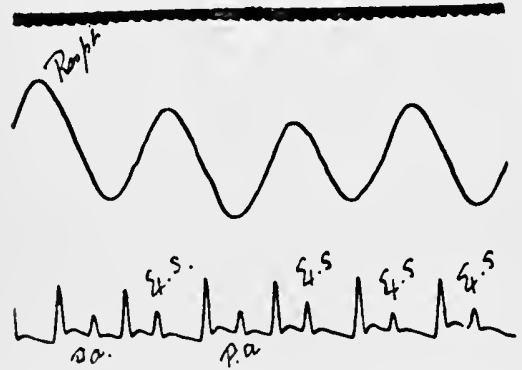

FIa. 111. Tracing of the radial artery, showing both pulsus alternans and extra-systoles.

beat is longer than that following the large one.

Prognosis. -W hen pulsus alternans occurs apart from a disordered cardiac mechanism, it is an indication of extreme exhaustion of the heart muscle; when the condition lasts only for a few cycles, this exhaustion may be only temporary, but even in these cases the prognosis should be guarded; when it is continuous, death may follow within a few months, or at most in a few years. When the condition is associated with tachycardia, it does not appear to have any serious significance in itself.

Treatment.-As pulsus alternans is usually a sign of great exhaustion of the heart muscle, complete and long-continued rest, both physical and mental, is urgently needed. 


\section{CHAPTER XV}

\section{AURICULAR FIBRILLATION}

THE discovery of auricular fibrillation is one of the greatest discoveries regarding the functional pathology of the heart which has ever been made. It is of the utmost practical importance that the condition should be recognised owing to its bearing on the diagnosis, prognosis, and treatment of cardiac affections. Auricular fibrillation is a specific clinical condition, which can be recognised with certainty. It is characterised in the vast majority of cases by complete irregularity of the arterial pulse, and also by absence of all signs of the normal contraction of the auricles. It accounts for approximately 50 per cent. of all cases of persistent irregularity of the heart, and it is found in from 60 to 70 per cent. of all cases of serious cardiac failure with dropsy. Mackenzie first described the condition as " nodal rhythm," " and the irregularity peculiar to the condition has been known variously as "pulsus irregularis," "pulsus irregularis perpetuus," and the " mitral " pulse.

Nature of Auricular Fibrillation.-By auricular fibrillation is meant a condition in which co-ordinate contraction in the auricle is replaced by inco-ordinate contraction; the individual fibres, instead of contracting in an orderly and simultaneous manner, doing so rapidly and independently

1 In what is termed nodal rhythm, the stimulus for contraction arises in some part of the auriculo-ventricular junctional tissues, giving rise to a simultaneous contraction of both auricle and ventricle, in which case the $a$ and $c$ waves are superimposed. 
of each other, with the result that systole of the chamber as a whole never takes place. When auricular fibrillation has once set in, in the great majority of cases it persists for the remainder of life. But, instead of this, it may appear for a few hours only and never return, or it may occur at infrequent intervals for weeks or months and then cease. Many cases of paroxysmal tachycardia are due to paroxysms of auricular fibrillation, and rnay last for a few scconds or may continue for months. The tendency to occurrence increases, however, until finally the condition usually becomes permanent.

Effect of Auricular Fibrillation on the Heart.--The cffect of auricular fibrillation on the heart's action is marked. Instead of the ventricle receiving stimuli from the auricle at regular intervals, it receives them at completcly irregular intervals. Further, the impulses which cscape are numerous, and, if the conducting tissues be able to transmit them, the ventricular rate rises. Thus, an irregular, and in the vast majority of cases a much too frequent, pulse is produced. Such a change in the action of the ventricle as a rulc produces a considerable effect on the heart's efficiency.

Etiology. - The majority of cases of auricular fibrillation fall within two groups: (1) those with a history of rheumatism, and (2) patients suffering from myocardial degeneration. In the case of the former, the condition occurs more commonly in early adult life, and the patients frequently have valvular disease-more often mitral than aortic disease, and more especially mitral stenosis. In the second group of cases, the condition is more common after middle age. Auricular fibrillation has been also found occasionally in pneumonia, diphtheria, infective endocarditis, and the terminal stages of various exhausting diseases. Full doses of digitalis may rarely produce it in predisposed cases. It is occasionally noted in aged people who exhibit no other symptoms referable to the heart, so long as the pulse-rate does not exceed the normal 
for their age. Its onset can now and again be traced to bodily effort, especially in the middle-aged or elderly.

Symptomatology. - Patients with auricular fibrillation very frequently complain of fluttering in the precordium, or of irregular action of the heart, or of both, possibly only on exertion. Apart from this, there is occasionally an absence of subjective symptoms. But, when the ventricular rate is much above the normal, symptoms as a rule are present, and these are the same as those of cardiac failure from other causes, such as shortness of breath, a sense of exhaustion, cyanosis, and dropsy. It would appear, however, that patients suffering from auricular fibrillation are exempt from definite attacks of angina pectoris. When the pulse-rate is very slow, the Adams-Stokes' syndrome may be present.

The degree of cardiac failure which arises as a result of auricular fibrillation may be slight or very severe. It depends upon $(a)$ the rate of the ventricular action, and (b) the degree of inherent muscle defect. As a rule, the onset of symptoms is gradual, but may ensue rapidly and the patient may become very ill within a few hours. Similarly, the cessation of auricular fibrillation may be accompanied by an equally rapid improvement in the patient's condition.

There is a wide variation in the ventricular rate, according as the pathway for impulses to the ventricle is free or interfered with, this ranging from 40 to 140 or even 180 per minute, the average rate being between 90 and 140 . The rate of the radial pulse does not necessarily represent the ventricular rate, for many beats of the heart may not be transmitted to the wrist, especially when the ventricle is beating rapidly; the ventricular rate, therefore, should be counted at the apex, either by palpation or auscultation. The character of the pulse is important. It is irregular, the irregularity in the majority of cases being continuous and persisting for the rest of the patient's life, in this respect differing from most other types of cardiac irregularity. A 
feature of even greater importance is the fact that the irregularity is complete (Figs. 112-115). In a sphygmogram,

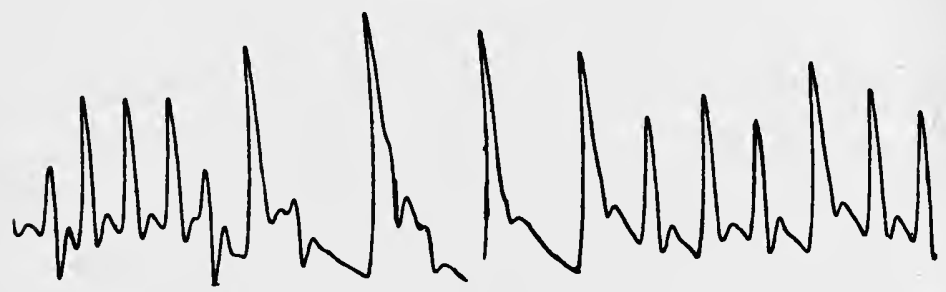

Fig. 112. Tracing of the radial artery, from a case of mitral stenosis with auricular fibrillation, showing complete irregularity of the pulse.

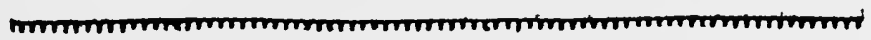

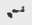

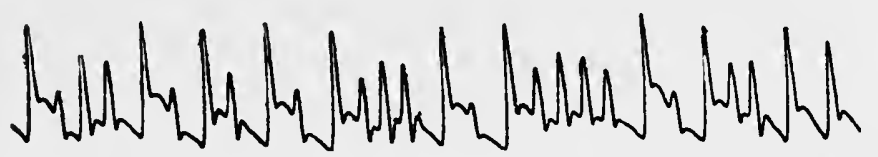

Fia. 113. Tracing of the radial artery, from a case of myocardial degeneration with auricular fibrillation, showing complete irregularity of the pulse.

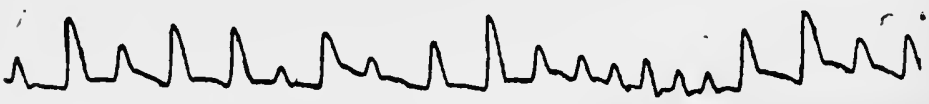

Fig. 114. Tracing of the radial pulse, from a case of auricular fibrillation, showing complete irregularity of the pulse.

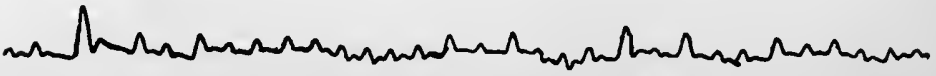

Fic. 115. Shows the same as Fig. 114.

it will be found that two beats of the same length or character rarely follow each other. There is often no rela- 
tion also between the length of a pause and the strength of the beat which follows it-i.e. a short pause may bc

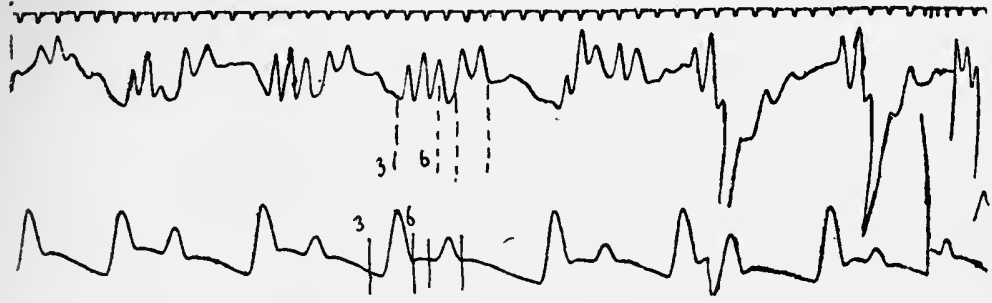

Fig. 116. Simultaneous tracings of the jugular and radial pulses, from a case of auricular fibrillation not under the influence of digitalis, showing coupled beats.
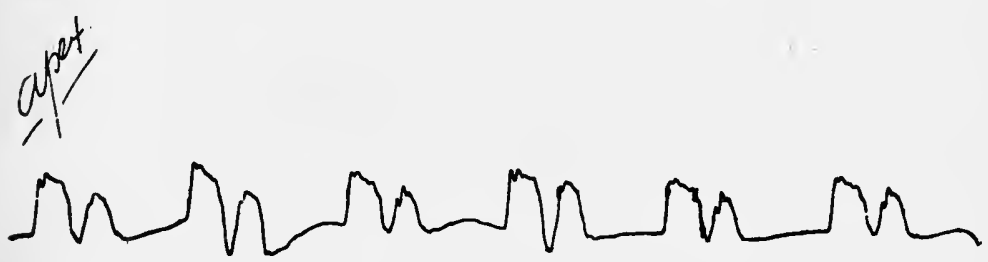

FIG. 117. Tracing of the apex-bəat, from a case of auricular fibrillation, fully under the influence of digitalis, showing coupled beats.

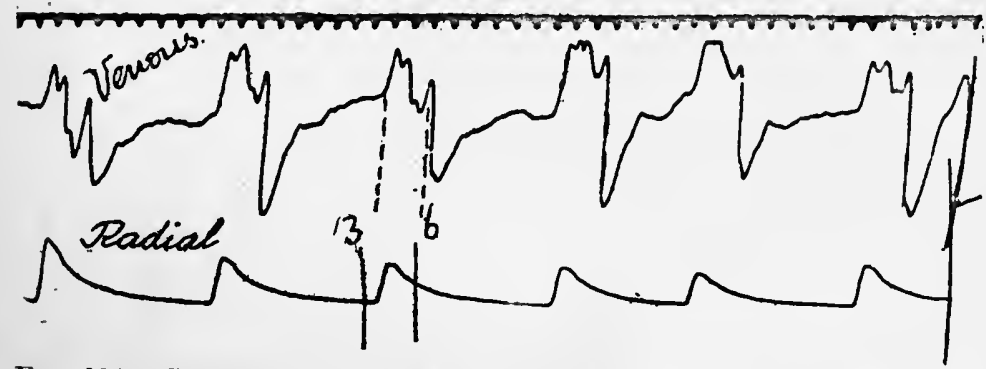

FIG. 118. Simultaneous tracings of the jugular and radial pulses, from a case of auricular fibrillation, showing the ventricular form of venous pulse, and an unusually slow radial pulse.

followed by a strong beat, and a long pause by a weak beat. The beats may be coupled for long or short periods, and this may occur independently of the action of digitalis (Fig. 116), although it is usually due to it (Fig. 117). 
When the ventricular rate is slow (Figs. 118 and 119), or very rapid, the irregularity may be only slight, and we may have to adopt careful measurements of the sphygmogram to detect it.

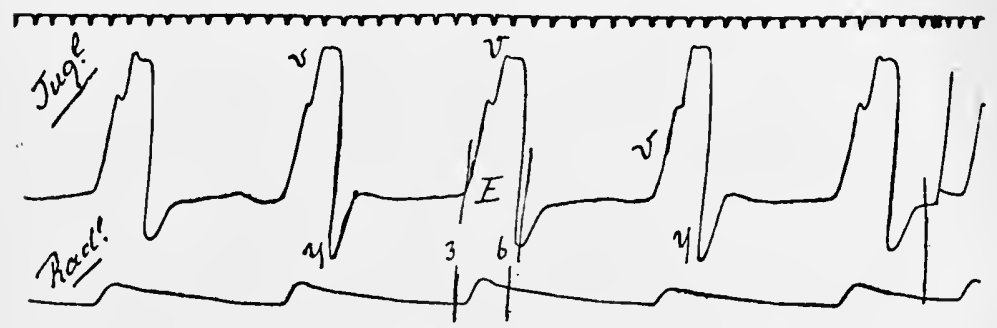

Fig. 119. Simultaneous tracings of the jugular and radial pulses, from a case of auricular fibrillation, showing the ventricular form of venous pulse, and an unusually slow radial pulse.

In cases of mitral stenosis in which a presystolic bruit, due to auricular systole, is present, this disappears with the onset of auricular fibrillation; while if a diastolic bruit be present it persists. The jugular veins may be so

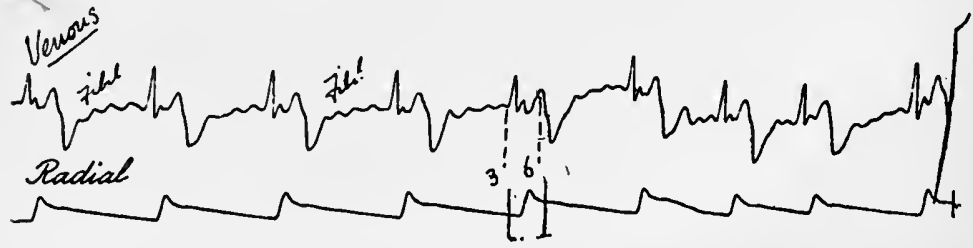

Fic. 120. Simultaneous tracings of the jugular and radial pulses, from a case of auricular fibrillation, showing multiple undulations (marked Fibl.) in the plebogram, caused by the fibrillating auricle.

distended that there is no visible pulsation in them. When pulsation is visible, however, it is of the positive type, only one wave being visible, this occurring during ventricular systole. In a tracing of the jugular vein, there is an absence of the normal $a$ wave; in other words, the ventricular form of venous pulse exists (Figs. 118-120). The normal $a$ wave cannot be present because the auricle 
does not contract. On the other hand, in cases in which there is infrequent cardiac action, multiple undulations, caused by the fibrillating auricle, may be present during diastole (Figs. 120, 128, 129). There is also an absence

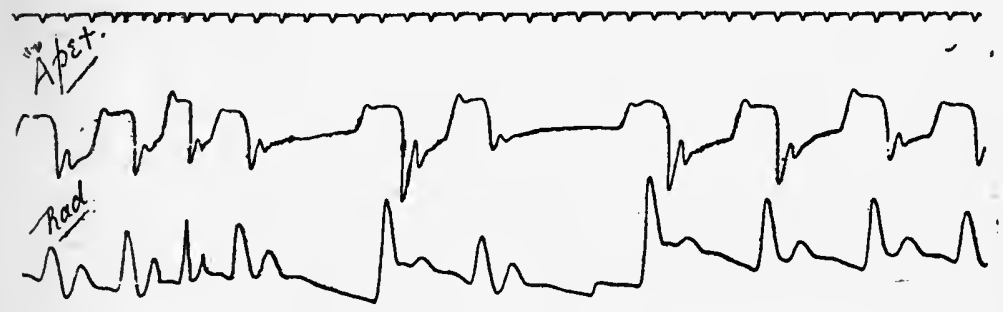

Fig. 121. Simultaneous tracings of the apex-beat and of the radial pulse, from a case of auricular fibrillation, showing absence of the normal $a$ wave in the cardiogram.

of the normal $a$ wave in a cardiogram (Fig. 121) and in a tracing of the liver pulse (Fig. 122).

Diagnosis.-Auricular fibrillation can be diagnosed with absolute certainty when complete irregularity of the

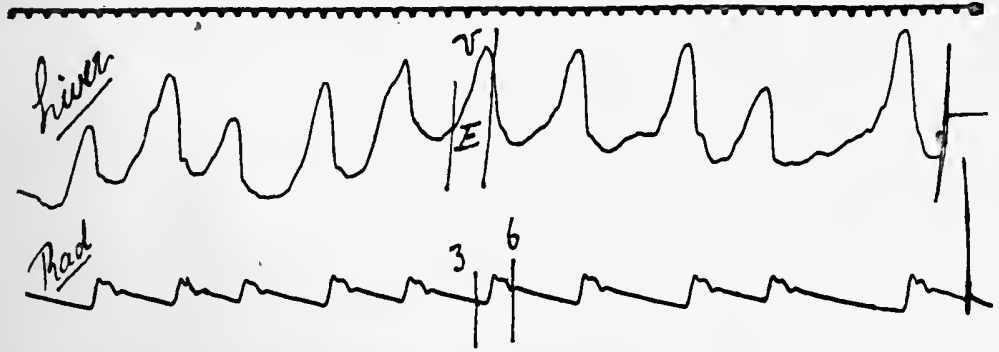

Fia. 122. Simultaneous tracings of the liver and radial pulses, from a case of auricular fibrillation, showing absence of the normal $a$ wave in the former.

arterial pulse, the ventricular form of venous pulse, and multiple undulations in the jugular pulse are present, together with an absence of all signs of an auricular wave in a tracing of the apex-beat and of the liver pulse; or on electro-cardiographic examination. The differential diagnosis between the $h$ or $b$ wave which is occasionally 
to be noted in polygraphic tracings of auricular fibrillation and the $a$ wave has been dealt with in Chapter $V$.

We may be sure also that auricular fibrillation is present when there is complete irregularity of the pulse in conjunction with the ventricular form of venous pulse; and, indeed, we may be reasonably certain of its existence from the mere presence of complete irregularity of the pulse.

Prognosis.-Auricular fibrillation is in the vast majority of cases indicative of some myocardial change. Furthermore, the rapidity and irregularity of the heart's action usually associated with the condition necessarily embarrass the functional efficiency of the organ. Put briefly, the prognosis of auricular fibrillation depends upon the capacity of the heart to carry on its work under the new rate and rhythm, and this in turn depends upon the integrity of the cardiac muscle. If this be relatively good, there may be for years little or no indication of cardiac failure. In the great majority of cases, however, this is not the case, and so heart failure results. This is especially the case when the pulse-rate is rapid and the heart is dilated. The rate of the pulse is of great prognostic importance, a persistent rate of 120 or over being usually of serious omen.

Occasionally, with the inception of auricular fibrillation grave cardiac failure may supervene with surprising rapidity, and death may follow within a few weeks, or even days. In endeavouring to form a prognosis, a point of the utmost importance is to ascertain how the heart responds to treatment. Cases of auricular fibrillation form a group quite by themselves in the way in which they respond to digitalis or one of its allies ; the majority respond in a striking manner (Figs. 74, and 123-128), and sometimes the drug is almost a specific, more especially in the rheumatic group. Another point of the utmost importance in weighing the prognosis is to ascertain how far the patient is able to modify his life so as to bring it within the limits of the heart's strength. 


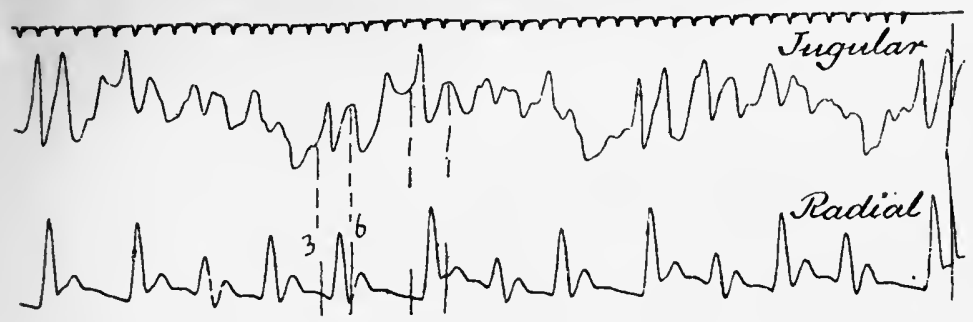

F19. 123. Simultaneous tracings of the jugular and radial puls 3 , from a case of auricular fibrillation, before the administration of digitalis. The pulse-rate is between 70 and 80 per minute.

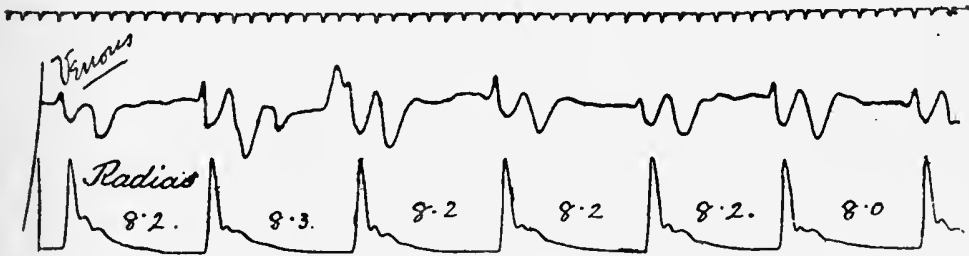

FIG. 124. Simultaneous tracings of the jugular and radial pulsas, from the same case as Fig. 123, when fully under the influence of digitalis. The pulse-rate is about 30 per minute.

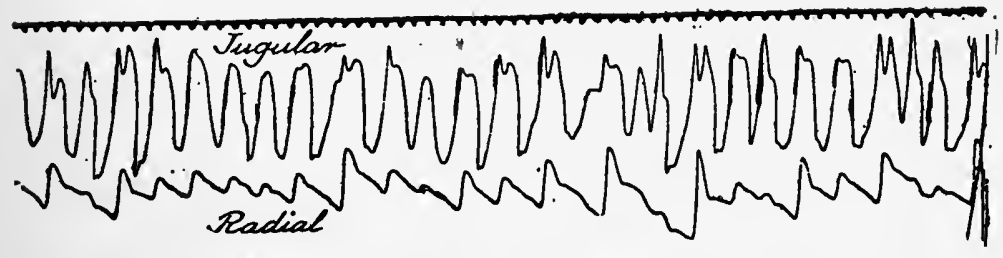

Fra. 125. Simultaneous tracings of the jugular and radial pulses, from a case of mitral disease with auricular fibrillation, before the administration of digitalis.

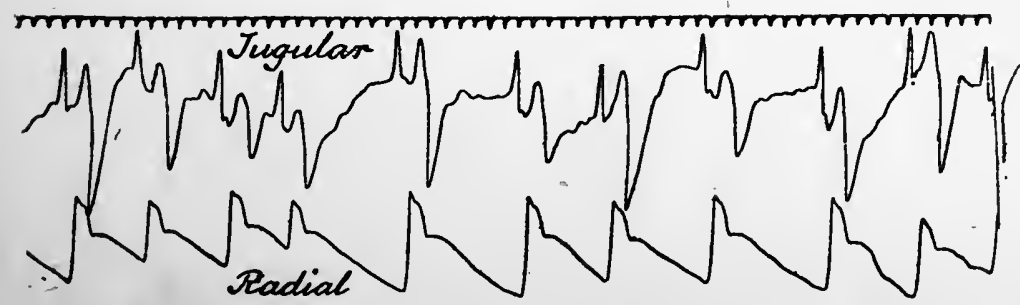

Fia. 126. Simultaneous tracings of the jugular and radial pulses, from the same patient as Fig. 125, when fully under the influence of digitalis. 
As has been pointed out, temporary attacks of auricular fibrillation usually have a tendency to become more frequent, until ultimately the condition becomes permanent.

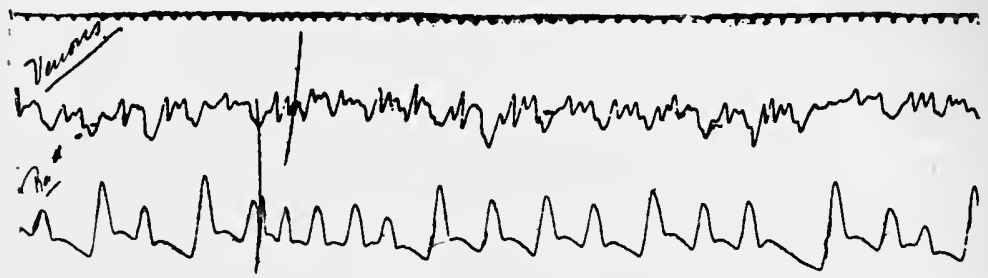

FIG. 127. Simultaneous tracings of the jugular and radial pulses, from a case of myocardial degeneration with auricular fibrillation, before the administration of digitalis.

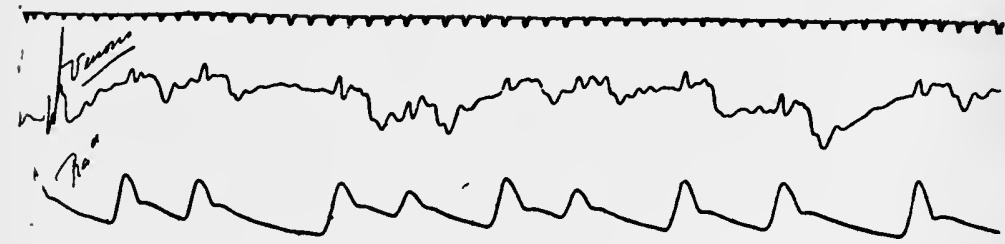

Fig. 128. Simultaneous tracinge of the jugular and radial pulses, from the same case as Fig. 127 , after the administration of full doses of
digitalis.

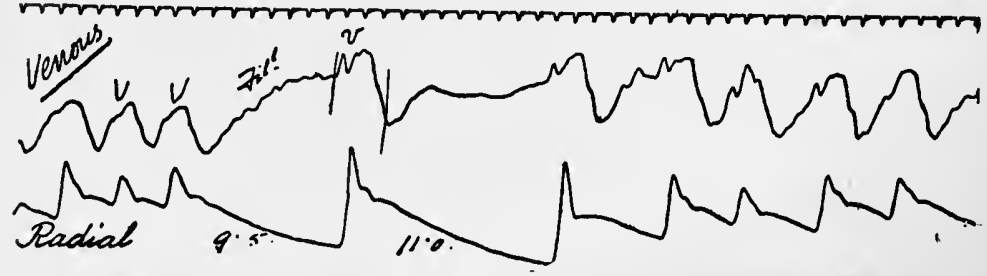

Fra. 129. Simultaneous tracings of the jugular and radial pulses, from a case of myocardial degeneration with auricular fibrillation, fully under the influence of digitalis. Note the occasional long pauses.

Treatment.-Whether there be mitral disease, aortic disease, or disease of the myocardium, if the ventricular rate be rapid, digitalis or one of its allies should be administered. The dosage, the choice of preparation, and the question of the great importance of the long-continued administration of the drug in most cases, have been fully dealt with in Chapter IX. 


\section{CHAPTER XVI}

AURICULAR FLUTTER

THE term " auricular flutter" was first used clinically by Jolly and Ritchie a few years ago, and signifies a morbid condition in which the rate of auricular contraction is extremely rapid. Auricular flutter was first induced experimentally by MacWilliam in 1887, and it is now recognised as a not uncommon clinical condition. Attacks of auricular flutter probably account for the great majority of cases of paroxysmal tachycardia with regular rhythm or with pulsus alternans. Ritchie has written an excellent monograph on the subject, to which I must refer those of my readers who wish a fuller description of the condition than is possible in a work of this kind.

Nature of Auricular Flutter.-As already noted, the contractions of the auricle are extremely rapid; the rate may range from 180 to 380 , perhaps being usually between 280 and 300 per minute. The ventricular rate varies considerably in different cases, this depending upon the auricular rate and the ability of the auricular-ventricular bundle to receive and transmit impulses sent by the auricle. Excepting in rare cases, each systole of the ventricle is the result of a stimulus for contraction received from the auricle in the normal fashion; rarely complete heart-block is present, as in the first case recorded by Jolly and Ritchie. In the vast majority of cases, the ventricle does not respond to each auricular contraction, partial heart-block being present. There is usually a definite ratio between the auricular and ventricular rate, this varying from 5:1 to $2: 1$; it would appear that the order 
of frequency is $2: 1,4: 1$, and 3:1. There may be a sudden and abrupt change from one ratio to another. In some cases, the response of the ventricle to auricular contraction is at irregular intervals, instead of being in definite ratio-this giving rise to irregularity of the pulse rhythm.

The commencement and termination of auricular flutter are sudden and abrupt. The duration of the condition varies in different individuals, as well as in different paroxysms occurring in the same individual. Auricular flutter may persist for only a few minutes or hours, and may never return; or it may last for days, weeks, or cven for years, recurring at intervals. It may be succeeded by the normal rhythm, or in many instances followed by auricular fibrillation: I have seen the latter occur during the administration of digitalis, but it may also occur apart from the use of the drug. There may be alternation of auricular flutter, the normal rhythm, and auricular fibrillation, the change taking place with the greatest abruptness and suddenness, and occurring from day to day or even cvcry few moments, one or other condition ultimately becoming permanently established. Sometimes auricular flutter may persist, but, as the result of the occurrence of partial heart-block, the ventricular rate may diminish, and this may take place during the administration of digitalis or apart from the use of this drug. When once a patient has suffered from an attack of auricular flutter, there is a tendency to its recurrence from time to time.

Effect of Auricular Flutter on the Heart.-The effect of auricular flutter on the heart depends upon the ventricular rate and the integrity of the myocardium. When the ventricular rate is rapid and the myocardium is considerably damaged, the condition produces a marked effect upon cardiac efficiency, frequently resulting in cardiac dilatation, dropsy, and other indications of heart failure. 
Etiology.-It is yet too early to speak with certainty of the etiology of this condition; possibly this is much the same as that of auricular fibrillation. It appears to be most common in advanced age, but has been found as early as five years of age. It seems to be of much more frequent occurrence in males than in females. In all probability auricular flutter is most commonly associated with myocardial degeneration. Not infrequently mitral stenosis is found to be present, while in some cases there is a history of rheumatism; the condition has also been met with during the course of some of the acute infectious diseases.

Symptomatology.-The symptoms after the onset of auricular flutter may come on gradually or rapidly, and the patient may or may not be conscious of the change in the cardiac action. He may complain of palpitation, but otherwise there may be an entire absence of subjective symptoms, this depending upon the ventricular rate, the degree of integrity of the myocardium, and the duration of the condition. If the myocardium be fairly healthy and there be not much increase in the ventricular rate, there may be for some time only a moderate degree of dyspnœa or other indications of cardiac distress on exertion; if, however, the rate of ventricular contraction be much increased, the efficiency of the heart is apt to be greatly impaired; while if the integrity of the myocardium be considerably involved and the ventricular rate be rapid, such indications of cardiac failure as cardiac dilatation and dropsy are apt to supervene.

Taking into consideration all cases of auricular flutter, in a majority in which the condition has existed for some time, the ordinary symptoms of cardiac failure supervene; or, if these were present before the onset of auricular flutter, they increase in severity, until ultimately indications of extreme cardiac failure may appear. When the ventricular acceleration is marked, as the amount of blood propelled from the ventricle at each systole is so 
small as to give rise to cerebral anæmia, the patient may complain of vertigo, and there may be from time to time attacks of faintness or even complete loss of consciousness. Cheyne-Stokes' respiration is also not an infrequent symptom when the condition has lasted for some time.

The rate and rhythm of the arterial pulse may vary greatly in different cases, and, it may be, at different times in the same case. If the ventricle respond to each auricular contraction, the ventricular rate is exceedingly rapid, and graphic methods are necessary to determine it. In the vast majority of cases, however, partial heart-block is present, and as 2:1 rhythm is the most usual a ventricular rate of 140-150 is frequently found. In other cases of partial heart-block, the ventricular rate may not be much increased, and, indeed, there may even be bradycardia, as in 4:1 or more partial heart-block-as well as in complete heart-block. When there is a constant and uniform ratio between the auricular and ventricular rate, the rhythm of the arterial pulse is regular ; in these cases, however, if the ventricular rate be above 150, pulsus alternans may be present. When, as is not infrequently the case, the response of the ventricle to auricular contraction is at irregular intervals, there is irregularity of the pulse rhythm, it may be even markedly so; indeed, in some of these cases the sphygmogram may resemble that from a case of auricular fibrillation, detailed measurements being necessary for the purpose of differential diagnosis.

On inspection of the neck, the jugular veins may be distended and no pulsation visible; or extremely rapid movements may be evident.

Evidences of cardiac dilatation, as, for example, enlargement of the area of percussion impairment, may be found. If murmurs of mitral disease were present prior to the onset of auricular flutter, they may become faint or almost disappear if the ventricular rate be greatly 
accelerated; this applies particularly to the case of a mitral presystolic bruit.

A tracing of the jugular pulse differs in outline according to whether the ventricle responds to each auricular

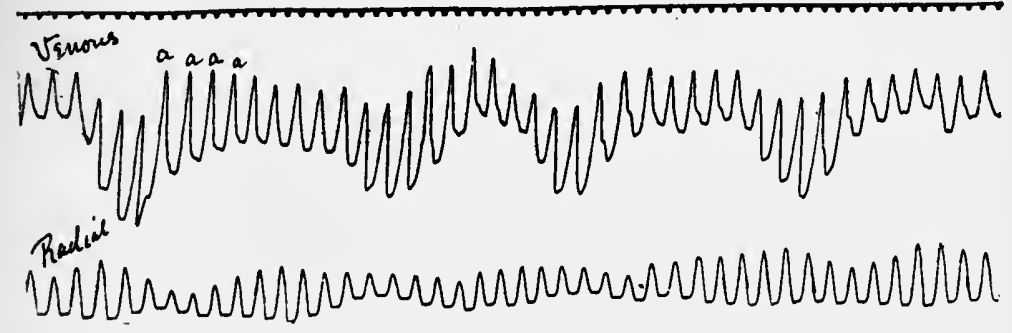

Fia. 130. Simultaneous tracings of the jugular and radial pulses from a case of auricular flutter, in which the ventricle responded to each auricular contraction. The rate is 188 per minute.

contraction, or to the degree of heart-block which may be present. In the former case, there is usually a single wave (Fig. 130). When the ventricle responds alternately to

\section{Sugular}

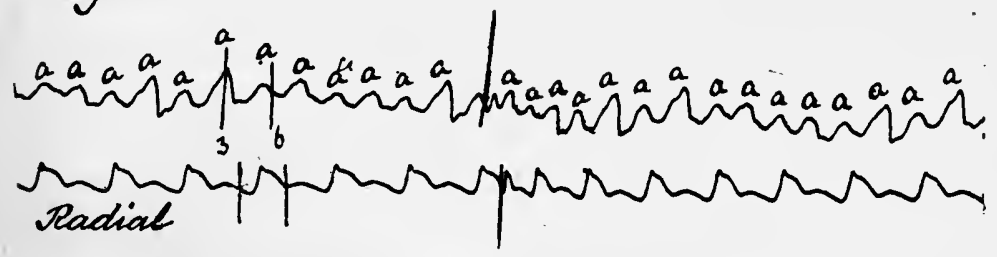

Fra. 131. Simultaneous tracings of the jugular and radial pulses from a case of auricular flutter, with $2: 1$ heart-block. The rate of the auricle is 186 per minute.

the auricle, there are two $a$ waves to each ventricular beat, and these usually fall within the limits of ventricular systole (Figs. 131 and 132). In 3:1 r. rhythm the character of the jugular pulse may be seemingly that of normal cardiac action, and it is particularly in these cases that an electro-cardiographic examination is required to estab- 
lish the diagnosis. As has been already noted, complete heart-block is rarely found.

Diagnosis. - The diagnosis of the condition rests on the detection of the extremely rapid contractions of the

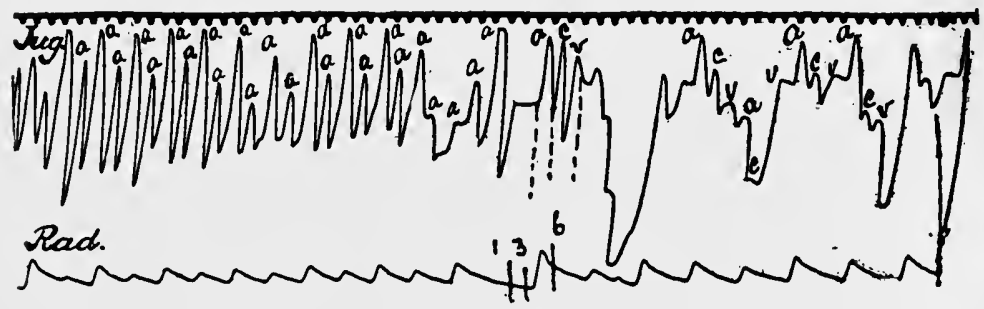

Fro. 132. Simultaneous tracings of the jugular and radial pulses from a patient with myocardial and arterial disease, showing the termination of an attack of paroxysmal tachycardia, due to auricular flutter. The first part of the tracing shows auricular flutter, with $2: 1$ heart-block. the rate of the auricle being 232 , and the ventricular rate 116 , per minute. The normal rhythm of the heart is resumed after the long pause with the beat $1,3,6$.

auricle, and this is not infrequently impossible without the employment of the polygraph or electro-cardiograph; indeed, in some cases a correct diagnosis cannot be made with certainty even though the polygraph be

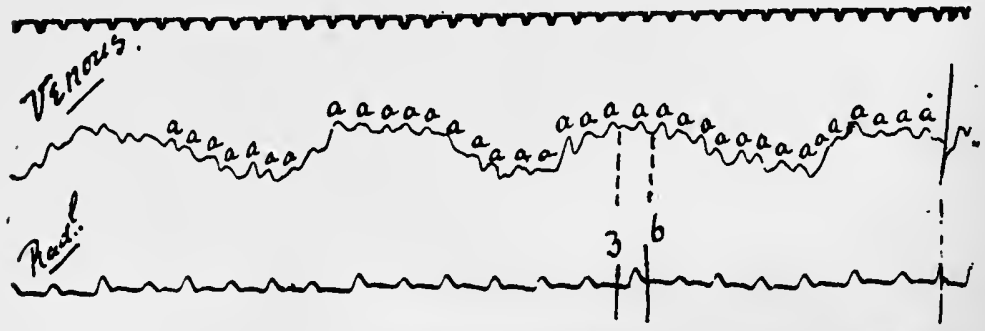

FrG. 133. Simultaneous tracings of the jugular and radial pulses from a case of auricular flutter. The rate of the auricle is 370 per minute.

employed, an electro-cardiographic examination being necessary.

Whenever an individual is conscious of a marked increase in the cardiac rate or complains of attacks of palpitation, occurring suddenly and without apparent 
cause, or suffers from dyspnœa on exertion and other indications of cardiac failure coming on rapidly, we should consider the possibility of auricular flutter, especially if the rhythm of the pulse be regular.

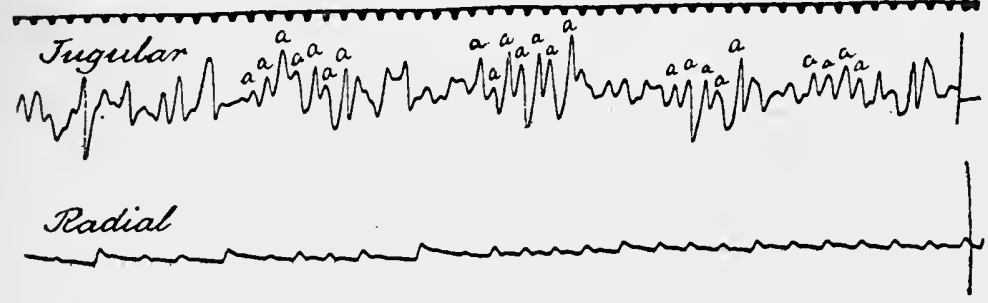

Fra. 134. Simultaneous tracings of the jugular and radial pulses from a case of auricular flutter. The rate of the auricle is 372 per minute.

Auricular flutter should be diagnosed from tachycardia associated with a normal rhythm. In a polygraphic tracing of the latter, the $a$ wave is superimposed upon the $v$ wave of the antecedent cardiac cycle, because of the

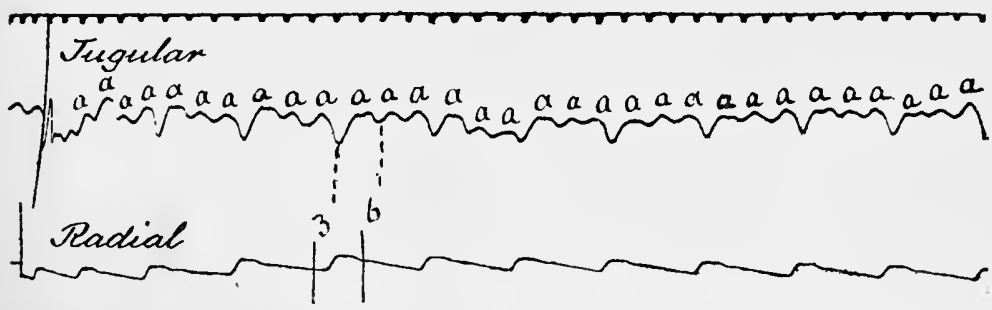

Frc. 135. Simultaneous tracings of the jugular and radial pulses from a case of auricular flutter, with partial heart-block. The rate of the auricle is 250 , and the ventricular rate 74 , per minute.

shortening of the ventricular diastole due to the greatly increased frequency of the cardiac rate. In these cases, if a presystolic bruit, due to auricular systole, be present, it disappears; it should, therefore, be remembered that marked pulsation of the jugular veins and disappearance of a mitral presystolic bruit in cases in which there is great exaggeration of the ventricular rate are not necessarily indicative of the supervention of an abnormal 
rhythm. If the tachycardia be due to an abnormal rhythm, and if the ventricular rhythm be regular, the case is probably one of auricular flutter; while if the rhythm be irregular, it is necessary to consider whether the case

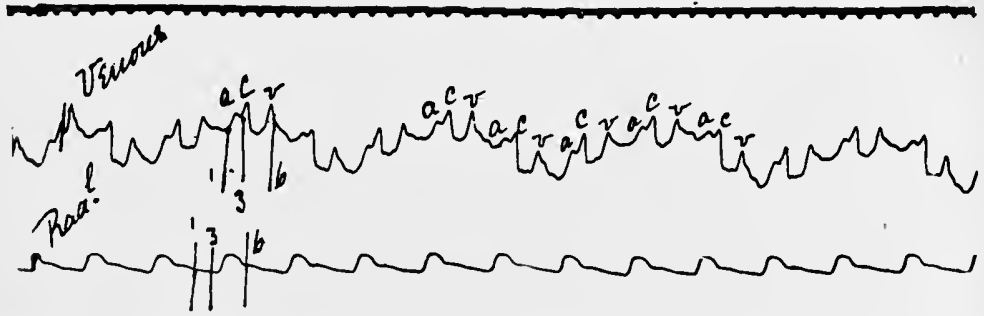

FIG. 136. Simultaneous tracings of the jugular and radial pulses from a patient subject to attacks of paroxysmal tachycardia, due to auricular flutter. The rhythm of the heart is normal and the pulse-rate is 83 per minute.

may not be one of auricular fibrillation. In this connection, if a sphygmogram from a case of auricular flutter be carefully studied, it will be found that even though the ventricular rhythm is markedly irregular the pulse-beats measure out

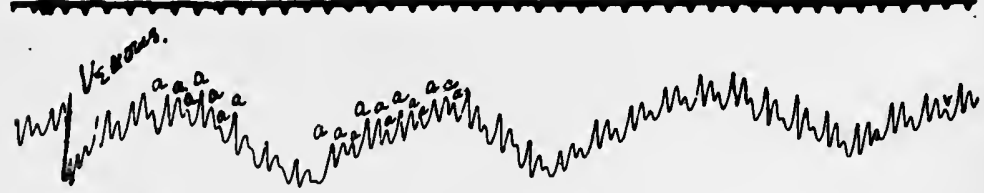
trin

FIG. 137. Simultaneous tracings of the jugular and radial pulses from the same patient as Fig. 136, after walking for about a quarter of a mile. The exertion induced an attack of auricular flutter. The auricular rate is 382 , and the ventricular rate 187 , per minute.

into groups of equal length, whereas this is never the case in auricular fibrillation. The polygraph and electrocardiograph are of notable assistance in the differential diagnosis of the two conditions. The diagnosis of auricular fibrillation with markedly exaggeratedliventricular rate in 
which the irregularity is only slight from auricular flutter should also be considered.

Cases of auricular flutter in which the auriculo-ventricular ratio is constantly $3: 1$ to $4: 1$, so that the ven-

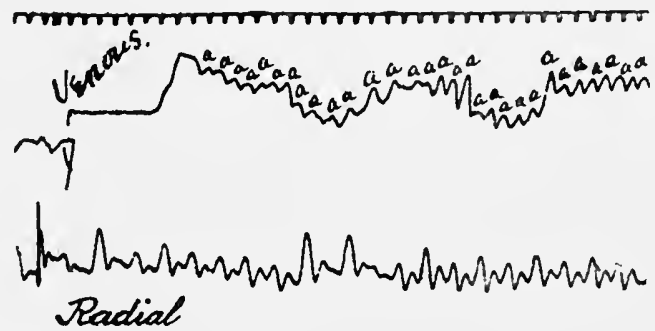

FIG. 138. Simultaneous tracings of the jugular and radial pulses from a case of auricular flutter. The auricular rate is 382 per minute. The radial pulse is about 185 per minute and shows alternation.

tricular rate is not much, if any, increased, and in which the ventricular rhythm is regular, are easily missed. The employment of the polygraph or electro-cardiograph is often necessary in such cases. Irregularity due to the

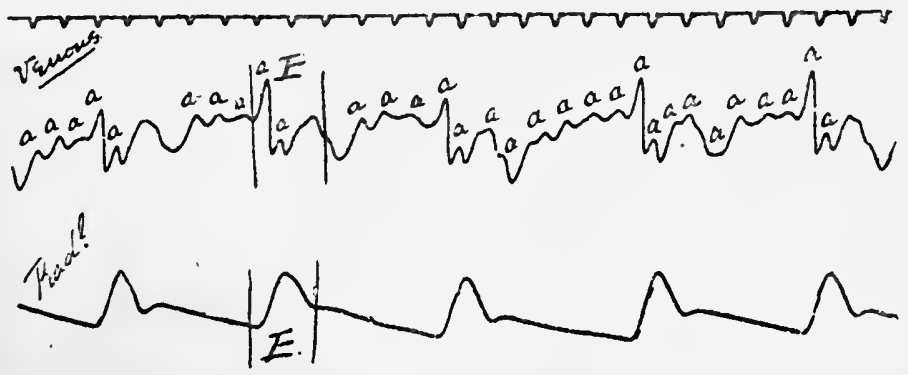

FiG. 139. Simultaneous tracings of the jugular and radial pulses from the same patient as Fig. 138, when fully under the influence of digitalis. The auricular rate is 382 , and the ventricular rate is 50 , per minute.

presence of extra-systoles should also be excluded; for their differential diagnosis, as well as for that of other conditions from auricular flutter, the reader is referred to the book on this subject by W. T. Ritchie which has already been mentioned.

The Action of Digitalis on Auricular Flutter.-The 
administration of full doses of the drug at first usually induces partial heart-block or an increase of the degree of block already existing, with a resulting diminution of the ventricular rate (Figs. 138 and 139). If the impulses passing from the auricles to the ventricles be blocked at irregular intervals, irregularity of ventricular rhythm results; while if $2: 1,3: 1$, or $4: 1$ ratio be induced, the rhythm is regular. Later on, the drug may induce auricular fibrillation. This may persist, or the physiological rhythm may be restored - either evcnt occurring during the administration of the drug or after it has been discontinued-or the normal rhythm may be interrupted from time to time by paroxysms of flutter, or after the cessation of the drug the flutter may return and continue.

In a hospital patient whom I had the opportunity of observing for some months, auricular fibrillation developed during the administration of the drug, and some time after the drug was stopped there was a return to the normal rhythm. In another case, which I have watched for some years, auricular fibrillation developed during the administration of the drug, and when this was discontinued auricular flutter returned. This occurred on several occasions, until at last the normal rhythm was established during the administration of the drug, and has persisted, except for brief attacks of flutter occasionally induced by exertion; while if the administration of digitalis be stopped, the flutter returns and persists until the drug is again used, when, after full doses have been taken for some days, the normal rhythm is again restored.

Prognosis.-The prognosis of auricular flutter depends upon the frequency and duration of the attacks, the ventricular rate, the condition of the valves, the myocardium, and the vessels, and the results of the administration of digitalis. When once a patient has suffered from an attack of auricular flutter, there is a tendency to its recurrence from time to time. The effect which an 
attack has upon the heart depends upon the ventricular rate and the degree of integrity of the myocardium; these have been already dealt with. Attacks of loss of consciousness, especially when they occur in the subjects of marked cardiac failure, are attended with danger.

Treatment.-When the attacks are transient, absolute rest is advisable, and the treatment is that of paroxysmal tachycardia. When they are of longer duration, or the condition has become permanently established, one of the digitalis series of drugs should be administered in large doses. We may employ the tincture of digitalis, commencing with a drachm, or in urgent cases with even $1 \frac{1}{2}$ or 2 drachms, per diem. These doses should be continued until the full physiological reaction is obtained. After this one of two plans may be adopted: (1) The dosage may be lessened, and afterwards the administration of the smallest dose necessary to control the ventricular rate continued in the hope that the flutter may cease; or (2) large doses may be continued, in the hope of inducing auricular fibrillation, after which the administration of the drug is discontinued for some days, in the hope of a return to the normal rhythm. The first plan is generally to be preferred. 


\section{CHAPTER XVII}

\section{PAROXYSMAL TACHYCARDIA}

Definition and Nature.-The term paroxysmal tachycardia is here employed to denote a condition, not uncommon, in which a marked acceleration of the cardiac rate occurs, which commences suddenly and abruptly and without apparent cause, lasts for a varying period, ccases suddenly and abruptly, and is due to an abnormal rhythm - the stimulus for cardiac contraction, instead of arising at the junction of the great veins with the auricle, having its origin at an abnormal point. The rcturn of the cardiac rate to what it was prior to the paroxysm is due to the reversion of the cardiac rhythm to the normal. From the definition given, it will be seen that paroxysmal tachycardia does not include acceleration of the cardiac rate associated with the normal or sinus rhythm.

It should be noted that the nature of the abnormal cardiac rhythm is not always the same; in other words, the condition which gives rise to the paroxysm of tachycardia varies. The point of origin of the new rhythm is usually situated in the auricle, and the ventricle responds to impulses received from that chamber; but it may rarely arise in the ventricle, and in this case the auricle responds to impulses received from the ventricle. When the former is the case, the two commonest causes are temporary auricular flutter (Figs. 132, 136 and 137), and auricular fibrillation (Fig. 140), but there may occasionally be other causes (Fig. 233).

The paroxysm of tachycardia may last only for a few beats, or may persist even for months ; as a rule, it lasts 
for some hours, and only exceptionally does it continue for several weeks, and rarely for months. The patient may experience one attack and never have another, or

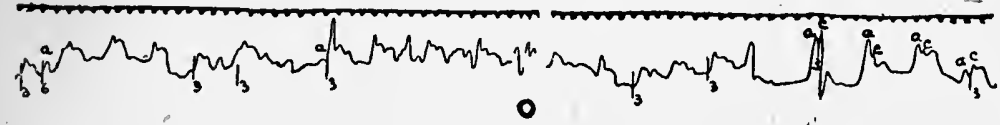

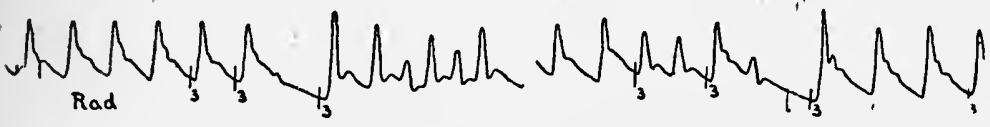

Fra, 140. Simultaneous tracings of the jugular and radial pulses, showing the commoncem nnt and termination of a short attack of paroxysmal tachycardia, due to auricular fibrillation. The first six beats and the last three in the sphygmogram are regular and due to a normal contraction of the heart. The beats between occur during a period of auricular fibrillation. At $O$ a period of fifteen seconds has been cut out (Mackenzie).

he may be subjected to many in the course of twentyfour hours, or the attacks may occur at varying intervals, frequent or long, for many years. Permanent auricular fibrillation or flutter may supervene.

Etiology.-The etiology of paroxysmal tachycardia is obscure. It may occur at any age after five, but usually first occurs during middle life. It appears to be more common in males, and this certainly agrees with my experience. A history of previous infection by rheumatism is not uncommon, and a fair proportion of cases are the subjects of valvular disease, particularly of mitral stenosis, or of myocardial degeneration; but no evidence of organic disease of the heart is found in a considerable proportion of cases. The condition has been noted in association with alimentary toxæmia, in reflex irritation, neurasthenia, and other morbid states. Among exciting causes are physical exertion (Figs. 136 and 137), emotional excitement, digestive disturbances-particularly flatulent distension of the stomach or colon, and in one of my own cases the adoption of a certain posture appeared to bring on the attacks.

Symptomatology.-In some cases there is limitation of 15 
the field of cardiac response between the attacks, such as dyspnœa and precordial distress on exertion. Other cases are absolutely free from subjective symptoms in the intervals, but in many cases auricular or ventricular extrasystoles are to be observed.

It has been already noted that there is a sudden, abrupt, and great acceleration of the cardiac rate, coming on without apparent cause, while at the end of the paroxysm there is a sudden and abrupt return to the rate and cardiac rhythm which preceded the attack. In some cases the patient is able to recognise the onset and termination of the tachycardia, while in others he is unable to do so. After the onset, the patient may be unconscious of the tachycardia, if the attack be brief; but, if it persist for any time, he usually becomes conscious of it, and gencrally complains of a fluttering sensation in the chest, this sensation being unlike that of palpitation. The patient may also experience a sensation of pulsation in the neck. His face is usually pale, and has an anxious expression. The cardiac rate is usually above 140 per minute, and may reach even 300 ; in a majority of the paroxysms it is between 150 and 190 ; it should be counted at the apex, either by palpation or auscultation-unless it is extremely rapid, when graphic methods are necessary. The pulsc is of smaller volume than normal, and its character may resemble that of the pulsus celer. It may be quite regular, or very irregular when the condition is due to auricular flutter in which the response of the ventricle to auricular contraction is at irregular intervals, or compl.tely irregular when the result of auricular fibrillation. A noteworthy feature is the frequent presence of pulsus alternans, but this can only very rarely be made out by the finger. The blood-pressure is generally lower during the attack. The area and force of the apex-beat may be increased. The jugular veins may be distended, and may show very rapid pulsation.

Analysis of the polygraphic tracings gives different 
results, according to the site from which stimuli for eontraction arise; in all probability, it will show auricular flutter or auricular fibrillation as the cause of the attack.

During the attack the common indications of cardiac failure may be present, their severity depending upon the cardiac rate, the duration of the paroxysm, and the integrity of the myocardium. In some cases, even when the paroxysm lasts for a day or two, there may be only some limitation of the field of cardiac response, with little, if any, cardiac dilatation, hepatic enlargement, or dropsy; while, in long-continued paroxysms, and even in some cases in which the paroxysms last for only a few hours, cardiac failure may be extreme. There may be dyspnœa on exertion, a feeling of discomfort, oppression, or actual pain in the chest, lassitude, weakness, or exhaustion; pallor of the skin, with undue prominence of the superficial veins, cold perspiration, an anxious expression of the face, and symptoms of cerebral anæmiasuch as giddiness and faintness; flatulence, nausea, and vomiting may also be present. Later on cyanosis, cough with expectoration, and even hæmoptysis and signs of œdema of the bases of the lungs may be present.

The area of cardiac impairment may become considerably enlarged-even within a few hours, the first sound of the heart shortened, sharp, high, and clear in pitch, the long pause is often shortened, while the rhythm of the sounds sometimes resembles that of a fœtal heart, and if murmurs were previously present they alter in character or even disappear. There may be tenderness of the precordium and hepatic area, the liver may be palpable and pulsating, ascites and general anasarca may occur, and the urine may become scanty, high-coloured, and albuminous.

A remarkable and characteristic feature of the condition is that with the sudden reversion of the rhythm to the normal there is an extraordinarily rapid recovery to the 
state in which the patient was prior to the paroxysm; within a few hours there may, indeed, be no evidence of cyanosis, distension of the veins, cardiac dilatation, or enlargement of the liver. In some cases the cessation of the attack is accompanied by the passage of large quantities of wind, and excessive eructations, while a large quantity of urine may be voided. Death during the attack is not common.

Diagnosis.-The diagnosis of paroxysmal tachycardia is usually not difficult. The most important points from the diagnostic point of view are (1) the cardiac ratc, and (2) the commencement and termination of the attack of tachycardia, the second of these being the more important. With regard to the cardiac rate, a persistent ventricular rate of over 160 is almost invariably due to an abnormal rhythm, and a persistent rate of over 140 may be due to the same cause. Tachycardia of a rather less severe grade must certainly not be presumed to be due to an abnormal rhythm. There are, however, distinguishing features between tachycardia due to a normal and that due to an abnormal rhythm. The suddenness and abruptness of the onset and termination of the tachycardia is a characteristic feature of the latter-the maximum rate is attained within a few seconds, and the return of the cardiaz rate to what it was prior to the attack takes place equally quickly, whereas in tachycardia associated with a normal rhythm, the onset and offset are gradual; and, further, as Lewis has pointed out, the cardiac rate is not influenced by change of posture or other forms of physical exertion, as is the case in tachycardia not associated with an abnormal rhythm. Polygraphic or electrocardiographic examination will put the diagnosis beyond all doubt.

Prognosis.-The difficulty in forming a prognosis in any case of paroxysmal tachycardia is great. The prognosis may be considered from two standpoints : (1) that of a particular paroxysm, and (2) the question of recurrence 
of the attacks. With regard to the former, it may be noted that death during an attack is comparatively infrequent, although this may occur when the duration of the attack is prolonged. The points which should be taken into account in considering the prognosis of an individual attack are the ventricular rate, the duration of the attack, and the degree of cardiac failure present. When the ventricular rate is not very high, and there is little or no cardiac dilatation, and an absence of oedema of the lungs, hepatic enlargement and anasarca, the outlook is good as far as the risk to life is concerned; while, on the other hand, if the clinical picture be the reverse, the outlook is uncertain, although it should be remembered that the paroxysm may cease at any time and there is no means of knowing when this may occur, and the patient improves with extraordinary rapidity.

With regard to the question of the recurrence of the attacks, it is impossible to give an answer. For, in some cases the patient may never suffer from a second attack; while, on the other hand, they may recur, even several times in the course of twenty-four hours, or at frequent intervals for many years, or, lastly, permanent auricular flutter or fibrillation may supervene even after a few attacks. An attempt should be made to estimate the degree of integrity of the myocardium between the attacks, and, as has been already pointed out, the best way to ascertain this is to find out how the heart responds to effort. If the field of cardiac response be not diminished, and the attacks be infrequent and transitory, the cardiac rate not being much increased, with an absence of or only a slight degree of cardiac failure during the paroxysm, the prognosis may be considered good.

Treatment.-The results of treatment of this disorder, in the great majority of cases, are very unsatisfactory. It is true that the paroxysm not infrequently ceases when various remedies are applied; thus, bringing up of 
wind, the act of vomiting, the adoption of a certain posture, pressure upon the vagus-particularly the right, friction of the chest-wall, and local applications in the form of an ice-bag, mustard leaves, or warmth to the precordium, may be followed by relief. But it should be remembered that the nature of the disorder is to stop suddenly, and, therefore, the question of post hoc and propter hoc always arises. As far as my observations have gone, the remedy which offers most encouragement is strophanthin injected intravenously. Failing this, digitalis should be given by the mouth and pushed to the full physiological reaction. If there be indications of heart failure, such as dyspnœa, cyanosis, or dropsy, treatment on the lines laid down elsewhere should be adopted.

Between the attacks, the general condition, and whatever appears to be the exciting cause of the paroxysms, should receive attention. Any gastro-intestinal disorder should always be corrected. Bromides may be tried. In one case-and in one only-in my experience, the continuous administration of digitalis proved effective. 


\section{CHAPTER XVIII}

CARDITIS : THE RHEUMATIC INFECTION OF THE HEART IN CHILDHOOD

By carditis is meant inflammation involving the endocardium, myocardium, and pericardium simultaneously.

Before dealing with endocarditis, myocarditis, and pericarditis individually, it may be advisable to discuss the rheumatic infection of the heart in childhood. This is a subject of the utmost importance, for by far the larger number of cases of heart disease under thirty years of age, and a considerable proportion of those of later life, are the direct result of this infection. And, notwithstanding the fact that this subject has received a great deal of attention, it is still not sufficiently realised that it is very easy to overlook a rheumatic infection in childhood, and, even when we are especially on the look-out for it, it is not infrequently very difficult to come to a definite conclusion whether the heart is affected or not. This is unfortunate, because the future welfare of the patient in large measure depends upon the adoption of sufficiently prolonged, careful, and adequate treatment during the stage of infection.

In all probability, the reason why infection of the heart by the rheumatic poison is so frequently overlooked is that the clinical picture of acute and subacute rheumatism was originally drawn from the disease as it appears in adult life, the result being that many practitioners do not even now realise that rheumatism as it appears in childhood presents many and important points of difference. 
It is important to bear in mind the following facts : In adult life arthritis is the chief manifestation of the rheumatic infection, and is looked upon as constituting a typical attack, while cardiac involvement is regarded as a complication. In children, on the other hand, arthritis is usually slight, and may be entirely absent, while affections of other parts are more frequent and constitute a more conspicuous feature in the disease.

Endocarditis, myocarditis, or pericarditis may accompany the slightest affection of the joints. Fewer joints are usually affected than in the case of the adult, there is often no perceptible swelling, and only a little pain, stiffness, and tenderness; acid pcrspirations are usually slight, and the pyrexia is in most instances moderate. Sometimes the affection is confined to the tendons or the fascia in the neighbourhood of the joint, as, for example, the tendons of the hamstring muscles under the knee, causing the child to walk on the tips of the toes, with the knces bent. This may be mistaken for incipient talipes equinus, or paresis. On the other hand, we should be careful to exclude infantile scurvy, acute anterior poliomyelitis in its early stages, syphilitic disease of the ends of the long bones, and other affections of the joints in children.

Endocarditis, myocarditis, and pericarditis are much more frequently a manifestation of the rheumatic infection in children than in adults, being indeed at least as usual an indication of the rheumatic infection as any other ; they are just as much part of the disease as is the affection of the joints, and should not be regarded as " complications." In childhood, subcutaneous nodules, certain exudative erythemata, and purpura rheumatica are also more frequent manifestations of the rheumatic infection; chorea is a common indication; and tonsillitis is also perhaps more commonly of rheumatic origin than in adult life. Cardiac involvement may occur in association with any of these, or with an indeterminate 
febrile attack, or it may occur alone-that is, it may be the only manifestation of rheumatism.

Endocarditis, myocarditis, and pericarditis occur more frequently in conjunction in children than in adults. While endocarditis may occur acutely in children as it does in adults, it often occurs in a subacute and insidious form. There is not infrequently a total absence of subjective symptoms, which if present are often masked by other manifestations of the rheumatic infection. The diagnosis, therefore, rests largely upon physical signs, and the existence of the condition is frequently unknown or unsuspected until the heart is examined. The physical signs, however, are not infrequently slight and vague in character, and the diagnosis is consequently often attended with extreme difficulty.

Now, if the foregoing be facts, it is clear that when a child complains of pain in the joints, muscles, or tendinous structures, or what are called "growing" pains; or has subcutaneous nodules in the tendons or round the joints, or subperiosteal nodules on the bones; or exhibits an erythematous eruption; or if there be a suspicion of chorea; or if he suffer from malaise and unexplained pyrexia ; or complain of pain in the chest, or shortness of breath, or present marked pallor, he should at once be put to bed, and a systematic and most careful examination of the heart be performed daily, and if there be any doubt whether the heart is involved or not, the patient should be kept in bed till this has been set at rest. 


\section{CHAPTER XIX}

\section{ENDOCARDITIS}

$\mathrm{By}$ endocarditis is meant inflammation of the lining membrane of the heart. In the great majority of cases the inflammation involves chicfly the endocardium of the valves, and is not infrequently so limited. The term "valvulitis" is applicd to endocarditis of the valves, while inflammation of the endocardium lining the cavities of the heart is referred to as mural endocarditis. The surface of the valves next the blood-stream, viz., the ventricular surfacc, of the semilunar valves and the auricular surface of the auriculo-ventricular valves, is affected. In adults the left side of the heart is far more commonly affected than the right - the mitral valve being more frequently attacked than the aortic; while, on the other hand, during fœtal life the oppositc is the case. Some degree of myocarditis is probably always present with endocarditis.

Varieties of Endocarditis.-A satisfactory classification of endocarditis is not easy, that most commonly adopted being as follows: (1) acute, (a) simple or benign, and (b) ulcerative, malignant, or infective ; and (2) chronic or sclerotic. It should be noted that it is impossible to draw a sharp line of distinction between these varieties on grounds of etiology, pathology, or morbid anatomy. Clinically, however, the distinctive features of each are fairly marked, although even in this case the distinction is not absolute. E. Libman, following Osler and Horder, has recently described a sub-acute variety, ulcerative in its character, and in these cases he has isolated a streptococcus, which he has called streptococcus mitis. This is apparently 
the streptococcus salivarius, or streptococcus fæcalis of Andrews and Horder.

\section{Acute, Simple or Benign Endocarditis}

In this form of endocarditis, the parts affected become swollen, as the result of œdema and connective tissue proliferation, and minute cauliflower-like or warty excrescences, called vegetations, make their appearance on the segments of the valve or on the mural endocardium. The valvular vegetations are usually not situated at the extreme margins of the cusps, but at those parts which come into apposition during closure, namely, a short distance from their margins. The vegetations consist of blood platelets, leucocytes, and fibrin. Carey Coombs has found foci containing large multi-nuclear cells in the inflamed valves. Various micro-organisms, including streptococci, staphylococci, pneumococci, gonococci, and more rarely tubercle bacilli, bacillus typhosus, bacillus anthracis, bacillus coli communis, bacillus diphtheriæ, meningococcus, and the diplococcus rheumaticus of Poynton and Payne, have been found on examination of the vegetations, but they are not present in large numbers in the acute simple form of endocarditis.

Fragments may bccomc detached from the affected valves, be carried by the blood to remote parts, and ultimately become impacted in a vessel; this process is known as embolism, and the impacted fragment as an embolus. This may result in obstruction of the circulation, and (1) necrosis, or hæmorrhage, or both, within the area of distribution of the occluded vessel, and the formation of infarcts, or (2) gangrene of the area supplied in the case of one of the larger arteries of the limbs. Infarction most generally occurs in the spleen or kidneys, although it is not infrequently found in the brain or its membranes, the retina, lungs (in right-sided endocarditis), intestines, and skin. In cerebral infarction, softening of the area supplied by the affected vessel takes place rapidly, 
while in pulmonary infarction localised pneumonia is a frequent sequel.

An attack of acute simple endocarditis may be followed by, (1) resolution, a rare event ; (2) organisation, which is the usual sequel; or (3) ulceration. When organisation occurs, it results in the formation of fibrous cicatricial tissue, which tends to contract as life advances, and ultimately to give rise to permanent stenosis, or incompetenee, or both.

Etiology.-Acute simple endocarditis occurs most commonly in childhood and adolescence, and is rarely, if ever, a primary discase. Acute rheumatism is responsible for the great majority of cases, the onset of endoearditis occurring usually about the end of the first week. The association between the rheumatic infection and acute endocarditis has been dealt with in Chapter XVIII. Chorca and searlet fever are common causes of acute simple endocarditis; less frcquently tonsillitis, pneumonia, smallpox, measles, ehickenpox, diphtheria, and other infectious diseases; while Bright's disease, gout, syphilis, diabetes, and pulmonary tuberculosis are also supposed to be responsible for a certain number of cases.

Acute simple endocarditis not infrequently occurs in individuals who are the subjects of the chronic or selerotic form of the disease-the so-called recurring endocarditis. Symptomatology.-It has already been noted that while endocarditis may occur acutely in children as in adults, it often occurs in a subacute and insidious form. There is not infrequently a total absence of subjective symptoms, or, when present, they are often masked by other manifestations of the causal infection. Sometimes the onset is aceompanied by a rise of temperature above that already existing, this increased pyrexia occurring without any increase in the arthritic symptoms in the case of rheumatism; the degree of pyrexia is usually not marked, but occasionally there is a sharp rise of temperature. There may be toxic rashes, in the form of urti- 
carial erythcma, erythema multiforme, and erythema nodosum.

The patient may suffer from palpitation, mild or severe; dyspnœa and precordial distress may be also present, the former being usually not pronounced, while the latter generally takes the form of an uncomfortable feeling in the precordium, not amounting to actual pain. Faintness, actual precordial pain, and oedema are rarely met with until the affection is far advanced. In cases of severe carditis, however, in which great and rapid dilatation occurs, symptoms of marked cardiac failure may be present even at the onset of the illness.

The pulse is usually increased in frequency, and is in some cases irregular. The apex-beat may be tumultuous in character, and displaced outwards, and there may also be evidence of enlargement of the area of cardiac impairment transversely; these signs may occur very early in the disease. The first sound at the mitral or aortic area, according to which of these valves is affected, may become slightly prolonged, or roughened, or may exhibit a lack of clearness; within 24 hours it may be accompanied by a distinct murmur, soft and blowing in character, the cardiac sound, however, not being abolished. The murmur is strictly limited to either area. In the case of mitral incompetence, the pulmonary second sound later on becomes accentuated, and may be reduplicated. In the case of the aortic valve, the second sound may become altered, and a soft, blowing, diastolic bruit may subsequently develop; the latter, however, is of much less frequent occurrence during the course of the illness. Reduplication of the second sound and slight accentuation of the first sound at the apex are believed to be early signs of mitral stenosis. A sof $t$, blowing, diastolic bruit in the mitral area is rarely heard during the course of the illness. A mitral presystolic murmur and a short sharp first sound do not usually make their appearance until some years after the onset 
of the rheumatic infection, the mitral orifice having become permanently stenosed.

It is not my intention here to discuss fully the symptoms of embolism, but it may be desirable to outline a few of these, according to the locality affected. When the spleen is the region attacked, the condition frequently remains latent; but there may be sudden sharp pain with tenderness in the splenic region, evidence of enlargement of the organ, and possibly friction fremitus, due to perisplenitis. When the kidney is the organ affected, here also there are often no symptoms to be noted, although there may be sevcre lumbar pain, tenderness on pressure, albuminuria, and at times hæmaturia. In cerebral embolism, the middle cerebral artery (usually the left), or one of its branches, is most commonly attacked. In such a case, aphasia, paralysis, delirium, or coma may result, the symptom-complex varying according to the region of the brain involved, and resembling that of cerebral hæmorrhage. In the case of embolism, however, there are no premonitory symptoms, the onset is sudden, coma is not so profound, and, indeed, consciousness is as a rule not entirely abolished. When the retinal vessels are affected, loss of vision, hæmorrhage, or optic neuritis may be the sequel. Pulmonary embolism may be immediately fatal. As a rule, however, there are sudden severe dyspnœa, pain in the chest, cough, with bloody expectoration, and sudden collapse; while if one of the smaller branches of the pulmonary artery be involved the symptoms may be only slight. On examination, impairment of the percussion note, absence of breath sounds, evidence of pleural friction, and, sometimes, the physical signs of pneumonia, may be.detected. When the larger arteries supplying the extremities are involved, there may be sudden severe pain, gangrene of the affected part, and complete or partial obliteration of the pulse below the site of occlusion. In the case of embolism of the superior mesenteric artery, there is sudden severe colicky pain in the abdomen, to- 
gether with tenderness and distension; loose, offensive, and tarry motions ; collapse, and death within a few days. Petechial hæmorrhages in the skin are supposed to be duc to minute emboli. When hepatic embolism occurs, symptoms are usually absent. Embolism of the coronary artery is rare; its symptomatology will be discussed later.

Diagnosis.-The diagnosis of acute simple endocarditis is often a matter of great difficulty. We should suspect its existence in those cases in which there is an increase in the degree of pyrexia without any aggravation of the joint symptoms during the course of acute rheumatism, or when pyrexia persists without any ascertainable cause ; or when there is palpitation, dyspnœa, acceleration or irregularity of the pulse, together with the existence of a murmur not previously present.

Increased frequency of the pulse-rate, provided other causes can be excluded, is of considerable diagnostic importance: The same can also be said of the sudden appearance of partial heart-block ; it is important, therefore, to be on the look-out for an intermittent pulse, and, if this be found, to ascertain whether it is due to the presence of extra-systoles which fail to reach the wrist or to partial heart-block, as the latter occurring during the course of infectious disease is a sign, and may be the only sign, of myocardial involvement, with which endocarditis is usually associated. The differential diagnosis between extrasystole and partial heart-block has been dealt with in Chapter XII.

When the apex-beat is tumultuous in character it is suggestive of involvement of the endocardium or pericardium. Displacement of the apex-beat and evidence of enlargement of the area of impairment transversely are not necessarily indicative of acute endocarditis, as a certain degree of cardiac dilatation is met with in most of the acute fevers, as the result of toxæmia or anæmia. Neither does a recently developed mitral systolic 
murmur necessarily indicate endocarditis, for it may be due to anæmia or to simple dilatation. A preliminary prolongation, roughening, or want of clearness of the first sound at the mitral or aortic area is strongly suggestive of valvulitis, as is also the early appearance of the murmur; a murmur which is due to anæmia or to simple dilatation of the left ventricle does not usually make its appearance in the early stages of rheumatism. An aortic systolic is not so suggestive of acute endocarditis as a mitral systolic murmur, while a pulmonary systolic murmur may be almost disregarded. In acute endocarditis, the murmur is definitely limited to the mitral or aortic area. The character of the murmur is of some diagnostic value, a soft blowing, in contrast with a harsh, murmur being in favour of endocarditis. Accentuation of the second sound in the pulmonary area later on in the illness is also suggestive of mitral incompetence.

An altered second sound, with later on a diastolic murmur, in the aortic area is indicative of involvement of the endocardium; the latter, however, is rarely heard during the course of an acute illness, and practically never during its early part. The early signs of mitral stenosis have already becn noted.

The differential diagnosis between endocardial murmurs and adventitious sounds which are due to pericarditis or pleurisy, as well as sounds which are cardio-pulmonary in origin, has been discussed in Chapter II.

It is necessary to distinguish the murmur of acute endocarditis from that due to previously existing valvular disease. The existence of subcutaneous nodules or pericarditis, the sudden appearance of partial heart-block (indicative of coincident acute myocarditis), or the appearance of new murmurs or the disappearance of old ones, is in favour of a fresh attack of acute endocarditis, whether simple or malignant. Embolism is not pathognomonic of acute endocarditis, as it may occur in chronic valvular disease, especially in mitral stenosis. In acute 
endocarditis, however, it is not infrequently multiple and recurrent, and is of more usual occurrence in the systemic than in the pulmonary circulation.

If acute endocarditis occur in an individual already the subject of valvular disease, the murmur is not of recent development, it is often loud or harsh, audible over a considerable area, and evidence of cardiac enlargement is usually present.

Prognosis.-The immediate prognosis of acute simple endocarditis is as a rule favourable, death being of rare occurrence; this may happen, however, if there be accompanying pericarditis or severe myocarditis. With regard to the ultimate prognosis, it has been pointed out that complete resolution in all probability rarely occurs. Organisation is the usual sequel, ultimately giving rise to permanent stenosis, or incompetence, or both; in a certain percentage of these cases, the early murmur may disappear, and a permanent murmur may not become established until much later on, even several years. It should be noted that the future prognosis of a case of acute simple cndocarditis largely depends upon whether convalescence is sufficiently prolonged. In a certain number of cases, ulceration of the valve results.

Treatment.-The management of a case of acute simple endocarditis is of great importance, especially with regard to the future of the patient. When there is no doubt that the heart is affected by rheumatism, suitable anti-rheumatic treatment should be adopted. I have tried the method of administering large doses of sodium salicylate in combination with large doses of bicarbonate of soda, as recommended by the late Dr. David Lees, and, comparing the results with those in which the drug was given by the ordinary method, am strongly inclined to believe that the former is the more efficacious. In the opinion of some, salicylates should be avoided when there is undoubted cardiac involvement, in the belief that when given for considerable periods they depress the heart and 
cause further dilatation. I myself do not share this view. Some prefer iodides, given in full doses over considerable periods. A vaccine of the diplococcus of rheumatism has been recommended. Cases have also been recorded in which the administration of antistreptococcus serum, particularly in the prolonged or recurring cases, has apparently been attended by beneficial results. It may be given in 10 c.c. doses subcutaneously once a day for a week. When the cause is other than rheumatism, such as diphtheria, this should be treated.

In all cases, whatever the cause, we should endeavour to arrest the morbid process as early as possible, and to give the heart the best chance of the fullest possible repair. Absolute rest in bed is indispensable. In grave cases, the foot of the bed may, with advantage, be raised to the extent of one or two feet. At no stage in the management of a case should the heart be allowed to work beyond the limit of its capacity. In the majority of cases, absolute rest in bed is necessary for three months, and in some cases the period should be even longer. The patient should remain in the recumbent posture for at least a month after the fever has subsided. If tachycardia, or irregularity indicative of partial heart-block or of auricular fibrillation, or if pulsus alternans, be present, this period should be prolonged, even to three or four months. During this time he should on no account be allowed to sit upright in bed or to get out of bed for an action of the bowels. Afterwards, the amount of exertion should be most carefully graduated. At first, an extra pillow may be allowed. A few days later, the back may be slightly raised, and this may be gradually increased until the patient is moved to a couch, to which he should be confined for a further period of three months. After the first two or three weeks of this period, massage, at first very gentle and for short periods only, and gradually increased, may be allowed; and during the 
latter six weeks, active movements may be enjoined, and the patient may be gradually allowed to sit up. Later, slight walking exercise may be permitted. For some months later, great care should be exercised with regard to exertion, and the patient should be given rules as to the amount and kind of exercise; for, on the one hand, exercise may do much harm unless carefully regulated, while, on the other, in moderation it is of great value. If, during any of these stages, exertion be accompanied or followed by breathlessness, palpitation, a sense of fatigue, precordial pain or distress, or undue increased frequency of pulse, the patient is doing too much and th amount of exertion must be reduced.

Excitement and worry of every kind should be avoided during an attack of acute endocarditis. The diet should be light, small in bulk, yet highly nutritious, and preferably solid. The daily amount of fluid taken by the mouth should be restricted to two pints per diem. Saline infusions per rectum, or in grave cases intravenously, may be used. Strict attention should be paid to the condition of the bowels.

The internal administration of iodide of sodium in the later stages is employed by some. Dr. Caton recommended small blisters applied repeatedly to the precordium; I myself have been disappointed with the results so obtained. Mustard leaves may be used in a similar manner. Alcohol, of which the best forms are good old brandy or champagne, should not be given unless there is much exhaustion, dysproca, cyanosis, or an unduly rapid pulse ; in these cases, oxygen may also be employed. The amount of alcohol required of course varies with each individual case'; it is well to begin with 2 or $3 \mathrm{oz}$. of brandy per day, gradually increasing the quantity as required, and leaving it off by degrees as the patient improves. Other diffusible stimulants, such as ether and ammonia, may be used. Camphor and tincture of musk are employed a good deal on the Continent. Strychnine has a great reputation 
in the treatment of cardiac failurc due to associated acute myocarditis. It may be tried when there is great cxhaustion. In my opinion, it is much better given hypodermically; in urgent cases up to $\frac{1}{80} \mathrm{gr}$, , or cven $\frac{1}{30} \mathrm{gr}$., every two hours. The results of the administration of the digitalis series of drugs are as a rule extremely disappointing; the response is usually very slight, if at all, whether auricular fibrillation is present or not. In suitable circumstanees quinine may be administcred, and, if anæmia be present, iron and arsenic may be preseribed. When the blood-pressure is very low, five minims of a 1 in 1000 solution of suprarenal extract, or pituitary (infundibular) extract, may be administercd by the mouth.

The treatment of symptoms has, in the main, been dealt with previously. For precordial pain or distress, a hot linseed poultice may be applied to the precordium; if this fail, the continuous application of an ice-bag may be tried, as recommended by Lees. Dover's powder (10 to $15 \mathrm{gr}$.), or, if necessary, morphia, may be given by the mouth. In less acute pain, a fly-blister is not infrequently efficacious. Sleeplessness and other symptoms should be treated as described elsewherc.

It is well to examine a patient who has suifered from acute endocarditis at regular intervals after the attack, and careful enquiry should be made as to his mode of life. In children, we should be especially on the look-out for any rheumatic manifestation, and, as soon as detected, the condition should be immediately and adequately treated.

\section{U'lcerative, Malignant, oR Infective Endocarditis}

While in ulcerative endocarditis the left side of the heart is more commonly affected than the right, the latter is attacked in a proportionately larger number of cases than in acute simple endocarditis. When the 
left side of the heart is involved, the affection is more commonly confined to that side; occasionally the right side of the heart only is involved. The mitral valve is more commonly affected than the aortic, but in smaller proportion in comparison with the acute simple variety; a frequent combination is disease of the aortic valve together with the anterior cusp of the mitral valve. ${ }^{1}$ The inflammation and structural changes are more intense and extend deeper than in the case of the acute simple form of the disease; the vegetations are larger, reaching even to the size of a hazel nut, form rapidly, and tend to undergo softening and disintegration; there is necrosis of the underlying tissue; and a tendency for the affection to spread to the adjacent endocardium. Besides the valves and neighbouring endocardium, the inter-ventricular septum and the wall of the left auricle are frequently affected. Micro-organisms in large numbers, among which the most common is one or other strain of streptococcus, are present on the surface and more or less deeply in the substance of the vegetations. Horder found that if certain methods of blood-culture were adopted, a positive result is obtained in the great majority of cases. Among the organisms most commonly found in the blood is the streptococcus, pneumococcus, Pfeiffer's bacillus influenzæ, the gonococcus, and the staphylococcus; while such organisms as the bacillus coli communis and bacillus typhosus are occasionally present. Horder found that the streptococci usually present were not of the type met with in suppurative processes, but were culturally, bio-chemically, and pathologically identical with those found in the alimentary canal.

1 Osler found that the valves were affected in the following order of frequency : Mitral alone, aortic alone, aortic and mitral together, cardiac wall, tricuspid, and pulmonary. In an analysis of 118 post-mortem examinations, Forder found that both the mitral and aortic valves were affected in 63 cases, the mitral valve alone in 38 cases, and the aortic valve alone in 22 cases. 
The valve may become almost completely destroyed, or there may be perforation of the segments, or aneurisms may form, which bulge in the direction of the bloodcurrent, in the case of the mitral valve into the left auricle, and in the case of the aortic valve into the left ventricle; these may rupture, giving rise to perforation of the valve. Perforation of the septum or of the heart wall may also occur. The mural endocardium and the aorta or puImonary artery may also become infected, either by direct extension or by contact with loose pieces of the thrombus, and undergo changes similar to those of the valves. Acute aneurism and rupture may result also in this case.

Embolism is much more common in the malignant than in the simple form of endocarditis; the same local results follow in both cases, but in the case of the former, as the embolus carries with it micro-organisms, inflammatory changes and suppuration may develop at the seat of the cmbolus, giving rise to metastatic abscesses. Among the organs which may be so affected are the spleen, kidneys, lungs, brain, and liver; pleurisy, empyæma, suppurative pericarditis, and multiple abscesses all over the body may occur. The arterial wall at the site of obstruction may yield, resulting in embolic aneurism.

Etiology.-Ulccrative endocarditis may occur on valves which were previously healthy, but is much more frequently met with in the case of already existing valvular disease. The condition may be primary, but is usually secondary to some other affection, and is due to bacterial infection. It may be due to acute rheumatism or chorea, but less frequently so than is acute simple endocarditis. Other causes are pneumonia, the eruptive fevers, influenza, gonorrhœa, puerperal fever, erysipelas, septicæmia from whatever cause (osteomyelitis, hepatic abscess, cystitis, open wounds on the surface of the body), and rarely diphtheria, enteric fever, malaria, tuberculosis, ordysentery. It has already been noted that the acute 
simple form of endocarditis may pass into the ulcerative variety.

Symptomatology.-The mode of onset, the symptoms, and the course of ulcerative endocarditis are very varied.

Not infrequently the disease is latent for a long time, the affection being masked by the symptoms of the primary cause; whilc in rare instances the disease is latent during the whole course, and is only discovered post-mortem. Usually, however, there is pyrexia, or the degree of pyrexia increases, the type of temperature also becoming altered. The pyrexia is most commonly of the irregular remittent type, but may be intermittent, or high and continued. These may be the only indications of the existence of malignant endocarditis, but in the majority of cases the patient also suffers from rigors, followed by profuse sweating; sweatings, however, may occur without the existence of rigors. The patient may complain of pains in the limbs or back, and as the case progresses there is an increasing degree of general weakness, loss of flesh, and profound anæmia. Septic embolism is of frequent occurrence, as also is albuminuria, while evidence of nephritis is not uncommon. Septic rashes, in the form of urticarial erythema, erythema multiforme, erythema nodosum, and occasionally a pustular rash may arise. Cardiac signs are usually to be noted. Jaundice and optic neuritis may occur. A leucocytosis is present, the polynuclear cells being relatively increased in number; the leucocyte count usually varies between 10,000 and 15,000 , but 20,000 or 30,000 are not infrequent in acute cases. In the advanced stages, low delirium not infrequently sets in, and ultimately the patient may become completely comatose. The duration of the disease is very variable. It may last for a few days or the patient may live for 6 , 12, or according to some even 18 months or longer.

Four types of the disease are commonly described, namely, (1) the cardiac, (2) the septic or pyæmic, (3) the typhoid, and (4) the cerebral. It should be remembered, 
however, that there is no sharp line of demarcation between these types, and, moreover, a case may, from time to time, present features representative of more than one type.

Cardiac Type.-This variety of endocarditis is frequently associatcd with the rheumatic infection; in the great majority of cases the patient has alrcady been the subject of valvular disease; and the case may have begun as an acute simple endocarditis. The symptoms of cardiac involvement predominate, palpitation, shortness of breath, and discomfort or even pain in the precordium being usually found early in the illness. There is increased frequency of the pulse, the beats reaching as many as $\mathbf{1 2 0}$ or even more, and in some cases there is irregularity of rhythm. The apex-beat may be tumultuous in character, and there may be also evidence of enlargement of the area of cardiac impairment transversely. On auscultation, a bruit can usually be detected, most commonly in the mitral or aortic area. As the disease advances, there is not infrequently evidence of involvement of another valve than the one originally attacked. A supposed characteristic feature is that sometimes the murmurs exhibit variations from time to time; pre-existing murmurs may change; a mitral presystolic murmur, for example, may disappear, or become replaced by a systolic bruit. The respiratory rate is increased, and bronchitis, pulmonary congestion or œdema, and general anasarca may develop.

Septic or Pyæmic Type.-In this type of the disease the patient has not so frequently suffered from previous cardiac disease, and it is rarely associated with acute rheumatism, being usually met with in septic conditions, e.g. puerperal fever. The right side of the heart is more often affected than in the case of the cardiac variety. The pyrexia is intermittent in type, and there may be a condition of hyperpyrexia. Rigors and sweatings are a prominent feature, and septic embolism and metas- 
tatic abscesses are most characteristic of this variety. The symptoms of cardiac involvement may be masked by the general condition; the heart may be only a little if at all enlarged, and murmurs may be only present in the pulmonary, though not infrequently they are found in the mitral or aortic area. If the patient live long enough, however, evidence of valvular involvement may become conspicuous. The joints may become inflamed and suppuration may occur.

Typhoid Type.-The temperature is high and more or less of a continued type. Headache, malaise, diarrhœathe motions often resembling typhoid stools-and abdominal tenderness are usually present. Cardiac symptoms are usually not conspicuous, and examination of the heart may reveal no abnormal physical signs, except in those cases suffering from previously existing valvular disease. A characteristic feature of this form of the disease is that early in its course the teeth become covered by sordes, the tongue becomes dry and brown, with rapid prostration, mental apathy, somnolence, low muttering delirium, and other evidence of the typhoid state, and finally coma.

Cerebral Type.-In this variety of the disease meningitis is also present. It is often found in association with pneumonia, occurring during the course or convalescence of that disease. The onset is usually abrupt, and is frequently marked by a rigor. The clinical picture may closely resemble basal or cerebro-spinal meningitis. Rigors are not usually present during the course of the disease, but hyperpyrexia and profuse sweating may arise. Severe headache, vomiting, convulsions, acute delirium, paralysis of one or more of the cranial nerves, general paralysis, and coma may also occur.

Diagnosis.-The diagnosis of ulcerative endocarditis may be very difficult. In its consideration the following points are of importance: The type of pyrexia, the presence of rigors, of severe constitutional symptoms, 
marked anæmia, and embolism; the results of an examination of the heart, of a blood count, and of a cultural examination of the blood revealing the presence or absence of miero-organisms. The types of pyrexia met with in the different varieties of the disease have been already fully described. The heart should invariably be examined in cases of pyrexia of uncertain origin ; in this connection, however, as has been noted, there may be an absence of abnormal eardiae physieal signs. The possibility of ulcerative endocarditis should also be borne in mind in any case in which rigors have occurred, as well as in a paticnt exhibiting severe constitutional symptoms together with the development of a recent murmur or murmurs at the mitral or aortie valve ; the sudden appearance of an aortic systolic murmur is of especial significance, while bruits audible over the tricuspid area are also of diagnostie importance. An irregular remittent, intermittent, or high and continued type of pyrexia together with an aortic diastolic bruit is very suggestive of ulcerative endoearditis. The occurrence of embolism, as evidenced by petechial homorrhages in the skin, enlargement of the spleen, or homaturia, is of great diagnostic importance, as also is the presence of micro-organisms as the result of a cultural examination of the blood. In an individual who is known to be the subject of valvular disease, the symptoms already enumerated, including constitutional prostration out of proportion to the cardiac lesion, is very convincing evidence of the existence of ulcerative endocarditis.

In the consideration of the diagnosis of this disease, it is necessary to bear in mind the differential diagnosis between it and acute simple endocarditis occurring during the course of rheumatic fever, recurring attacks of acute or subacute rheumatism in the subjects of valvular disease, pyæmia, enteric fever, meningitis, pneumonia, tuberculosis, malaria, and typhus. The differential diagnosis in the first of these is often very difficult. In favour of the 
ulcerative form of the disease is to be found the more severe constitutional symptoms, loss of weight, early, severe and progressive anæmia, and more prolonged and higher grade of pyrexia, the presence of rigors, and the more common occurrence of embolism ; and on examination of the blood micro-organisms are sometimes found, a negative cultural result after several examinations of the blood being strongly in favour of the simple form of the disease.

In pyæmia, rigors are of more frequent occurrence, and the skin is of an earthy colour. Those cases of ulcerative endocarditis in which petechial hæmorrhages, diarrhœa, abdominal tenderness, and the typhoid state are present simulate enteric fever, while, on the other hand, it is to be noted that emboli may also occur in enteric fever. In ulcerative endocarditis, the onset is more abrupt; the temperature is not so regular; rigors, dyspnœa, and other indications of cardiac distress, and the presence of cardiac murmurs (either from previous heart disease or of recent origin) are of common occurrence ; there may be optic neuritis or retinal hæmorrhages ; leucocytosis is present instead of leucopænia, and the agglutination test and Widal reaction is negative; rosecoloured spots and pea-soup stools are not found; there is no pronounced tenderness of the abdomen, and tympanitis is rarely present.

As has already been noted, pneumococcic endocarditis not infrequently accompanies or follows pneumonia. The existence of valvular disease, petechiæ, and emboli are in favour of ulcerative endocarditis.

The differential diagnosis of ulcerative endocarditis and acute generalised tuberculosis must be considered in cases in which there is continued pyrexia without obvious signs, since in cases of endocarditis which have persisted for some time there may be considerable loss of flesh. The existence of valvular disease, and of a leucocytosis, instead of a leucopænia, the results of a cultural examination of the blood, 
and of the sputum for tubercle bacilli, and the presence or absence of local signs of tuberculosis assist the diagnosis.

Cases of ulcerative endocarditis with a pronouncedly intermittent temperature and rigors with profuse sweating may closely simulate malaria; in the latter, the existcnce of leucopænia, instead of leucocytosis, the presence. of the malarial parasite in the blood, and the result of the administration of quinine will decide the question.

Prognosis. - The prognosis of ulcerative endocarditis is always grave. In the opinion of some, recovery may take place; but cven so it is an extremely rare event. Periods of quiescence or even apparent improvement may occur.

Treatment.-Absolute rest in bed is the first essential. The tceth should receive careful attention, and if pyorrhœa alveolaris be present it should be treated. The diet should be fluid and liberal in amount, and the bowels carefully regulated. Many drugs have bcen employed in the treatment of this disease, such as quinine, the sulphocarbolates, carbolic acid, mercury, arsenic, formalin, and the organic preparations of silver-such as protargol. Quinine is probably of value in lowering the temperature. In those cases in which leucocytosis is absent or slight, nuclein may be given by the mouth, subcutaneously, or even intravenously. If given subcutaneously, the dose should be 5-15 min. of a 10 per cent. solution once a day. Silver salts may be given in combination with nuclein.

Within recent years specific measures in the form of vaccines and antisera have been extensively employed in the treatment of this disease, but the results, on the whole, have not been encouraging. Some physicians combine the two methods. In the use of vaccines, better results are obtained from the patient's own micro-organisms than from stock vaccines. An attempt should be made to isolate any organisms which may be present in the blood, and if successful a vaccine should be prepared. The 
question of dosage and the determination of the proper intervals between successive doses is difficult. Formerly it was thought that frequent estimations of the opsonic index afforded a good guide. We now know that this is not the case, if for no other reason than the fact that in ulcerative endocarditis there is a constant fluctuation of the index. The clinical symptoms and temperature, however, are of service, and should be carefully noted. It is most important to avoid too large and too frequent dosage. We should begin with a small dose, and proceed very cautiously, both in regard to an increase in dosage and in lessening the intervals between the doses. If any dose be followed by pain in the limbs, headache, increase in the severity of symptoms, or rise of temperature, it is either too large, or the period of time that has elapsed since the administration of the previous dose has not been sufficiently long. In such a contingency, the next dose should be a smaller one-perhaps half or even less-and should not be increased for some little time, and, as a rule, at a longer interval. As an example of dosage and spacing of the doses, we may begin with 2 to 5 millions streptococci or 50 to 100 millions staphylococci, administering a second dose after an interval of three to five days, carefully watching the effects. During the time that the vaccine is being prepared from the patient's own microorganism, a stock preparation of the micro-organism may be employed. When streptococci are found, antistreptococcus serum-univalent or polyvalent-may be employed. Ten c.c. may be given subcutaneously, or 15 c.c. per rectum, daily for three or four days, after which there should be an interval of several days. If the administration be followed by an increase of symptoms or rise of temperature, small doses, such as $2 \frac{1}{2}$ c.c., should be substituted. When the condition is due to the pneumococcus, Merck's or Pane's antipneumococcus serum in full doses may be tried.

The daily administration of normal horse serum, 
either subcutaneously or intravenously, has recently been praised.

In cases with an unduly rapid pulse, great exhaustion, and cardiac dilatation, alcoholic stimulants and strychninc may be employed. The best method of reducing the temperature is tepid sponging of the body; of drugs the best is quinine. Other symptoms should be treated on the lines laid down elsewhere.

\section{Chronic or Sclerotic Endocarditis}

Chronic endocarditis is a sclerosis of the valve, and may be (1) secondary to the acute-especially the rheumatic form, which is the more common event, or (2) primary, as a result of degenerative changes.

Etiology and Morbid Anatomy.-When the condition is due to a previous attack of endocarditis, it is more apt to occur in earlier life, the mitral valve being more commonly affected, while if the result of degenerative processes it is more apt to occur in middle or later life, the aortic valve being more commonly affected than the mitral.

With regard to the etiology of primary chronic endocarditis, heredity is certainly a factor, and the condition is more common in males, and during or after middle life. Prolonged muscular or mental strain, over-indulgence in food or drink, syphilis, chronic metallic poisoning-particularly that due to lead, gout, and chronic renal disease are among the most common causes. When syphilis is the cause, the condition is apt to come on before middle life.

When organisation occurs in acute simple endocarditis, the result is the formation of fibrous tissue, which tends to contract as life advances, and as a result there may be thickening, shortening, puckering, chronic vegetations, coalescence of two or more segments, cartilaginous changes, or calcification of the valves; there may also be shortening and thickening of the chordæ tendineæ and apices of 
the papillary muscles. These structural changes ultimately give rise to permanent stenosis, or incompetence, or both, and there is a tendency in these cases to recurrent attacks of valvulitis, resulting in still greater deformity.

In primary chronic endocarditis, there is sclerotic thickening of the endocardium, often occurring in patches, and frequently associated with fatty or calcareous changes, to which the term atheroma is usually applied. There may be thickening, small nodular prominences, shortening, puckering, coalescence of two or more segments, and calcification of the valves; the chordæ tendinæ and apices of the papillary muscles may also be affected. These structural changes also ultimately give rise to permanent stenosis, or incompetence, or both.

The degenerative processes are frequently associated with arterio-sclerosis, which may be widespread. When they affect the aortic valve they may be limited to the valve, or, as is more frequently the case, may also affect the walls of the aorta, in which case dilatation of that vessel may follow. The neighbourhood of the mouths of the coronary arteries is also often involved, and there is frequently some occlusion of one or both of these vessels. When this is so, chronic myocardial degeneration is likely to ensue.

It may here be noted that fotal endocarditis is usually of the sclerotic form, the right heart being more commonly affected than the left.

Symptomatology.-Primary chronic endocarditis being a degenerative process produces no symptoms until a certain degree of structural changes in the valves has taken place, when stenosis, or incompetence, or both occur. 


\section{CHAPTER XX}

CIIRONIC VALVULAR DISEASE

By chronic valvular disease is meant a chronic affection of the cusps of the cardiac valves, or of the orifices, or both. Not only may the orifices and cusps be affected, however, but also the chordæ tendineæ and the musculi papillares. In many cases the cusps are only secondarily affected; to illustrate this it may be mentioned that incompetence of a valve may be duc entirely to dilatation of its orifice, or, in the case of the auriculo-ventricular valves, to an affection of the musculi papillares or chordæ tendineæ. Whatever the nature and form of the valvular lesion, it gives rise to stenosis or narrowing, which results in obstruction to the flow of blood; or to incompetence, which allows of regurgitation; or frequently to both of these together. Along with the valvular lesions there are usually coincident changes in the cardiac musculature, the aorta, or coronary arteries.

The Relative Proportion and the Combinations of the Different Valvular Diseases. - It should be noted that not infrequently more than one valve is involved at the same time, especially in the case of rheumatic valvular disease.

If we include what is termed the relative variety of incompetence, tricuspid incompetence will be found to be the most common chronic valvular disease. If this be not included, the mitral is the valve most commonly affected, accounting for half the number of the cases of chronic valvular disease, incompetence of this valve being more 
frequent than stenosis. When mitral incompetence results from a previous attack of acute endocarditis, there is usually some degree of stenosis as well; when it is due to causes other than lesions of the cusps, it is often associated with tricuspid incompetence. Pure mitral stenosis is comparatively infrequent; in the process of thickening and contraction, there is almost invariably some degree of incompetence.

In the majority of cases of aortic disease a double murmur is present; but this does not necessarily indicate the presence of stenosis as well as of incompetence, for there are other causes of an aortic systolic bruit besides aortic stenosis. Aortic regurgitation is very common; a greater or less degree of stenosis, however, not infrequently accompanies the incompetence. Pure aortic stenosis, on the other hand, is one of the rarest of valvular affections.

Tricuspid incompetence is the most common form of right-sided valvular affections. It is rarely primary, however, and then stenosis is usually present with it, and also mitral or aortic disease, particularly the former. Tricuspid incompetence is a common result of mitral disease, there being no lesion of the cusps, but relative incompetence of the valve. When tricuspid incompetence occurs alone, it is in all probability congenital. Tricuspid steno is is extremely rare.

Though murmurs with their area of maximum intensity at the pulmonary area are common, disease of the pulmonary valve is rare, and is usually of congenital origin. Pulmonary stenosis is one of the most frequent forms of congenital heart disease, and is usually associated with other lesions, such as a patent interventricular septum; as an acquired condition it is very rare. Pulmonary incompetence is the rarest of valvular lesions.

If relative tricuspid incompetence be included, the most frequent combination of valvular affections is mitral disease 
with tricuspid incompetence. The next most common is aortic incompetence with mitral incompetence; the latter may be the sequel of the aortic disease. These are followed in order of frequency by aortic incompetence with mitral obstruction, and aortic, mitral, and tricuspid incompetence. Double aortic and double mitral disease is fairly common. Aortic incompetence and mitral stenosis, and aortic stenosis with mitral stenosis, are somewhat rare combinations.

Etiology.-Chronic valvular disease may be of congenital origin (vide congenital heart disease), or may be due to a previous attack of one of the forms of acute endocarditis resulting in chronic endocarditis, or to degenerative processes, new-growths, or traumatism. This list does not include relative incompetence of a valve.

The causes of the various forms of acute endocarditis, and also of degenerative processes, have been fully dealt with in Chapter XIX. When chronic valvular disease is due to a previous attack of acute endocarditis, it is more apt to occur in early life, and when due to primary degenerative processes in middle or later life.

Neoplasms may arise from the walls of the heart or from the valves. They rarely cause obstruction. A traumatic lesion of a cusp or of the chordæ tendineæ, the result of excessive physical exertion, may cause stenosis or incompetcnce of a valve, and is of much more common occurrence in the case of the aortic than the mitral valve. It rarely takes place, however, in the absence of pre-existing disease of the valve.

In relative incompetence of a valve the cusps themselves are healthy. This is more common on the right than on the left side of the heart, and is almost unknown in aortic disease, and is not nearly so frequent as in the case of the mitral valve. The condition may occur from the following causes: (1) Dilatation of the cavity of the ventricle; this produces an alteration of the relation of the cavity and of the cusps, the segments of which 
cannot properly approximate, with consequent regurgitation through the orifice. (2) Enlargement of the orifice on account of relaxation of the surrounding muscular structures; in these cases the tonicity of the muscular ring is impaired, and the cusps do not completely come together. (3) Enlargement of the ventricular cavity and dilatation of the orifice combined, and (4) Owing to shrinkage or stretching of the musculi papillares or chordæ tendineæ, as a result of disease.

\section{Aortic Stenosis}

Aortic stenosis may be absolute or relative. In absolute stenosis the orifice is less than its normal size, while in relative stenosis the size is normal, but dilatation of the aorta just above the valve occurs.

Etiology.-Aortic stenosis is more commonly found in middle or later life, and in males. Absolute stenosis may be due to a previous attack of acute endocarditis, in which case the mitral valve is also as a rule similarly affected. It is, however, commonly due to degenerative processes, and is for this reason more common in middle or later life and in males. It may be congenital, and is rarely due to injury, direct or indirect.

Symptomatology.-When aortic stenosis is the result of a previous attack of acute endocarditis, the subjective symptoms are as a rule long delayed, and indeed the condition may be latent all through life. The most common subjective symptoms are giddiness, sleeplessness, a sense of oppression or constriction behind the sternum, pain, and dyspnoa on exertion. Later, dilatation of the left ventricle and relative insufficiency of the mitral valve, and ultimately dilatation of the right side of the heart, may occur. Even when the stenosis is the result of degenerative changes, so long as the lesion is slight, there arc usually no subjective symptoms; - when, however, 
this is considerable, the symptoms just enumerated may be present, and angina pectoris, or even Adams-Stokes' syndrome, may be super-added.

The pulse is sometimes very characteristic ; indeed, not infrequently the diagnosis of the lesion is greatly assisted by the peculiar character of the pulse. In moderate degrees of stenosis, the pulse may not show any striking abnormal features. As the lesion increases, however, it becomes infrequent, and the sphygmogram shows a pulse of smaller amplitude, the percussion wave is oblique, and the summit is gradual and slow, while to

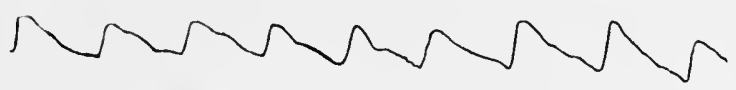

FIG. 141. Sphygmogram from a case of aortic stenosis.

the finger the pulse is full between the beats (Fig. 141). It may be anacrotic (Figs. 26 and 149) or of the bisferiens type (Figs. 27, 150 and 151). When in addition to stenosis aortic incompetence is present, which is not uncommon, the pulse which is characteristic of the former will be modified by the latter condition. The blood-pressure is variable; it tends to be subnormal, but is often supernormal, as the result of co-existing arterio-sclerosis or of chronic interstitial nephritis. When aortic stenosis is associated with arterio-sclerosis, as is frequently the case, the vessel wall may be thick and tortuous.

Almost always there are signs of hypertrophy of the left ventricle. There is sometimes a certain amount of bulging of the precordium. The apex-beat is displaced chiefly downwards-it may be as far as the 6th intercostal space or lower, but as a rule only slightly outwards. The area and force of the apex-beat are increased; it may be heaving or thrusting in character. A characteristic sign of aortic stenosis is a gradual, slow rise of the apexbeat-a "slow heave," which is sustained. A thrill, systolic in time, is often palpable; this has its maximum 
intensity in the aortic area, and may be felt over the whole precordium, and, indeed, sometimes all over the chest and the large vessels of the neck. On percussion, the area of deep cardiac impairment is almost always increased, the enlargement being chiefly downwards, and to a less extent to the left, while in uncomplicated cases the right border extends very little beyond the normal limit. The area of superficial cardiac impairment is as a rule unaffected. On auscultation, a systolic murmur is audible, with its point of maximum intensity in the aortic area, or over the manubrium sterni, or to the left of the sternum. The murmur, however, may be audible all over the chest, and even at the back, in which case it is loudest at the level of the fourth vertebra close to the left border of the spinal column. The direction of selective propagation is upwards along the vessels of the neck. It is usually loud, and has been heard several feet away from the patient. It is generally rough and grating, but may be musical, or soft and blowing. The first sound in the mitral area is sometimes long, sustained, low in pitch, and muffledas in hypertrophy of the left ventricle from other causes. The second sound in the aortic area varies considerably in loudness, largely according to the nature of the lesion of the valve (for example, the fusion of the cusps may be such as not to allow such apposition as is nccessary to cause an aortic second sound), and the presence or absence of arterio-sclerosis. It is usually diminished and may be practically absent, but occasionally may be louder than normal. Later, owing to dilatation of the ring supporting the mitral valve, relative mitral incompetence may occur, when enlargement of the left auricle, passive hyperæmia of the lungs, and changes in the right side of

- the heart are apt to supervene ; diminution of the force of the apex-beat and increase to the right of the area of cardiac impairment are among the other physical signs to be noted.

Diagnosis.-It has been pointed out that pure aortic 
stenosis is one of the rarest valvular affections, although with aortic incompetence it is not so very uncommon. Yet, Walshe, in spcaking of the relative frequency of valvular lesions, considered the affection to be more common than either aortic incompetence or mitral stenosis. Formerly, a diagnosis of aortic stenosis was usually made from the mere presence of a systolic murmur having its point of maximum intensity over the aortic area; and even at the present time, although more light has recently been thrown upon the subject, the same mistake is not infrequently made, and aortic stenosis is often diagnosed when no such lesion is present. It is not sufficiently appreciated that it is not justifiable to make a diagnosis of aortic stenosis from the mere presence of a systolic murmur over the aortic area, additional cvidence being required, as the murmur may be due to other causcs.

A diagnosis of aortic stenosis can readily be made when the characteristic pulse and apex-beat, an aortic systolic thrill and murmur, with the direction of selective propagation upwards along the vessels of the neck, and a diminished or absent second sound, are present. These features combined distinguish the condition from others that may give rise to a systolic bruit with its point of maximum intensity over the aortic area; when, however, only one or few of these characteristic features are present, difficulty with regard to the diagnosis may easily arise. An anacrotic pulse is characteristic, but not pathognomonic, since other conditions besides aortic stenosis may produce it; nevertheless, it is of greater diagnostic value than pulsus bisfereins. While the presence of a thrill is a valuable diagnostic sign, it should be remembered that it may also be found in dilatation of the aorta. A systolic murmur with the direction of selective propagation upwards along the vessels of the neck is at least strongly presumptive, and, in the opinion of some, is almost sufficient evidence.

Certain conditions must be excluded in considering the 
diagnosis of aortic stenosis; among these are bruits due to anæmia, cardio-pulmonary murmurs, pulmonary stenosis, patent ductus arteriosus, pressure upon the aorta by a mediastinal tumour where no definitc pulsation is present, mere roughening or stiffness of the aortic cusps or orifice, fusiform dilation of the aorta just above the valve---what is called "relative stenosis"-and aneurysmal dilatation of the ascending aorta.

The distinguishing features of bruits due to anæmia, and also of cardio-pulmonary sounds have been dealt with in Chapter II. When a systolic murmur due to aortic stenosis has its point of maximum intensity over the pulmonary area the condition may be mistaken for pulmonary stenosis. One point alone is sufficient for differential diagnosis ; in pulmonary stenosis the bruit is never propagated into the arteries at the root of the neck. Further, the character of the respective pulses differs materially, while in aortic stenosis there is evidence of hypertrophy of the left, and not of the right, ventricle, and the apexbeat is characteristic. The physical signs of patent ductus arteriosus are described clsewhere.

In the case of pressure upon the aorta by a mediastinal tumour where no definite pulsation is to be felt, the murmur is often audible over the vessels of the neck. An area of impairment may usually be detected, however, and there is rarely any alteration in the character of the second sound. When an aortic systolic bruit is due merely to roughening or stiffness of the aortic cusps or orifice, there is an absence of the characteristic pulse, of hypertrophy of the left ventricle in a young person who is free from kidney disease, and of the characteristic apex-beat. In fusiform dilation of the aorta just above the valve, the murmur is often heard over the vessels of the neck, but occasionally the area of impairment at the level of the second and third costal cartilages extends farther out to the right than the normal limit, and the second sound is not only accentuated, but is low-pitched, ringing, and 
usually audible over a much wider area. As a rule, there is very little difficulty in diagnosing aortic stenosis from aneurysmal dilatation of the ascending aorta.

Prognosis.-In the opinion of some, the prognosis of pure aortic stenosis is more favourable than that of any other chronic valvular lesion; others again place it after mitral incompetence. In my experience, it largely depends upon whether the affection is due to a previous attack of acute endocarditis, or is the result of degenerative processes. In the former case, the lesion is less serious than any other chronic valvular lesion, and the risk of sudden death is slight. In the latter case, however, the prognosis is less favourable than in mitral incompetence, as degenerative processes are apt to be progressive, and while these may be limited to the valve, more frequently they affect the walls of the aorta also. The neighbourhood of the mouths of the coronary arteries also is often involved, and partial occlusion of one or both of these vessels is of not infrequent occurrence; in this case, chronic myocardial degeneration is likely to ensue, and sudden death may result.

Treatment.-This (together with the trcatment of the other forms of chronic valvular disease) will be discussed at the end of the chapter.

\section{Aortic Incompetence}

Etiology.-Aortic incompetence is more commonly found in middle or later life, and in males. It may result from a previous attack of acute endocarditis, in which case the. mitral valve is as a rule similarly affected; a previous attack of acute endocarditis is the commonest cause of the condition in young subjects. But, taking all the cases together, and more particularly when it occurs at a later period of life, the condition is more commonly due to degenerative processes. Aortic incompetence is the mòst 
frequent cardiac lesion due to injury, and may follow sudden physical over-strain, causing a rupture of a valve segment; in such cases, it is apt to end fatally. The condition is extremely rarely of congenital origin. Relative incompetence of the aortic valve is almost unknown. In relative incompetence there is no affection of the valve segments, the incompetence being due to stretching of the fibrous aortic ring-in other words, to widening of the orifice itself. This may occur in great dilatation of the commencement of the arch of the aorta.

Symptomatology.-There may be an absence of subjective symptoms for some time, excepting that the patient may complain of dyspnœa on over-exertion. When the condition is due to degenerative processes, the onset of symptoms is usually very insidious. The face is usually pallid and anxious, although in some cases the colour is flushed; while if relative mitral incompetence supervene the facies resembles that of mitral disease. Cerebral symptoms are more apt to occur early than in mitral disease, the patient complaining of giddiness, syncopal attacks, headache, or sleeplessness. Precordial pain is usually more severe in aortic than in mitral disease. At first there may be merely a sense of oppression behind the sternum, becoming worse on physical exertion; or there may be more or less continuous pain. Ultimately, angina pectoris is apt to occur, especially when the lesion is due to degenerative processes. The attacks are usually brought on by exertion. They may be associated with, or alternate with, syncopal attacks. The patient may complain of palpitation, and breathlessness is also a prominent symptom of aortic incompetence when due to degenerative processes. There may be a sensation of beating in the head, nose bleeding is not a rare occurrence, and there may be loss of flesh.

Whether the lesion is post-rheumatic, or degenerative, increasing dyspnœa, cough, hæmoptysis, physical signs of pulmonary congestion and odema, especially of the 
bases, hepatic enlargement, dropsy, and other evidence of mitral incompetence and dilatation of the right heart, may supervene; while in the late stages mental symptoms, such as delirium, hallucinations, delusions, or even a suicidal tendency, may be present.

The typical pulse is very characteristic, and is called the collapsing, the water-hammer, or Corrigan's pulse. Its collapsing character may be intensified by raising the arm above the head, and this method of examina-
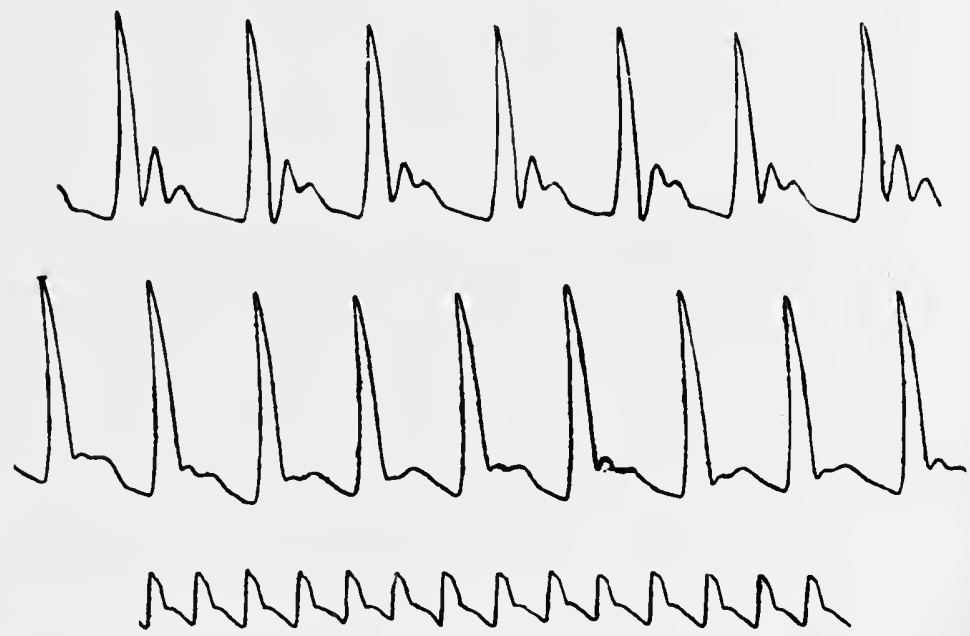

Figs. 142, 143 and 144. Sphygmograms from cases of aortic incompetence.

tion should always be employed in suspected aortic regurgitation. The typical pulse is one of great amplitude, with an abrupt upstroke and a sudden fall. The tidal wave may be considerable, but is maintained only for a short period, the dicrotic notch falls low on the downstroke, while the dicrotic wave may be well-marked, small, or altogether absent (Figs. 142-4). The artery is empty between the beats. When in addition to aortic incompetence stenosis is present, the characteristic pulse of the former will be modified by the latter condition; 
this is of great diagnostic importance in stenosis (Figs. 145151). A high pulse-pressure is almost invariably a feature of aortic incompetence; the minimum pressure is sometimes considerably below, and the maximum sometimes
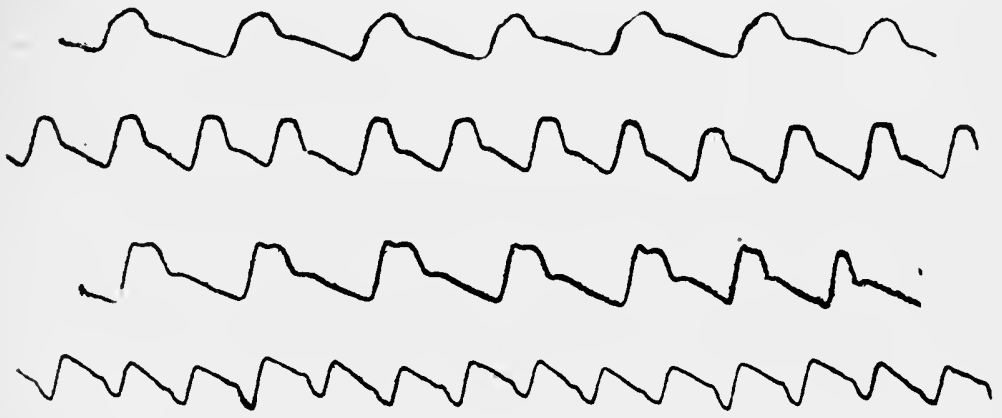

Figs. 145, 146, 147 and 148. Sphygmograms from cases of aortic stenosis and incompetence. The characteristic pulse of the latter condition is modified by the presence of stenosis.

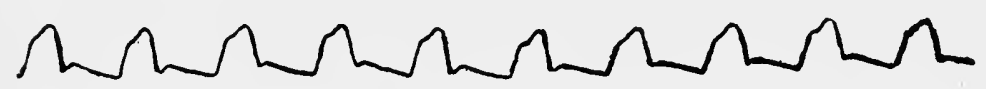

FIG. 149. Sphygmogram from a case of aortic stenosis and incompetence. It shows the anacrotic pulse.

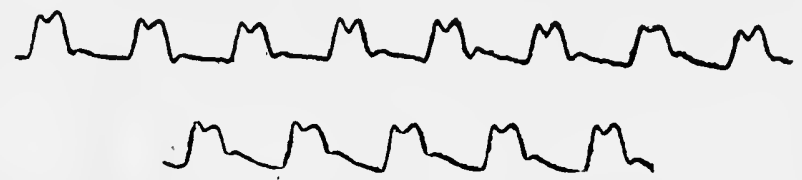

Frgs. 150 and 151. Sphygmograms from a case of aortic stenosis and incompetence. They show the pulsus bisferiens.

considerably above, the normal. When the condition is associated with arterio-sclerosis, as is frequently the case, the vessel wall may be thick and tortuous. A characteristic feature of aortic incompetence is exaggerated pulsation of the arteries; this may be especially noted in the carotid, temporal, and brachial arteries. Increased pulsation of the retinal arteries is sometimes to be noted with the ophthalmoscope. Pulsation in the superficial veins, 
especially in the veins of the back of the hand when hanging down, is sometimes seen. In the opinion of some writers, pulsation over the upper part of the precordium, and in the second and third right intercostal spaces, is occasionally to be noted, even in the absence of dilatation of the aorta.

In aortic incompetence signs of hypertrophy of the left ventricle are almost constant, and there is some bulging of the precordium-it may be to a considerable extent. The apex-beat is displaced, sometimes to a marked degree ; the displacement is to some extent outwards, but more markcdly downwards, sometimes reaching to the sixth or scventh interspace and the mid-axillary line. The extent and force of the beat are usually considerably increased, and it is frequently heaving or thrusting in character.

A diastolic thrill is rarely present; when it is, its maximum intensity is not infrequently at a lower level than the aortic arca, and may cven be felt towards the lower part of the sternum and in the neighbourhood of the apexbeat.

The area of deep cardiac impairment is usually increased downwards, it may be markedly so, and to a slight degree to the left. In uncomplicated cases the right margin of the heart extends only a little beyond the normal limit. On auscultation, a diastolic murmur is audible, which may continue for a variable distance into the long pause, bccoming fainter and fainter. Its point of maximum intensity is very variable, the typical position being over the inner end of the second right intercostal cartilage. Frequently, however, it is in the second left space, or immediately over the sternum. More rarely it is at a lower level than this, approaching even the level of the apex-beat, and in the writer's experience more often nearer the left than the right border of the sternum. The murmur has a tendency to be propagated downwards towards the lower end of the sternum. It is often heard over a wide area, and is usually soft and 
blowing, but may be musical, and more rarely rough and grating. An aortic systolic bruit is also usually present, but, as has been already pointed out, its presence does not necessarily indicate stenosis. The second sound may be audible or may be absent. The first sound at the apex is sometimes long, sustained, low in pitch, and muffled. Austin Flint believed that in certain cases of aortic incompetence a presystolic murmur is audible at the apex, and most writers agree with him. A diastolic murmur may be audible in the carotid, brachial, and femoral arteries; the last of these is referred to as the murmur of Duroziez. Physical signs of dilatation of the aorta are sometimes also present.

When aortic incompetence is due to degenerative processes, degenerative lesions in other organs, as shown by the presence of chronic renal disease or emphysema, may be present.

Later, dilatation of the left ventricle may occur, in which case the force of the apex-beat becomes diminished, and the left border of cardiac impairment is found to have extended still farther to the left. Owing to dilatation of the ring supporting the mitral valve, relative mitral incompetence may supervene, and then enlargement of the left auricle, passive hyperæmia of the lungs, and changes in the right side of the heart are apt to supervene, so that the area of cardiac impairment is also further displaced to the right; pulsation, diastolic in time, may be noted in the epigastrium in these circumstances.

The course of aortic incompetence is very uncertain. Gradual cardiac failure is the rule, but, on the other hand, death may occur suddenly, from syncope or angina pectoris.

Diagnosis.-There is little or no difficulty in recognising aortic incompetence in the great majority of cases. The characteristic radial pulse, the high pulse-pressure, the greatly exaggerated pulsation of the arteries, the physical signs of hypertrophy of the left ventricle, the characteristic 
murmur, capillary pulsation, and, rarely, visible pulsation in the superficial veins, present a definite clinical picture with regard to the diagnosis of which there should be no mistake. It should be remembered, however, that the phenomenon of the capillary pulse and pulsation of the superficial veins are occasionally also seen in other conditions. The murmur of Duroziez is pathognomonic of the lesion.

When the point of the maximum intensity of the murmur is in the pulmonary area, the condition may be mistaken for pulmonary incompetence. In this event, the points which should be remembered in considering the differential diagnosis are that pulmonary incompetence occurs more frequently in early life, that the pulse is not collapsing in character, that there is an absence of greatly exaggerated pulsation of the arteries and of capillary pulsation, that there is evidencc of hypertrophy of the right, but not of the left, ventricle, and that the accompanying murmur is very rarely propagated as far down as the apex of the heart, and is never audiblc over the carotid artery. Dyspnœa, cyanosis, and clubbing of the fingers are also more suggestive of pulmonary incompetence. It should be remembered, however, that both lesions may occur in the same individual, in which case there is usually a blending of the physical signs of both conditions. I have occasionally seen a diagnosis of mitral stenosis made in cases of aortic incompetence, when the point of maximum intensity of the diastolic bruit-especially when the bruit is only audible towards the latter part of the long pauseis as low as, or lower than, half-way down the sternum and on the left side of the middle line, and especially so when it is near the apex-beat. The points of differential diagnosis are the following: In mitral stenosis the pulse is markedly different from that of aortic incompetence, and, further, there is an absence of greatly exaggerated pulsation of the arteries and of capillary pulsation, there is evidence of hypertrophy of the right, but not of the 
left, ventricle, the murmur is harsher in character, the first sound is short, sharp, and clear, and the area of the thrill and murmur is usually very limited.

Prognosis.-The prognosis of aortic incompetence is usually serious. Sudden death is more frequent than in - any other form of chronic valvular disease, and may occur even when there are no indications of cardiac failure. In attempting to form a prognosis, we should endeavour, among other inquiries, to ascertain the extent of the lesion, and whether it is due to a previous attack of acute endocarditis or to degenerative processes, the condition of the myocardium and coronary arteries, and whether the affection is stationary or progressive. In the absence of cardiac failure, the pulse-pressure is an indication of the degree of aortic incompetence. When the lesion is the result of a previous attack of acute endocarditis, the prognosis is, other things being equal, much more favourable than when due to degenerative processes; indeed, if there be no indications of cardiac failure, and if the rate and character of the pulse, the pulse-pressure, and the size of the heart, exhibit little or no alteration, and if the murmur accompany, but do not replace, the second sound, the patient may live to an advanced age. If, on the other hand, the lesion be due to degenerative processes, and be progressive, the patient in all probability will not survive for more than four or five years.

It is a matter of vital importance to ascertain whether the myocardium and coronary arteries are also affected. In this connection, it may be noted that when the affection is due to a previous attack of acute endocarditis, the myocardium and coronary arteries may not be implicated for a long time and may even escape altogether; whereas, when due to degenerative processes, they are apt to be affected at an early period, especially if widespread arteriosclerosis exist. Needless to say, when aortic incompetence is due to rupture of a cusp, the prognosis is still graver, and a fatal termination may rapidly supervene. 
When the result of dilatation of the orifice, the affection is likely to be progressive. Syncopal attacks and attacks of angina pectoris are of grave significance. The gravity of the prognosis is in every case increased by widespread arterio-sclerosis, and kidney disease.

\section{Mitral Stenosis}

In mitral stenosis the commonest type of lesion is for the cusps to be united by their margins; but it may be situated elsewhere, as, for example, at the level of the auriculo-ventricular ring. When the lesion consists of a union of the margins of the cusps, two well-marked types of stenosis may result, (1) the "funnel-shaped mitral," which is more common in childhood, and (2) the " button-hole mitral," which is by far the more common in adults and persons of advanced years.

In the former, the united cusps form a funnel-shaped mcmbrane which projects downwards into the left ventricle, the wide mouth of the funnel being situated at the ring, and the smaller end being drawn down towards the apex of the heart, by shortening of the chordæ tendineæ and papillary muscles. In the case of button-hole stenosis, the united cusps form a diaphragm, perforated by a narrow slit-like aperturc, which almost closes the orifice. A combined form, that is, a funnel having a narrow slit at its lower end, instead of a circular aperture, has also been described.

Etiology.-Mitral stenosis is always organic. It commences much more commonly in early life, and is more than twice as common in females than in males. It is usually the result of a previous attack of acute and subacute endocarditis, due to rheumatism in one or other of its protean manifestations. It sometimes originates in adults as a result of degenerative processes, due to syphilis or in association with chronic interstitial nephritis. In a certain proportion of cases its etiology is obscure.

Symptomatology. - In mitral stenosis there is not very 
infrequently an absence of subjective symptoms for many years, except that dyspnoa, palpitation, or precordial pain may accompany any sudden severe demand made on the cardiac muscle. Later, the heart muscle is liable to fail, and dilatation may result. Dyspnœea is induced by less and less effort, cough is a frequent symptom, the patient is liable to attacks of bronchitis and bronchopneumonia, palpitation and precordial pain may be present, and epistaxis, hæmoptysis, and infarction may occur ; embolism is of more frequent occurrence in mitral stenosis than in any other variety of chronic valvular disease. There is often a persistent dusky flush over the cheeks, and clubbing of the finger-ends, gradual loss of flesh, and, in the young, stunted growth are sometimes to be noted. Later, there may be lividity of the lips, ear-tips, and cheeks, and puffiness around the ankles in the evening. Ultimately, dyspnoa may become continuous, and therc may be orthopnœa and cardiac asthma, especially at nighttime, sleeplessness and restlessness, evidences of congestion and oedema of the lungs and hydrothorax, a sallow hue of the skin, watery bile-stained conjunctivæ, marked cyanosis, loathing of food, vomiting, enlargement of the liver and spleen, ascites, general anasarca, and albuminous and sometimes smoky urine. Sudden death is rare, the fatal termination being usually the result of gradual cardiac failure.

The subjects of mitral stenosis are liable to subacute and acute rheumatic attacks, and chronic renal disease may be a complication.

Usually the pulse is small, the upstroke being short and soon attaining its maximum, the pulse-wave is long, and dicrotism is absent (Figs. 152-6). The maximum blood-pressure is usually rather below the normal, but the minimum pressure is rather high, so that the pulse is full between the beats. When cardiac failure supervenes, both the systolic and diastolic pressure fall. The complete irregularity of the pulse which is associated with 
auricular fibrillation is often found in mitral stenosis with cardiac failure.

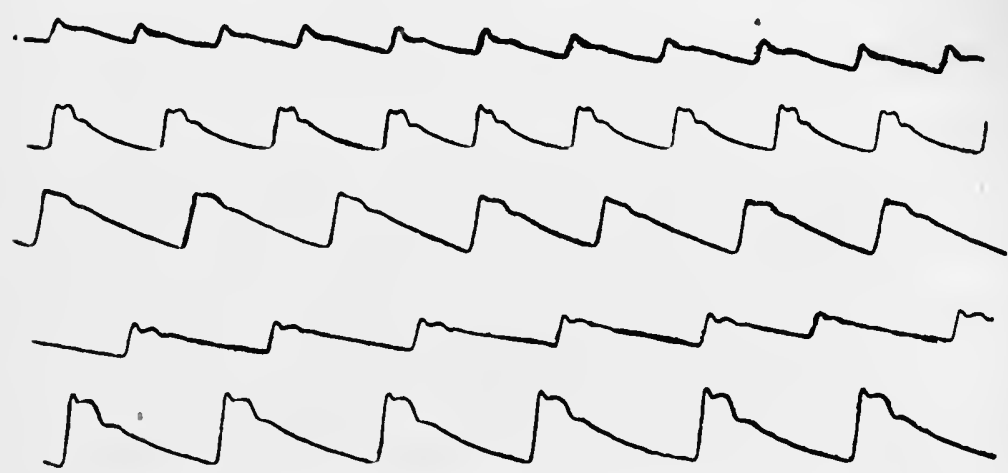

Figs. 152, 153, 154, 155 and 156. Sphygmograms from cases of mitral stenosis.

On inspection and palpation, the following points may be noted in cases of pure mitral stenosis. Sometimes, more especially in children, there is a certain amount of bulging of the precordium in the region of the ensiform cartil.ge, due to enlargement of the right ventricle. The apex-beat may be in the normal position or displaced. In pure mitral stenosis the displacement is usually directly to the left, it may be beyond the nipple line; less frequently it is also somewhat downwards. The apex-beat may be normal, diffuse, or indefinite. On palpation, there is sometimes a peculiar sudden tap communicated to the fingers, which is very characteristic. Not infrequently there is pulsation in the epigastrium, diastolic in time. There may be a heaving impulse in the epigastrium and over the lower sternum and costal cartilages when marked hypertrophy of the right ventricle exists; in these cases, this is the chief impulse, being more marked than that of the left ventricle. Occasionally there is systolic pulsation in the third or second left interspace, near the sternum; this may be due to enlargement of the conus arteriosus or of the pulmonary artery. 
In many cases there is a thrill, having its maximum intensity at the apex or somewhat nearer the parasternal line. This may be absent at some periods and present at others ; it may be absent when at rest, and in evidence after exertion or when the arms are extended. Examination for the characteristic thrill and murmur of mitral stenosis should be conducted with the patient lying down, sitting up and inclined forwards, standing up with the arms extended, and after gentle exercise. The thrill is usually presystolic in time, but may be diastolic. When presystolic, on palpation of the precordium there is a sensation communicated to the hand resembling that produced by the purring of a cat. Sometimes an impulse may be made out at the base of the heart, coincident with the closure of the semilunar cusps, with its maximum intensity in the second left intercostal space.

On percussion, the area of cardiac impairment may be found to be unaltered when the obstruction is slight, but usually the transverse diameter is increased, especially to the right. The left border may be displaced outwards, even beyond the nipple line. The upper border of the area of impairment is sometimes found to be higher than normal, and may reach as high as the second costal cartilage, and an extension of the area of impairment outwards along the third and fourth intercostal spaces, duc to enlargement of the right auricle, may be occasionally detected.

On auscultation, the physical signs are very characteristic. In the large majority of cases a murmur is present. This may be audible at some times and not at others. It is heard at the apex, or between this point and the left border of the sternum. The murmur is usually presystolic in rhythm; as in the case of the presystolic thrill, it is due to the systole of the auricle driving blood through a narrowed orifice, and disappears with the onset of auricular fibrillation. The murmur is almost invariably rough; it may be harsh and vibrating, and 
this quality of the murmur is almost pathognomonic; Le ennec compared the sound to the rasping noise made by a file. The first sound which terminates the murmur is altered in a characteristic way, to be described later. The locality of the murmur is almost always very limited, and the area may not be larger than one inch in diameter; this should be particularly noted, because it accounts for the fact that the lesion is so often unrecognised. Instead of presystolic, the bruit may be diastolic in time. Diastolic murmurs tail off, and do not have, as a rule, the harsh and vibratory character of the presystolic bruit; they are, instead, soft and blowing in type, and are due to the passive inflow of blood from auricle to ventricle.

There may be a combination of two murmurs; there may be a long murmur beginning immediately after the second sound, and continuing all through ventricular diastole and auricular systole, the early part being descendo and the latter part crescendo. Or, at one time there may be a presystolic, and at another time a diastolic, murmur.

The first sound becomes short, sharp, and clear, like a sudden snap. It may resemble the second sound so closely as to be mistaken for it, especially when the second sound is not well heard at the apex. As mitral stenosis is almost invariably accompanied by incompetence, a mitral systolic murmur is usually audible; indeed, in the opinion of some writers, this is the murmur most commonly present in mitral stenosis. Others believe, however, that in a certain percentage of cases where a systolic bruit is heard in mitral stenosis the cause depends on secondary dilatation of the right side of the heart and incompetence of the tricuspid valve.

The second sound is usually accentuated, particularly in the pulmonary area, and may be reduplicated, the effect resembling a " postman's knock." According to the late Sir William Broadbent, in a later stage of the disease the second sound at the apex may disappear, the short, sharp first sound may become louder, and the murmur 
become more frequently diastolic in time. In the absence of a diastolic, as well as of a presystolic, murmur, all that is audible at the apex now is the altered first sound.

When cardiac failure supervenes, the pulse-rate increases, the apex-beat becomes diffuse and less forcible, the transverse area of cardiac impairment increases, and all the signs of tricuspid incompetence may be present.

Diagnosis. - When the characteristic physical signs of mitral stenosis-the pulse, the thrill, evidence of hypertrophy of the right ventricle, the murmur, the short, sharp, and clear first sound, and the reduplicated second sound-are present, the diagnosis is easy. The thrill is almost pathognomonic. Some writers believe that a similar thrill may be present in aortic incompetence, and therefore that this affection should always be first excluded. The thrill must be distinguished from a mere vibration of the chest-wall which may be occasioned by a forcibly acting heart when the ribs are rigid. In this case, by separating the fingers and placing them in the intercostal spaces the osseous vibrations are not felt.

The murmur is sometimes not detected, on account of the area over which it is audible being very limited; or, in some cases there is from time to time a complete absence of a thrill and murmur. In the latter case, we are justified in inferring that in all probability mitral stenosis is present from the character of the pulse and first sound, together with evidence of hypertrophy of the right ventricle, and accentuation or reduplication of the second sound.

Some writers are of opinion that a rumbling presystolic bruit is sometimes heard in the case of adherent pericardium in children. Even if this be so, the bruit is not rough and vibratory in character, neither does it terminate abruptly at the first sound, nor is the first sound modified in the way already described, and, further, there are usually definite physical signs of adherent pericardium. As it is generally believed that a presystolic murmur at the apex 
is heard in certain cascs of aortic incompetence, the question whether mitral stenosis is present in addition to aortic incompetence has occasionally to be considered. In this connection, it may be noted that Flint's murmur has not the harsh and vibrating character which is commonly found in a presystolic bruit due to mitral stenosis, and if a thrill be present it is not " purring " in character. It should be borne in mind that both lesions may be present at the same time, and, therefore, it is sometimes difficult to say whether the murmur is that described by Flint or one due to the presence of mitral stenosis. In coming to a decision, the character of the murmur, the thrill, and the pulse-the characteristic collapsing pulse of aortic incompetence being modified if mitral stenosis be present as well-will be of assistance.

Mitral stenosis is sometimes unrecognised in cases in which there is associated incompetence; the presystolic murmur, instead of ending in the first sound, ends in a systolic murmur, and a diagnosis of a long systolic murmur is erroneously made. There is, however, a difference in character between the former and the latter parts of this continuous bruit, the earlier portion being rough and vibratory in character, and the latter blowing or musical. Further, we can observe the position in the cardiac cycle of the two parts of this continuous murmur by placing the fingers upon the apex-beat or over the carotid artery.

When the second sound at the apex has disappeared, the presystolic murmur may be mistaken for a systolic murmur, and the modified first sound for the second sound. In this event, a differential diagnosis can be made from the difference in character of the two murmurs, from the fact that a presystolic murmur ends, while a systolic murmur begins with an accent, and by timing the murmur in the manner already indicated.

When in addition to the absence of a second sound at the apex, there is also no bruit present, mitral steniosis 
may not be diagnosed, and a diagnosis of dilatation of the left ventricle made; in dilatation, however, a second sound is audible.

The differential diagnosis between aortic incompetence and mitral stenosis has already been discussed.

A differential diagnosis may have to be made between tricuspid and mitral stenosis. The former is extremely rare, and the point of maximum intensity of the thrill and murmur is in the tricuspid area.

Occasionally a mere reduplication of the first sound is mistaken for mitral stenosis, but no careful observer should make this mistake.

Prognosis.-Mitral stenosis ranks after aortic incompetence in gravity among the chronic valvular lesions, and the prognosis is much less favourable than in the case of mitral incompetence. According to an analysis of the post-morten records of $\mathbf{5 3}$ cases admitted to St. Mary's Hospital made by Broadbent, the average age at death was found to be $\mathbf{3 3}$ years in the case of males, and $\mathbf{3 7}$ to 38 in the case of females. It should be borne in mind, however, that the death-rate in hospital cases is probably higher than the average, owing to the social status of the patients.

There is apparently a tendency for mitral stenosis to be progressive, pulmonary complications are frequent, chronic renal disease may also accompany it, and embolism is relatively frequent. When the lesion occurs in the early years of life, physical and mental development are apt to be retarded, and the patient is liable to fresh attacks of rheumatic valvulitis. The prognosis depends upon, among other things, the degree of stenosis, which when considerable is almost invariably accompanied by incompetence. It has been pointed out that in a later stage of the disease the second sound at the apex may disappear, the short, sharp first sound become louder, and the murmur more frequently diastolic; these phenomena, therefore, signify a downward grade. Sudden death is of 
rare occurrence, a fatal termination being usually the result of gradual cardiac failure, which in most cases is associated with the onset of auricular fibrillation.

\section{Mitral Regurgitation}

Mitral regurgitation may be organic or relative, the latter being also termed functional.

Etiology. - When organic, by far the most common cause is a previous attack of acute endocarditis, but sometimes it is due to degenerative processes. Rarely it is due to trauma, in which case there is usually a rupture of a chorda tendineæ or of a cusp. Relative incompetence is a somewhat common cause of mitral regurgitation. It may be due to simple overstrain of the heart, wasting states, toxic conditions-such as alcoholism, lead, pneumonia, and diphtheria-acute febrile affections, anæmias, Graves' disease, acute myocarditis, fatty or fibroid degeneration of the myocardium, super-normal blood-pressure, chronic Bright's disease, or aortic incompetence.

Symptomatology.-In by no means an inconsiderable proportion of cases, the disease is latent throughout the whole course. When symptoms are present, the clinical picture resembles that of mitral stenosis; it should be noted, however, that hæmoptysis and embolism are of less common occurrence.

When the lesion is slight, the pulse as a rule scarcely differs from the normal. When the lesion is considerable, the amplitude is apt to be smaller than normal, the upstroke rather steep, there is often very considerable dicrotism, the pulse being easily compressible and not felt between the beats, and the blood-pressure is low. The complete irregularity which is associated with auricular fibrillation is often found in mitral incompetence with cardiac failure.

In mitral incompetence due to organic valvular disease, 
there is sometimes, more especially in children, a certain amount of bulging of the precordium. The apex-beat is usually displaced outwards, it may be even as far as the anterior axillary line. As there is hypertrophy of the left ventricle, the apex-beat may be also displaced somewhat downwards, and usually its area and force are greater than normal; it may even be heaving in character. Sometimes there is pulsation in the epigastrium, diastolic in time; and when there is marked hypertrophy of the right ventricle, there may be a heaving impulse in the epigastrium and over the lower sternum and costal cartilages. Occasionally there is visible pulsation in the second or third left intercostal space, and more rarely a thrill, systolic in time, which has its maximum intensity at the apex. A shock, accompanying the second sound, may be occasionally detected, particularly in the pulmonary area.

On percussion, the area of cardiac impairment is found to be enlarged. Its transverse diameter is increased, the left border being displaced outwards, even as far as the anterior axillary line, and the right border as much as an inch-and-a-half or more to the right of the sternum. The upper border of cardiac impairment may be as high as the second rib, and an extension of the area of impairment outwards along the third and fourth intercostal spaces, due to enlargement of the left auricle, may occasionally be detected.

On auscultation a systolic murmur is audible. This usually commences with the first sound, which it may entirely replace or merely accompany-usually the latter-and may extend all through the short pause; rarely it follows the first sound. Its point of maximum intensity is at or near the apex-beat, and its direction of selective propagation towards the axilla and the angle of the scapula. Occasionally it can be heard over the whole chest. The area of conduction of slight mitral systolic murmurs is frequently not large. A mitral sys- 
tolic murmur is usually soft and blowing, occasionally musical, and more rarely rough and grating. It is usually louder when the patient lies down, and becomes feebler in the sitting or standing posture ; in any doubtful case, therefore, the patient should be examined in these several positions. The second sound at the pulmonary area is, in the great majority of cases, accentuated, and may be reduplicated.

When cardiac failure supervenes, the pulse-rate increases, there is usually present an increased degree of dilatation of the left ventricle-the apex-beat becoming more diffuse and its force lessened, and the left cardiac border being found still further to the left-and in addition dilatation of the right heart, while all the signs of tricuspid incompetence may be present.

In relative mitral incompetence, the apex-beat may be displaced outwards, but not downwards, and instead of being more forcible than normal it is usually less so. The systolic murmur is as a rule not propagated beyond the mid-axillary line, and there is often no accentuation of the pulmonary second sound.

Diagnosis.-At the outset, it is necessary to point out that a systolic bruit audible over the apex of the heart is not infrequently erroneously interpreted as indicative of organic disease of the mitral valve, when in reality the murmur is functional. In order to say that a mitral systolic murmur is indicative of organic mitral incompetence, we must satisfy ourselves that certain other physical signs are present. With this object in view, it is advisable to note (1) the position and character of the apex-beat, (2) the area of propagation of the murmur, and (3) the existence or absence of accentuation of the pulmonary second sound. In the case of a mitral systolic murmur due to organic valvular disease, owing to the presence of hypertrophy of the left ventricle, the apex-beat is usually displaced downwards as well as outwards. When the degree of valvular disease is so slight that no definite 
downward displacement of the apex-beat is present, its force is almost invariably increased, whereas in functional conditions it is apt to be less forcible than normal.' If the murmur be propagated towards the angle of the scapula beyond the mid-axillary line, we may be practically certain that it denotes the existence of organic disease. A definite accentuation of the pulmonary second sound, provided there is no condition of the lungs to account for it, is in favour of the view that we are dealing with organic disease. The diagnosis is further strengthened by evidence of the existence of mitral stenosis. The diagnosis of relative mitral incompetence due to chronic Bright's disease or to aortic disease from organic mitral incompetence is particularly difficult, since chronic nephritis and aortic disease in themselves give rise to hypertrophy of the left ventricle. In these cases the history of the case is of importance.

In addition to the points of differential diagnosis just enumerated, murmurs due to anæmia and cardio-pulmonary bruits have other distinguishing features. These have been discussed in Chapter II. Cardio-pulmonary murmurs are particularly apt to be misleading when left-sided pulmonary tuberculosis, thickening or adhesion of the pleura, fibrosis of the lung, or right-sided pleurisy with effusion exist, because in these conditions the apex-beat may be displaced outwards; it is rarely, however, displaced downwards.

Prognosis.-The prognosis of mitral incompetence is more favourable than that of aortic stenosis or incompetence, or mitral stenosis. If the lesion be slight and the conditions of life favourable, the individual may even live to the normal span of life without experiencing much, if any, inconvenience. When, on the other hand, the lesion is severe, as indicated by marked cardiac enlargement, and more especially when symptoms of cardiac failure are present-more particularly pulmonary symptoms - the patient may live only for a few years, especially 
when the lesion is accompanied by pericardial adhesions. It should be remembered, however, that even when considerable or marked cardiac failure exists, if rest and other suitable therapeutic measures be adopted, the patient may improve for a considerable time. In attempting a prognosis, we should note, among other things, the pulserate, the degree of displacement of the right margin of cardiac impairment, and the existence or otherwise of any indications of venous stasis.

With regard to the prognosis of mitral regurgitation duc to relative incompetence, this depends upon the cause.

\section{Mitral Stenosis and Regurgitation (Double Mitral Disease)}

It has becn already explained that when mitral incompetence results from a previous attack of acute endocarditis, there is usually some degree of stenosis as well, and also that pure mitral stenosis is comparatively infrequent, as it is almost invariably accompanied by incompetence. We thus get the double lesion.

Etiology.-Double mitral disease is always organic; it is usually due to a previous attack of acute endocarditis, although in elderly people it may be due to degenerative processes.

Symptomatology. - The symptoms partake of the
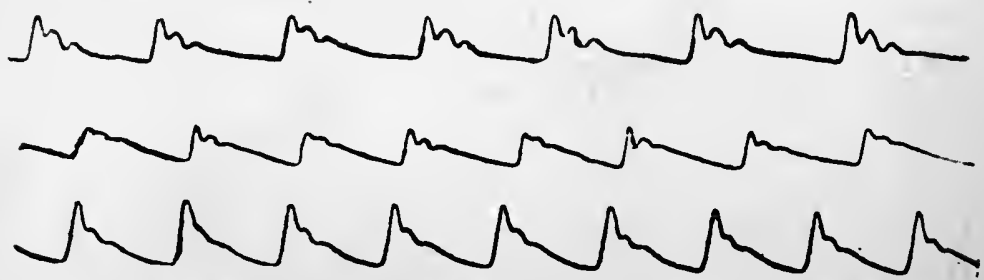

FıGs. 157, 158 and 159. Sphygmograms from cases of mitral stenosis and regurgitation.

character of both lesions (Figs. 157-9). Auricular fibrillation, cardiac failure, and tricuspid incompetence 
are more likely to supervene than in the case of a single lesion. There is usually considerable enlargement of the heart, and on auscultation the combined murmurs may be heard, but not infrequently a mitral systolic is the only murmur present. Accentuation of the pulmonary second sound is usually more marked than in the case of a single lesion.

\section{Pulmonary Stenosis}

Pulmonary stenosis may be due to a lesion of the cusps, of the conus arteriosus, or of the pulmonary artery.

Etiology.-The disease is almost always congenital; indeed, it is perhaps the most common congenital cardiac affection. When it occurs as an acquired condition, it may be the result of a previous attack of acute endocarditis, of which rheumatism and the eruptive fevers arc the principal causes; and when this is so, it is usually associated with tricuspid stenosis. It has been supposed that the condition sometimes results from atheroma of the pulmonary artery, or from pressure of an aortic aneurism. In some cases the etiology is obscure.

Symptomatology. - The symptoms of the congenital form of the disease will be discussed in Chapter XXIV. In the acquired form, the degree of dyspnœa is, as a rule, not great, and some authorities are of opinion that there is little tendency to cyanosis in these cases ; similarly, there is a difference of opinion as to the existence of clubbing of the fingers and toes. The tendency to chronic venous stasis is not great, and as a consequence œdema of the lower extremities and ascites are not prominent features, although there may be tenderness over the liver, hepatic enlargement and pulsation, and the urine may become scanty, high-coloured, exhibit an excess of urates, and may contain albumin or even blood.

Prognosis and Treatment.-These will be discussed in Chapter XXIV. 


\section{Pulmonary Incompetence}

Pulmonary incompetence is the most rare of all valvular lesions.

Etiology.-The condition occurs more commonly in carly life, and with about equal frequency in both sexes. It is frequently a congenital affection, and is then usually accompanied by pulmonary stenosis. In the majority of cases, it is the result of a previous attack of acute endocarditis, of which rheumatism and the eruptivc fevers are the principal causes. It is believed by some that the condition may be caused by degenerative processes, and some authorities are of opinion that relative incompetence may also occur; that is, the incompetence may be due to dilatation of the pulmonary artery and its ring, as a result of long-standing disease of the left side of the heart, more especially mitral disease.

Symptomatology.-There may be a certain degree of cyanosis and dyspnœa, usually only present on exertion, whilc not infrequently there is also clubbing of the fingers. At a late stage, cyanosis becomes marked, dyspnœa occurs apart from exertion, there may be anasarca of the dependent parts, catarrh of the mucous membranes, enlargement of the solid abdominal viscera, transudation of fluid into the serous sacs, the urine may become scanty, highcoloured, exhibit an excess of urates, and may be albuminous or even bloody, while affections of the respiratory system are especially apt to occur. There may be evidence of chronic venous congestion and odema of the lungs, particularly of the bases, and more rarely pulmonary infarction occurs.

The pulse may be small and feeble, and the bloodpressure is, as a rule, subnormal. Occasionally there is visible pulsation in the third and fourth intercostal spaces to the right of the sternum. There is also frequently some bulging of the precordium in the region of the ensiform cartilage, due to enlargement of the right ventricle, while 
the apex-beat is usually displaced to the left. There may be pulsation in the epigastrium, diastolic in time, and when there is marked hypertrophy of the right ventricle there is frequently a heaving impulse in the epigastrium, and over the lower sternum and costal cartilages. Distension or pulsation of the jugular veins may be present. Rarely a diastolic thrill is present, having its point of maximum intensity over the pulmonary area. On auscultation, a diastolic murmur, with its point of maximum intensity in the pulmonary area, usually soft and blowing in character, is audible, sometimes over a considerable area, its direction of selective propagation being downwards towards the lower end of the sternum.

Diagnosis. - The diagnosis of pulmonary incompetence is frequently a matter of considerable difficulty. The lesion with which it is most likely to be confounded is aortic incompetence, because the point of maximum intensity of the thrill and murmur of the latter is not infrequently to the left of the sternum. The differential diagnosis between these two conditions has been discussed under aortic incompetence. It should be noted that both lesions are rarely present in the same individual. The symptoms of pulmonary incompetence and patent ductus arteriosus resemble each other; in the case of the latter the murmur occupies both systole and diastole.

- Prognosis.-When due to organic disease and when unaccompanied by any other valvular lesion, the prognosis in pulmonary incompetence may not be unfavourable; instances, indeed, have been recorded in which life has been prolonged almost to the normal span. In relative incompetence, on the other hand, the prognosis is always grave. Death in either case may result from right-sided cardiac failure, bronchitis or broncho-pneumonia, or pulmonary tuberculosis. 


\section{Tricuspid Stenosis}

Etiology.-Tricuspid stenosis occurs much more commonly in males than in females-in a proportion of at least three to one. While a considerable proportion of cases arc congenital (in which case there is usually also some other cardiac lesion), the majority arise after birth. The acquired form is usually associatcd with mitral stenosis, and is due to a previous attack of acute endocarditis caused by rheumatism, chorea, or more probably one of the acute specific fevers. In many cases, however, the cause cannot be ascertained.

Symptomatology.-In the acquired form of the disease, cyanosis is the most conspicuous symptom, and may be very marked ; dyspnœa is less evident, and may be only present on exertion. The skin temperature of the extremities is often subnormal, and the individual complains of feeling cold and is very susceptible to any fall of temperature. The cercbral functions are not infrequently affected. CEdema about the ankles is sometimes present, and at a later stage may become general, together with tenderness over the liver, hepatic enlargement, and pulsation, ascites, splenic enlargement, and scanty, high-coloured urine, which may contain albumin and even blood.

The radial pulse may show no abnormality, or, its rate may be increased, and its volume diminished. The apex-beat may be displaced to the left, and there is usually distension, and it may be pulsation, of the jugular veins. There may be a presystolic, or more rarely a diastolic thrill, with its point of maximum intensity in the tricuspid region. On percussion, the right border of the heart is found to be displaced to the right, on account of enlargement of the right auricle, and the left border may be found to be displaced outwards. On auscultation, a murmur may be present, limited in extent, having its point of maximum intensity in the tricuspid area. It is usually presystolic, but may' be diastolic. 'The 
presystolic murmur is almost invariably rough; it may even be harsh and vibratory in character. The diastolic murmur, on the other hand, is usually soft and blowing. In some cases no murmur of any kind is audible. In certain instances, some writers have recorded a short sharp first sound in the tricuspid area. When pulsation of the liver is present, the pulsation is of the auricular and not of the ventricular type.

Diagnosis. - In by no means an inconsiderable proportion of cases of tricuspid stenosis the lesion is entirely overlooked, no doubt on account of the absence of any murmur. In the opinion of Mackenzie, we may infer that tricuspid stenosis in all probability exists when pulsation of the liver is found to be auricular in type.

The differential diagnosis of tricuspid and mitral stenosis is often a matter of great difficulty. The early appearance of marked cyanosis and of extensive dropsy are features in favour of the existence of the former lesion. When the point of maximum intensity of the murmur is strictly in the tricuspid area, we can be fairly certain of the diagnosis. It should be remembered that both lesions may be present; in this case a murmur with its point of maximum intensity midway between the tricuspid and mitral areas, or two murmurs with separate points of maximum intensity, may be detected. The value of the auricular type of hepatic pulsation has been noted.

Prognosis.-The prognosis in this affection is by no means easy. It largely depends upon whether mitral stenosis also exists, and if present, in what degree; if this be present the prognosis is usually very unfavourable.

\section{Tricuspid Incompetence}

Etiology.-It should be remembered at the outset that the reflux of a certain amount of blood through the tricuspid orifice may occur under physiological conditions. In severe muscular exertion, for example, where the intra- 
cardiac pressure on the right side of the heart is excessive, a natural function of the tricuspid valve is to provide for a certain amount of regurgitation, and thus afford relief to the temporary embarrassment due to distension of the right ventricle.

Pathological tricuspid incompetence is rarely congenital, and when it is is usually associated with pulmonary stenosis. Apart from those of congenital origin, there are two classes of cascs, (1) those in which the incompetence of the valve is primary, that is, due to a lesion of the cusps themselves - a rare condition; and (2) cases of relative tricuspid incompetence, in which the cusps are normal, the incompetence being due to dilatation of the right ventricle. The latter constitute the great majority of cases.

With regard to the first group, the lesion may be the result of a previous attack of acute endocarditis, in which case the tricuspid incompetence rarely occurs alone; there is usually tricuspid stenosis, and also some other valvular lesion, more espccially some structural alteration of the mitral cusps. Uleerative cndocarditis may affect the tricuspid valve alone.

Relative incompetence is always consequent to some other condition. It may result from chronic Bright's diseasc or aortic diseasc, resulting in dilatation of the left ventricle and venous stasis in the lungs, mitral disease, and affections of the lungs which cause embarrassment. in the pulmonary circulation, such as emphysema, cirrhosis, chronic fibroid tuberculosis, and chronic diseases of the bronchi. Among other causes are pulmonary stenosis or regurgitation, wasting states, toxic influences (such as alcoholism, lead, pneumonia, and diphtheria), acute febrile conditions, anæmia, acute myocarditis, and myocardial degeneration.

Symptomatology.-There is always a tendency to cyanosis, which may be marked, while dyspnœa on exertion is almost always present. Headache and giddiness are of 
common occurrence, while insomnia or disagreeable dreams, hallucinations, and even insanity may occur. Digestive disturbanees and even jaundiee may be present, and in rare instances hæmatemesis and melæna. GEdema about the ankles may also be noted. These patients are very liable to attacks of bronehial eatarrh, while, later, there may be evidence of chronic venous congestion and œedema of the lung,s, partieularly of the bases, hæmoptysis, and hydrothorax, the latter being more likely to oeeur in trieuspid ineompetence than in affections of the left side of the heart. Hepatic enlargement and pulsation, ascites, general anasarea, and albuminous and even bloody urine may be also present in the later stages of the disease.

The pulse is usually small. In children a certain amount of bulging of the preeordium may be present in the region of the ensiform cartilage, due to enlargement of the right ventricle, and there may also be pulsation in the epigastrium, diastolie in time. A powerful heave in the epigastrium may be sometimes noted when the right ventriele is hypertrophied, and less eommonly also visible pulsation in the fourth and fifth right intercostal spaces near the sternum. More rarely a systolie thrill is present in the trieuspid area. Visible distension and pulsation of the jugular veins may exist. If the vein be emptied from below by means of the finger, and its upper extremity kept elosed by pressure, it rapidly fills from below. The pulsation of the jugular vein is usually of the ventrieular form, but oeeasionally is of the auricular type. The liver may be enlarged and pulsating, the pulsation being strietly systolic in time--unless tricuspid stenosis also exists. The area of eardiac impairment is inereased to the right, sometimes to a considerable extent.

On auseultation, a murmur, systolic in time, having its point of maximum intensity in the tricuspid area, and usually soft and blowing in charaeter, is usually, but by no means always, audible. It is condueted towards the 
right, but as a rule its area of conduction is slight. The pulmonary second sound is diminished in cases of tricuspid incompetence uncomplicated by a mitral or pulmonary lesion.

As relative incompetence of the tricuspid valve is always consequent upon some other condition, the physical signs of the primary affection are of course present.

Diagnosis.-When after being emptied from below by means of the finger the jugular vein rapidly fills from below, and when pulsation of the liver occurring synchronously with the apex-beat, evidence of enlargement of the right side of the heart, a soft blowing systolic murmur having its point of maximum intensity in the tricuspid area, and diminution of the pulmonary second sound are present, no doubt need arise regarding the diagnosis. In marked tricuspid incompetence the murmur may be absent, in which case a positive diagnosis can be made when the first three signs mentioned are present; most writers, indeed, agree that the first sign is sufficient for a diagnosis. In slight cases, a systolic murmur audible in the tricuspid area may be the only existing physical sign; but we should makc certain the murmur is not propagated from any other area. Lastly, it has been pointed out that on severe muscular exertion a natural function of the tricuspid valve is to provide for a certain amount of regurgitation, and thus a tricuspid systolic murmur may appear under physiological conditions.

Prognosis.-The prognosis of tricuspid incompetence depends upon the cause and associated condition. When the affection is due to organic disease, the outlook is serious.

With regard to the prognosis of tricuspid regurgitation due to relative incompetence, when this is the result of aortic or mitral disease it is usually serious; it may be the precursor of a fatal termination in the near future. When, on the other hand, it is due to intercurrent pulmonary affections, wasting states, toxic 
conditions, acute febrile states, or anæmia, the prognosis may be good.

\section{Treatment of Cirronic Valvular Disease}

If there be no indications of cardiac failure, no direct treatment is necessary. But, explicit and detailed instructions with regard to his manner of life-as fully described in Chapter IX-should invariably be given to the patient. The importance of always living within the limits of the heart's strength, and avoidance of sudden and violent effort, is of special importance in aortic disease. Horder has pointed out that the question of oral sepsis is an important one, and that this should receive attention, with the object of diminishing the risk of malignant endocarditis. In mitral disease the patient is liable to attacks of bronchitis and bronchopneumonia, and in both mitral and aortic disease in children and young adults there is a liability to recurrent acute and subacute rheumatic attacks; preventive measures should, therefore, be adopted, and if notwithstanding an attack of either occur it should be immediately and adequately treated.

When there are indications of cardiae failure, such as dyspnoa, it is imperative that the work of the heart should be lessened by rest. If the cardiac failure be slight in degree, continuous rest in bed is often not necessary; it is often advisable, however, to begin the treatment by keeping the patient in bed for a short time. Other measures which may be adopted in addition to rest, as well as the treatment of symptoms, have been dealt with in Chapter IX. 


\section{CHAPTER XXI}

DISEASES OF THE MYOCARDIUM AND CORONARY AR'TERIES

Affections of the myocardium are of profound importance. It has been pointed out in Chapter VII that valvular defeets, diseased conditions of the blood vessels, and disturbances of the cardiac mechanism should bc regarded from the point of view which they bear to the myocardium, and not so much as specific affections in themselves. It has also been noted that, along with valvular lesions, coincident changes in the cardiac musculature, the aorta, or eoronary arteries are as a rule present. We are, however, now concerned with affections of the myoeardium occurring independently of valvular disease, and of these inflammation of the myocardium or myocarditis is undoubtedly of most importance.

\section{Myocarditis}

There are two ehief varieties of myocarditis, the acute and the chronic. The latter will be discussed under chronic myocardial disease.

\section{Acute Myocarditis}

Acute myocarditis may be general, or limited to one or more portions of the cardiac wall. Acute degenerative processes, especially fatty degeneration, of the musclefibres are usually found; the interstitial connective tissue, on the other hand, may show little or no change, or may exhibit considerable cellular infiltration. It has been noted by Romberg and others that this cellular in- 
filtration is often focal, the foci being from $0 \cdot 1-0 \cdot 2 \mathrm{~mm}$. in diameter, and situated in the neighbourhood of the mitral orifice; these foci have a homogeneous centre, surrounded by a zone of giant cells, and are believed by some to be pathognomonic of the rheumatic infection. Some writers recognise two forms of myocarditis, the parenchymatous and the interstitial ; in most cases, however, both the parenchyma and interstitial tissue are affected. Dilatation of one or more chambers of the heart usually supervenes. Abscess formation, a condition known as suppurative myocarditis, is of rare occurrence, and is usually pyæmic in origin; . in these cases, which are usually fatal, perforation of the ventricular wall may occur.

Acute myocarditis may be followed by (1) complete resolution, in which no permanent changes supervene; or (2) organisation, with the formation of connective tissue, which tends to contract as life advances, and which may occur in limited areas or may involve the whole myocardium. The latter condition is known as chronic myocarditis.

Etiology.-The affection is usually due to one of the acute specific fevers, especially acute rheumatism, diphtheria, influenza, pneumonia, scarlet fever, and septicæmia or pyæmia. Some degree of myocarditis is always present with pericarditis, and probably also with endocarditis.

Symptomatology.-The clinical picture in acute myocarditis varies considerably in different cases; not infrequently it is indefinite, and, indeed, in rare instances the condition may remain latent during its whole course and is only discovered post-mortem, death being due to the severity of the primary illness. The onset may be insidious, or there may be a definite change in the clinical picture of the causal disease. If pyrexia be already present, there may be a further rise of temperature, or if there have been no pre-existing pyrexia it may develop; it may be 
signalised by a rigor, and be slight or considerable in degree. Not infrequently dyspnœea and cough are present, respiration shallow and more rapid, while CheyneStokes' breathing may be occasionally noted. There may be great prostration, and syncopal attacks may occur if the affection be severe. There is pallor or lividity of the face, according to the degree of severity of the disease. Vomiting not infrequently occurs, and, indeed, may be the first symptom. Jaundice has been noted in some cases. Dropsy may develop, and the urine contain albumin and blood; in the severe form of diphtherial myocarditis, albuminuria is generally present. As the illness progresses, the patient may sink low in bed, complain of headache, with restlessness, insomnia, convulsions, muttering delirium, stupor, and even coma. The severity of the subjective symptoms may be out of all proportion to the physical signs.

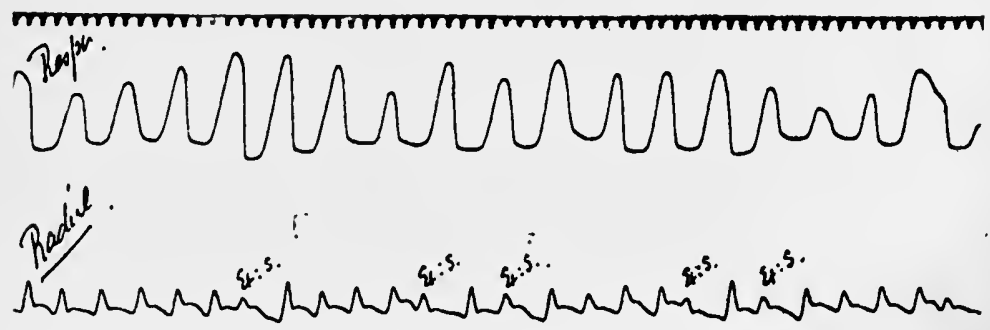

FIG. 160. Tracing of the radial pulse, from a child suffering from croupous pneumonia, showing extra-systoles. Note the slight pulsus alternans following the extra-systoles.

The pulse is feeble and soft; it is usually, but by no means always, increased in frequency, and, indeed, bradycardia may be present. Bradycardia may be met with during the convalescent stage of diphtheria, influenza, and pneumonia, and occasionally during the febrile stage, and may be due to irritation of the vagus, or sometimes to partial heart-block. In some cases there is irregularity of rhythm, due to extra-systole (Fig. 160), or partiàl 
heart-block (Fig. 161), or auricular fibrillation; exceptionally, complete heart-block is present. The apexbeat, which may or may not be displaced, is diffuse and diminished in force, and even abolished in severe

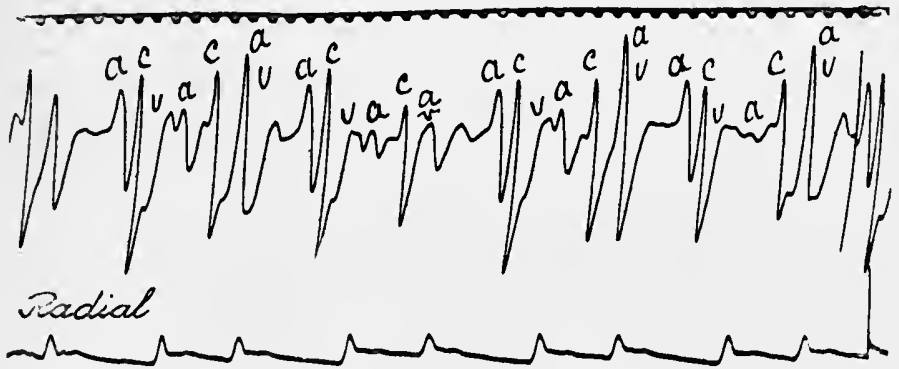

FIG. 161. Simultaneous tracings of the jugular and radial pulses, from a case of acute myocarditis occurring during diphtheria, showing partial heart-block. Every third stimulus from the auricle fails to reach the ventricle.

myocarditis. The area of cardiac impairment is usually increased transversely, both to the left and right, as the result of dilatation, which may sometimes come on rapidly.

On auscultation, the first sound is weak and short, and in the case of rapidly acting hearts the fotal type of cardiac action may be approximated. The second sound over the pulmonary area is sometimes accentuated, and occasionally reduplicatcd. The gallop rhythm may be present. The first sound may be clear, but a systolic murmur is not infrequently heard over the mitral, and sometimes over the tricuspid, area, due to functional inefficiency of the valves; a systolic bruit is perhaps more commonly noted over the base of the heart. Not infrequently physical signs of odema of the lungs, bronchitis, or effusion of fluid into the pleural sac are to be found.

In diphtherial and influenzal myocarditis, the symptoms may appear either during the febrile stage of the disease or during convalescence; this applies also, but less frequently, when some other cause of myocarditis 
is present. When the symptoms appear during convalescence, they are not so acute or so severe. Among them may be noted anorexia, languor, and pallor; dyspnœa, fatigue, precordial distress or actual pain, and, possibly, syneopal attacks on exertion; tachycardia, subnormal blood-pressure, an enfeebled cardiac impulse, evidence of slight cardiac dilatation, a short first sound at the apex, an aeentuated pulmonary second sound, and sometimes a mitral systolic bruit.

The diphtherial form of myocarditis may be mild or severe. There may be bradyeardia; in the severe form there is usually albuminuria, and a fatal termination may take place rapidly from cardiac failure. A severe form of myocarditis may also oceur as the result of influenza. A grave form of myoearditis has been met with in septicamia and in severe eases of enteric fever.

Diagnosis. - The diagnosis of aeute myocarditis is often extremely diffieult, the eondition not infrequently presenting an indefinite clinical pieture, and at times, indeed, remaining latent; we may often suspect or infer its existence, although it may not be possible definitely to affirm it. It is necessary at the outset to emphasise the importance of being on the look-out for the occurrence of partial heart-block during the course of an infectious disease, for it is a sign, and may be the only sign, of myocardial damage. The presence of severe myocarditis may be presumed when there are indications of marked cardiac failure eoming on rapidly during the course of an infectious disease, and out of proportion to the severity of the latter - cyanosis, vomiting, and increase of the area of cardiac impairment being perhaps especially significant. A diagnosis of the mild form of myocarditis occurring during or after the febrile stage of an infectious disease may be arrived at from a consideration of the symptoms already enumerated. It may be necessary in a given case to difierentiate myocarditis from acute endocarditis, and this is often a matter of extreme difficulty, 
for it should be remembered both conditions may coexist, and a mitral systolic murmur may be due to either.

Prognosis.-The prognosis of the severer forms of acute myocarditis is extremely grave, the mortality being high ; the course of the disease is rapid, a fatal termination occurring usually within a week, and in some cases even within twenty-four hours, although life may be prolonged for several weeks. Death is usually due to cardiac failure, which may occur suddenly; in rare instances to rupture of the wall of the heart. Sudden death is particularly prone to occur in diphtherial myocarditis, and may takc place even when the patient is considered convalescent. Vomiting is a symptom of grave significance. When a severe attack of acute myocarditis occurs in an individual who is a subject of heart disease, or in the elderly, the outlook is very grave. In the young and robust, complete recovery is the rule; this may be slow, and even in some of these cases there is only partial recovery, dyspnœa, palpitation, and other indications of cardiac impairment persisting for months or even years, being probably due to diffuse fibrosis with dilatation.

The prognosis of acute myocarditis as a whole is largely influenced by the causal factor. When due to the rheumatic infection, it is often mild in character and may end in complete recovery; in the graver types of rheumatic infection, however, the condition is almost hopeless. In diphtherial myocarditis, the prognosis of the severer forms is always grave; a fatal termination may take place rapidly. Among the symptoms of bad omen are marked pallor, restlessness, syncopal attacks, vomiting, a greatly accelerated pulse of low tension, bradycardia, and a modification of the heart sounds resembling the fotal type. In the milder forms, however, recovery is the rule if early and adequate therapeutic measures be adopted. If influenza occurring in the aged be the cause, the prognosis is particularly bad; recovery, however, is the 
rule, although it is slow, and there is a tendency to relapses; fatal syncope has been known to occur after the influenzal attack has disappeared.

Treatment.-The treatment of acute myocarditis is of great importance, and is the same as that of acute simple cndocarditis.

\section{Diseases of the Coronary Arteries}

Before dealing with the various forms of ehronic myoeardial disease, it may be advisable to discuss the diseases of the coronary arteries, as they bear an important causal relation to some of thcm, coronary disease being usually aceompanied by morbid changes in the cardiac musculature.

Among discases of the coronary arteries, the two most important are (1) cndarteritis obliterans, and (2) atheroma or endarteritis deformans.

Eitlier of these may affect the orifices of both coronary arteries; or the vessels may be affected beyond the orifices; or one of the branches only may be implicated; or the larger vessels may entirely cscapc, the smaller arteries alone being attacked.

By endarteritis obliterans is meant a chronic inflammatory change of the inner coat of the vessel, with organisation, but not degeneration. This inflammatory change leads to thickening, which is often uniform, and is due to a proliferation of the endothelium and the sub-endothelial connective tissue. The middle coat is usually not much affected, while the outer coat may show fibrous thickening. Aortitis may be associated with endarteritis obliterans, and the former may partially or wholly occlude the orifices of the coronary arteries.

By atheroma we mean a chronic inflammatory change, with early degeneration, but not organisation. There are two forms of atheroma, namely, (1) the sclerous, diffuse, or generalised form, in which thickening of the arterial 
wall is uniform, and (2) the nodular form, in which it occurs more or less in patches. The nodular form may affect the aorta and the coronary arteries, the left coronary being more frequently attacked than the right. In atheroma of the aorta, the neighbourhood of the coronary arteries is often involved, giving rise to partial occlusion of one or both of these vessels ; indeed, atheroma of the coronary arteries may be secondary to that of the aorta. Atheroma of the coronary arteries is often associated with the sclerous form of atheroma of the renal vessels (granular contracted kidney), and with degenerative processes of the valves, giving rise to chronic valvular disease, especially of the aorta. In the nodular form of atheroma, thickened patches, yellow and opaque, and raised above the surface, may be seen in the inner coat. The essential change occurs in the inner coat, and consists of a cell proliferation of the endothelial and sub-endothelial connective tissue. Degeneration occurs early in the deeper part of the thickened coat ; this may cause rupture of the internal elastic lamina and involve part of the adjacent muscular coat. Later on, the raised patches may undergo calcification, or soften and break down-leading to what is called an atheromatous ulcer.

Etiology of Coronary Disease.-Endarteritis obliterans is usually due to syphilis. With regard to the etiology of atheroma, heredity is certainly a factor, and the condition is more commonly found in males after middle life. Prolonged muscular or mental strain, over-indulgence in food and drink, syphilis, chronic metallic poisoning-particularly that due to lead-gout, and chronic renal disease, are among the most common causes. When syphilis is the cause, the condition is apt to come on before middle life.

Results of Coronary Disease.-If the lumen of a coronary artery or one of its main branches be completely and abruptly closed, either as the result of thrombosis or embolism, infarction follows. This usually occurs near 
the apex of the left ventricle, the infarct later becoming surrounded by dense fibrous tissue, from which strands radiate between the adjacent muscular bundles, resulting finally in scar formation. The result of this infarction is a weakening of the cardiac wall, which may lead to aneurism or rupture. Thrombi may form within the aneurism or before it has formed, and result in embolism and consequent infarction in the brain, kidneys, spleen; or elsewhere.

Complete and abrupt closure of a coronary artery is usually fatal. Its occurrence may be signalised by sudden dyspnoa, collapse, and other indications of rapid cardiac failure, and sometimes acutc anginal paroxysms, with a rapidly fatal termination. Or, death may occur suddenly, this, indeed, bcing one of the common causes of sudden death. If, on the other hand, the vessel be small, the patient may recover, but the condition is one which is recognised with difficulty during life.

In those cases in which closure of a coronary artery or one of its main branches is gradual, or is only partial, infarction docs not take place; local anæmia results, and consequent interference with the nutrition of the myocardium, inducing fatty or fibroid degeneration, which, thereforc, are usually found together in coronary diseasc.

\section{Chronic Myocardial Disease}

Of the various forms of chronic myocardial disease, the three most common are fatty infiltration or cardiac lipomatosis, fatty degeneration, and chronic interstitial myocarditis; the last of these has also been variously termed chronic myocarditis, fibrous myocarditis, sclerotic myocarditis, cardio-sclerosis, fibroid infiltration, and fibroid degeneration. Fatty degeneration and fibroid degeneration often co-exist, and to this condition the name of fibro-fatty heart is sometimes given. 


\section{Fatty InfiLtration}

Fatty infiltration consists in an excess of fat in those situations .in which it is naturally found, and also in its deposit between the fasciculi of the cardiac musculature, the chief distribution being along the arterial branches, and often causing atrophy of the muscle-fibres by compression. The affection is usually most marked over the right ventricle. The deposit of fat commences in the sub-epicardial fat, and may even extend to the sub-endocardial tissue. In some instances, the atrophied musclefibres become the seat of true fatty degeneration, due to interference with their nutrition. In fatty infiltration, more or less dilatation of the cardiac chambers is usually present, and is often accompanied by hypertrophy.

Etiology.-Heredity is certainly a factor, and the condition is more common in males, and during or after middle life, being rare in early life, and comparatively rare before middle age. It is usually associated with general obesity, a generous appetite, and lack of exercise and fresh air. It is more especially found in connection with over-indulgence in carbohydrates, fats, and alcohol, particularly malt liquors and sweet wines, and faulty metabolism is generally present. It is also apt to occur in individuals suffering from affections of the lungs which cause embarrassment of the pulmonary circulation, such as emphysema, cirrhosis, and chronic fibroid tuberculosis.

Symptomatology.-The patient is usually stout and flabby, and the subjective symptoms are those of an enfeebled circulation. Exertion is frequently accompanied by dyspnœa, a sense of oppression in the chest, and slight cyanosis, with a tendency to perspire easily ; the association of cyanosis with dyspnoa on exertion is one of the most usual features of the affection. The patient is easily fatigued by physical or mental effort, and there is often giddiness or faintness. The digestive functions are as a 
rule little, if at all, impaired; on examination, however, there may be evidence of gastric dilatation and of hepatic enlargement. The functions of the nervous system are often affected ; there is frequently impairment of memory, and not infrequently the patient is soporose, although, on the other hand, he may complain of insomnia. CheyneStokes' respiration is occasionally to be noted. The urine may be large in quantity, pale in colour, and of low specific gravity. The characteristics of the pulse vary considerably; as a rule the rate is increased, it is weak, the vessel is empty, there is hypo-tension, and the vessel wall is not thickened. On the other hand, the vessel may be full, the blood-pressure super-normal, and the wall thickened. The pulse is usually regular, but may exhibit one of the forms of irregularity. The cardiac impulse is usually feeble, unless hypertrophy is also present, when its area and forcc may be increased, and pulsation in the neck may be noted. The area of cardiac impairment is generally increased, as the result of dilatation or hypertrophy. On auscultation, the heart sounds, especially the first, are found to be weak, low in tone, and muffled; indeed, in the advanced stage the first sound may be almost inaudible; this may be due not only to an enfeebled left ventricle, but also to the unusual thickness of the thoracic parietes in these cases. Murmurs are usually not present, but a soft mitral or tricuspid bruit may be audible, owing to cardiac dilatation.

Later on, there may be evidence of œdema of the lungs and of the dependent parts, while the urine may be scanty, high coloured, and albuminous.

Diagnosis.-Fatty infiltration may be diagnosed from the indications of an enfeebled circulation, together with evidence of weakness of the cardiac impulse and sounds, occurring in a person who is the victim of obesity.

Prognosis.-In considering the prognosis, the family history, the age of the patient, his personal habits, and his willingness to carry out the necessary treatment have 
all to be considered. If appropriate therapeutic measures be carried out, the prognosis is usually favourable; otherwise the condition is apt to be progressive. Sudden death, due cither to syncope or rupture of the heart, while of less common occurrence than in fatty degeneration, has been known to occur, and a fatal issue may also ensue in the course of an acute illness or as the result of a surgical operation. As has been already noted, if fatty infiltration persist for some time, true degeneration is apt to arise.

Treatment.-This consists mainly in dieting, systematic elimination, and abundance of fresh air, while carefully regulated exercise and other measures which have for their object the reduction of bodily fat should also be employed. The diet should be strietly moderatc in amount, and sugars and starehes, especially bread and root vegetables, should be proportionally diminished; the diet, therefore, should be mainly nitrogenous. The food should be eaten slowly and thoroughly masticated. The amount of fluid with meals should be restricted, and taken at the end of the meal, while, on the other hand, a considerable quantity of fluid between meals is advisable. I generally recommend a pint of warm water slowly sipped an hour before breakfast, a tumblerful on going to bed and perhaps also an hour before the midday meal. It is better to avoid alcohol altogether, but, if this be not possible, brandy or whisky, well diluted, Moselle, or claret is much preferable to malt liquors, Burgundy, port, or champagne.

Regulated exercise in the open-air, such as walking, riding, golfing, and cycling, is valuable. Exercise indoors, as, for example, the use of dumb-bells, is of benefit as an adjunct. Graduated exercises are of special value in cases of slight cardiac failure due to fatty infiltration. In my opinion, the simple forms of movements are, certainly in the majority of cases, better than the resistance exercises as practised at Nauheim, and I am inclined to favour 
Swedish exercises. These movements may be indulged in even when the patient is confined to bed; but, other things being equal, it is better that they should be practised in the opcn-air. With regard to any form of physical exertion, care should be taken that it is at first limited in amount, and only gradually increased, while it should in evcry case stop short of producing any indications of cardiac distress. Systematic elimination, by means of purgatives, Turkish baths, or a visit to some Spa, is sometimes useful.

\section{Fatty Degeneration}

In fatty degeneration the muscle-fibres are more or less replaced by fat, and the affection may be general or local in its distribution. When the eausal factor is acute, as, for example, in pernicious anæmia, the condition is usually general; but when chronic, as in coronary discase, it is usually local; in both cases, but especially in the latter, the ventricles are affected to a greater degree than the auricles, and the left ventricle more than the right. The heart may or may not be enlarged; indeed, in pure degeneration, it may be even smaller than normal; fatty degeneration may accompany or succeed dilatation or hypertrophy, while, on the other hand, dilatation may follow degeneration.

Etiology.-Heredity is certainly a factor, and the condition is more common in males. While it may occur at any period of life, it is far commoner during middle life and in the elderly. Coronary disease is perhaps the most common cause. The condition is found also in altered states of the blood, especially pernicious anæmia and leucocythemia, while it has been noted in scurvy, purpura, hrmophilia, and diabetes. The chemical poisons, especially alcohol, phosphorus, arsenic, antimony, and lead, and the acute infectious fevers, especially typhus, enteric fever, diphtheria, erysipelas, smallpox, septicæmia, 
pneumonia, and influenza, as well as cachectic states the result of cancer, tuberculosis, long-continued suppuration, and other wasting diseases, are also responsible factors in the production of the disease. Among other causes are the retention of waste products in chronic gout and nephritis; chronic venous congestion due to mitral disease and those affections of the lungs which cause embarrassment of the pulmonary circulation; longstanding valvular disease with dilatation; and as a sequel to fatty infiltration. It may be noted that phosphorus, even in small doses, may cause fatty degeneration within a few days.

Symptomatology. - The clinical picture is in most cases indefinite; in rare instances, indeed, the disease is latent during its whole course, until a fatal termination occurs, due to syncope or angina pectoris, or from some other cause.

The general appearance of the patient varies, which may be due to the causal factor, e.g. pernicious anæmia. The countenance not infrequently is pale and waxy, but, on the other hand, it may be ruddy, or even cyanotic; cyanosis, however, is rarely seen unless there is much cardiac dilatation. Arcus senilis, coldness of the extremities, and a subnormal temperature may be present. The most common subjective symptoms are dyspnœa, together with some degree of cyanosis of the face, and perhaps giddiness and acceleration of the pulse-rate on exertion-dyspnœa on exertion being an early and persistent symptom. In some cases there is a sense of uneasiness, and even actual pain, in the precordium on exertion, but it should be noted cardiac pain is not usually a prominent feature of the disease except in those cases in which angina pectoris accompanies it; the latter, however, is by no means uncommon. Complaint is sometimes made of debility and general lassitude. The digestive functions are usually impaired, as evidenced by anorexia, drowsiness after meals, atonic dyspepsia, with a tendency 
to flatulent distension of the stomach, torpidity of the functions of the liver and bowel, and occasionally gastrointestinal catarrh.

Cerebral symptoms, due to a diminished supply of blood to the brain, are often present and of great diagnostic importance. Slight and transient feelings of giddiness and faintness are of common occurrence. There may be diminution of intellectual power, early fatigue on mental effort, impairment of memory, a persistent tendency to drowsiness, and a lack of the sense of refreshment after sleep, or, on the other hand, insomnia, mental irritability, or even symptoms of marked mental derangement.

Syncopal attacks or transient epileptiform attacks closely resembling petit mal may occur. The former are of frequent occurrence, and may result in complete unconsciousness, which may be protracted, thus simulating a transient apoplectiform seizure; there is, however, no succeeding paralysis. Marked bradycardia is associated with some of these syncopal or epileptiform attacks, in other words the Adams-Stokes' syndrome. There may be a persistent tendency to sighing or yawning, while occasionally Cheync-Stokes' respiration is present; this, however, is usually not met with until an advanced stage of the disease, when it is not infrequent, and occurs for the most part during sleep.

The urine is usually of low specific gravity, and not infrequently contains a trace of albumin and tube casts. There is sometimes a certain amount of œdema about the ankles, but general dropsy is rare, unless there is much cardiac dilatation.

The pulse is almost always altered, the rate varying considerably in different cases; it may be increased, especially in the more acute forms of the disease. On the other hand, there may be bradycardia, even of marked degree, in the more chronic forms, especially in the senile variety. The pulse is usually small, ill-sustained, and the blood-pressure is almost always subnormal; but, on 
the other hand, its volume may be full. It may be perfectly regular, or irregular. The vessel wall may be normal in the acute forms, or thickened, as occurs in a large proportion of cases, of the senile type.

The apex-beat is frequently not visible, and may not be palpable; if present at all, it is usually extremely weak, giving the impression to the hand of a short feeble tap, and there is usually an absence of pulsation in the epigastrium. Stokes pointed out that the impulse may only be felt in the neighbourhood of the left border of the lowcr end of the sternum. The apex-beat is as a rule not much displaced outwards. The area of cardiac impairment is generally enlarged, but usually not to any great extent, unless much dilatation has supervened or hypertrophy exists.

On auscultation, the first sound is usually found to be feeble, muffled, and short, and approximates in character that of the second sound; indeed, in the advanced stage of the disease, it may be almost, if not entirely, inaudible at the apex, although faintly heard in the neighbourhood of the left border of the lower end of the sternum. In the case of rapidly-acting hearts, the fœtal type of cardiac action may be approximated. A mitral and tricuspid systolic murmur may be present, especially when the cause is anæmia or one of the infectious fevers.

Diagnosis.-The diagnosis of fatty degeneration of the heart is often extremely difficult, as the clinical picture is in most cases indefinite, and, indeed, in rare instances the disease is latent during its whole course until the occurrence of a fatal termination. We may often suspect the existence of fatty degeneration without being able to confirm it; a positive diagnosis, indeed, is in the opinion of some not possible.

The presence of arcus senilis is in itself of no diagnostic importance, but is of some value when accompanied by other symptoms. Cheyne-Stokes' respiration is not 
pathognomic of fatty degeneration, since it is also to be met with in fibroid degeneration, aortic disease, uræmia, and cerebral conditions.

The following points are of importance in a consideration of the question of diagnosis: a causal factor, an absence of valvular disease, the presence of dyspnœa and cyanosis on exertion, the occurrence of angina pectoris, cerebral symptoms, especially syncopal and epileptiform attacks, a pulse of small volume and of low tension, of enfeebled cardiac impulse, general cnlargement of the area of cardiac impairment, and an alteration of the cardiac sounds as already noted.

In the opinion of some the differential diagnosis between fatty and fibroid degeneration is impossible, and it should also be remembered that they often occur in association. The existence of constant torpor, CheyneStokes' respiration, and apoplectiform attacks, and the occurrence of a fatal termination before marked cyanosis, general anasarca, hepatic enlargement, and other indications of a failing right heart occur, are more suggestive of fatty degeneration. Further, in the fibroid form of myocardial degeneration, the pulse is usually more frequent, stronger, and of normal or super-normal tension, while the vessel wall is thickened, the enlargement of the area of cardiac impairment is usually greater, and the aortic second sound accentuated. It should, however, be borne in mind that fatty degeneration may supervene upon the hypertrophy of valvular disease.

In the differential diagnosis from valvular disease with cardiac failure, the history may be helpful, as also the characteristic murmurs of the various forms of valvular disease. In mitral disease, for example, hypertrophy is more marked, and, if cardiac failure be present, the usual clinical picture is one of chronic venous stagnation-a condition which is comparatively rare in fatty degeneration-and the degree of cardiac dilatation is greater.

Prognosis.-Takingthe cases as a whole, the prognosis 
of fatty degeneration is grave, as it is one of the commonest diseases in which sudden death occurs. The duration of life, however, may vary from a few months to many years. In considering the probable duration of life, among the points which should be considered are the following: when the affection supervenes upon fatty infiltration the patient may live for many years. In the acute forms, as, for example, when due to infectious diseases or anæmia, the immediate prognosis is graveespecially if cardiac failure make its appearance at an early stage of the disease-but the ultimate prognosis is not infrequently favourable; the outlook in diphtheritic cases is, however, uncertain. In the chronic forms, as, for example, in coronary disease, the immediate prognosis is more favourable, but the ultimate prognosis is not so good; and yet even in these cases life may be prolonged for some years, if the patient be able to live a carefully regulated life.

A fatal termination may result from gradual or rapid cardiac failure, or from sudden death, the latter occurring perhaps in a majority of cases, and may be due to syncope, angina pectoris, or rupture of the heart. Rupture of the heart is perhaps not so common in fatty as in fibroid degeneration.

Treatment.-The treatment of fatty degeneration is the same as that of chronic interstitial myocarditis.

\section{Chronic Interstitial Myocarditis}

By chronic interstitial myocarditis is meant an increase of the interstitial fibrous tissue of the cardiac wall, as a consequence of which atrophy and degeneration of the muscle-fibres result. When the condition has continued for some time and the increase of fibrous tissue is extreme, the affected muscle-fibres may be entirely replaced by 
dense scar-like tissue. The affection may be general or local in its distribution, the latter being usually due to coronary disease. When the affection follows acute myocarditis, a more or less diffuse fibrosis, usually most marked beneath the pericardium and endocardium, is found. In both forms, the auricles are little, if at all, affected, and the left side of the heart is much more commonly attacked than the right, especially in the neighbourhood of the apex and the lower part of the interventricular septum. When the condition is the result of chronic venous congestion, however, it is more in evidence on the right side of the heart. When the coronary arteries are diseased, the aortic cusps are not infrequently the seat of degenerative processes, and there may also be widespread arterio-sclerosis and cirrhosis of the kidneys.

An increase of fibrous tissue leads to an increase in the thickness of the cardiac wall-the so-called false hypertrophy - and ultimately to dilatation of its chambers.

Etiology.-Heredity is certainly a factor, and the condition is more common in males in about the proportion of two to one, and during or after middlc life. It is to be found in occupations involving prolonged muscular or mental strain. Coronary disease constitutes the most important cause. The condition may follow acute myocarditis, and also be the result of chemical poisons, e.g. alcohol. Syphilis may induce fibroid degeneration, through gummatous deposits acting as foci of irritation, or by causing obliterative endarteritis of the coronary arteries. Conditions of defective metabolism, such as chronic gout, and increased peripheral resistance, as, for example, in arterio-sclerosis and granular kidney, also play a part in the etiology of the disease. Among other causes are chronic venous congestion, due to mitral disease and those affections of the lungs which cause embarrassment of the pulmonary circulation; old standing valvular disease ; the chronic adherent form of pericarditis ; acute infectious fevers; and perhaps altered states of the blood. 
Symptomatology. - The clinical picture of fibroid degeneration is a varied one; if the morbid condition be general or extensive, the disease may be difficult to distinguish from fatty degeneration, with which it often co-exists. In very rare instances, the disease is latent during its whole course and is only discovercd on post-mortem examination. The onset of the symptoms is gradual, and the clinical features are those of chronic heart failure. The most common symptoms are progressive dyspnœa on exertion, together with slight cyanosis of the face; there may also be giddiness, a sense of oppression in the precordium, and not infrequently pain on exertion, and slight odema about the ankles, especially at night. There may be a sensation of fulness and throbbing in the head, noises in the ears, and flashes of light before the eyes. The urine is usually diminished in quantity and of high specific gravity, and may contain albumin, which is frequently present in the later stages of the disease. The patient may suffer from attacks of cardiac asthma, especially at night, and angina pectoris is of common occurrence. There may be cerebral symptoms, due to a diminished supply of blood to the brain; of these the two most important are syncopal attacks and transient epileptiform attacks closely simulating petit mal. The former may result in complete unconsciousness, which may be so protracted as to resemble a transient apoplectiform seizure, but there is no succeeding paralysis. Cheyne-Stokes' respiration is sometimes found. The usual indications of failure of the right side of the heart may supervene. Ultimately the patient is apt to suffer from marked dyspnœa on the slightest exertion, orthopnœa, and the most distressing cardiac asthma, while the quantity of urine steadily diminishes, and anasarca increases.

The pulse may be of normal rate, increased in frequency, or bradycardia may be present, the last of these being relatively common. The character of the pulse varies considerably. It is usually fuller than normal, with height- 
ened pressure, although the latter is not pronounced, unless cirrhosis of the kidney or some such affection exists ; when cardiac failure is present, however, the pulse may present the opposite characteristics. The wall of the radial artery is usually thickened. When the affection is the result of chronic venous congestion or of chronic adhesive pericarditis, the pulse may be feeble, of subnormal pressure, with no evidence of thickening of the radial wall.

The apex-beat is displaced downwards and to the left, its area and force are increased, the area, however, being proportionately greater, and the force of the apex-beat proportionally less, than in pure hypertrophy. The area of cardiac impairment is increased in all directions. When the affection is due to mitral disease or emphysema, the impulse is most obvious near the ensiform cartilage, and the increased area of impairment to the right of the sternum.

On auscultation, the first sound is prolonged and muffled at the apex, with often a soft systolic murmur; the first sound is barely audible at the base, and may be entirely absent. The second sound in the aortic area is accentuated and may be reduplicated; when due to mitral disease, however, the second sound in the pulmonary area may be accentuated, but at the same time is less ringing in character than in the earlier stages of valvular disease. A tricuspid systolic bruit is sometimes heard.

Diagnosis.-An absolute diagnosis of chronic interstitial myocarditis is not infrequently a matter of extreme difficulty. The differential diagnosis between this condition and fatty degeneration has been already dealt with on. page 310. It is also necessary to distinguish the affection from the cardiac failure of valvular disease, as, for example, relative mitral incompetence occurring in fibroid. degeneration from mitral incompetence due to primary. organic disease of the valve, a problem of the utmost 
difficulty. In the former case, there is no history of valvular disease, the subjective.symptoms are frequently out of proportion to the physical signs, and angina pectoris and syncopal and epileptiform attacks are of more frequent occurrence.

Prognosis.-The prognosis of the affection closely rcsembles that of fatty degeneration. Here also the duration of life may vary from a few months to many years. A fatal termination may take place as the result of gradual or sudden cardiac failure. In estimating the prognosis, among other things which should be taken into consideration are the presence or absence of the pulsus alternans, angina pectoris, and syncopal attacks.

Treatment.-It is of great importance the patient should curtail his physical and mental activities so as not in any way to exceed the limited strength of the heart. His daily life should be considered with great care and in detail. Carefully regulated exercise in the fresh air is permissible, but violent and sudden efforts should be strictly forbidden. The amount and kind of exertion should be adapted to each individual according to the strength of the heart. Such exercise as walking against a wind and cycling uphill are contra-indicated; on the other hand, quiet walking on the level, or even slow walking up an incline, quiet cycling, croquet, and even riding or golf may sometimes be allowed in suitable cases. In all cases, exertion should stop short of producing breathlessness, palpitation, fatigue, a sense of tightness across the chest, or precordial pain or distress. If these precautions be not taken, there is real danger. Even when there are no indications of cardiac failure, periods of complete rest are often advisable. High altitudes are better avoided. Only warm bathing should be allowed. The clothing should be warm. Particular care should be taken with regard to diet; it should be most carefully regulated on the lines previously laid down. The starchy and fatty elements of the diet should be proportionately diminished. Gastric and intestinal dyspepsia, 
especially with flatulence, should be avoided, or treated when present. In advanced cases, gastric or intestinal dyspepsia is occasionally the exciting cause of a fatal seizure. What fluid is taken with meals should be taken at the end. It should not be forgotten that people who lead a comparatively inactive life require a smaller amount of food than those in full work. Overloading the stomach should be avoided. Rest before and after food, and in the afternoon, should be enjoined. The state of the bowels should receive most careful attention. In these cases, it is sometimes of advantage to diminish the body weight. If there be super-normal blood-pressure, it should be regulated by the methods described in Chapter IV. If anæmia bc the cause or an accompaniment of the condition, it should bc adequately treated.

When there are indications of cardiac failure, rest, proportionate to the degree of failure present, is imperative ; other therapeutic measures also, as previously described, should be adopted. The indications for one of the digitalis series of drugs have already been discussed.

\section{Hypertrophy of the Heart}

In cardiac hypertrophy there is an increase in the thickness of the walls of one or more of the cavities of the heart, due to an increase in the actual size, and possibly also in the number, of the muscle-fibres. The hypertrophy may include the musculi papillares, columnæ carneæ, and musculi pectinati. The ventricles are more affected than the auricles, for the walls of the latter contain more fibrous tissue.

It is customary to divide hypertrophy of the heart into three kinds, namely, (1) the concentric, in which with the increased thickness of the walls the cavities are diminished in size; (2) the simple, in which the cavities 
are normal in size; and (3) the excentric type, in which the cavities are increased in size; in other words, there is hypertrophy with dilatation.

In the opinion of some, the concentric variety only exists as a post-mortem change. The excentric variety may give rise to enormous enlargement of the organ, in which case the heart is often spoken of as a "beefy heart" or a "cor bovinum." This is especially apt to occur in aortic valvular disease.

Hypertrophy may be general or local. In the former case, all the chambers of the heart are fairly equally involved. In the latter, which is much the more common, while all the chambers are not affected, there is as a rule hypertrophy of more than one, though in varying degree. The following is the comparative order of frequency : left ventricle, right ventricle, left auricle, right auricle. When the left ventricle is affected, the apex of the heart is accentuated, and the whole organ is elongated and rather conical in form. When the right ventricle, on the other hand, is concerned, the apex is less pronounced, and the whole organ is broader and somewhat quadrate in form.

Etiology.-Hypertrophy is apt to occur when the heart has been subjected to an excessive amount of work. It is more common, therefore, in men, on account of their greater exposure to long-continued excess of muscular effort and to mental strain. Among other causes are super-normal blood-pressure and arterio-sclerosis independent of kidney disease, nephritis (especially the chronic interstitial form), valvular disease, congenital atresia of, or pressure upon, the aorta, adherent pericardium, habitual excesses in food or drink, over-indulgence in tobacco, lithæmia, certain affections of the nervous system causing persistent increased cardiac rate, hyper-thyroid secretion, chronic diseases of the lungs (such as emphysema, chronic interstitial pneumonia, and fibroid tuberculosis), arterio-sclerosis of the pulmonary artery, and pregnancy. 
Cirrhosis of the kidneys, aortic disease, and drinking large quantities of beer may give rise to enormous hypertrophy.

The hypertrophy is likely to be general in the case of long-continued over-indulgence in food, alcohol, or tobacco, excessive muscular or mental strain, affeetions of the nervous system, and hyper-thyroid secretion; in arterio-sclerosis, nephritis, congenital atresia of, or pressure upon, the aorta, and in pregnancy, on the other hand, the left ventricle is more especially affected; whereas in chronic affections of the lungs and arterio-sclerosis of the pulmonary artery the right ventricle is principally involved.

For the development of full hypertrophy, an adequate blood-supply is necessary, and in this connection the state of the general nutrition, as well as the anatomical and physiological condition of the coronary arteries, is of eonsiderable importance.

Symptomatology. - The symptoms of the morbid condition which is the cause of the hypertrophy, whether of the left or right side of the heart, may be present. With regard to the hypertrophy itself, therc may either be a complete absence of subjective symptoms, or, on aecount of inereased tension in the cerebral circulation, a sensation of fulness and throbbing in the head, headaehe, hebetude, flushings, noises in the ears, and flashes of light before the eyes. Somctimes patients complain of discomfort or even of actual pain in the precordium, especially when lying on the left side, and this sensation is aggravated by exertion. Actual pain, however, is not common, unless perhaps in the case of neurasthenics and arising from tobacco poisoning. Another symptom of which the patient may complain is palpitation. When cardiac failure supervenes, complaint may be made of dyspnœa on exertion and other symptoms.

Hypertrophy of the left ventricle is characterised by a full strong pulse, of prolonged duration, and by a blood- 
pressure above the normal. The pulse-rate, on the other hand, is apt to be rather lower than normal. There may be some bulging and widening of the intercostal spaces in the precordial area to the left of the sternum, especially when the hypertrophy occurs during the growing period of life. The apex-beat is displaced downwards and outwards ; it may be as low as the sixth, seventh, or even eighth intercostal space. Its extent and force are increased, and its character "heaving " or " thrusting," the beat being slower and longer than normal. This heaving or thrusting of the apex-beat is the most characteristic feature of the condition. The area of impairment is increased, both from above downwards and transversely; it may even extend downwards to the eighth interspace, and transversely to the anterior axillary line. On auscultation, the first sound in the mitral area is frequently long, sustained, low in pitch, and muffled; the short pausc may be lengthened, and the long pause shortened; while the second sound in the aortic area is frequently accentuated. Sometimes the first sound is reduplicated, and then is usually best heard rather to the inner side of the apex-beat. The aortic second sound also occasionally is reduplicated.

When there is considerable dilatation with the hypertrophy, occurring either in the first instance or subsequently in association with cardiac failure, the pulse increases in frequency, the blood-pressure becomes lower, the apex-beat becomes more diffuse, weaker, abrupt and rapid, and is of a tapping character. The transverse area of impairment becomes enlarged, and the first sound may be accentuated, sharp, clear, high in pitch, and considerably shortened. Other indications of cardiac failure, such as oedema, may also supervene.

In hypertrophy of the right ventricle, there may be, more especially in children, a certain amount of bulging in the region of the ensiform cartilage, while not infrequently there is pulsation in the epigastrium, diastolic 
in time. When the condition is marked, there may be a heaving impulse in the epigastrium, and over the lower sternum and costal cartilages, and in such cases is more marked than that of the left ventricle. The apex-beat is displaced chiefly to the left and only slightly downwards, and may be normal, diffuse and indefinite, or altogether invisible. When the right ventricle is much enlarged, that chamber may displace the left ventricle backwards, so that the clinical apex-beat is formed entirely by the right, instead of the left, ventriele; in these cases systolic rccession over the lower part of the precordium is sometimes observed. Systolic recession in the $3 \mathrm{rd}$, 4th, and 5 th intercostal spaces betwcen the margin of the sternum and the parasternal line on the left side, or in the 4th and 5th intercostal spaees on the right side, may be present. The area of eardiac impairment is usually increased, especially to the right; this may reach as much as two inches or even morc from the mid-stcrnal line. The left border may also be displaced outwards, but rarely extends beyond the nipple line. The first sound in the tricuspid area is not infrequently louder than normal, and the second sound in the pulmonary area is usually accentuated and may be reduplicated. Later, the symptoms of tricuspid incompetence may supervene.

When hypertrophy of the aurieles occurs, it is accompanied by dilatation. In hypertrophy of the left auriele, the area of impairment may be higher than normal. When the right auriele is affected, there may be an extension outwards of the area of impairment in the third and fourth right intercostal spaces.

Diagnosis. - A diagnosis of hypertrophy of any chamber or chambers of the heart should not be made merely from the presence of pulsation or percussion impairment in an abnormal area, for these may be due to causes other than hypertrophy, as, for example, morbid conditions of the lungs or pleuræ. The most reliable 
sign of hypertrophy of the heart is increased force of action of the part involved.

With regard to the diagnosis of hypertrophy of the left ventricle, we should note whether there is a thrusting or heaving apex-beat. A full, strong, and prolonged pulse, super-normal blood-pressure, displacement downwards and outwards, and increased area of the apex-beat, with accentuation of the aortic second sound, afford corroborative evidence. Occasionally emphysema of the left lung masks these signs, in which case the diagnosis is not easy. It should be remembered that increase of force of the apexbeat is sometimes met with in nervous subjects, especially in the young whose chest-walls are thin; but in these cases there is no displacement of the apex-beat, the impulse is sharp and short, the area of impairment is not increased, and the sounds of the heart are not altered in the characteristic manner described. I have seen an incorrect diagnosis of hypertrophy of the left ventricle made in cases of retraction of the left lung; in these cases there is an increased area over which the healthy heart comes into direct contact with the chest-wall. 'The apex-beat, however, is displaced upwards, is not heaving or thrusting in character, and there are signs of a pulmonary lesion. Hypertrophy of the right ventricle can be diagnosed when the objective signs already mentioned are present. The diagnosis of hypertrophy of the left auricle is exceedingly difficult; hypertrophy of the right auricle is more easily detected.

Prognosis.-This depends upon, among other things, the cause, the degree of hypertrophy, the degree of dilatation present, the condition of the systemic and coronary arteries, and of the myocardium. With regard to the first, the prognosis depends very largely upon whether this is serious and irremediable, as when renal cirrhosis is present, in which case dilatation is apt to supervene.; or whether it is merely transitory, as, for example, when the result of a period of excessive muscular strain, in 
which case it seems clearly established that hypertrophy may disappear when the cause no longer exists. Longcontinued hypertrophy is apt to be followed by dilatation, which adds to the gravity of the condition as a whole, although in cases of super-normal blood-pressure and arterio-sclerosis it has the advantage of lessening the danger of rupture of the walls of the systemic vessels.

With regard to the condition of the coronary arteries, as has already been pointed out, an adequate blood-supply to the cardiae musculature is necessary for the full development of hypertrophy, and in this connection the anatomical and physiological condition of the coronary arteries, as well as the state of the general nutrition, is of considerable importance. It need only be pointed out that anginal attacks in all probability point to the existence of coronary disease, in which case the supply of blood to the hypertrophied heart will be insufficient, with a resultant tendency to degeneration. Moreover, if the degree of hypertrophy be excessive, the coronary arteries, although healthy, may not be able to supply an adequate amount of nourishment to the cardiac musculature. With regard to the integrity of the myocardium, the importance of testing the cardiac response has already been fully considered.

Treatment.-The hypertrophy itself does not need treatment. It is always advisable, however, to consider the cause, and, in some cases, as for example when chronic Bright's disease, super-normal blood-pressure, or arterio-sclerosis is present, it should be suitably treated. In order to provide a good supply of food to the heartmuscle, we should see that the patient obtains plenty of fresh air, and an adequate supply of nutritious and easily digestible food, and any dyspepsia or anæmia present should receive attention. Undue physical or mental strain should be avoided. If cardiac failure supervene, it should be treated as described elsewhere. 


\section{Dilatation of the Heart}

In cardiac dilatation there is an increase in the capacity of one or more of the cavities of the heart.

Looking at the condition from the pathological standpoint, the muscular walls of the chambers may be of normal thickness, which is not common; more usually they are either thinner or thicker than normal. The last is by far the most common; i.e. in the great majority of cases there is some hypertrophy with the dilatation; this is called excentric hypertrophy, and has already bcen described. It should be remembered that when dilatation has existed for a long time, the walls become thin, although, perhaps, in the first instance thickened.

Looking at the subject from the clinical standpoint, however, dilatation means an increase in the capacity of one or more of the cavities of the heart as the result of impairment of the function of tonicity; this form of dilatation is associated with cardiac failure. From this point of view, dilatation with hypertrophy is only included when the dilatation is in excess of the hypertrophy. Hypertrophy is apt to be followed by dilatation, and the latter, therefore, may be secondary to the former.

Dilatation rarely affects one chamber alone, all of them being usually affected in some degree, although unequally so. The heart becomes larger, and when the condition is more or less general the organ approaches the globular form, particularly when the right side is affected. With the increase in the capacity of the ventricles, the dimensions of the auriculo-ventricular orifices also increase, and this results in what is known as relative incompetence. In dilatation of the auricles there is usually enlargement of the venous channels.

In dilatation a larger quantity of blood exists in the affected chamber than normal, while the propulsive power is diminished; the chamber, therefore, cannot empty itself. As the dilatation progresses the contraction of 
the chamber becomes less effective, and the amount of residual blood progressively increases.

Etiology.-Cardiac dilatation is more common in men, on account of their greater exposure to long-continued excess of muscular effort and to mental strain; this is especially so in certain occupations. General cardiac dilatation may occur in any of the following conditions : severe or prolonged pyrexia, more especially in the case of pneumonia, scarlet fever, typhoid or typhus fever; toxæmias, as influenza and diphtheria, alcoholism, tobacco poisoning, and overdosage by the salicylates; various forms of anæmia; cachectic conditions ; myocardial degeneration; rheumatic carditis; and affections of the coronary arteries causing defective blood supply. In the case of toxæmia of influenza and diphtheria, as well as in the acute carditis of rheumatism, dilatation may be acute.

Dilatation may occur secondarily to hypertrophy, especially when the nutrition is defective. One of the most common causes of secondary dilatation is some form of mechanical obstruction, slowly produced, either in the blood-vessels or in the valves. Some of the causes of hypertrophy may produce acute dilatation by acting quickly, before there is time for hypertrophy to occur; this is especially the case when the muscular fibres are already impaired. Thus, whereas prolonged and continued excess of muscular effort tends to produce hypertrophy, a sudden physical strain is apt to produce acute dilatation, especially if the individual be not in training (see primary cardiac overstrain) ; further, in cases of completely established hypertrophy, dilatation may be produced with apparent suddenness by a comparatively trivial ailment, such as bronchitis, influenza, or any febrile attack.

It should be particularly noted that the most common clinical condition in which acute cardiac dilatation occurs is the onset of a new heart rhythm, such as auricular fibrillation. 
In addition to arterio-sclerosis of the pulmonary artery, and the chronic pulmonary affections enumerated in the etiology of hypertrophy, acute dilatation of the right side of the heart may occur in pneumonia, œdema of the lungs, and in pleurisy with effusion.

Symptomatology.-The symptoms of the cause are sometimes to be noted, and in addition there is usually languor, and weakness and fatigue on physical exertion, whilc strain is liable to induce a sensation of faintness, or even actual syncope. Shortness of breath and palpitation on exertion are of common occurrence, while there may be an uncomfortable feeling, amounting to actual pain, in the precordium. Some degree of cyanosis of the facc may also be noted. The digestive functions are usually impaired, as shown by anorexia, drowsiness after meals, atonic dyspepsia with a tendency to flatulent distension of the stomach, torpidity of the functions of the liver and bowel, and occasionally gastro-intestinal catarrh. Cerebral symptoms, such as giddiness, lack of power of concentration, early fatigue on mental effort, a tendency to drowsiness, disagreeable dreams, nervousness, and even symptoms of mental derangement may be noticeable. Jaundice is not infrequently present, and also vomiting, hepatic enlargement, œdema of the feet, and sometimes splenic enlargement. The urine may be scanty, and contain albumin, and even blood and tube casts. There is a tendency to chronic congestion and œdema of the lungs, particularly of the bases, and ascites and pleural effusion are occasionally present. As the case progresses, dyspnœa may occur on the slightest exertion, and orthopnœa, cardiac asthma, anasarca, and a still further diminution in the amount of urine.

The pulse is usually found to be more frequent, of smaller amplitude, and weaker, while the blood-pressure is lower than normal. Distension and pulsation of the jugular veins may take place.

Sometimes, particularly in children, in dilatation of the 
right ventricle there is a certain amount of bulging of the precordium in the region of the ensiform cartilage. The apex-beat is displaced chiefly outwards. It is diffuse, and often difficult or even impossible to locate, but if it can be determined is found to be weaker than normal and tapping in character. In dilatation of the left ventricle a diffuse wavy impulse may be present in the anterior axillary linc. In enlargement of the right ventricle, the clinical apex-beat may be formed by the r.ght, instead of the left, ventricle, in which case there may be a more diffuse systolic recession over the lower part of the precordium, in place of the normal apex-beat; also systolic recession in the $3 \mathrm{rd}, 4 \mathrm{th}$, and 5 th intercostal spaces between the margin of the sternum and the parasternal line on the left side, or in the 4 th and 5 th intercostal spaces on the right side, may be present. The area of cardiac impairment is increased transversely, and is more marked to the left when the left ventricle is chiefly involved, and to the right in the ease of the right ventricle. In dilatation of the left auricle the area of impairment may be higher than normal, and in dilatation of the right auricle it may extend outwards in the 3rd and 4th right intercostal spaces. On auscultation, both cardiac sounds are found to be weaker; and, further, the first sound is considerably shortened, sharp, high, and clear in pitch, resembling the second sound in character. The long pause is often diminished, on account of a:celeration of the rate of the heart, and sometimes the rhythm resembles that of a foetal heart or the ticking of a watch-in which case each sound and each pause arc of about the same duration. Sometimes a triple rhythm is present, three sounds being heard. A mitral, and in a still larger proportion of cases a tricuspid systolic bruit, may be present - on account of relative incompetency of these valves.

The physical signs of dilatation enumerated above may be modified by those of the cause of the condition, or of accompanying hypertrophy. In the case of valvular 
disease, the original murmurs may become weaker or even inaudible when dilatation occurs.

The symptoms of cardiac dilatation may come on rapidly in the acute forms, or more gradually in the more chronic cases. In many of the cases associated with the onset of a new cardiac rhythm the dilatation takes place so rapidly that in the space of even a few hours there are urgent dyspnœa, extreme hyperæsthesia of the chestwall, evidence of œdema of the lungs, and enlargement and pulsation of the liver. When dilatation is due to a sudden physical strain, faintness passing into actual syncope, together with vomiting, and even fatal syncope, may occur before the signs of venous stasis have had time to develop.

Diagnosis. - A diagnosis of cardiac dilatation can readily be made when the physical signs described-the altered character of the pulse, cardiac sounds and pauses, the displacement and characteristic alteration of the apexbeat, along with evidence of enlargement of the heartare present.

The diagnosis of dilatation from hypertrophy is easy on a comparison of the physical signs of the two conditions. When hypertrophy and dilatation co-exist in the same individual, there is a blending of the signs of both; it is, however, often difficult to estimate the relative degree of each; but other indications of dilatation, such as cyanosis, distension and pulsation of the jugular veins, evidence of pulmonary engorgement, enlargement of the liver, and dropsy may present themsclves. It is sometimes difficult to diagnose great dilatation, especially of the right ventricle, from pericardial effusion. The points relating to the diffcrential diagnosis will be fully discussed in Chapter XXII. Cardiac dilatation must also be distinguished from those cases of mitral stenosis in which there is an absence of a presystolic murmur at the time of examination. It is, further, sometimes necessary to exclude a right-sided pleural effusion displacing the heart 
to the left, in which case signs of fluid in the right pleural sac will be found.

When dilatation of the heart occurs very rapidly as the result of the inception of a new cardiac rhythm, the case may be mistaken for one of broncho-pneumonia or capillary bronchitis.

Prognosis.-The prognosis of cardiac dilatation depends, among other things, upon the cause, the degree of dilatation and whether this is out of proportion to the cause, the degree of any co-existing hypertrophy, the severity of the symptoms, the rate and force of the pulse, the vigour of the apex-beat or of the impulse of the right ventricle, and the size of the heart. Taking cases of cardiac dilatation as a whole, the prognosis is unfavourable.

In acute dilatation from temporary causes, such as sudden physical strain or acute disease, if immediate danger can be tided over, there is a prospect of complete recovery, provided an adcquate pcriod of rest is available. The same applies to those cases due to the inception of a new cardiac rhythm, if the rhythm return to the normal. In chronic dilatation from a permanent cause, e.g. chronic valvular disease, complete recovery is improbable; it may take place, however, in some instances under suitable treatment. The prognosis is very unfavourable when dilatation supervenes upon marked arterio-sclerosis or myocardial degeneration, and this applies also to cases of Bright's disease.

Treatment.-The first thing that requires consideration is the recognition of the cause of the condition, whatever its nature, and suitable therapeutic measures should be employed in this connection. Added to this is the treatment of the affection itself, which is that of cardiac failure, and consists of an adequate period of rest, dieting, perhaps the administration of cardiac tonics or stimulants, and symptomatic treatment. Rest is imperative, and in acute cases must be absolute. Graduated exercises may be of 
service in a small group of cases, as, for example, in fatty infiltration. With regard to the administration of cardiac tonics, such as digitalis, the reader is referred to the appropriate section for their indications, dosage, and method of administration. When dilatation is due to bacterial poisoning, digitalis appears to be ineffective. In acute cases, strychnine, alcohol, ether, and ammonia, by the mouth or subcutaneously, may be given. When venous engorgement and other indications of distension of the right chambers of the heart are present, purgatives, diuretics, and blood-letting by means of leeches or venesection, are indicated. The spccial indications for the practice of venesection in these cases, as well as the treatment of gastro-intestinal and other symptoms, have already been noted. 


\title{
CHAPTER XXII
}

\author{
AFFECTIONS OF THE PERICARDIUM
}

\section{Pericarditis}

Varieties.-The varieties of pericarditis which may be met with are the fibrinous or dry, sero-fibrinous, purulent, hæmorrhagic, and chronic adhesive, i.e. adherent pericardium. Some writers describe a chronic form of pericarditis; this, however, is practically synonymous with chronic adhesive pericarditis.

Pericarditis may be general or local ; if the latter, the base of the heart, close to the origin of the great vessels, is as a rule principally affected.

The fibrinous form of pericarditis consists in a transudation of lymph, which covers the free surface of the pericardium, coagulates and forms a fibrinous exudate, most marked at the base of the heart close to the great vessels. In these cases there is little or no effusion of serum into the pericardial sac. In the sero-fibrinous form, on the other hand, there is a considerable amount of effusion, in some cases a very large quantity. The rate at which fluid is poured out varies from a few days to several weeks. The fluid may be clear and transparent, with only a few flakes of exudate, or - as is more often the case-slightly turbid and opaque, containing uncoagulated fibrinogen and also a good deal of fibrin. Purulent pericarditis is due to the presence of a large number of leucocytes in the exudate, which is quite opaque, and has a greenish colour; the pus is as a rule sweet, but it 
may have an offensive odour, due to decomposition. In hæmorrhagic pericarditis, the fluid is bloody.

In the dry variety of pericarditis, as well as in the sero-fibrinous form, complete resolution of the fibrin and, in the latter case, absorption of the effusion, may take place, so that no trace of the inflammation is left. When, however, resolution of the fibrin and absorption of the fluid occur slowly, which is the rule in the sero-fibrinous form of the disease, organisation takes place. This may give rise to white patches of thickening (" milk spots") upon the pericardium, or may result in its two layers becoming adherent by permanent fibrous adhesions; in the latter case, chronic adhesive pericarditis or adherent pericardium is produced. In purulent pericarditis the pus rarely perforates the pericardium, and has been known to become absorbed, leaving bchind a pultaceous mass.

It may be noted that some degree of myocarditis is always present with pericarditıs.

\section{Acute Pericarditis}

Etiology.-It is questionable if pericarditis be ever primary or idiopathic. The affection is more common in children, especially those of the second decade, and in males. By far the commonest cause, especially in young people, is rheumatism, under which chorea is to be included, the pericardial inflammation usually appearing during the second half of the first week or the first part of the second week of the rheumatic attack. The relation between rheumatism and pericarditis has been already discussed in Chapter XVIII. The terminal stages of Bright's disease, especially of the two chronic forms, and in particular the chronic interstitial variety, and pneumonia, more especially affecting the left side, are frequent causes of pericarditis ; in pneumonia, infection may take place by means of the blood-stream or by extension. Other causes of pericarditis are scarlet fever, measles of 
severe type, small-pox, enteric fever, influenza, diphtheria, tuberculosis, gonorrhœa, and other acute infectious conditions ; constitutional diseases, such as gout and diabetes ; disordered blood states, such as leucocythæmia, lymphadenoma, purpura, and scurvy ; new growths; affections of the neighbouring parts, as, for example, the pleuræ, lungs, mediastinum, ribs, vertebræ, and peritoneum; injuries to the chest wall; and in conjunction with myocarditis.

When pericarditis is due to Bright's disease, there is a tendency to the purulent, hæmorrhagic, or chronicadhesive variety of the disease. The pericarditis which occurs in connection with malignant disease is usually of a chronic type, and is generally accompanied by the effusion of fluid, which is usually hæmorrhagic, but may be purulent or serous. Purulent pericarditis is usually due to pyæmia, septicæmia, as in the exanthematous fevers, or suppuration in the neighbourhood of the pericardium, as, for example, empyema, or the rupture of a pulmonary vomica. It may also occur in Bright's disease, or in tuberculous deposits in the pericardium. Among the micro-organisms which may be found are staphylococci, streptococci, pneumococci, and gonococci. Hamorrhagic pericarditis is more frequently met with in the aged, in Bright's disease, in acute infectious conditions, such as smallpox, tuberculosis, scurvy and purpura, malignant disease, either by direct extension or secondary, wounds of the heart, and in leakage from an aneurism.

Symptomatology.-The symptoms of the primary affection are generally to be noted. The severity of the symptoms of the disease itself exhibits great variation, and there may be a complete absence of subjective symptoms throughout the whole course; on auscultation, however, pericardial friction is usually found.

If pyrexia be already present, there is usually a further slight rise of temperature, although this may not be noted, and, in the opinion of some, there may even be a slight fall. This applies equally where there has been no 
pre-existing pyrexia. When effusion has occurred, there is, as a rule, no increase of temperature, and, indeed, if the pyrexia have been markcd, there is a tendency for it to become lower. Occasionally the onset of the disease is marked by a rigor.

The patient is usually pale, and sometimes cyanotic; when effusion has occurred, these features become more pronounced, and he usually wears an anxious expression. Precordial uneasiness, or even pain, is frequently present, and is the most conspicuous symptom; the pain may be located in the epigastrium, the left scapular or interscapular region, instead of in the precordium, and varies greatly in severity and character. It may be constant and dull, or recurring, sharp, and stabbing: anginal attacks are rarely to be noted. When pain is present, there is usually accompanying hyperæsthesia. Sometimes palpitation and breathlessness are complained of, and there may be faintness; the respiratory rate may be increased, and there may be even cardiac asthma or Cheyne-Stokes' respiration. Interference with the respiratory functions is more pronounced when effusion has occurred, and may be greatly aggravated, unless the effusion of fluid is gradual. Not infrequently a dry cough is present. Dysphagia is of uncommon occurrence, being more marked in cases of effusion and when the patient is in the recumbent posture. When the recurrent laryngeal nerve is implicated, the voice becomes affected, while if the phrenic nerve be involved the patient may suffer from hiccough.

There may be headache, restlessness, insomnia, convulsions, mild nocturnal delirium, or, less frequently, noisy delirium, stupor, and even coma. Hepatic enlargement is of comparatively common occurrence. A certain amount of œdema may become evident; it is usually general and is more marked in the dependent parts. Not infrequently pulmonary œdema occurs. The urine may become scanty and albuminous. 
The pulse is more frequent in rate and smaller in volume than normal. After a shorter or longer period, the blood-pressure becomes lower. At first, the apex-beat may be excited and its force considerably incrcased; later it becomes diminished in force, sometimes tapping in character and displaced outwards. Friction fremitus can sometimes be noted in fibrinous pericarditis. Its area of maximum intensity does not absolutely correspond with that of a thrill due to valvular disease. As a rule friction fremitus accompanies ventricular systole and diastole, having a to-and-fro character, but sometimes it is only systolic; in any case it is not exactly synchronous with either systole or diastole. It gives the impression to the hand of being superficial. After a shorter or longer period, the area of deep cardiac impairment may become enlarged to the left, upwards, and to the right. Auscultation reveals the most characteristic and reliable physical sign, namely, that of pericardial friction. It may accompany every phase of the cardiac cycle. As a rule, it accompanics ventricular systole and diastole, is of a to-and-fro character, with usually a short pause between the two portions. Sometimes it is only ventriculosystolic, while at other times, when the inflammation involves the auricle, it accompanies auricular systole, ventricular systole, and ventricular diastolc, giving rise to a triple rhythm. In any case, the different portions of the friction sounds do not absolutely coincide either in rhythm or duration with any period of the cardiac cycle, being rather later than any of them and usually heard during portions of more than one period. The sound is usually first audible over the base of the heart, but later on may become audible over the whole precordium. The area of maximum intensity does not absolutely correspond with that of any of the valvular areas. A characteristic feature is that as a rule the sound is either not at all or only slightly conducted, and there is no definite selective direction of propaga- 
tion. It is of a rubbing quality, either fine and creaking, or coarse and grating, and is superficial, giving the impression of being produced immediately beneath the stethoscope, while moderate additional pressure with the stethoscope usually causes an increase in its intensity. The friction may not be so well heard when the patient is lying down as when he is sitting up, and its loudness may be also influenced by respiration, being as a rule louder during inspiration-in contra-distinction to pleural friction.

The heart sounds are usually audible. There may be doubling of the first sound, giving rise to a triple rhythm, while a mitral systolic murmur is not infrequently present, due either to co-existing endocarditis or to cardiac dilatation.

As the exudate is absorbed the friction gradually disappears, but it usually remains, unless the quantity of fluid is great, and even then it may persist-this being in marked contrast with friction occurring as the result of pleuritis. If the friction persist, it occupies a higher level than before. When pericarditis is secondary to pleurisy or pneumonia, there is often pleuro-pericardial friction along the left border of the heart, where the pleura lies in front of the pericardium. This also occurs in cases of pericarditis in which the outer surface of the pericardium and the opposing pleural surface become involved.

The early physical signs of sero-fibrinous pericarditis are those of the fibrinous variety. The pulse becomes still more frequent, smaller in volume, and the bloodpressure lower. At times, the pulse is somewhat collapsing in type. The pulsus parodoxus (Fig. 31) may be present. Not infrequently, there is prominence of the precordium, and, if so, the intercostal spaces may be widened or even bulge, as well as the ribs, and there may also be prominence of the epigastrium. The apex-beat is often displaced upwards, and is usually found in the 
fourth, but sometimes in the third, interspace, in which case it is not the normal apex of the heart, but a point nearer to the base-line, and the impulse is more diffuse than normal ; it may be to the left of the nipple line, and sometimes is more mobile than normal. It is diffuse and its force is diminished. It is more distinct in the recumbent posture than when the patient sits upright, on account of the tendency of the fluid to gravitate backwards when lying down, and forwards when sitting up. As the amount of effusion increases, the apex-beat becomes less and less distinetly felt, until it may be quite imperceptible. Frietion fremitus may be present, but not in the same degree as in the fibrinous variety. The most charactcristic physical signs are those obtained by percussion. The areas of superficial and deep cardiac impairment are increased, in some cases considerably; this increase takes place in all directions, and is progressive. Some observers believe that in the early stage of pericardial effusion, impairment of the percussion note may be found in the fifth right intercostal space, between the costal cartilages, where the right margin of the heart forms an angle with the liver (Roch's sign). When the effusion is considerable, the form of increased area of cardiac impairment is characteristic, its outline as a rule elosely resembling that of a pear hanging downwards by its stalk. The area of relative impairment often extends well to the left of the apex-beat; it may be almost to the left mid-axillary line. The right border may be found considerably to the right of the sternum, even as far out as the nipple line. The extension upwards may be as high as the left clavicle, the upper lobe of the left lung being compressed. The lower limit may reach the lower margin of the sixth rib. In the recumbent posture the area is smaller than when the patient sits upright, on account of the tendency of the fluid to gravitate backwards in the one case and forwards in the other. The degree of impairment is absolute. 
On auscultation, the heart sounds are found to become progressively indistinct and muffled, and may be lost, though sometimes even when the quantity of fluid is large they may persist, especially at the base; this also holds good with regard to friction. The pressure of the effusion upon the lungs may result in the production of dulness and diminished breath sounds at the extreme left base close to the spinal column; as well as skodaic resonance, tubular breathing, and œgophany, not only over a patch in the neighbourhood of the angle of the left scapula, but also over the borders of the lungs antcriorly; the upper limit of the arca of impairment posteriorly may shift on change of position. The liver and spleen also may be pushed downwards. The extent of the effusion, as well as the displacement of the organs, may be recognised by $\mathbf{X}$-ray examination. Finally, physical signs of tricuspid incompetence, including enlargement of the liver, may supervene.

As the fluid becomes absorbed, the area of cardiac impairment diminishes, the heart sounds gradually return to the normal, and, as the two surfaces of the pericardium come once more into contact, friction may reappear.

The symptoms of purulent pericarditis are those of the scro-fibrinous variety, except that rigors, a high and fluctuating temperature, profuse perspirations, great wasting, pallor, rapid prostration, marked rapidity of the pulse-rate, a subnormal blood-pressure, a smaller area of cardiac impairment, and an absence of pericardial friction, are more likely to occur. It should be noted, however, that the temperature may be almost normal, and cases have been reported in which it was even subnormal ; there may also be little alteration in the frequency of the pulse or respiration. Sweatings may occur without the existence of rigors. An examination of the blood usually reveals a leucocytosis. When pus is present from the first, the subjective symptoms may be obscured by those of the associated condition, and only the physical 
signs of pericarditis with cffusion may be noted; though even then there may be no increased area of impairment, and an absence of friction.

Course.-The duration of acute pericarditis exhibits great variation. In most cases, improvement is to be noted in rather more than a fortnight. The rate at which fluid absorbs varies greatly; it may disappear within a week, but usually takes from two to thrce weeks, while in rarc instances the effusion remains and the condition becomes chronic. When adhesions occur, time alone will reveal their presence.

Diagnosis. - It has been pointed out elsewhere it is extremely important to be on the look-out for an insidious or masked attack of pericarditis.

The diagnosis of the fibrinous variety of pericarditis is not as a rule difficult, and rests not upon subjective symptoms, but upon physical signs. Of these the most characteristic sign is the presence of friction sounds of the type alrcady described. This is pathognomonic of fibrinous pericarditis, or of tuberculous, cancerous, or gummatous deposits, and should be differentiated from an endocardial murmur (cspecially if the friction sound be single), and from pleuro-pericardial friction. The differential diagnosis in cach case has been discussed in Chapter II.

Sero-fibrinous pericarditis can be diagnosed from the physical signs described, such as a progressive enlargement of the area of cardiac impairment, synchronous with a progressive muffling of the cardiac sounds.

When the left border of the area of relative impairment is outside the apex-beat, in the absence of any morbid condition of the lung or pleura, the cause will be found to be pericardial effusion, in which case the right border will be found to be displaced considerably to the right. If the impairment in the left parasternal line be found to extend upwards into the second interspace or higher, and we are sure the heart as a whole is not displaced 
upwards by pressure from the abdomen, and disease of the lung or pleura, and the presence of aneurism, newgrowth, and enlarged glands can with certainty be excluded, the condition is in all probability due to pericardial effusion or great enlargement of the right ventricle.

In the diagnosis of sero-fibrinous pericarditis, we should be careful to exclude dilatation of the heart, hydro-pericardium, left localised pleural effusion, pulmonary consolidation, thoracic aneurism, new-growth, enlarged glands, and abscess of the mediastinum. In each case, the causation, mode of onset, and physical signs should be considered.

It is sometimes difficult to diagnose pericardial eifusion from great dilatation of the heart, especially of the right ventricle. In pericardial effusion there is often bulging of the precordium, with obliteration of the intercostal spaces; the apex-beat is internal to the left margin of percussion impairment, and may be displaced upwards to the fourth or even third interspace; the area of cardiac impairment may extend to the second left costal cartilage, the extension often coming on rapidly, and in form is pear-shaped, instead of increased transversely ; the angle formed by the heart and liver impairment is obtuse, whereas in the case of an enlarged right heart it is acute or is at most a right angle; the percussion note is absolutely dull and the sense of resistance greatly increased; and there may be cvidence of displacement of the liver and spleen. On auscultation, the heart sounds are muffled, whereas in cardiac dilatation the characteristic physical signs already described are present.

Hydro-pericardium may be detected by noting the cause, the absence of friction during any period of the illness, and the presence of general anasarca, ascites, or pleural effusion.

A positive diagnosis of purulent pericarditis may be made by an exploratory puncture. 
Prognosis.-In most cases, the immediate prognosis of pericarditis is not unfavourable. It depends upon, among other things, the age of the patient, the cause, the severity of the subjective symptoms, the amount and nature of any existing effusion, the degree of involvement of the myocardium, and whether the endocardium is implicated or not. The prognosis is more unfavourable at the extremes of life, and with a history of alcoholism. When the affection is due to rhcumatism, the immediate prognosis is favourable in the great majority of cases; a fatal termination, however, sometimes occurs at. the extremes of life. When due to Bright's disease, pneumonia, tuberculosis, acute infectious conditions, and newgrowth, the prognosis is unfavourable; a fatal termination is the rule in the pneumococcal form of the disease, and is almost invariable in tuberculosis, while the presence of a new-growth makes the prospect hopeless. With regard to the severity of the subjective symptoms, a severe degree of dyspnœa, or of cyanosis, or pronounced mental symptoms are of unfavourable omen.

If the effusion be serous and moderate in degree, recovery is the rule, although adhesions result; if, on the other hand, the amount of fluid be large, the termination may be fatal, and this usually occurs during the second or third week. When the effusion is purulent, the prognosis is grave, especially if the pus be putrid, and a fatal termination may occur within a few days. The outlook is likewise grave when the effusion is hæmorrhagic in character. Death may be due to asphyxia, sudden syncope, especially on exertion while in bed, and more rarely asthenia.

With regard to the ultimate prognosis of pericarditis, time is necessary to determine whether adhesions have resulted, and, if so, their site and degree, and whether the myocardium has been permanently damaged and to what extent.

Treatment.-The primary condition should be treated, 
and if rheumatism be the cause, the procedure suggested in Chapter XIX should be carried out. If the pyrexia continue notwithstanding the administration of salicylates, the best way of reducing the temperature is by tepid sponging of the body; if drugs be employed, the best of these is quinine, although some place reliance upon antipyrine, phenacetine, and antifebrine, but they are not advisable except for occasional use. In septic cases, the sulphocarbolates and quinine may be administered.

In all cases, whatever the cause, we should endeavour to arrest the morbid process as early as possible, and to give the heart the best chance of the fullest possible repair. Absolute rest in bed is essential ; excitement and worry of every kind should be avoided. The diet should be light, and during the acute stage it may be advisable to restrict it only to milk. An aperient should be administered at the outset, and during the illness strict attention should be paid to the state of the bowels. The internal administration of iodides is recommended by some. If there be much exhaustion, dyspnœa, cyanosis, or an unduly rapid pulse, alcohol may be given, and oxygen employed, the quantity of alcohol varying with the individual requirement. Other diffusible stimulants, such as ether and ammonia, may be administered, especially in cases of sudden collapse. Strychnine may be tried when there is great exhaustion; it is better withheld, however, in the early stages of the illness when the cardiac action is excited.

For precordial pain and distress, a hot linseed poultice or hot fomentations may be applied to the precordium, or, if these fail, the continuous application of an ice-bag may be of service; other remedies, such as dry or wet cupping, mustard leaves, the application of the Paquelin cautery, or leeches to the precordium, are recommended. In some of these cases there may be indications for the use of venesection; this has been referred to in 
Chapter IX. Internally, Dover's powder (10 to 15 gr.), or, if necessary, morphia, may be administered. In the less acute cases, a fly blister is not infrequently efficacious. If insomnia or other symptoms be present, they should be treated on the lines previously laid down.

In cases of moderate effusion, the former treatment should be continued. If, however, the effusion become considerable, counter-irritation, the administration of iodide of potassium, purgatives, and diuretics, and limitation in the amount of fluid taken, are indicated. If the amount of effusion do not diminish, or if there be marked dyspnœa, cyanosis, and a small rapid pulse, paracentesis pericardii should be performed. The site chosen for this procedure varies. It may be performed in the fifth left intercostal space just inside the mammary line; or quite near the margin of the sternum in the same space; or in the angle between the ensiform cartilage and the left costal margin of the sternum, near the lower end of the body of the sternum, and passing upwards and inwards behind it into the pericardial sac. Formerly a trochar and cannula, or a fine aspirating needle, was employed, but the use of an aspirator is now found to be preferable.

The stage of convalescence requires careful management, on the same lines as those of acute endocarditis. Tonics, such as quinine and arsenic, may be administered with advantage.

The treatment of purulent or hæmorrhagic pericarditis consists in free incision and drainage of the pericardial sac, unless the state of the patient is too grave to allow of it-in which case paracentesis may be performed, and, if improvement occur, incision and drainage may be subsequently permissible-or unless the condition is associated with a hopeless primary cause.? 


\section{Chronic Adhesive Pericarditis or Adherent Pericardium}

In this form of pericarditis the two layers of the pericardium are bound together by permanent fibrous adhesions. The degree varies greatly. It may amount to only a few fine bands, most commonly situated at the base of the heart close to the great vessels, crossing the pericardial sac; or, in more severe cases, it may result in the formation of a layer of connective tissue, occasionally of considerable thickness, which more or less completely unites the two layers of the pericardium. The formation of partial synechiæ, however, is a much more common result than obliteration of the sac. Sometimes extra-pericardial adhesions exist. In this way adhesions may form between the pericardium anteriorly and the anterior chest wall ; laterally with the pleuræ and lungs ; posteriorly with the aorta, œsophagus, or vertebral column; and below with the diaphragm. In rare in-. stances, extra-pericardial adhesions are found in the absence of adhesions between the two layers of the pericardium. In advanced cases, pericardial adhesions may become more or less completely calcified, owing to the deposition of lime salts.

In a large proportion of cases, cardiac, especially ventricular, hypertrophy, usually accompanied by dilatation, exists, even in the absence of co-existing valvular disease. The degree of cardiac enlargement varies with the position and nature of the adhesions; it may be small or altogether absent, even when the sac is completely obliterated, provided the sac is not thickened and there is an absence of extra-pericardial adhesions; whereas it may be very great if the reverse be the case. Fatty or fibroid degeneration of the myocardium may occur.

The openings of the large vessels entering and leaving 
the heart may be eonstrieted, and this may interfere with the influx and egress of blood.

Etiology.-The condition usually results from a previous attack of acute periearditis, especially if accompanied by effusion, or it may follow a primary chronic inflammation; the latter may be due to an extension of inflammatory proeesses from the neighbouring parts.

Symptomatology. - The condition may be latent throughout its whole course. If symptoms be present, they may exhibit considerable variation in different cases. Dragging pain in the precordium, sometimes extending considerably beyond it, is not an uncommon symptom, and anginal attacks have been known to occur; there may be also palpitation, dyspnœa, and cyanosis. Edema is present in a certain proportion of cases; ascites and hydrothorax are occasionally to be noted, as also hepatic and splenic enlargement, and scanty and even albuminous urine. The pulse may exhibit the characteristics of pulsus paradoxus (Fig. 31). The precordium may be prominent; less eommonly there is flattening. The apexbeat is usually displaced, and its position does not vary with change of posture-a faet which also applies to the area of cardiac impairment. The area of pulsation is usually greatly increased, and may even extend from the second to the sixth intercostal space, and almost from the right parasternal line beyond the left mammillary line; it may be wave-like and undulatory in character. If adhesions between the pericardium and chest wall exist, indrawing of the apex-beat may occur, also with systolic recession of the intercostal spaces on either side of the sternum, of the lower end of the sternum and adjacent costal cartilages, and in the epigastrium; while systolic retraction of the lateral and posterior walls of the left side of the chest, upon the latter of which Broadbent has laid great stress as indicating adhesion between the pericardium and diaphragm, is occasionally to be noted. A diastolic rebound or shock, 
after the systolic retraction, is rarely present at the apexbeat, or over a larger area of the precordium, or even over the left lateral or posterior aspect of the chest; this is supposed to be pathognomonic of the affection. The position of the apex-beat and the area of cardiac impairment may be unaltered on deep respiration. Diminished expansion of the left side of the chest, and incomplete descent of the left half of the diaphragm during inspiration, as shown by diminished movement of the upper part of the abdominal wall on the left side, may be noted. Collapse of the veins of the neck during diastole -Friedreich's sign-may occasionally be observed.

The area of cardiac impairment is usually much increased, both to the right and to the left. Bruits indicative of relative incompetence both of the mitral and tricuspid areas may be present. Some authorities are of opinion that a rumbling mitral presystolic bruit is sometimes heard in adherent pericardium in children; this bruit, however, is not rough or vibratory in character, nor does it terminate abruptly in the first sound, as in the case of mitral stenosis. Lastly, there may be physical signs of co-existing heart disease.

Diagnosis.-The diagnosis may be a matter of considerable difficulty. A positive diagnosis should not be made from the presence of one sign only, except when diastolic shock or rebound exists, and perhaps also in those cases in which systolic recession of the sternum itself is present. It should be noted the pulsus paradoxus, indrawing of the apex-beat, and systolic recession in the intercostal spaces on either side of the sternum, or in the epigastrium, or even of the lateral or posterior walls of the left side of the chest, do not necessarily indicate the existence of chronic adhesive pericarditis; and further, that an absence of alteration of the position of the apex-beat and of the area of . cardiac impairment with change of posture or deep respiration may also be present in pleural adhesions. When, 
however, several or all of the physical signs of the affection are present a positive diagnosis is warranted, especially if there be indications of right-sided cardiac enlargement or failure in the absence of mitral or pulmonary disease. A history of acute pericarditis or of acute rheumatic infection is of additional assistance, although, on the other hand, it should be remembered that the affection may be chronic from the beginning.

Prognosis.-This depends upon the situation, extent, and nature of the adhesions, the size of the heart, the existenec or otherwise of valvular disease, the degree of integrity of the myocardium, and the degree of cardiac failure which may be present. The prognosis may be good, and, indeed, the patient's longevity may not be affected; or, on the other hand, it may be grave, especially in early life.

Treatment.-The treatment of the condition consists in counter-irritation externally, and the internal administration of iodide of potassium during the later stage of the attack of acute pericarditis as a prophylactic. When adhesions have formed, detailed instructions should be given to the patient to curtail his physical and mental activities so as not in any way to exceed the limited strength of the heart. When cardiac failure supervenes, it should be treated on the lines previously laid down.

It may be necessary to consider the advisability of performing the operation of "cardiolysis" in some cases of severe adhesions between the pericardium and the chest wall. This operation was introduced by Brauer in 1902 , and is indicated when there is also evidence of cardiac failure and ordinary treatment has failed; but the operation should not be unduly delayed. It is usually not necessary during childhood or adolescence, because of the flexibility of the chest wall. The operation consists in the resection of the $4 \mathrm{th}, 5 \mathrm{th}$, and 6 th ribs on the left side, and, where possible, freeing the adhesions. 


\section{Chronic Pericarditis}

It has been already noted that some writers describe a chronic form of pericarditis. This, however, is practically synonymous with chronic adhesive pericarditis or adherent pericardium, although it sometimes happens that in acute pericarditis with effusion, especially when due to tuberculosis, the effusion may persist for an indefinite period and the condition become a chronic pericarditis, resulting in considerable thickening of both layers of the pericardium. Further, but less frequently, pericarditis may be chronic from the first, especially in the aged, in alcoholism, and in nephritis.

When the result of acute pericarditis, the symptoms are those of that stage of the disease at which it became chronic. When chronic from the first, the affection is not infrequently latent throughout its whole course; thcre may, however, be dyspnœa, a sense of oppression, and rarely pain, in the precordium, an increase in the area of cardiac impairment, feeble cardiac sounds, and sometimes pericardial friction.

\section{Tuberculosis of the Pericardium}

Tuberculosis of the pericardium may be primary, or part of an acute general tuberculosis, or secondary to that of the lungs, the pleuræ, the mediastinal or bronchial glands. It may give rise to, (1) tuberculous pericarditis, in which the effusion may be clear, purulent, or bloodstained, and in which tuberculous nodules with giant cells are sometimes found in the fibrous tissue consequent upon organisation; (2) miliary tubercles, which may be seen along the course of the small blood-vessels in the epicardium, especially in the sulci; and (3) larger tubercular masses in the epicardium, situated particularly at the base of the heart, and presenting a yellow caseating 
centre. The two latter conditions are much less common than the first. There is a greater tendency to the chronic type of pericarditis in the case of tuberculosis than when due to other causes, the condition persisting for several wceks or even months, and not infrequently giving rise to a layer of connective tissue of considerable thickness, more or less completely uniting the two layers of the pericardium; there may or may not be fluid, which is frequently blood-stained, and is occasionally purulent.

Symptomatology.-The affection (1) may be entirely latent throughout its whole course; (2) may present the symptoms of acutc pericarditis, with, in addition, loss of weight and strength, a fluctuating temperature, a tendency to chronicity, and ending in effusion of fluid or the formation of adhesions; (3) may be a chronic adhesive pericarditis, with consequent hypertrophy and dilatation, and ultimatcly indications of cardiac failure; or (4) may present the symptoms of acute tuberculosis.

Diagnosis. - This can be made from an absence of any history of rheumatism or other cause of pericarditis, and, further, there may be evidence of tuberculosis elsewhere.

Prognosis.-This is very grave.

Treatment.-The treatment is that of pericarditis due to other causes, together with that indicated in ordinary tuberculosis, such as an abundance of fresh air, a generous diet, and the administration of cod-liver oil.

\section{HydRopericardium}

A certain amount of fluid is usually found in the pericardium after death; in hydropericardium or dropsy of the pericardial sac, this is in excess. The fluid is clear, pale amber in colour, and has a specific gravity of about 1015.

Etiology.-The condition is always secondary: It may occur along with ascites, hydrothorax, and general anasarca, 
in valvular or myocardial disease or in nephritis. Or, it may occur alone, as the result of pressure upon the veins of the pericardium or of the heart by aneurism, enlarged glands, or new-growth of the heart, pericardium, or mediastinum; from constriction of the veins by adhesions; and in various cachetic conditions, scarlet fever, and some purpuric conditions.

Symptomatology.-The symptoms of the primary affection are usually to be noted. The condition in itself does not give rise to pyrexia or precordial pain. There may, however, be a sense of oppression in the chest, dyspnœa, cyanosis, attacks of faintness or even unconsciousness, œedema, hepatic enlargement, and scanty urine. In some cases there is drowsiness or mild muttering delirium. The pulse is soft, and of subnormal pressure. On examination of the heart, the physical signs are found to be those of pericarditis with effusion, except that there is an absence of friction on auscultation.

Diagnosis.-A diagnosis may be arrived at by a consideration of the cause, signs of fluid in the pericardial sac, and an absence of friction.

Prognosis.-The prognosis is that of the primary affection.

Treatment.-This is based upon the causal condition, and, in addition, a lessened intake of fluid, together with the use of purgatives and diuretics are indicated. If urgent symptoms appear to demand it, paracentesis may be performed ; this, however, is rarely necessary.

\section{Pneumopericardium}

By pneumopericardium is meant the presence of air in the pericardial sac. It is a very rare condition. Pericarditis is always present, with serous (pneumohydropericardium), purulent (pneumo-pyopericardium), or hæmorrhagic (pneumo-hæmopericardium) effusion. 
Etiology. - The affection may be due to wounds through the chest wall, or the result of communication with an air-containing cavity, such as occurs in abscess or gangrene of the lung, malignant disease of the œsophagus, gastric ulcer, and hepatic abscess. It is further believed by some that gas may be spontancously generated within the pericardial sac intra vitam.

Symptomatology.-Breathlessness, precordial discomfort or even pain, faintness, and cyanosis are the most constant symptoms. There may be a certain amount of bulging of the precordium, and the apex-beat is diminished in force or even absent. On percussion, a tympanitic note is to be observed, the arca of which changes with an alteration of posture. In the recumbent position it may be heard over most of the precordium, while if the patient be sitting upright the lower part of the precordium becomes impaired and the upper part hyper-resonant. On auscultation, metallic and splashing sounds, resembling those produced by a water-wheel or a churn, synchronous with the movements of the heart, are audible; these sometimes become more pronounced on shaking the patient, and occasionally may be heard at a considerable distance. Pericardial friction is sometimes also to be noted.

Diagnosis.-The diagnosis is not difficult when the physical signs cnumerated are present. Care, however, should be taken to exclude a large pulmonary vomica in the ncighbourhood of the heart, a left-sided pncumothorax, and dilatation of the stomach.

Prognosis.-The majority of cases die within the course of a few days. Traumatic cases, however, have been known to recover; the wound may heal and the air be absorbed.

Treatment.-In traumatic cases, paracentesis of the pericardial sac, with free incision and drainage if the fluid be purulent, is advisable. In other cases, treatment is usually only palliative. 


\section{HEMOPERICARDIUM}

By this is meant the presence of blood in the pericardial sac, occurring apart from pericarditis.

Etiology.--It may be due to punctured wounds, perforation of the esophagus by a dental plate, rupture of the aorta, of one of the coronary arteries, or of the heart, or as the result of purpura or scurvy.

Symptomatology.-Death may take place suddenly, or there may be sudden collapse, marked pallor, perspiration, or syncope, with a rapidly fatal termination. On examination, the pulse will be found to be feeble, and of low tension, with, perhaps, increased area of cardiac impairment.

Prognosis.-Except in some traumatic cases, where surgical interference is possible, the outlook is always hopeless.

Treatment.-This is limited to immediate surgical operation in certain cases. 


\section{CHAPTER XXIII}

LESS COMMON DISEASES OF THE HEART AND PERICARDIUM

\section{Aneurism of the Heart}

ANEurism of the heart may occur in its valves, or walls, or in the coronary arteries.

Acute aneurism of the valves may occur in malignant endocarditis, the semilunar being more commonly affected than the auriculo-ventricular valves. The aneurism bulges in the direction of the blood current; rupture may take place, giving rise to perforation and valvular incompetence.

Acute aneurism of the walls of the heart may take place in acute mural malignant endocarditis, usually in the interventricular septum, in the neighbourhood of the undefended space. A diagnosis of this affection is scarcely possible.

Chronic aneurism of the walls of the heart occurs as the result of fibroid degeneration, and is more frequent in males. In the majority of cases, it arises in the left ventricle near its apex. The aneurism consists of a sac or localised depression, communicating with one or more chambers of the heart. It is lined by thickened endocardium, and the wall is formed of fibrous tissue and muscle-cells, in varying proportion, according to the length of time it has been in existence. Occasionally a certain amount of calcarcous matter is present. The visceral layer of the pericardium may be thickened, and its parietal layer also involved. The aneurism generally contains laminated fibrinous coagulum, and its 
size may vary from that of a small marble to an orange or even larger. The symptoms are those of general heart failure. The area of cardiac impairment may be increased, and occasionally a tumour in the region of the apex may be detected. Sudden death is not of uncommon occurrence, although rupture is comparatively rare.

Aneurism of the heart may also result from a stab.

Aneurism of a coronary artery occurs rarely, and is usually due to atheroma.

\section{Wounds of the Heart}

Wounds of the heart are not of very uncommon occurrence. They may be the result of stabbing, bullet wounds, or the passage of foreign bodies from the œsophagus. Recovery occurs in a fair proportion of cases, especially when due to stabbing.

Treatment of these cases is purely surgical.

\section{Rupture of the Heart}

Rupture of the heart is a rare event, and is usually due to fatty degeneration or infiltration of the myocardium. It is occasionally the result of abscess or aneurism of the myocardium, or of traumatism. The left ventricle is most commonly involved, usually its anterior wall. Rupture of the heart occurs nearly always during physical exertion. It may result in sudden death, or its occurrence may be indicated by sudden agonising pain, intense dyspnœa, pallor, and collapse; in the latter case, the patient may live for some hours or even days.

\section{Abscesses in the Myocardium}

In pyæmia, large abscesses in the myocardium are of rare occurrence; they may perforate into the ventricle or pericardium. Minute abscesses, on the other hand, 
are not infrequent, as, for example, in ulcerative endocarditis.

Syphilitic Affections of the Heart and Pericardium

Syphilis may affect the cardiac valves, the myocardium, or the pericardium.

With regard to the valves, it has been pointed out that syphilis may be a cause of degenerative processes, which may result in chronic valvular disease, more particularly of the aortic valve. In addition, acute aortitis may involve the aortic valve with resultant incompetence, and give rise to severe anginal attacks.

With respeet to the myocardium, as has been noted, syphilis may eause endarteritis obliterans or atheroma of the coronary arteries, the consequences of which have already been described. Gummatous deposits also occur in the cardiae wall, more especially at the apex of the left ventricle and the interventricular septum. The size of these gummata varies from a small pea to a considerable tumour. Softening of the centre may take place, with surrounding fibroid degeneration, or they may undergo fibroid change with resulting scar formation; ultimately rupture or aneurism may occur. Symptoms may be absent, or angina pectoris, syncopal or cpileptiform attacks, and tachycardia may be present. There may be no cardiac enlargement. When in the case of a young adult who is known to have had syphilis, but has not suffered from rheumatism, the foregoing symptoms are present, cardiac syphilis should always be suspected.

Syphilis of the pericardium is of rare occurrence, and is usually late secondary, or tertiary, though it may be congenital. The disease more commonly takes the form of an infiltration, with consequent formation of fibrous tissue, resulting in adhesions between the two layers of the pericardium, while in some cases there is effusion of fluid into the pericardial sac. Gummata are of rare occurrence. The affection is usually latent. 
A diagnosis is sometimes possible from the discovery of the clinical features of pericarditis in a known syphilitic subject. The prognosis depends mainly upon how early a diagnosis is made and treatment undertaken. Small gummata, when treated early, respond to adequate therapeutic measures, but when fibrosis has occurred the condition is irremediable.

The treatment of cardiac syphilis consists in rest, the administration of iodide of potassium, and in some cases also of mercury.

\section{New-growths of the Heart and Pericardium}

Neoplasms may arise from the walls of the heart or from the valves. They, as well as tuberculous deposits, are usually secondary. Primary carcinoma and sarcoma of the pericardium are extremely rare, and secondary deposits are also of unusual occurrence; in the latter case they may be secondary to those of the œsophagus, mediastinal glands, lungs, or pleuræ; more rarely from distant parts. Instances of hydatid cysts and actinomycosis of the myocardium and pericardium have occasionally been noted post mortem; they are not diagnosable during life.

The symptoms of new-growths of the pericardium are those of pericarditis, especially of the chronic type, together with the cachexia characteristic of the primary cause. The diagnosis rests upon the recognition of these features, with possibly evidence of malignant disease elsewhere. The prognosis is hopeless, and treatment at the best is only palliative. 


\section{CHAPTER XXIV}

CONGENITAL HEART DISEASE

Development of the Heart.-Before dealing with congenital heart disease it may be advisable to give a short account of the development of the heart, and of the fotal circulation.

During the first few weeks of embryonic life, two lateral tubes in the anterior part of the embryo become fused to form a singlc tube, which is continuous in front with the two primitive aortæ, and bchind with the veins (Fig. 5). Slight constrictions of the tube then appear, dividing it into four portions, namely, the sinus venosus, the auricle, the ventricle, and the bulbus arteriosus. Rather later, the tube becomes bent upon itself, and the auricular portion comes to lie behind and to the left, and the ventricular portion in front and to the right. The constriction betwcen auricle and ventricle now becomes elongated into a short canal, and, later, two cushionsthe endocardial cushions-develop from the walls of the canal; these eventually meet, and the right and left auriculo-ventricular orifices are thus formed.

The cavity of the heart subsequently becomes divided into a right and left side by the development of the septa. The inter-auricular septum is the result of the coalescence of two separate septa, the primary and the secondary septum. The former takes its origin from the upper part of the undivided auricle, and grows downwards ; it is at first sickle-shaped, but ultimately reaches the canal already referred to, and unites with the partition in the 
canal. The central part of the primary septum becomes perforated by small apertures, resulting in a lattice-like membrane, through which the blood passes from right to left. At a later date, all these apertures become closed, with the exception of one, which enlarges. The secondary septum likewise takes its origin from the upper surface of the auricle, a little to the right of the primary septum, and grows downwards to the centre of the cavity. It is incomplete, although the aperture does not corresponid with the aperture in the primary septum. The se two septa now fuse together, leaving an oblique opening, which is called the foramen ovale. The inter-auricular septum is not completed until the fifth month. The foramen ovale is fully open until the fourth month, when a valve develops, which allows the passage of blood from the right to the left auricle, but prevents its return. The foramen becomes completely closed after birth.

The inter-ventricular septum arises in the lower part of the ventricle. A separate septum also develops in the bulbus arteriosus, which ultimately divides the bulb into the pulmonary artery and the aorta, each communicating with its respective ventricle. The inter-ventricular septum grows upwards towards the cushions already referred to, and towards the septum which is developing in the bulbus arteriosus. The fused cushions, the inter-ventricular septum, and the septum in the bulbus arteriosus together. form the pars membranacea or undefended space, which in the fully developed heart is situated at the extreme upper end of the inter-ventricular septum, immediately below the level of the auriculo-ventricular groove. The inter-ventricular septum is completed about the eighth week, and the septum of the bulbus arteriosus a few days earlier. Free communication exists between the aorta and the pulmonary artery by means of the ductus arteriosus, which does not become obliterated until after birth.

In fotal life, the blood is aerated in the placenta, and 
passes to the foetus through the umbilical vein, ultimately reaching the inferior vena cava, which enters the right auricle. Here most of the blood is deflected by the Eustaehian valve through the foramen ovale to the left auriele. It then passes to the left ventricle, and thence to the aorta, whenee part of it is distributed to the head and neek and upper extremities, the remainder passing onwards to the deseending aorta. The venous blood from the head and neek and upper extremities is conveyed from the superior vena eava to the right auriele, thenee to the right ventricle, and then to the pulmonary artery. Only a small portion of the blood passes through the lungs to the left auricle, the greater part passing onward through the duetus arteriosus to the deseending aorta, where it becomes mixed with the blood which has been conveyed from the left ventricle, and is distributed to the trunk and lower extremities, and ultimately through the umbilical arteries to the placenta. It will thus be seen that the blood supplied to the head and neck and upper extremities is more highly aerated than that supplying the trunk and lower limbs.

When at birth the function of respiration commenees the placental circulation ceases. The blood now passes from the right auricle to the right ventricle, thence through the pulmonary artery to the lungs, and the foramen ovale and the ductus arteriosus soon become elosed.

Etiology and Pathology of Congenital Heart Disease. - Congenital heart disease is more common in males than in females. It may be the result of some developmental defect, or of fœtal endocarditis-due to rheumatism or one of the other causes of acute endocarditis affecting the mother during pregnancy. Syphilis is said to be a eausal factor. Both developmental defects and fotal endocarditis are more common on the right than on the left side of the heart, as the result of a relatively higher pressure. The pulmonary orifice is more commonly 
affected than the tricuspid. The lesion of congenital heart disease may give rise to stenosis, or incompetence, or both. The endocarditis is usually of the sclerotic type, although vegetations may occur. It may be noted that there is a predisposition to endocarditis in the fœtus the subject of some developmental error, and this increases the primary malformation; endocarditis may also occur after birth.

Varieties of Congenital Heart Disease.-There are many varieties of this condition, and I shall not attempt to deal with them all. As a rule, several lesions co-exist, a single lesion being uncommon.

In ectopia cordis the heart is situated outside the thoracic cavity ; it may lie in the neck (ectopia cervicalis), or outside the chest-wall (ectopia pectoralis), or in the abdominal cavity (ectopia abdominalis). Other malformations are usually also present. In meso-cardia the heart occupies the mesial position of the thoracic cavity, the apex-beat being in the epigastrium.

In dextro-cardia or dexio-cardia, the heart lies on the right side of the thoracic cavity ; this variety of congenital heart disease is usually associated with transposition of the viscera (Fig. 245).

More or less complete absence of the pericardium is rarely found.

The development of the heart may become arrested at the stage when only an auricle, a ventricle, and a bulbus arteriosus exist, a comparatively rare condition, to which the term "bilocular" heart has been applied. Another comparatively rare form is a complete absence of the inter-ventricular septum, in which case the heart consists of two auricles and one ventricle, and is said to be "trilocular." The tricuspid and mitral orifices are usually present, and the bulbus arteriosus is usually divided into a pulmonary artery and an aorta. Other abnormalities frequently also exist. Another rare condition is complete absence of the inter-auricular septum, 
in which case the heart consists of an auricle and two ventricles ; this is usually associated with other abnormalities. One of the most frequent forms of congenital heart disease is an incomplete septum ventriculorum, due to an arrest of development; it is a much commoner condition than complete absence of the septum. The aperture of communication between the two ventricles is usually situated in the region of the pars membranacea. It is not common to find this condition existing alone, and is frequently associated with pulmonary stenosis or atresia, and hypertrophy of the right ventricle; less frequently with other defects. When co-existing with pulmonary stenosis or atresia, the blood can pass from the right to the left ventricle. When not so associated, the acrated blood after birth passes from the left to the right ventricle, and the walls of both chambers undergo hypertrophy. There may be complete abscnce of the septum of the ductus arteriosus, or this may be incomplete, with resultant communication between the pulmonary artery and the aorta; in these cases, the right ventricle is generally hypertrophied.

The scptum between the auricles may be incomplete, due to arrest of development, and is much commoner than complete absence of the septum. The defect is usually situated at the foramen ovale, due to maldevelopment of the membrane of the fossa ovalis. It should be noted that the absence of an absolute closure of the foramen ovale is not pathological.

Patent foramen ovale may exist without any other form of congenital heart disease. The majority of cases, however, are associated with pulmonary stenosis, or a defect in the ventricular septum, or some other congenital defect. Some writers believe that in the larger number of cases a patent foramen ovale results from pulmonary stenosis, while others hold a different view. Whichever view be correct, pulmonary or tricuspid lesions are often found associated with it, and occasionally lesions 
at the aortic or mitral orifice. In the case of a patent foramen ovale, the blood passes from the right to the left auricle, instead of into the right ventricle and thence to the lungs.

Pulmonary stenosis is perhaps the commonest form of congenital heart disease. Other lesions are usually associated with it, a patent inter-ventricular septum being present in the great majority of cases, and a patent foramen ovale is of not infrequent occurrence, while the ductus arteriosus may remain patent. Further, owing to increased pressure in the cavity of the right ventricle, there is sometimes a deviation of the inter-ventricular septum to the left; in this case, the right ventricular cavity is enlarged, and the aorta takes its origin, either completely or partially, from this chamber, the left ventricle being small and atrophied, and communicating with the right ventricular cavity. In pulmonary stenosis the blood accumulates within the right ventricle, and is compelled to take a new channel. If the defect have occurred before the ventricular septum is completed, the septum remains incomplete, and blood passes through the opening and thence to the aorta, the right ventricle being the chief agent in carrying on the systemic circulation. Owing to the increase in pressure in the right heart, the right ventricle and auricle hypertrophy. If the lesion be severe, much of the blood to the lungs must reach them from the aorta through the ductus arteriosus, which does not, therefore, close after birth. If the inter-ventricular septum have completely closed before pulmonary stenosis takes place, the pressure in the right auricle becomes increased; if the foramen ovale be patent, the blood passes from the right to the left auricle, and in this way a patent foramen ovale is not infrequently found. The right auricle is small and atrophied, and the tricuspid orifice becomes smaller. The cavity of the left ventricle enlarges, and its walls hypertrophy, as it carries on both the systemic and pulmonary circulation. In almost all these 
cases the blood is carried to the lungs by the patent ductus arteriosus. It will thus be seen that the circulation in cases of pulmonary stenosis depends upon the patency of the foramen ovale or inter-ventricular septum, and of the ductus arteriosus.

Pulmonary atresia or obliteration of the pulmonary artery is much less common. When complete, and the inter-ventricular septum is closed, the right ventricle is small and atrophied, the right auriculo-ventricular opcning ultimately eloses, and the heart becomes triloeular.

Stenosis and atresia of the aorta are of much less common occurrence than of the pulmonary artery; cither of them may occur alone or with other lesions. Stenosis may exist at one of the following sites: (1) at the orifice, (2) above the entranee of the ductus arteriosus, and (3) just bclow the cntranee of the duct. In the first of thesc, the ventricular and auricular septa do not elose, and the left ventricle remains undeveloped. Most of the systemic circulation is earried on by the pulmonary artery, through the ductus arteriosus. The second, or new-born type, is often associated with other malformations, as, for example, pulmonary stenosis. The ductus arteriosus remains open, and most of the systemic circulation is carried on by the right ventricle, through the duct. The third or adult type of stenosis is more common.

By coarctation of the aorta is meant stenosis of the aorta at or just below the junetion of the duetus arteriosus with the aorta, i.e. in the region of the aortic "isthmus." The condition is more common in males than in females. The ductus arteriosus may remain patent or may close. When the condition is present in adults and in children who have survived the period of infancy, it is supposed to have arisen gradually after birth, in association with the closure of the ductus arteriosus. Usually in such cases an elaborate system of collateral arterial circulation has developed, so that the arterial blood from the upper part 
of the body-that is to say, from the great arteries given off by the aorta above the site of the obstruction-can pass by enlarged anastomosing branches to the lower part of the trunk and the lower extremities.

Hypoplasia of the aorta, or narrowing of the lumen of the vessel throughout its whole extent, with diminished size of the heart, was first described by Virchow, and there is sometimes accompanying chlorosis. Other malformations of the heart are occasionally also present.

The condition known as double aorta is a form of congenital heart disease very rarely found.

Persistence of the ductus arteriosus occurring alone is also a rare condition. It is usually associated with other congenital defects, being a common accompaniment of pulmonary stenosis or atresia, when a patent inter-ventricular septum is also present; it may also accompany aortic stenosis or atresia, and congenital disease of the auriculo-ventricular valves.

Premature closure of the ductus arteriosus and of the foramen ovale are of rare occurrence.

Transposition of the pulmonary artery and the aorta may be found, the aorta arising from the right ventricle and the pulmonary artery from the left. Other lesions are usually also present, such as a patent foramen ovale, a patent septum ventriculorum, and a patent ductus arteriosus. Other forms of malposition of the pulmonary artery and of the aorta have been recorded, such as both trunks taking their origin from the left ventricle.

A communication between the pulmonary artery and the aorta has been known to occur.

Supernumary or defective cusps of the cardiac valves have been noted; the number of cusps may be increased or decreased, and in the latter case incompetence of the valves may result.

Congenital lesions of the auriculo-ventricular valves may occur. Stenosis or atresia of the tricuspid or mitral orifice is much less frequent than of the pulmonary artery 
or of the aorta. A considerable proportion of cases of tricuspid stenosis are congenital; in this case, there is usually some other cardiac lesion. Tricuspid incompetence is rarcly congenital ; it may bc associated with pulmonary atresia. Congenital lesions of the mitral orifice are of much rarer occurrence than those of the tricuspid orifice.

Symptomatology.-The subjcctive symptoms of congenital heart disease vary in their intensity according to the site and degree of the lesion. It may be laid down as a broad principlc that septal defects have a less disturbing effect upon the circulation than stenosis or atresia; indeed, in some of the former cases, there may be a completc absence of subjective symptons throughout the whole course, and the affection is only discovered on physical examination or post-mortem. The results of physical examination also show variation.

Cyanosis and dyspnoa on exertion are the two chief

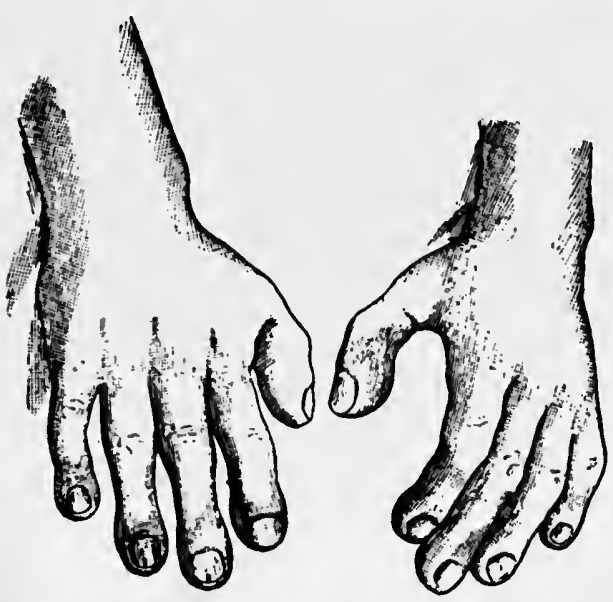

Fia. 162. Clubbing of the fingers, from a case of congenital heart disease. symptoms. The former may be prescnt within a few wceks of birth, or may only make its appearance after sóme years. It is a more conspicuous feature of this form of cardiac disease than of any other, the term "morbus cœruleus" being, indeed, frequently used for congenital heart disease. This is most marked in the lips, nose, the malar region, and the fingers and toes, being especially noticeable in the nails, and may be confined to these 
localities, or may be more general. The degree of cyanosis varies up to a deep purple, and is increased by exertion. The lips and nostrils may exhibit thickening, and clubbing of the ungual phalanges of the fingers (Fig. 162 ) and toes (Fig. 163) is usually present, due to chronic venous engorgement. There may be an absence of dyspnœa while at rest, but it may make its appearance on exertion, while in some cases paroxysmal dyspnœa occurs.

While the temperature taken in the mouth or rectum is usually normal, the skin temperature is usually subnormal. The subject of congenital heart disease often

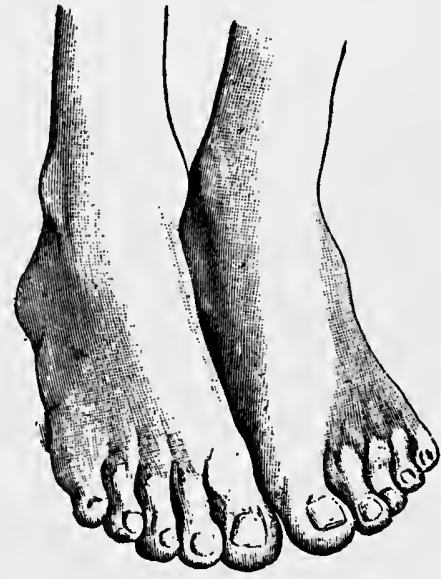

Frg. 163. Clubbing of the toes, from a case of congenital heart disease. complains of feeling cold, is very susceptible to any fall of temperature, and is liable to attacks of bronchitis. On examination, the blood is found to have an increased viscosity, and its specific gravity may be as high as $\mathbf{1 0 7 0}$. There is a large increase in the number of the erythrocytes, which may even reach $9,000,000$ per cubic millimetre, the hæmoglobin is increased, and may be as high as $160 \%$, while the leucocytes count may show an average of 15,000 per cubic millimetre. There is usually retardation of growth, and in some cases wasting; the mental condition is backward, and the patient is frequently dull and lethargic. There may be syncopal attacks, even resulting in prolonged unconsciousness, while epileptiform attacks may also occur. In certain instances there is a tendency to hæmorrhage from the nose, gums, and less frequently from the lungs. Edema is sometimes present, which at a later stage may become general, and 
hæmoptysis, hepatic enlargement, albuminuria, and transudation of fluid into the serous sacs may also occur. Not infrequently other bodily malformations are to be noted.

The veins of the retina are almost always dilated and tortuous, the lumina appearing in some places to be unduly wide, and in others abnormally narrow; both arteries and veins are much darker than normal.

The pulsc is usually quiekened in rate. There is usually evidence of cardiac enlargement, although perhaps not of severc grade, the right side of the heart being particularly affected. There is not infrequently some bulging of the precordium in the region of the ensiform cartilage, due to enlargement of the right ventriele, while the apexbeat is usually displaced to the left. There may be pulsation in the epigastrium, diastolic in time, and when there is marked hypertrophy of the right ventricle there is frequently a heaving impulse in the same region, and over the lower sternum and costal cartilages. Distension or pulsation of the jugular veins may also be present. Sometimes a thrill and frequently a murmur, usually systolie in time, are to be noted. The point of maximum intensity may be over the pulmonary area, but it may be difficult or impossible to define. The murmur is usually rough and grating, and, pcrhaps, extremely loud; on the other hand, it is somctimes very faint. The pulmonary second sound may be normal, diminished, or loud and ringing.

The subjeet of congenital heart disease may ultimately die from gradual cardiac failure-the clinical picture resembling that of mitral diseases, infective endocarditis, bronchitis, broneho-pneumonia, pulmonary tuberculosis, one of the acute infectious diseases, cerebral thrombosis or abscess, or convulsions-while instances of sudden death have been known to occur.

Passing now to a consideration of the symptomatology of the individual lesions, in dextro-cardia the 
apex-beat is on the right side of the thorax, and the point of maximum intensity of the aortic second sound is on the left, instead of the right, side. When patency of the inter-ventricular septum exists alone, the subjective symptoms are usually slight. A certain amount of dyspnœa and cyanosis on exertion may occur, but cyanosis is not a prominent feature, and there is usually no clubbing of the ungual phalanges of the fingers and toes. There may be evidence of enlargement of the right side of the heart. In some instances a systolic thrill and a loud and harsh murmur over the precordium are present. Writers also differ in regard to their point of maximum intensity; according to some, e.g. Roger, it is in the median line in the upper third of the precordium, while in the opinion of others it is near to the left margin of the sternum in the third left interspace or even as low down as the xiphisternum. There is a diversity of opinion also in respect of the question of the direction of selective propagation of the bruit, some believing that there is none, and that the bruit is not audible over the carotid artery, whereas others affirm the opposite. Some writers say that the second sound in the pulmonary area is accentuated. When patency of the inter-ventricular septum is secondary to other lesions, the symptoms of the primary affection predominate.

When patency of the inter-auricular septum exists alone, there is not infrequently an absence of subjective symptoms, and the condition, therefore, is not discovered during life. In others, however, they are present, the results of physical examination in such cases exhibiting considerable variation. A murmur or murmurs may be present over the precordium, being in some cases systolic, in others diastolic, or, according to some writers, even characteristically presystolic, and having the point of maximum intensity over the base of the heart.

In congenital pulmonary stenosis, cyanosis occurs in the vast majority of cases, and usually within a few weeks 
of birth, but in rare instances it only makes its appearance after some years. Clubbing of the fingers and other symptoms of congenital heart disease are usually present. The pulse may present no special peculiarity, or it may be small and cmpty. Sometimes a thrill, systolic in time, and with its point of maximum intensity over the pulmonary area, is present. On percussion the left border of the heart is often, and the right border is almost always, found to be displaced outwards. The most characteristic physical sign is to be found on auscultation; this is a murmur, with its point of maximum intensity in the pulmonary area, systolic in time, usually rough and grating, and, it may be, extremely loud. The murmur may bc heard over a considerable area of the chest; occasionally it is quite distinct as high as the first rib; sometimes, indeed, all over the chest. As a rule, it has no direction of selcctive propagation, and in the vast majority of cases is not audible over the vessels of the neck. The pulmonary second sound is usually diminished or even absent; sometimes there is a pulmonary diastolic bruit present, owing to incompetence of the valve.

The physical signs of congenital stenosis at the aortic orifice are the same as those of the acquired form of the discase. In stenosis above the entrance of the ductus artcriosus, cyanosis is usually present, but the physical signs are indefinite; systolic and diastolic bruits may be audible over the front and back of the thorax. In typical eases of the "adult" type of coarctation of the aorta, a number of thickened tortuous arteries can be both seen and felt pulsating beneath the skin of the back and front of the trunk.

When a patent ductus arteriosus occurs alone, there may be no subjective symptoms, or they may be only slight, such as slight cyanosis and dyspnœa on exertion. The area of cardiac impairment is usually displaced to the right, also sometimes to the left, and other evidence of enlargement of the right heart, such as systolic retraction, 
may also be present. According to the late G. A. Gibson, there is sometimes a certain amount of fulness, a thrill, and a murmur, having their point of maximum intensity in the second left intercostal space at least two inches from the middle line. The murmur is prolonged, and occupies both systole and diastole; it commences shortly after the beginning of the first sound, and extends into a considerable part of the diastole; indeed, it may persist almost throughout the whole cardiac cycle. It is not propagated to the vessels of the neck. The second sound in the pulmonary area may be accentuated. Other writers have noted the presence of systolic pulsation, a small area of impairment of percussion note, and a shadow on skiagraphic examination.

In congenital lesions at the tricuspid orifice, cyanosis, dysproa, and clubbing are not conspicuous features, and the latter is not infrequently entirely absent. The physical signs are the same as those of the acquired form of the disease.

Diagnosis. - While it is usually not difficult to say that congenital heart disease exists, it is a much more difficult task to diagnose the form of the disease, and, further, it should be remembered that several lesions often co-exist. In considering the question of diagnosis, the history .of the case, the subjective symptoms, the existence or otherwise of evidence of enlargement of the right side of the heart, and the time of occurrence, the point of maximum intensity, and direction of selective proragation of any existing murmur are all points which should be taken into account.

With regard to the diagnostic value of the various symptoms, it may be remarked that cyanosis, even of marked degree, may be present during an attack of acute bronchitis in infancy and early childhood, especially in anæmic and rickety subjects, and also in cretinism; and that clubbing of the fingers may exist in the acquired form of heart disease, more especially in mitral stenosis, 
while it may be absent in the slighter forms of congenital disease. It is also necessary to point out that in some cases of congenital heart disease the right side of the heart may be only slightly enlarged. The murmurs are usually systolic in time and loud, and their point of maximum intensity and direction of selective propagation are not so typical as those of the acquired form of heart disease. In cases of a non-functional systolic murmur having its point of maximum intensity over the pulmonary area, we should think of the possibility of congenital heart disease. It may, however, be exceedingly difficult, and indeed impossible, to distinguish functional basic murmurs from those of congenital heart disease during infancy and childhood by means of auscultation alone.

In those subjects of heart disease who have attained to adult life, and even in the case of young ehildren, it is difficult to say whether the lesion is congenital or acquired. If there be a history of marked cyanosis soon after birth, and there have been known to be clubbing of the fingers, evidence of enlargement of the right side of the heart, and a systolic thrill and murmur whose point of maximum intensity and direction of selective propagation be not so typical as those of the acquired form of heart disease present in early life, a positive diagnosis of the congenital form of heart disease is not difficult.

With regard to the question of the diagnosis of individual lesions, meso-cardia and dextro-cardia may be mistaken for extrinsic causes of displacement of the apex-beat to the right, such as occurs in left-sided pleural effusion and pneumo-thorax, and as the result of right-sided adherent pleura, chronic interstitial pneumonia, and fibroid pulmonary tuberculosis. In cases of patency of the inter-ventricular septum, the absence of marked cyanosis; and the physical signs already described, are points of diagnostic value. In patency of the foramen ovale occurring alone, the presence of subjective symptoms and of a murmur or murmurs as described, in association with an 
absence of evidence of hypertrophy of the right ventricle, are in favour of a positive diagnosis. With regard to the diagnosis of congenital pulmonary stenosis, if there be cyanosis, clubbing of the fingers, evidence of enlargement of the right side of the heart, a systolic thrill and murmur having their point of maximum intensity in the pulmonary area, and if the murmur be not audible over the vesscls of the neck, the diagnosis is easy; whereas the point of maximum intensity of the thrill and murmur is a mattcr of little importance, the fact that the murmur is not transmitted to the vessels of the neck is of the greatest value in differential diagnosis. Functional murmurs should always be excluded. The diagnosis of pulmonary from aortic stenosis presents great difficulties, for, as has been pointed out, a systolic murmur due to aortic stenosis may have its point of maximum intensity in the pulmonary area, and when this is the case the condition may well be mistaken for pulmonary stenosis. The differential diagnosis has been dealt with in Chapter XX. The clinical features of pulmonary stenosis may resemble those of patent ductus arteriosus, and the two conditions may co-exist. In patency of the ductus arteriosus, the point of maximum intensity of the murmur is further to the left, and occupies both systole and diastole.

The diagnosis of stenosis at the aortic orifice has been discussed in Chapter XX. A diagnosis of stenosis of the aorta above the entrance of the ductus arteriosus is rarely possible. A diagnosis of patency of the ductus arteriosus may be arrived at from a consideration of the characteristic physical signs previously described. It should be noted that a prolonged thrill and murmur occupying both systole and diastole may also be met with in arterio-venous aneurism. The diagnosis of lesions of the tricuspid and mitral orifices has been dealt with in Chapter XX.

Prognosis.-The prognosis of congenital heart disease 
exhibits wide variation, depending upon, among other things, the site, the nature and severity of the lesion, the foree of the right ventricular impulse, the size of the heart, the degree of integrity of the myocardium, the severity of the subjective symptoms, and the existence or otherwise of trieuspid incompetence.

If the defect be slight, as, for example, a patent foramen ovale or a small opening in the inter-ventrieular septum, a considerable proportion of cases live to an advanced agc without experiencing subjective symptoms; whereas if the defect be serious, the duration of life is brief in a large proportion of eases, and, indeed, the individual may die within a few hours. The degree of cyanosis is not absolutely reliable. The occurrenee of paroxysms of dyspnœa, syneopal attacks, and convulsions is a very unfavourable omen.

Meso-cardia, dextro-eardia, and absence of the pericardium are conditions compatible with long life. On the other hand life eannot exist with a bilocular heart. Trilocular heart is eompatible with life for some years, and, indeed, instances have been recorded in which individuals have lived to adult age; usually, however, the period of life is short. Persons suffering from septal defeets not infrequently live to an advaneed age. When patency of the inter-ventricular septum oceurs alone and the opening is not large, the individual may live to past middle life ; when, however, it occurs in association with other lesions, the prognosis depends upon the latter. The prognosis of an individual suffering from pulmonary stenosis depends upon, besides those points already mentioned, whether there is a patent inter-ventricular septum to afford relief to the over-distended right heart. Death may occur on the one hand within a few hours after birth, while, on the other, in the case of lesions of slighter grade life may be prolonged for 5,10 , or 20 years, and occasionally even to middle life ; indeed, if a patent inter-ventricular septum 
be present in these slighter lesions, cases have been recorded in which patients have lived to the age of 60 . Taking cases of pulmonary stenosis as a whole, however, most of the patients die at an early period of life. Pulmonary atresia is a much more serious condition, and life is prolonged for only a brief period. Cases of stenosis at the aortic orifice do not survive birth, and in those in which stenosis exists above the entrance of the ductus arteriosus life is prolonged at the outside for a few months only, but when just below the entrance of the duct it may be prolonged till middle age. Persistence of the ductus arteriosus is not infrequently compatible with adult life, and individuals have been known to have lived even to old age. Cases of transposition of the pulmonary artery and the aorta do not as a rule survive infancy, but instances have been recorded in which these patients have lived to middle age.

Treatment.-The general measures laid down in Chapter IX are applicable also to this affection. It is particularly important that the patient should be kept warm, and it is imperative that careful and detailed measures should be adopted for the prevention of bronchitis; if bronchitis should occur, adequate treatment should be adopted without delay. When cardiac failure supervenes, it should be treated on the lines previously laid down. 


\section{CHAPTER XXV}

\section{PRIMARY CARDIAC OVERSTRAIN}

By primary cardiac overstrain is meant a cardiac disorder which is the immediate result of excessive physical exertion in an individual whose heart has been sound, or in whom the heart has becn already somewhat impaired. It includes the condition known as "soldier's heart" or the "irritable heart of soldiers," first described by Da Costa and Myers (see *, p. 378). It is necessary to point out, however, that in the light of recent discoveries many of the cases described by these writcrs were undoubtedly cases of auricular fibrillation, the onset of the latter condition having in many cases been associated with undue physical exertion. The rcader is referred to the writings of Clifford Allbutt on the subject of primary cardiac overstrain for a fuller description of the malady.

Etiology.-The affection is the direct result of excessive physical exertion, and is most frequently met with in persons of athletic habits and in soldiers. It is more apt to occur in those who undergo excessive physical exertion while not in training; indeed, it is questioned by some whether the affection can occur in persons with previously sound hearts who have been properly trained. It is also more likely to occur in older persons, and where there is a history of rheumatism or of some other acute infection, in anæmia, and in those who have indulged in excesses of any kind. In the case of soldiers, the excessive physical exertion may be long continued, while tight belts and closely fitting uniforms appear to embarrass 
the action of the heart, and thus predispose to the condition.

Symptomatology. - When the malady is due to sudden physical strain, as in boat-racing or Alpine climbing, there may be lividity or cyanosis of the face, giddiness, faintness passing into actual syncope, together with vomiting and even fatal syncope, and possibly evidence of acute dilatation of the right heart. Later on, there may be collapse, cyanosis, coldness of the skin, precordial uncasiness and even actual pain, quick and shallow respiration, and a quickened, small and thready pulse; while later, in severe cases, there may be evidence of œdema of the lungs. These acute symptoms may be followed by general lassitude, impaired digestive functions, an increase of rectal temperature, and a pulse of subnormal pressure, although at the same time the apex-beat may be forcible and tumultuous; tachycardia, and a forcible and tumultuous apex-beat being especially noticeable on the least exertion. For some weeks, dyspnœea and an increase of cardiac rate from 30 to 40 per minute above the normal on even slight physical exertion, nervousness, languor, and a sense of fatigue on mental exertion arc apt to occur.

In the chronic form of the affection, such as occurs in soldiers or in older people, among the symptoms are palpitation, even of severe degree, a feeling of oppression or of constriction of the chest, and sometimes actual pain over the precordium, extending to the left shoulder, and occasionally down the arm; as a rule there is no sense of impending death, although cases of soldiers collapsing during a battle apparently because of the pain have been recorded. Among other symptoms which may be noted are dyspnœa and faintness on exertion, tremors, nervousness, insomnia, noises in the ears, flashes of light before the eyes, vertigo, a dull headache, hebetude, and languor, while the digestive functions are often impaired. There may be some degree 
of cyanosis. These symptoms may pass away with rest, only to reappear on exertion. The pulse-rate is usually increased, but is rarely over 120 per minute while at rest, although exertion causes a further increase-up to as much as 140 or 150 . The pulse is usually small, weak, and of subnormal pressure, whilc the apex-beat may be diffuse and diminished in force or even absent, but on cxertion may become unduly forcible and tumultuous. The arca of eardiac impairment is usually increased to the left, and not infrequently also to the right. The cardiac sounds may be feeble, or short and sharp, and may be reduplicated, or a mitral or tricuspid systolic murmur may be audible, while the sccond sound in the pulmonary area may be accentuated.

Diagnosis.-The diagnosis in large measure resolves itself into the question whether the case is one of primary cardiac overstrain, or whether the heart has been previously damaged; in the latter case, excessive physical exertion is merely the exciting cause. In considering this problem, the presence or absence of a previous history of one of the acute infections and the state of the general health, and whether there is evidence or otherwise of artcrial, myocardial, or valvular disease, are points of material importance.

Prognosis.-The prognosis of primary cardiac overstrain exhibits a wide variation, ranging from a slight illness on the one hand to a severe and even irremediable affection on the other. The prognosis is, as a rule, better when the malady is due to physical over-exertion in persons of athletic habits than in the case of soldiers. In the less severe degree of acute cases occurring in the young and previously sound and vigorous, recovery is the rule; the patient may be well in a few days or weeks, although, on the other hand, progress may be slow, and a long time may elapse before the individual is capable of an ordinary amount of work. If tricuspid regurgitation be present, this, unlike mitral regurgitation, usually passes 
off quickly. But if the heart have been previously affected by rheumatism or by some other infection, and in the case of older persons, the prognosis is serious ; recovery may be long delayed, or the patient may be always compelled to lead a sedentary life, and, in some cases, even a fatal termination may result.

The prognosis among soldiers is, generally speaking, not good; indeed, a majority are not able to return to their occupation. In all cases of primary cardiae overstrain a diminishing increase of pulse-rate on exertion is of favourable omen.

Treatment.-In the acute cases, Michell reeommends a very hot bath, the patient being lifted out of it in a blanket, or a hot pack, together with the administration of one or two fifteen-grain doses of diuretin. A strietly limited amount of food, both solid and liquid, is indicated in the early stages, and no tea, coffee, alcohol, or tobacco. Oxygen may be required in some cases; morphia and strychnine are rarely necessary. Later on, tea and coffee may be allowed in limited quantities, but the patient should still abstain from alcohol and tobacco. The bromides are often extremely useful in persons who suffer from an unduly excitable nervous system or from insomnia. When the patient is able to get up, extreme caution should be enjoined as to the amount and character of any exertion. The use of electricity is contra-indicated. Warm baths, followed by a cold douche, may in individual cases be tried, if followed by a reaction. Such general tonics as iron, arsenic, and quinine may be indicated.

In the more severe or chronic cases, prolonged physical and mental rest may be necessary, although massage may be employed at an early stage with advantage, provided it is at first very gentle and at all times stops short of causing fatigue. The diet should be small in quantity, nutritious, and easily digestible, and if atonic dyspepsia be present it should be treated. 
* Since these remarks were written, considerable attention has been paid to the subject of "soldier's heart," owing to the very considerable number of cases answering this deseription which have arisen, apparently as a result of the conditions attendant upon the life of the soldier in modern warfare. In this connection, the reader is referred to a discussion which took place at the Royal Society of Medicine. It would almost appear as if the terrible strain which has proved to be peculiar to active service at the present time have brought to light certain factors in the production of cardiac overstrain which have hitherto, if indeed they existed, been unrecognised.

Speaking broadly, so far as present investigations have gone, it may be assumed that in a large proportion of the cases of soldiers invalided home, beeause of so-called heart affections, the heart is not the "fons et origo" of the malady, and the circulatory symptoms are but a manifestation of the general state.

On enquiry, it has been found that in many of these cases there has been a history of some slight illness, such as an antecedent diarrhœa, or a febrile attack pointing to some infection, and that the most prominent symptom is one which has been described as a sense of extreme exhaustion or collapse.

In an excellent summary of these eases, Sir James Mackenzie has laid down some fundamental distinctions in their symptomatology from that of primary cardiac disease. As already stated, the sense of exhaustion after effort is very prominent. There are often also giddiness and faintness, which may disappear on lying down. There is a sense of profound depression, often aceompanied by irritability of temper, while vaso-motor instability in varying forms is also a marked feature of the condition. When cardiac uneasiness or pain is complained of, it does not necessarily follow exertion, and very frequently comes on when the patient is at rest.

In those who suffer from chronic heart disease, on the other hand, the first subjective symptoms are primarily cardiac, and usually arise as a result of effort. Of these, breathlessness, precordial uneasiness or even pain, often associated with palpitation, are the most prominent; the nervous and vasomotor symptoms, so eharacteristic of the poisoned heart, are little, if at all, in evidence. 
While there is much to be said for the theory that these cases are the result of toxic poisoning following some microbic invasion, the practical evidence is, so far, too vague for dogmatism; and much further investigation, from a bacterial point of view, would be necessary before it can be accepted as proved. But what the toxins may be or how produced, does not so far invalidate the results of the treatment advocated by Mackenzie, and clinical results, even if $\mathrm{cm}-$ pirical, are often in advance of established scientific data.

The treatment is mainly based on the assumption that the root of the malady lies in the central nervous system, the cardiac symptoms being merely incidental.

The method of treatment emphasised by Sir James Mackenzie aims briefly at the elimination of any toxic content of the blood, and the restoration of the patient's general bodily and mental health. To this end he advocates fresh air, and judicious exercise, especially in the form of games to begin with, laying special stress on any form of recreation which the patient may individually enjoy. By healthy and pleasurable preoccupation of this kind, the sense of depression and exhaustion so characteristic of these cases is removed, and the outlook for an early return to ordinary exercise and duty, than would otherwise be the case, is much enhanced. 


\section{CHAPTER XXVI}

\section{ANGINA PECTORIS}

The term angina pectoris is usually employed to denote a paroxysmal attack of pain in the region of the heart, with a tendeney to radiate in eertain directions, and often accompanied by a feeling of constriction within the ehest and a sense of impending death.

Etiology.-Heredity is a factor, for it is well known that certain families are more prone to degenerative proeesses of the arterial system and of the heart than others. The affeetion is mueh more frequent in males than in females, and is found more particularly in those who are passing from middle into elderly life. It is much more prevalent in those classes of socicty who are subject to excessive mental wear-and-tear, whether from overwork, worry, or anxiety, and is commoner in those who have been subjected to long-continued physical overstrain. The other important predisposing causes are gout, syphilis, rheumatism, some of the acute infectious diseases, especially influenza and enteric fever, and toxic chemical substances, such as alcohol, and perhaps tea, coffee, and tobacco. In persons under 40, syphilis is an important causal factor.

The commonest structural changes found in angina pectoris are disease of the coronary arteries, acute or chronic aortitis, chronic disease of the aortic and much less commonly of the mitral valves, adherent pericardium, fatty or fibroid degeneration of the myocardium, fatty infiltration, arterio-sclerosis, fusiform dilatation of the aorta, and sacculated aneurism. Narrowing or occlusion of one 
or both coronary arteries is believed to be the characteristic lesion in the gravest cases, and there are some who believe that coronary disease constitutes the essential pathology of angina pectoris. Arterio-sclerosis, from whatever cause, predisposes to the disease and is present in a majority of patients, and in many there is in addition super-normal blood-pressure.

Among the exciting causes which induce attacks, the most common is physical exertion, such as walking uphill or hurrying to catch a train, especially after meals and against a cold wind; sometimes even the effort of dressing, or stooping to lace the boots, is sufficient to precipitate an attack. Other exciting causes may be cold in the sense of getting chilled from whatever cause, emotional excitement, less frequently mental effort, and dyspepsia-particularly those forms attended with flatulent distension of the stomach or colon. It must be remembered, however, that flatulent distension is also a frequent concomitant of the paroxysm. Only rarely do attacks occur apart from some exciting causc; occasionally the attack comes on whilst the patient is in bed, but this happens usually at the end of a fatiguing day.

Symptomatology.-As has been already stated, angina pectoris is characterised by a paroxysmal attack of pain in the region of the heart, with a tendency to radiate in certain directions, and often accompanied by a feeling of constriction within the chest and a sense of impending death.

Pain is the cardinal feature, and its essential character is its paroxysmal nature. It is always sudden in onset. In some cases the attack attains its maximum with extraordinary suddenness, while in other instances it happens gradually.

The pain is felt most commonly over the lower sternal region, rather to the left side. Less frequently it is felt over the upper sternal region, and still less frequently in one side of the chest or the other; when the latter is the 
case it is usually on the left side. I have seen two cases in which the pain was strictly limited to the epigastrium. The pain, as stated, has a tendency to radiate in certain directions. It frequently spreads to the left.shoulder and armpit; occasionally to both sides; in many cases down the arm, generally on the ulnar side, usually not beyond the elbow, although it may reach as far as the finger tips. It sometimes strikes through to near the angle of the scapula, on one or both sides, or to a wide area on the back of the chest. Oecasionally it extends to the neck and occiput, or to the jaw, and rarely it radiates in a downward direction, being felt even in the lower part of the trunk and lower limbs. The pain may begin in the left arm, in the jaw, the left pectoral muscle, the left leg, or in the abdomen - the so-called angina abdominisand remain localised; in the case of the last of these being sometimes mistaken for gastralgia. The severity of the pain varies from slight discomfort to intense anguish; it is no guide to the degree of the structural changes present.

The feeling of constriction in the chest already referred to is described in different terms by different individuals ; in some cases the sensation is as if the chest were held in a vice, in others as though a heavy weight were pressing on the chest. When the sensation of impending death is present, this overshadows the most severe pain. It is possible that this feeling is always present in some degree in every paroxysm of angina pectoris, and it may be present without the pain-the "angina sine dolore" of Cairdner.

When an attack occurs, the patient, if in motion, stops short and rests, the expression of the face being even one of anguish or intense anxiety. The skin is usually pallid or even cadaveric in hue, and there is frequently a clammy sweat. The breathing is shallow, and the patient is afraid to make an effort to breathe normally. There may be a sensation of numbness, more particularly 
of the arm ; this may be present only during a paroxysm, while in other cases it is a constant symptom. Among other subjective phenomena which may be present are tingling and giddiness, while hyperæsthesia over the painful area may often be noted. In almost every grave case, the patient complains of a sensation of sinking, and of a feeling as if the heart were stopping, but rarely loses consciousness, unless fatal syncope occurs. The pulserate seems to vary in different cases; in some it is increased, while in others it is diminished. Very considerable changes are frequently found in the character of

Drug. Hour. Hlood-pressure. Sensations.

\begin{tabular}{|c|c|c|c|c|}
\hline Drug. & Hou & & Blood-pressure & Sensations. \\
\hline & $\begin{array}{l}1.23 \mathrm{r} \\
1.25\end{array}$ & $\mathrm{~m}$. & $\begin{array}{l}120 \mathrm{~mm} \text {. } \\
142\end{array}$ & $\begin{array}{l}\text { Before attack. } \\
\text { Pain fairly severe. }\end{array}$ \\
\hline ( & 1.26 & , & 142, & 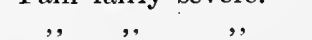 \\
\hline & 1.28 &, & 144 & , \\
\hline & 1.29 & , & 152 & Pain worse. \\
\hline & 1.30 & , & 150 & \\
\hline & 1.31 &, & $150,$, & \\
\hline myl nitrite, min. v. $\gamma$ & 1.32 & ,", & 154 & Says pain is no better. \\
\hline & 1.33 & 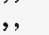 & 140 & \\
\hline & 1.34 & , & 130 & Pain egsier \\
\hline . & 1.35 & , & 130 & Pain easier. \\
\hline & 1.36 & , & 128 & Still better. \\
\hline & 1.37 & , , & 128 & Pain nearly gone. \\
\hline & 1.45 & & 120 & No pain. \\
\hline
\end{tabular}

Fic. 164. Shows the rise of blood-pressure during an attack of angina pectoris, and the typical result of the inhalation of amyl nitrite.

the pulse in different cases; at times it appears to be quite unaffected; at others it may become irregular, and different forms of irregularity have been observed, but there does not seem to be anything definitely known on the subject. The blood-pressure is often considerably increased at the crisis of the attack (Fig. 164), but it should be remembered that this is not by any means a universal rule. Some writers have stated that the area of cardiac impairment has been found to be considerably increased, and the hcart sounds are said to be occasionally altered in character. The patient may yawn continuously, and there may be hiccough, flatulence, retching, or vomiting. 
The attack as a rule passes off gradually, but sometimes ccases suddenly; as it subsides the patient may belch up a large quantity of air, and it is often followed by the passage of abundant pale urine. After the attack the paticnt may be much exhausted, and may feel ill for some days. Death may occur at the height of an attack, and this may take place even in the first.

The attack may last for a few seconds, a few minutes, or longer; so frequently may they recur that for hours or cven days the patient appears to be suffering from one continuous attack-status anginosus.

The above description is that of a typical and severe attack, but there are many grades and modified forms. For cxample, there may be on physical exertion or cmotion only a little discomfort or pain felt over the sternum, radiating to the arm and neck, and accompanied by a feeling of uneasiness and distress, which may at first disappear as soon as the existing cause ccases, but at a later stage may continue for some minutes after its cessation.

Diagnosis.-A correct diagnosis of angina pectoris is practically always possible if full advantage be taken of all the avenues of investigation in each case. The age, sex, and previous history of the patient, the site of the pain, its paroxysmal character, its severity, its tendency to radiate in certain directions, the fact that it is often first noticed during or after exertion, that it is often accompanied by a feeling of constriction within the chest and a sense of impending death, and evidence of cardio-vascular disease, together go to form a typical clinical picture.

Among the conditions which should be excluded are pseudo-angina, aneurism, pleurodynia, acute pleurisy, cervico-brachial neuritis, and colic, whether of gastric, hepatic, or renal origin. The differential diagnosis between the true or grave form of angina pectoris and pseudoangina will be discussed later.

In pleurodynia, the pain is not paroxysmal in character, 
its distribution is unilateral, its severity is often increased by deep respiration, and pressure along the dorsal vertebræ and in the course of the intercostal nerves frequently clicits tenderness.

It has been already pointed out that the distribution of the pain of angina pectoris sometimes resembles that of gastric colic, namely in the epigastrium, left hypochondrium, and left interscapular region, and it should be further borne in mind that relief in both complaints is often accompanied by gaseous eructations. In hepatic and renal colic, the pain is often sudden in onset and extremely severe, and in some cases it radiates in the same direction as that of angina pectoris. Among the points which should be taken into consideration in the differential diagnosis of hepatic colic and angina pectoris are the following: In hepatic colic, the pain is situated in the right hypochondrium or epigastrium, and is of longer duration; the patient, instead of being rendered immobile, cannot keep still; the tendency of the pain is to radiate rather to the right shoulder and never down the left arm; there is an absence of the sense of impending death, nausea and vomiting occur, and perhaps jaundice; and there may also be tenderness over the gall-bladder, while there is no evidence of heart disease. In renal colic, the site of the pain is in the flank, radiating to the genitalia, its duration is longer, and is accompanied or followed by changes in the urinc, with no evidence of cardiac disease.

Prognosis.-This is very difficult. While the proportion of cases in which the immediate outlook is grave is not large, and while the great majority of other cascs respond to treatment to a very considerable extent, the prognosis is usually serious, the occurrence of sudden death being not uncommon.

In trying to forecast the future, it is necessary to consider (1) the nature and severity of the underlying cause, 
and (2) the frequency and severity of the attacks. With regard to the first, naturally the outlook is much better when the disorder is due to fatty infiltration than when associated with coronary disease, fatty or fibroid degeneration of the myocardium, or general arteriosclerosis. In trying to decide whether the latter group of causes is present, the importance of the functional efficiency of the heart has been noted; in this connection, it is necessary to point out that obvious signs of heart discase are absent in a certain proportion of cases in which the coronary arteries are extensively involved. When there is no doubt that the case should be placed in the latter group, the outlook is usually grave, and is still more so if in addition there be aortic incompetence or chronic renal disease. At the same time, much may sometimes be done by competent treatment. A point of great importance is to ascertain how far the patient is able to modify his life so as to bring it within the limits of the heart's strength, and also the response the paticnt makes to treatment. Thus, patients under 40, with a history of syphilis, may completely recover with appropriate treatment. Again, if the blood-pressure be high, and the attacks be associated with vaso-motor spasm, as these can sometimes be suitably treated, the outlook is better than when such is not the case; experience will show whether the high blood-pressure and the vaso-motor spasm can be relieved by treatment.

Secondly, it is obvious that the more frequent and severe the attacks, and if provoked by slight exciting causes, the worse the prognosis. In the severer forms, the prognosis is usually grave, and there is always the probability of recurrence, with liability to sudden death. When the attacks occur with increasing severity and upon slight exertion in an elderly subject, there is a great risk that death will occur in an attack; nevertheless, even in these cases, suitable treatment may sometimes be rewarded with comparative success. Very rarely is the 
disease fatal in the first attack. Apparently there is a tendency for the paroxysms to become less frequent and less severe if dilatation of the heart supervene.

Treatment.-The treatment resolves itself into two parts : (1) prevention, if possible, of the recurrence of the paroxysm ; and (2) an endeavour to relieve it.

With regard to the first of these, we should try to improve the general health, more especially the circulatory and nervous systems ; and since the disease occurs usually in association with exhaustion of the cardiac muscle, a cardinal indication is to try to remedy this condition, and to prevent its recurrence. In this connection, it is advisable first of all to review the etiology, and to endeavour to find out the cause of this exhaustion, and for this purpose it will be necessary to inquire in detail into the past and present life of the patient.

In all cases the clear indication is to enjoin rest, proportionate to the degree of cardiac failure, and with this object in view it may be necessary to order complete rest in bed for some time, and even to give sedatives, such as chloral or opium. Morphine in most cases of this kind is more useful than opium. After a period of rest, explicit instructions should be given that in future the patient should live within the limits of the heart's strength, the daily life, physical and mental, being carefully regulated. He should avoid exertion which produces breathlessness, palpitation, or fatigue; as well as hurry, sudden effort, walking or hurrying against a cold wind, and cold in the sense of getting chilled. Cold sheets at night, and a cold bath in the morning, should be prohibited. It should not be forgotten that the nervous system may be a factor in the production of angina pectoris, and when there is reason to believe that this is the case, appropriate measures should be employed. When the patient has suffered from a period of mental strain or sleeplessness, and, in- 
decd, whenever there is any excitability or irritability of the nervous system, the administration of bromide of ammonium, in doses of 10-20 grains three times a day, is often of great value. Emotional excitement, gastrointestinal dyspepsia-particularly that form which is accompanicd by flatulent distension-constipation, and all other possible exciting causes of an attack should be carefully treated. Over-indulgence in food, alcohol, or tobacco should be avoided. Flannel should be worn next to the skin, both summer and winter. It is sometimes advisable to improve the general health by the administration of tonics, such as iron, arsenic, quinine, cod-liver oil, and the hypophosphites. When there is reason to believe that syphilis is the cause, appropriate and energetic anti-syphilitic treatment should be employed. Similarly, if high tension be present, this should be treated on the lines laid down in Chapter IV. In fact, we should revicw the whole of the causes of angina pectoris, predisposing and exciting, with a view to treatment.

Of drugs which may be given with the hope of preventing an attack, the most hopeful are iodide of potassium and nitroglycerine. The former is probably in most respects the best, and is particularly indicated when arteriosclerosis is present, more especially if there be a history of syphilis. In some cases, 5 gr. doses are sufficient, while in others the drug should be pushed. Sometimes, syrup of hydriodic acid, in doses of from $\frac{1}{2}$ to 2 drachms, well diluted with water, is better tolerated. Nitroglycerine may be given in the form of tablets, or the 1 per cent solution of trinitrin. The usual dose is $\frac{1}{10}$ grain ; this may be given three or four times a day, and gradually increased to $\frac{1}{20}$ grain or even $\frac{1}{10}$ grain. Either drug should be given intermittently for two or three weeks at a time, while in some cases it is good to alternate them. Sodium nitrite, erythrol tetranitrate, mannitol nitrate, and nitrite of potash are other drugs which may occasion- 
ally be employed. Taken altogether, the nitrites are not so valuable as the iodides.

For the paroxysm, the most efficacious remedy in most cases is the inhalation of nitrite of amyl ; the action of the drug is almost immediate, and the relief is usually complete, this being particularly the case with widespread arterial constriction (Fig. 164). It is best administered in the form of the glass capsules or perles, each containing from 2-5 min., which the patient should carry about with him. This dose may have to be repeated. A similar effect may be obtained by nitroglycerine, but its action is not so rapid, although its effect is more prolonged. The liquor trinitrini may be given, beginning with one, and increasing, if necessary, to $5 \mathrm{~min}$. doses, in a little water ; or, the patient may nibble one or more of the tabellæ nitroglycerini until the pain disappears. If flatulent distension be present, a draught of carminative mixture, or, if this be not available, some very hot water with a little essence of peppermint or brandy, sipped slowly, may be given with the amyl inhalation or the nitroglycerine drops. In cases in which there is pronounced heart failure, brandy or ether, preferably the latter, in doses of 20 to $60 \mathrm{~min}$., in which 1-2 min. of a 1 per cent solution of trinitrin may be dissolved, may be injected subcutancously. Erythrol tetranitrate and sodium nitrite are not so suitable.

If the drugs enumerated fail, chloral by the mouth, hypodermic injections of morphine, or inhalations of chloroform, should be employed. Twenty grains of chloral hydrate may be tried. As a rule it is necessary to give morphine in large doses $-\frac{1}{4}-\frac{1}{3}$ grain. Nitroglycerine may be combined with the morphine. The nitrites and chloroform are not of such value when there is no decided arterial spasm as in cases in which it is present. Where there is cyanosis or asthma, oxygen inhalations may be tried, particularly when morphine is found necessary. 
If the drugs enumerated are not at hand, hot drinks, water containing whisky, brandy, capsicum, or spirits of chloroform, or a carminative mixture, may do good by the relief of flatulence.

\section{Pseudo-angina}

The term pseudo-angina is used by some writers to denote a paroxysmal attack of pain in the region of the heart, independent of cardio-vascular lesions. While, however, this is the ease, it should be remembered that it may be met with in individuals who are the subjects of valvular disease.

The malady is much more frequent in females than in males, and while it is found more particularly between the ages of twenty-five and fifty, it may oecur at any age. It is not infrequently associated with hysteria, neurasthenia, or toxic eauses. While the exeiting eauses are the same as those of the true form of angina pectoris, physical exertion does not play such an important part; cold, emotional excitement, and perhaps dyspepsia, are, on the other hand, more important factors.

The pain is usually less severe than in the true form of angina, and is usually accompanied by a fecling of distension instead of constriction. The patient may complain of shortness of breath, palpitation, and a feeling of faintness. The pulse is small, and its frequeney is usually inereased. The attack sometimes lasts for an hour or longer, and may be followed by the passage of abundant pale urine, the patient often complaining also of a sense of fatigue.

Pseudo-angina includes at least two groups of cases, namely, (1) the neurotic, and (2) the vaso-motor or toxic. The first group includes the hysterical and neurasthenic cases, while the toxic group includes cases which are due to the excessive use of tobacco, and perhaps tea or coffee, and those which follow the acute infections-particularly influenza.

In the neurotic cases the attacks are not infrequently 
periodic and nocturnal and induced by emotional excitement. Instead of becoming or remaining immobile, in a number of cases the patient moves about in an agitated manner, and even rushing to the window for air. Associated nervous symptoms may be present; in hysterical subjects there may be points of hyperæsthesia, and anxsthetic areas. The paroxysms show a tendency to recur for some hours, or even days, following the attack.

In the vaso-motor or toxic variety, the individuals are not infrequently the subjects of vaso-motor disturbances, such as numbness, coldness, and pallor or lividity of the extremities; while in those cases associated with the excessive use of tobacco, symptoms of nicotine poisoning, such as tobacco amblyopia, may be present.

Diagnosis.-The differential diagnosis between true and pseudo-angina is as a rule not difficult. In its consideration, the age, the sex, the previous history, the presence or absence of evidence of cardio-vascular lesions, the exciting cause and character of the attack, its response to treatment, as well as the other distinguishing features already described, should all be taken into account.

Prognosis.-The prognosis with regard to life is good, the attack never proving fatal, and usually having little effect on the duration of life. Further, with suitable management and treatment, there is a good chance of complete cure ; the neurotic forms, however, tend to recur. In those cases of toxic origin, it is essential that the causal factor should be removed; when abuse of tobacco is responsible, however, the complaint often persists for a long time.

Treatment.-Our first duty is to reassure the patient. We should review the etiology of the malady, and measures appropriate to each case, as, for example, when the condition is associated with hysteria, neurasthenia, or the excessive use of tobacco, should be adopted. Further, all possible exciting causes should be prevented or treated. Anti-neuralgic therapeutical agencies are more likely to be efficacious than the use of vaso-dilators. 
For the paroxysm, very hot water, sipped slowly, may be tried; while, if this be not successful, diffusible stimulants may be employcd, although their frequent use is not to be encouraged. If these be of no avail, use may have to be made of morphia. It may be of service for the patient to carry about with him a carminative mixture, a dose of which may be taken when an attack is threatened. 


\section{CHAPTER XXVII}

\section{CLINICAL ELECTRO-CARDIOGRAPHY ${ }^{1}$}

IT has been known for a considerable time that changes in electric potential take place in muscle when it contracts, and, further, that a record of these changes may be obtained by connecting the muscle with a sensitive galvanometer by means of electrodes.

A. D. Waller, in 1887, employed a capillary electrometer to register the changes in electric potential in the human heart during contraction. He demonstrated that these changes were distributed through the body, and he used the moist skin surfaces of the arms and legs as leads, connecting them with a galvanometer.

Einthoven employed the string galvanometer to register the changes in electric potential in the human heart. $\mathrm{He}$ modified this instrument, the Einthoven string galvanometer being now generally employed in physiological and clinical investigations. ${ }^{2}$ In the Einthoven galvanometer there is an exceedingly fine silvered glass fibre, which is suspended in a box, and placed vertically between the poles of a powerful electro-magnet. When a current passes through this fibre, minute movements of the fibre take place. The shadow of the oscillating fibre is magni-

1 The electro-cardiograms chosen for the purpose of illustration were considered the most representative of those taken from about 15,000 cases.

Horizontal lines; $1 \mathrm{~cm} .=1 / 1,000$ volt.

Vertical lines; thick $=1 / 5$ sec., thin $=1 / 25$ sec.

In most of the figures, the lead is marked in the upper left-hand corner.

2 The electro-cardiograph supplied by the Cambridge Scientific Instrument Co., Ltd., is, in my opinion, the most satisfactory. 
fied, and projected on to a photographic sereen, and in this way a photograph of these movements is obtained. The sensibility of the fibre can be adjusted by altering the tension, the standard generally adopted being that of Einthoven, i.e. that when a difference of potential of one millivolt is passed through the fibre, this gives a deflection on the plate of $1 \mathrm{~cm}$. in amplitude.

Following Einthoven, the eommon practice is to accept
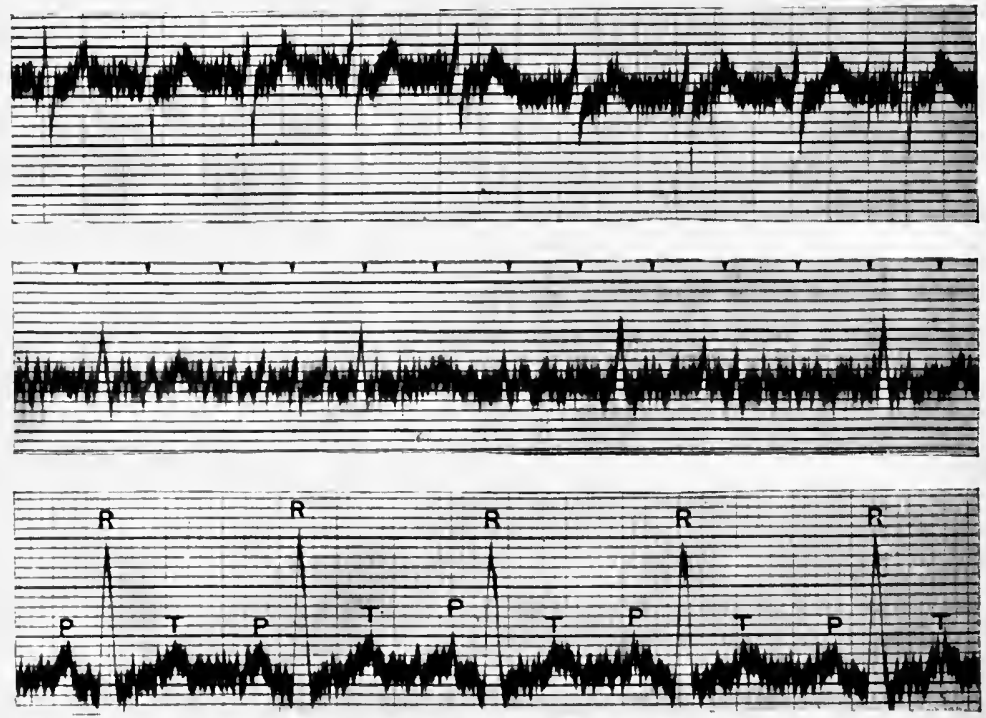

Figs. 165, 166, and 167. Electro-cardiograms from three different subjects, showing oscillations resulting from tremors of the somatic muscles.

three leads and the following nomenclature, i.e. lead I, or the transverse, a lead from the right and left hands; lead II, or the axial, from the right hand and left foot; lead III, or the left lateral, from the left hand and left foot.

In taking an electro-cardiographic record, it is important that the patient should not move, that the skeletal muscles should be relaxed, and breathing should not be deep. Muscular contractions of the hands or feet, or 
such movements as coughing or sneezing, cause coarse and irregular vibrations of the string; while oscillations may accompany deep breathing, or may result from tremor of the somatic muscles (Figs. 165-7). It may be here noted that those oscillations which accompany deep breathing are much slower than those due to cardiac action. Oseillations resulting from tremor of the somatic muscles somewhat resemble those due to auricular fibrillation (Figs. 209-219) ; the differential diagnosis will be discussed later.

Before proceeding to take a record, it may be advisable to take a short control before connecting the instrument with the patient, in order to exclude vibrations of the string due to extraneous causes.

The record of the changes in eleetric potential which

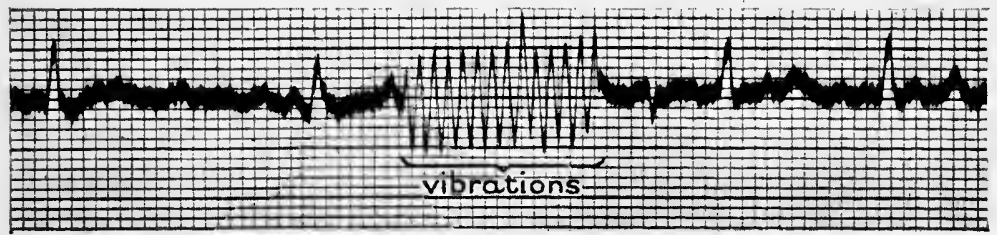

FIG. 168. Electro-cardiogram, showing oscillations, due to telephone bell ringing.

take place in the heart during contraction is ealled an electro-cardiogram.

If a normal electro-cardiogram be studied, certain upward and downward deflections are seen in each eardiae cycle, the former being the more important (Figs. 169 and 170). These deflections may exhibit considerable variations in amplitude and form. The lettering of the peaks adopted in this volume is that of Einthoven.

The first deflection, $\boldsymbol{P}$, is due to the contraction of the auricles; the others are the result of the contraetion of the ventricles, and are termed $Q, R, S$, and $T$, in some instances $T$ being followed by $U . R$ and $T$ are the most constant deflections, the former more especially so; $\boldsymbol{Q}$ and $S$ are not infrequently absent; while the $U$ deflec- 
tion is of very uncommon occurrence. $\boldsymbol{P}$ is a small, blunt, and rounded, upward deflection, $Q$ and $S$ are downward, steep, defleetions, usually of small amplitude. $\boldsymbol{Q}$ passes

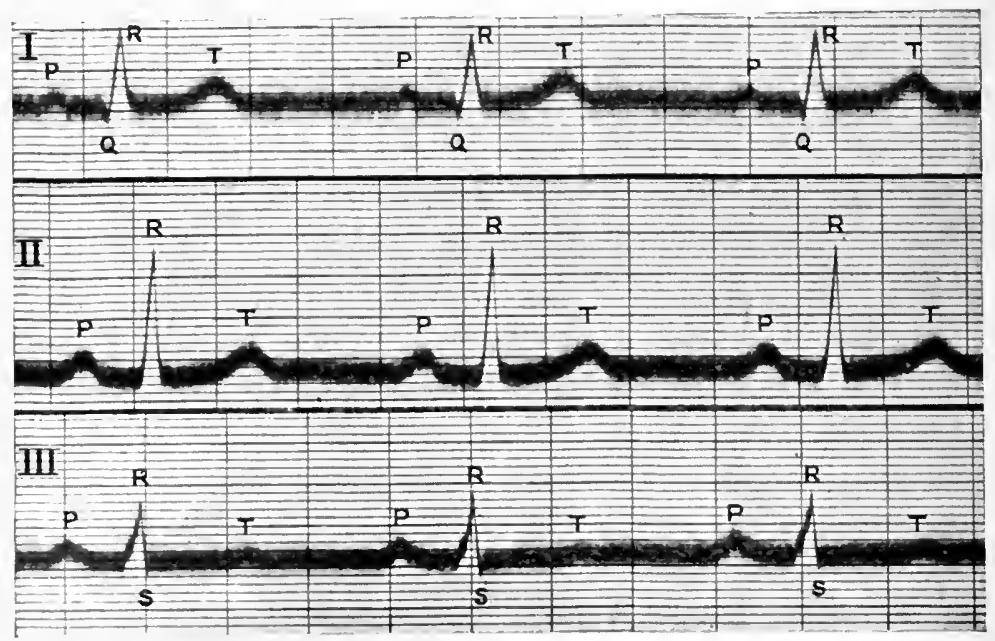

Fig, 169. Electro-cardiogram of a normal subject. The first deflection, $P$, is due to the contraction of the auricles; the others are the result of the contraction of the ventricles, and are termed $Q, R, S$, and $T$. In normal subjects the amplitude of all the defloctions is usually greatest in lead II, especially the deflection $R$.

at once into $\boldsymbol{R}$, which is an upward sharp spike, and of greater amplitude than any of the other deflections.

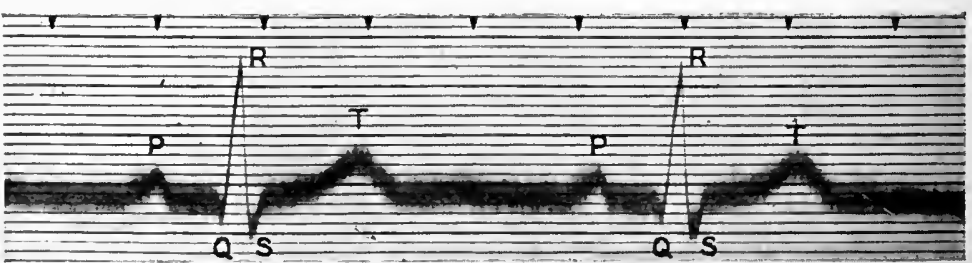

Fig. 170. A normal electro-cardiogram.

If $S$ be present, it follows immediately upon $R$. During the interval between $R$ or $S$, as the case may be, and $T$, the string remains at zero. $T$ is a prominent broad, blunt deflection. It is an upward deflection in lead II; it. is 


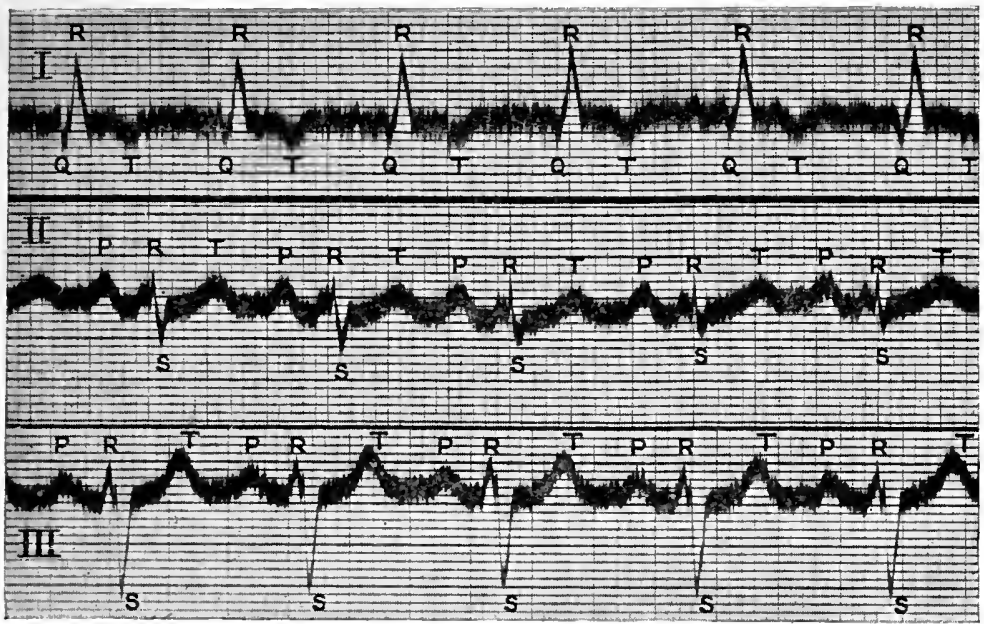

FIG. 171. Electro-cardiogram showing inversion of $T$ in lead I. There is also left-sided preponderance.

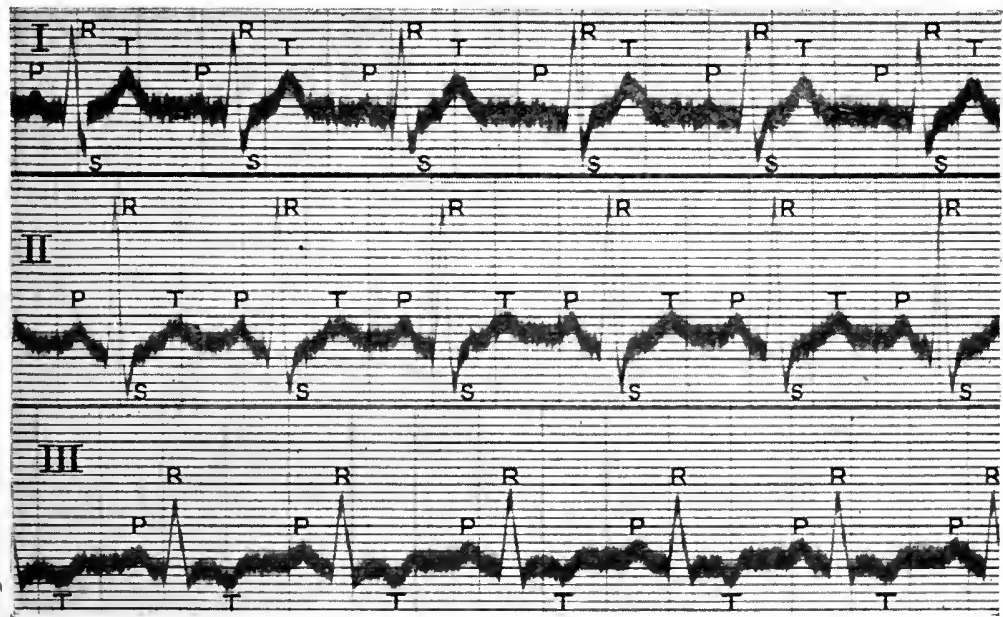

FrG. 172. Electro-cardiogram showing inversion of $T$ in lead III.

almost always an upward deflection, but may be inverted in lead I (Figs. 171, 173, 190, 193, 194, 195, and 219); and is not infrequently inverted in lead III (Figs. 172, 173, 202, and 238). It is said that inversion of $T$ in lead II (Figs. 

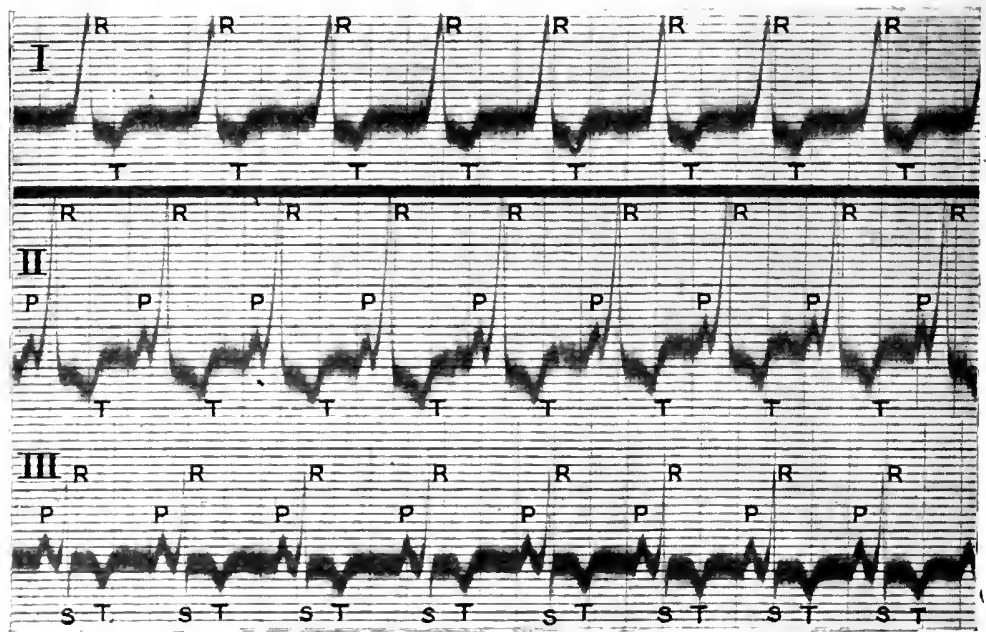

FIa. 173. Electro-cardiogram showing inversion of $T$ in all the leads.

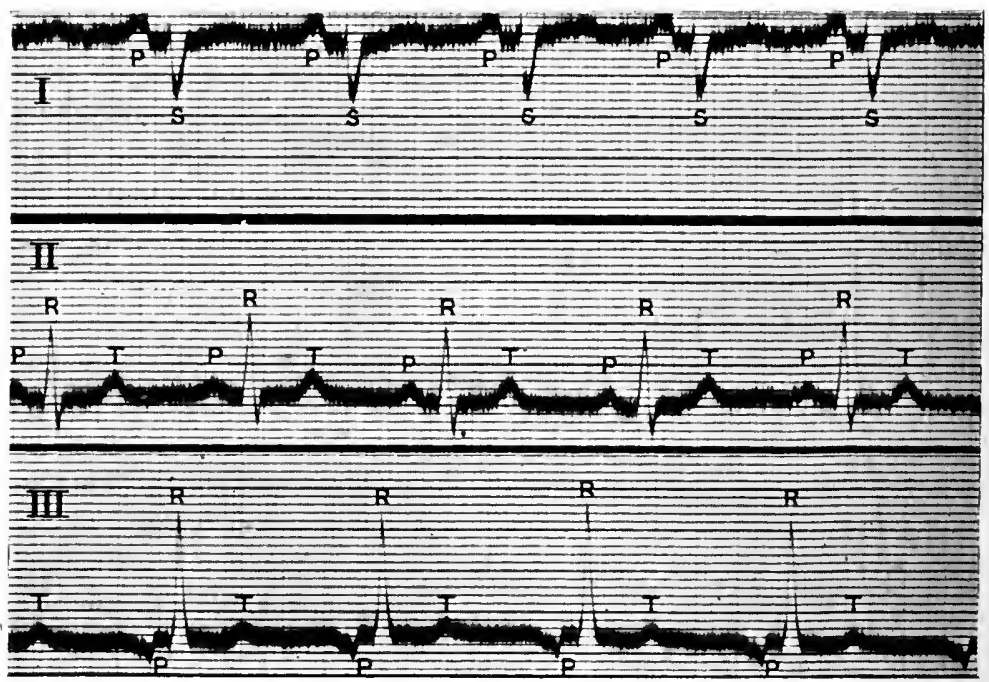

Fra. 174. Electro-cardiogram showing inversion of $P$ in lead III. There is also right-sided preponderance.

173, 219, and 238) is always pathological, and in lead I nearly always so. The amplitude of the deflection $U$ is 


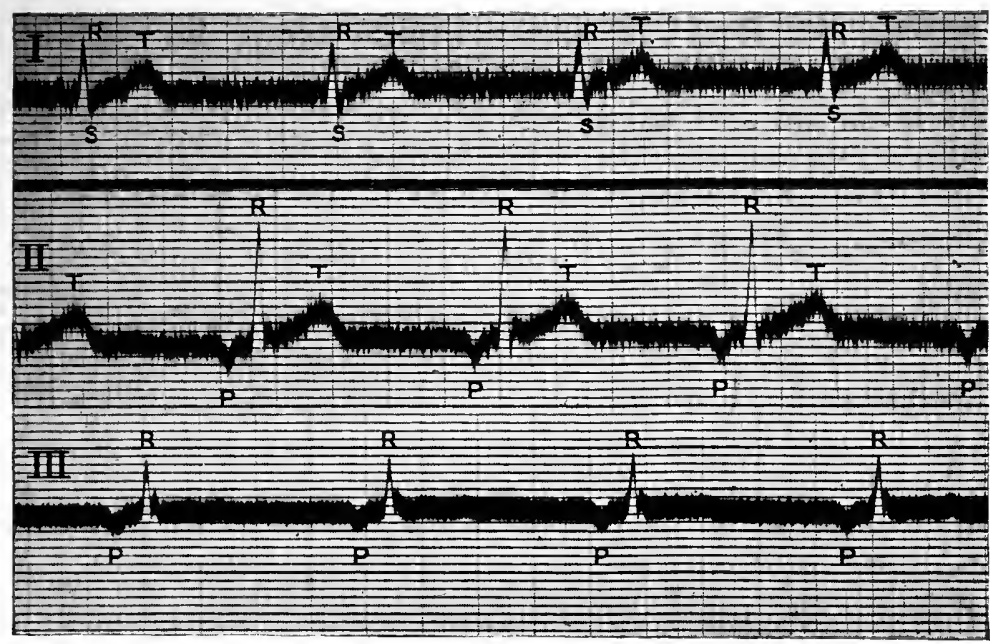

FIG. 175. Electro-cardiogram showing inversion of $P$ in leads

II and III.

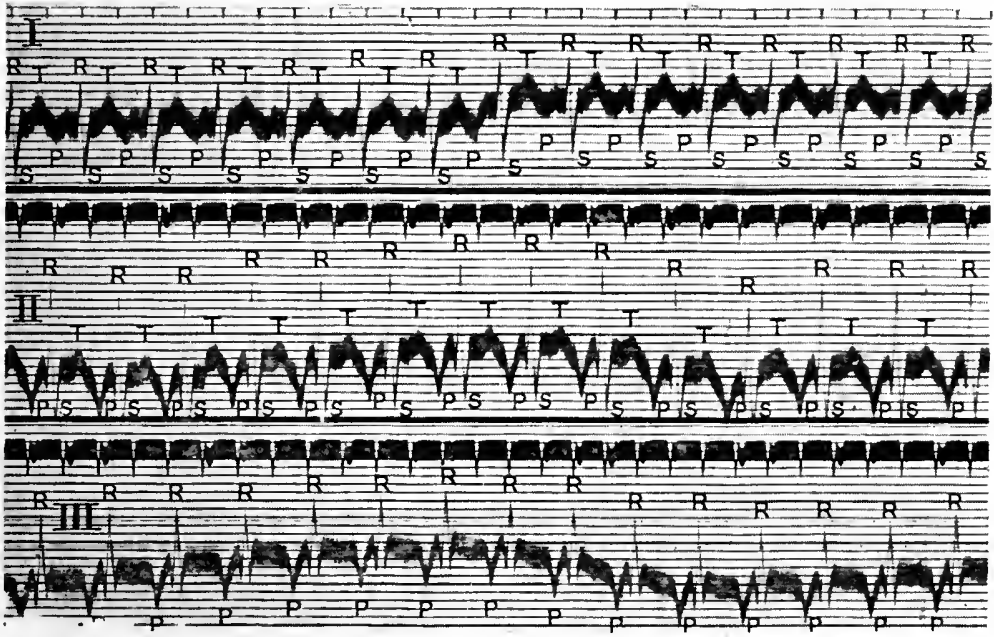

FIG. 176. Electro-cardiogram showing inversion of $P$ in all the leads. There is also right-sided preponderance.

considerable. If $P$ (Figs. 174, 175, 176, and 206) or $R$ be a downward deflection in lead I, II, or III, it is abnormal, Bifurcation of $R$ (Figs. 177, 178, and 179) and $S$ is 
occasionally found in normal individuals, and of $\boldsymbol{P}$ (Fig. 180) more rarely.

In normal subjects the amplitude of all the deflections
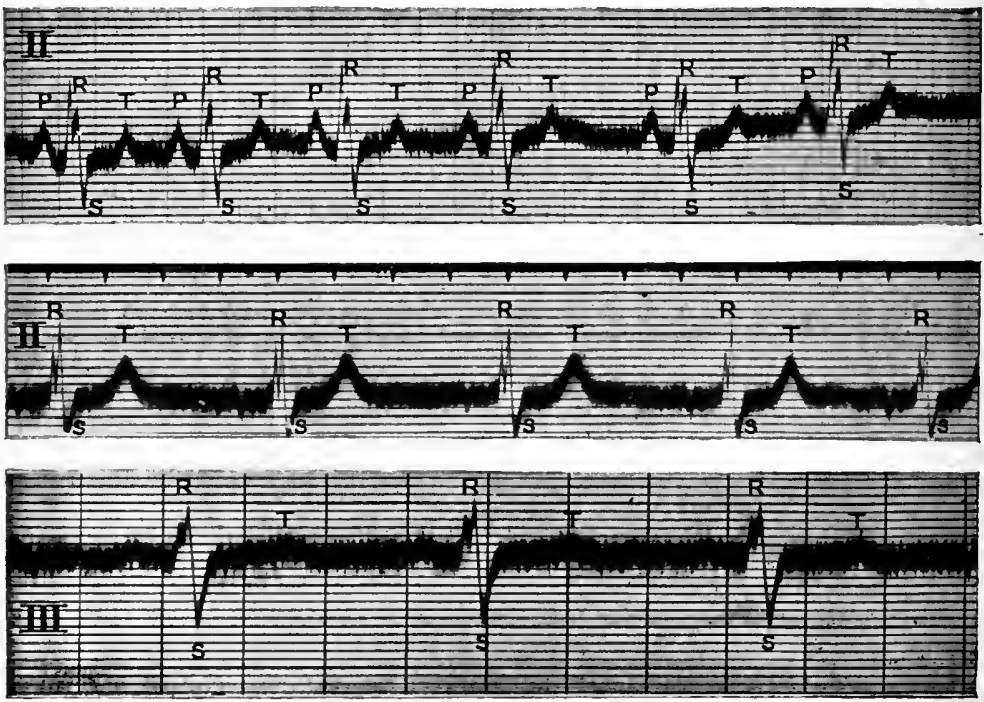

Figs. 177, 178, and 179. Electro-cardiograms from three different:" subjects, showing bifurcation of $R$.

is usually greatest in lead II, espccially the deflection $R$, while the deflections in lead III are not infrequently of

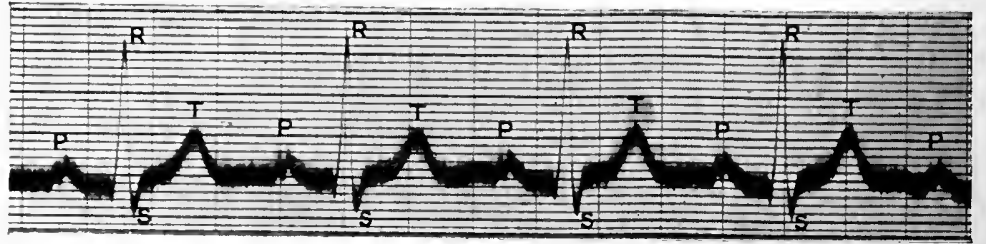

Fra. 180. Electro-cardiogram showing bifurcation of $P$.

small amplitude. When it is not possible to take more than one lead, lead II should be chosen.

The time-distance between the beginning of $\boldsymbol{P}$ and the commencement of $\boldsymbol{Q}$, or between $\boldsymbol{P}$ and $\boldsymbol{R}$, as the case 
may be, is an index of the $A s-V s$ interval, that is, the interval separating the commencement of auricular and ventricular contraction, and is a measure of the function of conductivity of the auriculo-ventricular bundle; it is called the $\boldsymbol{P}-\boldsymbol{Q}$ or $\boldsymbol{P}-\boldsymbol{R}$ interval. The rule is to employ the $P-R$ interval, on account of the frequent absence of the $Q$ deflection. In normally acting hearts this interval does not measure more than one-fifth of a second (Figs.

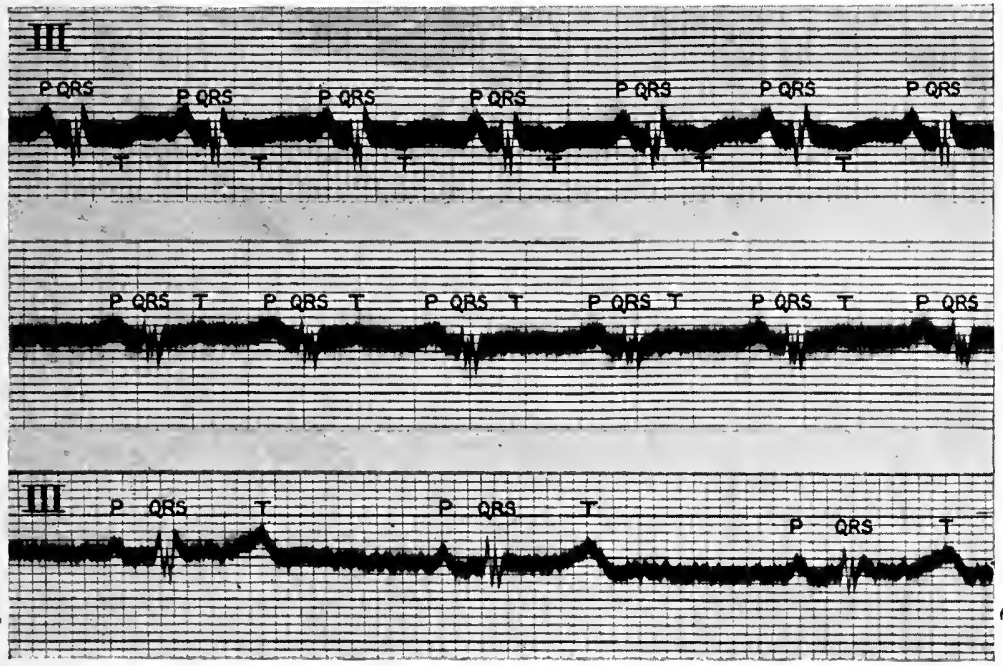

FIGS. 181, 182, and 183 . Electro-cardiograms from three different subjects, showing bizarre types of $Q R S$ group of deflections.

169 and 170). If, therefore, it exceed one-fifth of a second (Figs. 195, 202, and 207), we may conclude that there is delay in the conduction of the stimulus from auricle to ventricle; this means that the function of conductivity is depressed, and that probably there is some affection of the auriculo-ventricular bundle. The $\boldsymbol{P}-\boldsymbol{R}$ interval is in all probability a more reliable indication of the rate of conduction of the stimulus for contraction than the $A-C$ interval in a polygraphic tracing, because the presphygmic interval and the period between the opening of the aortic valves and the carotid pulse are not included. 
The period of time occupied by the $Q, R, S$ group of deflections is normally not more than $\frac{1}{10}$ second. When the cardiac rate is unusually frequent the duration of the
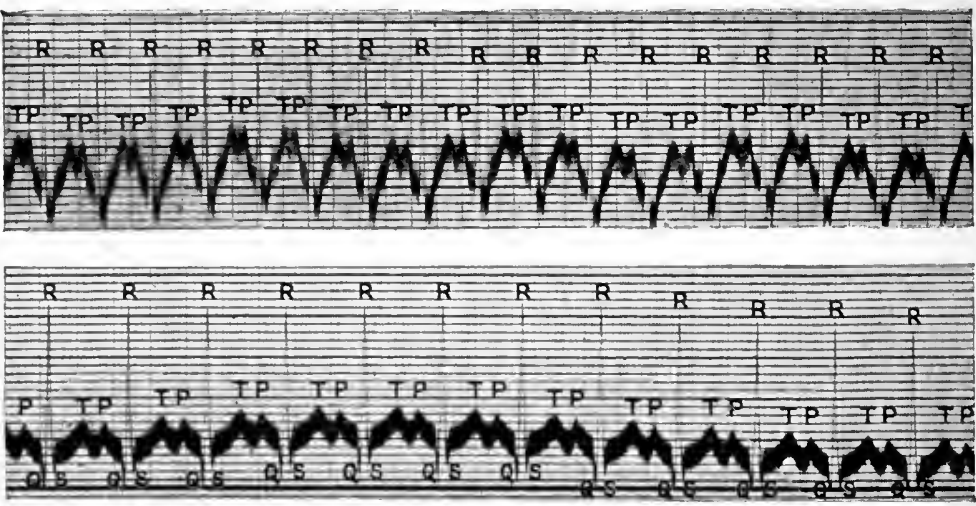

FIGS. 184 and 185. Electro-cardiograms from two different subjects, in which the interval between the deflection $T$ and the following $P$ is diminished.

diastolic interval is shortened, so that the deflection $T$ approaches more and morc to the following $\boldsymbol{P}$ (Figs. 184 and 185), and $P$ and $T$ may even coincide and superimpose (Fig. 186).

Electro-cardiograms of a number of healthy persons all

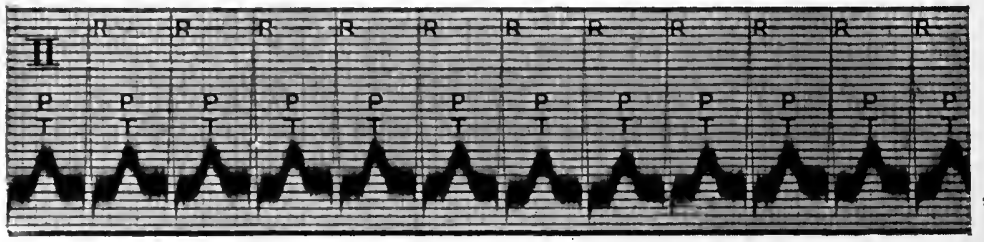

FIG. 186. Electro-cardiogram in which $P$ and $T$ coincide and superimpose.

present different features, but the curve in each case will present individual characteristics which are constant. The form of electro-cardiographic curves depends upon the point of origin and path of conduction of the stimulus for contraction; any departure from the normal in respect 
of either of these will, thcrefore, rcsult in a corrcsponding alteration in the form of the electro-cardiogram. A normal $\boldsymbol{P}$ signifies that the stimulus for contraction arises in the remains of the sinus venosus at the orifices of the grcat veins, and that from this point the stimulus spreads over the whole of the auricles in the usual manner. When $P$ is abnormal in form as, for instance, inverted or diphasic, it is believed that the contraction of the auricle has commenced at some site other than the sino-auricular node; for example, the deflection may be diphasic in cases of auricular flutter.

Sinus Irregularity.-The electro-cardiogram of this
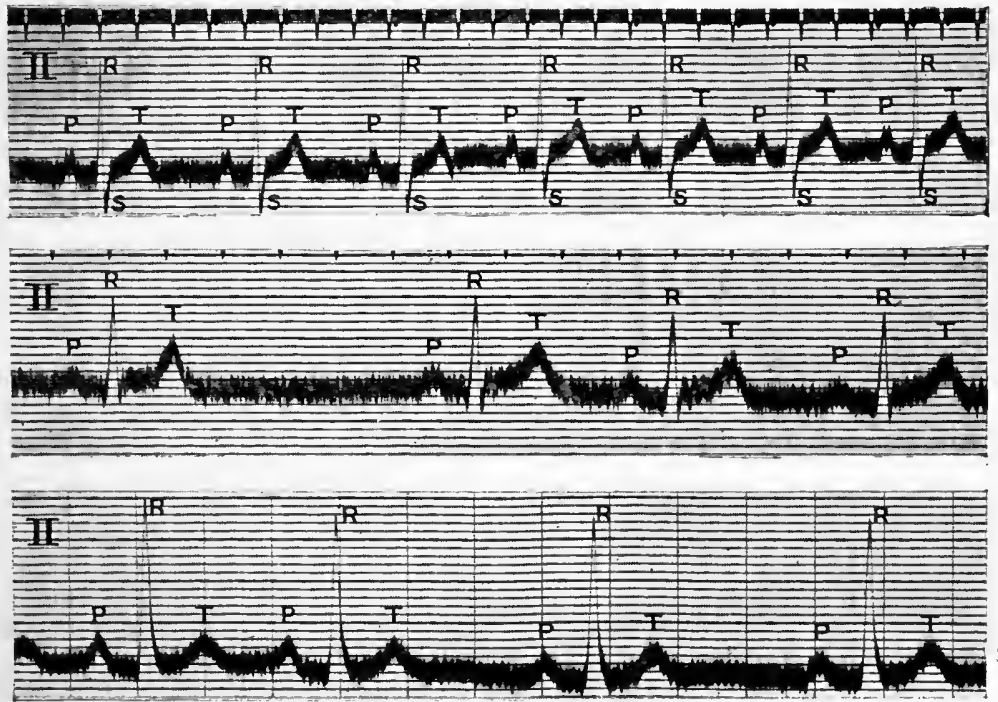

Figs. 187, 188, and 189. Electro-cardiograms from three different subjects, showing sinus irregularity. The auricular and ventricular deflections are of normal form, but there are variations in the length of the interval between $T$ and $P$.

rhythm may readily be identified (Figs. 187, 188, and 189). As the stimulus for contraction takes its origin at the physiological site, and from this point pursues a normal course, the deflections due to the contraction of the auricles 
and ventricles are of normal form, and the ventricular complexes follow the $\boldsymbol{P}$ deflections after a normal interval. As, however, there is a variation in the length of the diastolic interval, variations are found in the length of the interval between $\boldsymbol{T}$ and $\boldsymbol{P}$.

Extra-Systoles.-Extra-systoles may be rcadily re-

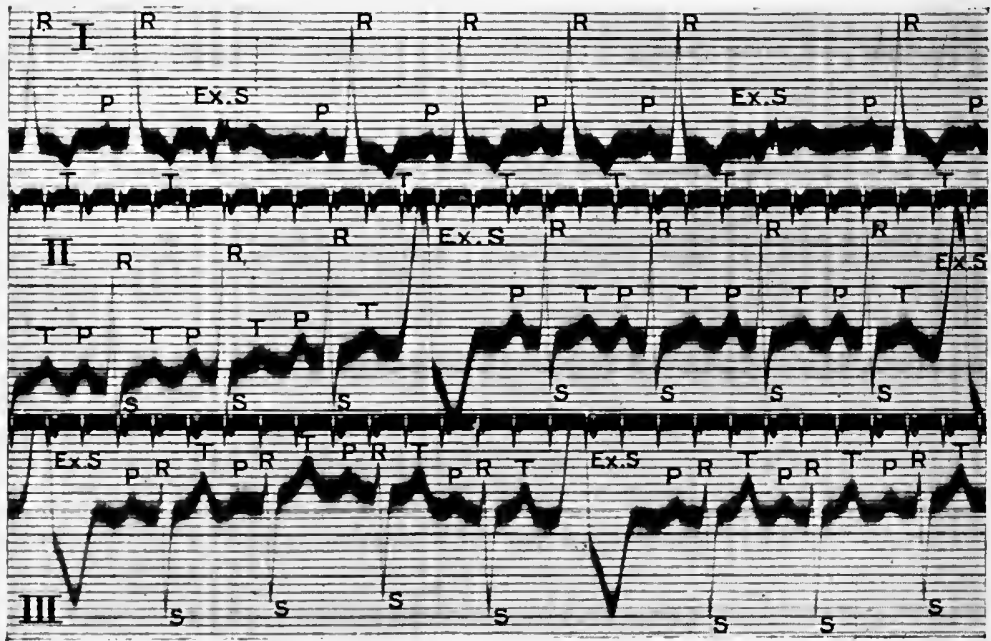

FiG. 190. Electro-cardiogram showing extra-systoles, marked Ex.S, which arise in the right or basal portion of the ventricle. The direction of the deflections is the same in leads II and III, but is reversed in lead $I$. The corresponding $P$ deflections are imbedded in the diphasic variations. There is also inversion of $T$ in lead $I$, and leftsided preponderance.

cognised by means of the electro-cardiograph, and usually their site of origin.

In the ventricular variety (Figs. 190-199), when an extrasystole occurs, the ventricular complex occurs earlier than the anticipated time, and, as the path of conduction of the stimulus for contraction is abnormal, its form is abnormal, but is of the same duration as that of the rhythmic ventricular contraction. As the auricle maintains its usual rhythm and contracts as the result of the normal stimulus arriving from the sinus, the corresponding $\boldsymbol{P}$ deflection ap- 


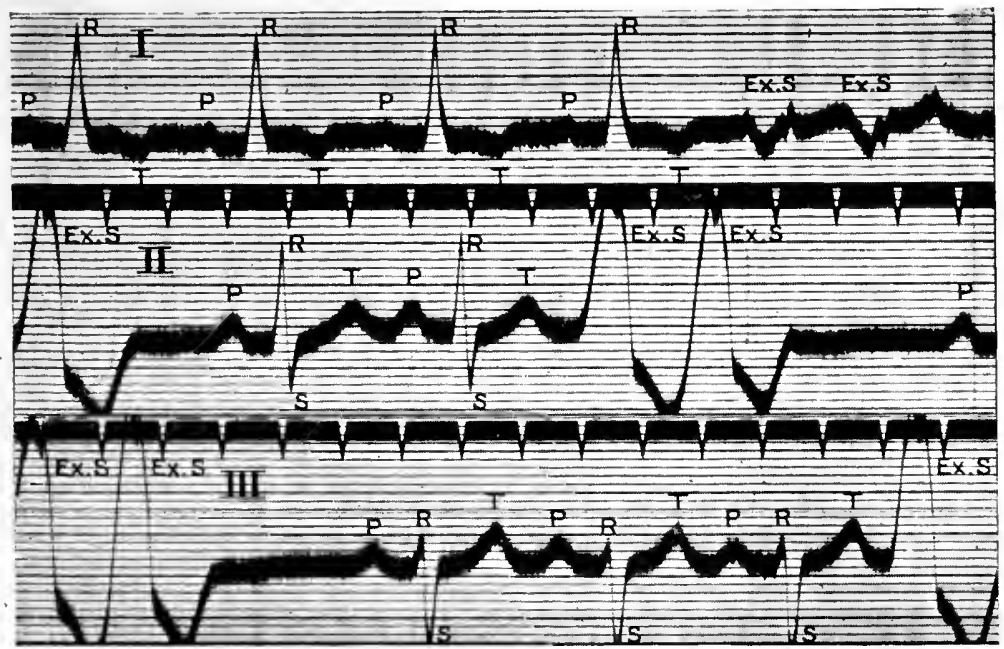

FIG. 191. Shows the same as Fig. 190.

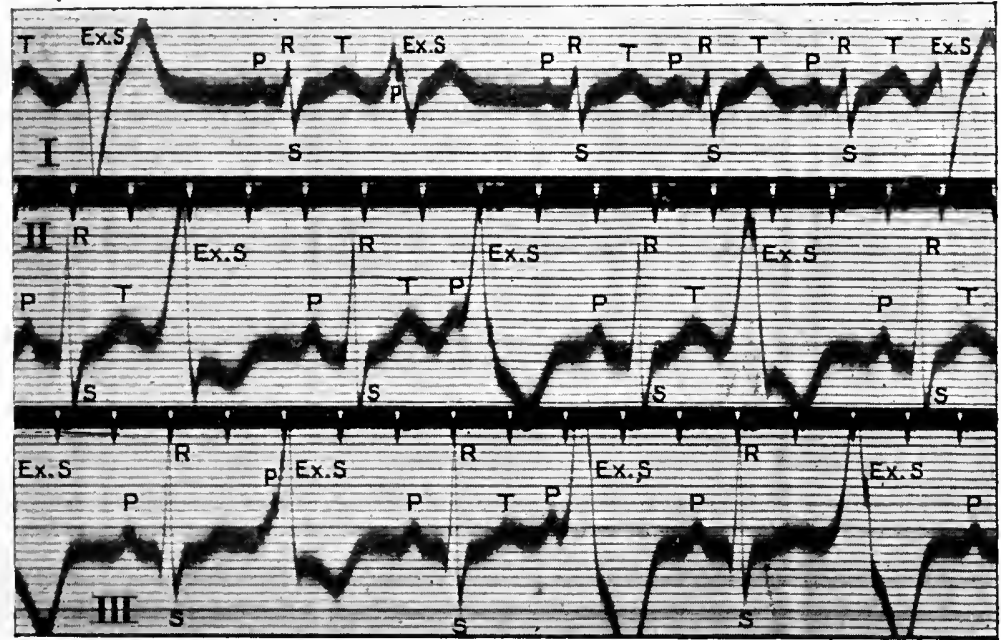

FrG. 192. Electro-cardiogram showing extra-systoles, which arise in the right or basal portion of the ventricle. They do not, however, arise from quite the same focus ; the point of origin of the second extrasystole in lead I and possibly the second in lead III is not quite the same as that of the others-being probably in or near the auriculoventricular node. The direction of the deflections is reversed in lead I in the case of two of the extra-systoles, but" not in those in leads II and III. In the case of some of the extra-systoles the corresponding $P$ deflections are imbedded in the diphasic variations, while in the case of the others they can be detected, and in one case (in lead III) $P$ is almost separate. 
pears at the antieipated time and is normal in form; but it is usually embedded in the diphasic variation, although it may be deteeted in this part of the electro-cardiographic curve in some cases, and occasionally is nearly separate.

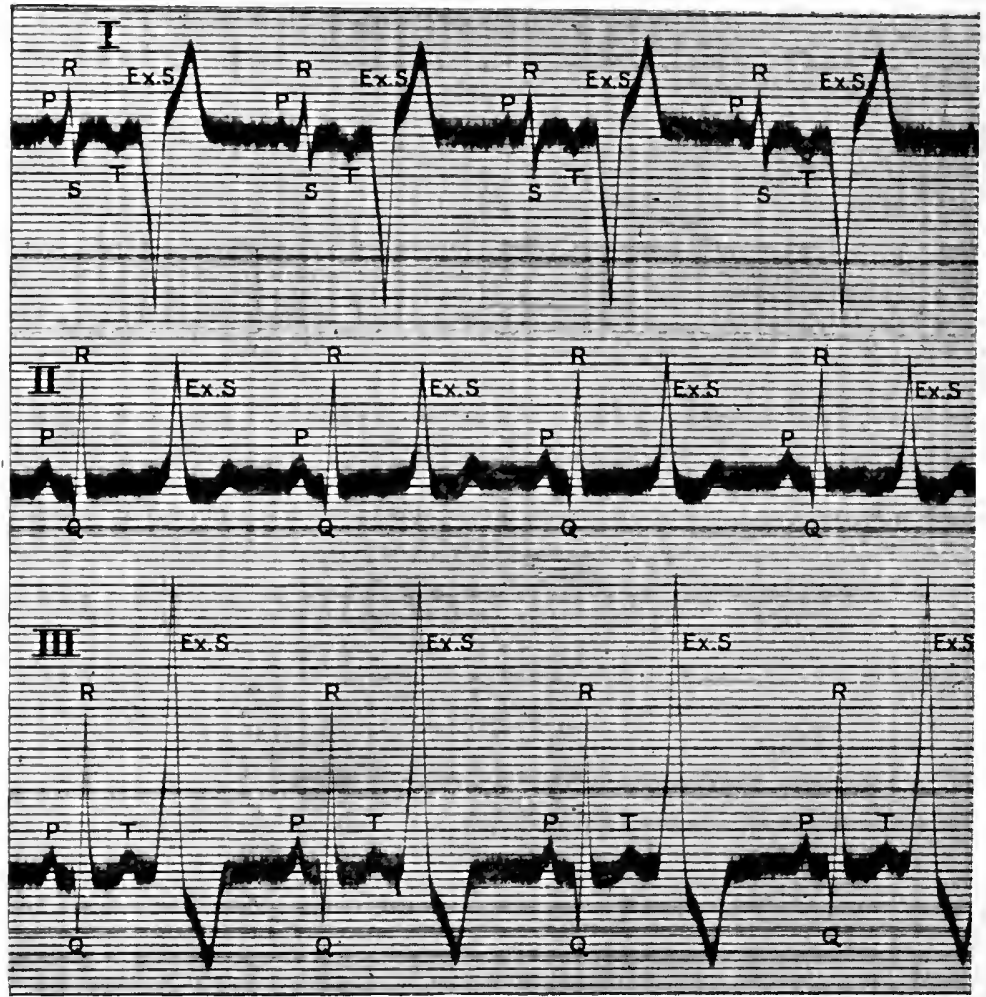

Fig. 183. Elcctro-cardiogram showing an extra-systole with its succeeding compensatory pause occurring regularly after each normal beat. The extra-systoles arise in the right or basal portion of the ventricle. The direction of the deflections is reversed in lead I. There is also inversion of $T$ in lead $I$.

There are two main varieties of ventricular extrasystoles, and it is possible to differentiate between them by means of the electro-cardiograph. One variety has its origin in the basal or right portion of the ventricle, in which case the ventricular complex consists of an 


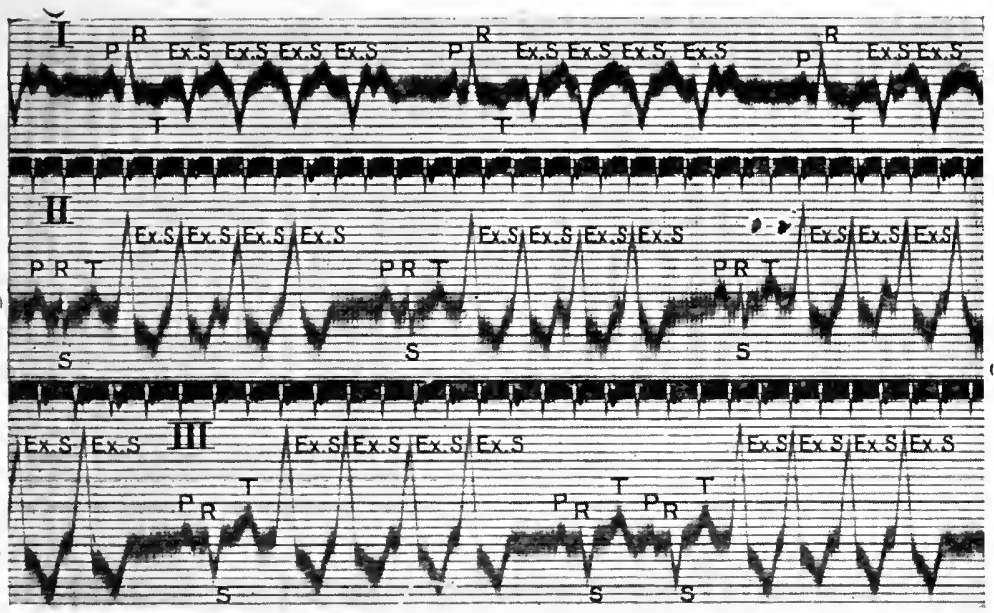

FrG. 194. Electro-cardiogram showing short paroxysms of extra-systoles, which arise in the right or basal portion of the ventricle. The direction of the deflections is reversed in lead $I$. There is also. inversion of $T$ in lead $I$ and left-sided preponderance.

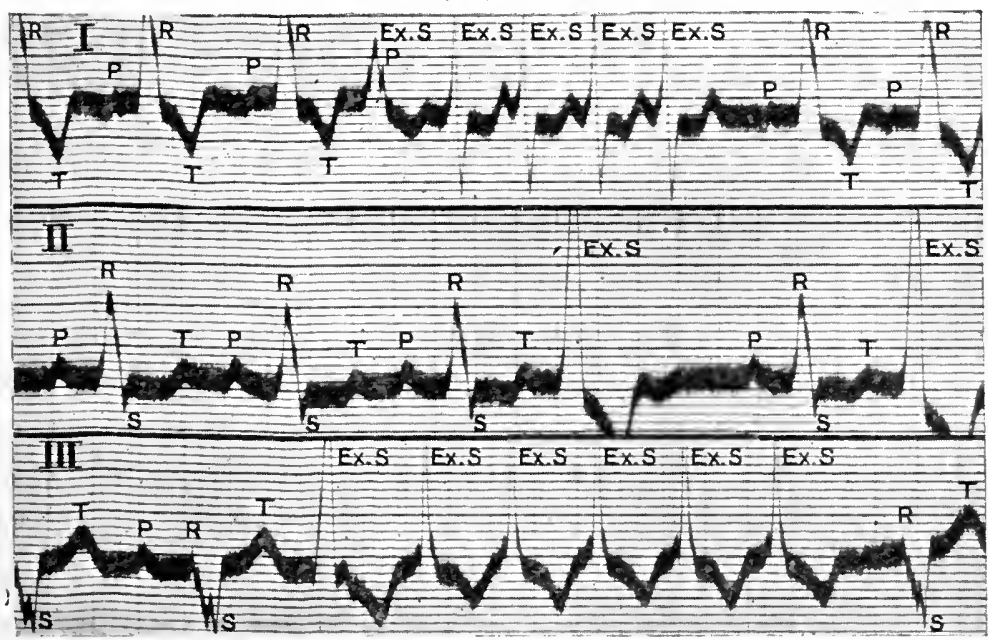

FrG. 195. Electro-cardiogram showing : in lead I, a single extra-systole, followed by a short paroxysm of four extra-systoles; in lead II, two single extra-systoles; in lead III, a short paroxysm of six extrasystoles. The two paroxysms of extra-systoles arise from the right or basal portion of the ventricle, but not from the same focus as the point of origin of the single extra-systole in lead I, while it is difficult to say whether the two extra-systoles in lead II correspond to the single extra-systole in lead $I$ or to the paroxysms. The "normal" $R$ ' $s$ are wide and of unusual form in all leads; there is probably a right-sided bundle lesion. The $P-R$ interval is increased, being $7 / 25$ sec. There is also inversion of $T$ in lead $\mathrm{I}$. 
upward, tall, and pointed deflection, and then of a downward and broader deflection; and in the other in the apical or left portion of the ventricle, in which case the ventricular complex consists of a downward, deep, and pointed deflection, and then of an upward and broader deflection. It should be noted that in any given case, while the dircction of the deflections is usually the

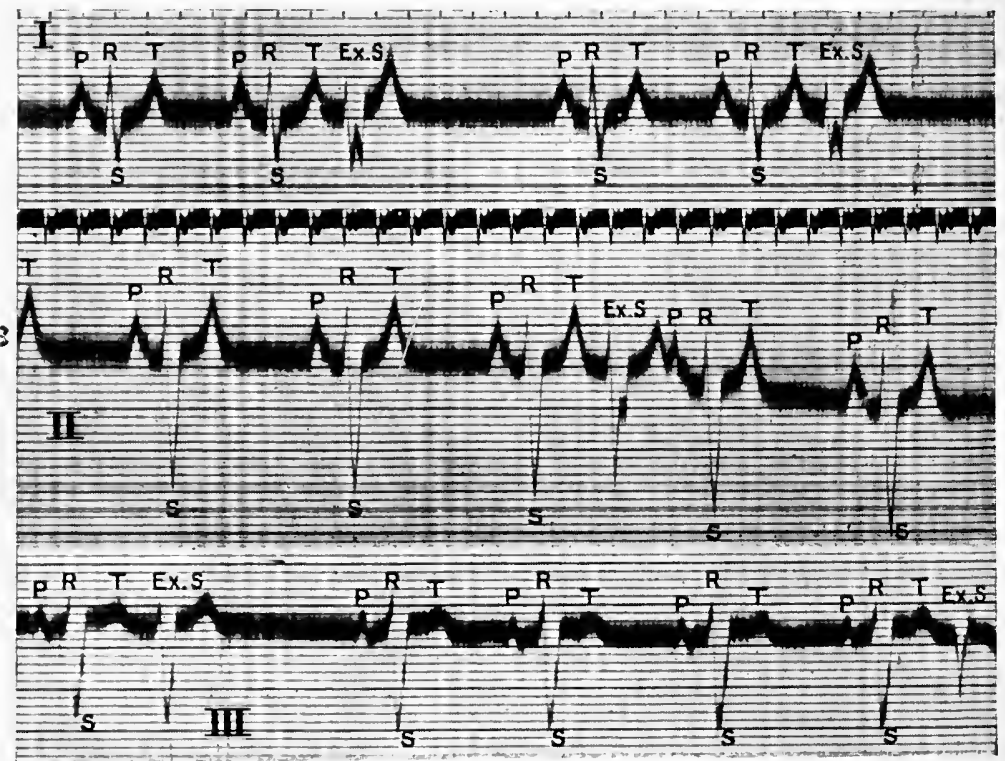

FIG. 196. Electro-cardiogram showing extra-systoles, that in lead II being interpolated. They probably arise very near the auriculoventricular node.

same in leads II and III, it is generally reversed in lead I ; this means that lead I of the first variety usually gives the same form of electro-cardiogram as leads II and III of the second variety, and that lead I of the second variety presents the same form of electro-cardiogram as leads II and III of the first variety.

If the ventricular extra-systole take place after the normal auricular contraction, and the wave of contraction 


\section{CLINICAL ELECTRO-CARDIOGRAPHY 409}
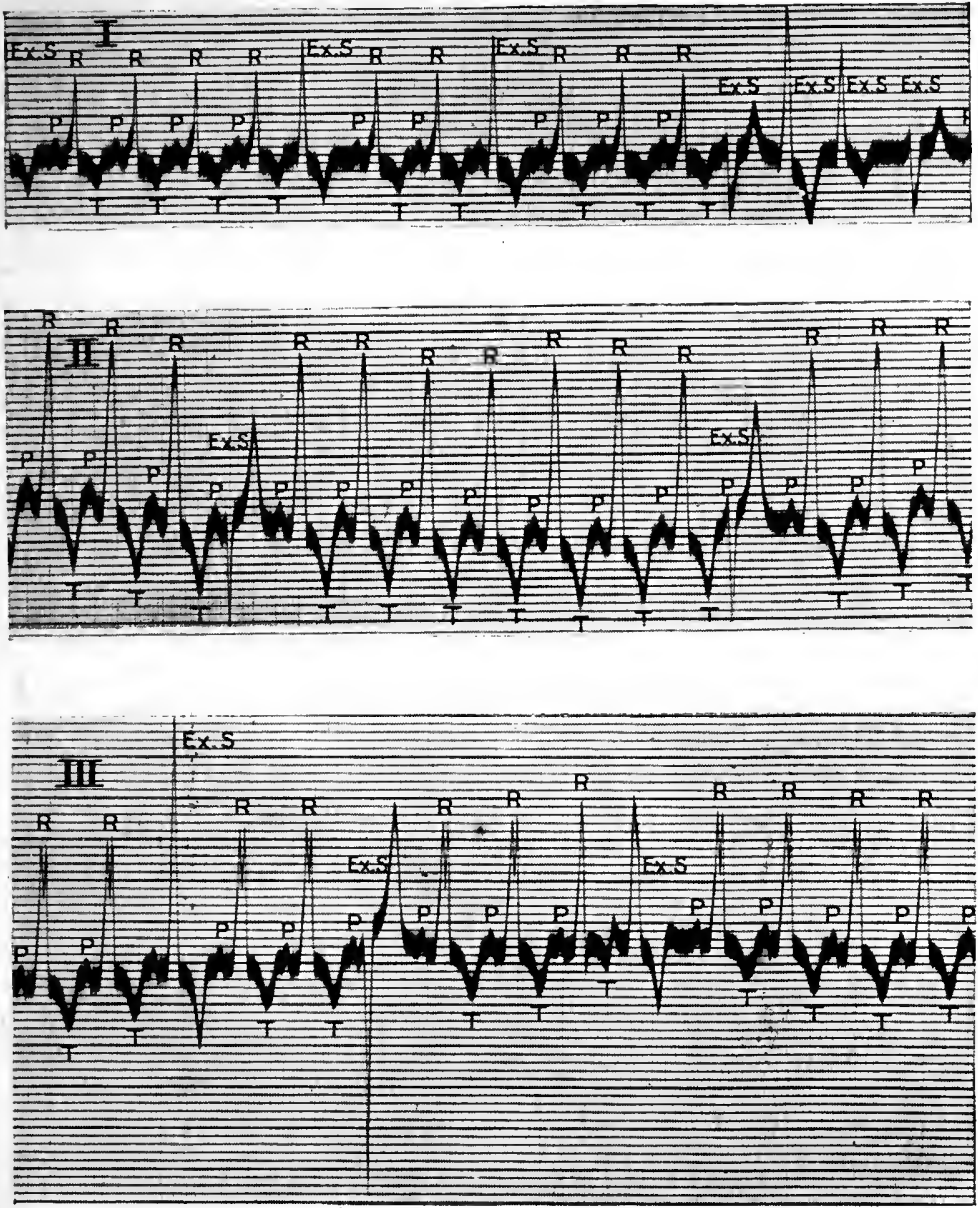

FIG. 197. Electro-cardiogram showing ventricular extra-systoles. In lead $I$, they are of two types, and there is a short paroxysm of extrasystoles at the right hand of the figure. In lead II, there are two extra-systoles, arising from the left or apical portion of the ventricle. In lead III, there are three extra-systoles, the first and the third being right basal and the second being apical. There is inversion of $T$ in leads $I, I I$, and III. 

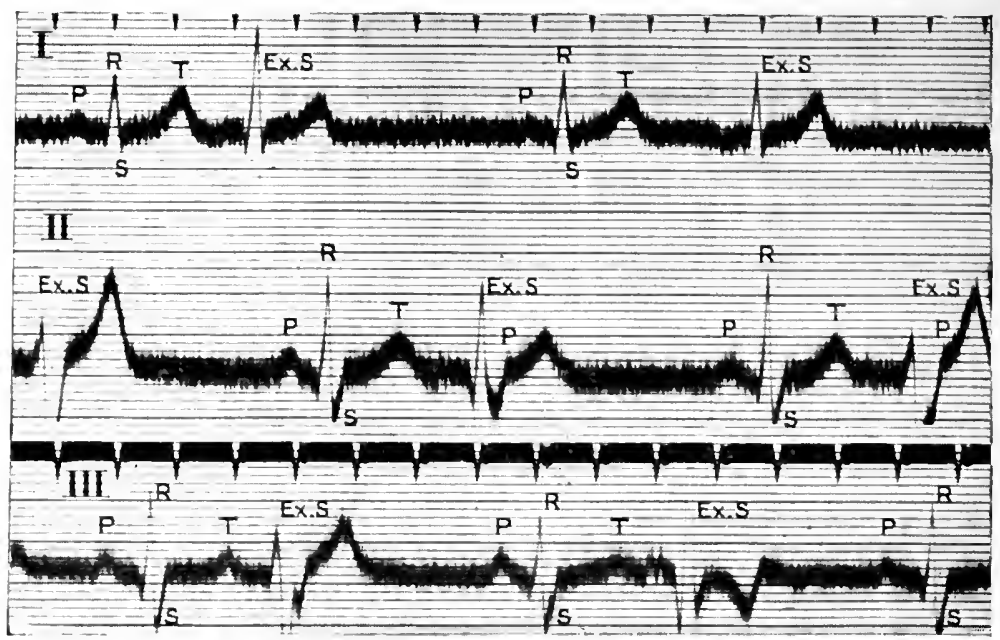

FıG. 198. Electro-cardiogram showing ventricular extra-systoles. The first and third in lead II and the first in lead III arise in the left of apical portion of the ventricle. The second in lead II, the second in lead III, and those in lead I arise in the right or basal portion of the ventricle, probably high up near the auriculo-ventricular node.

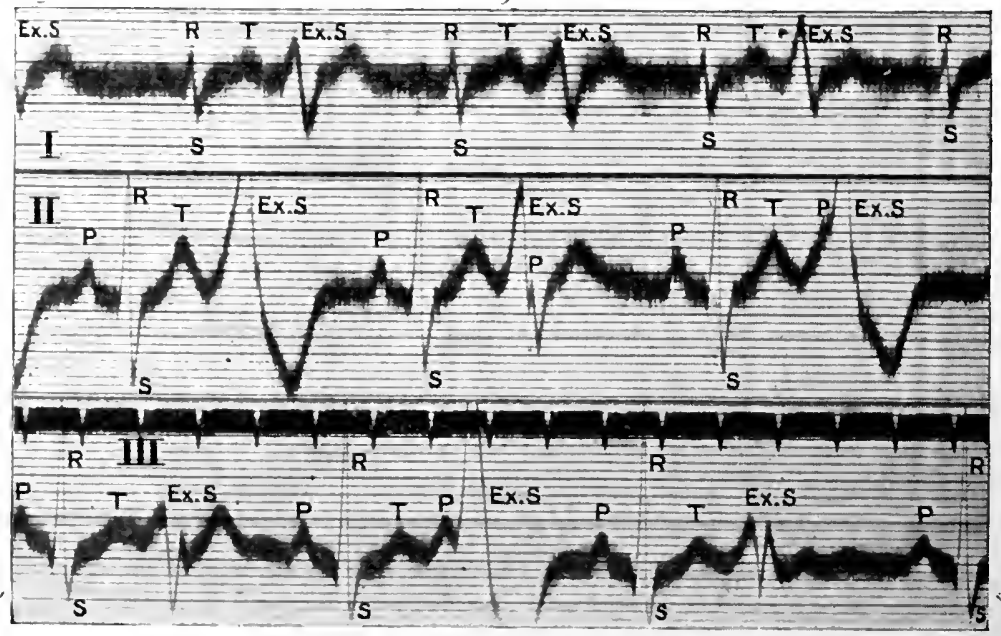

FIG. 199. Electro-cardiogram showing a ventricular extra-systole after each normal beat. Those in leads I and II arise from the right or basal portion of the ventricle, from the same focus or two foci which are near each other. In the third load, the second extra-systole arises in the right or basal portion of the ventricle and the first and third from the left or apical portion of the ventricle. 
from the auricle have reached the ventricle and met that of the premature ventricular contraction in the ventricular wall, the deflections of the premature contraction of the ventricle will present both normal and abnormal features.

The site of origin of an extra-systole may occasionally be at one time the basal or right portion of the ven-

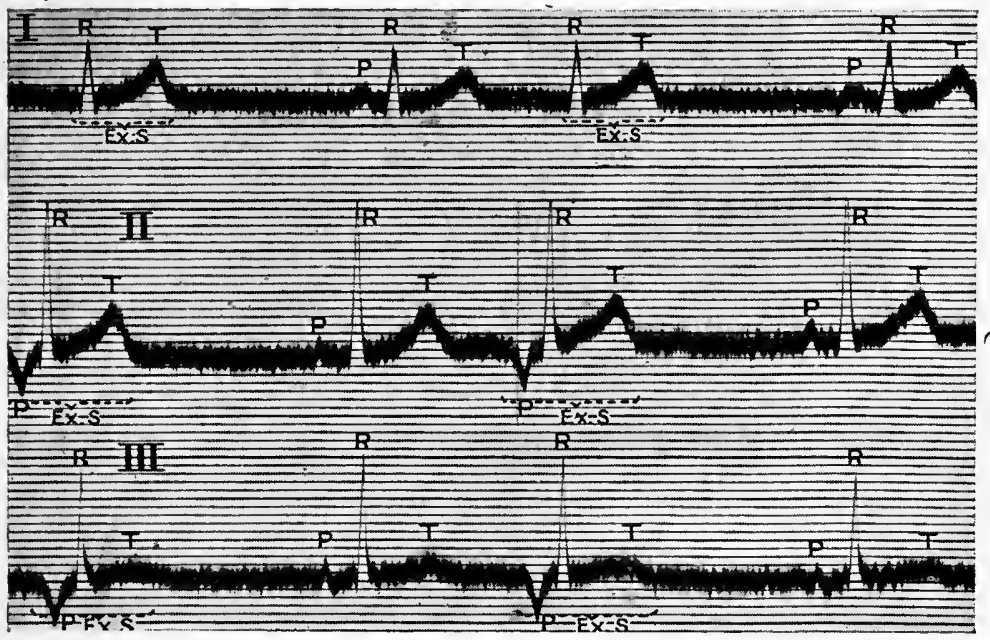

FIG. 200. Electro-cardiogram showing an auricular extra-systole after each normal beat.

tricle, and at another the apical or left portion,' in the same subject.

The departure from the normal form of electro-cardiogram is not nearly so pronounced in auricular as in ventricular extra-systole. In this variety (Fig. 200), when an extra-systole occurs, the $\boldsymbol{P}$ deflection takes place before the anticipated time, and, as the point of origin of the stimulus for contraction is abnormal, it is usually abnormal in form and often inverted; the $\boldsymbol{P}$ deflection is followed by premature ventricular deflections, which are usually of normal form, since the path of conduction of the 
stimulus for contraction is normal. The premature contraction of the auricle may take place so early as to coincide with the ventricular contraetion of the preeeding cardiac cycle, in which case $\boldsymbol{P}$ and $\boldsymbol{T}$ superimpose.

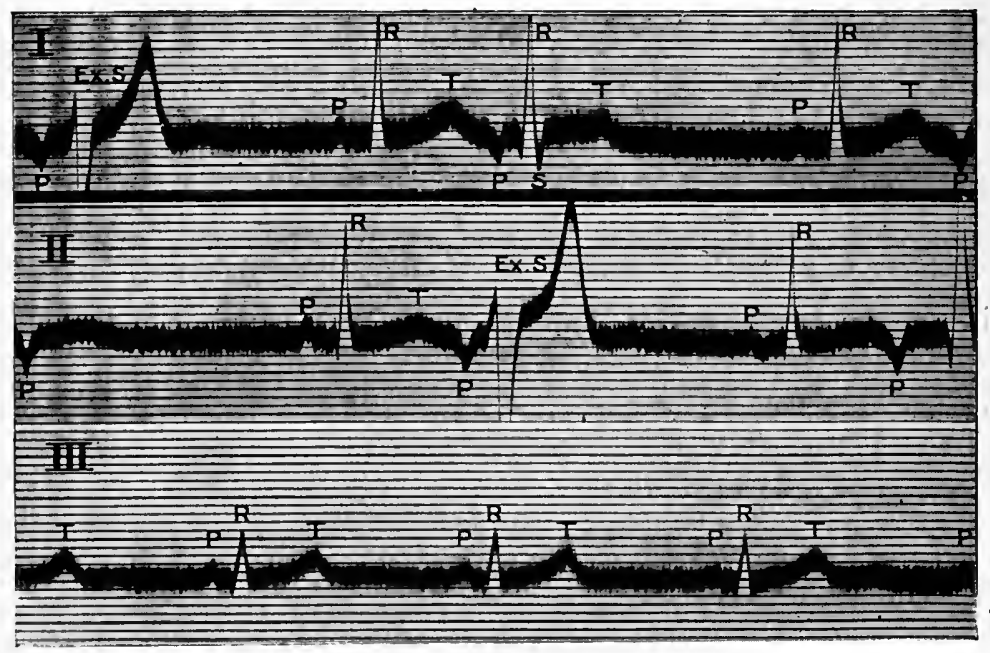

FIG. 201. A rare form of clectro-cardiogram. Reading from the left to right of the figure: In lead $\mathrm{I}$, inverted $P$, indicating an abnormal auricular contraction, but instead of being followed by a normal ventricular contraction it is succeeded by a ventricular extra-systole; after this appear a normal beat, an auricular extra-systole, and a normal beat. In lead II, what looks like an auricular contraction arising from an abnormal focus, not followed by any ventricular contraction; then a normal beat, followed by an auricular extrasystole, but instead of the latter being succeeded by a normal ventricular contraction it is followed by a left apical extra-systole; then a normal beat; then an auricular extra-systole, followed after a long interval by a right basal extra-systole-only part of the latter being included in the figure.

Auriculo-Ventricular Block.--In the first degree of depressed conductivity (leads I and III of Fig. 202), there is merely an increase of the $\boldsymbol{P}-\boldsymbol{R}$ interval. In the seeond degree, the $\boldsymbol{P}$ deflections are found at regular intervals and arc normal in form, but more frequent than the ventrieular complexes-even twice (lead II of Fig. 202), threc times, or more, according to the degree of bloek; 
in other words, sometimes the $\boldsymbol{P}$ deflections are not followed by deflections due to ventricular systole. But,

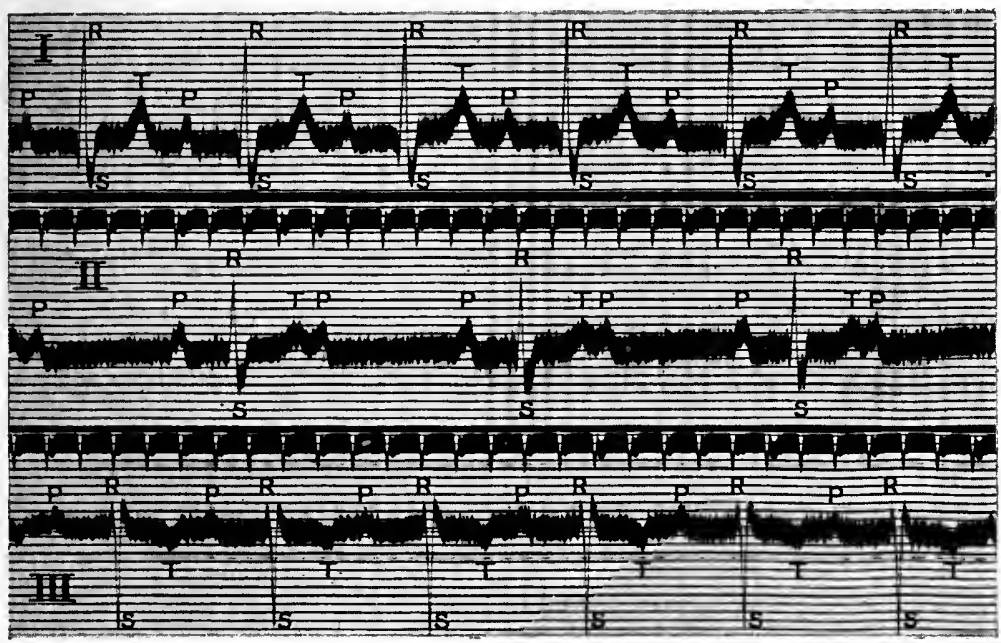

FIG. 202. Electro-cardiogram showing partial heart-block. In each lead there is an increase of the $P-R$ interval, and in lead II there is also continuous 2:1 rhythm, every other stimulus from the auricle failing to reach the ventricle. There is also inversion of $T$ in lead III and left-sided preponderance.

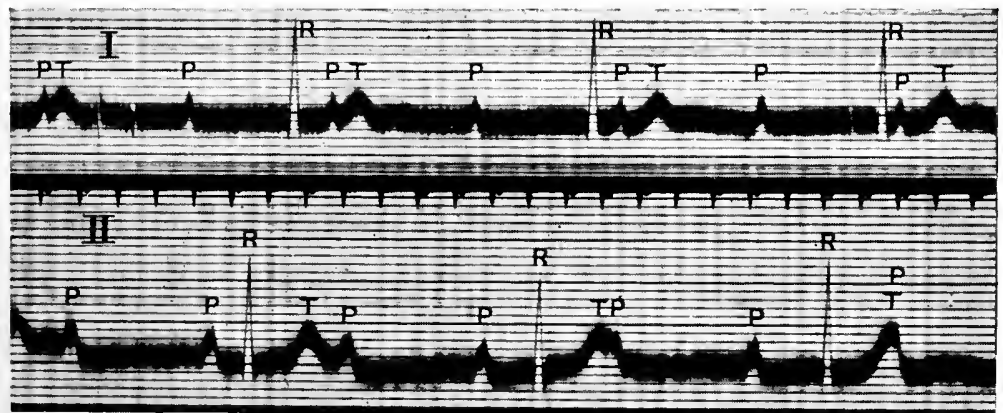

FIG. 203. Electro-cardiogram of leads I and II, showing complete heartblock, or disassociation of the auriculo-ventricular rhythm, the auricles and ventricles beating independently of each other.

unlike complete heart-block, on each occasion the ventricular complex is preceded by a $\boldsymbol{P}$ deflection. In com- 


\section{$4: 14$ \\ DISEASES OF THE HEART}

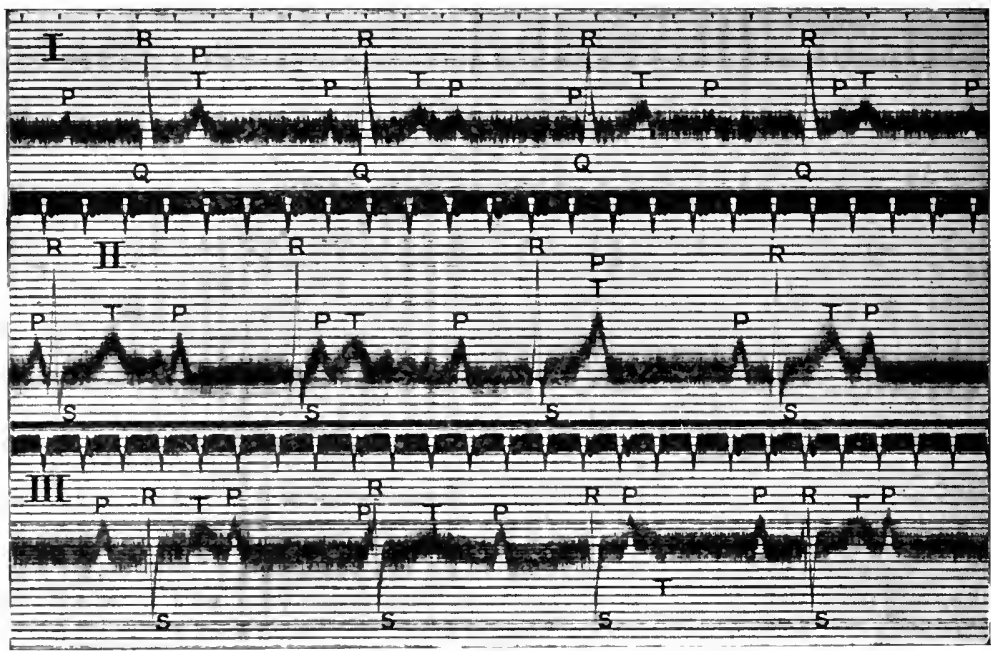

FIG. 204. Electro-eardiogram showing complete heart-block, with also left-sided"preponderance.

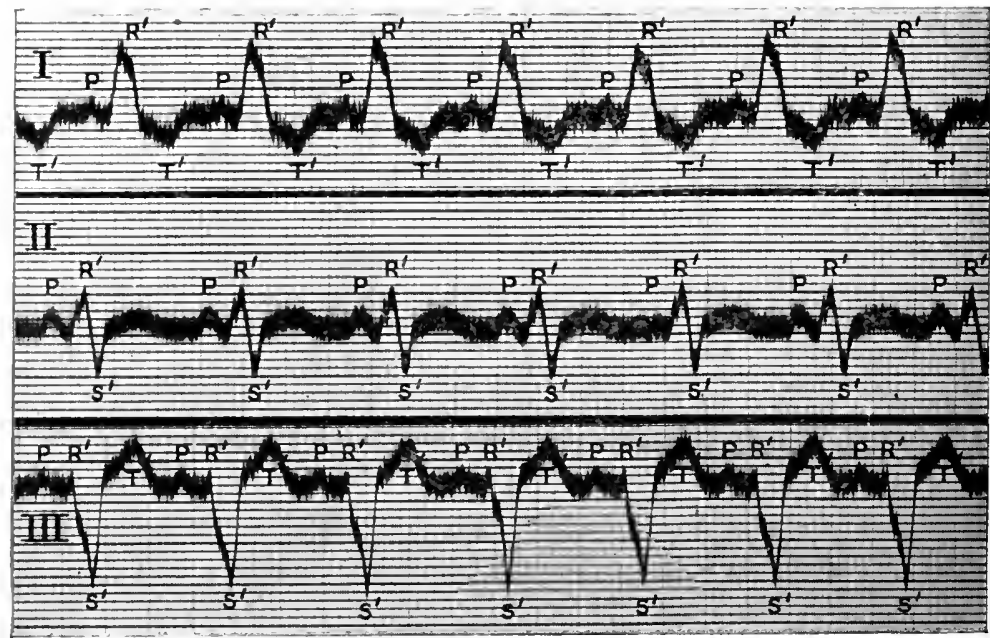

FIG. 205. Electro-cardiogram showing a lesion of tha right min brans.h of the auriculo-ventricular bundlo.

plete heart-block (Figs. 203 and 204), the $\boldsymbol{P}$ deflections are found at regular intervals and are normal in form, and besides being more frequent than the ventricular 
complexes, the time-relation between the $\boldsymbol{P}$ deflections and the ventricular complexes is a constantly varying one--the $\boldsymbol{P}$ deflection at one time preceding, at another following, and sometimes again coinciding with the ventricular complexes. The ventricular deflections are of normal form.

It is believed that a lesion of either the right or left main branch of the auriculo-ventricular bundle may be recog-

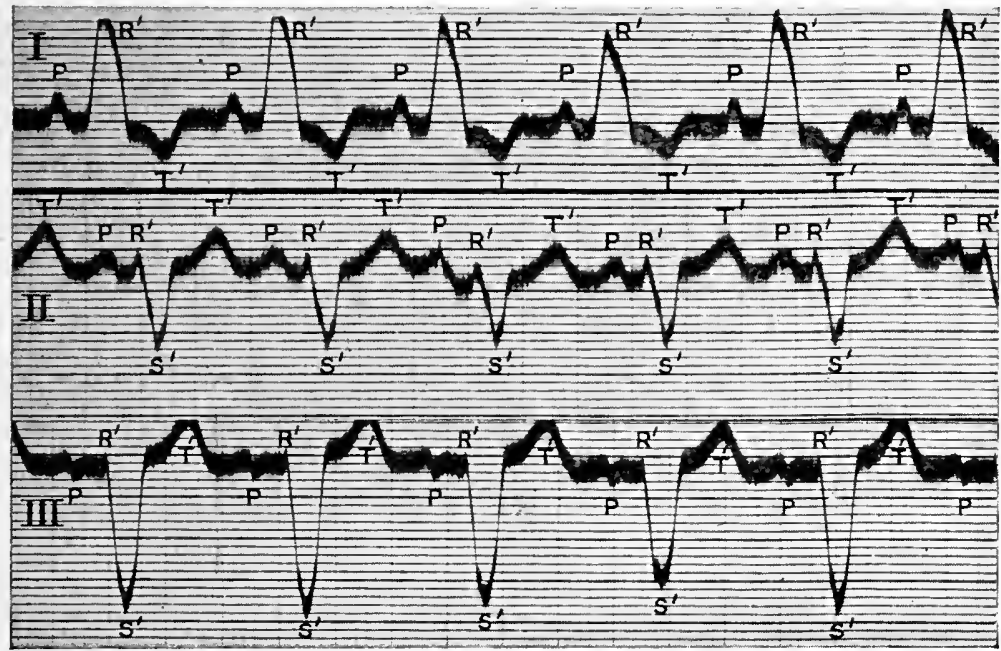

F1G. 206. Electro-cardiogram showing a lesion of right main hranch of the auriculo-ventricular bundle. There is also inversion of $P$ in lead III.

nised by means of the electro-cardiograph. Each ventricular complex is preceded by a $\boldsymbol{P}$ deflection, but the ventricular complexes are abnormal in form; their amplitude is increased, and the period of time occupied by the $Q, R, S$ group of deflections is increased, exceeding one-tenth of a second. When the right branch is involved, the stimulus for contraction from the auriclc travels to both ventricles along the left branch alone: in lead I the deflection $R$ is tall and broad, and in lead III the deflection $S$ is deep and broad (Figs. 205, 206, and 


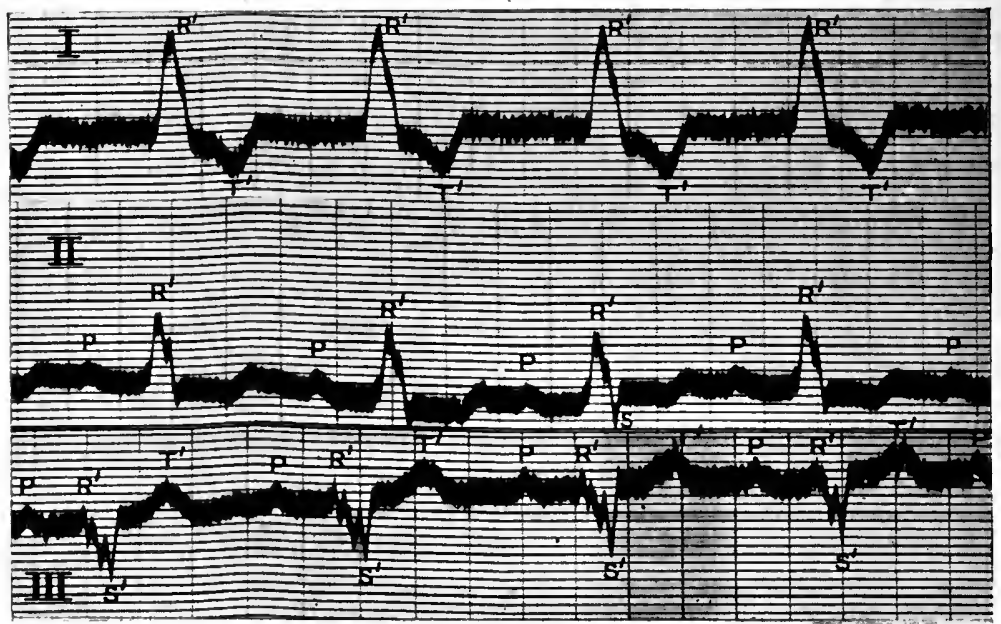

FIG. 207. Electro-cardiogram showing a lesion of the right main branch of the auriculo-ventricular bundle. The $P-R$ interval is increased.

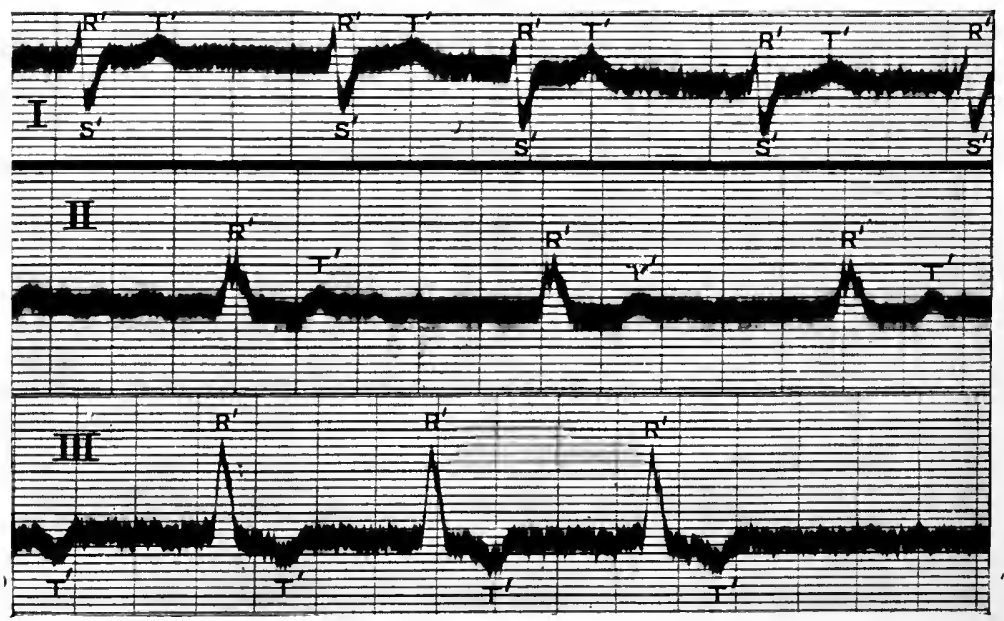

FIG. 208. Electro-cardiogram showing a lesion of left main branch of the auriculo-ventricular bundle. There is also auricular fibrillation.

207). When the left branch is involved, the stimulus for contraction from the auricle travels along the right branch alone; in lead $I$ the deflection $S$ is deep and broad, and in lead III the deflection $R$ is tall and broad 
(Fig. 208). It will thus be seen that when the right branch is involved the deflections of largest amplitude point away from each other, whereas in the case of the left branch the opposite holds good. It is necessary to distinguish between the electro-cardiograms of a lesion of the right or left main branch of the auriculo-ventricular bundle and hypertrophy of the left or right ventricle respectively. This will be referred to later.

Alternation of the Heart.-This may be sometimes recognised by means of the electro-cardiograph, by an alternation in the amplitude of the deflections due to the contraction of the ventricle (Fig. 234). Both the $R$ and $T$ waves may be affected, or one more than the other. It should be noted that alternation of the heart is sometimes shown in a sphygmogram without any corresponding evidence in an electro-cardiogram, while rarely the opposite holds good. It should be further noted that the alternation in a sphygmogram and electro-cardiogram do not always correspond; i.e. the smaller ventricular deflections correspond with the larger pulse-wave.

Auricular Fibrillation.-In auricular fibrillation the electro-cardiogram is characteristic (Figs. 208-219). The ventricular complexes are separated by irregular intervals; intervals of the same length rarely follow one another, and the pauses show no relationship to each other. The amplitude of the $R$ deflections also varies from cycle to cycle. Further, there is often no relationship between the length of a pause and the amplitude of the $R$ deflection which follows it-i.e. a short pause may be followed by an $\boldsymbol{R}$ deflection of large amplitude and a long pause by one of small amplitude. When the ventricular rate is slow, the irregularity may be only very slight, and the amplitude of the $\boldsymbol{R}$ deflections is almost constant. As the ventricle receives stimuli from the auricle through the normal channel, the ventricular deflections are of normal form; $T$, however, may show inversion (Fig. 219). 
There is an absence of $\boldsymbol{P}$ deflections; these cannot be present bccausc the auricle does not contract. There
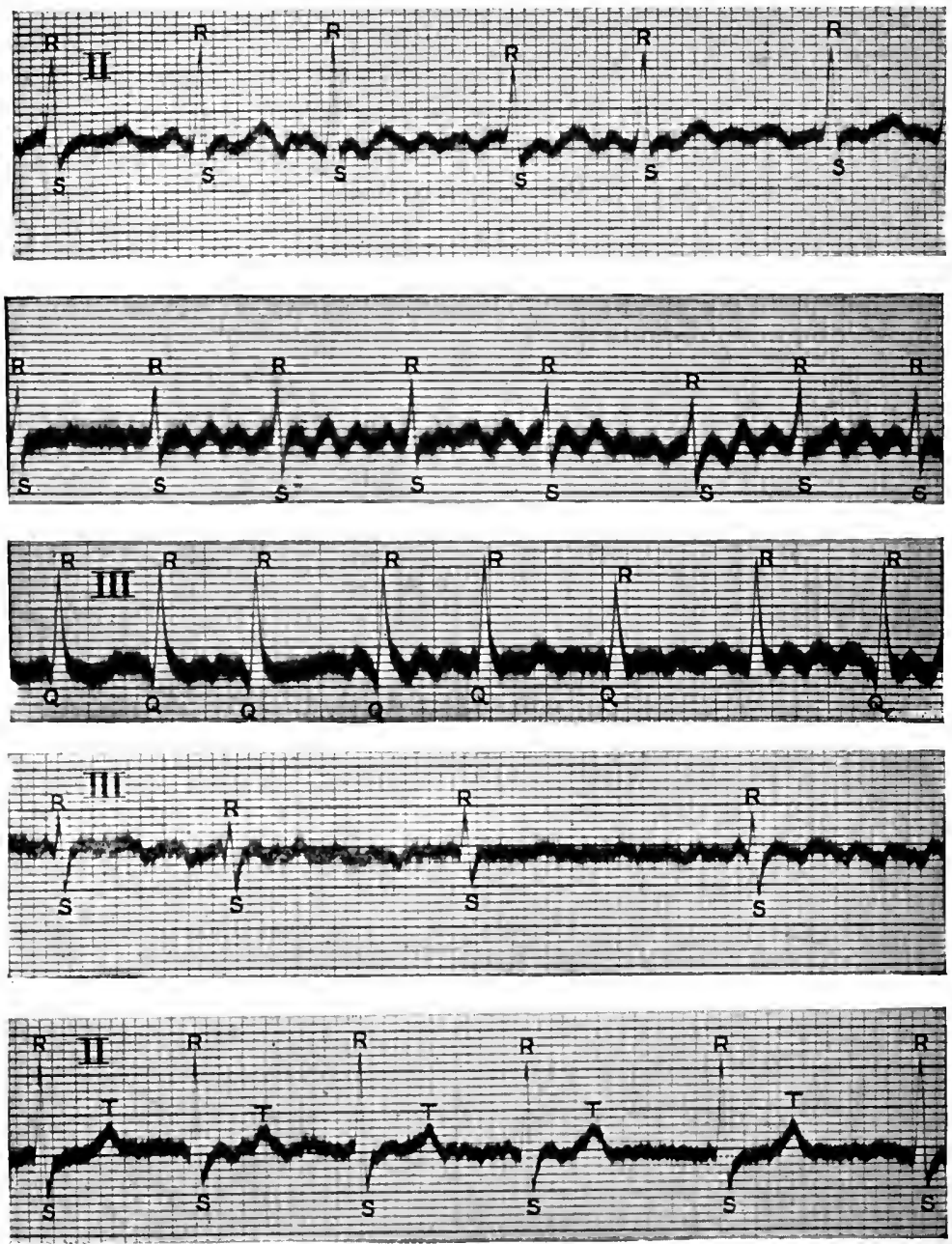

Figs. 209-213. Electro-cardiograms from cases of auricular fibrillation.

are, on the other hand, oscillations during diastole caused by the fibrillating auricle, occurring at the rate of from 400 to 600 per minute, and irregular in time and form. 
These are most evident in cases of slow cardiac laction, whereas if the action be frequent it is often difficult to

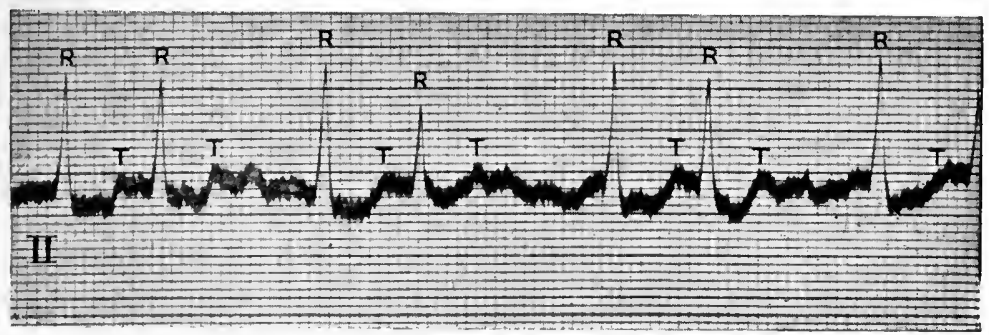

Fic. 214. Electro-cardiogram from a case of auricular fibrillation. Note the coupling of the beats.

detcct them. Their size varies, being sometimes very minute and sometimes of considerable size. They may

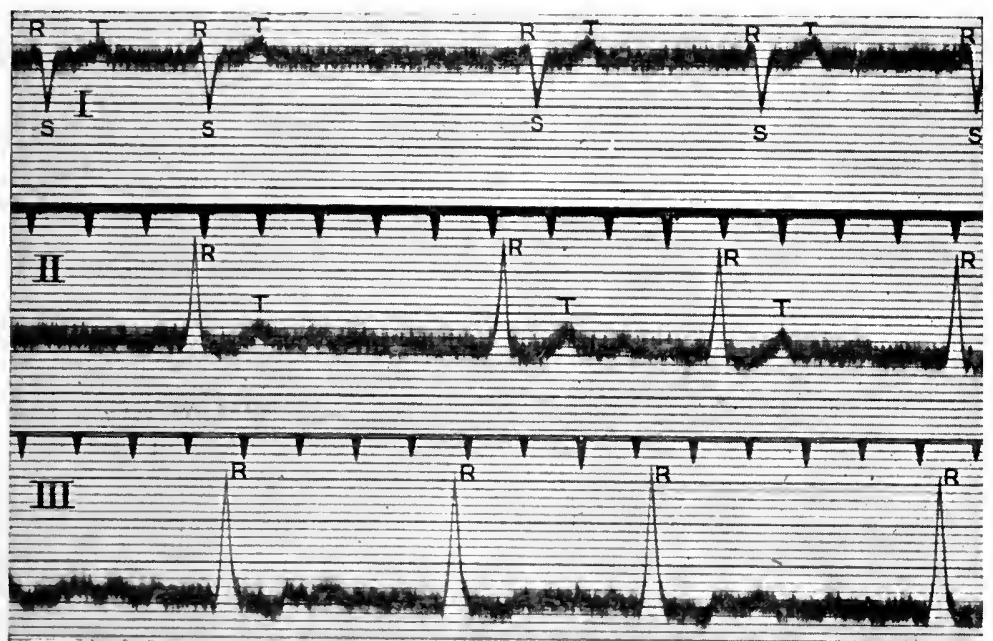

Fig. 215. Electro-cardiogram from a case of auricular fibrillation, with also right-sided preponderance.

coincide with the $T$ deflections, in which case the outline of the latter is altered.

These oscillations should be distinguished from those which occur as a result of tremor of the somatic muscles 


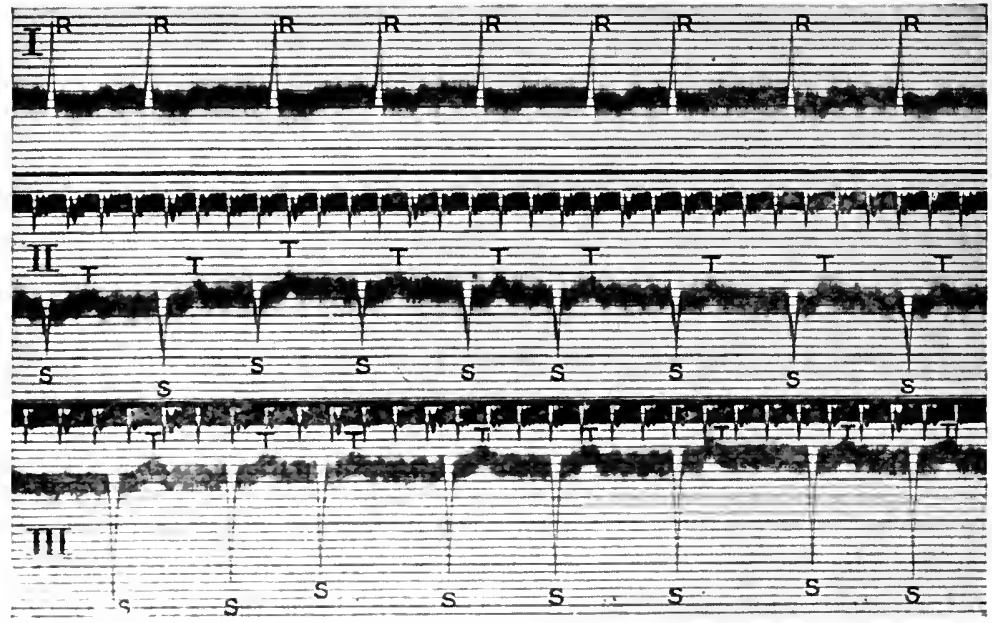

FIG. 216. Electro-cardiogram from a case of auricular fibrillation, with also left-sided preponderance.

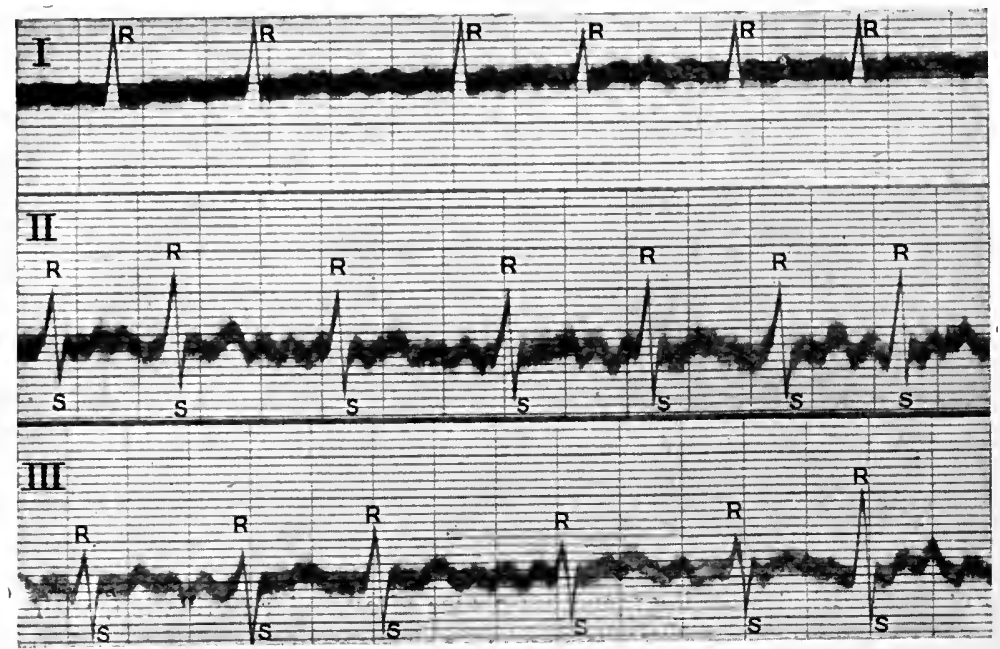

Fra. 217. Electro-cardiogram from a case of auricular fibrillation.

(Figs. 165, 166, and 167). These are of frequent occurrence in the subjects of exophthalmic goitre, and may be of considerable size. Oscillations due to auricular fibrilla- 


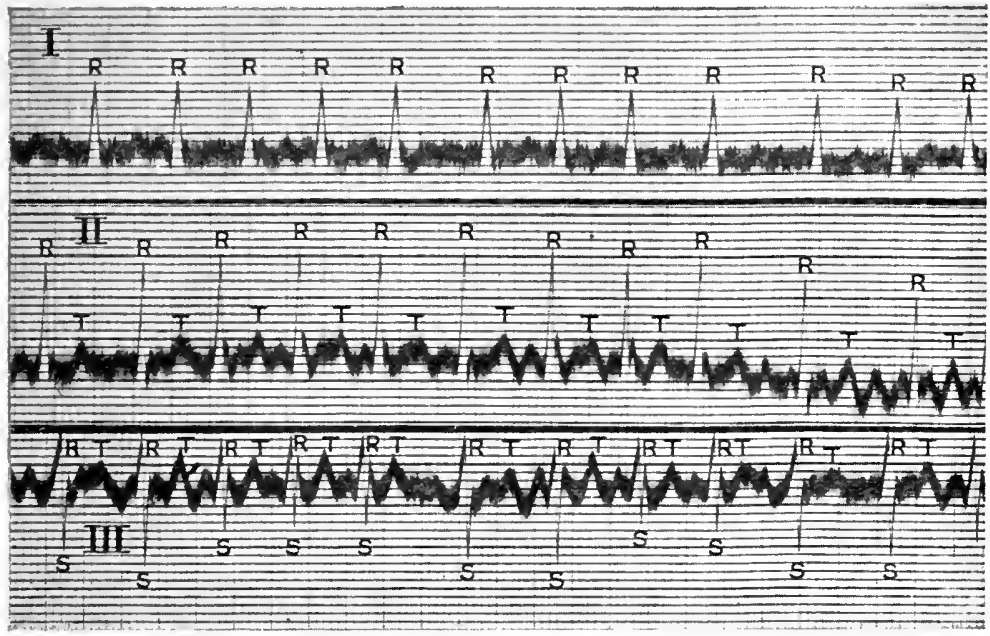

FIG. 218. Electro-cardiogram from a case of auricular fibrillation.

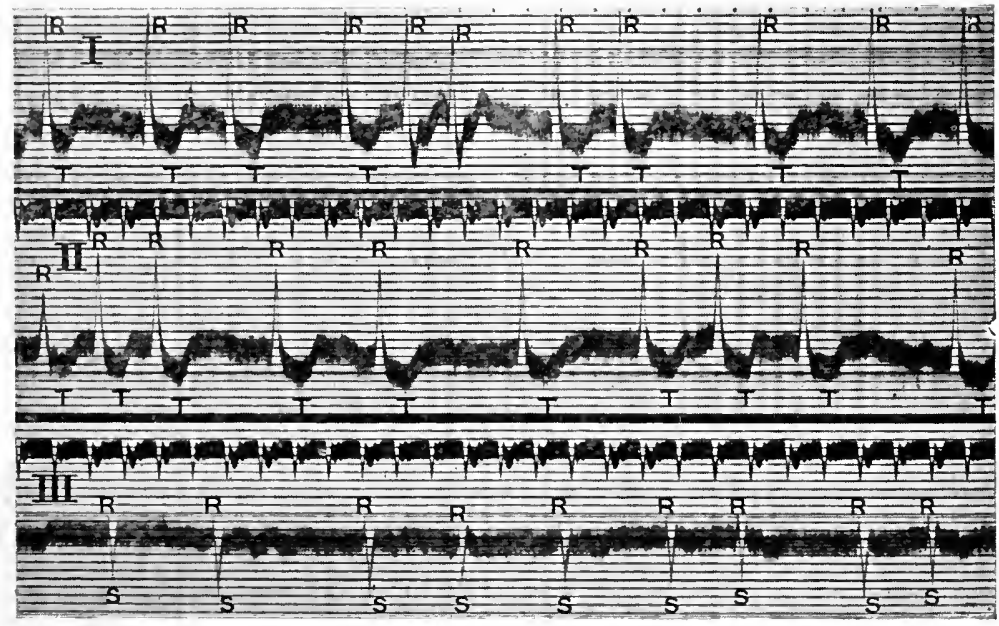

FIa. 219. Electro-cardiogram from a case of auricular fibrillation. There is inversion of $T$ in leads $I$ and $I I$ and left-sided preponderance. The fifth and sixth $R^{\prime} s$ in lead $I_{-}^{s}$ strongly suggest extra-systoles.

tion may be distinguished from those resulting from tremor of the somatic muscles by the fact that in the latter case they are of more frequent occurrence-about 
50 per second-and occur at regular intervals, the deflection $\boldsymbol{P}$ or some indication of it may be noted, and the ventrieular complexes do not occur at irregularly irregular intervals.

It is further necessary to distinguish electro-cardiograms of auricular fibrillation from those of auricular flutter. This will be referred to later.

Auricular Flutter.-In this condition it is of especial importanee to analyse the three leads (Figs. 220-224,

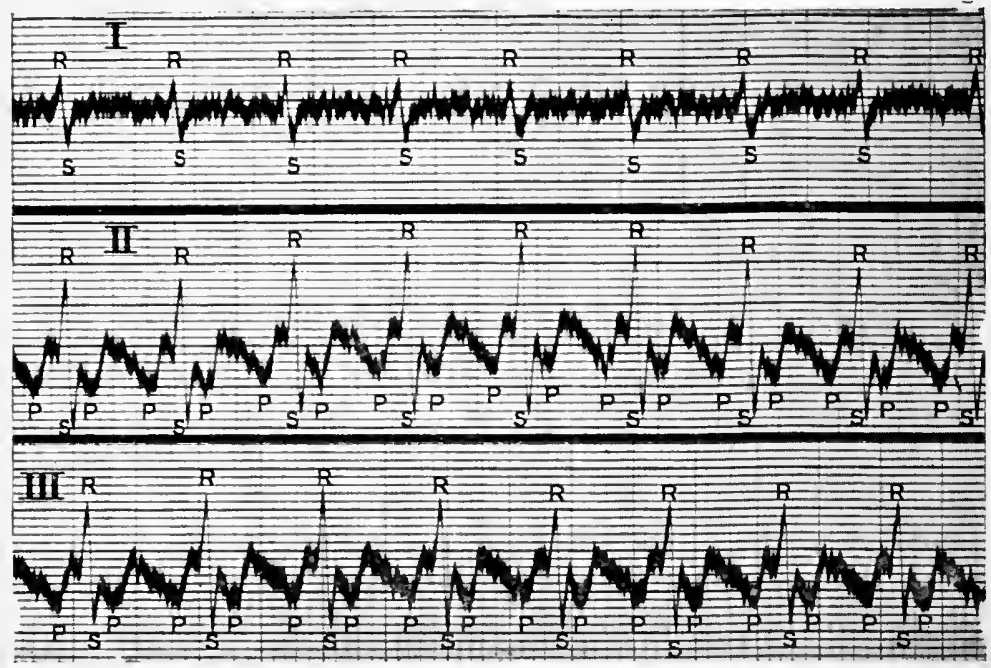

FIG. 220. Electro-cardiogram from a case of auricular flutter, with 2:1 heart-block. The rate of the auricle is between 320 and 330 per minute.

and 226-232).* The auricular deflections may range from 180 to 380 per minute. They are rhythmic in all leads, and in each lead of any given case are almost invariably of constant form. In lead I their amplitude is comparatively small. In leads II and III their amplitude is greater, . and they arc frequently either diphasic or inverted. The

* Most of the electro-cardiograms of auricular flutter are from cases kindly sent to me for electro-cardiographic examination by Sir James Mackenzie. to whom I wish to express my thanks. 


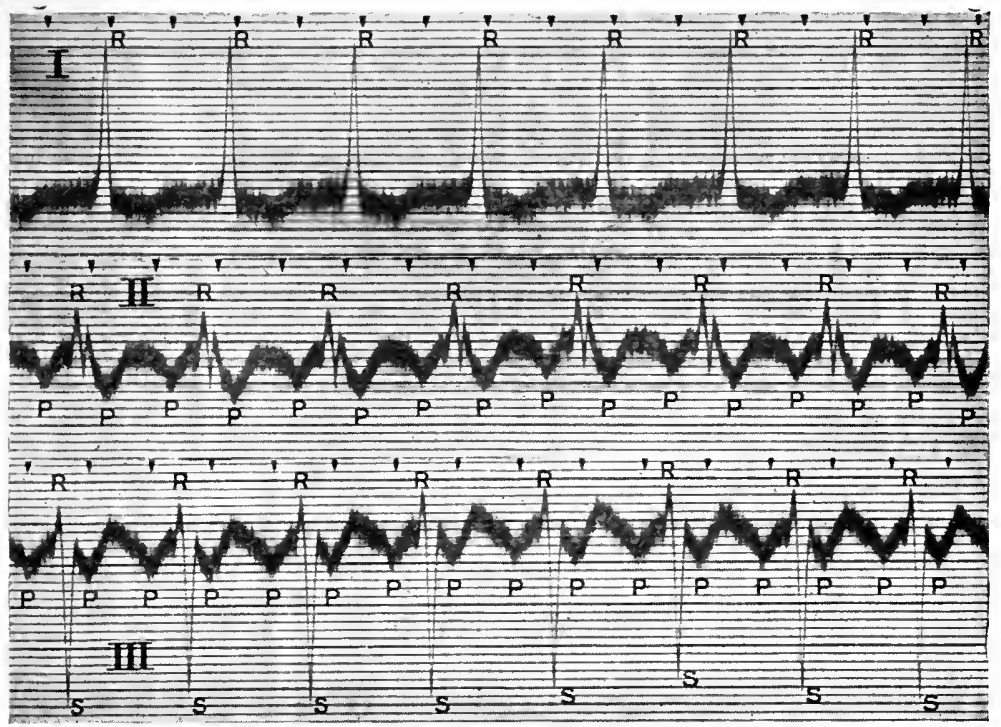

FIG. 221. Electro-cardiogram \from a case of auricular flutter, with 2:1 i heart-block. The rate of the auricle is about 300 per minute. There is also left-sided preponderance.

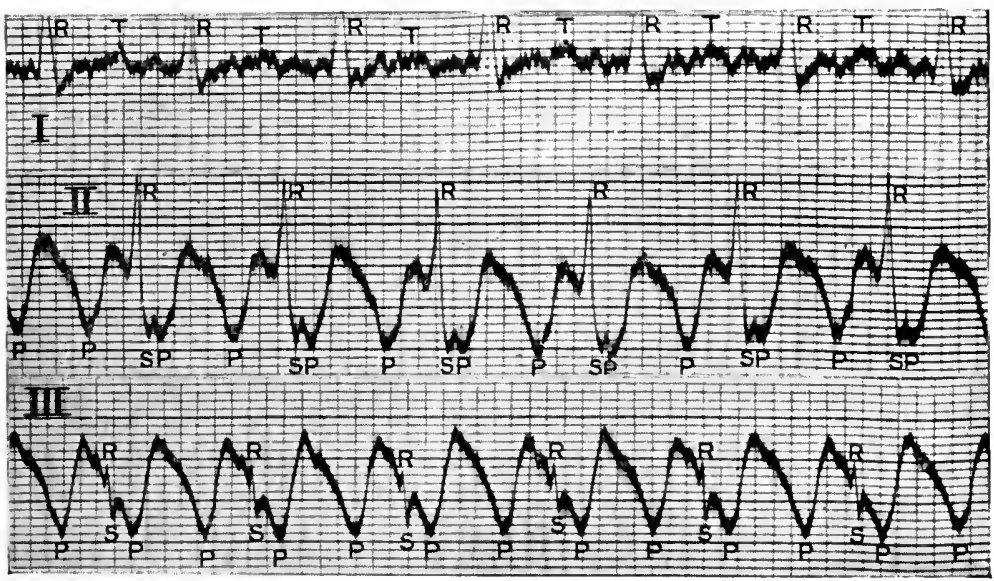

F13. 222. Electro-cardiogram from a case of auricular flutter, before the administration of digitalis. The rate of the auricle is about $\mathbf{3 4 0}$ per minute, and there is $2: 1$ heart-block. 


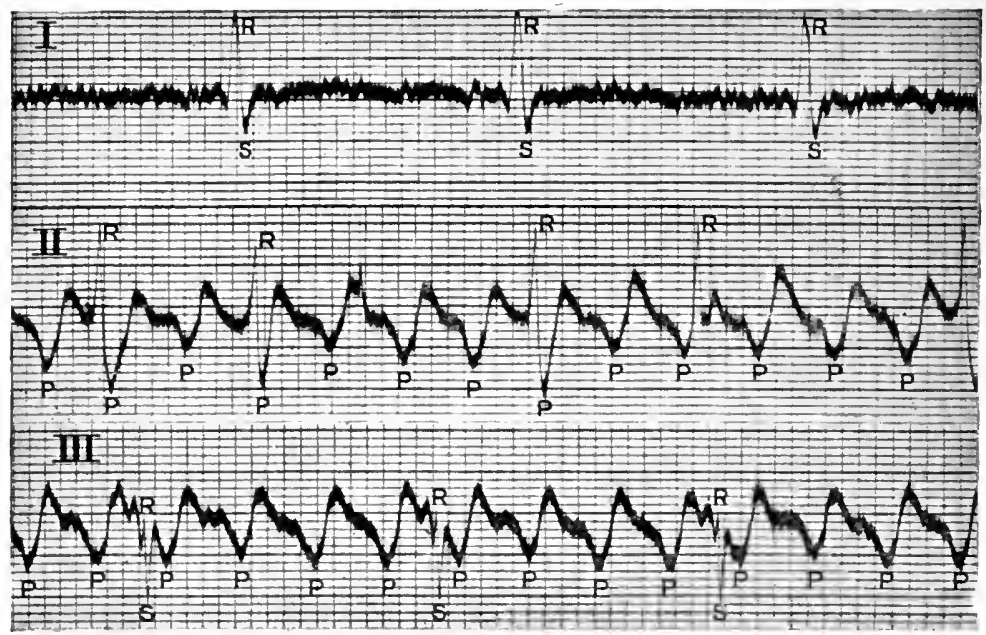

FIG. 223. Electro-cardiogram from the same case as Fig. 222, when fully under the influence of digitalis. There is an increase of the degree of heart-block, with a resulting diminution of the ventricular rate. In leads I and III there is continuous $4: I$ heart-block, and in lead II there is alternate $4: 1$ and $2: 1$ auriculo-ventricular rhythm.

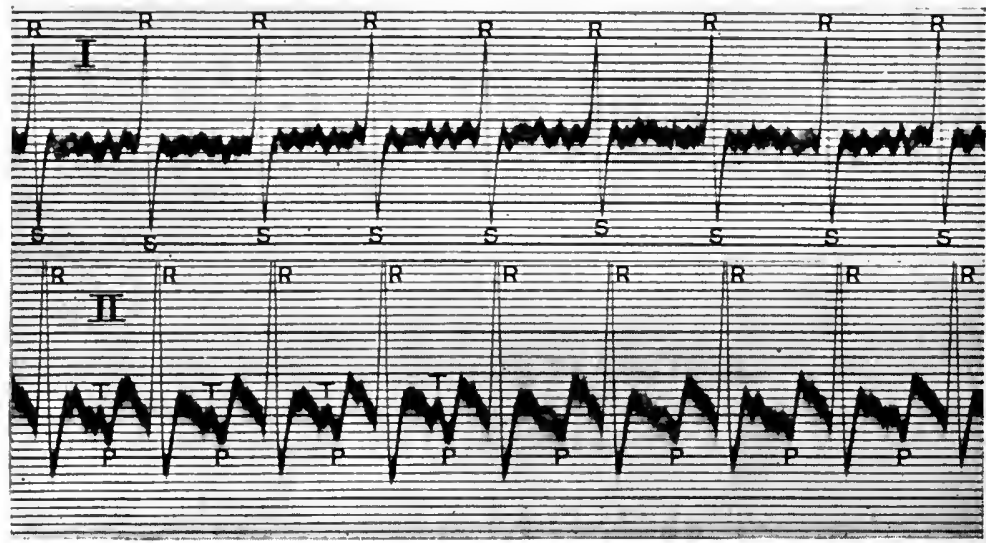

FIG. 224. Electro-cardiogram from a case of auricular flutter, with 2:1 heart-block.

relative number of ventricular complexes depends upon whether the ventricle responds to each auricular contrac- 
tion, or to the degree of auriculo-ventricular block which may be present. The ventricular deflections are of normal form, being of supra-ventricular origin.

As has just been noted, it is necessary to distinguish

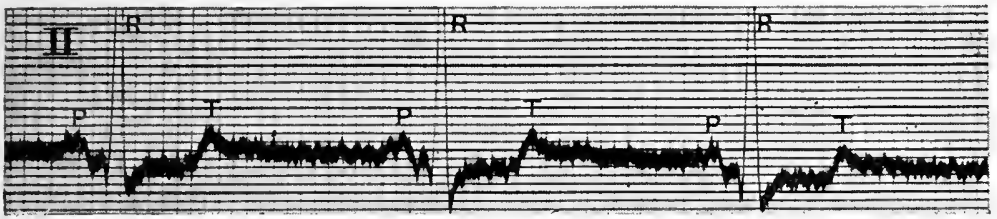

FIG. 225. Electro-cardiogram from the same case as Fig. 224, after the administration of full doses of digitalis. The rhythm of the heart is normal and the ventricular rate is between 60 and 70 per minute.

the electro-cardiograms of auricular flutter from those of auricular fibrillation. In the case of the latter, the $\boldsymbol{R}$ deflections occur at irregularly irregular intervals; the amplitude of the $R$ deflections varies from cycle to cycle ;

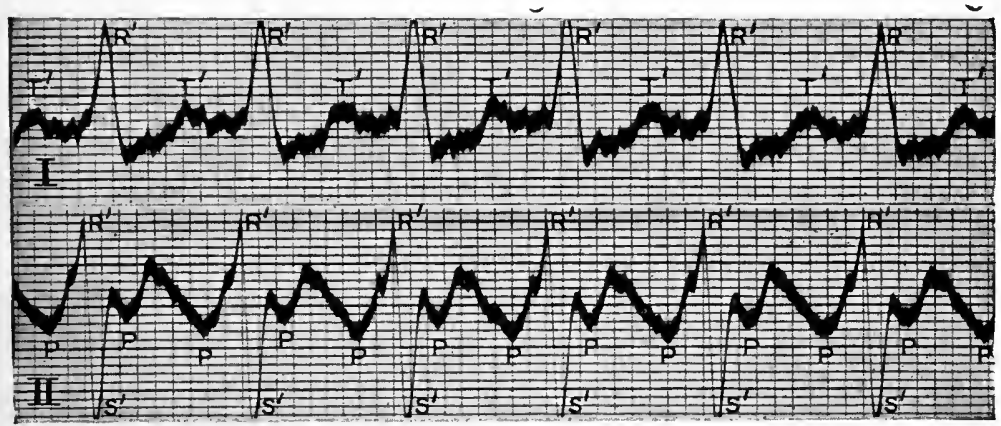

FIG. 226. Electro-cardiogram of leads I and II from a case of auricular flutter, with 2:1 heart-block. The rate of the auricle is about 330 per minute. There is also probably a lesion of the right main branch of the auriculo-ventricular bundle.

and there is often no relation between the length of a pause and the amplitude of the deflection $R$ which follows it, i.e. a short pause may be followed by an $\boldsymbol{R}$ deflection of large amplitude, and a long pause by one of small amplitude. In the case of auricular flutter, it will be 


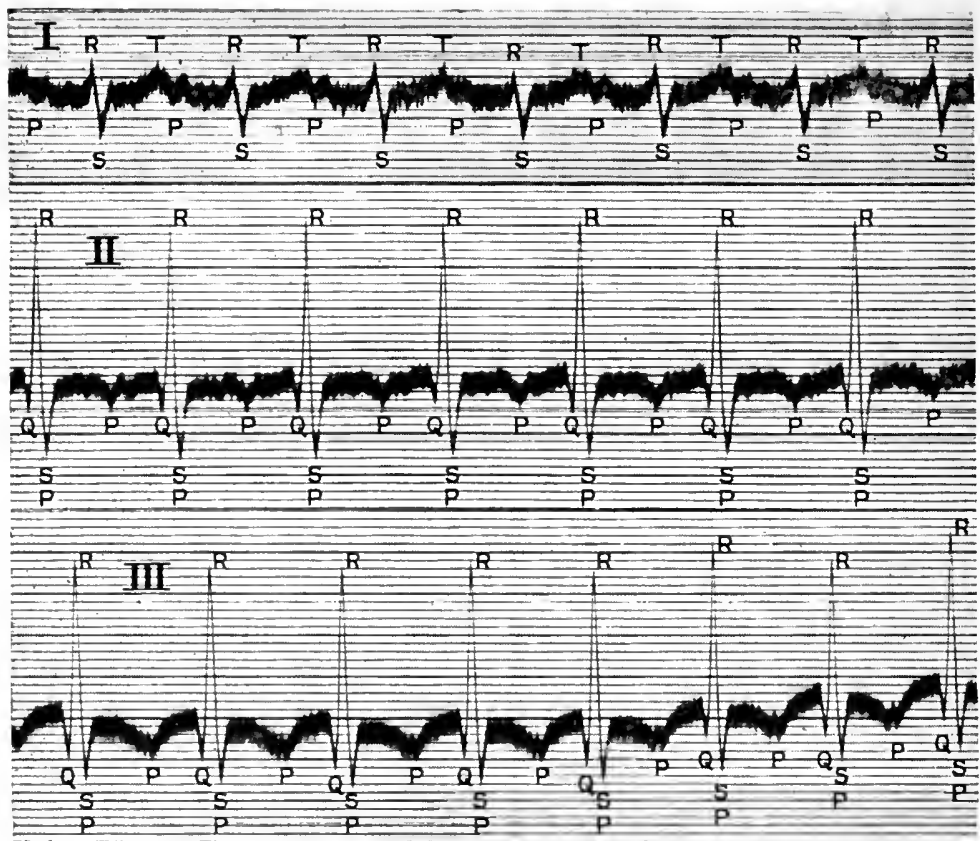

FIG. 227. Electro-cardiogram from a case of probable auricular flutter, with $2: 1$ heart-block. The auricular rate is 320 per minute. There $E$ is also right-sided preponderance.

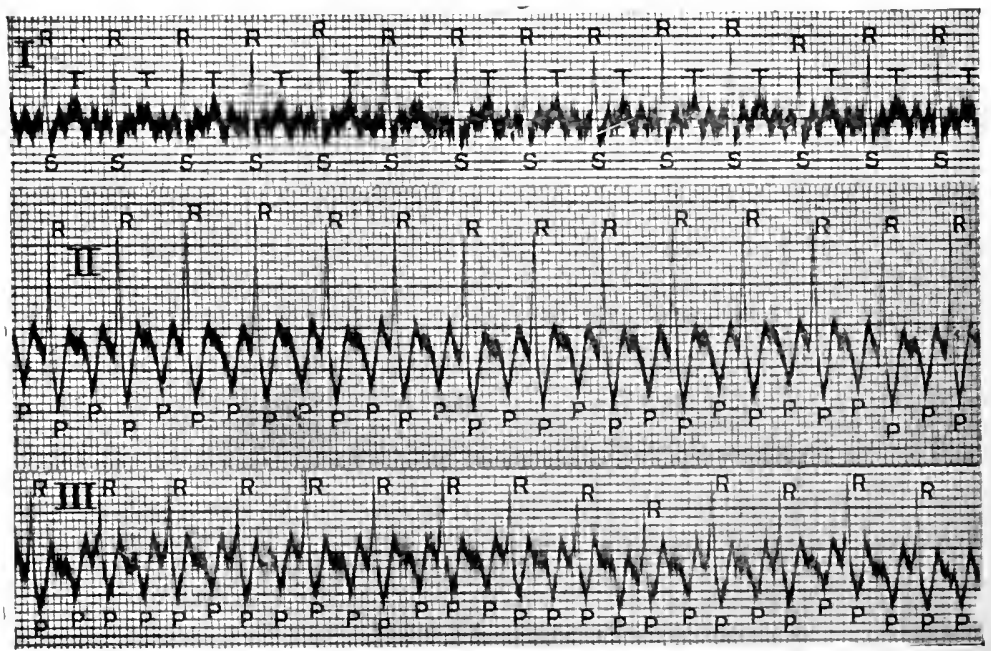

FIG. 228. Electro-cardiogram from a case of auricular flutter, before the administration of digitalis. The rate of the auricle is about 280 per minute, and there is $2: 1$ heart-block. 
found that these features are not present, even though the ventricular rhythm is markedly irregular. Further,

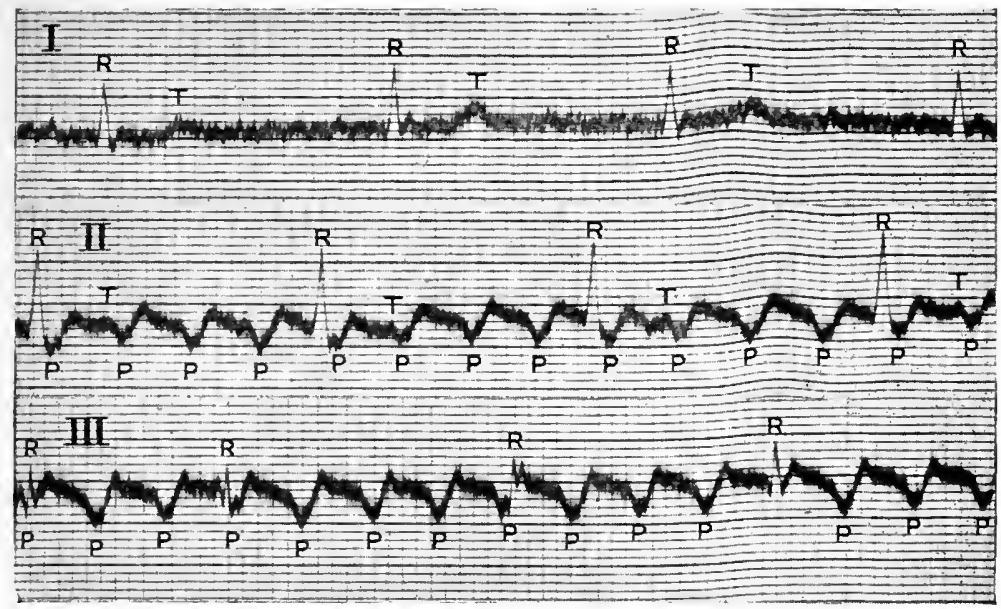

Fig. 229. Electro-cardiogram from the same case as Fig. 228, 14 days after the administration of full doses of digitalis. There is an increase in the degree of heart-block, the auriculo-ventricular ratio being continuously $4: 1$.

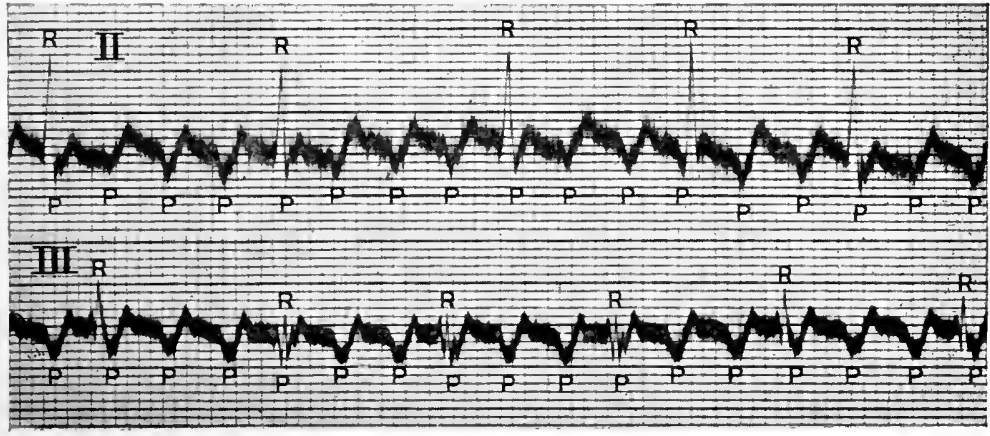

FIG. 230. Electro-cardiogram from the same case as Fig. 229, 14 days later, smaller doses of digitalis having been administered. In lead II, the auriculo-ventricular ratio is at first $4: 1$ and later $3: 1$; in lead III it is $3: 1$.

the deflections due to auricular systole in cases of auricular flutter may be distinguished from the oscillations 

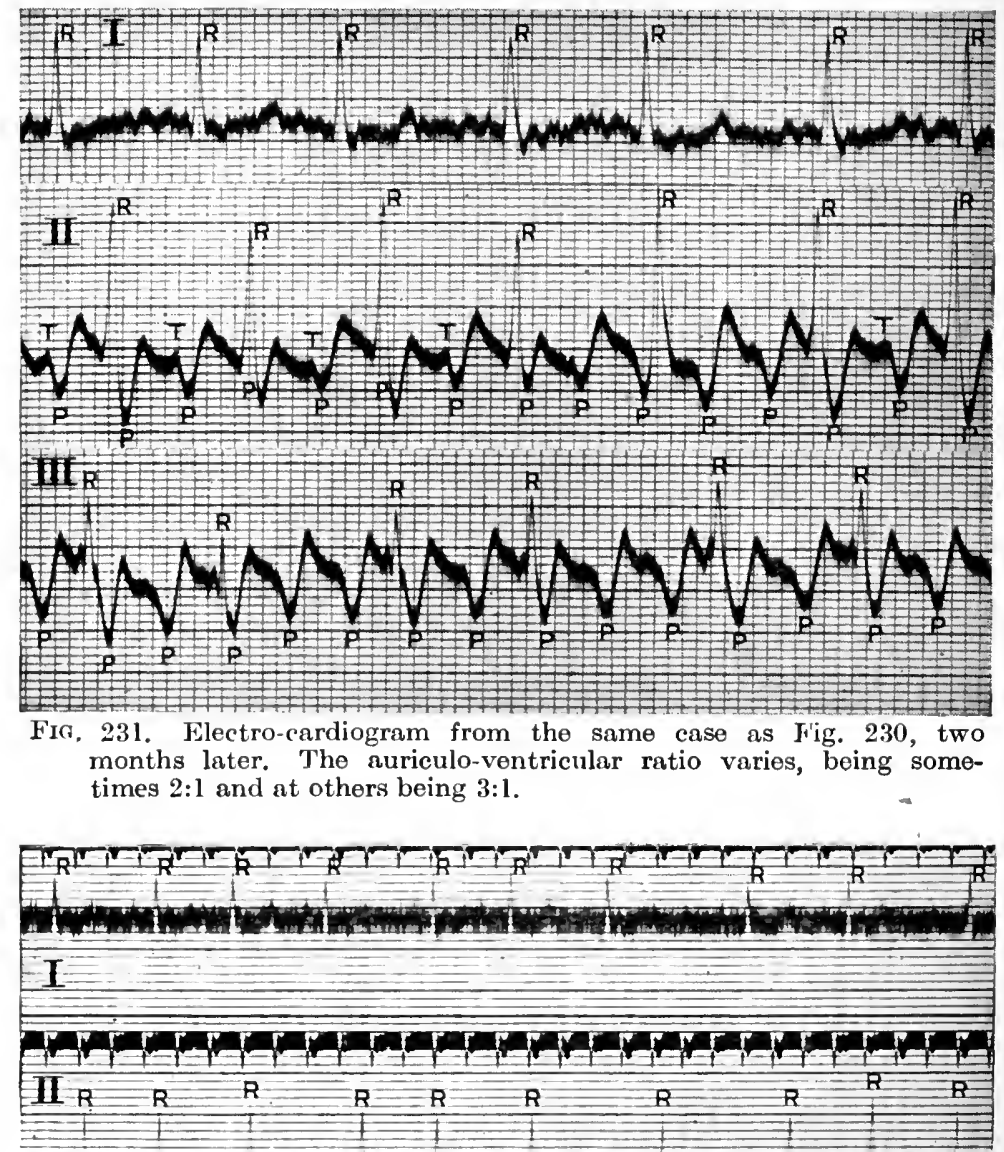

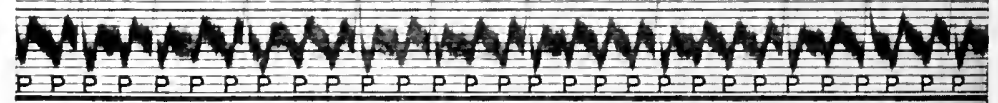

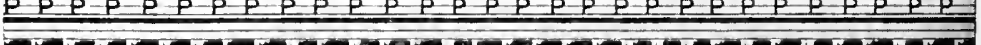

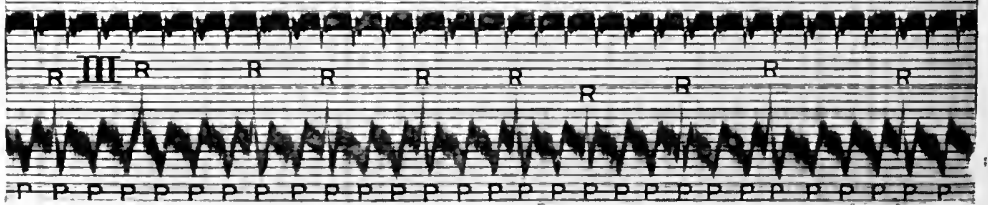

FIG. 232. Electro-cardiogram from the same case as Fig. 231 two years later. The auriculo-ventricular ratio is sometimes $2: 1$, at others $3: 1$, and at others again $4: 1$. The response of the ventricle to auricular contraction being at irregular intervals, there is irregularity of the ventricular rhythm. 
found in auricular fibrillation. In the case of the former, they are less frequent, rhythmic, and of constant form in each lead; whereas in the latter they are more numerous, irregular in rhythm, inconstant in form in each lead, and smaller in size.

Paroxysmal Tachycardia.-As might be inferred from

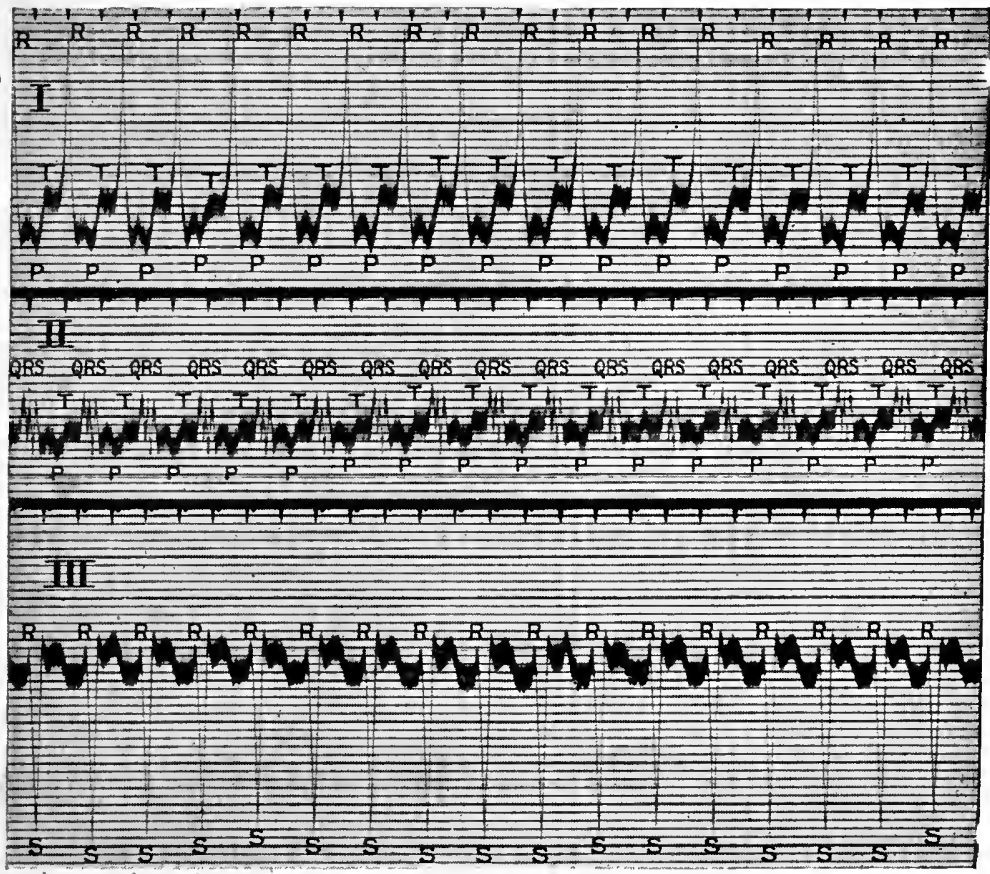

FrG. 233. Electro-cardiogram from a case of paroxysmal tachycardia. The point of origin of the new rhythm is situated in the auricle. The $P$ deflection is inverted, and coincides with the ventricular contraction of the preceding cardiac cycle. The rate is between 180 and 190 per minute.

what has already been stated in Chapter XVII, electrocardiograms present different features, according to the point of origin and path of conduction of the stimulus for contraction. In all probability they are those of auricular fibrillation or auricular flutter; but they may be indicative of auricular or ventricular (Figs. 194, 195, and 197) extra-systoles, or other causes (Fig. 233). 
Cardiac Hypertrophy.-In predominant hypertrophy of the right ventricle, $R$ is small or absent in lead $\mathrm{I}$, being smaller than $R$ in lead III, while $S$ is deep ; in lead III, $\boldsymbol{R}$ is well-marked, and $\boldsymbol{S}$ is quite small, being smaller than $S$ in lead III (Figs. 174, 176, 215, 227, and 236). The deflcctions of greatest amplitude in leads I and III, therefore, point towards each other. In predominant hypertrophy of the left ventricle, the electro-cardiogram is the

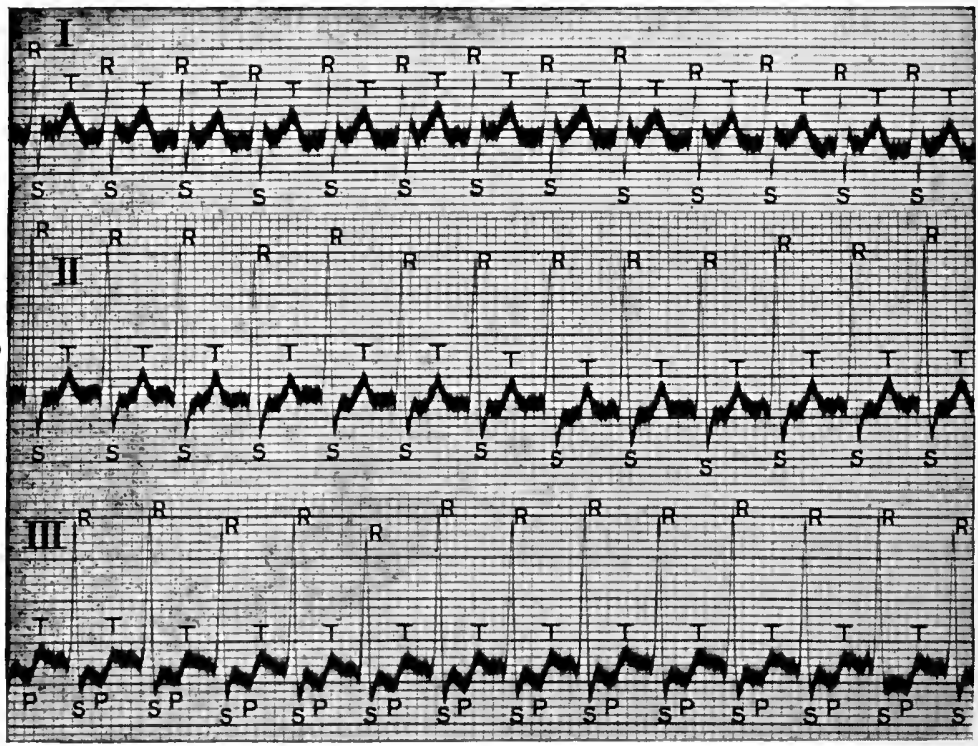

F19. 234. Electro-cardiogram from a case of paroxysmal tachycardia. The point of origin of the new rhythm is situated in the auricle. The $P$ deflection is inverted, and coincides with the ventricular contraction of the preceding cardiac cycle. It might be regarded as auricular flutter, in which the ventricle responds to each auricular contraction. The rate is between 190 and 200 per minute. There is alternation in the amplitude of the $R$ deflections.

reverse of that of the right ventricle (Figs, 171, 190, 191, 194, $202,204,216,219,221,237$, and 238). In lead I, $R$ is large and may be very large, being larger than $R$ in lead III, while $S$ in lead $I$ is small or non-existent, being smaller than $S$ in lead III; in lead III, $R$ is small or absent, and 


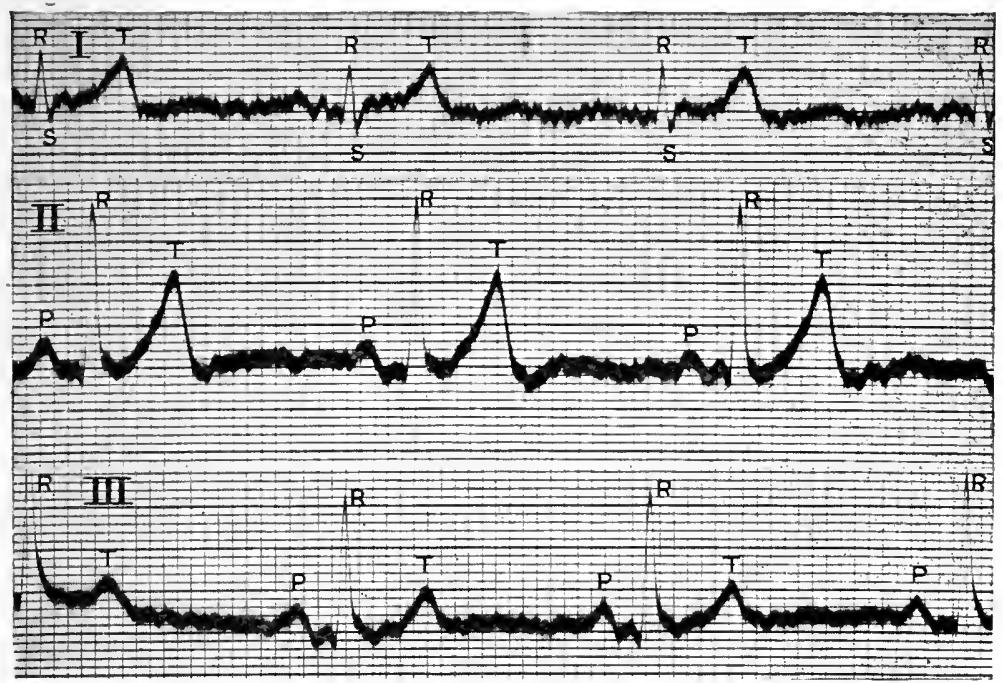

Fia. 235. Electro-cardiogram from the same case as Fig 234, after the administration of full doses of digitalis. The rhythm of the heart is normal and the ventricular rate is between 60 and 70 per minute.

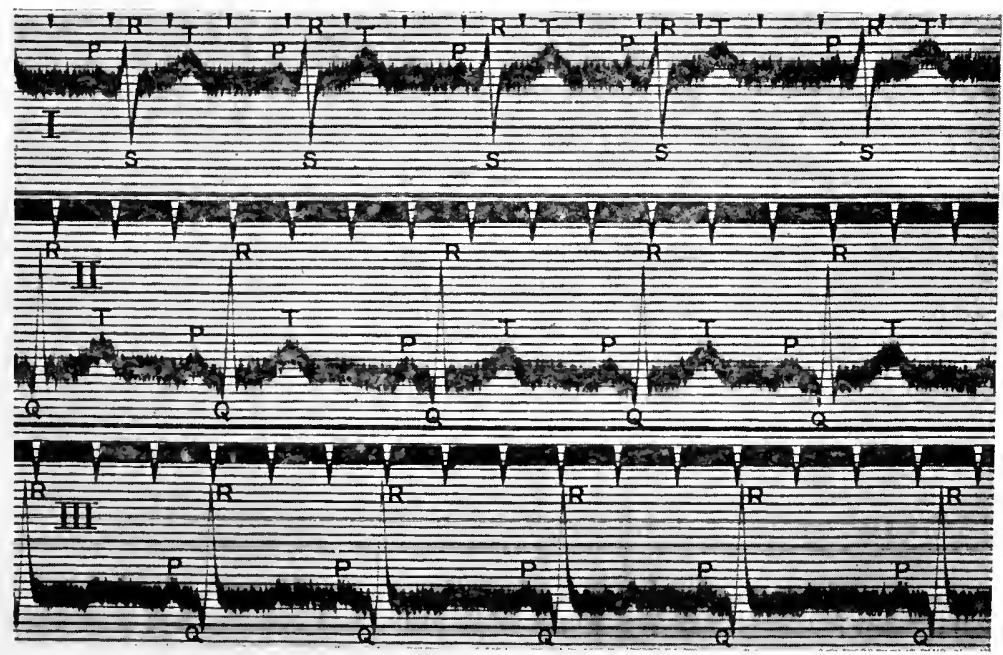

Fia. 236. Electro-cardiogram showing predominant hypertrophy of the right ventricle. 


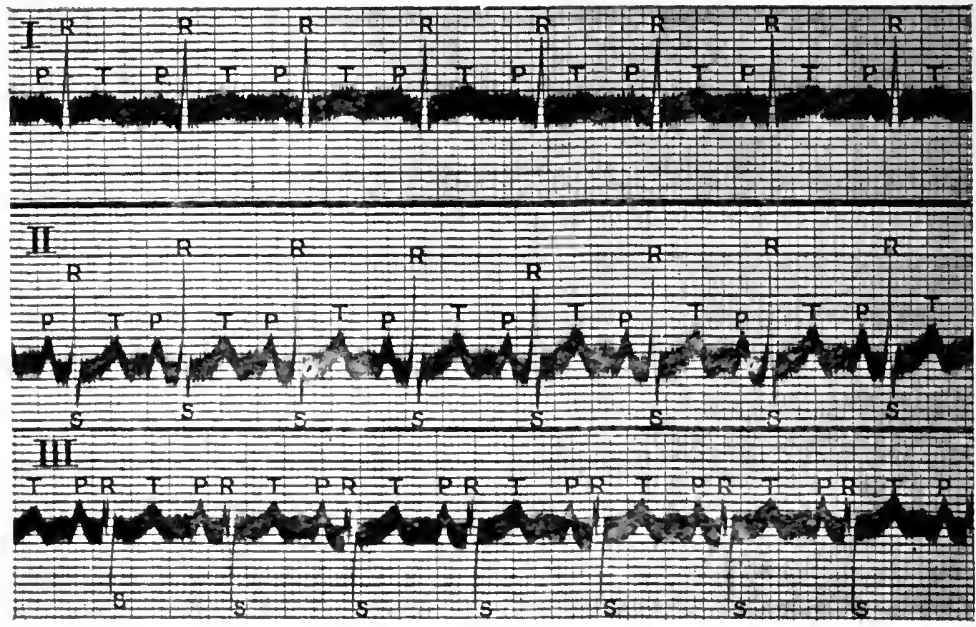

FIG. 237. Electro-cardiogram showing predominant hypertrophy of the left ventricle.

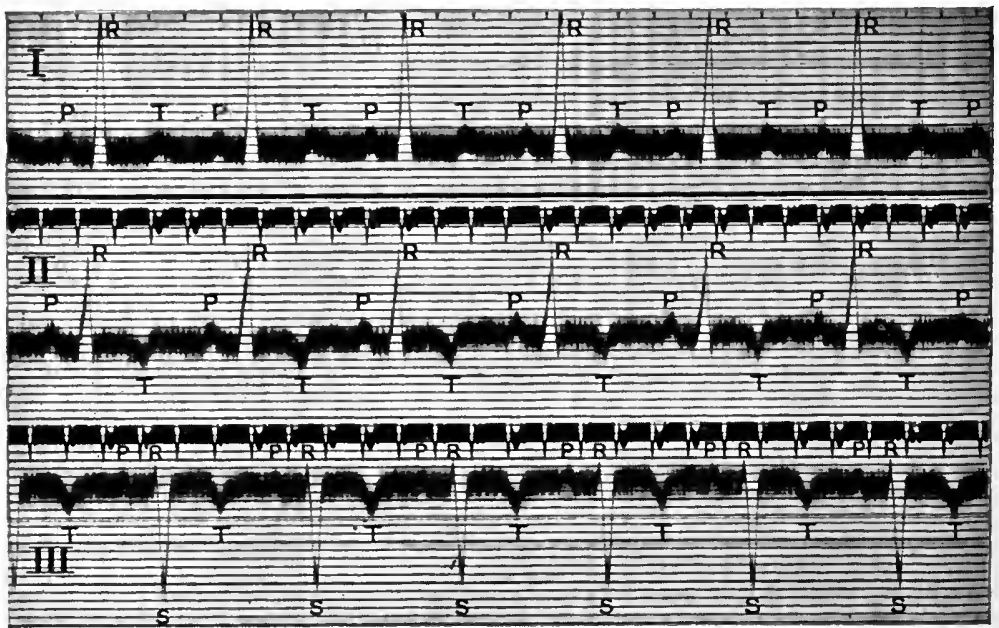

FIG. 238. Electro-cardiogram showing predominant hypertrophy of the left ventricle. There is also inversion of $T$ in leads II and III.

$S$ is large and Imay be very large. The deflections of greatest amplitude in leads I and III, therefore, point away from each other. 
The distinguishing feature between a lesion of the right or left main branch of the auriculo-ventricular bundle
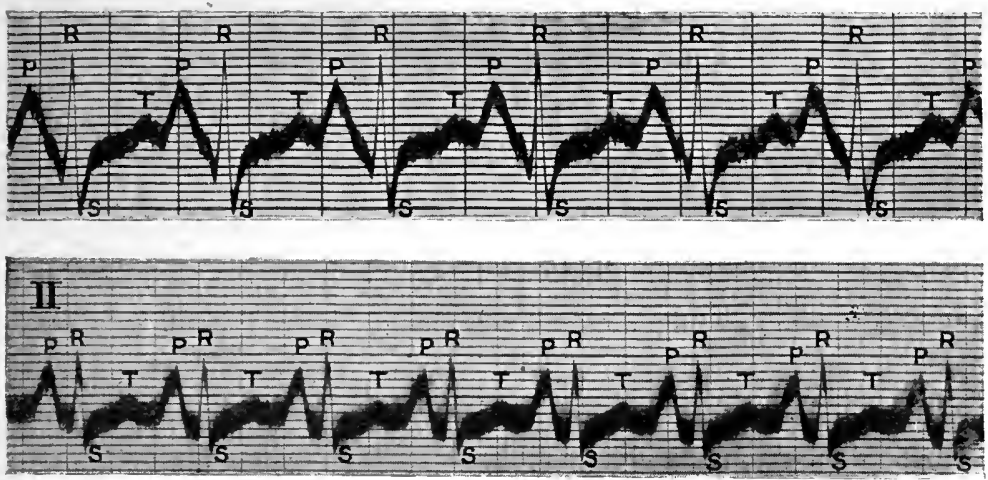

FrGs. 239 and 240. Electro-cardiograms from two different subjects, showing increased amplitude of the deflection $P$.

and predominant hypertrophy of the left or right ventricle respectively is that in the case of the former the period of time occupied by the $Q, R, S$ group of deflections is increased.
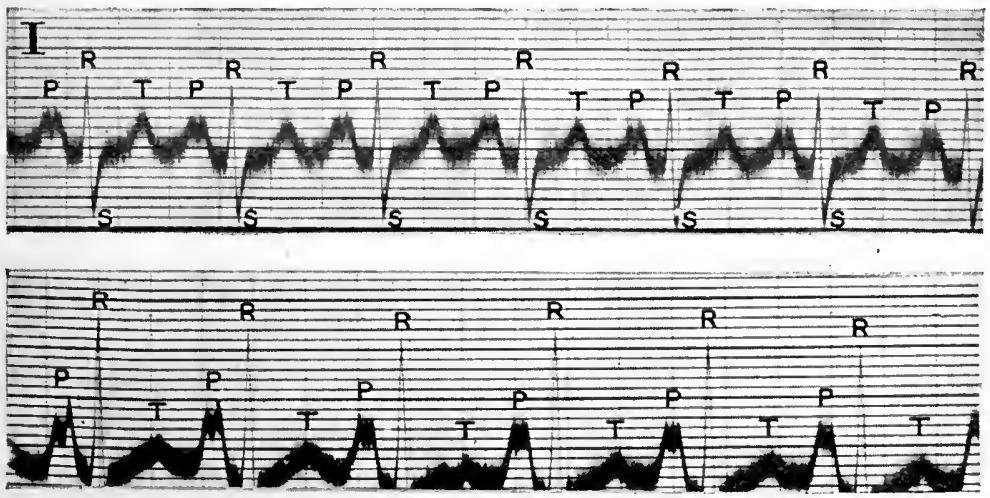

Figs. 241 and 242. Electro-cardiograms from two different cases of mitral stenosis. The deflection $P$ is increased in amplitude, and is also broad, has a flat top, and is bifurcate.

It is said that the amplitude of $\boldsymbol{P}$ is increased (Figs. 239 and 240 ), or cven doubled, in hypertrophy of the auricles. 28 


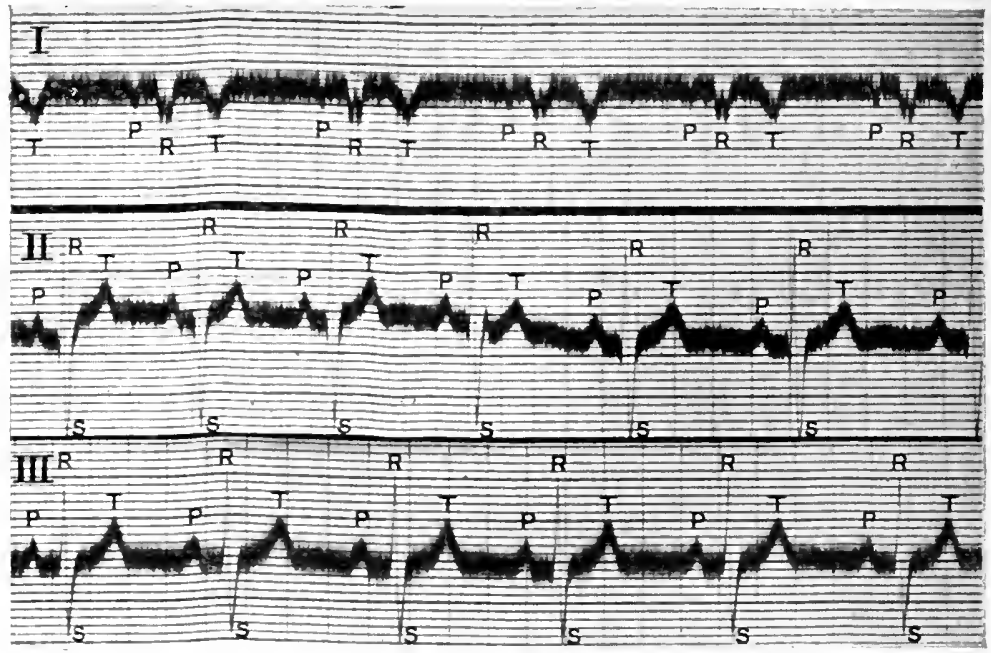

Fig. 243. Electro-cardiogram from a case of transposition of the heart. All the deflections in lead I are inverted.

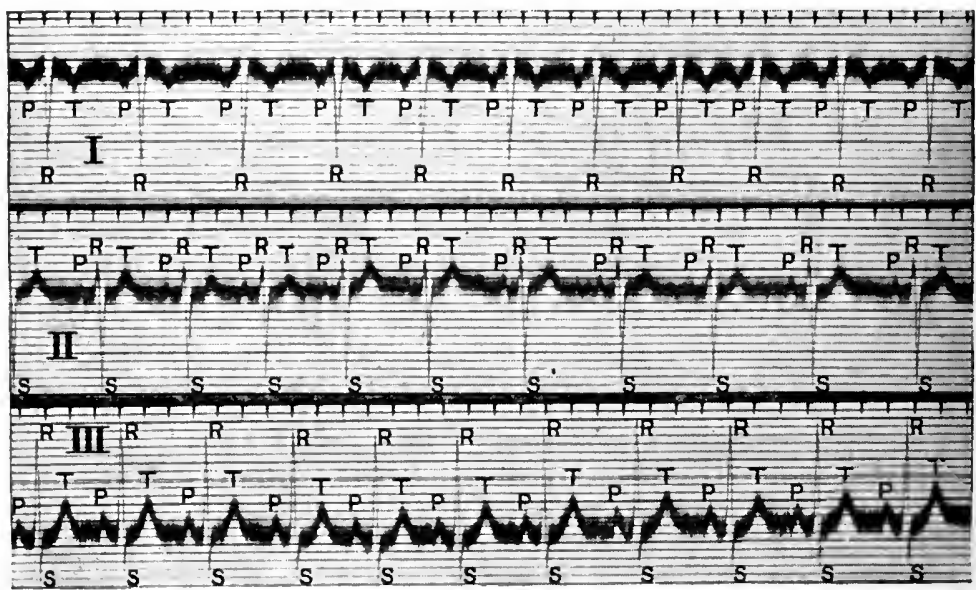

FIG. 244. Shows the same as Fig. 243.

Some writers, however, state that this is occasionally to be met with in normal hearts.

Chronic Valvular Disease.-In mitral stenosis, the 


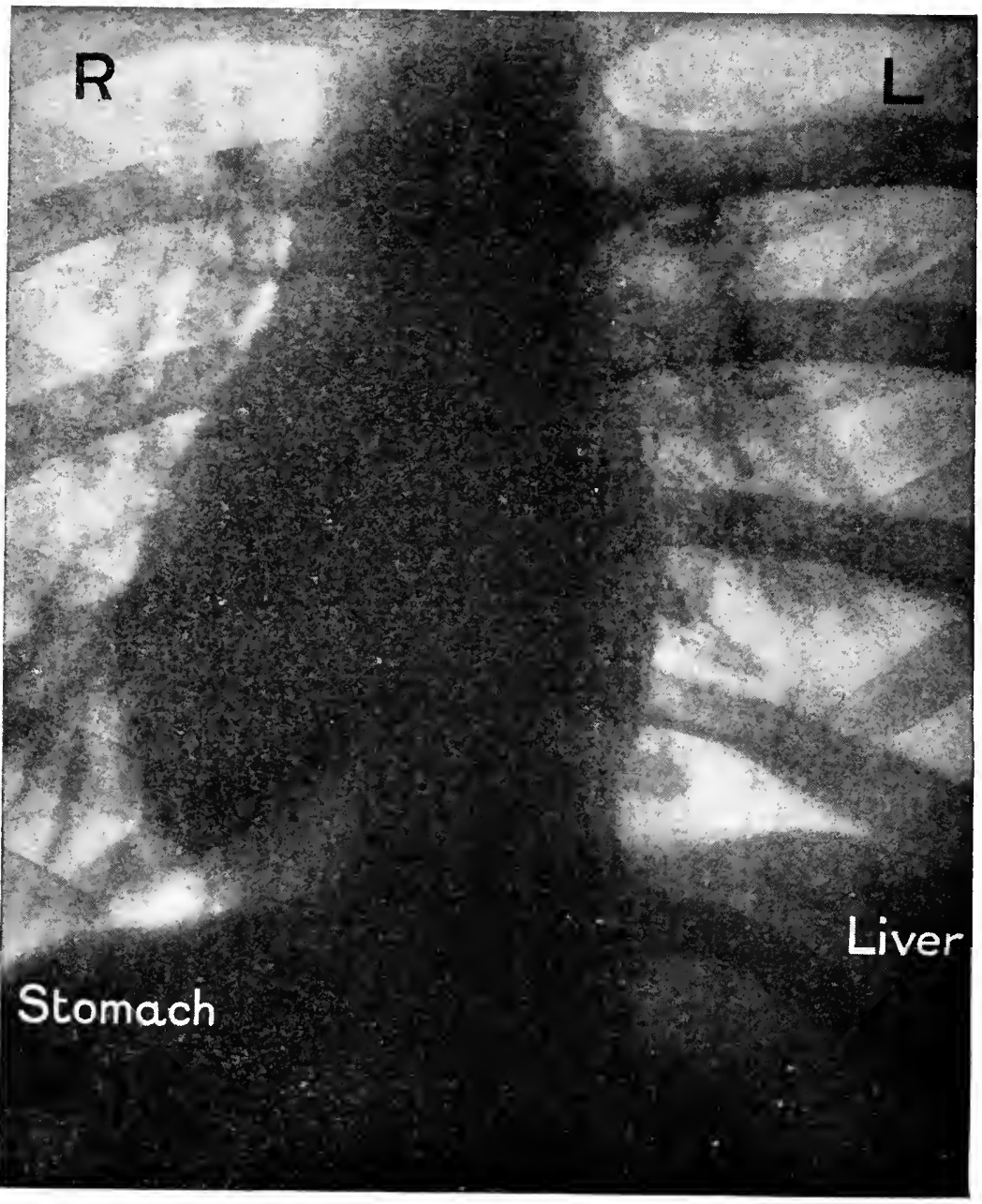

Fia. 245. Radiogram from the same case as Fig. 244. The photograph was taken from the front. The heart lies on the right side of the thoracic cavity, and there is also transposition of the liver and stomach. ('There is considerable thickening of the hilar glands, with infiltration of surrounding lung tissue.)

amplitude of the deflection $\boldsymbol{P}$ is often markedly increased, and not infrequently is also broad, has a flat top, and is 
bifurcate (Figs. 241 and 242). Lewis believes that these features are of diagnostic value. While a marked increase in the amplitude of the deflection $\boldsymbol{P}$ is especially found in mitral stenosis in which there is hypertrophy of the auricles, in my opinion it is not justifiable to diagnosc the lesion from this alone. In many cases of mitral stenosis, the ventricular complexes are the same as those of predominant hypertrophy of the right ventricle. When auricular fibrillation supervenes in mitral stenosis, the curves will present features characteristic of that condition. In many cases of aortic incompetence, the ventricular complexes are the same as those of predominant hypertrophy of the left ventricle.

Congenital Heart Disease.-It is said that an increase in the amplitude of the deflections is often found in congenital heart disease.

Transposition of the Heart.-In this condition, all the deflections of a curve from lead $I$ are inverted (Figs. 243 and 244). It is believed that this form of electrocardiogram is found in no other condition. 


\section{N DE X}

Abscesses in the myocardium, $\mathbf{3 5 3}$

Accelerator nerve, action on heart-rate and muscle functions, 15

$a-c$ interval, in cases of heart-block, 184-6, 188

Aconite, action on the heart, 138

experimental administration, $r$, sult of, 138, 139

Acupuncture, in treatment of dropsy, 155

Adams-Stokes' syndrome, diagnos is in heart-block, 196 extra-systoles simulating, 180 in auricular fibrillation, 205 in fatty degeneration, 308 in heart-block, characteristics, 194

in heart failure, 121

symptoms, compared with those of heart-block, 194

Adherent pericardium, see Pericarditis, chronic adhesive

Adrenalin,

in treatment of low blood-pressure, 80

Afferent nerves,

Age,

in relation to blood-pressure, 70

in relation to prognosis of heart

Agurin, disease, 126

action of, 147

Alcohol,

blood-pressure in relation to, 84

cardiac affections in relation to, 133

in treatment of acute, simple endocarditis, 243

heart failure, 148

Alternation of the heart,

in a sphygmogram, 417

in an electro-cardiogram, 417
Ammonium salt,

for nervous symptoms of cardiac disease, 133

in treatment of sleeplessness, 152

Amyl nitrite,

for paroxysm of angina pectoris, 389

in treatment of heart-block, 197

rise of blood-pressure in angina pectoris due to, 383

Anacrotic pulse,

definition, 60

diagnostic of aortic stenosis, 60 , 61,262

in aortic stenosis and incompetence, sphygmogram showing, 267

Anæmia,

accompanying heart disease, treatment, 135

capillary pulse observed in, 30

pernicious, cause of fatty degeneration, 306

Aneurism, 352-3

aortic systolic thrill indicating, 32

arterial pressure in, 81 .

blood-pressure in, $\mathbf{8 1}$

causes, characteristics, 352,353

conditions in which it occurs, 352

diastolic shock a reliable physical sign of, 28

Aneurism of thoracic aorta,

difference in the two radial pulses in, 68

sphygmogram showing right and left radial pulses, 68

Aneurismal dilatation, character of murmurs in, 53

Aneurismal varix, centripetal venous pulse observed in, 31

Angina abdominis, 382 
Angina pectoris, accompanying fatty degeneration, 307

age and sex incidence, 380

auricular fibrillation in relation to, 205

blood-pressure and pulse rate in, 383

conditions associated with, 380 , 381

conditions simulating, 384, 385

definition of, 380

diagnosis, 384

due to aortic incompetence, 265

duration of the attack, 384

effect of amyl nitrite on bloodpressure during a ttack of, 383 etiology, 380,381

grades and modified forms of, 384

nature of the pain, $381,382,384$, 385

patient's general condition in, 382 prognosis, 385, 386

relationship of pulsus alternans to, 201

structural changes and conditions associated with, 380,381

symptomatology, 381

treatment, 387-90

drugs, 388-9

general measures, 387,388

typical clinical picture of, 384

see also Pseudo-angina

"Angina sine dolore" of Gairdner, 382

Ankles,

œdema of, in tricuspid incompetence, 291

œdema of, in tricuspid stenosis, 288

Antiserum treatment of ulcerative endocarditis, 252

Antistreptococcus serum,

in treatment of rheumatic infection, 242

Aorta,

and pulmonary artery, conditions associated with transposition of, 363

and pulmonary artery, prognosis in cases of transposition of, 373

aneurismal dilatation of, area of cardiac impairment in, 38

aneurismal dilatation of, differential diagnosis of aortic stenosis from, 263, 264
Aorta (continued),

atheroma of, characteristics, 301

coarctation of, adult type, 368

coarctation of, cause and characteristics, 362

congenital affection of, 361

double, 363

hypoplasia of, 363

position of, 1

stenosis and atresia of, forms of congenital heart disease, 362

Aortic accentuation,

conditions in which it occurs, 41

Aortic arch,

aneurism of, characteristics of pulsation due to, 26

Aortic area,

systolic murmur over, diagnosis of aortic stenosis in relation to, 262

systolic pulsation in, characteristics, 26

Aortic disease,

and mitral, prognosis compared, 127

and mitral, tracing showing partial heart-block in, 186

patient's appearance in, 122

relative frequency among males and fermales, 126

syncope during, 121

treatment, 293

Aortic incompetence, accompanying aortic stenosis, 260

accompanying mitral stenosis, differential diagnosis, 278

administration of digitalis in, 141 blood-pressure in, 81

centripetal venous pulse observed in, 31

character of murmurs in, 54

conditions associated with, 265, 266,267

death frequent in, 128

diagnosis, 269, 270

differential diagnosis from pulmonary incompetence and mitral stenos is, 270, 287

electro-cardiogram in, $\mathbf{4 3 6}$

etiology, 264-5

exaggerated pulsation of arteries in, 30

frequency of, 262

prognosis, 271

relative characteristics and rarity of, 265

relative frequency of, 258 . 
Aortic incompetence (continued), sphygmograms from cases of, 266,267

stenosis frequently associated with, 257

symptomatology, 265-9

venesection contra-indicated in, 150

Aortic obstruction,

aortic systolic thrill indicating, 32

Aortic orifice, position of, 4

stenosis a.t, grave nature of, 373

Aortic regurgitation,

apex-beat and jugular vein tracings from a case of, 97

Aortic stenosis,

anacrotic pulse from a case of, 60

anacrotic pulse and pulsus bisferiens diagnostic of, 60,61

and incompetence, sphygmograms from cases of, 267

diagnosis, 261-3

differential diagnosis from mitral stenosis, 270

differential diagnosis from pulmonary incompetence, 270

differential diagnosis from pulmonary stenosis, 263

etiology, 259

form of congenital heart disease, 362

prognosis, 264

sites and characteristics, 362

sphygmogram from a case of, 260

symptomatology, 259

types of, 362

Aortic stenosis, congenital,

above and below entrance of ductus arteriosus, prognosis, 373

above entrance of ductus arteriosus, diagnos is rarely possible, 371

diagnosis, 371

differential diagnosis of congenital pulmonary stenosis from, 371 physical signs, 368

Aortic systolic murmur, causes of, 257

Aortic systolic thrill, significance, 32

Aortic valve, area of sound, 5 degenerative processes giving rise to valvular disease, 116 structure and characteristics, 4
Aortitis,

associated with endarteritis obliterans, 300

Apex-beat,

age in relation to position of, 4

and radial pulse, simultaneous tracings, $56,64,67$

and radial pulse, tracings showing positive cardiogram, 94, 95

characteristics, 3

definition, 2

diminution or disappearance of, not necessarily indicative of disease, 25

displacements, conditions causing, 24

extent of, conditions increasing, 24,25

extrinsic causes of displacement of, 370

faint or absent, percussion in cases of, 35

force of, conditions increasing and diminishing, 25

" heaving" or "thrusting" of, 319,321

in acute pericarditis, 334, 335, 336

in acute, simple endocarditis, 239

in aortic incompetence, 268, 269

in aortic stenosis, 260,262

in cardiac dilatation, 326

in cardiac failure, 124

in cardiac hypertrophy, 319,320

in chronic interstitial myocarditis, 314

in congenital heart disease, 366 , 367

in fatty degeneration, 309

in "irritable heart," 164

in mitral regurgitation, '281, 282

in mitral stenosis, 274,278

in pericardial effusion, 336, 339

meso-cardia and dextro-cardia simulating displacement of, 370

radial pulse tracing more reliable than that of, 90

systolic recession of, characteristics and cases in which observed, 27

tracing, how obtained, 91, 92 tracings in case of greatly enlarged heart, 97, 98, 99

Arcus senilis,

presence in fatty degeneration, 309 
Area of cardiac impairment,

in adherent pericardium, 344,345

in aortic incompetence, 268

in aortic stenosis, 261, 263

in cardiac dilatation, 326

in congenital heart disease, 366, 367,368

in hypertrophy of the heart, 319 , 320

in mitral regurgitation, 281

in mitral stenosis, 275

in palpitation, 160

in paroxysmal tachycardia, 227

in pericardial effusion, 336,338 , 339

in tricuspid stenosis, $\mathbf{2 8 8}$

Area of deep or relative impairment, how determined, 34

Area of superficial or absolute impairment,

how determined, 36

Arsenic,

as a cardiac tonic, 148

Arterial circulation, mechanism of the, 69,70

Arterial pressure,

action of digitalis on, 139 action of drugs on, 72, 86

clinical data to be considered in estimating the, 71,72

diastolic, definition, 70

diastolic, how estimated, 74-6

diastolic, normal range of, 77

effect of digestion on, 78

effect of exercise on, 78

effect of pain on, 77

effect of sensory stimulation on, 77

effect of sleep on, 78

effect of tobacco on, 78

factors increasing the, 69

factors on which dependent, 69 , 70

fall of, following stimulation of divided vagus, 15

in angina pectoris, 383

in aortic stenosis, 260

in complete heart-block, 190

in heart failure, 124

in mitral stenosis, 273

in nervous patients, 77

in paroxysmal tachycardia, 226

increase in angina pectoris, 383

maximum and minimum, how estimated, 67,68

mean, definition, 70

methods of estimating, 70

normal, character of the pulse, 61
Arterial pressure (continued), normal standard of, 77

range of variation in different individuals, 71

sphygmomanetric method of estimating, observations and technique, 71-6

systolic, definition, 70

systolic, fluctuations in, 72

systolic, how estimated, 74-6

systolic, in acuto and chronic nephritis, 80, 81

systolic, normal standard as measured in brachial artery, 77

variations in, under physiological conditions, 77

variations under pathological conditions, 78-87

vaso-motor system regulating, 70

Arterial pressure, subnormal,

character of pulse in, 61

conditions in which seen, 79

factors in causation, 78,79

treatment, 79

Arterial pressure, super-normal, advantages of sphygmomanometer in, 71

aortic accentuation in, 41

causation and conditions in which seen, 80,82

character of the pulse in, 62

importance of early detection of, 71

nitrites in, 149

symptomatology, 81

treatment, 82-7

vaso-dilators in, 142

venesection in, value and effects of, 69

Arterial pulsation,

characteristics and causes, 28 , 29,99

Arteries,

auscultation of, method, 53, 54 .

condition of the rall of, how determined, 67

drugs causing dilatation of, 86

elasticity of walls of, $69,70,76$

exaggerated pulsation of, conditions in which observed, 30

exaggerated pulsation of, in aortic incompetence, 267 , 269

size of, how determined, 67

Arterioles,

muscularity of, 70 
Arterio-sclerosis,

accompanying angina pectoris, 381

administration of iodides in, 148

aortic accentuation in, 41

aortic incompetence associated with, 267

aortic stenosis associated with, 260

brachial artery to be examined in all suspected cases of, 67

degenerative processes of chronic endocarditis frequently associated with, 255

predisposing to angina pectoris, 381

Arterio-venous aneurism,

murmurs in, 50

thrill and murmur of, 371

Arthritis,

chief manifestation of rheumatic infection of the heart in adult life, 232

in childhood, in relation to rheumatic infection of the heart, 232

Atheroma,

associated with granular contracted kidney, 301

etiology, 301

nature of the condition, 300

nodular, blood-pressure in, 81

nodular, characteristics, 301

sclerous form, characteristics, 300,301

Atropine,

hypodermic injection in sinus irregularity, 166

in cardiac affections, 148

in heart-block, 197

in partial heart-block, 189

Auricle,

and ventricle, movements recorded by clinical polygraph, 88

dilatation of, 323

formation in primitive cardiac tube, 9,356

hypertrophy of, 316,320

incomplete and complete a.bsence of septum between, 360

indications of failure of, 124

jugular pulse affording information regarding the action of, 90

normal contraction, how indicated, 106, 107

period of contraction of, 11
Auricle (continued),

position of, 2

sound produced by contraction of, 185

stimulation and contraction of, order and course, 11

stimulus from, to ventricle, 184 , 401

systole of, in the cardiac cycle, 16

Auricular and ventricular contraction,

interval separating the commencement of, 100,101

Auricular extra-systole, see Extrasystole

Auricular fibrillation, 203-12

administration of digitalis in, 140 , $141,143,145$

associated with acute cardiac dilatation, 324, 327

auricular wave in, 100

cases mistaken for primary cardiac overstrain, 374

causing paroxysmal tachycardia, 224

conditions associated with, 203, 204

degrees of heart failure resulting from, 205

diagnosis, 209

difforential diagnosis from auricular flutter, 220

differential diagnosis from partial heart-block, 104, 105

duration of, 204

effect of digitalis in, 142, 143, 145, 210,212

effect of digitalis in, tracings showing, 211, 212

effect on the heart, 204

electro-cardiograms showing, 417421

electro-cardiograms to be distinguished from those of auricular flutter, 425,426

etiology, 204

following digitalis in auricular flutter, 222

general observations, 203

$h$ or $b$ wave in, 104, 105

jugular and radial pulse tracings from case of, 106, 107

nature of the condition, 203

nature of the murmurs in, 49

nature of the pulse in, 66

prognosis in, 129

prognosis, 210

pulse tracings in, 206-9, 211, 212 
Auricular fibrillation (continued),

recent advances in treatment of, 130

rest in, 136

rheumatism in relation to, 204

symptomatology, 205

treatment, 212

ventricular venous pulse in, 29 , 107

Auricular flutter,

age and sex incidence, 215

auricular fibrillation following, 214

conditions associated with, 215, 216

definition and introduction of the term, 213

diagnosis, 218-9

differential diagnosis from auricular fibrillation, 220

differential diagnosis from tachycardia with normal rhythm, 219,220

duration of, 214

effect of digitalis in, 214, 221, 222

effect on the heart, 214,223

electro-cardiograms of, 422-6

electro-cardiograms to be distinguished from those of auricular fibrillation, 425

etiology, 215

heart failure following, 215

jugular and radial pulse tracings in, 217-21

nature of the condition, 212

paroxysmal tachycardia due to, character of the pulse in, 226

paroxysmal tachycardia with regular rhythm or pulsus alternans chiefly due to, 213

partial heart-block present in majority of cases of, 213

prognosis, 222

pulsus alternans associated with, tracings showing, 200

symptomatology, 215

temporary, causing paroxysmal tachycardia, 224

treatment, 223

with partial heart-block, pulse tracing in, 219

Auricular liver pulse, 111

Auricular systole, occurrence of, 10, 18 period of completion, 19

Auricular venous pulse, sec Jugular pulse
Auricular wave,

in a cardiogram, tracingsshowing, 95,96

in a phlebogram, how determined, 109

in the jugular pulse, 98, 99, 100, $101,105,106,107$

Auriculo-ventricular block, auricular flutter with, tracings illustrating, 217, 218

complete, tracings showing, 190 , 191,192

diagnosis, 195

electro-cardiogram showing, 412

extra-systoles with, tracingss howing, 192

present in majority of cases of auricular flutter, 216, 222

pulse rate in, 195

tracings illustrating, 187, 188

Auriculo-ventricular bundle,

compression producing various degrees of heart-block, 12

depressed conductivity indjcating some affection of, 101

dissections showing origin of (illustration), 7, 8

electro-cardiogram showing lesion of right and left branch of, 414-7

excitability of, 10

lesion in heart-block, 193

measure of function of conductivity of, in an electro-cardiogram, 401

of Kent and His, position and characteristics, 9

position of, 7

probable remains of primitive cardiac tube, 10

starting-place of heart's contraction, 10

Auriculo-ventricular node, excitability of, 10

position, 7

probable remains of primitive cardiac tube, 10

Auriculo-ventricular orifices, lowered state of tonicity causing dilatation of, 14

Auriculo-ventricular rhythm, disassociation, 185

disassociation, electro-rardiogram showing, 413

disassociation in complete heartblock, 190

Auriculo-ventricular valves, congenital lesions of, 363 
Auscultation, 39-54

diagnosis of extra-systoles by, 180 how performed, 39

of arteries, method, 53, 54

of veins, method, 54

Baillie's pill,

in treatment of cardiac dropsy, 154

Baths and spa treatment, 85, 137

Beefy heart, 317

Bilocular heart, nature of the condition, $\mathbf{3 5 9}$ prognosis, 372

Blocked auricular extra-systoles, 176

Blood, circulation in the fœtus, 357,358 , 360,361

condition in congenital heart disease, 365

condition in ulcerative endocarditis, 247, 252

elimination of toxic content of, in "soldier's heart," 379

Blood-letting, see Venesection

Blood-pressure, see Arterial pressure

Blood-supply,

of the heart, 6

Blood-vessels, see Arteries, Veins

Brachial artery, examination in all cases of suspected arterio-sclerosis, 67 normal standard of systolic bloodpressure as measured in, $\mathbf{7 7}$

Brachial pulsation, exaggerated, 30

Bradycardia, during fatty degeneration, 308 etiology, 162

in acute myocarditis, 296

nature of the condition, 162 occurrence in heart failure, 124 paroxysmal form of, definition

Brain, and character, 184

diminished blood supply to, in heart failure, 120, 121

Breathing, deep, oscillations accompanying, slower than those due to cardiac action, 395

Bright's disease,

as cause of pericarditis, 331,332

indications of failing heart muscle in, 42

Bromide of ammonium,

for excitability and irritability of nervous system, 388
Bromides,

use in heart affections, 148

Bronchitis, cases of congenital heart disease

liable to attacks of, 365

occurrence in cardiac failure, 123

occurrence during congenital heart disease, 373

Broncho-pneumonia, cardiac dilatation simulating, 328 occurrence in cardiac failure, 123

"Bruit du diable," 53, 54 see also Murmurs

Bulbus arteriosus, formation in the fotus, 356,357

"Button-hole" type of mitral stenosis, 272

Caffeine, action of, 147

administration in cardiac disease, 147

Capillary bronchitis, cardiac dilatation simulating, 328

Capillary electrometer, electric changes in human heart registered by, 393

Capillary pulse, conditions in which observed, 30

Carbohydrates, heart affections in relation to, 134

Carcinoma of the heart, 355

"Cardiac asthma," nature of the condition, 119

Cardiac cycle, and sounds, 16-20

auricular wave in a, 106

characteristics and mechanism, 16-20

diagrammatic representation of events in $a, 16$

position of the $a$ wave in, 100, 105 position of thrills in, 31

relation of the different events in, as recorded in a sphygmogram, cardiogram, carotid pulse tracing, and phlebogram, 105

rhythm described, 19

systole of the auricles, 16

time necessary for completion, 19 time of appearance of each wave in, how determined, 108

Cardiac dilatation, see Dilatation

Cardiac distress, on exertion, conditions other than heart disease causing, 117 
Cardiac hypertrophy, see Hypertrophy

Cardiac overstrain, see Primary cardiac overstrain

Cardiac pain,

causes and characteristics, 160

severity not necessarily indicative of severity of morbid changes, 127

treatment of, 157

Cardiac syphilis, 354-55

Cardiac tonics, 148

Cardiac type of ulcerative or malignant endocarditis, 248

Cardiogram, analysis and interpretation of $a$, 108

$A s-V_{s}$ interval in a, 100,101

definition, 93

events in cardiac cycle recorded by, in relation to those of sphygmogram, carotid pulse tracing, and phlebogram, 105

method of taking a, 93

nature of, 93

negative, characteristic features, 97,98

negative or inverted, significance of, and how obtained, 93

negative, tracings showing, 97, 98

positive, 93

positive, tracings showing, 94, $95,96,98$

positive, what it consists of, 94, 95,97

post-sphygmic period in $\mathrm{a}, 96$

sphygmic or pulse period in a, 93-5

Cardiolysis,

indications for, in chronic ad-

Cardioptosis, hesive pericarditis, 346

downward displacement of apexbeat in, 24

Cardio-vascular system, articles of food stimulating, 79 meat stimulating, 83

Carditis,

definition of the term, 231

C'arotid artery,

relation of internal jugular vein to (illustration), 21

tracing, analysis and interpretation of, 108, 109

tracing, how obtained, 91, 92

Carotid pulse,

compared with venous pulse, 29
Carotid pulse (continued),

exaggerated, conditions in which observed, 30

tracing, events in cardiac cycle recorded by, in relation to those recorded by sphygmogram, cardiogram, and phlebogram, 105

Carotid wave,

in a phlebogram, how determined, 109, 110

in the jugular pulse, 99, 100, 105

"Catch in the breath," 180

Centripetal venous pulse, conditions in which observed, 31

Cerebral anæmia,

in arterio-sclerosis and myocardial degeneration, 120

symptoms, 121

symptoms during paroxysmal tachycardia, 227

Cerebral embolism, during acute endocarditis, characteristics and signs, 238

Cerebral infarction, during acute endocarditis, 235

Cerebral symptoms, during chronic interstitial myocarditis, 313

during fatty degeneration, 308

in aortic incompetence, 265

of cardiac dilatation, 325

of heart failure, 120

Cerebral type of ulcerative or malignant endocarditis, characteristics, 249

Chest,

"constriction" of, in angina pectoris, 382

position of the heart in relation to, 1

(illustration), 3

systolic recession of walls o1, 28

Cheyne-Stokes' respiration,

as symptom of auricular flutter, 216

as symptom of cardiac failure, 119 conditions in which seen, 310

Childhood,

cardiac dilatation in, 325

chronic adhesive pericarditis of, 345

congenital heart disease of, diffculty of diagnos is, 370

endocarditis of, cause, 236

mitral stenosis of, characteristics, 274

pericarditis of, 331 
Childhood (continued),

rheumatic infection of the heart in, characteristics, diagnosis, and symptoms, 23l-3

tricuspid incompetence of, 291 see also Congenital heart disease

Chloralamide,

in treatment of sleeplessness, 152

Chloral hydrate,

in treatment of angina pectoris, 389

in treatment of insomnia, 152

Chordæ tendineæ, character of murmurs in, 51

Circulation,

effect of digitalis on, 144

enfeebled, diagnosis of fatty infiltration from, 304

fœetal, development of, 356, 357, 361

mechanism of, $17,69,70$

septal defects less disturbing than stenosis or atresia, 364

when most efficiently carried on, 14

Clubbing,

of fingers and toes in congenital heart disease, 364, 365, 368

Coarctation of the aorta, adult type, 368

cause and nature of the condition, 362

Coffee,

cardiac affections in relation to, 133

Collapsing pulse, 61

Compensatory changes, 126

Compensatory pause, definition and occurrence of, 169 , $172,174,177$

Concentric cardiac hypertrophy, 316,317

Conductivity,

definition, 11, 13

degrees of, 184

second degree, nature and characteristics, 186-8

depressed, definition and nature of, 101

first degree, nature and characteristics, 185

how observed, 13

period when exercised to fullest extent, 15

refractory period of, 15

vagus nerve depressing function of, 15
Congenital heart disease, cause of death in, 366

condition of the blood in, 365

conditions due to, 359

cyanosis and dyspncea in, 364, 365

diagnosis, 369-71

electro-cardiogram in, 436

etiology and pathology of, 358

forms of, 257

in children and adults, diagnosis, 370

prognosis, 371-3

pulmonary incompetence, 286

pulmonary stenosis probably the commonest, 285

symptomatology, 364-9

syncopal attacks in, 365

treatment, 373

tricuspid incompetence, 290

varieties of, 359

Constipation,

avoidance in cardiac affections, 134

Contractility, definition, 13

of heart muscle, how measured, 14

primitive cardiac tube initiating, 10

vagus nerve depressing function of, 15

Contraction,

stimulus for, power of originating, 11

stimulus for, starting-places, 11

Convulsions,

in congenital heart disease, grave nature of, 372

Cor bovinum, 317

Coronary arteries,

abrupt closure of lumen a cause of sudden death, 128

abrupt closure, signs of, 302

aneurism of, cause and rarity, 353

blood supplied to heart by, 6

in relation to cardiac hypertrophy, 322

involvement in aortic stenosis, 264

position of, 6

Coronary disease,

accompanying cardiac hypertrophy, 322

accompanying fatty degeneration, 311

angina pectoris in relation to, 381 , 386 
Coronary disease (continued), chronic interstitial myocarditis following, 312

chronic myocarditis in relation to, 300,301

common cause of fatty degeneration, 306

etiology, 301

results of, 301

with obvious signs of heart disease, 386

see also Endarteritis obliterans, Atheroma

Corrigan's pulse, 61

in aortic incompetence, 266

Cough,

treatment of, $155-6$

Crehore's method of recording heart sounds, 114

Croupous pneumonia,

radial pulse tracing showing extra-systoles, 296

Cyanosis,

as diagnostic aid in congenital heart disease, 369,370

in congenital pulmonary stenosis, $367,368,370$

of congenital heart disease, occurrence and characteristics, 364,365

oxygen inhalations in, 149

Death,

in aortic incompetence, frequency of, 271

in heart affections, causes, 128

Deflections,

in an electro-cardiogram, explanation of, 395-402

Degenerative changes, prognosis in, 127,128

Depressed conductivity, see Conductivity

Dextro-cardia,

causing displacement of apexbeat, 24

diagnosis, 370

effect on heart's position, 39

nature of the condition, 359

prognosis, 372

symptomatology, 366, 367

Diastole,

characteristics and significance, 17,18

definition, 17

Diastolic blood-pressure, see Arterial pressure
Diastolic shock, conditions in which present, 28 definition, 28

Diastolic thrill, in aortic incompetence, 268

Dicrotic wave, 57 in aortic incompetence, 266 sphygmogram showing, 59

Dicrotism, conditions in which found, 59

Diet, during acute endocarditis, 243 during high blood-pressure, 83 in cardiac affections, 133, 134 in fatty infiltration, 305

in heart failure, 151 in primary cardiac overstrain, 377 in subnormal blood-pressure, 79

Digestion, effect on blood-pressure, 78

Digestive disturbances in heart failure, 122

Digestive functions, impairment in cardiac dilatation, 325

Digestive system, effect of digitalis on, 144

Digitalis, action on the heart and vessels, $139,140,141$

advantages and disadvantages of, 141,142

blood-pressure not raised by, 139 cases contra-indicating administration of, 141, 142, 144, 145 causing sinus irregularity, 167

dosage, 143,144

effect in auricular fibrillation, 210,212

effect in auricular flutter, 214, 221-223

electro-cardiogram of case of auricular flutter under and after administration of, 424 , 425,427

in auricular fibrillation, tracings showing effect of, 211,212

indications for administration of, 140

inducing heart-block, 192

infusion in treatment of dropsy, 154

in heart-block, 197

in low blood-pressure, 79, 80

in paroxysmal tachycardia, 230

in vomiting, 156

results of treatment by, 139,140

supply of, and preparations, 146 
Dilatation of the heart,

apex-beat and radial artery tracings from severe. cases of, $97,98,99$

cause, 13,14

character of the apex-beat in, 25

conditions associated with, 324

diagnosis, 327

differential diagnosis from hypertrophy, 327

differential diagnosis of pericardial effusion from, 339

etiology, 324 .

nature of the condition, 323

power of, to what due, 15

prognosis, 328

symptomatology, 325

treatment, 328, 329

Diphtherial myocarditis,

characteristics, symptoms, 298

grave nature of, 299

Diuretics,

cardiac affections in which usc-

Diuretin, ful, 149

action of, 147

Double aorta, 363

Double mitral disease,

etiology and symptomatology, 284

Dover's powder, acute pericarditis, 342

relief of precordial pain by, 244

Dropped beats, 174, 195

cause and frequency, 186, 187, 188

sino-auricular block causing, 198

Dropsy, see CEdema

Drugs,

action of, 138

action on blood-pressure, 72,82

general observations, 137

in treatment of

angina pectoris, 388

cardiac dilatation, 329

heart-block, 197

heart diseaso, 137-49

high blood-pressure, 86

sleeplessness, 152, 153

ulcerative endocarditis, 252

Ductus arteriosus,

complete absence of the septum of, 360

condition in coarctation of the aorta, 362

persistence alone, conditions associated with, 363
Ductus arteriosus (continued),

persistence of, prognosis, 373

premature closure rare, 363

see also Patent ductus arteriosus

Duroziez,

murmur of, 269,270

Dysphagia,

occurrence in acute pericarditis, 333

Dyspnoea,

early and important symptom of cardiac failure, 118

in dextro-cardia, 367

in heart failure, 123

of congenital heart disease, occurrence and characteristics, 364 , 365

on exertion, more pronounced in mitral than in aortic disease, 119

on exertion, an early symptom of mitral disease, 119

oxygen inhalations in, 149

paroxysms in congenital heart disease, grave nature of, 372

symptom of affections other than heart disease, 118

symptom of fatty degeneration, 307

treatment of, 153-4

Ectopia cordis,

characteristics of the condition, 359

Efferent nerves, in relation to blood-pressure, 70

Einthoven,

method of recording heart sounds', 113

Einthoven galvanometer, characteristics and use, 393, 394

Electric changes, in human heart and muscle, how recorded, 393

Electricity, contra-indicated in primary cardiac overstrain, 377

Electro-cardiograph, nature of information obtained by, 114

Electro-cardiogram, a rare form of, 412

definition and nature of, 395 deflections explained, 395-402

from case of transposition of the heart, 434, 436

in congenital heart disease, 436

method of taking a, 394, 395 
Electro-cardiogram (continued), nature and mechanism of, 394401

of auricular fibrillation, 417-21

of auricular flutter, 422-6

of auriculo-ventricular block, 412

of cardiac hypertrophy, $\mathbf{4 3 0}$

of chronic valvular disease, 436

of mitral stenosis, 433

of paroxysmal tachycardia, 429, 430

of partial heart-block, 413

oscillations accompanying deep breathing slower than those due to cardiac action, 395

showing complete heart-block, 413

showing extra-systoles, 404-12

showing lesion of right and left branches of auriculo-ventricular bundle, 414-7

showing sinus irregularity, 403

Embolism,

during acute, simple endocarditis, $235,238,240$

during ulcerative endocarditis, 246,250

occurrenco in cardiac failure, 123

Embryo,

development of the heart in, $356,360,361$

Embryocardia, 43

Emphysema,

area of apex-beat diminished in, 25

downward displacement of apexbeat in, 24

heart sounds in, 40, 41

Endarteritis deformans, see Atheroma

Endarteritis obliterans, nature of the condition, 300

Endocardial murmurs, characteristics and causes, 44-5

Endocarditis,

aneurism of heart occurring in, 352

as manifestation of rheumatic infection of the heart, 232

classification and varieties of, 234

congenital heart disease causing, 359

definition and general observations, 234

fœtal, 358

in childhood, 232, 233

structural changes following, 116
Endocarditis, acute, acute myocarditis accompanying 116

aortic incompetence following, 271

aortic incompetence resulting from, 264

aortic stenosis following, 259, 264 chronic valvular diseases following, 258

differential diagnosis of acute myocarditis from, 298

mitral stenosis resulting from, 272 prognosis less serious than in degenerative changes, 127

Endocarditis, acute simple,

character of the pulse in, 237, 239

characteristics and nature of the condition, 235

diagnos is, 239, 240

differential diagnosis from ulcerative type, 250

embolism and infarction during, 235,240

etiology, 236

prognosis, 24!

sequelæ of, 236,241

sounds and murmurs in, 237,240

symptomatology, 236

treatment, 241

vegetations and micro-organisms during, 235

Endocarditis, chronic or sclerotic, characteristics, 254

etiology and morbid anatomy, 254 symptomatology, 255

Endocarditis, rheumatic, danger of, 126

Endocarditis, ulcerative,

cardiac type of, 248

cerebral type, 249

diagnosis, 249-51

differential diagnosis from the simple type, 250

duration of, 247

etiology, 246

nature of the condition, 244-6

prognosis, 252

septic or pyæmic type, 248

sequelæ of, 246

symptomatology, 247

treatment, 252

typhoid type, 249

Enlargement of the heart, see Heart enlargement

Epigastrium, pulsation in, characteristics, 27 systolic recession in, 27 
Epileptiform attasks,

during chronic interstitial myocarditis, 313

transient, during fatty degeneration, 308

Ergot,

in treatment of low blood-pressure, 80

Erythrol tetranitrate, action on blood-pressure, 86,87

Eserine,

in treatment of low blood-pressure, 80

Excentric hypertrophy, 317, 323

Excitability,

heart rate in relation to, 13

increased, results, 13

vagus nerve depressing function of, 15

where most highly developed, 13

Exercises,

cases in which contra-indicated, 136

effect on blood-pressure, 78

in chronic interstitial myocarditis, 315

in treatment of

cardiac affections, 136

fatty infiltration, 305

high blood-pressure, 82, 83

Exocardial sounds, characteristics and causes, 44

Extra-systoles,

age and sex incidence, 179

and infrequency of pulse rate due to heart-block, differential diagnosis, 181

and partial heart-block, differential diagnosis, 64

and pulsus alternans, tracing showing, 202

auricular blocked, 176

auricular, definition, 169

auricular, diagnosis, 181,182

auricular, el ectro-cardiogram showing, 411

auricular, nature and mechanism of, 175-7

auricular, tracings showing, 175-6 auriculo-ventricular block with, tracings showing, 192

compensatory pause following, $169,172,174,177$

compensatory pause following, nature of, 172

conditions in which seen, 179

conditions inducing, 13

definition of the tern, 169
Extra-systoles (continued),

diagnosis, 180

differential diagnosis, 181, 182

etiology, 179

frequent cause of irregularity of the pulse, 169

in croupous pneumonia, tracing, 296

interpolated, definition, 169

interpolated, nature of, 173

multiple, nature and occurrence of, 178

nodal, definition, 169

nodal, diagnosis, 182

nodal, nature and mechanism of, 177-8

nodal, tracings showing, 177, 178

occurrence in complete heartblock, cause and characteristics, 189

prognosis, 182

pulse wave in, 174

pulsus bigeminus in, 174

pulsus bigeminus in, diagnosis, 181

pulsus bigeminus due to, 201, 202

pulsus trigeminus in, 174

pulsus trigeminus in, diagnosis, 181

simulating Adams-Stokes' syndrome, 180

sinus rhythm in, 170

sounds in, 173,180

starting-point of stimulus for contraction, 169,170

subjective symptoms, 179

tracings showing, 170

treatment, 183

ventricular, definition, 169

ventricular, diagnosis, 181, 182

ventricular disappearance of, cause, 174

ventricular, electro-cardiograms showing, 404-10

ventricular, nature and mechanism of, 170-4

ventricular, tracings showing, 171-4

ventricular, two varieties differentiated by electro-cardiogram, 406-10

Fainting attacks, see syncope

Faintness,

treatment of, 154

False hypertrophy, 312

Family history of the patient, in relation to prognosis, 126 
Fatty degeneration,

cause of death in, 311

conditions associated with, 306

diagnos is, 309

$\therefore$ differential diagnosis from fibroid degeneration, 310

differential diagnosis from valvular disease with cardiac failure, 310

differential diagnosis of chronic interstitial myocarditis from, 310

duration of, 310

etiology, 306

nature of the condition, 306

persistent infiltration causing, 305

prognosis, 310

symptomatology, 307

treatment, 311

Fatty infiltration,

cases of angina pectoris due to, 386

cause of death in, 305

diagnosis, 304

etiology, 303

nature of the condition, 303

prognosis, 304

symptomatology, 303

treatment, 305

Febrile states,

centripetal venous pulse observed in, 31

Fibro-fatty heart, 302

Fibroid degeneration, see Myocarditis, chronic interstitial

Fingers, clubbing of,

in acquired heart disease, 369

in congenital heart disease, 364 , 365,370

in congenital pulmonary stenosis, 368

in pulmonary stenosis and incompetence, 285,286

Flatulence,

accompanying angina pectoris, 381

Flint's murmur, 278 :

Fœtal circulation, development of, $356,357,361$

Fœtal endocarditis, 255

congenital heart disease resulting from, 358

Fœtal heart,

action of, 356-8, 360, 361

arrested development of, characteristics and conditions due to, 359,360

Fotal rhythm, 43
Food, see Diet

Foramen ovale, formation and growth of, 357 premature closure rare, 363 see also Patent foramen ovale

Frank, method of recording heart sounds, 114

Friction sounds, characteristics and causes, 44

Functional efficiency of the heart, estimation of degree of impairment, 116, 117

"Funnel-shaped " mitral stenosis, 272

Galvanometer,

changes in electric potential in heart and muscle reoorded by, 393,394

Einthoven, characteristics, 393, 394

Gastralgia,

angina abdominis frequently mistaken for, 382

pain of angina pectoris sometimes mistaken for, 382

Gastric colic,

pain of angina pectoris frequently resembling that of, $\mathbf{3 8 5}$

Giddiness,

common manifestation of cerebral anæmia, 120

Granular contracted kidney, atheroma of coronary arteries frequently associated with, 301

Graphic records of heart sounds, 113

Grave's disease, heart sounds in, 40

" Gripping in the throat," 180

" Growing pains," in childhood, 233

Gummatous deposits, in the cardiac wall, 354

Gums, hæmorrhage from, in congenjtal heart disease, 365

Guy's pill, in treatmentof cardiac drojsy, 154

Hæmatemesis, in heart failure, 122

Hæmopericardium, etiology, 351 prognosis and treatment, 351 symptomatology, 351 
Hæmoptysis, in heart disease, 123 treatment of, 156

Heart, ability to undergo compensatory changes, 126

action of digitalis on, $139-46$

action of strychnine on, 147

anatomical structure of, 2

area of absolute or superficial impairment, conditions increasing, 39

how determined, 36

area of relative impairment, conditions in which diminished or abolished, 38

area of relative or deep impairment, conditions increasing, 37

how determined, 34

arrested development of, characteristics and conditions due to, 359,360

blood-supply of, 6

changes in size and shape of, $\mathbf{3 2 3}$

contractile power dependent on rest, 136

development of, 356

diminution of apex-beat not necessarily indicative of disease of, 25

displacement forwards, 38

effect of auricular fibrillation on, 204

effect of auricular flutter on, 214 , 223

innervation of, 6

less common diseases of, 352-5

morbid changes in, pain not necessarily indicative of severity of, 127

new-growth of, 355

orifices of, areas in which character of sounds are ascertained, 5

position, 4

position of, 1, 33

age and $o^{t}$ her conditions affecting, 1, 2

(illustration), 3

rheumatic infection in childhood, characteristics, diagnosis, and symptoms, 231-3

rheumatic infection of, cause, 126

rupture of, 353

size of, how determined, $\mathbf{3 3}$

structure of, 6
Heart (continued),

syphilitic affections of, see Syphilis

valves of, position, 4

sounds of, areas over which heard, 5

(illustration), 5

wounds of, 353

Heart, alternation of,

in sphygmogram and electrocardiogram, 417

Heart, aneurism of, see Aneurism

Heart bcat,

action of aconite on, 138

rate in normal heart, 19

sounds accompanying, characteristics, 19

Heart-block, 184-98

$a-c$ interval in, 184, 185, 188

age and sex incidence, 192

analysis of polygraphic tracing of, 188,189

and Adams-Stokes' syndrome, 121,194

as indication of myocardial lesion, 196

blood-pressure during, 190

cardiac failure during, grave nature of, 196

cause and mechanism of production, 12

character of pulse and sounds in, 64

compression of auriculo-ventricular bundle producing, 12

diagnosis, 195, 196

electro-cardiogram from case of auricular flutter with, 425, 426

etiology, 192

first degree of conductivity, 185

infrequency of pulse rate due to, and extra-systole, differential diagnosis, 181

infrequency of pulse rate in, 195

jugular pulsation in, 29

morbid anatomy, 193

prognosis, 196

symptomatology, 193

syncopal attacks during, significance of, 196

syphilis causing, 197

treatment, 197

Heart-block, complete,

definition, 184

electro-cardiogram showing, 413, 414

nature of the condition, 189 
Heart-block, complete (continued), occurrence of extra-systole in, 189

pulse rate in, 189

sounds in, 190

sphygmogram illustrating, 189

tracings showing, 190, 191, 192

Heart-block, partial,

and complete, differential diagnosis, 195, 196

accompanying auricular flutter, 216

auricular flutter with, pulse tracings in, 219

the $a$ wave of, 105

definition and cause, 184

differential diagnosis of auricular fibrillation from, 104, 105

digitalis in auricular flutter producing, 222

electro-cardiogram showing, 413

extra-systole and, differential diagnosis, 64

important sign of myocardial damage in infectious disease, 298

in aortic and mitral disense, tracing illustrating, 186

in case of acute myocarditis during diphtheria, 297

intermittent pulse due to, diagnos is, 180

of neurogenic origin, lessened or abolished by atropine, 189

present in majority of cases of auricular flutter, 213

prognos is, 196

pulsus bigeminus due to, sphygmogram showing, 66

tracing showing, 188

Heart dilatation,

accompanying aortic stenosis, 259

area of deep or relative impairment increased by, 37

associated with myocardial degeneration, point in diagnosis, 35

in auricular flutter, 216

Heart displacement, pulsation due to, characteristics, 26

Heart efficiency, functional

degree of impairment, how estimated, 117

Heart enlargement,

in congenital heart disease, 366 , 370
Heart enlargement (continued),

in dextro-cardia, 367

prominence of precordium associated with, 23

signs of, 38

with patent ductus arteriosus, 368

Heart failure,

accompanying fatty degeneration, grave nature of, 311

accompanying mitral incompetence, 283,284

Adams-Stokes' syndrome in, 121 administration of digitalis in, 140 administration of strychnine in, 146

aortic incompetence generally resulting in, 269

area of cardiac impairment in, 124

auricular fibrillation causing, 210 auricular fibrillation with, prognosis, 129

auricular flutter leading to, 215

bradycardia in, 124

"cardiac asthma" in, characteristics, 119

cardiogram in, 94

causation, 115

causes, 15

causes of sudden death in, 128

cerebral symptoms of, 120

changes in heart muscle in, 115

Cheyne-Stokes" respiration in, 119

condition of urine in, 123

death from, in congenital heart disease, 366

degrees of, resulting from auricular fibrillation, 205

diffusible stimulants in temporary attacks, 148

digestive disturbances in, 122

digitalis in, results, $140,142,144$

dilatation associated with, 323

distension and pulsation of jugular veins in, 124

drugs in, 137-49

during heart-block, grave nature of, 197

during paroxysmal tachycardia, 227

dyspnœa an early and important symptom of, 118

dyspnœa in, 123

embolism in, 123

first indications of, 117, 118

following mitral stenosis, 277 
Heart failure (continued),

heart rate in relation to, 14

hepatic disease accompanying, 122

icteric change of skin in, 122

impairment of functional efficiency, how estimated, 116, 117

nature of principles underlying, 115

objective signs, 118

œdema an important manifestation of, 122

oxygen administrations in, indications for, 149

pain in precordium a frequent symptom of, 120

pain in, to what due, 120

palpitation in, 119

patient's appearance in, 122

physical exertion in relation to, 117

prognosis, 128

rapid onset in course of infectious disoase, 298

rest in, importance of, 135

value demonstrated, 15

right side, indications of, 124

sense of exhaustion in, 119

structural changes and degenerative processes causing, 116

symptomatology, 116-24

syncope during, characteristics, 121

systematic exercises in, 136

tracing showing complete auriculo-ventricular block, 191

treatment, 150, 151

with dropsy, percentage of cases due to auricular fibrillation, 203

Heart, fatty infiltration of, see Fatty infiltration

Heart, functional disorders of, 158-65

Heart hypertrophy, see Hypertrophy

Heart irregularity, classification of causes, 163 general observations, 163

nature of the pulse in, 57

persistent, percentage of cases due to auricular fibrillation, 203

Heart, irritable,

definition, symptomatology, diagnosis and treatment, 164, 165
Heart muscle, cardiac failure due to changes in, 115

exhaustion of, treatment, 387

failing, indications of, 42

importance of, in relation to treatment in general, 131

involvement in heart-block of severe degree, 196

nature of changes in, in heart failure, 115

pulsus alternans as indication of extreme exhaustion of, 202

Heart muscle fibres, activity and functions of, 12-15 atrophy by compression, 303 contraction and stimulation of, mechanism, 14

function of conductivity, 13,15

function of contractility, 13

function of excitability, 13

function of stimulus production, 13,14

function of tonicity, 13

nervous control of functions of, 15

rest after exercise of each function of, 15

Heart overstrain, see Primary cardiac overstrain

Heart rate,

action of digitalis on, in case of auricular fibrillation, diagram illustrating, 143

diminished, causes, 13

effect of physical exertion on, 161

in paroxysmal tachycardia, 226 , 228

increased blood-pressure not raised by, 69

increased, causes, 13

increased, heart failure in rela. tion to, 14

inhibition and acceleration, to what due, 16

normal, 20, 161

on what dependent, 13, 20

regular, when stimulus production and excitability are equal, 13

response to abnormal or irregular stimuli, 13

vagus nerve decreasing, 15

variations in healthy individuals, 20,21

ventricular, how lessened, 13

Heart sounds, see Sounds 
Heart, transposition of, electro-cardiogram from case of, 434,436

Heart, "weak," use of the term, 165

Heart's action, in auricular fibrillation, 210 increased, nature of murmurs in, 49 with auricular fibrillation, 204

Heart's contraction, irregularities in stimulus for, 184

power of, to what due, 15

rate of travel, 13

starting place and course $0_{1}, 10$, 11

stimulus for, how conveyed, 11 , 184

Heart's strength,

habits and mode of life in relation to, 131-3

standard varying in different individuals, 117

Henbane,

in treatment of sleeplessness, 152

Hepatic colic,

characteristics of the pain of, 385

pain of, compared with that of angina pectoris, 385

Hepatic embolism, during acute endocarditis, 239

Hepatic pulsation,

in diagnosis of tricuspid stenosis, 289

in tricuspid incompetence, 291, 292

\section{Heredity,}

and angina pectorns, 380

factor in causation of angina pectoris, 380

His' Bundle, 9

Huerthle, method of recording heart sounds, 113

Hydriodic acid, syrup of, in treatment of angina pectoris, 388

Hydropericardium, characteristics, 348

detection of, 339

diagnosis, $\mathbf{3 4 9}$

etiology, 348

symptomatology, 349

treatment, 349

Hydrothorax, causing dyspncea, 153
Hypertension, see Arterial pressure, super-normal

Hypertrophy of the heart,

and "irritable hэart," differentil diagnosis, 164

area of relative or deep impairment increased by, 37

character of the apex-beat in, 25

co-existing with chronic adhesive pericarditis, 343

coronary disease accompanying, 322

diagnosis, 320, 321

differential diagnosis of dilatation from, 327

dilatation accompanying, 323 , $324,326,327$

dilatation secondary to, 323,324

electro-cardiograms of, 430

etiology, 317

nature of the condition, 316,317

prognosis, 321

sounds in, 43

symptomatology, 164, 165, 318

treatment, 322

types of, 316

ventricles, displacement of apexbeat in, 24

Hypnotics,

in insomnia of heart disease, 152

Hypoplasia of the aorta,

characteristics and occurrence of, 363

Ifypotension, see Arterial pressure, subnormal

Icteric change of skin in heart disease, 122

Infarction,

during acute, simple endocarditis, 235

following coronary disease, 30l, 302

Infective diseases,

hoart-block during, treatment, 197

heart-block in cases of, 192,193

Influenza,

prophylactic measures in cases of cardiac disease, 135

Influenzal myocarditis, characteristics and symptoms, 297, 298

grave nature of, 299

Insomnia, see Sleeplessness

Inter-auricular septum, absence of, characteristics of the condition, 359, 360 
Inter-auricular septum (contin'ed), formation and growth of, 356, 357

Intercostal spaces, -systolic recassion of, to what due, 27

Intermittent pulse, 174,182

definition, character, and causation of, $63,174,182$

duo to extra-systole or partial heart-block, differential diagnosis, 180

sino-auricular block causing, 198 sphygmogram showing, 63,64

Interpolated extra-systole, definition and occurrence of, I69, 173

Inter-ventricular septum,

absence of, characteristics of the condition, 359,363

complete closure of, 361

formation and growth of, $\mathbf{3 5 7}$

see also Patent inter-ventricular septum

Intra-cranial hæmorrhage, systolic blood-pressure in, 81

Iodide of potassium,

in treatment of

angina pectoris, 388

cardiac syphilis, 355

chronic adhesive pericarditis, 346

Iodide of sodium,

in treatment of acute endocarditis, 243

Iodides,

action on blood-pressure, 87

cases in which beneficial, 148

in treatment of rheumatic infection of the heart, 242

more valuable than nitrites in

Iron, angina pectoris, 389

as a cardiac tonic, 148

Irritable heart,

symptoms, diagnosis, and treatment, 164, 165

Irritable heart of soldiers, 374,376 , 377

Joint affections,

and rheumatic infection of the heart, 232

in childhood, 232

Jugular pulse, action of the auriclo indicated by, 90

and arterial pulse, sinultaneous tracings by polygraph, 90
Jugular pulse (continued),

and radial pulse, simultaneous tracings of, $61,62,66,109$, 110

tracings in sinus irregularity, 168

auricular form of, characteristics, $29,106,107$

tracings showing, 101, 102, 103, 105

auricular wave in the, 98, 100, $105,106,107$

how recognised, $109,110,111$

carotid wave in the, 99, 100, 105

how recognised, 109,110

character in auricular fibrillation, 208210

tracings, 206-9, 21 I, 212

character in auricular flutter, 216

tracings, 217-21

characteristics and causes, 28

compared with radial, in heartblock, 196

conditions to which due, 29, 30

how best observed, 29,30

how recorded, 98-104

in complete heart-block, 190

in heart failure, 124

in tricuspid incompetence, 291

tracings, how interpreted, 108, 109,110

how obtained, 90-2, 98-105

tracings in auricular fibrillation, 206-9, 211,212

tracings in heart-block, 185-8, 190-2

tracings in paroxysmal tachycardia, 225

tracings in sinus irregularity, 168

tracings showing extra-systoles, 170-6.

tracings showing pulsus alternans, 199-201

tricuspid regurgitation in relation to, 30

ventricular form of, characteris:tics and significance, 29, 107

tracings showing, 106, 107, 208

ventricular wave in the, 100,101 , $102,103,105$

how recognised, 110, 111

see also Phlebogram

Jugular vein,

and apex-beat, tracings showing: cardiogram, 96, 97

trasings from case of enlarged, heart, 98 
Jugular vein (continued),

distension of, conditions causing, 29

external, structure and charac. teristics, 22

internal, relation to carotid and subclavian arteries and sterno-mastoid (illustration), 21

structure and characteristics, 22

Kent, Stanley, auriculo-ventricular bundle, 9

Kidneys, endarteritis deformans frequently associated with atheroma of, 301

involvement in acute, simple endocarditis, 238

see also Renal

Leucocyte count, in congenital heart disease, 365 in ulcerative endocarditis, 247

Life and habits, in relation to cardiac treatment, 131-3

Lips, in treatment of heart-block, 197 condition as sign of congenital heart disease, 364, 365

Liver,

affections accompanying heart disease, 122

Liver pulse, and radialpulse, tracings, 111,112 characteristics and significance, 111,112

in auricular fibrillation, 209

tracing, how obtained, 90, 91, 92

Lungs, see also Hepatic pulsation

affections of, in cardiac dilatation, 325

disorders associated with tricuspid incompetence, 291

fatty infiltration apt to occur in cases of affections of, 303

fibrosis of, effect on heart's position, 39

left emphysema of, simulating hypertrophy of left ventricle, 321

lesions increasing area of relative cardiac impairment, 38

œdema of, causing dyspnœa, 153 treatment, 156
Lungs (continued), position of the heart in relation to, 1

resonance, diminished, significance of, 34

retraction of extent of apex-beat increased in, 25

see also Pulmonary

Massage,

in treatment of cardiac affections, 137

Meat,

blood-pressure in relation to, 83 , 84

stimulation of cardio-vascular system by, 79

Melæna,

in heart failure, 122

Menorrhagia, accompanying heart failure, 122

Mental condition, in congenital heart disease, 365

Mercury, cases in which benoficial, 148

in treatment of high blood-pressure, 85

Mercury-digitalis combination, in treatment of dropsy due to cardiac failure, 154,155

Mesenteric artery, superior, embolism of, 238

Meso-cardia,

characteristics of the condition, 359

diagnosis, 370

prognosis, 372

Metrorrhagia, accompanying heart failure, 122

Micro-organisms,

in ulcera tive endocarditis, 245

of acute pericarditis, 332

of acute, simple endocarditis, 235

Microphone, use in recording heart sounds, 113

Milk,

in treatment of vomiting, 156

"Milk spots"

upon the pericardium, 331 .

Mitral disease, accompanying auricular flutter, 216

and aortic disease, prognosis compared, 127

and aortic, partial heart-block in, tracing showing, 186

dyspncea on exertion, an early symptom, 119 
Mitral disease (continued),

frequency of, 256,258

hæmoptysis in, cause, 123

patient's appearance in, 121, 122

relative frequency among males and females, 126

treatment, 293

use of digitalis in, 140, 141

with auricular fibrillation, tracings showing effect of digitalis, 211

with cardiac failure, simulating fatty degeneration, 310

Mitral incompetence, conditions associated with, 283

degree of stenosis usually associated with, 257

due to aortic stenosis, 261

organic, diagnosis, 282, 283

prognosis, 283

prognosis compared with that of aortic stenosis, 264

relative and organic, differential diagnosis, 283

relative frequency of, 258

symptomatology, 280, 281

Mitral orifice, congenital lesions rare, 364 position of, 4

Mitral pulse,

auricular fibrillation described as, 203

Mitral regurgitation, conditions associated with, 283 diagnosis, 282, 283

etiology, 280

mitral systolic thrill indicating, 32

prognosis, 283

symptomatology, 280

Mitral stenosis,

accompanying auricular fibrillation, 204, 208

acquired tricuspid stenosis usually associated with, 288

and aortic incompetence, differential diagnosis, 278

and tricuspid, differential diagnosis, 279, 289

associated with auricular flutter, 215

a-c interval in case of, 185

" button-hole," characteristics, 272

conditions complicating, 279

congenital cases, 363

diagnosis, 32, 277

differential diagnosis from aortic incompetence, 270, 277
Mitral stenosis (continued),

differential diagnosis from mitral incompetence, 278

differential diagnosis from tricuspid stenosis, 279

differential diagnosis of dilatation from certain cases of, 327

electro-cardiograms of, 433,435 , 436

etiology, 272

" funnel-shaped," characteristics, 272

heart failure following, 277

heart sounds in, 40,43

jugular pulse and apex-beat tracings in, 185

mitral presystolic or diastolic thrill indicating, 32

nature of murmurs in, 48

œdema accompanying, 123

point of maximum intensity of murmurs due to, 50

prognosis, 279

sphygmograms from cases of, 274

symptomatology, 272-6

thrill and murmur of, examination for, 32

tracing showing irregularity of radial pulse, in, 186

unaccompanied byauricular fibrillation, sounds in, 185

Mitral stenosis and regurgitation, etiology, 284

sequelæ, 284, 285

sphygmograms from cases of, 284

symptomatology, 284

Mitral systolic murmur, 276

Mitral valve,

acute infection of endocardium of, 116

area of sound, 5

organic disease of, diagnosis, 282

structure and characteristics, 4

Morbus cœruleus,

use of the term, 364

Morphia,

hypodermic administration in cardiac failure, 153

Morphine,

in treatment of angina pectoris, 389

Mouth,

hygiene of, in treatment of chronic valvular disease, 293

Movements of the heart, starting-point and course of, 16

Multiple extra-systoles, definition, 169 


\section{Murmurs,}

aortic systolic, causes, $\mathbf{2 5 7}$

auriculo-systolic, characteristics, 49

" bruit du diable," 53, 54

cardio-pulmonary, characteristics, 53

in mitral incompetence, 283

to what due, 53

classification of, 44

diastolic, characteristics, 46

conditions indicated by, 48-9

definition, 46

diagram illustrating rhythm of, 46,47

in aortic incompetence, 268 , 269,270

due to mitral stenosis, cxamination for, 32

where best heard, 50

in acute, simple endocarditis, 237,240

in adherent pericardium, 345

in aneurismal dilatation, 53

in aortic incompetence, 268,269 , 270

in auricular flutter, 219

in cardiac dilatation, 326

in chronic interstitial myocarditis, 314

in congenital heart disease, 366 , $367,368,369,370$

in dextro-cardia, 367

in fatty degeneration, 309

in mitral stenosis, $275,276,277$

in patent inter-auricular septum, 367

in pulmonary incompetence, 287

in tricuspid incompetence, 291

in tricuspid stenosis, 288

in ulcerative or malignant endocarditis, 248,250

indicating valve defects, 48

inorganic or functional, 44, 45

characteristics and point of maximum intensity, 52

loudness of, variations in, and their significance, 52

mitral systolic, indicating organic mitral incompetence, 282

mitral and tricuspid systolic, to what due, 48

musical, cases in which found, 51 obstructive, point of origin, 52 of congenital aortic stenosis, 371 of congenital heart disease, 370 of congenital pulmonary stenosis, 368
Murmurs (continued),

of patent ductus arteriosus, 287, 369

organic. 44,45

over the precordium, 45

point of maximum intensity and direction of selective propagation of, 50

points in investigation of, 45

presystolic,

characteristics, 46

conditions indicated by, 47-9

definition, 46, 49

diagrams illustrating rhythm of, 46,47

in mitral stenosis, 275,276 , 277,278

presystolic and auriculo-systolic, terms not synonymous, 49

prolonged, occupying both systole and diastole, 50

regurgitant, point of origin, $\mathbf{5 2}$

rough and grating variety, characteristics, 51, 52

soft and blowing variety, characteristics, 51, 53, 54

systolic,

characteristics, 46

conditions indicated by, 47-9

definition, 46

diagnosis of aortic stenosis, from, 262, 263

diagrams illustrating rhythm of, 46,47

in acute myocarditis, 297

in aortic stenosis, 261,262

in congenital pulmonary and aortic stenosis, 371

in mitral regurgitation, 281

in tricuspid incompetence, 292 time of occurrence, how ascer-

Muscle, tained, 45, 46

electric changes in, how recorded, 393

somatic, electro-cardiograms showing oscillations resulting from tremors of, 394-5

see also Heart muscle

Myocardial degeneration,

as cause of auricular fibrillation, 204

auricular fibrillation usually indicative of, 210

auricular flutter commonly associated with, 215

cardiac asthma and CheynoStokes' respiration in, 119 
Myocardial degeneration (continued), cerebral symptoms of, 120

dilated heart associated with, point in diagnosis, 35

jugular and radial pulse tracings in, 185

result of digitalis administration in, 143

resulting from aortic stenosis, 264 syncope accompanying, 121

with auricular fibrillation, tracings showing effect of digitalis, 212

Myocardial lesions,

heart-block as sign of, 196

invariably present in heart-block, 193

Myocarditis,

as manifestation of rheumatic infection of the heart, 232

degree of, always present with pericarditis, 331

in childhood, 232, 233

symptomatology, 295

Myocarditis, acute,

accompanying acute endocarditis, 116

conditions usually associated with, 297

diagnosis, 298

differential diagnosis from endocarditis, 298

duration of, 299

during diphtheria, tracings showing partial heart-block, 297 etiology, 295

heart-block an important sign during infectious disease, 298

nature of the condition, 294, 295 prognosis, 299

structural changes following, 116 treatment, 300

Myocarditis, chronic,

coronary disease in relation to, 300,301

see also Fatty Degeneration, Fatty Infiltration

Myocarditis, chronic interstitial, chronic aneurism of walls of heart resulting from, 352

diagnosis, 314

differential diagnosis from fatty degeneration, 310

differential diagnosis from heart failure of valvular disease, 314

etiology, 312

nature of the condition, 311,312
Myocarditis, chronic interstitial (continued),

prognosis, 315

symptomatology, 313

treatment, 315

Myocarditis, diphtherial,

prognosis in, 299

symptoms, 297, 298

Myocarditis, influenzal,

prognosis in, 299

symptoms, 297, 298

Myocarditis, interstitial, 295

Myocarditis, parenchymatous, 295

Myocarditis, suppurative, 295

Myocardium,

abscesses in, 353,354

cardiac distress on exertion indicating, 117

conditions causing heart failure in relation to, 115

degree of integrity during paroxysmal tachycardia, how ascertained, 229

diagnos is, 40

heart sounds in, 40

serious nature of, 128

fatty or fibroid degeneration as cause of angina pectoris, 386

in relation to cardiac trcatment in general, 131

involvement in acute endocarditis, 239

structure and characteristics, 6

syphilis of, characteristics and symptoms, 354

Neck,

pulsation at the root of, characteristics and causes, 28, 29

Neoplasms,

cause and occurrence of, 258

Nephritis, aortic accentuation in, 41 systolic blood-pressure in, 80, 81

Nerves, of the heart, characteristics, 6

Nervous strain, effect on blood-pressure, 77

Nervous system, excitability and irritability during angina pectoris, treatment, 388

frequently affected in cases of fatty infiltration, 304

unduly excitable, in cardiac disease, treatment, 133

Neurotic types, of pseudo-angina, 390 
New-growths of the heart and pericardium, 355

“ Night starts," 119

Nitrites,

cases in which beneficial, 149

less valuable than iodides in angina pectoris, 389

see also Amyl nitrite

Nitrogenous diet,

in treatment of fatty infiltration, 305

restriction in treatment of cardiac affections, 133, 134

Nitroglycerine,

in treatment of angina pectoris, 388,389

Nodal extra-systole,

definition, 169

nature and mechanism of, 177-8.

Nodal rhythm,

auricular fibrillation described as, 203

Nose,

condition as sign of congenital heart disease, 364

hæmorrhage from, in congenital heart disease, 365

Numbness,

as symptom of angina pectoris, 382,383

\section{Edema,}

accompanying heart failure, characteristics, 122

treatment of, 154-5

Orthopncea,

in heart failure, 118

Oxygen,

inhalations in cardiac failure, 149

in treatment of heart-block, 197

"Pace-maker,"

of the heart, 10

Pain,

characteristics, 120

effect on blood-pressure, 77

in acute, simple endocarditis, relief of, 244

of acute pericarditis, 333,341

of angina pectoris, $381,382,384$

of aortic incompetence, 265

Palpitation,

as symptom of cardiac failure, 119

causal treatment, 156

definition, 158

diagn :sis of extra-systoles by, 180

etiology, 159
Palpitation (continued),

prognosis, 160

pulse rate in, 159

restriction of the term, 158

symptomatology, 159

with and without organic disease, 159

Paracentesis pericardii, site for, 342

Paraldehyde,

in treatment of sleeplessness, 152

Parietes,

tumour causing undue prominence of precordium, $\mathbf{2 3}$

Paroxysmal tachycardia,

age and sex incidence, 225

area of cardiac impairment in, 227

cardiac rate in, 226,228

conditions associated with, 225

definition, 224

diagnosis, 228

duo to auricular fibrillation, pulse tracings, 225

du' to auricular flutter, pulse tracings in, 220

electro-cardiograms of, 429,430

etiology, 225

frequently due to paroxysms of auricular fibrillation, 204

nature of the condition, 224

prognosis, 228

recurrence of the attacks, 229

sudden cessation of the attack, 227,228

sudden onset of, 228

symptomatology, 225

treatment, 229,230

ventricular venous pulse in, 29

with regular rhythm or pulsus alternans, chiefly due to auricular flutter, 213

Partial heart-block, see Heart-block

Patent ductus arteriosus,

clinical features of congenital

pulmonary stenosis resem-

bling, 371

diagnosis, 371

murmurs of, 50,287

symptomatology, 368, 369

Patent foramen ovale,

causes and conditions associated with, $360,361,363$

diagnosis, 370

prognosis, 372

Patent inter-ventricular septum, 361,363

diagnosis, 370 
Patent inter-ventricular septum (continued),

duration of life in cases of, 372,373 prognosis, 372

symptomatology, 367

uncomplicated, prognosis, 372

Percussion,

how performed, 33-5

object of, 33

Percussion wave, nature of, 58

sphygmograms illustrating, 58

Pericardial effusion,

causing increase in area of relative cardiac impairment, 37 , 38

causing undue prominence of precordium, 23

characteristics, diagnosis, 335-7, 339

differential diagnosis from great cardiac dilatation, 339

displacement of apex-beat in, 24

paracentesis pericardii in, $\mathbf{3 4 2}$

prognosis, 340

treatment, 341, 342

Pericardial friction, 332, 334, 335, 336

conditions in which it occurs, 44

Pericarditis,

as manifestation of rheumatic infection of the heart, 232

course and duration of, $\mathbf{3 3 8}$

in childhood, 231-3

thrills due to, characteristics, 31

varieties of, compared, $\mathbf{3 3 0}$

Pericarditis, acute,

conditions usually associated with, 332

diagnosis, 338, 339

etiology, 331, 332

nature of the condition, 331

prognos is, 340

symptomatology, 332

treatment, 340,341

Pericarditis, chronic,

nature of the condition, 347

symptoms, 347

tuberculosis of pericardium leading to, 348

Pericarditis, chronic adhesive,

cardiolysis in, 346

diagnosis, 345

etiology, 344

nature of the condition, 343

prognosis, 346

symptomatology, 344, 345

treatment, 346
Pericarditis, fibrinous or dry,

diagnosis, 338

nature of, 330,331

Pericarditis, hæmorrhagic, 332

conditionsusually associa ted with, 332

prognosis, 340

treatment, 342

Pericarditis, purulent, cause, 332

nature of, 330, 331

symptoms, $\mathbf{3 3 7}$

treatment, 342

Pericarditis, sero-fibrinous, diagnosis, 338, 339

nature of, 330

physical signs, 335-7

Pericardium,

absence of, prognosis, 372

less common diseases of, 352-5

new-growths of, diagnesis and symptoms, 355

syphilis, characteristics and diagnosis, 354, 355

Pericardium, adherent, see Pcricarditis, chronic adhesive

Pericardium, tuberculosis of, conditions due to, 347

diagnosis, 348

nature of the condition, 347

symptomatology, 348

treatment, 348

Petechial hæmorrhages,

during acute endocarditis, 239

Phlebogram,

analysis of a, 98-104

the $a$ wave in a, 98,100, 105

the $a-c$ interval in a, 100, 101

how determined, 111

analysis and interpretation of $a$, 108-11

the $c$ wave in a, 99, 100,105

definition, 93

events in cardiac cycle recorded by, in relation to those recorded by sphygmogram, carotid pulse tracing, and cardiogram, 105

$h$ or $b$ wave in a, 103, 104

tracing showing, 105

method of taking a, 98-104

onset and termination of ventricular systole in a, 110

sphygmic period in a, how determined, 109, 110

the $v$ wave in $a, 100,101,102,103$, 105 
Phosphorus, causing fatty degeneration, 307

Physical exertion, as cause of angina pectoris, 381 cardiac dilatation in relation to, 325

cardiac distress on, causes, 117

causing angina pectoris, 381

excessive, primary cardiac overstrain resulting from, 374

exhaustion following, a symptom of cardiac failure, 119

fatty infiltration in relation to, 306

heart-rate increased by, 21

prognosis of cardiac affections in relation to, 126

symptoms of cardiac distress on, causes, 117

Pituitary extract,

in treatment of low blood-pres. sure, 80

Pleuræ,

lesions increasing area of relative cardiac impairment, 38 position of heart in relation to, 1

Pleural effusion,

associated with cardiac dilatation, 327,328

causing undue prominence of precordium, 23

displacement of apex-beat in, 24

effect on heart's position, $\mathbf{3 9}$

oscurrence in cardiac disease, 123

Pleurisy,

thrills due to, characteristics, 31

Plcurodynin,

differential diagnosis from angina pectoris, 384,385

nature of the pain of, 384

Pleuro-pericardial friction, characteristics and causes, 44

Pneumo-hæmopericardium, 349

Preumonia,

as a cause of pericarditis, 331

chronic interstitial, displacement of apex-beat in, 24

Pneumopericardium, conditions associated with, 349

diagnosis, 350

etiology, 350

prognosis, 350

symptomatology, 350

treatment, 350

Pneumo-pyopericardium, 349

Pneumothorax, displacement of apex-beat in, 24
Polygraph,

clinical, characteristics and use, 88

(illustration), 88

ink, characteristics and method of using, 89-92

(illustrations), 89, 91

kind of information supplied by, 90

tracing of partial heart-block, analysis of, 188

Polygraphic records, analysis of, 108, 109

tracings illustrating, 109, 110

interpretation of, 108, 109

Polygraphic tracing,

analysis in complete heart-block, 190,191

Potassium iodide, see Iodide of potassium

Precordial pain, relief of, 244

Precordium,

audible murmur over, significance, 45

changes in form of, conditions causing, 23

pain in, prognosis in relation to, 127

a frequent symptom of cardiac failure, 120

pulsation in region of, causes and chardcteristics, 26, 27

structure and characteristics, 2

Primary cardiac overstrain,

cases of auricular fibrillation mistaken for, 374

diagnosis, 376

etiology, 374

nature of the condition, 374

prognosis, 376

symptomatology, 375

treatment, 377

Primitive cardiac tube,

(illustration), 9

age modifications, 10

characteristics and functions, 911

development of, 356,357

oxcitability of, 10

in relation to stimulus for contraction, 11

Prognosis, 125-9

age in relation to, 126

factors to be considered in, 125

family history of patient in relation to, 126

general observations on, 125,129 
Prognosis (continued),

habits and mode of lif $e$ in relation to, 126,127

in acute endocarditis and degenerative changes compared, 127, 128

in males and females compared, 126

in mitral and aortic disease compared, 127

Pseudo-angina,

age and sex incidence, 390

characteristics and symptoms, 390

definition of the term, 390

diagnosis, 391

neurotic cases, 390

pain compared with that of true angina, 390

prognosis, 391

treatment, 391

vaso-motor or toxic cases, 390 , 391

Pulmonary accentuation, conditions in which it occurs, 41

Pulmonary affections, accompanying acute dilatation, 325,327

Pulmonary area,

pulsation in, characteristics and causes, 26

Pulmonary artery,

and aorta, conditions associated with transposition of, 363

and aorta, prognosis in cases of transposition of, 373

Pulmonary atresia, frequency of, 362

grave nature of, 373

incomplete septum ventriculorum frequently associated with, 360

Pulmonary diastolic thrill, significance of, 33

Pulmonary embolism, during acute endocarditis, 238

Pulmonary incompetence, diagnosis, 287

differential diagnosis from aortic incompetence, 270,287

etiology, 286

prognosis, 287

rarity of, 257

symptomatology, 286

Pulmonary infarction, during acute endocarditis, 236

Pulmonary œdema, treatment, 156 ;
Pulmonary orifice,

position of, 4

Pulmonary stenosis, common form of congenital heart disease, 257, 361

course of the blood in, 361, 362

differential diagnosis from aortic stenosis, 263

etiology, 285

incomplete septum ventriculorum associated with, 360

patent foramen ovale associated with. 360

symptomatology, 285, 367

Pulmonary stenosis, congenital,

and aortic, differential diagnosis, 371

clinical features resembling those of patent ductus arteriosus, 371

diagnosis, 371

prognosis, 372,373

Pulmonary systolic thrill, diseases in which found, 33

Pulmonary valve, area of sound, 5

rarity of disease of, 257

structure and characteristics, 4

Pulsation,

at the root of the neck, characteristics, causes, 28, 29

carotid form of, characteristics, 29

carotid and venous compared, 29

varieties, causes and characteristics, 26

see also Carotid; Jugular ; etc.

Pulse,

anacrotic, from case of aortic stenosis, 60

arterial, character in auricular flutter, 216

arterial, and venous, simultaneous tracings by polygraph, 90

character in acute myocarditis, 296

character in acute pericarditis, 334,335

character in acuts, simple endocarditis, 237, 239

character in angina pectoris, 383

character in aortic incompetence, 266,267

character in aortic stenosis, 260

character in auricular fibrillation, 204-9

tracings, 206-9, 211, 212 
Pulso (continued).

character in auricular futter, 216 tracings, 217-21

character in auriculo-ventricular block, 187, 195

character in cardiac dilatation, 325

character in chronic interstitial myocarditis, 313

character in completeheart-block, 189

character in congenital heart disease, $366,367,368,369$

character in fatty degeneration, 308

character in fatty infiltration, 304

character in fibroid degeneration, 310

character in heart-block, tracings, $185-8,190-2$

character in heart failure, 123, 124

character in mitral regurgitation, 280,282

character in mitral stonosis, 273 , 274,278

character in palpitation, 159

character in paroxysmal tachycardia, 226

character in partial heart-block, 64

character in primary cardiac overstrain, 376

character in pulmonary incompetence, $270,286,287$

character in sinus irregularity, 167

character in tricuspid incompetence, 291

character in tricuspid stenosis, 288

character in ulcerative or malignant endocarditis, 248

collapsing, "water hammer," or Corrigan's pulse, 61

coupling of the beats, restriction of the term, 66

digitalis in relation to rate of, 140,141

extra-systoles a frequent cause of irregularity of, 169

hard, definition, 67

how recorded, 55

increased rate an indication for digitalis administration, 140 , 142

infrequent, causes, 162

in heart block, 195
Pulse (continued),

intermittent, see Intermittent pulse

of high blood-pressure, 62

of low blood-pressure, 61

of normal blood-pressure, 61

radial, and jugular, in heart-block compared, 196

radial, irregularity in mitral stenosis, tracing, 186

radial, rate in auricular fibrillation, 205, 208

rate of the, 56

rhythm of the, 63

soft, definition, 67

symmetry of the two pulses, 68

systolic, in mitral stenosis, 274 $276,277,278$

systolic and diastolic portions of the arterial, 58, 59

volume of the, 57

wave, character of, 57

see also Intermittent pulse, Jugular pulse, etc.

Pulsus alternans,

accompanying auricular flutter, 216

age incidence, 200

and extra-systoles, tracing showing, 202

as indication of extreme exhaustion of heart muscle, 202

associated with an attack of auricular flutter, tracings showing, 200

conditionsusually associa ted with, 199

definition, 199

depressed contractility probably indicated by, 13

detection by the finger, 201

diagnosis, 201

differential diagnosis from pulsus bigeminus due to extrasystoles, 201, 202

etiology, 200

nature of the condition, 199

presence frequent in paroxysmal tachycardia, 226

prognosis, 202

relationship to attacks of angina pectoris, 201

symptomatology, 200

tracings showing, 199, 200

treatment, 202

Pulsus bigeminus, due to extra-systoles, differential 
Pulsus bigeminus (continued), diagnosis of pulsus alternans from, 201, 202

due to partial heart-block, sphygmogram showing, 66

heart-block rosulting in (tracing), 187

in extra-systole, diagnosis, 181

nature of the condition, 174

radial pulse tracing showing, 201

restriction of the term, 65

sphygmogram showing, 65

Pulsus bisferiens,

definition and character of, 60

diagnostic of aortic stenosis, 60 , 61

in aortic stenosis and incompetence, sphygmograms showing, 267

in the sphygmogram, 60

Pulsus celer, definition and character of, $6 \mathrm{I}$ sphygmogram showing, 61

Puisus dicroticus, nature of the, 59

Pulsus irregularis, auricular fibrillation described as, 203

Pulsus paradoxus,

definition and character of, 62 sphygmogram showing, 62

Pulsus tardus, conditions usually associated with, 61

definition and nature of, 61 sphygmogram showing, 61

Pulsus trigeminus, definition and character of, 66 in extra-systole, diagnosis, 181 nature of the condition, 174 sphygmogram showing, 66,67

Purgatives,

cardiac affections in which beneficial, 149

in treatment of œdema, 154

in treatment of high bloodpressure, 85

Purkinje fibres, 9

Pyæmic type of ulccrative or malignant endocarditis, char-

Pyrexia, acteristics, 248

capillary pulse observed in, 30

in acute pericarditis, treatment, 341

of acute pericarditis, 332,333

30
Quinine,

in treatment of acute pericarditis, $34 \mathrm{I}$

Radial artery,

and apex-boat, simultaneous tracings showing positive cardiogram, 94, 95

tracings in auricular fibrillation, 206

tracings in case of greatly enlarged heart, 99

Radial pulse,

and apex-baat, simultaneous tracing, 67

and jugular pulse, simultaneous tracings of, 61,62

tracing in sinus irregularity, 168

tracings showing auricular form of venous pulse, $10 \mathrm{I}, 102,103$

character in auricular flutter, 216 tracings, 217-21

in aneurism of thoracic aorta, sphygmogram showing, 68

in tricuspid stenosis, 288

right and left, difference in, 68

showing extra-systoles in croupous pneumonia, 296

simultaneous tracings of the apex-beat and, 56

tracings, analysis and interpretation of, 108

tracings illustrating, 109, 110

tracings in aneurism of thoracic aorta, 68

tracings in auricular fibrillation, 206-9, 211, 212

tracings more reliable than those of apex-beat, 90

tracings showing extra-systoles, $170-6$

tracings showing heart-block, 185 $8,190-2$

tracings showing intermission of pulse, 64

tracings showing pulsus alternans, 199-201

tracings showing sinus irregularity, 167, 168

tracings showing paroxysmal tachycardia, 225

Regurgitation, murmurs indicating, 48

Relative aortic incompetence, 265

Relative mitral incompetence, symptomatology, 282

Relative stenosis, 263 
Renal colic,

characteristics of the pain of, 385

pain of angina pectoris compared with that of, 385

Respiration,

sinus irregularity in relation to, 167

Respiratory curve, tracing obtained by polygraph, method, 90, 92

Respiratory functions,

impairment in acute pericarditis, 333

Respiratory movements, method of recording, 92

Respiratory system, affections in heart disease, 123

Rest,

contractile power of heart dependent on, 136

in treatment of

acute, simple endocarditis, 242

heart failure, 135

high blood-pressure, 82

subnormal blood-pressure, 79

value in heart failure demon-

Retina, strated, 15

dilation of veins of, in congenital heart disease, 366

"Reversed rhythm,"

definition, 12

Rheumatic infection of the heart, 126

arthritis chief manifestation in adult life, 232

in childhood, characteristics, diagnosis, and symptoms, 231-3

myocarditisdue to, characteristics, 299

treatment, 241

Rheumatism,

acute, relation to acute, simple. ondocarditis, 236

as cause of auricular fibrillation, 204

avoidance in valvular disease, 135

in childhood and adult life compared, 231, 232

Rhythm of the pulse, 63

Rupture of the heart, causes and characteristics, 353

Salicylates,

in rheumatic infection of the heart, 241.
Saline infusions,

in treatment of acute endocarditis, 243

Salt diet,

blood-pressure in relation to, 84

Salt-free diet,

in cardiac affections, 134

Sarcoma,

of the heart, 355

Scoliosis,

causing prominence or bulging of precordium, 23

Septal defects,

less disturbing effect on circulation than stenosis or atresia, 364

prognosis, 372

Septic type of ulcerative or malignant endocarditis, characteristics, 248

Septum ventriculorum,

incomplete, cause and characteristics, 360

Sino-auricular block, characteristics and rarity of, 198

constitution of, 10

probablestarting place of stimulus for contraction, 10

structure and characteristics, 6

Sinus irregularity,

atropine abolishing, 166

causes and characteristics, 166

diagnosis, 168

electro-cardiograms showing, 403

jugular and radial pulses in, tracing, 168

prognosis, 168

respiration in relation to, 167

simulating dropped beats due to partial heart-block, 195

symptomatology, 167

tracings of respiration and radial pulse in, 167

Sinus venosus,

in mammalian heart, 10

in the fœtal heart, 356

rhythm governing that of remaining segments of heart, 10

stimulation and contraction of, Skin, order and course, 10, 11

icteric change in cardiac disease, 122

Sloep,

effect on blood-pressure, 78

Sleeplessness,

treatment, 152-3 
Sodium nitrite,

in treatment of high blood-pressure, 86,87

Soldier's heart, 374,376

etiology, 379

general observations on, 378, 379

prognosis, 377

symptomatology, 378

treatment, 379

Sounds of the heart,

adventitious, of endocardinlorigin, $44,45,46$

adventitious, of exocardial origin, 43,44

alterations in, 39-43

areas of, characteristics, 5

illustration, 5

diminished intensity, conditions causing, 40, 41

friction, characteristics and causes, 44

friction, in acute pericarditis, $332,334,335$

gallop rhythm, 42

graphic records of, 113

in acute myocarditis, 297

in acute pericarditis, 334,335

in acute, simple endocarditis, 237 , 240

in aortic incompetence, 269

in aortic stenosis, 261, 263

in cardiac dilatation, 326

in cardiac failure, 124

in cardiac hypertrophy, 319,320

in chronicinterstitialmyocarditis, 314

in complete heart-block, 190

in congenital heart disease, 367

in congenital pulmonary stenosis, 368

in emphysema, 40

in extra-systoles, 173, 180

in fatty degeneration, 40, 309

in fatty infiltration, 304

in Graves' disease, 40

in hypertrophy of right ventricle, 41

in "irritable heart," 164

in mitral stenosis, $40,276,277$

in palpitation, 160

in partial heart-block, 64

in patent ductus arteriosus, 369

in pericardial effusion, 337

in pneumo-pericardium, 350

in sinus irregularity, 167

increased intensity, conditions causing, $39,40,41$

method of listening for, 19, 20
Sounds of the heart (continued), occurrence and characteristics, 19,20

produced by contraction of auricle, 185

reduplication of, 42,43

triple rhythm, conditions giving rise to, 43

Southey's tubes, use in treatment of dropsy, 155

Spa treatment, 137

Sphygmogram,

analysis and interpretation of a, 108,109

conditions affecting, 62

definition, 93

events in cardiac cycle recorded by, in relation to those recorded by cardiogram, carotid pulse tracing, and phlebogram, 105

normal, 57

showing anacrotic pulse, from case of aortic stenosis, 60

showing dicrotism and hyperdicrotism, 59

showing intermission of the pulse, 63

showing jugular and radial pulses, 61,62

showing pulsus bigeminus, 65,66

showing pulsus bisferiens, 60

showing pulsus paradoxus, 62

showing simultaneous tracings of apex-beat and radial pulse, 67

showing simultaneous tracings of jugular and radial pulses, 66

starting-place in a, 93

Sphygmograph,

advantages and use of, 55,56

(illustration), 56

Sphygmomanometer, advantages and value of, 71

kinds recommended, 73

method of estimating bloodpressure by, 71-6

points in the use of, 71

(illustration), 74

Spleen,

enlargement in cardiac disease, 122

involvement in acute, simple

Squills, endocarditis, 238

action on the heart, 145,146

Status anginosus, 384 
Stenosis, valvular lesions giving rise to, 256

Sterno-mastoid, pulsation outside, characteristics, 28

relation of internal jugular vein to (illustration), 21

Sternum,

position of the heart in relation to, 1,2

systolic recession of, significance, 27

Stimulus production, definition, 13

heart rate in relation to, 13

variable nature of, 13

where most highly developed, 13

Streptococcus initis, 235

Streptococcus salivarius, 235

Strophanthin,

intravenous injections, results, 146,147

in treatment of paroxysmal tachycardia, 230

Strophanthus, action on the heart, 145,146

Strychnine,

administration in cardiac affections, 147

in treatment of, acute pericarditis, 341 acute, simple endonarditis, 244 dyspnœa, 154

heart-block, 197

low blood-pressure, 80

Subclavian arteries, relation of internal jugular vein

Sulphur, to (illustration), 21

action on blood-pressure, 85

Superficial veins, pulsation in, when best observed, 31

Symmetry of the two radial pulses, 68

Sympathetic nerve,

heart's activity in relation to, 15

Syncopal attacks,

in chronic interstitial myocarditis, 313

in congenital heart disease, 365 , 372

in fatty degeneration, 308

in heart-block, 196

occurrence in heart disease, 120 , 121
Syphilis,

as a causal factor of angina pectoris, 380,386

causing heart-block, 197

endarteritis obliterans usually due to, 301

fibroid clegeneration resulting from, 312

Syphilitic affections of the heart and pericardium, 354

Systolic blood-pressure, see Arterial pressure

Systolic recession,

in cardiac dilatation, 326

in cardiac hypertrophy, 320

in the epigastrium, $\mathbf{2 7}$

of the apex-beat, cases in which observed, 27

of chest walls, 28

of the intercostal spaces, cause, 27

of the sternum, 27

Tachycardia,

associated with normal rhythm, differential diagnosis of auricular flutter from, 219 , 220

causes, 157

definition of the term, 161

ctiology, 161

treatment, 157, 161

with low blood-pressure, pulsus ccler in case of, 61

see also Paroxysmal tachycardia

Tea,

in relation to cardiac disease, 133

Temperaments,

effects on blood-pressure, 77

Theocin-s odium-acetate, 147

Thrills,

area of maximum intensity, 32

causes and characteristics, 31

conditions indicated by, 32,33

due to aortic incompetence, 268

due to antic stenosis, 260,261 , 262

due to mitral stenosis, 275,277 , 278

due to pulmonary incompetence, 287

in congenital pulmonary stenosis, 368

in dextro-cardia, 367

of congenital heart disease, 366 , $367,368,369,370$

of tricuspid incompetence, 291

of tricuspid stenosis, 288 
Thrills (continued),

systolic, diastolic, and presystolic, $31,32,33$

where palpable, 31

Tic-tac heart beat, 43

Tidal wave,

definition, 57

in aortic incompetence, 266

increased and diminished intensity of the, 60

\section{Tobacco,}

as cause of pseudo-angina, 391

effect on blood-pressure, 78,85

Toes,

clubbing of, in congenital heart disease, 364, 365

in pulmonary stenosis, 285

Tonicity,

cardiac dilatation following impairment of function of, 323

definition, 13

vagus nerve depressing function of, 15

Tonics,

in treatment, 135

Toxic content of the blood, elimination of, 379

Treatment,

baths and spa, 137

body-warmth in, 134, 135

diet in, 133, 134, 151

drugs in, 137-49, 152

general observations on, 130

heart muscle in relation to, 131

importance of, 130

instructions regarding mode of life, 131-3

massage in, 137

objects of, 130

of acute myocarditis, 300

of acute pericarditis, 341,342

of acute, simple endocarditis, 241-4

of angina pectoris, 387-90

of auricular fibrillation, 212

of auricular flutter, 223

of cardiac dilatation, 329

of cardiac failure, 150,151

of cardiac hypertrophy, 322

of cardiac pain, 157

of cardiac syphilis, 355

of chronic adhesive pericarditis, 346

of chronicinterstitial myocarditis, 315

of chronic valvular disease, 293

of congenital heart disease, $\mathbf{3 7 3}$

of cough, 155-6
Treatment (continued),

of dyspnoea, 153

of extra-systoles, 183

of faintness, 154

of fatty degeneration, 311

of fatty infiltration, 305,306

of hæmopericardium, 351

of hæmoptysis, 156

of heart-block, 197

of hydropericardium, 349-

of œdema, 154-5

of palpitation, 156

of paroxysmal tachycardia, 229 , 230

of partial heart-block of neurogenic origin, 189

of pneumo-pericardium, 350

of primary cardiac overstrain, 377

of pseudo-angina, 39l

of pulsus alternans, 202

of rheumatic infection of the heart, 241.

of sleeplessness, 152

of soldier's heart, 379

of subnormal arterial pressure, 79

of super-normal arterial pressure, 82-7

of tachycardia, 157

of tuberculosis of pericardium, 348

of ulcerative endocarditis, 252

of vomiting, 156

physical exercisc in, 132,133

rest in, importance of, 135

systematic exercises in, 136

tonics in, 135

Tricuspid incompetence,

conditions associated with, 290

diagnos is, 292

etiology, 257, 289

frequency of, 257

most common valvular disease, 256

of congenital origin, 290

prognosis, 292

rarely congenital, 364

relative, conditions causing, 290

signs of, following pericardial effusion, 337

symptomatology, 290, 291

when congenital, 257

Tricuspid orifice, congenital lesions at, 369

Tricuspid regurgitation, accompanying primary cardiac overstrain, 376

and the jugular pulse, 30 
Tricuspid regurgitation (continued), due to relative incompetence, prognosis, 292

Tricuspid stenosis, accompanying tricuspid incompetence, 290

congenital cases, 363, 364

diagnosis, 289

differential diagnosis from mitral stenosis, 289

et iology, 288

nature of murmurs in, 48

prognosis, 289

pulmonary stenosis usually associated with, 28i

symptomatology, 288

Tricuspid thrills, rarity, 33

Tricuspid valve, area of sound, 6

(illustration), is

function of, 290

structure of, 4

Trilocular heart, cause of, 362

nature of the condition, 359, 362 prognosis, 372

Trinitrin,

in treatment of angina pectoris, 388,389

Tubs, cardiae, see Primitive cardiac tube

'T'uberculosis,

differential diagnosis of ulcerative cndocarditis and acute generalised, 251

of the pericardium, diagnosis, symptoms, and treatment, 347,348

pulmonary, displaccment of apexbeat in, 24

Typhoid type of ulcerative or malignant endocarditis, characteristics, 249

\section{Urine,}

condition in angina pectoris, 384 condition in cardiac failure, 123

condition in fatty infiltration, 304

condition in pulmonary incompetence, 286

condition in pulmonary stenosis, 285

Vaccines,

in treatment of ulcerative endocarditis, 252, 253
Vagus nerve,

affection in heart-block, 193

blood-pressure in relation to, 15

characteristics, 6

heart's activity in relation to, 15

Vagus origin,

of sinus irregularity, 166

Valvular cusps,

character of murmurs in, 51

Valvular disease,

avoidance of rheumatism in, 135

chronic, definition of the term, 256

etiology, 258

fatal cases, 279

general observations on, 256

relative incompetence, causes, 258

syphilis resulting in, 354

treatment, 293

with indications of cardiac failure, 293

conditions associated with, 115

degenerative processes of aortic valve giving rise to, 116

differential diagnosis of clironic interstitial myocarditis from cardiac failure of, 314

electro-cardiograms in, 435,436

evidences of cardiac failure in, 118

fatal cases, frequency of, 271

frequent combinations of, 257, 258

invariably present in heart-block, 193

nature of murmurs indicating, 48 prognosis in, 127

relative frequency of, 262

relative incompetence, 48

relative proportion and the combinations of the different, $256,257,258$

supernumary or defective cusps, 363

traumatic lesions of cusps, 258

with cardiac failure, differential diagnosis of fatty degeneration from, 310

Valvular incompetence,

aneurism giving rise to, 352

Valvular vegetations,

in acute, simple endocarditis, 235

Valvulitis, 234

Vaso-dilators,

in cases of super-normal bloodpressure, 142

in treatment of paroxysmal dyspnœea, 154 
Vaso-motor or toxic types, of pseudo-angina, 390

Vaso-1notor system, general blood-pressure and local blood supply regulated by, 70

Vegetations, in ulcerative endocarditis, 245

Veins, auscultation of, method, 54 superficial, pulsation in aortic incompetence, 267, 268, 270

Venesection,

class of cases in which suitable and unsuitable, 149, 150

in treatment of high blood-pressure, 85

value and effects in super-normal arterial pressure, 69

Venous congestion,

chronic interstitial myocarditis resulting from, 312,314

Venous engorgement, chronic, conditions due to, in congenital heart disease, 365

Venous pulsation, see Jugular pulse

Ventricles,

action in the fœ'

and auricles, movements recorded by clinical polygraph, 88

contraction of, 18

contraction of, cause and effects, 16,17

development of, from primitive cardiac tube, 9

dilatation of, 323

displacement of apex-beat in dilatation and hypertrophy of, 24

formation in the fotus, 356

hypertrophy,

co-existence with adherent pericardium, 343

in aortic stenosis, 263

in mitral stenosis, 274, 277

in the fotus, 361

in relation to stimulus for conleft, traction, 11

dilatation, 326

dilatation in aortic incompetence, 269

electro-cardiogram of hypertrophy of, 430,432

emphysema of left lung simulating, 321

enlargement, indications, 37
Ventricles (continued), hypertrophy of, characteristics, 317,318

hypertrophy of, diagnosis, 321

hypertrophy of, heart sounds in, 40

indications of failure of, 124

"reversed rhythm" due to stimulus for contraction proceeding from, 12

right,

dilatation of, 326

electro-cardiograms of hypertrophy of, $430,431,433$

enlargement, pulsation in, 26

enlargement, systolic recession in, 28

hypertrophy of, characteristics, $317,318,319,320$

hypertrophy of, condition in which generally found, 360 , 361

lypertrophy of, diagnosis, 321

hypertrophy of, heart sounds in, 41

position of, 2

stimulation and contraction of, order and course, 11

stimulus from auricle to, 184 , 401

Ventricular diastole,

beginning and end of, 19

period of completion, 19

time of occurrence, 17,18

Ventricularextra-systoles, sce Extrasystoles

Ventricular form of liver pulse, 111

Ventricular form of venous pulse, characteristics, 29,107

conditions to which due, 107

in auricular fibrillation, tracing showing, 106, 107, 208, 210

Ventricular rate,

halving of, cause, 186, 187, 195

in auricular fibrillation, 204, 205

in auricular flutter, $213,214,215$. 216

in paroxysmal tachycardia, 228

not accelerated by drugs, 197

Ventricular rhythm,

definition, characteristics, 184

Ventricular systole,

beginning and end of, 19

onset and termination in a phlebogram, how determined, 109,110

period of completion, 19 
Vontricular systole (continued), post-sphygmic period, characteristics, 18,19 pre-sphygmic period, 18 quickness or abruptness of, 58 sphygmic or pulse period, characteristics, 18,19 time of occurrence, 18

Ventricular wave, in a phlebogram, how determined, 110.111 in the jugular pulse, 100, 101, $102,103,105$

\section{Vomiting,}

in acute myocarditis, 299
Vomiting (continued), treatment, 156

Water-hammer pulse, $6 \mathrm{l}$

Weak heart, usc of the term, 165

Weiss, method of recording heart sounds, 114

Wounds of the heart, 353

X-ray examination, value and advantages of, 113 

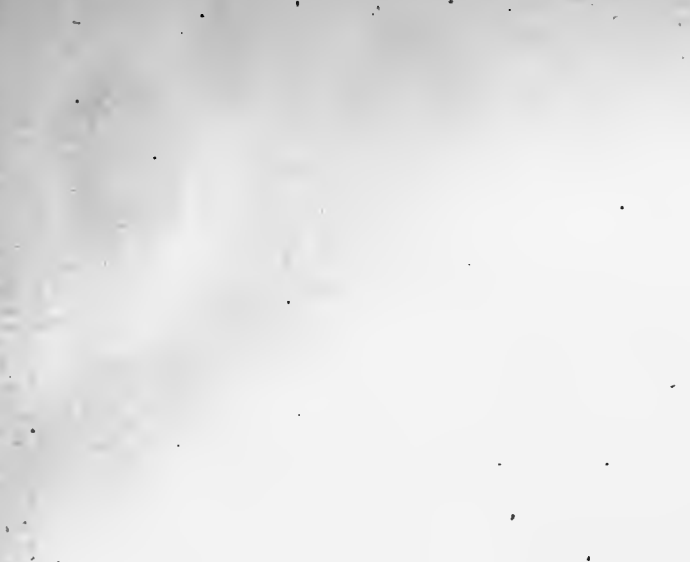



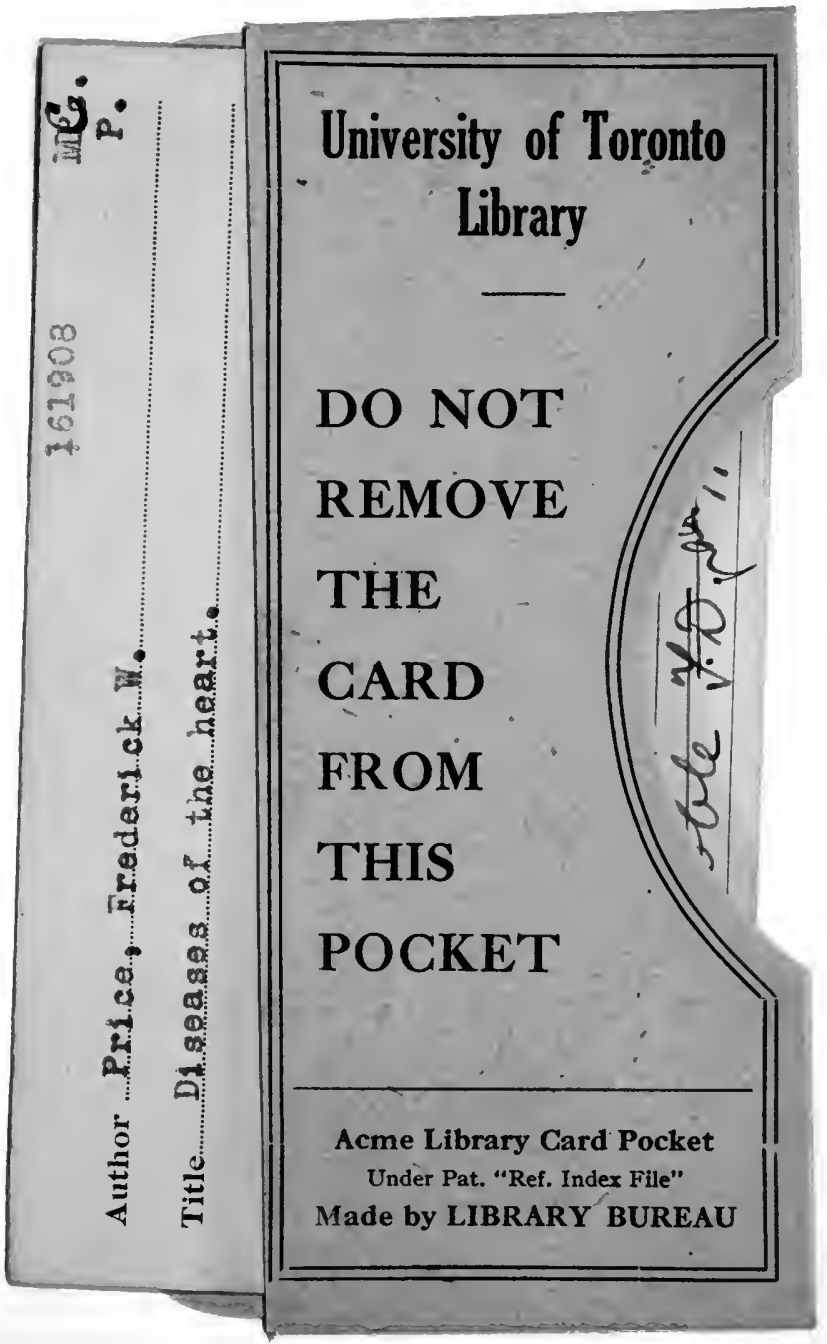


